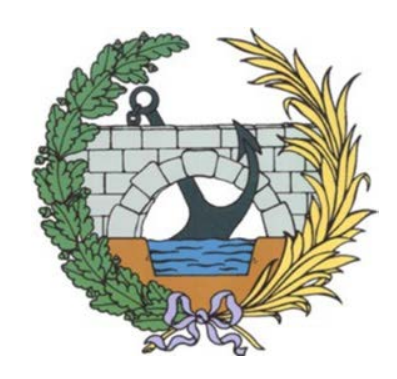

UNIVERSIDAd POLITÉCNICA DE MADRID

DOCTORAL THESIS

\title{
Energy Harvesting Materials Based on Carbon Nanotube Fibre for Tough Electronics
}

Author:

Alfonso MOnREAL BERNAL
Supervisor:

Dr. Juan J. VILATELA

A thesis submitted in fulfillment of the requirements for the degree of Doctor of Philosophy

$$
\text { in the }
$$

Higher Technical School of Civil Engineers 



\title{
Abstract \\ Energy Harvesting Materials Based on Carbon Nanotube Fibre for Tough Electronics
}

\author{
by Alfonso Monreal Bernal
}

The Carbon Nanotube Fibres (CNTf) present high electrical conductivity, high mechanical properties (strength, elastic modulus and toughness), large specific surface area and electrochemical stability among others. This combination of properties makes them an interesting material that can simultaneously act as electrode, current-collector and mechanical reinforcement. This thesis is centred in the fabrication and study of new materials based on the CNTf used as current-collector for new energy conversion devices with augmented mechanical properties. Used as Counter-Electrode (CE) in Dye Sensitised Solar Cells (DSSC), CNTf leads to efficiency of $9 \%$, comparable to Pt reference. Some insights are discussed about the catalytic nature of this material and the CNTf/electrolyte interaction (based on iodide/triiodide redox couple). New device architectures are proposed enabled by the properties and scalable process of the CNTf, such as 1 metre photovoltaic module, bifacial devices or free-standing flexible current-collector DSSC. Moreover, large $\mathrm{CNTf} /$ semiconductor hybrid materials have been produced by different techniques, such as sol-gel/hydrothermal method, atomic layer deposition or doctor blading commercial semiconductor pastes. It is demonstrated that they behave as electronic heterojunctions, and also, that there is charge transfer produced by photo-excitation of the semiconductors. The semiconductor dimension, morphology and synthesis method have a strong influence over the properties of the hybrid materials. Last but not the least, the implementation of these hybrids as photoanodes in DSSC is presented. Several routes are investigated for reducing the strong electrolyte recombination observed in presence of the CNTf. The passivisation of the CNTf electrode with metal oxides by ALD in combination with semi-transparent CNTf currentcollectors drastically reduced the recombination for CNTf photoanodes. The results here presented are a first step towards the fabrication of a full carbon photovoltaic device with augmented mechanical properties. 



\title{
Resumen \\ Materiales Para Captación de Energía Basados en Fibra de Nanotubos de Carbono y su Utilización Como Electrodos Tenaces
}

\author{
por Alfonso MONREAL BERNAL
}

La fibra de nanotubos de carbono (CNTf) tiene, entre otras propiedades, alta resistencia, módulo elástico y tenacidad, alta conductividad electrónica, elevada superficie específica y buena estabilidad electro-química. La combinación de estas propiedades hacen que la fibra de CNT sea un material interesante para utilizarlo simultáneamente como electrodo, colector de corriente y refuerzo mecánico. Esta tesis se centra en la fabricación y estudio de nuevos materiales basados en la fibra de CNT como conductor para nuevos dispositivos de conversión de energía con propiedades mecánicas aumentadas. Usada como contraelectrodo (CE) en celdas solares orgánicas DSSC, se han obtenido eficiencias del $9 \%$, comparable al material de referencia, el Platino. También se discute sobre la naturaleza de las propiedades catalíticas de este material, así como de su interacción con electrolitos con yoduro/ triyoduro como par redox. Se proponen nuevos diseños de dispositivos en base a las propiedades y al método de fabricación de la fibra CNT. Entre otros diseños se destaca el módulo solar basado en tejidos fibra CNT contínua superiores al metro de longitud, dispositivos bifaciales o el uso de la fibra CNT directamente como conductor flexible sin otro tipo de substrato. Por otro lado, en la presente investigación se han fabricado materiales híbridos de fibra CNT/ semiconductor por varios métodos de síntesis: por el método sol-gel/ hidrotermal, por deposición de capas atómicas (ALD), así como mediante la técnica "doctor blade". A través de los híbridos obtenidos, se ha observado que la morfología, el tamaño y el método de síntesis tienen una gran influencia sobre sus propiedades. Posteriormente, usando estos materiales como fotoánodos en celdas solares DSSC, se ha podido concluir que el balance entre los procesos de recombinación e inyección gobiernan el funcionamiento del dispositivo basado en CNTf. Se han investigado varios métodos para reducir la recombinación en estos dispositivos cuando se usa la fibra CNT como conductor en el fotoánodo. Entre ellos, la combinación de utilizar fibra semitransparente y pasivar su superficie con capas conformales de óxidos metálicos por ALD, han dado resultados prometedores. Las conclusiones que se extraen de esta tesis asientan los primeros pasos para la fabricación de una celda solar enteramente de carbono con propiedades mecánica mejoradas. 



\section{Acknowledgements}

I would like to start by expressing my gratitude to my Ph.D supervisor, Dr. Juan J. Vilatela. For the last six years I have received his help always that I have asked. This work would not have been possible without his academic support. It has been a true privilege to work under his guidance.

In addition, I thanks my external reviewers and committee members for their time and for playing a critical role at the last stage of this thesis.

I would like to extend my gratitude to the members of the Multifunctional Nanocomposite Group - past and present; it has been a pleasure to share the ups and downs of research. I am particularly thankful to Dr. Juan Pedro Fernández and Dr. Belén Alemán for his continuous help and advice.

I want to thank Dr. Rubén Costa and his former group at FAU University for welcoming me to Germany and showing me the depth of their scientific knowledge, especially grateful to Dr. Fabian Lodermeyer, Oliver Langmar, Andreas Kunzmann and Rubén Casillas, their help and friendship during that time are priceless memories.

I am thankful to the collaborators Prof. Dominik Eder and Dr. Nina Kemnade as well as Dr. Daniel Granados and Dr. Manuel Rodríguez, for fabricating and discussing the ALD materials studied during this work. My thanks also go to Prof. Ernesto Pérez from ICTP-CSIC for providing access to different experimental techniques.

I am indebted to a lot of people at IMDEA Materials Institute but among them special thanks to Dr. Miguel Castillo, for his constant help with electron microscopy techniques; and also to the past and present laboratory technicians of the institute, always providing smart solutions to the intrinsic issues of the experimental research.

I would like to acknowledge Dr. John Hammersley, Overleaf platform cofounder, for providing me unlimited free-access during the time I spend writing this thesis.

This work has been financially supported by the Madrid regional government (S2013/MIT-3007 MAD2D project).

Finally, special thanks to my girlfriend Paula, this thesis would never have been possible without her unconditional emotional support. 



\section{Contents}

Abstract iii

Acknowledgements vii

$\begin{array}{llr}1 & \text { Introduction } & 1\end{array}$

2 Background 3

2.1 Energy harvesting and other multifunctional materials . . . . . . 3

2.2 Carbon nanotubes . . . . . . . . . . . . . . . . 5

2.2.1 Structure, properties and synthesis . . . . . . . . . . 5

2.2.2 Macroscopic assemblies: CNT dispersions and CNT fibre . . . . 7

2.2.3 CNTs and CNTf as current-collector in tough electronics . . . . 14

2.3 Dye-Sensitised Solar Cell: working principle and associated processes 15

Electrical modelling of a DSSC . . . . . . . . . . . . . 17

Interfacial electron transfer processes and kinetics . . . . . . 19

2.4 Roles of nanocarbons and state-of-the-art of CNTs in DSSC . . . . . . . 21

2.4 .1 CNT counter-electrode for DSSC . . . . . . . . . . . . 22

2.4 .2 CNT-doped electrolytes . . . . . . . . . . . . . . 30

2.4.3 CNT/semiconductor hybrids as photoanodes . . . . . . . . 35

2.5 Objectives of the present work . . . . . . . . . . . . . . 40

3 Experimental Techniques $\quad 43$

3.1 Synthesis and fabrication of materials and devices . . . . . . . . . 43

3.1.1 Synthesis of Carbon Nanotube Fibres (CNTf) . . . . . . . . . . . 43

3.1.2 Semiconductor synthesis methods . . . . . . . . . . . . 45

Sol-gel synthesis method . . . . . . . . . . . . . 45

Hydrothermal synthesis method . . . . . . . . . . . . . 45

Atomic Layer Deposition synthesis method (ALD) . . . . . . 45

3.1.3 CNTf/polymer composite fabrication . . . . . . . . . . . 47

3.1.4 Fabrication of symmetric electrochemical cells and Dye Sensitised Solar Cells (DSSC) including control experiments . . . . . 47

Pt symmetric cell study and construction parameters influence 48

3.1.5 Architectures according to the number of $\mathrm{TiO}_{2}$ layers: transparent and reflective photoanodes ... . . . . . . 50

3.2 Physical characterisation . . . . . . . . . . . . . . . . . . 52

3.2.1 Profilometry . . . . . . . . . . . . . . . . . 52

3.2.2 Scanning and Transmission Electron Microscopy (SEM, TEM) . 52

3.2.3 Energy Dispersive X-ray Spectroscopy (EDX) . . . . . . . . . . . . . 52

3.2.4 X-ray Photoelectron Spectroscopy (XPS) . . . . . . . . . . . . . . 52

3.2.5 Powder X-ray Diffraction (XRD) . . . . . . . . . . . . 53

3.2 .6 Raman spectroscopy . . . . . . . . . . . . . . . . . 53

3.2.7 Ultraviolet-Visible spectroscopy (UV-VIS) . . . . . . . . . . . . . 53

3.3 Mechanical properties characterisation . . . . . . . . . . . . . 55 
3.4 Electrical properties characterisation . . . . . . . . . . . . . . 55

3.4.1 Linear Sweep Voltammetry (LSV) . . . . . . . . . . . . . . 55

Resistivity measurements . . . . . . . . . . . . . 55

Tafel plot . . . . . . . . . . . . . . . . . . 56

3.5 Photovoltaic devices: photo-electro-chemical characterisation . . . . . 57

3.5.1 Light source and Air Mass (Solar simulator) . . . . . . . . . . 57

3.5.2 Current-density-Voltage curves for DSSC $(J-V) \ldots \ldots \ldots$

Series resistance characterisation . . . . . . . . . . . 59

3.5 .3 Cyclic Voltammetry $(C V) \ldots \ldots$. . . . . . . . . . 60

3.5.4 Electrochemical Impedance Spectroscopy $($ EIS $)$. . . . . . . . . . 60

4 CNTf Counter-Electrodes for DSSC $\quad 61$

4.1 CNT fibre properties: motivation for DSSC

counter-electrodes/current-collectors . . . . . . . . . . . . . . 61

4.2 Study of the catalytic activity of CNTf: symmetric electrochemical cells 66

4.2.1 Electrolyte diffusion and reactivity at the CNTf electrode . . . . 66

4.2.2 Electrochemistry in presence of nanocarbon porous membranes: EIS study . . . . . . . . . . . . . . . . 73

4.3 CNTf counter-electrode/current-collector: photovoltaic performance . 78

4.3.1 CNTf-CE thickness optimisation and influence over the performance of the device . . . . . . . . . . . . . 78

4.3.2 CNTf-CE towards high efficiency DSSC . . . . . . . . . . 85

4.3.3 CNTf-CE/ $/ \mathrm{TiO}_{2}$-photoanode microcontacts: spacer-free DSSC . 95

4.4 Summary and conclusion . . . . . . . . . . . . . . 997

5 Fundamental Properties of CNTf/MOx Heterojunctions 99

5.1 Chemical synthesis route of $\mathrm{ZnO} / \mathrm{CNTf}$ based heterojunctions . . . . . 99

5.1.1 In-situ double step sol-gel/hydrothermal method . . . . . . . . 100

5.1.2 Morphology and crystal structure of the CNTf/ZnO-NWs . . . 101

5.1.3 Electrical characterisation of the CNTf/ZnO-NWs heterojunction . . . . . . . . . . . . . . . . . . 107

5.1.4 Charge transfer at the interface: photocurrent measurements . . 114

5.1.5 Electromechanical behaviour of CNTf/ZnO flexible electrodes and sensing applications . . . . . . . . . . . . . . 116

5.2 Physical synthesis route of CNTf/MOx heterojunctions . . . . . . . . 117

5.2.1 Atomic layer deposition synthesis technique . . . . . . . . . . 117

5.2.2 Morphology, structure and interfacial characterisation of the CNT fibre/ ZnO-ALD material . . . . . . . . . . . . . . . . 121

5.2.3 Chemical environment and electronic structure of the CNTf/ZnO-

ALD material . . . . . . . . . . . . . . . . . . . 130

5.2.4 Electrical characterisation and photocurrent measurements of the CNTf/ZnO-ALD heterojunction . . . . . . . . . . . 134

5.2.5 Charge transfer study of the interface . . . . . . . . . . . . . 137

5.3 Summary and conclusion . . . . . . . . . . . . . . . . . . 139

6 CNTf/MOx Photoanodes for DSSC 141

6.1 CNTf/Dye semiconductor-free photoanodes . . . . . . . . . . . . . 142

6.2 CNTf scaffolds: high surface area photoanodes by ALD . . . . . . . . . 145

6.3 CNTf-photoanodes: increasing oxide thickness and dye loading . . . . 155

$6.4 \mathrm{CNTf} / \mathrm{MO}_{\mathrm{x}}-\mathrm{ALD}$ as photoanode blocking layers . . . . . . . . . . . . 159

$6.4 .1 \mathrm{CNTf} / \mathrm{ZnO}-\mathrm{NWs}$ photoanodes . . . . . . . . . . . . . 162 
6.5 CNTf as transparent photoanode current-collector . . . . . . . . . . 164

6.6 Summary and conclusion . . . . . . . . . . . . . . 166

7 CNTf Electrodes for DSSC: Engineering Aspects 169

7.1 Progress towards innovative DSSC architectures . . . . . . . . . . . 169

7.1.1 Electrolyte infiltration . . . . . . . . . . . . . . . . . . 169

7.2 Towards Glass/TCO-free electrodes . . . . . . . . . . . . . . . . 171

7.3 PVDF/CNTf-CE nanocomposite for tough electronics DSSC devices . 174

7.4 Overcoming issues with transparency enabled by mechanical properties 176

7.5 Electrical aspects: proof-of-concept mini-module . . . . . . . . . . . 178

7.5.1 Photoanode size-dependence effect of planar CNTf-CE DSSC . 178

7.5.2 CNTf-CE mini-module: resistive losses and size-dependence . 180

7.6 Summary and conclusion . . . . . . . . . . . . . . 183

8 Conclusions and Future Directions $\quad 185$

$\begin{array}{lr}\text { A CNTf/Polymer Nanocomposites } & 191\end{array}$

A.1 CNTf/PVDF nanocomposite fabrication . . . . . . . . . . . . . . . 191

A.2 CNTf/PVDF nanocomposite infiltration and mechanical properties . . 193

$\begin{array}{ll}\text { Bibliography } & 199\end{array}$ 



\section{List of Figures}

2.1 Power density output for flexible energy harvesting technologies . . . 3

2.2 Single- and multi- wall CNTs structure. . . . . . . . . . . 6

2.3 Percolation and surface area. Relation with surface area in hybrids and nanocomposites. . . . . . . . . . . . . . . 8

2.4 Nanocomposite elastic modulus effective reinforcement vs CNTs volume fraction . . . . . . . . . . . . . . . . . . . . 9

$2.5 \mathrm{CNT} / \mathrm{TiO}_{2}$ interaction depending on the number of walls of CNTs. . . 10

2.6 CNTf structure, from molecular to macroscopic level . . . . . . . . . . 11

2.7 CNTf as-made material and important physical characteristics . . . . . 12

2.825 years of performance evolution in the photovoltaic field . . . . . . 16

2.9 Schematic of the working principle of a n-type DSSC . . . . . . . . 17

2.10 Energy bands diagram schematic of a n-type DSSC . . . . . . . . . 17

2.11 Diode equivalent circuit for a solar cell . . . . . . . . . . . . . 18

2.12 Electron transfer processes in a DSSC . . . . . . . . . . . . 20

2.13 Schematic of a DSSC incorporating CNTs as CE. . . . . . . . . . . . 22

$2.14 C V$ and $L S V$ plots for symmetric cells with different CNTs contents . . 23

2.15 Impedance analysis of CNT symmetric cells . . . . . . . . . . . . . . 24

$2.16 \mathrm{~J}-V$ curves and Bode plots comparing Pt and CNT catalyst for DSSCs. . 25

2.17 Possible origins of nanocarbon's catalytic activity. . . . . . . . . . 26

2.18 Processing methods of CNT-CE DSSCs . . . . . . . . . . . . . . . 28

2.19 CNTf-based fiber-like DSSC . . . . . . . . . . . . . . . . . . . . . 29

2.20 Dual-role of dispersed CNTs in DSSC electrolytes. . . . . . . . . . . . 32

2.21 Schematic of a DSSC incorporating CNTs in the photoanode part of the device . . . . . . . . . . . . . . . . 35

2.22 Schematics of charge collection and electronic band structure of $\mathrm{TiO}_{2}$ photoanodes with and without $\mathrm{CNTs} \ldots \ldots . \ldots 37$

2.23 Processing methods of $\mathrm{CNT} / \mathrm{TiO}_{2} \mathrm{DSSC} \ldots \ldots . \ldots . \ldots 39$

$2.24 V_{o c}$ origin in the DSSC under illumination . . . . . . . . . . . . 42

3.1 CVD reactor and sample appearance . . . . . . . . . . . . . . . . . 44

3.2 Schematic process of CNTf symmetric cell and DSSC fabrication . . . . 48

3.3 Pt symmetric cells EIS control experiments . . . . . . . . . . . . . . . . 49

3.4 Transparent and reflective $\mathrm{TiO}_{2}$ layers for DSSC. . . . . . . . . . . . . . 51

3.5 Conductivity and contact resistance characterisation geometries. . . . . 56

3.6 Tafel representation of the $J-V$ curve and extracted parameters . . . . . 57

3.7 Solar cell $J-V$ curve under light, power output and main characteristic 58

3.8 DSSC series resistance calculation methods . . . . . . . . . . . . . 59

4.1 CNTf composition and thermogravimetrical analysis. . . . . . . . . . 62

4.2 Structure and morphology of CNTf used as CE . . . . . . . . . . . . 63

4.3 CNTf longitudinal and transversal conductivity . . . . . . . . . . 65

4.4 CNTf symmetric cell schematic representation and digital photograph 67 
4.5 Catalytic activity of the CNTf by EIS and $C V \ldots \ldots 9$

4.6 Electrochemical stability of the CNTf by $C V \ldots \ldots$. . . . . . . . 70

4.7 Triiodide/pentaiodide Raman signal in presence of CNTf . . . . . . . . 72

$4.8 \mathrm{CNTf} \mathrm{I}_{2}$ electrical doping effect . . . . . . . . . . . . . . 73

4.9 EIS study of Pt and CNTf symmetric cells . . . . . . . . . . . . . . 74

4.10 EIS study of Pt-CE with a mesoporous membrane separator . . . . . . 75

4.11 CNTf with non-catalytic active electrolyte . . . . . . . . . . 76

4.12 CNTf- and Pt- symmetric cells at $100{ }^{\circ} \mathrm{C} \ldots \ldots$. . . . . . . . . 77

4.13 CNTf DSSC schematic and digital image . . . . . . . . . . . . . . . 79

4.14 CNTf-CE thickness optimisation . . . . . . . . . . . . . 80

$4.15 \mathrm{~J}-\mathrm{V}$ curves of CNTf ozone treated counter-electrode . . . . . . . . 82

$4.161 \mu \mathrm{m}$ thick CNTf-CE DSSC device light soaking experiment . . . . . . . 83

4.17 CNTf- $v$ st-CE light soaking experiment . . . . . . . . . . . . . . 85

4.18 Influence of the $\mathrm{TiO}_{2}$ thickness over the CNTf-CE DSSC performance . 86

4.19 CNTf- $v$ s Pt-CE EIS analysis and equivalent circuit. . . . . . . . . . 88

4.20 CNTf-CE vs Pt light power dependence . . . . . . . . . . . . . . . . 990

4.21 CNTf-CE DSSC performance variability . . . . . . . . . . . . . . 93

4.22 Stability of Pt- and CNTf-CE DSSCs after 6 months . . . . . . . . . . 94

$4.23 \mathrm{Pt}$ - and CNT-CE DSSC geometry and dimensions after assembly . . . . 96

4.24 Electrical behaviour of $\mathrm{CNT} / \mathrm{TiO}_{2}$ heterojunction. . . . . . . . . . . 96

$5.1 \mathrm{ZnO}$ nanoparticles sol-gel synthesis optimisation. . . . . . . . . . . . 100

5.2 CNTf/ZnO hybrid morphology by sol-gel/hydrothermal process. . . . 101

5.3 Hybrid $\mathrm{ZnO} / \mathrm{CNTf}$ interface . . . . . . . . . . . . . . . . . . . 102

5.4 TEM and EDX analysis at the hybrid CNTf/ZnO interface . . . . . . 103

5.5 WAXS analysis of the $\mathrm{ZnO}$ nanoparticle seeds . . . . . . . . . . . 104

5.6 XRD study of the hybrid material . . . . . . . . . . . . . . 105

5.7 TEM of the ZnO-NW and CNT bundle . . . . . . . . . . . . . . . . 106

5.8 Raman spectra of the CNTf/ZnO hybrid. . . . . . . . . . . . . . 107

5.9 Electrical measurements details of the CNTf/ZnO heterojunction . . 108

5.10 Electrical considerations and control measurements . . . . . . . . . . 109

$5.11 \mathrm{I}-V, \mathrm{C}-\mathrm{V}$ measurements of the CNTf/ZnO-NWs heterojunction. . . . 110

5.12 Cheung analysis of the $\mathrm{CNTf} / \mathrm{ZnO}$ heterojunction . . . . . . . . . 111

5.13 Schematic of the electronic band structure at the junction . . . . . . . 113

$5.14 I-V$ curves under illumination . . . . . . . . . . . . . . . . . . . . 114

5.15 Photocurrent experiment of the CNTf/ZnO-NWs heterojunction. . . . 115

5.16 Electromechanical behaviour of the CNTf/ZnO-NWs interface. . . . . 117

$5.17 \mathrm{CNTf} / \mathrm{MO}_{x}$ heterojunctions by ALD technique. . . . . . . . . . . 118

$5.18 \mathrm{ZnO}$ ALD optimisation on top of the CNTf substrate . . . . . . . . . . . 119

5.19 SEM images of CNTf/ZnO with different $\mathrm{MO}_{x}$ thicknesses . . . . . . . 120

$5.20 \mathrm{SEM} / \mathrm{TEM}$ comparison between $\mathrm{ZnO}$ and $\mathrm{TiO}_{2} \ldots \ldots . . . \ldots . .122$

5.21 TEM structural analysis of the CNTf/ZnO-ALD herterojunction. . . . . 123

5.22 TEM-EDX analysis of 20 and $100 \mathrm{~nm}$ thickness ZnO-ALD coatings . . . 124

5.23 XRD/WAXS analysis of the CNTf/ZnO-ALD herterojunction. . . . . . 125

5.24 Raman spectra of the CNTf/ZnO-ALD heterojunction. . . . . . . . . 127

5.25 SEM images at the fracture surface of the CNTf/ZnO-ALD . . . . . . . 128

5.26 CNTf 2D and G Raman modes after the ZnO-ALD. . . . . . . . . . . . 129

5.27 $\mathrm{MO}_{x}$-ALD layer growth mechanism and the predicted residual stresses.130

5.28 Survey XPS spectra of the CNTf and CNTf/ZnO-ALD . . . . . . . . . . . 131

5.29 XPS C1s core level of CNTf substrate and CNTf/ZnO-ALD . . . . . . . 132

5.30 XPS O1s core level of CNTf substrate and CNTf/ZnO-ALD . . . . . . . 133 
5.31 XPS Zn2p core levels and Auger Zn LMM lines of CNTf/ZnO-ALD . . 133

$5.32 I-V$ curves electrical variation as a function of pressure. . . . . . . . 135

$5.33 I-V$ curves electrical variation as a function of the layer thickness. . . . 136

$5.34 \mathrm{CNTf} / \mathrm{ZnO}$-ALD UV-photocurrent vs ALD thickness . . . . . . . . . 138

6.1 Road-map towards CNTfs photoanodes. . . . . . . . . . . . . . . . . 142

6.2 Schematic of a CNTf decorated with N719 as photoanode . . . . . . . 143

6.3 Confirmation of N719 dye molecules decorating CNTf photoanodes by Raman . . . . . . . . . . . . . . . . . . . . . . . . . . 143

6.4 Schematic and $J-V$ curvesof the CNTf/N719 . . . . . . . . . . . . . . . 144

6.5 Representation of the structure between $\mathrm{CNTf}^{\mathrm{MO}_{x}}$ by ALD . . . . . . 146

$6.6 \mathrm{CNTf} / \mathrm{MO}_{x}$-ALD photoanodes schematic . . . . . . . . . . . . . . . 147

6.7 As-made $\mathrm{CNTf} / \mathrm{TiO}_{2}$-ALD analysed by TEM, EDX and XRD . . . . . . . 148

6.8 DSSC performance with $\mathrm{CNTf} / \mathrm{TiO}_{2}$-ALD photoanode . . . . . . . . 149

6.9 UV-VIS and Raman N719 dye detection . . . . . . . . . . . . . . . 150

$6.10 \mathrm{TEM}$ and XRD of recrystallised $\mathrm{CNTf} / \mathrm{TiO}_{2}$-ALD . . . . . . . . . . 151

6.11 Absorption spectra for N719 solution before and after dye loading . . . 152

$6.12 \mathrm{~J}-V$ curves of recrystallised $\mathrm{TiO}_{2}$-ALD photoanodes under illumination 153

$6.13 \mathrm{SEM}$ analysis of the $\mathrm{CNTf} / \mathrm{TiO}_{2}$-ALD cross-section . . . . . . . . . . . 154

6.14 SEM and EDX analysis of the CNTf/ZnO-ALD cross-section . . . . . 155

$6.15 \mathrm{CNTf} / \mathrm{TiO}_{2}$-paste photoanodes for DSSC . . . . . . . . . . . . . . . 156

$6.16 \mathrm{CNTf} / \mathrm{TiO}_{2}$-diluted paste photoanodes for DSSC. . . . . . . . . . . 158

6.17 Summary of the $J-V$ curves of the different photoanode configurations 159

$6.18 \mathrm{CNTf} / \mathrm{MO}_{x}-\mathrm{ALD} / \mathrm{TiO}_{2}$-paste photoanodes for DSSCs. . . . . . . . . . 160

6.19 CNTf/ZnO-NWs photoanodes produced by hydrothermal method for DSSCs. . . . . . . . . . . . . . . . . . . . . 163

6.20 Sheet resistance $R_{s q}$ and transmittance T vs CNTf thickness . . . . . . 165

6.21 Semi-transparent Glass/CNTf/ $\mathrm{TiO}_{2}$ photoanodes for DSSC. . . . . . . 166

6.22 Recombination mechanism vs CNTf surface exposure in photoanodes 167

7.1 Schematics showing the electrolyte flow inside the cell. . . . . . . . . . 170

7.2 Optical images showing the electrolyte flow inside the cell . . . . . . . 171

7.3 Free-standing CNTf-CE membrane DSSC fabrication process. . . . . . 172

7.4 Free-standing CNTf-CE DSSC characteristics. . . . . . . . . . . . . . . . 173

7.5 Flexible free-standing CNTf/PET CE . . . . . . . . . . . . . . 173

7.6 CNTf nanocomposite and CF composite counter-electrodes for DSSC. . 174

7.7 CNTf nanocomposite CE for DSSC: $J$ - $V$ curves and EIS. . . . . . . . . 175

7.8 CNTf-CE unipolar DSSC new architecture. . . . . . . . . . . . . 177

7.9 Photoanode area $v s$ the DSSC characteristics . . . . . . . . . . . . 178

7.10 Series resistance and power loss $v$ s photoanode area. . . . . . . . . . 179

7.11 Larga area mini-module CNTf-CE DSSC. . . . . . . . . . . . . . . 181

7.12 Larga area CNTf-CE DSSC connection influence over the parameters. . 182

A.1 CNTf/PVDF composites: manufacturing process and materials. . . . . 192

A.2 CNTf/PVDF nanocomposite consolidation by hot-press technique. . . 193

A.3 Morphological study of the CNTf/PVDF composite by SEM . . . . . . 194

A.4 X-ray tomography of the CNTf/PVDF nanocomposite. . . . . . . . . 195

A.5 CNTf/PVDF nanocomposte tensile test experiment and properties. . . 196 



\section{List of Tables}

2.1 Properties of CNTf compared to other high performance dry fibres. . . 14

2.2 CNTf and related materials in energy harvesting applications with augmented mechanical properties. . . . . . . . . . . . . . . . 15

2.3 Efficiency (PCE) records of CNT-CE for different processing routes. . . 29

2.4 Efficiency (PCE) obtained after integration of CNTs in different electrolytes for DSSC. Efficiencies have been rounded to the first decimal place. . . . . . . . . . . . . . . . . 33

2.5 PCE records of CNT-Photoanodes for different processing routes. . . . 40

4.1 Obtained parameters from $C V$ and $L S V$ for symmetric cells. . . . . . 70

4.2 DSSC characteristics of CNTf-CE different thicknesses. . . . . . . . . 81

4.3 DSSC photovoltaic characteristics for Pt- and CNT-CE with different thicknesses of titania layer. . . . . . . . . . . . . . . . 86

4.4 EIS parameters of DSSC with Pt- and CNT-CE. . . . . . . . . . . . . 89

5.1 Extracted parameters for the CNTf/ZnO Schottky heterojunction. . . . 114

5.2 Extracted parameters for the CNTf/ZnO-ALD hybrids heterojunction. 136

6.1 Shunt resistance comparison before and after $\mathrm{TiO}_{2}-\mathrm{ALD}$ recrystallisation treatment. . . . . . . . . . . . . . . . . . 153

6.2 Shunt resistance $\left(\Omega \mathrm{cm}^{2}\right)$ comparison of low and high temperature recrystallised $\mathrm{TiO}_{2}$ diluted paste. . . . . . . . . . . . . 157

$7.1 V_{o c}, J_{s c}, F F, P C E, P_{R}$ and $R_{s, l i g h t} A$ characteristics of different size photoanode DSSC devices. . . . . . . . . . . . . . . . . . . 180

A.1 Mechanical properties of CNTf/PVDF nanocomposite. Specific values included in brackets. . . . . . . . . . . . . . . . . . . . . 197 



\section{List of Abbreviations}

$\begin{array}{ll}\text { ALD } & \text { Atomic Layer Deposition } \\ \text { AM } & \text { Air Mass } \\ \text { BMII } & \text { 1-Butyl-3-Methyl Imidazolium Iodide } \\ \text { ca. } & \text { around (Latin: circa) } \\ \text { CC } & \text { Current-Collector } \\ \text { CE } & \text { Counter-Electrode } \\ \text { CTE } & \text { Coefficient Thermal Expansion } \\ \text { C-V } & \text { Capacitance-Voltage (measurement) } \\ \text { CVD } & \text { Chemical Vapour Deposition } \\ \text { CV } & \text { Cyclic Voltammetry } \\ \text { DSSC } & \text { Dye Sensitised Solar Cell } \\ \text { EDX } & \text { Energy Dispersive X-ray } \\ \text { e.g. } & \text { for example (Latin: exempli gratia) } \\ \text { EIS } & \text { Electrochemical Impedance Spectroscopy } \\ \text { FF } & \text { Fill Factor } \\ \text { FTO } & \text { Fluorine Tin Oxide } \\ \text { HAADF } & \text { High-Angle Annular Dark-Field } \\ \text { i.e. } & \text { in other words (Latin: id est) } \\ \text { I-V } & \text { Current-Voltage (measurement) } \\ \text { J-V } & \text { Current density-Voltage (measurement) } \\ \text { LHE } & \text { Light Harvesting Efficiency } \\ \text { LSV } & \text { Linear Sweep Voltammetry } \\ \text { MPP } & \text { Maximum Power Point } \\ \text { OCP } & \text { Open Circuit Potential } \\ \text { PCE } & \text { Power Conversion Efficiency } \\ \text { PV } & \text { Photovoltaic } \\ \text { SAXS } & \text { Small Angle X-ray Scattering } \\ \text { SEM } & \text { Scanning Electron Microscopy } \\ \text { SSA } & \text { Specific Surface Area } \\ \text { TCO } & \text { Transparent Conductive Oxide } \\ \text { TEM } & \text { Transmission Electron Microscopy } \\ \text { WAXS } & \text { Wide Angle X-ray Scattering } \\ \text { XPS } & \text { X-ray Photoelectron Spectroscopy } \\ \text { XRD } & \text { X-Ray Diffraction } \\ & \end{array}$





\section{Chapter 1}

\section{Introduction}

Energy is one of the biggest issues that concerns the entire world population this century. Furthermore, we are more worried about the environment and the sustainability of everything that surrounds us. We have put a lot of efforts in the last fifty years into decreasing our dependency on fossil fuels and that goal has been the engine for the development of many green energy sources. Among them, the solar energy has attracted a special interest not only of industry and scientific community, but also of all the society.

Solar energy field, precisely photovoltaic energy harvesting, is a very broad field. Although the most developed and standardised technology is based on the silicon technology, there are plenty of alternative technologies that are competing for providing potentially as efficient technologies at lower prices. Traditionally, the Si solar cells are known as first generation; among the alternatives that have appeared in the last thirty years we can find the named second generation, based on thin film technology, and the combination of more inexpensive organic/inorganic materials, known as third generation. In this thesis, the attention is focused in one type of hybrid photovoltaics known as Dye Sensitised Solar Cells (DSSC).

In the quest for new multifuntional applications and devices, new materials are required. There is a growing interest in electronic devices that can bear loads and deformations during working conditions. To these new applications that combine materials performing electrical functions while also having more mechanical tolerance than traditional ones, we referred as tough electronics.[1] New technologies for energy harvesting, energy storage or sensor applications are emerging along those lines. One shared need across all these proposed new multifunctional devices is a tough electrode able to fit into these new architectures. This material must be light, chemically and mechanically stable and obviously, to present high conductivity. Other properties are desirable as flexibility, high porosity or transparency. In some extent, all this properties are brought together by macroscopic assemblies of nanocarbons, as for instance the one studied in this thesis, Carbon NanoTube fibre (CNTf).

This thesis has been structured as follows: first of all, Chapter 2 presents the general motivation of this work, the different energy harvesting technologies and the CNTs and CNTf structure and properties. Later, the photovoltaic is introduced, the working principle of the DSSC, the different roles of nanocarbons in this device architecture and a literature review of the best performances of the field. 
The different material synthesis techniques and fabrication methods are detailed in the Chapter 3, as well as the experimental and characterisation techniques and conditions.

The Chapter 4 shows the CNTf implementation into the DSSC devices playing a double role: counter-electrode (CE) and current-collector (CC). For that, the catalytic properties of the CNTf towards the iodide/triiodide redox mediator was investigated. New insights into the electrochemistry interpretation of the nanocarbon electrodes are proposed. Finally, the CNTf electrodes have been optimised until eficiencies in the state-of-the-art range range have been obtained in the field nanocarbons, outperforming the standard Pt-CE material.

Heterojunctions between CNTf and metal oxides $\left(\mathrm{MO}_{x}\right)$ are investigated in the Chapter 5. Sol-gel, hydrothermal and Atomic Layer Deposition (ALD) methods have been explored for producing new large area heterojunctions between CNTf and Zinc Oxide $(\mathrm{ZnO})$ or titanium dioxide $\left(\mathrm{TiO}_{2}\right)$. The structure, morphology and electrical properties are related to the physical dimension of the semiconductor layers. The heterojunction resulted in a Schottky rectifying junction and the charge transfer properties of the interface are confirmed by photocurrent experiments.

This hybrid interfaces between $\mathrm{CNTf} / \mathrm{MO}_{x}$ are later implemented into solar cells schemes as photoanodes (Chapter 6). Several different configurations are proposed to study the impact on the balance between photogenerated carrier injection and recombination mechanisms. CNTf/ $\mathrm{MO}_{x}$ passivisation layers by ALD technique resulted promising materials as blocking layers for three dimensional currentcollectors as is the case of the CNTf. Finally, semi-transparent DSSCs are fabricated with semi-transparent CNTf-based electrodes, which have confirmed some strategies for diminishing the recombination mechanism in CNTf-DSSC.

In the Chapter 7 some engineering aspects regarding the CNTf implementation into the DSSC, such as the electrolyte filling in presence of CNTf membranes, are discussed. Later, exploting the combination of properties of the CNTf, a self-stanting $\mathrm{CE}$ with embedded electrolyte is investigated. The integration into a polymer matrix improves the stability and mechanical performance towards flexible and tough devices. A new bifacial-DSSC architecture is proposed to tackle the transparency issue when thick and opaque CNTf electrodes are used as CE. Finally, the scaling-up of the CNTf-DSSC is investigated by fabricating a prototype DSSC mini-module $\left(10 \mathrm{~cm}^{2}\right)$. The resistive losses associated to size effects are discussed.

The last Chapter 8 summarises the conclusions extracted during this thesis. Some experiments are proposed as future work to clarify some of the open questions that remains without answer at the end of this thesis. 


\section{Chapter 2}

\section{Background}

\subsection{Energy harvesting and other multifunctional materials}

Energy harvesting applications very often go hand in hand with light weight and augmented mechanical properties, such as in wearable,[2] self powered sensors,[3] medical devices,[4-6] ultraflexible[7] or tough solar cells,[8, 9] structural generators.[10]

In this context, some of the energy harvesting processes studied recently include: thermal gradients, piezoelectrics, tribologic/electrostatic effects or photovoltaic devices. Comparing different harvesting devices from different technologies is a key issue. Among the different metrics proposed,[11] power density (power per device volume) is an easy and convenient figure of merit.

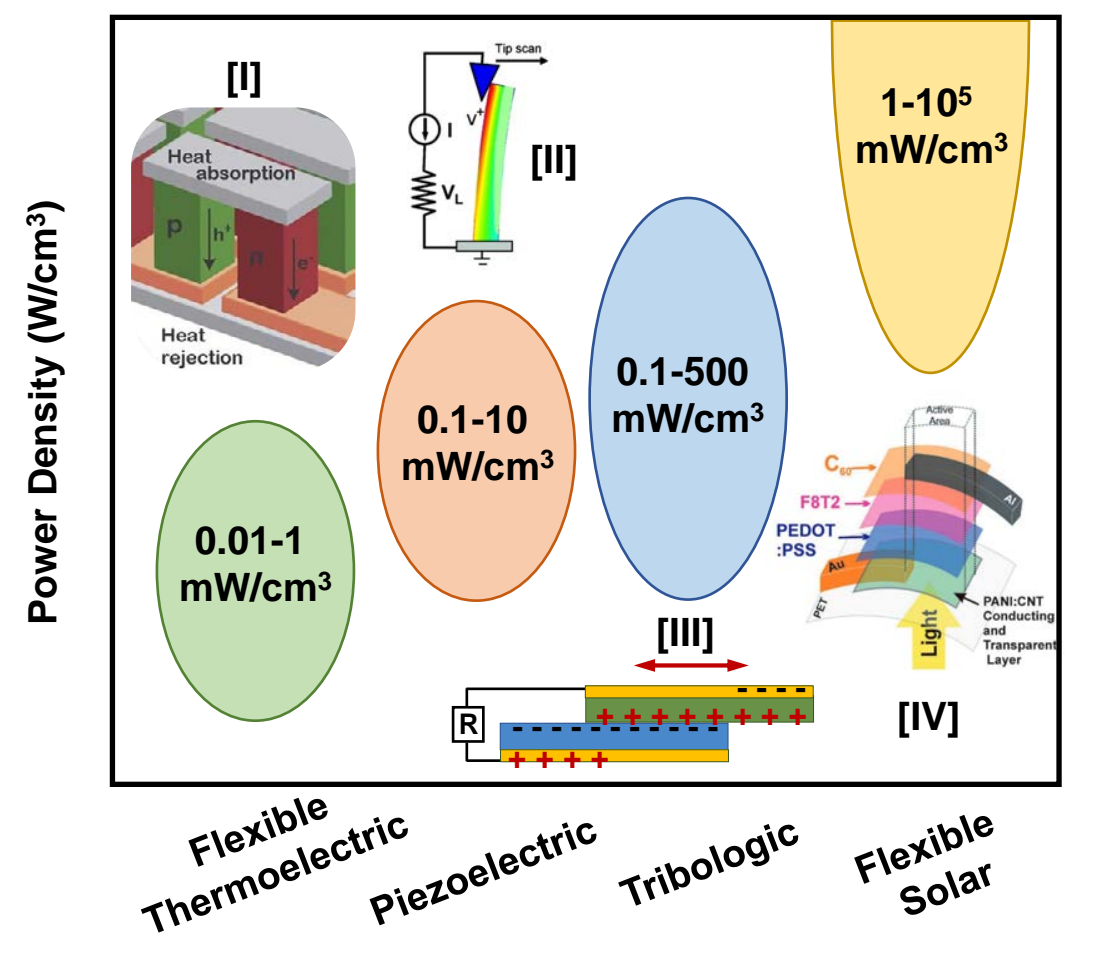

FIGURE 2.1: Power density output for some flexible energy harvesting technologies: [I] thermal energy,[12] [II] piezoelectric nanogenerators,[13] [III] tribologic harvesters[14] or [IV] solar energy.[15] 
Thermal energy harvesters (TEH), also known as thermoelectric generators, are solid-state devices that convert thermal energy (heat) into electricity. These devices, although they are not very efficient, are becoming very competitive for small applications, because their compact size, simplicity and scalability. Thermoelectric systems can be easily designed to operate with small heat sources and small temperature differences.[12] Thermoelectrics produces electrical power from the voltage created by the driven free carriers (electrons or holes) from hot to cold end (Figure 2.1[I]). The resulting voltage is proportional to the temperature difference and the Seebeck coefficient of the material. By connecting an electron conducting (ntype) and hole conducting (p-type) material in series, a net voltage is produced that can be driven through an external load. This technology has recently attracted great attention, especially, flexible thermoelectric materials for wearable human body energy harvesting applications.[16] TEHs result on a power output in the range of $0.01-1 \mathrm{~mW} \mathrm{~cm}^{-3}$ or 10-100 $\mu \mathrm{W}$ in absolute power per device from recent reported results.[6, 16]

The very first article where piezoelectric nanogenerators (PNG) were proposed was almost a decade ago by Z. L. Wang.[17] This technology is based in the well known piezoelectric property of some materials. It is the property of generating a potential under a mechanical stress (deformation) applied. Some popular piezoelectric materials are the quartz, lead zirconate titanate (PZT), zinc oxide $(\mathrm{ZnO})$ and polyvinylidene fluoride (PVDF). All them have been used for fabricating energy harvesters based on the piezoelectric effect. A piezoelectric nanogenerator is a device that harvest the mechanical energy and convert it into electrical energy (Figure 2.1[II]). Typical cantilever configurations where the piezoelectric undergoes cycles of tension and compression through external vibrations or cycle forces are used to harvest mechanical energy.[18] Other configurations as for instance, integrating them in other stretchable materials[19] or composites materials,[10] have been recently proposed enabling the integration in multitude of applications. The actual problems that faces piezoelectric harvesters is the limited frequency range in which this devices can operates due to resonant behaviour for providing a constant and reasonable energy output. These previous materials, with the common configurations for flexible or wearable devices, provides a power output in the range of $0.01-10 \mathrm{~mW}$ and power densities of $0.1-10 \mathrm{~mW} \mathrm{~cm}^{-3} .[6,11]$

Tribologic nanogenerators (TENG) were also proposed by Z. L. Wang during the last years.[20] The fundamental principle is based on the electrostatic phenomenon of accumulating charges at the surfaces of materials when they are rubbed. Although this is a very old phenomenon, the mechanism behind triboelectrification is still under study.[14] This phenomenon occurs when two materials come into contact, some charges move from one material to the other to balance their electrochemical potential. When separated, one of the materials may keep some extra charges generating on dielectric surfaces a driving force for electrons that flow in the electrode to compensate the electric potential drop created. The designs to exploit this mechanism mainly consist on two dissimilar materials facing each other and when the two materials moves relatively to each other by external forces, they create a potential variation. The charges will flow through an external load to compensate this electric field. Thus, a periodic load or movement would generate an AC output (Figure 2.1[III]). Exist multiple configurations, for instance, vertical separation of the materials, lateral sliding (by linear, cylindrical or disc rotation)[21, 22] or freestanding separation.[23] These tribologic energy harvesters result on a power output in the range of $0.01-100 \mu \mathrm{W}$ and power densities of $0.01-500 \mathrm{~mW} \mathrm{~cm}^{-3} \cdot[6,11,14,24]$ 
Finally, there are different proposed solar energy harvesting systems, e.g. solarthermal, concentrated solar power or photovoltaic. Among them, solar cells are one of the most widespread common technologies (Figure 2.1[IV]). A photovoltaic (PV) cell is used to convert solar radiation into electric power. In a photovoltaic device electrons and holes are separated inside the material, flowing in opposite direction and creating an electrical current when connected to electrical load. Due to the high power of the sun light, these devices have a large amount of input energy to harvest, in contrast to previous described technologies (mechanical vibrations or thermal fluctuations). Furthermore, solar radiation, in a short time span, is a constant source of radiation, on the contrary mechanical harvesters are quite limited to intermittent vibration and frequency compatibility of the device. Flexible, light weight or enhanced toughness are often required properties for novel solar devices applications, as for instance, wearables,[25, 26] aerospace applications[27] or smart buildings.[28] Flexible photovoltaic devices result on a power output in the range of $0.1-10 \mathrm{~mW}$ and power densities of $1-10^{5} \mathrm{~mW} \mathrm{~cm}^{-3}$.[29]

One common factor of all these type of technologies towards novel applications is that, in general, they need a tough, flexible, highly conducting electrode (current-collector, CC).

This work focuses on the materials service aspects of using electrodes/CC based on fibres of carbon nanotubes for energy harvesting. It focuses particularly on their use in solar energy harvesting devices, which as show in Figure 2.1, have substantially higher power density.

\subsection{Carbon nanotubes}

\subsubsection{Structure, properties and synthesis}

Among different nanocarbons, carbon nanotubes (CNTs) have been widely researched during the last three decades. They consist on a hollow tube made of carbon atoms in hexagonal arrangement, each $C$ sharing a covalent bond with its three neighbouring atoms (sp2 bonds). This type of hybridisation produce three $\sigma$ bonds shifted out of plane and delocalisation of the $\pi$ orbital outside the tube. This surface electronic structure makes the CNTs a complete different material to other type carbons, i.e. graphite, and it is responsible of remarkable electronic, optical and mechanical properties.[30] This carbon allotrope is typically synthesised by chemical vapour deposition (CVD) due to the higher purity of the material and better control of material properties, but other methods are also widely used, e.g. laser ablation, electric arc discharge, among others.[31]

Among the characteristics of the CNTs, it is important to distinguish the number of layers and the chirality of the tubes. If CNTs are seen as a graphene sheet rolled over itself forming a tube, several concentric tubes define a double-, triple- or multi-wall CNT (MWCNT), with an intertube separation distance of $\approx 0.34 \mathrm{~nm}$ [32] (see MW- and SW-CNT sketches in Figure 2.2a and b respectively). Apart from the number of layers, each CNT can be defined using the chiral vector $C_{h}$ (or helicity vector) and the angle of helicity $\theta$ (referring to Figure 2.2c):[33]

$$
C_{h}=O A=n a_{1}+m a_{2} \equiv(n, m)
$$




$$
\cos \theta=\frac{2 n+m}{2 \sqrt{n^{2}+m^{2}+n m}}
$$

where $n$ and $m$ are the integers of the vector $O A$ considering the unit vectors $a_{1}$ and $a_{2}$. Accordingly, the diameter of a CNT can be calculated from the chiral indices. The chiral vector is perpendicular to the tube axis $(T)$ and defines the type of CNT. For $m=0$, the graphene sheet is rolled-up as zigzag CNT $(2.2 \mathrm{~b}[\mathrm{i}])$ and for $n=m$ an armchair CNT is obtained(2.2b[ii]). The chirality of the tubes also defines the electrical properties,[33] and the condition for obtaining metallic behaviour is

$$
2 n+m=3 q
$$

where $q$ is an integer.

The typical diameter of a SWCNT ranges 1-4 nm, whereas for a MWCNTs is 5-50 $\mathrm{nm}$. In contrast the length of CNTs is several orders of magnitude higher than their diameter, leading to aspect ratios (proportion of length and diameter) as high as $10^{5}-10^{8} \cdot[34]$
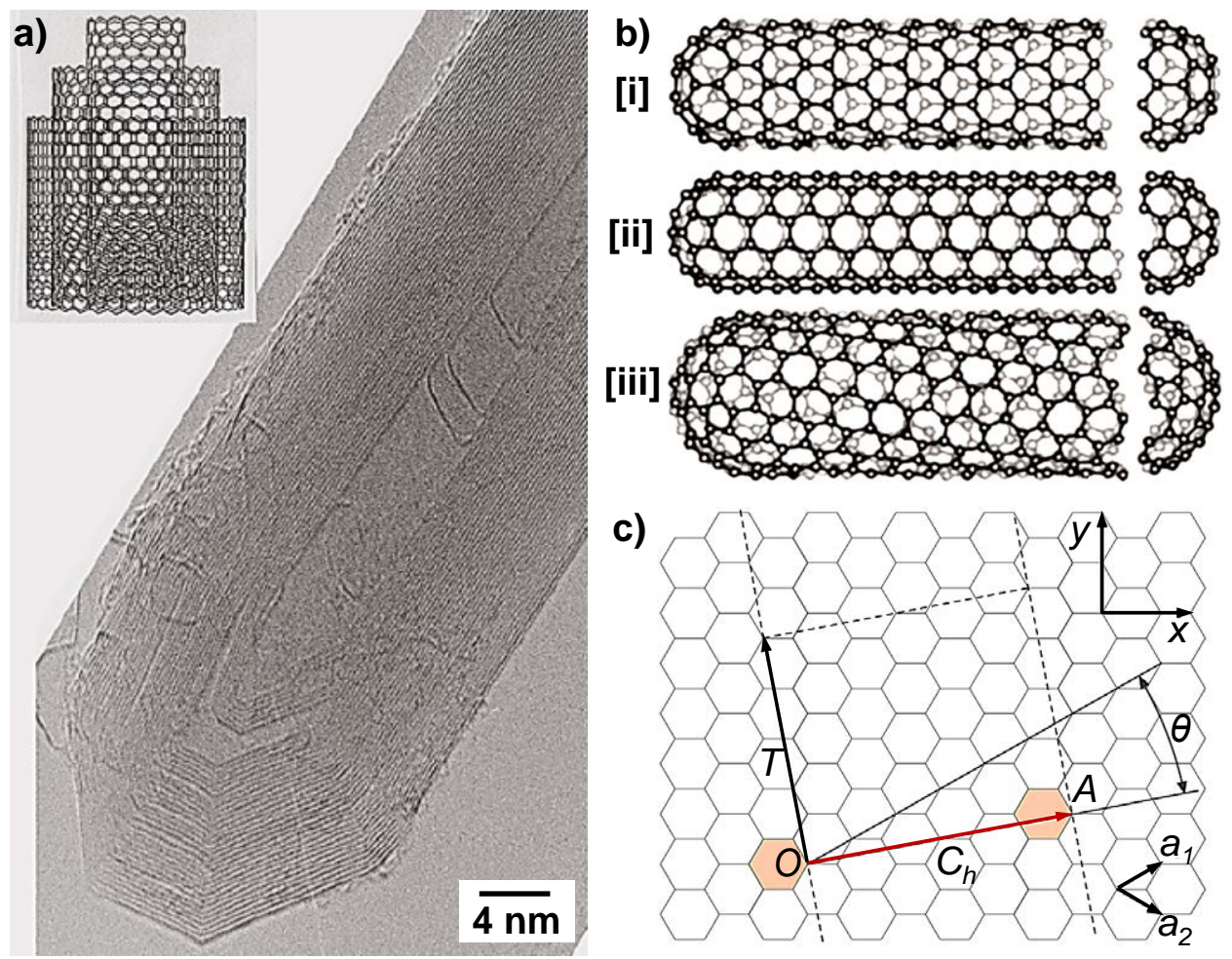

FIGURE 2.2: a) HRTEM image of a concentric MWCNT. In the insert, sketch of the concentric CNTs. b) Sketches of three different SWCNT structures as examples for [i] zig-zag, [ii] armchair and [iii] helical CNTs. c) Schematic of the the CNT structure starting from a graphene sheet. Reproduced from Bhushan et al.[31]

On account of their unique structure, the unidimensional nature of CNTs and small number of defects allow the electron propagation over long distances without scattering (ballistic transport). The carrier mobilities is in the range of $10^{5} \mathrm{~cm}^{2} / V s$,[35] 
the lowest resistivity reported for MWCNT is $10^{-6} \Omega \mathrm{cm}[36]$ and they can stand current densities of $10^{9} \mathrm{~A} / \mathrm{cm}^{2}$.[37] The thermal conductivity is $\approx 6000 \mathrm{~W} / \mathrm{mK}$ [38], comparable to that of a single, isolated graphene layer.

The CNTs are also characterised by their excellent mechanical properties. The experimental measured value of tensile modulus is $\approx 1 \mathrm{TPa}$,[39] highest tensile strength is $\approx 150 \mathrm{GPa}[40]$ and tensile strain of $12 \%$.[39]

Moreover, due to the high temperature of the synthesis reaction $\left(400-1200{ }^{\circ} \mathrm{C}\right.$ by CVD technique), CNTs are stable in air atmosphere up to $600^{\circ} \mathrm{C}$ and much higher stability in inert atmosphere, up to $1800{ }^{\circ} \mathrm{C}$ in $\mathrm{N}_{2}$.

Finally, CNTs in powder form, due to their small size and low density, results in lightweight porous structures with large specific areas. Values as high as $1000 \mathrm{~m}^{2} / \mathrm{g}$ for SWCNT and 50-200 $\mathrm{m}^{2} / \mathrm{g}$ for MWCNTs have been predicted.[41]

Regarding the synthesis of CNTs, catalytic CVD technique is the predominant method due to its high degree of control and scalability. It involves the catalytic decomposition of a carbon containing source on small metal particles or clusters. This is an heterogeneous process if a solid substrate has a role or an homogeneous process if the reaction and growth takes place in the gas phase.[31]

Heterogeneous process basically consist in passing a gaseous flow containing a given proportion of hydrocarbon (e.g. $\left.\mathrm{CH}_{4}, \mathrm{C}_{2} \mathrm{H}_{2}, \mathrm{C}_{2} \mathrm{H}_{4}, \mathrm{C}_{6} \mathrm{H}_{6}\right)$, usually as a mixture with either $\mathrm{H}_{2}$ or inert gas such as $\mathrm{Ar}$ or $\mathrm{N}_{2}$, over small metal catalyst particles (generally $\mathrm{Fe}, \mathrm{Co}$ and $\mathrm{Ni}$ ) previously deposited onto a inert substrate. This reaction takes place within a tubular furnace previously heated up to the desired temperature. The resulting material grows in contact to the substrate which can be later removed.

The homogeneous route, also called "floating catalyst method" differs from the previous method because it does not require any solid phase in the reactor. In this case, the nanometric catalyst particles are formed directly in the reactor. Metalorganic compound decomposes in the first zone of the reactor to generate the metallic particles that can catalyse the the nanotube formation. In the second part of the reactor, the carbon source is decomposed to atomic carbon which is then responsible for the formation of CNTs. By this process, CNTs produced without substrate are later collected and processes for its use.

\subsubsection{Macroscopic assemblies: CNT dispersions and CNT fibre}

During long time, lots of efforts have been made to exploit the properties of CNTs (and nanocarbons in general) at the macroscopic scale. For that, the conventional solution is to disperse the nanocarbons into different matrices for producing nanocomposites that shows the properties of the nanoscopic materials.

When CNTs are used for preparing hybrid materials or nanocomposites, thanks to their high aspect ratio, they present an important advantage against other nanocarbons. With increasing the number of particles in the matrix, at certain concentration, these nanoparticles create a critical network that spans the whole matrix. This concentration is known as the percolation threshold $\left(p_{c}\right)$ and the formation of this critical network is explained by the percolation theory. 

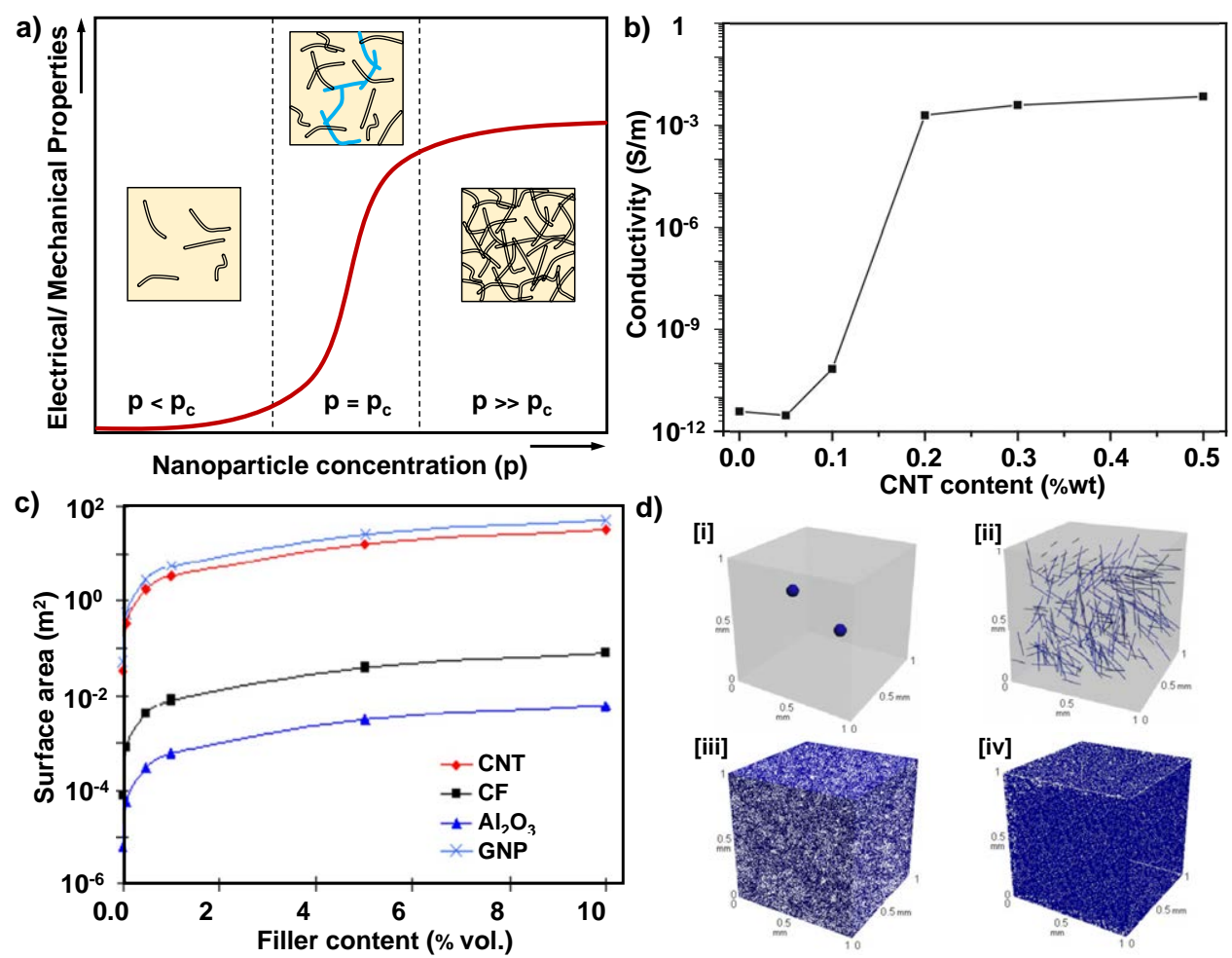

FIGURE 2.3: a) Sigmoidal curve followed by the enhancement of mechanical or electrical properties in nanocomposites as a function of the nanocarbon concentration. Critical path marked with a blue line representing the onset of the percolation threshold. b) Conductivity of MWCNT/polymer composite prepared by sonication. Image adapted from Martone et al.[42] c) Surface area of fillers in composites with varying filler contents. d) Distribution of micro- and nanoscale fillers of the same $0.1 \%$ vol. in a reference volume of $1 \mathrm{~mm}^{3}([\mathrm{i}]$ $\mathrm{Al}_{2} \mathrm{O}_{3}$, [ii] carbon fibre (CF), [iii] graphene nanoplatelets (GNP) and [iv] CNTs. Images c,d adapted from Ma et al.[43]

A schematic of the percolation process is presented in the Figure 2.3a. High aspect ratio leads to lower percolation threshold concentrations in the range of 10100 -fold lower in comparison with other nano- and micro- fillers but it is also very dependent on the matrix and dispersion methodologies. An experimental electrical percolation curve[42] is presented as example in the Figure $2.3 \mathrm{~b}$ showing several orders of magnitude increment of conductivity when the concentration of CNTs exceeds the percolation threshold $\left(p_{c} \approx 0.1\right)$.

Percolation mechanism helps understanding later applications of nanocarbons as dopants in electrolytes or increasing the conductivity of hybrids materials, as well as, the high interface developed by electrolyte/nanocarbon counter electrodes at low CNTs concentrations.

Moreover, comparing different nanomaterials, thanks to the aspect ratio and low density, the CNTs presents 10 to 500 times higher surface area for the same $\%$ vol. fraction. In the Figure $2.3 \mathrm{c}, \mathrm{d}$ it is compared the surface area of commonly used fillers taking into account their typical dimensions. Also, it is presented a visual comparison of $1 \mathrm{~mm}^{3}$ of material with $0.1 \%$ vol. dispersed nanoparticles, being [i] $\mathrm{Al}_{2} \mathrm{O}_{3}$, [ii] carbon fibre (CF), [iii] graphene nanoplatelets (GNP) and [iv] CNTs.[43] 
Although dispersion is a good strategy, typically the properties increase for mass fractions below 5-10 \% but, it is still a challenge to obtain high-loading nanocomposites reflecting a significant performance in mechanical properties with reproducible methods and results. [44, 45] The effective elastic modulus reinforcement, $\Delta E / \Delta V_{f}$, (see Figure 2.4) provides a measure of how efficiently the properties of the nanofiller are exploited in the composite, but also enables the comparison with the level of reinforcement achieved using other nanofillers.[46]

The data presented in Figure 2.4 corresponds to a different matrix nanocomposites, types of tubes and orientations reported in literature. There is a large scattering of the data evidencing the differences in experimental conditions and testing methods, but it is clearly observed the decrease in CNT reinforcement with increasing volume fraction.

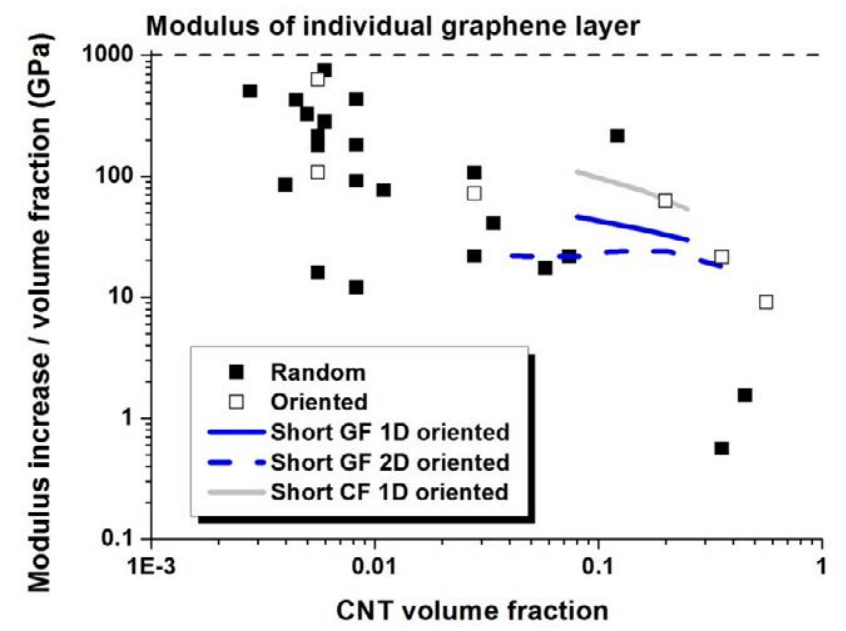

FIGURE 2.4: Nanocomposite elastic modulus effective reinforcement vs CNT volume fraction.[46]

An alternative approach is to directly assemble the CNTs as building-blocks during the synthesis process into a macroscopic material. CNT fibres (CNTf) are an example of macroscopic assembly fabricated by different bottom-up or top-down techniques. CNTfs are typically produced from CVD and macroscopically collected directly from the synthesis reactor[47] (Figure 2.5a) or from a vertically aligned forest of CNTs directly from substrate (Figure 2.5b).[48] These methods are known as dry-spinning processes. There are also CNTf produced by wet spinning $[49,50]$ but this material is not commonly used for photovoltaics applications. The different methods for producing this macroscopic assemblies of CNTs are summarised in the Figure 2.5. As shown, the obtained CNTfs by these methods are similar in appearance but their properties differ depending on the synthesis route.

The wet spinning process refers to basically two different methods, liquidcrystalline spinning[51] (Figure 2.5c) and polymer/ CNT coagulation route (Figure 2.5d).[49] In the first, the CNTs are dispersed in high concentration in superacids obtaining a liquid crystal solution of solvated tubes in equilibrium with a dilute isotropic phase. Under anhydrous condition, the liquid crystalline phase can be processed into highly aligned fibres of pure CNTs without the aid of any surfactants or polymers. The CNT dispersion can be extruded through a small capillary tube into a coagulation bath (for instance, diethyl ether, water or $5 \%$ wt aqueous sulfuric 
acid).[51] For the second method, a CNT/surfactant dispersion is gently injected into a stirred polymer solution, which acts as a coagulation bath. The excess of solvent is later evaporated. These nanotube fibres can be strongly bent without breaking, and their elastic modulus is 10 times higher than the modulus of high-quality bucky papers.[49]

The dry spinning methods are direct processing routes of the material obtained by heterogeneous and homogeneous CVD (explained in the previous section). The direct spinning from arrays of aligned CNTs[48] (heterogeneous CVD) consist on drawing and twisting the individual nanotubes into a macroscopic fibre. Because of the Van der Waals forces between nanotubes, the fibre can be continuously spun (see Figure 2.5b). This synthesis method allows a high degree of control over the properties of the CNTf by controlling the type on CNTs.

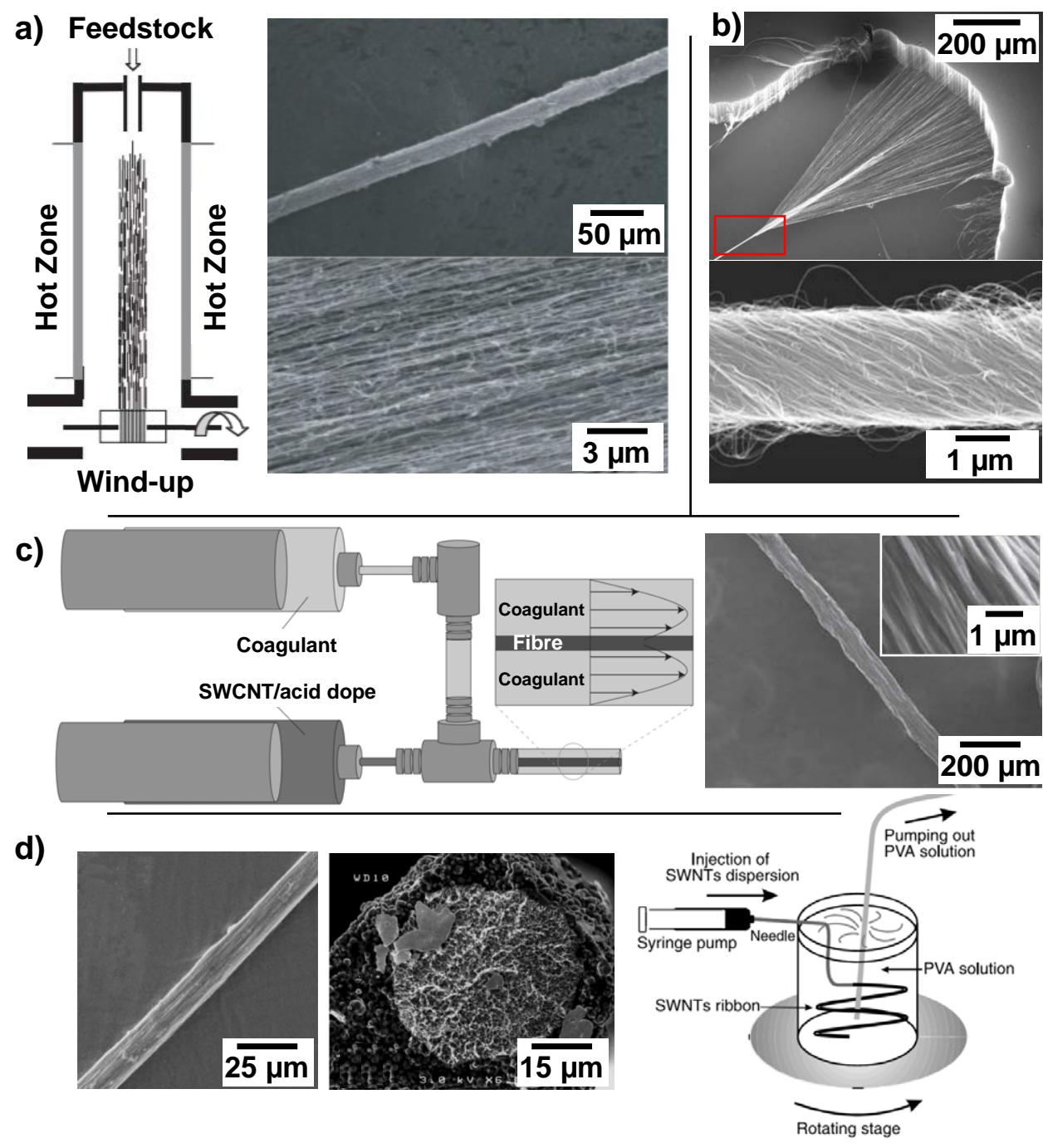

FIGURE 2.5: CNTf: schematic of the synthesis process and SEM images of the as-made fibres by different methods. Dry-spinning: a) from direct catalyst floating CVD (adapted from Li et al.[47]), b) from direct spinning of vertically aligned CNTs (adapted from Zhang et al.[48]). Wet-spinning: d) from liquid-crystalline spinning (adapted from Davis et al.[52]) and d) from polymer/CNT coagulation route

(adapted from Vigolo et al.[49]) 
Finally, the direct spinning from CVD reactor[47] (homogeneous CVD) allows the continuous withdrawal of CNTf by mechanically drawing from the gaseous reaction zone with a rotating spindle (see schematic in the Figure 2.5a). Apart from the parameters that affect the CVD reaction (carbon source, catalyst, promoter, temperatures, gas flow, injection rate) other parameters are critical for the final properties of the material, for instance, winding rate or post-treatment in-situ densification process. As said before, the content of sulphur compound in the chemical reaction, acting as promoter of the reaction, enhances the growth rate of the CNTs. Winding rates in the range of 5-100 $\mathrm{m} / \mathrm{min}[53,54]$ are common, enabling the production of dozens of kilometres of material per day, and tailoring the mechanical properties of the final fibre.

The CNTfs used in this thesis are produced by the direct spinning process (catalyst floating CVD process),[47] and the structure and properties described in this section will refer mainly to this type of material.

The CNT fibres, sometimes referred to as yarns or threads, consist of long and thin assemblies of axially aligned CNTs packed in parallel into CNT bundles. Thousands of this bundles are arranged showing a high degree of alignment forming an individual CNTf monofilament i.e. single fibre, typically with a diameter of several millimetres when comes out of the reactor, and 1-10 $\mu \mathrm{m}$ after condensation. In these assemblies the individual CNTs and CNT bundles agglomerate due to Van der Walls interactions, friction forces and local entanglements.[55] This structure is summarised in the Figure 2.6.

A large amount of fibres can be collected through this method for obtaining CNTf sheets, also referred to as films. For instance, a bobbin of the as-made macroscopic CNTf continuously spun for $10 \mathrm{~min}$ is presented in the Figure 2.7a. This flat 2D sheets (Figure 2.7b) are made of a network of $m m$-long CNTs packed into bundles with their axial dimensions arranged in the plane of the sheet (Figure 2.7b).[56]

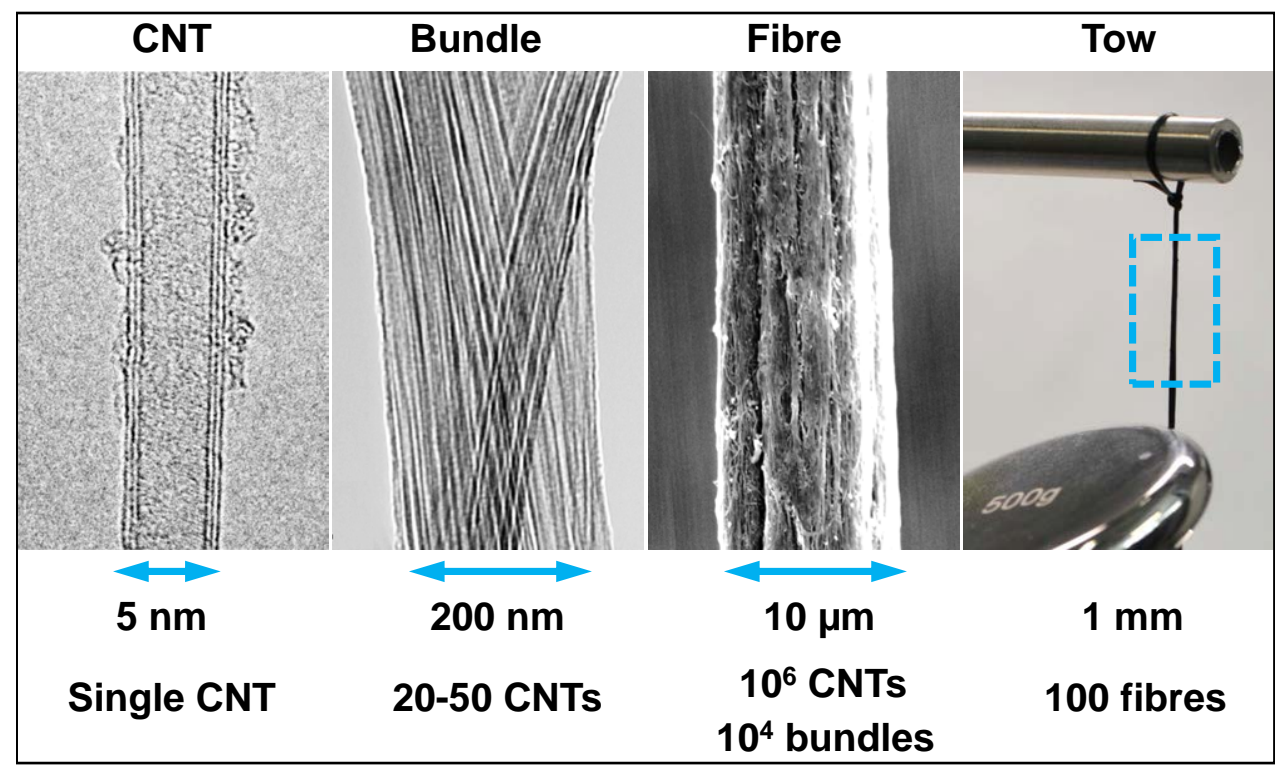

FIGURE 2.6: CNTf structure, from molecular to macroscopic level: single CNT,[57] bundle, fibre and a macroscopic tow of CNTf are presented with their dimensions and approximate number of units. 
The CNT bundles network creates a 3D structure of open and interconnected porosity (Figure 2.7d). The pore network of different materials is typically analysed by gas adsorption/desorption methods. Figure $2.7 \mathrm{~g}$ shows the isotherm obtained for a CNTf sample. According to the Brunauer-Deming-Deming-Teller (BDDT) classification, the shape of the isotherm is characteristic of macroporous materials with a wide distribution of pore sizes $(10-50 \mathrm{~nm})$ but no presence of micropores (Figure 2.7g).[58] The specific surface area (SSA) by Brunauer-Emmett-Teller BET for CNTf ranges $75-500 \mathrm{~m}^{2} / \mathrm{g}$ with a total pore volume of $0.89 \mathrm{~cm}^{3} / \mathrm{g}$.[58]
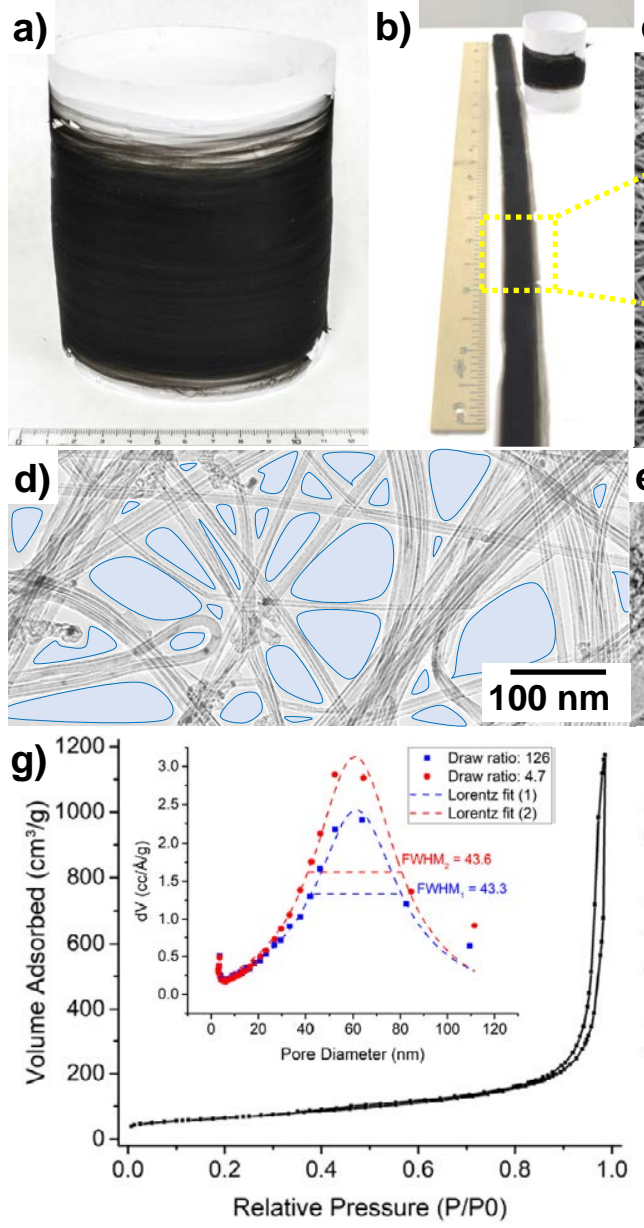

b)

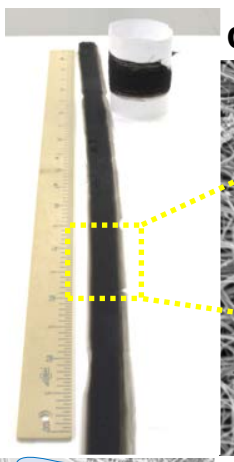

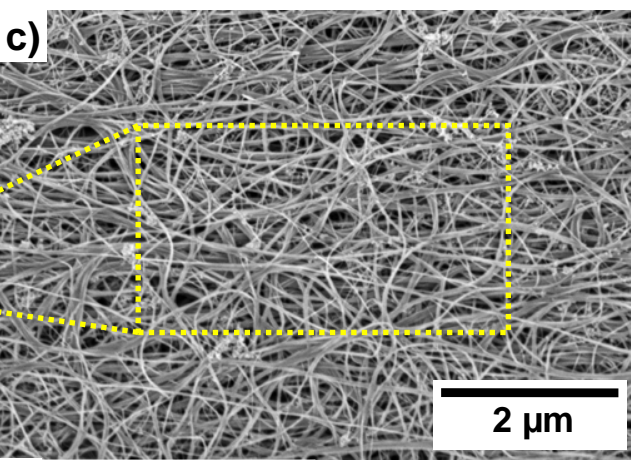
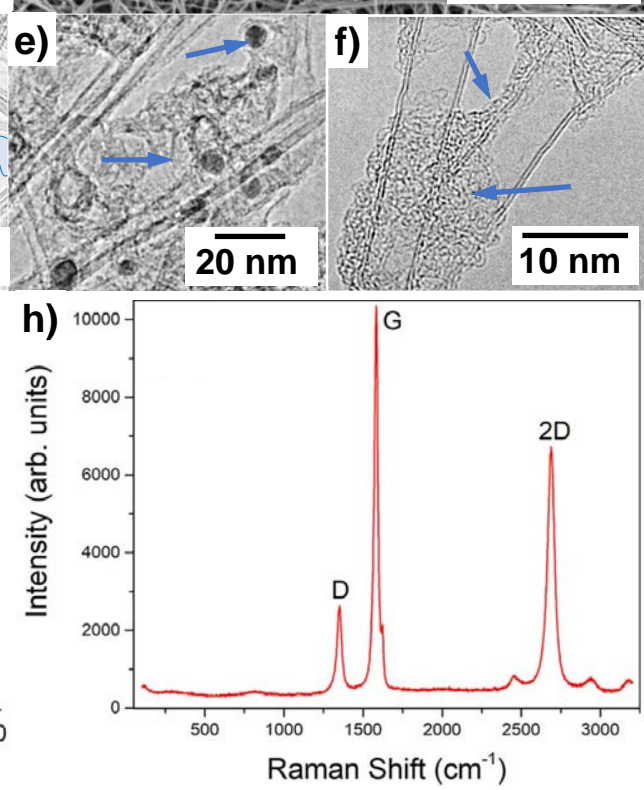

FIGURE 2.7: a) Digital photograph of CNTf bobbin. b) Large scale CNTf for electrode application. c) SEM image of the CNTf bundles forming the open and interconnected porosity of the material. d) TEM image with a graphic representation in $2 \mathrm{D}$ of the $3 \mathrm{D}$ porous formed between bundles. e) Encapsulated residual catalyst in in poorly graphitised material. f) CNT bundle coated with carbonaceous layer. g) BET analysis and pore size distribution of the CNTf (Courtesy of Dr. Evgeny Senokos). h) Raman spectrum of CNTf showing the characteristic features of few-layer CNTs.[59]

Note that, the high porosity as well as the high specific surface area allows the CNTf to be infiltrated with different materials (e.g. polymers, metal oxides, electrolytes) resulting in a huge interface to interact between materials.

The predominant organic impurities present in the CNTf are the non-graphitic 
or amorphous carbon and volatile hydrocarbons adsorbed on the surface of CNTs (Figure 2.7e). This contaminant can be removed by heat treatment, and it has been demonstrated the high impact over the mechanical properties of the fibre.[60]

Typically, there is also catalyst residue that appears encapsulated by poorly graphitised material[56] (Figure 2.7f). These impurities might present catalytic behaviours interfering with different processes when using the CNTf as electrode, for instance for batteries, energy storage or solar energy harvesting (DSSC). There have been many works studying the purification of CNTs[61] and most recently of CNTf.[54] The purification of the CNTf by sonication in acetone has resulted in improved mechanical and electrical properties, reducing the overall content of impurities by $42 \%$.[54]

The Raman spectrum of standard few-layer CNTf (Figure 2.7h) shows the characteristic $G$ band at $\approx 1580 \mathrm{~cm}^{-1}$, corresponding to planar vibrations of the $C-C$ bonds, the $\mathrm{D}$ band at $\approx 1350 \mathrm{~cm}^{-1}$, indicative of structural defects present in the $s p 2$ carbon lattice, and overtone modes in the range of at $2400-3000 \mathrm{~cm}^{-1}$.

The D/G intensity ratio is commonly used as an indicator of the quality of the material, providing the density of defects (sp3 impurities or broken $s p 2$ bonds) over the graphitic nature of the material. Moreover, the $\mathrm{G}$ band is strongly resonant with the number of layers of the CNTs, resulting in very intense signal when the optical transition of SWCNTs are excited.[59] Thus, $\mathrm{I}_{D} / \mathrm{I}_{G} \leq 0.1$ is indicative of SWCNT fibres. Standard MW-CNTf typically presents a $\mathrm{I}_{D} / \mathrm{I}_{G} \approx 0.3-0.4$, and defective fibres, as for instance functionalised by ozone treatment ranges from $\mathrm{I}_{D} / \mathrm{I}_{G} \approx 0.6 \mathrm{up}$ to 2.[62]

It is the combination of mechanical and electrical properties of the CNTf in addition to its low density what makes the material unique. But the properties of the CNTf material really stands out when they are normalised by their specific gravity (specific gravity is the density referred to the density of $\mathrm{H}_{2} \mathrm{O}, S G$ )

In the Table 2.2 the properties of the CNTf are compared to other high performance commercial fibres. For the shake of clarity, not all the properties of the CNTf have been included in the Table 2.2. For instance, the elongation at break of the CNTf is in the range 5-10\%, 3-5 times higher than that of carbon fibres (CF, 2.4 \%) [63, 64] and kevlar-K29 (3.6\%).[65] Consequently, the toughness (fracture energy) of CNTf almost twice compared with the rest of high performance fibre materials.[66] The thermal conductivity of CNTf sheets have been reported as $759 \mathrm{~W} / \mathrm{mK}$,[67] this is twice the thermal conductivity of copper $(400 \mathrm{~W} / \mathrm{mK})[63], 100$ times higher than $\mathrm{CF}[63]$ and four orders of magnitude higher than glass fibre $(0.04 \mathrm{~W} / \mathrm{mK})$.[65]

Moreover, something characteristic of the CNTf is its high surface area $(\approx$ $270 \mathrm{~m}^{2} / \mathrm{g}$ ).[58] This feature is hard to find in combination with the previous properties, and it can be exploited in multitude of applications, e.g. energy storage, catalytic processes, sensors, composites materials, etc.

The CNTf has demonstrated a high stability to harsh chemical conditions. Comparative immersion test of the CNTf during 30 days in several concentrated acids, saturated sodium chloride and deionised water has shows no no damage for the CNTf, in contrast, under this conditions, copper wires get severely corroded or completely dissolved.[68] They also showed the use of CNTf as circuit wiring to power up and LED being the CNTf immersed in concentrated acid. This experiment 
indicates the electrochemical stability of the material, although no specific studies on this regard has been found in literature.

In a similar work, they have shown the stability of the material and electrical performance at high temperature, confirming the stability of the material in air up to $450{ }^{\circ} \mathrm{C}$.

TABLE 2.1: Properties of CNTf compared to other high performance dry fibres.

\begin{tabular}{llllll}
\hline Material & $\begin{array}{l}\text { Tensile } \\
\text { strength } \\
(\mathrm{GPa} / \mathrm{SG})\end{array}$ & $\begin{array}{l}\text { Elastic } \\
\text { modulus } \\
(\mathrm{GPa} / \mathrm{SG})\end{array}$ & $\begin{array}{l}\text { Electrical } \\
\text { conductivity } \\
(\mathrm{S} / \mathrm{m})\end{array}$ & $\begin{array}{l}\text { Volumetric } \\
\text { density } \\
\left(\mathrm{g} \mathrm{cm} \mathrm{cm}^{-3}\right)\end{array}$ & Ref. \\
\hline CNT fibre & 1.5 & 50 & $3 \times 10^{5}$ & $0.4-1$ & {$[66]$} \\
Carbon Fibre (AS4) & 2.4 & 129 & $3 \times 10^{2}$ & 1.79 & {$[64]$} \\
Kevlar (K29) & 2.1 & 49 & - & 1.44 & {$[65]$} \\
E-Glass fibre & 0.8 & 30 & - & 2.55 & {$[69]$} \\
Copper & 0.023 & 12.3 & $5.8 \times 10^{7}$ & 8.93 & {$[70]$} \\
\hline
\end{tabular}

The great achievement of this material is that directly reflects the properties of the individual CNTs as a macroscopic material, taking into account that it is almost entirely composed by CNTs (mass fraction close to 1).

Comparing the electrical or mechanical properties of CNT nanocomposites with pristine CNT macroscopic assembles (CNTf), no matter the synthesis method, the last presents for instance electrical properties $10^{2}-10^{5}$-fold higher than the dispersed CNT nanocomposites.[44, 55]

\subsubsection{CNTs and CNTf as current-collector in tough electronics}

The previous sections show that CNTs and in particular their macroscopic assembles, i.e. CNTf, present a very interesting combination of characteristics for engineering applications as tough electronics. They combine the characteristics of a great electrical conductor material (very high thermal and electrical conductivity and very high current density capacity), with those properties of a high performance structural fibre, high strength, modulus and low density, in addition to other properties as great specific surface area, high open and interconnected porosity and electrochemical stability.

In relation to the energy harvesting application with augmented mechanical properties, novel applications are continuously being proposed. Some of them are summarised in the Table 2.2. 
TABLE 2.2: CNTf and related materials in energy harvesting applications with augmented mechanical properties.

\begin{tabular}{|c|c|c|c|}
\hline Application & $\begin{array}{l}\text { Energy } \\
\text { Performance }\end{array}$ & $\begin{array}{l}\text { Mechanical } \\
\text { Performance }\end{array}$ & $\begin{array}{l}\text { Year } \\
\text { Ref. }\end{array}$ \\
\hline $\begin{array}{l}\text { CNT-network/PANI } \\
\text { (Thermoelectrics) }\end{array}$ & $\mathrm{P}=5 \mu W m^{-1} K^{-2}$ & $\begin{array}{l}\text { Mechanical tolerance } \\
\text { and flexibility }\end{array}$ & $\begin{array}{l}2014 \\
{[71]}\end{array}$ \\
\hline $\begin{array}{l}\text { Kevlar/ZnO-NWs } \\
\text { (Piezoelectric) }\end{array}$ & $4-16 \mathrm{mWcm}^{-2}$ & High flexibility & $\begin{array}{l}2008 \\
{[72]}\end{array}$ \\
\hline $\begin{array}{l}\text { CNTf/ZnO-NWs } \\
\text { (Piezoelectric) }\end{array}$ & $V_{\text {piezo }}=60 \mathrm{mV}$ & $\begin{array}{l}\text { High flexibility and } \\
\text { toughness, } 10 \mathrm{~J} / \mathrm{g}\end{array}$ & $\begin{array}{l}2014 \\
{[73]}\end{array}$ \\
\hline $\begin{array}{l}\text { CFRP/ZnO-NWs } \\
\text { (Piezoelectric) }\end{array}$ & $4 n W /$ sample & Structural material & $\begin{array}{l}2016 \\
{[10]}\end{array}$ \\
\hline $\begin{array}{l}\mathrm{CF} / \mathrm{ZnO}-\mathrm{NWs} \\
\text { (Triboelectric) }\end{array}$ & $0.001 \mathrm{mWcm}^{-2}$ & High flexibility & $\begin{array}{l}2014 \\
{[74]}\end{array}$ \\
\hline $\begin{array}{l}\text { Kevlar-textile } \\
\text { (Triboelectric) }\end{array}$ & $1.1 \mathrm{~mW} /$ device & $\begin{array}{l}\text { Mechanical robust- } \\
\text { ness and flexible }\end{array}$ & $\begin{array}{l}2015 \\
{[2]}\end{array}$ \\
\hline $\begin{array}{l}\text { CNTf/n-doped-Si } \\
\text { (Schottky solar cell) }\end{array}$ & $\mathrm{PCE}=3 \%$ & $\begin{array}{l}\sigma=1 G P a, E=10 G P a, \\
67 \mathrm{~J} / g\end{array}$ & $\begin{array}{l}2012 \\
{[75]}\end{array}$ \\
\hline $\begin{array}{l}\text { CNTf/Polymer/Ti wire } \\
\text { (Polymer solar cell) }\end{array}$ & $\mathrm{PCE}=1.8 \%$ & $\begin{array}{l}\text { Mechanical tolerance } \\
\text { and flexibility }\end{array}$ & $\begin{array}{l}2014 \\
{[71]}\end{array}$ \\
\hline $\begin{array}{l}\mathrm{CNTf}-\mathrm{CE} / \mathrm{TiO}_{2}-\mathrm{CNTf} \\
\text { (fibre-like DSSC) }\end{array}$ & PCE $=3 \%$ & $\begin{array}{l}\text { Mechanical tolerance } \\
\text { and flexibility }\end{array}$ & $\begin{array}{l}2012 \\
{[76]}\end{array}$ \\
\hline $\begin{array}{l}\text { CNTs/CF-textiles } \\
\text { (DSSC) }\end{array}$ & $\mathrm{PCE}=8.9 \%$ & $\begin{array}{l}\text { High performance } \\
\text { mechanical properties }\end{array}$ & $\begin{array}{l}2017 \\
{[77]}\end{array}$ \\
\hline $\begin{array}{l}\text { CNTf/perovskite/Ti } \\
\text { foil (PSC) }\end{array}$ & $\mathrm{PCE}=8.3 \%$ & $\begin{array}{l}\text { High flexibility, trans- } \\
\text { parent electrode }\end{array}$ & $\begin{array}{l}2015 \\
{[78]}\end{array}$ \\
\hline $\begin{array}{l}\text { CNTf/perovskite } \\
\text { (fibre-like PSC) }\end{array}$ & $\mathrm{PCE}=3 \%$ & $\begin{array}{l}\text { Mechanical tolerance } \\
\text { and flexibility }\end{array}$ & $\begin{array}{l}2015 \\
{[79]}\end{array}$ \\
\hline $\begin{array}{l}\text { CNTf/enzymes/redox } \\
\text { polymer (Biofuel cells) }\end{array}$ & $2.18 \mathrm{mWcm}^{-2}$ & $\sigma=70 \mathrm{MPa}, 1.4 \mathrm{~J} / \mathrm{g}$ & $\begin{array}{l}2014 \\
{[80]}\end{array}$ \\
\hline
\end{tabular}

\subsection{Dye-Sensitised Solar Cell: working principle and associ- ated processes}

DSSC are one of the last invented solar technologies, known also as hybrid photovoltaics, where the combination of inorganic/organic materials have shown very promising results during the last two decades. The Figure 2.8 shows the certified photovoltaic efficiency evolution in which the emerging hybrid photovoltaics have achieved values higher than $20 \%$ in a short period of time.[81] Firstly, with the emergence of the dye sensitised solar cells (DSSC) up to $13 \%$ [82] and secondly, during the last half decade, the perovskite sensitised solar cells (PSC) as an evolution of the DSSC technology.

Nowadays PSC is providing very promising results in terms of achieved efficiency,[81] but this technology still presents big challenges to be overcome, for instance, short term stability. In contrast, DSSC is a mature enough technology for seeking new materials that potentially can improve their performance and implementation. 


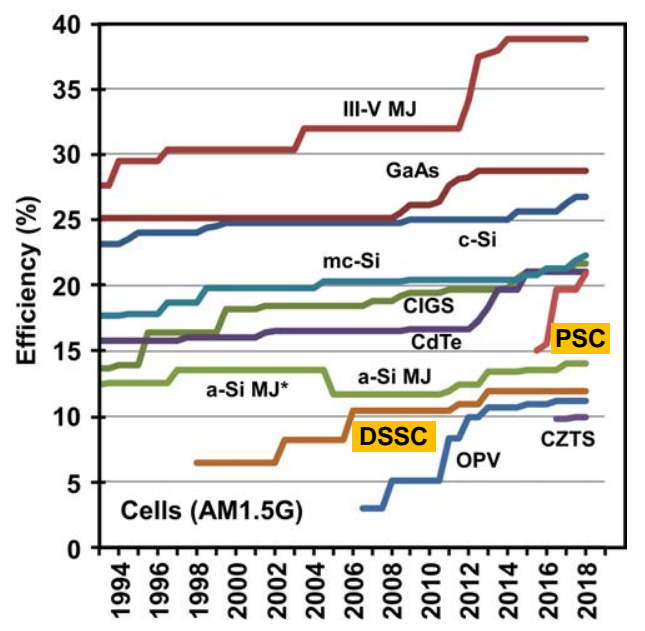

\author{
III-V MJ - Multi-Junction III-V semiconductor \\ GaAs - (Single-Junction) Gallium Arsenide \\ c-Si - (Mono) Crystalline Silicon \\ mc-Si - Multicrystalline Silicon \\ Thin Film Technology: \\ a-Si MJ - Amorphous Silicon Multi-Junction \\ CIGS - Copper Indium Gallium Selenide \\ CdTe - Cadmium Telluride \\ CZTS - Copper Zinc Tin Sulfide \\ OPV - Organic Photovoltaics \\ Hybrid Photovoltaics: \\ PSC - Perovskites Solar Cell \\ DSSC - Dye-Sentsitised Solar Cell
}

FIGURE 2.8: 25 years of progress in photovoltaic cells. Highest confirmed efficiencies for $>1 \mathrm{~cm}^{2}$ area cells for the shown technologies.

Adapted from Green et al.[83]

Nanocarbons have established themselves as one of the leading alternatives for this task. Their good electrical properties exceed the requirements for this applications. They have shown great chemical stability in many different environments. Moreover, their huge surface area is beneficial for the different charge transfer processes occurring in these devices.

Dye sensitised solar cells (DSSCs) have been under research over the last 25 years, since their invention by O'Regan \& Gratzel.[84] The rapid development of this technology with the achieving of photo-conversion efficiencies (PCE) higher than $13 \%[82]$ has position this technology as a promising alternatives to the Si-PV.

A conventional DSSC is built from a mesoporous $\mathrm{TiO}_{2}$ photoanode sensitised (i.e. loaded) with organic molecules that absorb visible light (typically N719) and photogenerates charges. The counter-electrode $(\mathrm{CE})$, typically $\mathrm{Pt}$, is the part of the DSSC where the catalytic reaction takes places regenerating the redox couple. Both materials are deposited onto transparent conductive oxides (TCO), allowing for front and back illumination for energy generation. The cell is filled by an iodide/triiodide redox couple based electrolyte. The schematic of a typical DSSC is depicted in the Figure 2.9.

The working principle of a DSSC can be understood following the electron transfer/flow through the materials inside the device, with the assistance of a simplified diagram of the equilibrium energy levels, as presented in Figure 2.10. When the cell is illuminated, the sensitiser (dye molecule) absorbs light and promotes one electron from its ground state $\left(S_{0}\right)$ to an excited state $\left(S^{*}\right)$, oxidation process. That photo-generated charge is injected into the nanocrystalline titania layer, where the electron diffuses through trap states close to the conduction band of the semiconductor driven by diffusion laws to the TCO current-collector. The photoanode is connected through an external load and separated from the cathode or counterelectrode. Pt nanoparticles deposited on the counter-electrode are responsible for reducing triiodide $\left(I_{3}^{-}\right)$molecules in the electrolyte to iodide $\left(I^{-}\right)$. When triiodide is reduced to iodide, it gains the electron responsible of giving back the dye molecules to its ground state restarting the cycle. Firstly, $I^{-}$is oxidised and $S^{*} \rightarrow S_{0}$, then it 
is reduced at the $\mathrm{CE}, I_{3}^{-}+2 e^{-} \rightarrow 3 I^{-}$. This part of the process involves diffusion of the iodide/triiodide species for the regeneration of the dye molecule.

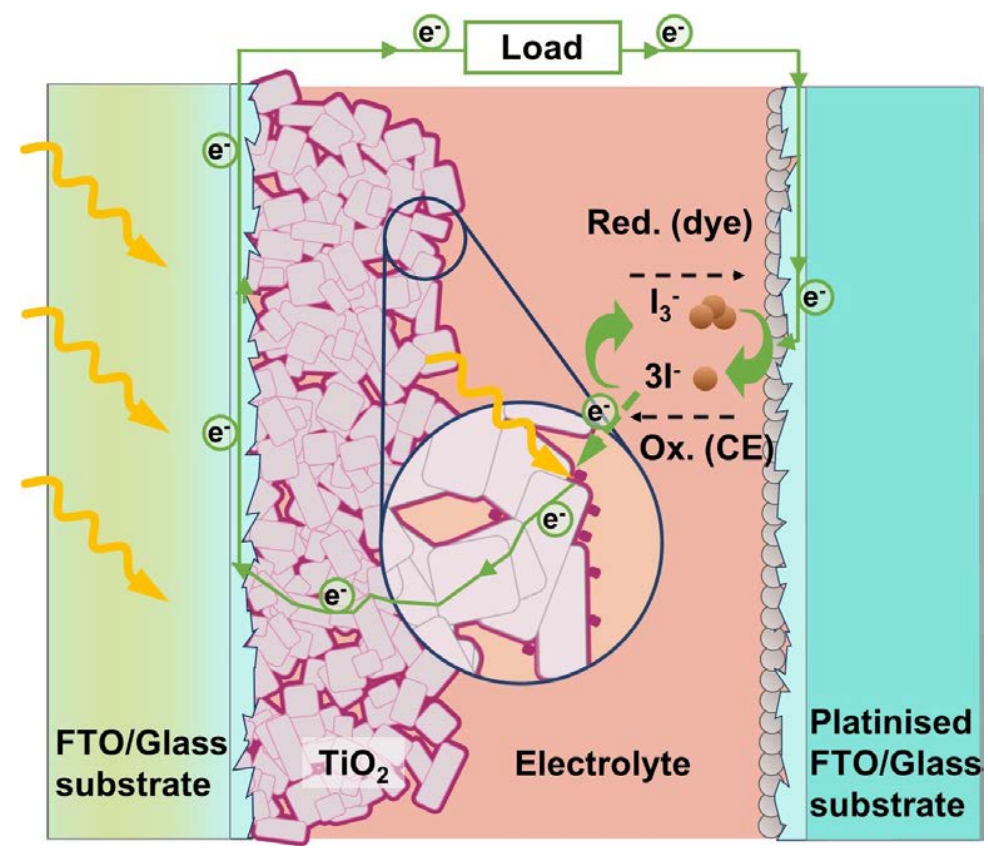

FIGURE 2.9: Schematic of the working principle of a n-type DSSC.

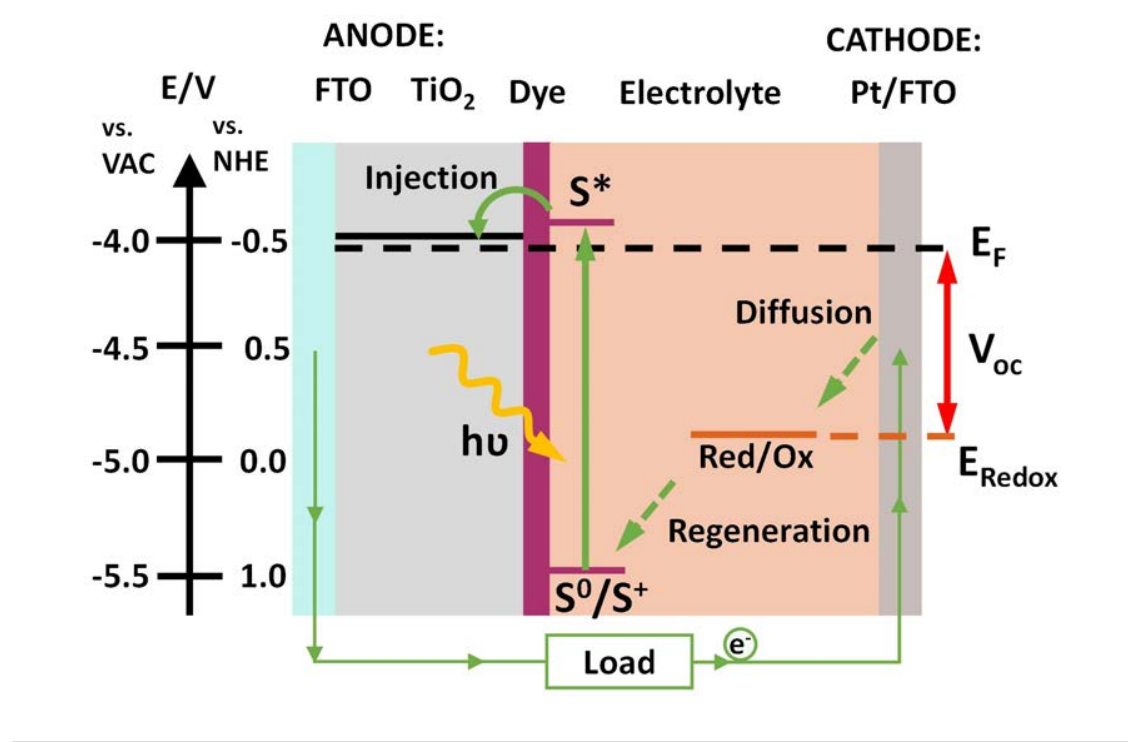

FIGURE 2.10: Energy bands diagram schematic of a n-type DSSC.

\section{Electrical modelling of a DSSC}

A solar cell can be modelled by a photocurrent source $\left(I_{\text {photo }}\right)$ in parallel with a diode, and two resistances, one in series $\left(R_{s}\right)$ and other in parallel (called shunt resistance, $R_{s h}$ ). Traditionally this model is known as single-diode exponential model. The electric circuit of this model is presented in the Figure 2.11a. This circuit is an approximation of the interaction of several physical processes.[85, 86] Typically, the 
solar cell modelling with a diode and a photocurrent source is derived from the application of the Shockley equation for ideal p-n junctions. In a first approximation it can be used for describing also DSSC.

In this model, $R_{s}$ represents the internal resistance of the cell (materials) and the resistance of the contacts and it should be as low as possible. The parallel resistance $\left(R_{s h}\right)$ represents the alternative paths or reactions to the desired $\mathrm{TiO}_{2}$ conduction, for instance by recombination sites at the anode, and it should be as high as possible. For DSSCs several unclear processes are involved and contribute to the drop of the $R_{s h}$ decreasing the performance.
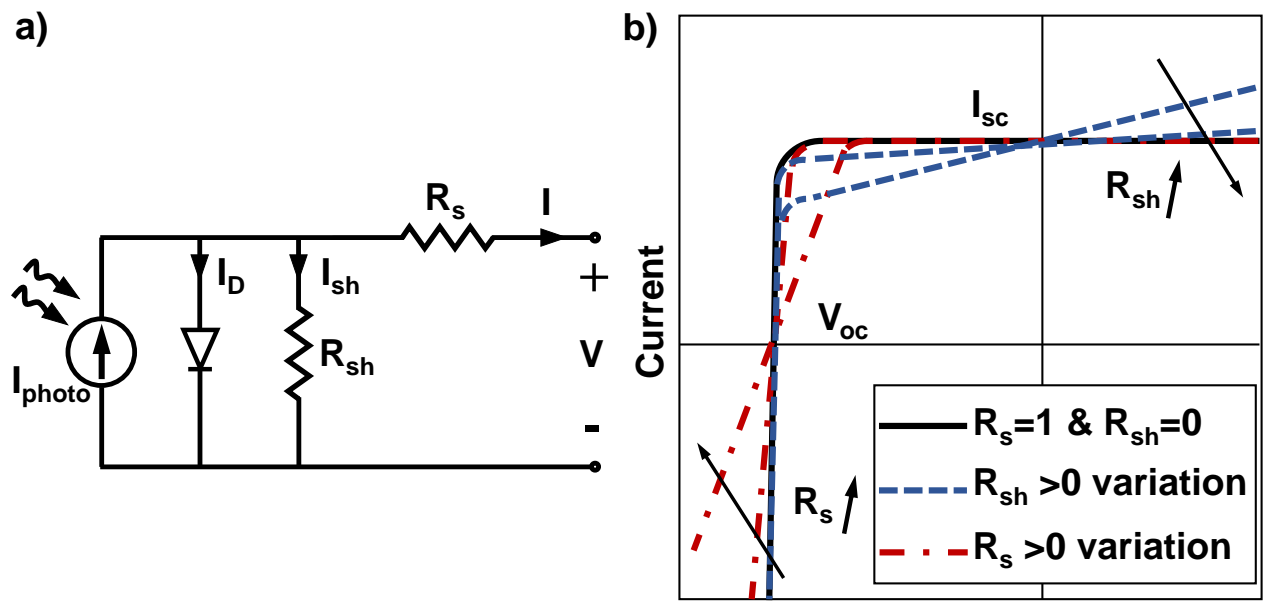

Voltage

FIGURE 2.11: a) Electric circuit of the single-diode exponential model for a solar cell. b) Influence of the series resistance $\left(R_{S}\right)$ and shunt resistance $\left(R_{s h}\right)$ on the $I-V$ curve. Adapted from $W$. Tress.[86]

The variation of how these resistances influence the $I-V$ curve is schematically presented in the Figure 2.11b. The $R_{S}$ of the DSSC lowers the operating voltage of the cell by $I R_{s}$, and the parallel $R_{s h}$ increases the leakage current of the solar cell.

Solving the equivalent circuit, the characteristic equation describing the the behaviour of the solar cell is obtained:

$$
I=I_{\text {photo }}-I_{0}\left[\mathrm{e}^{\left(\frac{q\left(V+I R_{s}\right)}{n k T}\right)}-1\right]-\frac{V+I R_{s}}{R_{s h}}
$$

where $I_{\text {photo }}$ is the photogenerated current, $I_{0}$ is the reverse saturation current, $V$ is the output voltage of the solar cell, $n$ is the ideality factor, $q$ is the elementary charge, $k$ is the Boltzmann constant, $T$ the absolute temperature.

In this equation, the first term represents the extracted current from the solar cell. The second term is the current flowing through the diode, described by the Shockley diode equation. And the last term relates to the losses due to the series and shunt resistance.

There are several important characteristics that define the performance of a solar cell. The fill factor $(F F)$ is defined as the ratio between the maximum obtained 
power (real) and the power obtained for short-circuit current and open-circuit voltage (ideal or theoretical, $P_{t h}$ ), in other words, the squareness of the $I-V$ curve. When $R_{s}$ increases, the $F F$ of the $I-V$ decreases. On the contrary, increasing $R_{s h}$ leads to increasing the FF.

From the equation 2.4 all characteristic parameters that define a solar cell can be defined. If the cell is operated at open circuit voltage $(I=0)$, the voltage across the output terminals is defined as open-circuit voltage $\left(V_{o c}\right)$. Assuming enough high shunt resistance to neglect the last term of the Equation 2.4, the $V_{o c}$ is given by:

$$
V_{o c} \approx \frac{n k T}{q} \ln \left(\frac{I_{\text {photo }}}{I_{0}}+1\right)
$$

In the same way, if the cell is under short circuit conditions $(V=0)$, the current measured through the terminals is defined as short-circuit current $\left(I_{s c}\right)$. If the cell presents good FF, i.e. low $R_{S}$ and high $R_{s h}$, the $I_{s c}$ is given by:

$$
I_{s c} \approx I_{\text {photo }}
$$

For any given bias voltage, the power output is the product of the measured cell current and the voltage, $P(V)=I(V) V$. Finally, the efficiency $(\eta)$, also called photoconversion efficiency $(P C E)$, is defined as the ratio of the maximum power extracted $\left(P_{m p p}\right)$ to the incident power on the surface of the solar cell:

$$
\eta=P C E=\frac{P_{m p p}}{P_{\text {in }}}=\frac{P_{t h} F F}{P_{\text {in }}}=\frac{I_{s c} V_{o c} F F}{P_{\text {in }}}
$$

$V_{o c}$ and $I_{s c}$ are important characteristics because they define and limit the performance of the device. Thus, increasing both parameters is desirable for enhancing the overall efficiency.

The origin of the $V_{o c}$ in DSSC is detailed in the Appendix at the end of this chapter, but abbreviating the explanation, it corresponds to the increase in band population under light conditions, and is defined as the difference between the redox potential of the electrolyte and the quasi-Fermi level of the semiconductor. This means that this characteristic directly related to new semiconductor or redox couple materials.

The evaluation of photovoltaics devices is performed by comparing their characteristics under illumination conditions. For that purpose, standard measurements were developed in the past. Essentially, these specify that the temperature of the cell should be $25^{\circ} \mathrm{C}$ and an illumination intensity of 1 sun (power equal to $1000 \mathrm{~W} / \mathrm{m}^{2}$ ) with a power distribution defined as "Air Mass (AM) 1.5G". This distribution corresponds to the spectral power observed when the sun's radiation is coming from an angle of $48^{\circ}$ to zenith position.

\section{Interfacial electron transfer processes and kinetics}

In DSSC the $I^{-} / I_{3}^{-}$has become the standard redox couple used in electrolytes, although some others, such as cobalt mediators, have been proved to provide better 
performance in presence of carbon materials.[87-89] Its function is acting as holetransport medium. In this thesis only $I^{-} / I_{3}^{-}$redox couple has been tested and only this redox couple is summarised along this chapter.

The are other types for p-type semiconductors or more complex organic conductors, but this work focuses on the system previously described and used as standard for this technology.

At open circuit potential conditions $(O C P)$, when the device is illuminated, the net rate of electron injection into the mesoporous $\mathrm{TiO}_{2}$, must be balanced by i) the net rate of electron transfer to $I_{3}^{-}$ions in the electrolyte $\left(I_{3}^{-} \rightarrow I^{-}\right.$reduction) and ii) the regeneration of the oxidised dye. For a standard device, where the N719-dye is efficiently regenerated by the $I^{-} / I_{3}^{-}$redox couple, this factor can be neglected.

Therefore, the limiting process in this system is the electron transfer to $I_{3}^{-}$. For an appropriate electrolyte regeneration, the requirements in the $\mathrm{CE}$ are high catalytic activity or specific surface area, high conductivity and electrochemical stability. The system leads to energy generation when this electron transfer happens at the counter-electrode, but it can also occur (a) between injected charges into the $\mathrm{TiO}_{2}$ and excited dye molecules, (b) at the interface between the $\mathrm{TiO}_{2}$ surface and the electrolyte or (c) at the electrolyte exposed areas of the anode current-collector (normally FTO). These undesired mechanisms are called recombination processes and they are the main cause of current and efficiency losses in DSSC devices. A summary of the electron transfer and recombination processes is presented in the Figure 2.12.

The recombination of the electrolyte at the $\mathrm{TiO}_{2}$ surface is minimised by using additives and by ensuring extensive dye loading of the mesoporous oxide surface. Recombination at the anode current-collector is avoided by using a compact layer of $\mathrm{TiO}_{2}$ a few $n m$-thick, known as blocking layer.

The improvement of charge injection leads to higher $J_{s c}$ and avoiding recombination reactions between the photogenerated electrons and the oxidised redox mediator leads to better $V_{o c}$ and $J_{s c}$.

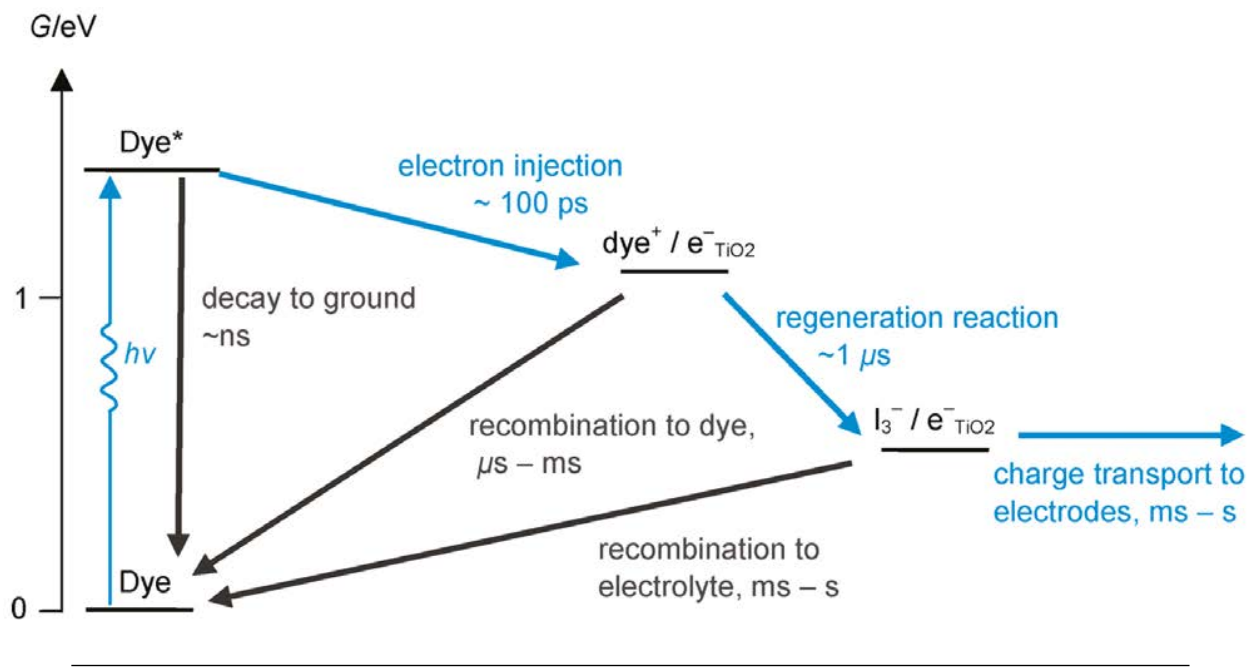

FIGURE 2.12: Electron transfer processes and kinetics in a DSSC. Blue path represents the desired processes, and black arrows the undesired recombination processes. Reproduced from Lisorti et al.[90] 
The application and analysis of the typical characterisation techniques, regarding the catalytic properties of materials and the photovoltaic performance, is described in detail in the work of Pazoki et al.[91] The accepted model and theory for the correct interpretation of this information was developed a decade ago in several works, Kern et al.,[92] Bisquert et al.,[93, 94] and Adachi et al.[95] This referenced theory and characterisation methods are the ones followed during this thesis and explained in the following sections and chapters.

By $C V$ and $L S V$ measurements the current response as function of the voltage applied is obtained. In contrast, EIS provides the response of an alternative current signal while varying the frequency. This techniques are typically applied to characterise DSSC devices or symmetric cells. A symmetric cell is fabricated using the same material as cathode and anode in a two-electrode electrochemical cell. Depending on the analysis applied, these techniques provide a wealth of information about the charge transfer, catalytic properties of CE materials, electrolyte diffusion characteristics and the performance of the final device.

From the $C V$ analysis the reaction potentials and currents are extracted. The redox peaks, corresponding to the reduction or oxidation reactions, show a faradaic current that is proportional to the rate of the reaction taking place at the surface of the electrode. The current itself is dependent on the rate at which material diffuse from the bulk of solution to the electrode, known as mass transport. The potential of the reaction is the driving force of the mass transport, and will shift to higher or lower potentials to compensate the mass transfer when it is disrupted (for instance when the diffusion in the electrolyte is slower).

The Tafel plot is the semi-logarithmic representation of an $L S V$ measurement. It is widely used tool for investigating the electrocatalytic activity of CE for DSSCs. The Tafel curve, can be divided in three zones: at low potential $(|U|<120 \mathrm{mV})$ is the polarisation zone. At middle potential (with a steep slope) is the Tafel zone. At higher potential (close to horizontal) is the diffusion zone. From the Tafel zone, the exchange current density $\left(J_{0}\right)$ is obtained, and the steeper the slope the higher the $J_{0}$. An increase in the $J_{0}$ leads to a higher catalytic activity towards the triiodide reduction. From the diffusion zone, the diffusion limiting current $\left(J_{\text {lim }}\right)$ is obtained. This current is limited from the ability of the ions to diffuse in the electrolyte. The higher the $J_{\text {lim }}$ the faster the diffusion of the redox couple in the electrolyte, which means improved catalytic activity of the CE. The relation with the diffusion coefficient is given by Equation 3.7,

$$
D_{\text {diff }}=\frac{\delta}{2 n\left[I_{3}^{-}\right] F} J_{l i m}
$$

where $J_{\text {lim }}$ is the polarisation diffusion-limited current, $\delta$ is the distance between electrodes of the symmetric cell, $n$ the number of transferred electrons in the $I_{3}^{-}+2 e^{-} \rightarrow 3 I^{-}$redox reaction, $\left[I_{3}^{-}\right]$is the equilibrium concentration of triiodide ions in the electrolyte and $F$ is the Faraday constant.

\subsection{Roles of nanocarbons and state-of-the-art of CNTs in DSSC}

New allotropes of the carbon, in form of different shape structures, have demonstrated to be excellent candidates for replacing standard materials in many different 
applications for energy conversion and storage. Among different nanocarbon, the attention is focused in the carbon nanotubes (CNTs). Besides the exceptional electrical conductivity, mechanical properties, high surface area, high chemical and thermal stability previously described, they are catalytic active towards different redox mediators typically used in DSSC schemes.

Nanocarbons have been incorporated in all its different forms to the DSSC. There are recent general reviews $[96,97]$ and more focused on specific nanocarbons, e.g. carbon nanotubes,[98] graphene materials[99] or carbon nanohorns.[100]

Similar to other nanocarbons, CNTs have been used in DSSC following three main strategies: i) as replacement of the $\mathrm{Pt}$ as catalytic active material towards the redox mediator (counter-electrodes), ii) as a conductive filler in the electrolyte, and iii) as conductive filler in the semiconductor $\left(\mathrm{TiO}_{2}\right)$ (photoanode or working electrode).

Note that, although in this thesis the CNTs are not specifically used as electrolyte dopant, some important aspects of this system are relevant for CE and photoanodes applications.

In view of these multiple roles and the complex charge dynamics operating in a full cell, it is of interest to produce not only highly efficient devices, but also welldefined cells with controlled properties and material's chemistry in order to clarify the effect of nanocarbons.

\subsubsection{CNT counter-electrode for DSSC}

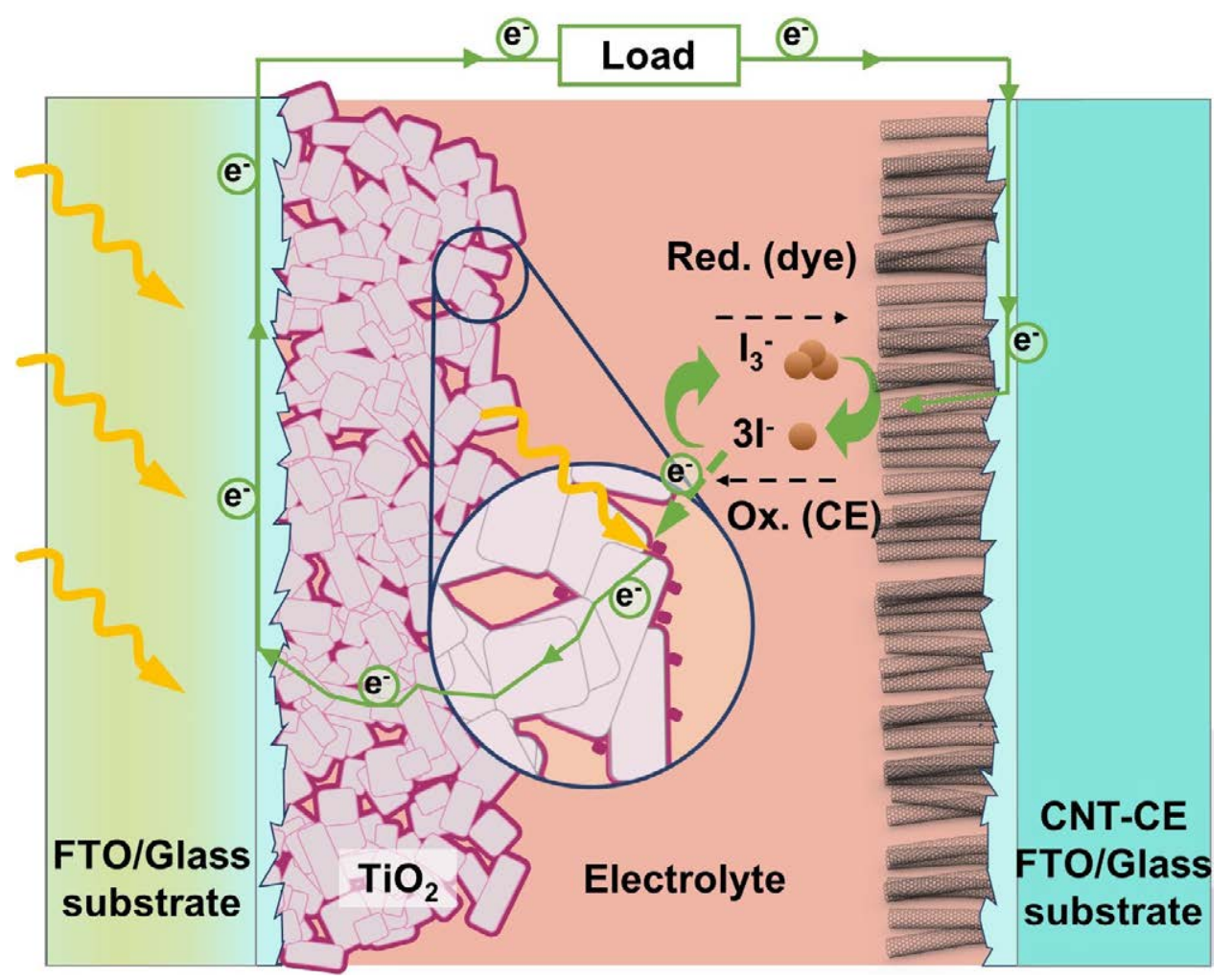

FIGURE 2.13: Schematic of a DSSC incorporating CNTs as CE. 
$\mathrm{Pt}$ is the most widely used material for the reduction of the triiodide molecule but, its degradation and elevated price make it a non-suitable material for large scale or industrial applications. In that sense, nanocarbons, being produced from one of the most earth abundant elements, are suitable materials for replacing the Pt.

As has been mentioned before, good CE materials requires high catalytic activity towards DSSC redox mediators, high specific surface area, electrical conductivity and chemical stability but, there are other desired features like cost or sustainability that will facilitate its industrial implementation. All these properties are present in CNTs.

The representation of the CNTs integrated as CE in the DSSC is presented in the Figure 2.13. The iodide/triiodide regeneration reaction at the CNTs surface is depicted, as well as the photocarrier injection from the dye into the mesoporous $\mathrm{TiO}_{2}$ layer.

\section{Catalytic activity of CNT as CEs}

Demonstrating the catalytic properties of CNTs and its high performance for DSSC counter-electrodes have been the subject of many of scientific works during the last decade.[96, 98] In-depth studies of the catalytic properties of CNTs have settle the basis towards the interpretation of CNTs effects in DSSC by electrochemical impedance spectroscopy $(E I S)$, cyclic voltammetry $(C V)$ and linear sweep voltammetry $(L S V) \cdot[101]$

In this section, example of the previous characterisation techniques and analysis methods to evaluate the contributions of nanocarbons to DSSC performance are discussed.

Yue et al.[102] performed CV for Pt and different concentrations of CNTs as $\mathrm{CE}$ in a standard symmetric device (Figure 2.14a). From these curves the peak-peak potentials separations are obtained, resulting in $E_{p-p}=0.25 \mathrm{~V}$ and $0.35 \mathrm{~V}$ for the best performed CNT- and Pt-CE, respectively. Also, the maximums for the peaks of the reduction potential of $I^{-} \rightarrow I_{3}^{-}$are located at $c a$. $0.2 \mathrm{~V}$ for the CNT- and Pt$\mathrm{CE}$ - iodide concentrations below $0.1 \mathrm{mM}$. For the oxidation potentials, 0.45 and $0.55 \mathrm{~V}$ are reported for the CNT- and Pt-CE respectively. The lower peak separation and higher peak currents indicates the better mass transfer and therefore, higher catalytic activity.
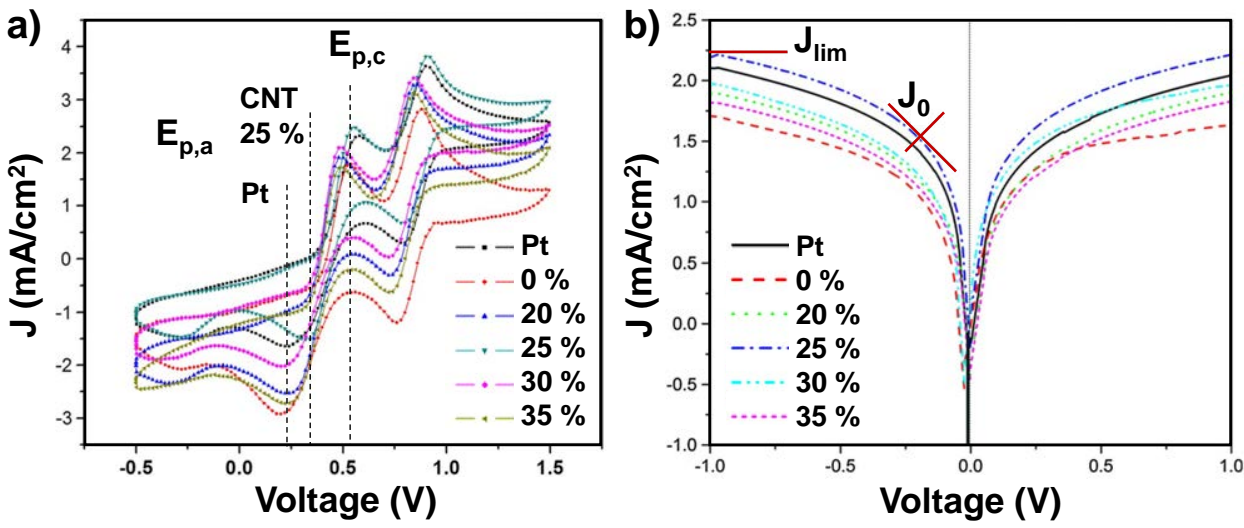

FIGURE 2.14: a) $C V$ and b) LSV plots for symmetric cells with different CNTs contents at the CE. Reproduced from Yue et al.[102] 
The Tafel plot, presented in Figure 2.14b, shows the improved catalytic properties reporting 33.8 and $27.7 \mathrm{~mA} \mathrm{~cm}^{-2}$ exchange current density $\left(J_{0}\right)$ for CNT- and Pt-CE respectively, as well as, better diffusion limiting current $\left(J_{\text {lim }}\right)$.

Generally, lower $E_{p p}$ and higher $J_{0}$ indicate a better catalytic performance of the $\mathrm{CE}$ material and electron transfer at the CE/electrolyte interface. Also, Jlim indicates the improved diffusion velocities of ions in the electrolyte in the presence of carbon material in an optimised configuration.

It is expected that nanocarbons have a high catalytic activity on account of their high SSA, $[103,104]$ in contrast with a typical Pt-CE, in which the electrode is decorated with isolated nanoparticles of $\mathrm{Pt}$ and much less accessible area for the electrolyte to interact with. This, however implies that nanocarbons $\mathrm{CE}$ are porous, which can reduce the diffusivity $\left(D_{\text {diff }}\right)$ of the electrolyte and thus efficiency.

CNT high frequency charge transfer: EIS analysis
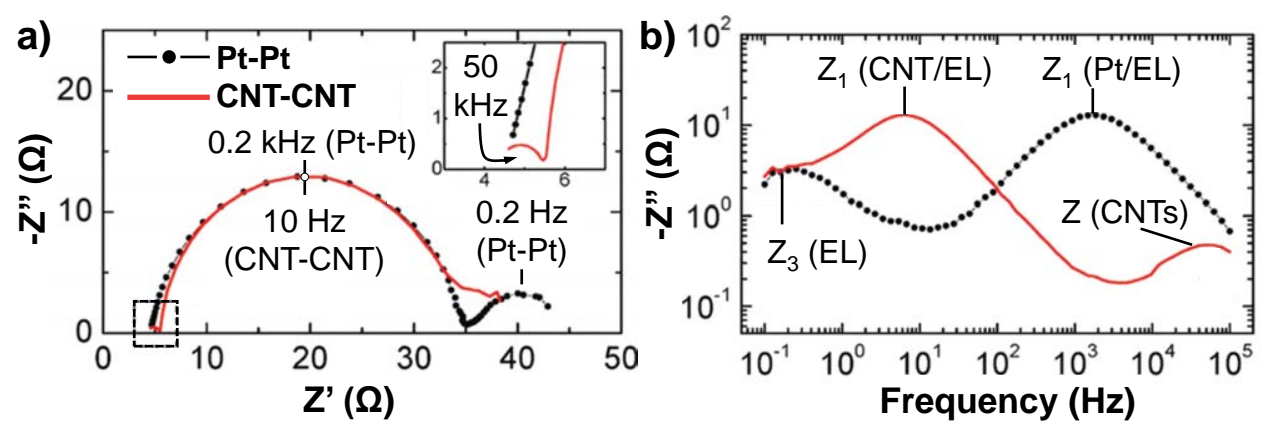

FIGURE 2.15: Nyquist plot a) and Bode phase plot b) of CNT/CNT symmetric cells. Images reproduced from Seo et al.[101]

The catalytic behaviour of nanocarbon-CE is typically further studied by EIS. The maximum frequency of the process as well as the charge transfer resistance are dependent on geometrical parameters of the system, i.e. separation between electrodes or effective area, and also on physical properties of the electrolyte, as for instance, viscosity. However, it is possible to discriminate different processes occurring in the cell.

Figure 2.15a shows the Nyquist plot for a $\mathrm{Pt} / \mathrm{Pt}$ and CNT/CNT symmetric cell. Two clear semicircles appear, one at low frequencies centred at $0.2 \mathrm{~Hz}$ corresponding to the bulk diffusion of the electrolyte and other at high frequency, $2 \mathrm{kHz}$, related to the $\mathrm{Pt} /$ electrolyte charge transfer.[101] On the contrary, for the CNT/CNT symmetric cell, different behaviour is usually reported. The Nerstian diffusion, i.e. bulk diffusion, is convoluted with a predominant mid-frequency peak centred at $10 \mathrm{~Hz}$ (see red line in Figure 2.15a). This is attributed to charge transfer process between CNTs and electrolyte. At the same time, a very fast process appears at $50 \mathrm{kHz}$ (small semicircle in the inset). This characteristic peak of the CNT material has been attributed to different phenomena, e.g. charge transfer in solid state CNTs, catalytic reaction with the redox mediator, and more recently, to a trapping-detrapping process at the mesoporosity of the carbon nanotubes.[105-109] Thus, it is clear that the assignation of this peak remains unclear. 

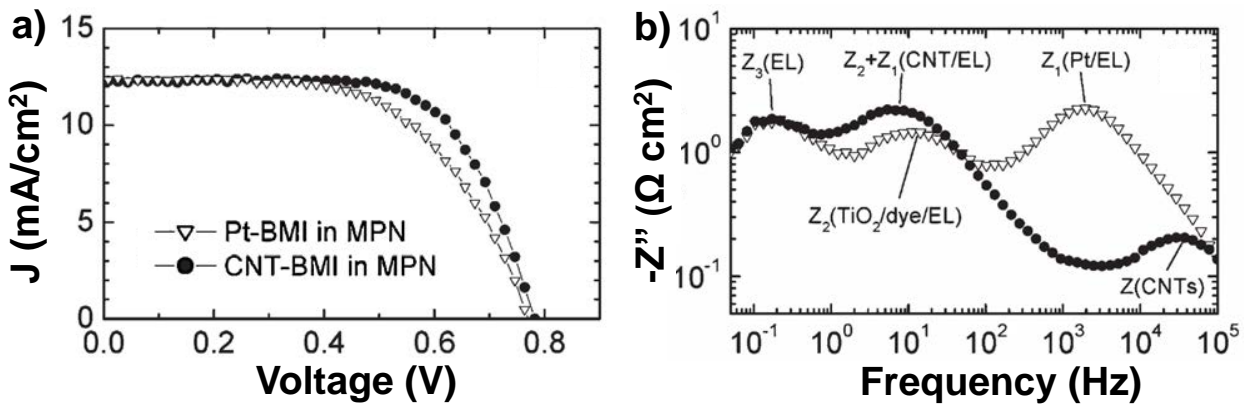

FIGURE 2.16: a) $J-V$ curves and b) Bode plots comparing Pt and CNT catalyst for DSSCs. Images reproduced from Seo et al.[101]

The impedance spectra of DSSC is shown in the Figure 2.15b. The Bode plot is very useful to understand the charge transfer processes that are present in the system. Under one full sun illumination (AM 1.5G), the standard device with $\mathrm{Pt}$ catalyst and $I_{3}^{-} \rightarrow I^{-}$redox couple shows three peaks. The peak at low frequency $\left(2 \times 10^{-2} \mathrm{~Hz}\right)$ the peak corresponds to Nernstian (i.e. bulk) diffusion whitin the electrolyte $\left(Z_{3}\right)$. The mid-frequency peak, centred at $10 \mathrm{~Hz}$, is related to the charge transfer at the $\mathrm{TiO}_{2} /$ dye/electrolyte interface $\left(Z_{2}\right)$. Finally, the peak at higher frequency, typically at $10^{3}-10^{4} \mathrm{~Hz}$ corresponds to the charge transfer at the catalyst/electrolyte interface $\left(Z_{1}\right)$. When CNTs are used as catalyst, although the $J-V$ curves are quite similar as shown in Figure 2.16a, the impedance behaviour is totally different. For CNT-CE the convolution between the high-frequency and mid-frequency domain is frequently observed $\left(Z_{1}+Z_{2}\right)$. Similarly to the previous CNT symmetric cell, a very fast process is observed at $50 \mathrm{kHz}(\mathrm{Z}(\mathrm{CNTs}))$, confirming that it is not a specific phenomena of the symmetric cells, and with no clear explanation of the nature of this interface.

Therefore, the interaction of high SSA electrodes with the electrolyte is unclear. Unresolved interfaces are still under debate for nanocarbon $\mathrm{CE}$ ( $50 \mathrm{kHz} \mathrm{Z}(\mathrm{CNTs})$ ). Moreover, for CNT materials is difficult to analyse EIS results due to the convoluted contributions of the CNT/electrolyte interface and the $\mathrm{TiO}_{2}$ /dye/electrolyte (CNT/EL and $\mathrm{TiO}_{2} /$ dye/EL).

\section{Possible nature of nanocarbon's catalytic activity:}

Although the catalytic activity general of carbon materials has been widely studied in the past understanding the nature of the active sitesin different nanocarbons remains an active topic of research.[110,111] Overall, the view is that the charge localisation surrounding defects induces the charge transfer required by the catalytic reactions.[112] This is suggested by observation that the edge plane of highly oriented pyrolytic graphite has an electrochemical reactivity several orders of magnitude higher than its basal plane, attributed to a large amount of defects at the edge that produce a high density of defect states near the Fermi level.[113-115]

Several types of carbon defects can promote catalytic active sites are summarised in the Figure 2.17a, e.g. carbon vacancies in graphitic surface, doping by either substitutional or superficial interaction, metal molecules or clusters decorating the surface, functional groups or the exposed carbon edges (zigzag or armchair). 
In contrast to the traditional sp3 carbons in which only convalent bonds or weak Van der Waals forces can be established with substrates or reagents, CNTs or graphene are suitable to adsorb and interact with molecules by relatively strong $\pi$ interaction.[116] This strong interaction can promote different catalytic reactions.[117, 118]
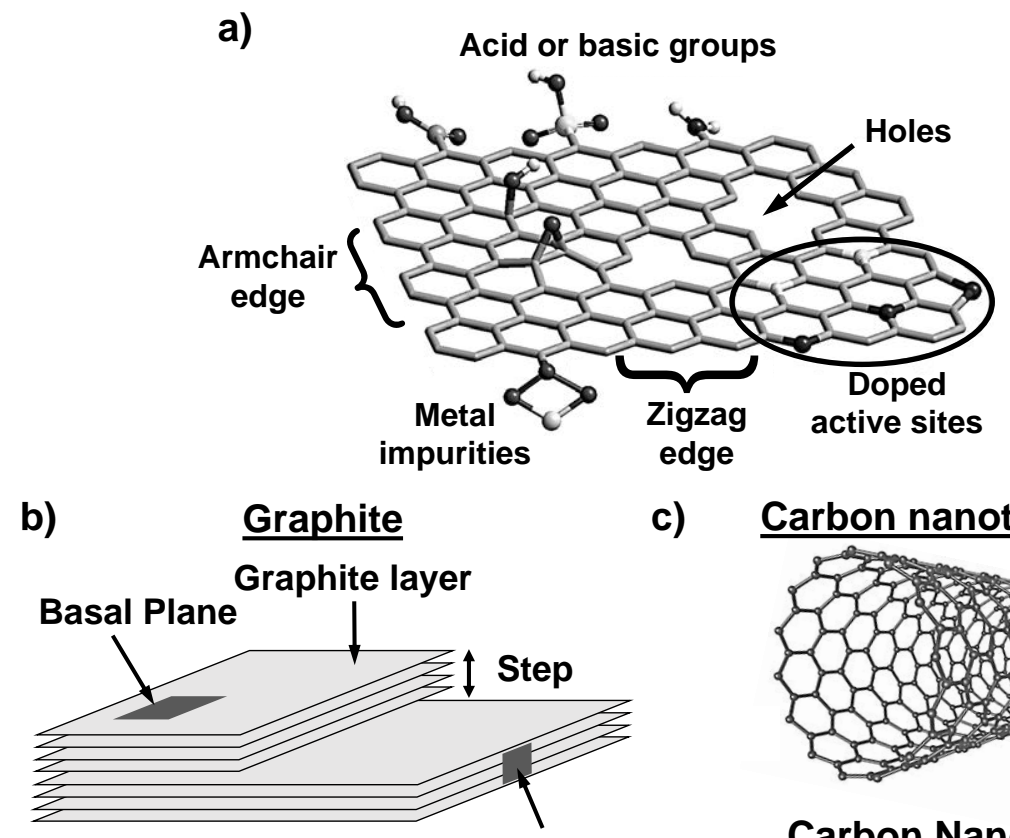

c) Carbon nanotubes
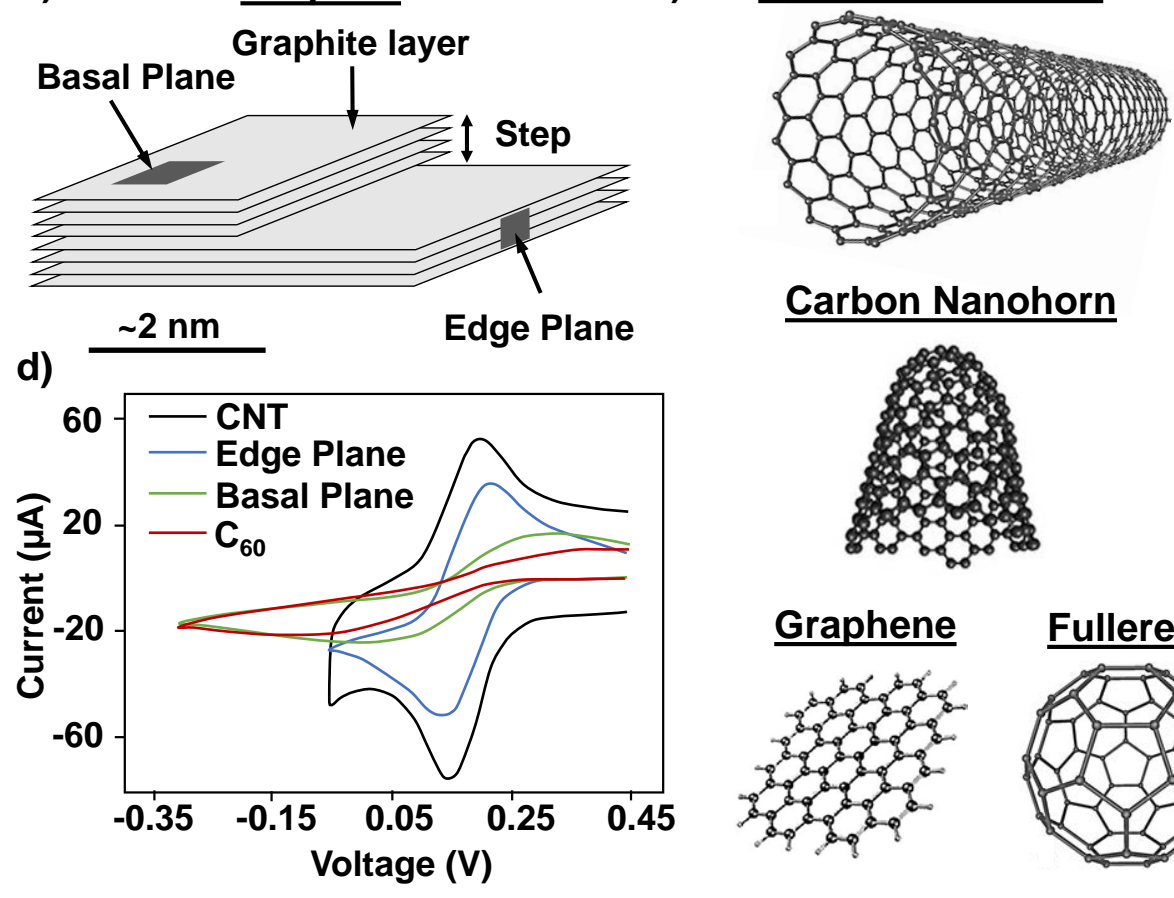

\section{Carbon Nanohorn}
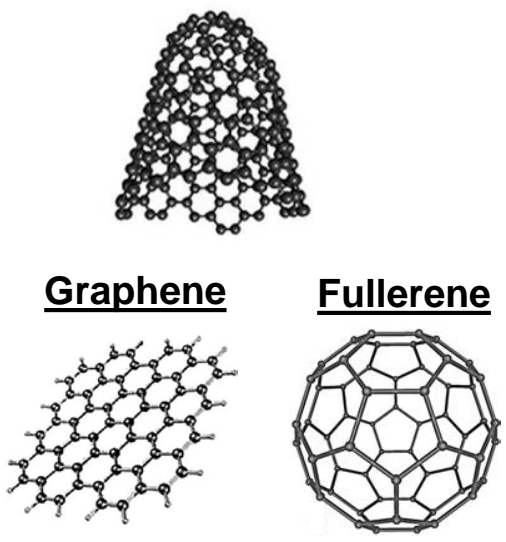

FIGURE 2.17: Possible origins of nanocarbon's catalytic activity: a) Illustration of the types of defects of the ideal graphene structure that can become active sites in catalysis. Reproduced from Primo et al.[112] b) Schematic of pyrolytic graphite electrodes exposing edge or basal planes. Adapted from Banks et al.[115] c) Schematics of different types of nanocarbons exposing different amount of carbon edges. d) $C V$ curves of carbon materials showing different catalytic performance. Adapted from Banks et al.[113, 114]

In addition to $\pi$ interactions, nanocarbon present other features that make their catalytic activity dependent on other factors besides defect density. In fact, nanocarbons can often show higher catalytic activity than more defective materials. 
In the graph Figure $2.17 \mathrm{~d}$ the catalytic activity towards ferricyanide (iron compound) is compared for different materials. The higher anodic diffusive currents are obtained for CNT materials, and decreases reducing the number of edge sites present on the material. CNTs presents a large proportion of graphite basal plane, and apart from surface defects, every CNT shows exposed carbon edge at least in one of its ends. Notice the low reactivity of pyrolitic carbon basal planes as well as the pristine fullerene molecules. Although there are material with higher density of carbon edge per carbon molecule, e.g. carbon nanohorns (Figure 2.17c), the conductivity of the material also plays a key role. This is because the charges has to reach and travel through the catalyst particles.

Although it is clear the catalytic nature of carbon defects and exposed edges, there are also other contributions to take into account. For instance, amorphous carbon produced as subproduct during the synthesis of the nanocarbon materials, or remaining catalyst particles of the synthesis of nanocarbons.

An often overlooked factor is the presence of these metal particles, which could be responsible of catalytic reactions, for instance hydrogenation of carboncarbon bonds by graphene material,[119] and even the reduction iodide/triiodide redox couple.[120] Note that in many cases metallic particles are added to CNT to increase their catalytic activity.[121, 122]

Finally, the interaction between graphene with metallic underlaying substrates has been suggested to drastically improved the catalytic properties. This other mechanism has to be considered as contribution mechanism to the origin of catalytic activity in some cases, for instance, the interaction between CNT walls and underlaying catalytic particles.[123]

Although there are some clear indications of carbon material catalysing many different reactions, also the metal or metal oxide particles present in the material must be considered in every case as origin of the catalytic effect, more so since the CNTs usually present a large proportion of metal particles incorporated inside the tubes that is difficult to remove.

\section{Different implementation routes of CNTs as DSSC-CE}

The main implementation routes in nanocarbons are depicted in this subsection. From the wide variety of combinations of materials that can be used as counterelectrode, the ones that strictly use CNTs as catalytic material are reviewed here. A complete view of the alternative CE-materials, i.e. metals, metal oxides, polymers, and other nanocarbons, has been recently reviewed by Yun et al.[97]

Several implementation methods has been reported in the past. The most used are: i) direct in-situ growth, ii) dispersion and spray deposition, iii) dispersion and doctor blading, iv) macroscopic assemblies (buckypapers and CNT fibres).

These typical processing methods and relevant state-of-the-art results are presented in the Figure 2.18. Besides, the record reported efficiency for every processing technique is presented in the Table 2.3.

Nam et al.[124] synthesised vertically-aligned CNT forest by CVD using directly FTO conductive substrates. The CNTs act as CE material, and with this configuration they reported the record efficiency obtained up to the day, PCE $=10 \%$, presented in the Figure 2.18a,b. Alternatively, Ramasamy et al.[125] applied an alcoholbased solution of MWCNT by spray coating directly over the FTO (see Figure 2.18c) 
and an efficiency of $P C E=7.6 \%$ was obtained. The advantage of these technique is that produces fast, reproducible and scalable layers. Another method proposed by Hashmi et al.[126] is the deposition by doctor blading CNT-based pastes, presented in Figure 2.18e,f. This method leads to an efficiency of $6 \%$ but with some interesting applications for flexible substrates.
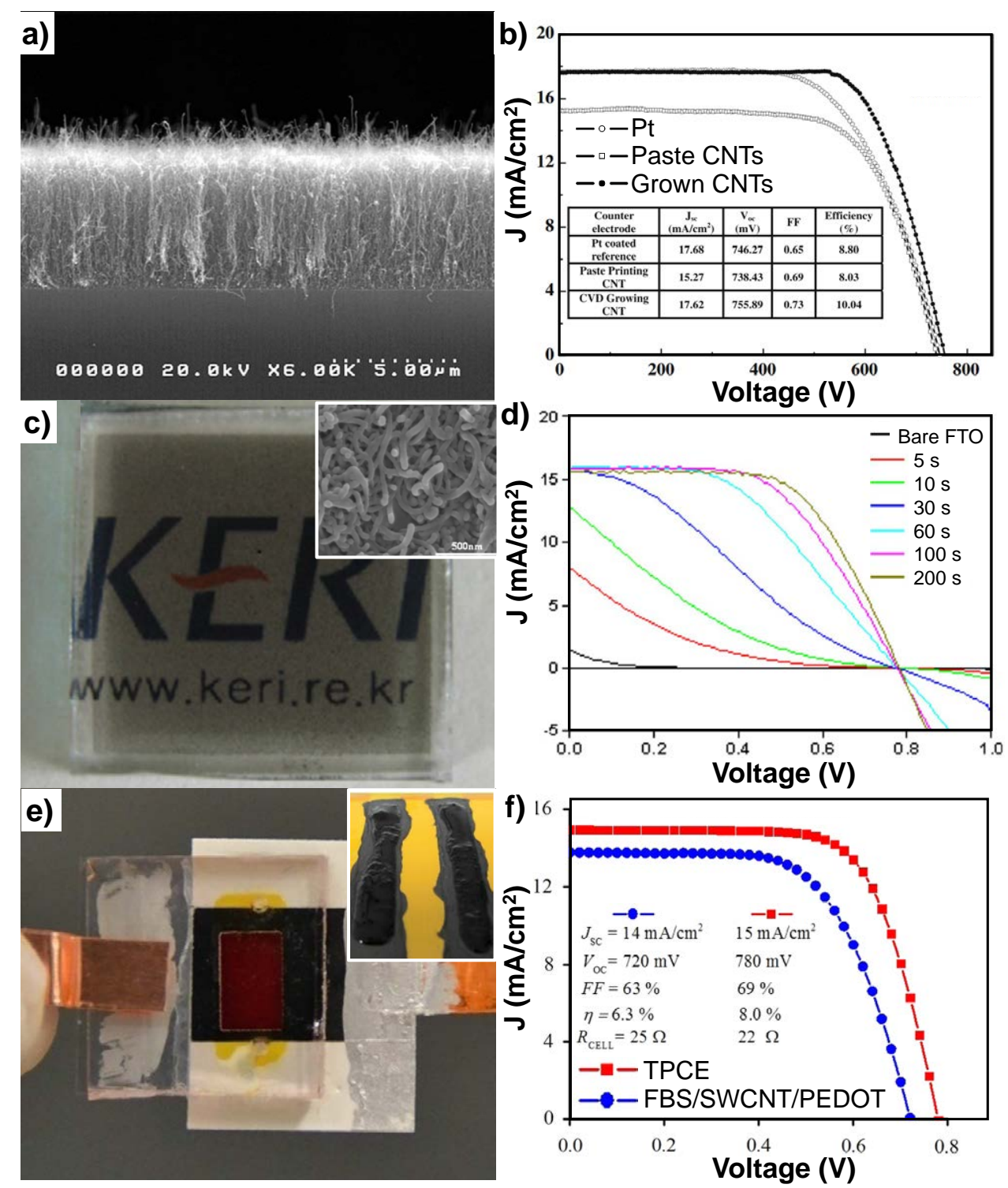

FIGURE 2.18: a) Cross-sectional SEM image of CNT forest used as CE directly grown by CVD on FTO current-collector and $b$ ) figures of merit of the CNT-DSSC and Pt-CE reference. Reproduced from Nam et al.[124] c) Spray coated CNT-CE with SEM image of the CNTs (inset) and d) $J-V$ curves function of the deposition time. Reproduced from Ramasamy et al.[125] e) CNT-CE DSSC produced by doctor blading a mixture of CNT and polymeric binders. Doctor blade CE presented as inset of the same image. f) Comparison of the CNT and Pt DSSC J- $V$ curves. Reproduced from Hashmi et al.[126] 
TABLE 2.3: Efficiency (PCE) records of CNT-CE for different processing routes.

\begin{tabular}{llll}
\hline Processing Route & CNT Type & PCE (\%) & Ref. \& Year \\
\hline Self-assembled CNT fibre (CVD) & MWCNT & 9.5 & This work, 2018 \\
Directly grown CNTs (CVD) & MWCNT & 10.0 & Nam et al. [124] 2010 \\
Spray deposition & MWCNT & 7.6 & Ramasamy et al.[125] 2008 \\
Doctor blading CNT-paste & SWCNT & 6.0 & Hashmi et al.[126] 2014 \\
Buckypapers (CVD+filtering) & SWCNT & 4.0 & Roy et al.[127] 2012 \\
\hline
\end{tabular}

a)
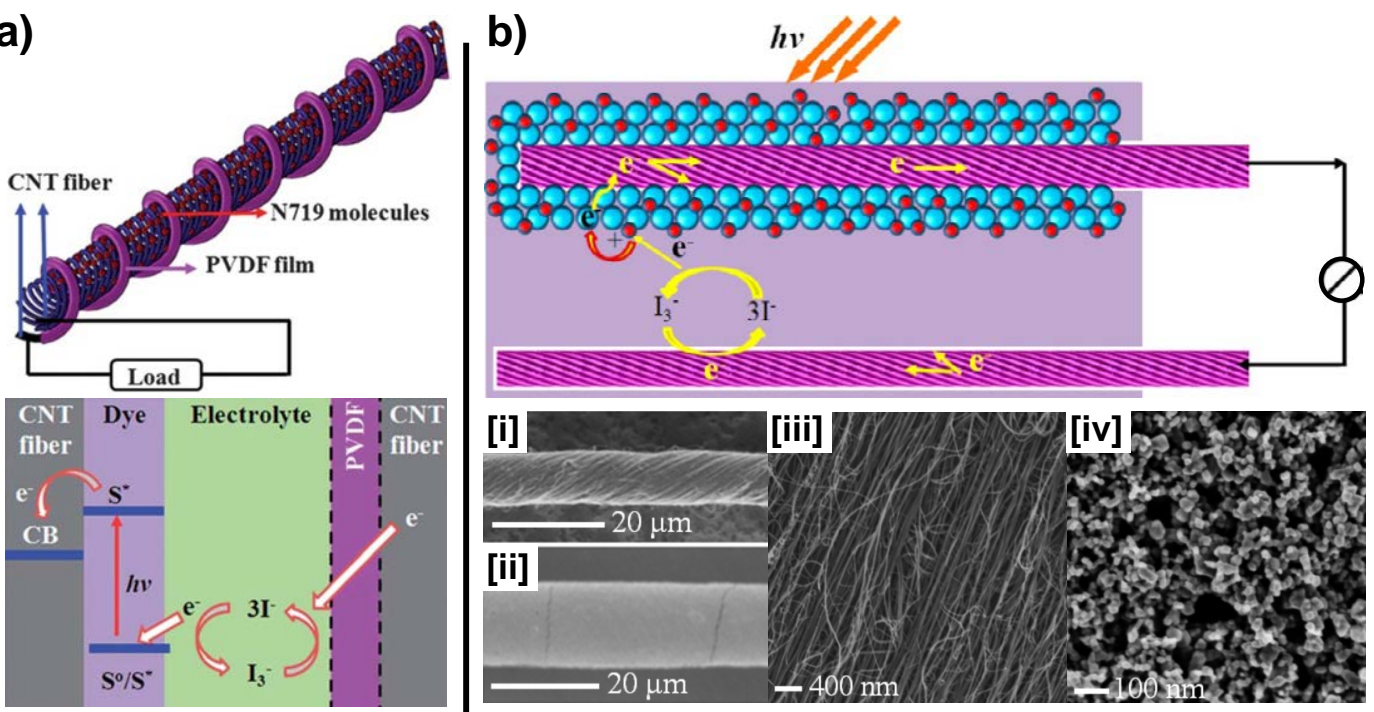

c)
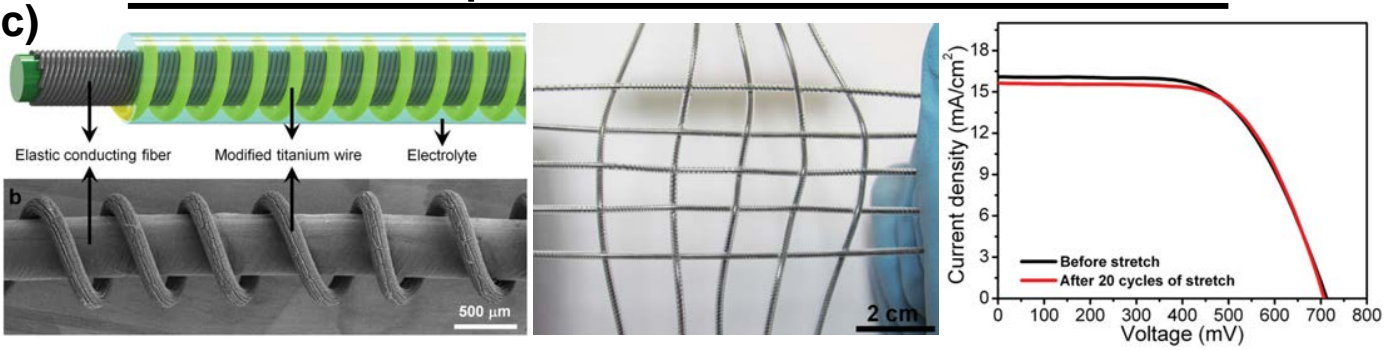

FIGURE 2.19: a) Full CNT-fibre fibre-like DSSC: device schematic (up) and electronic band structure (down). Adapted from Cai et al.[128] b) $\mathrm{CNT}$ fibre- $\mathrm{TiO}_{2} / \mathrm{CNT}$ fibre-CE fibre-like DSSC: device schematic (up) and SEM images (down) of a pristine CNT fibre and a $\mathrm{CNT} / \mathrm{TiO}_{2}$ composite fibre ([i] pristine $\mathrm{CNT}$ fibre, [ii] $\mathrm{CNTf} / \mathrm{TiO}_{2}$ composite fibre and [iii], [iv] high-resolution images of [i] and [ii] respectively). Adapted from Chen et al.[76] c) Flexible CNTf-based DSSC for wearable applications. Left: schematic and SEM image of an individual fibre-like DSSC; centre: proof-of-concept device of a flexible and stretchable array of fibre-like devices; right: $J-V$ curves before and after 20 cycles of stretch. Adapted from Yang et al.[129]

Other options for planar CNT-CE are based on macroscopic assemblies of CNTs. There are different methods of assembly CNTs into macroscopic materials, 
for instance, buckypapers or assembling them into fibres. Using buckypapers obtained by filtering of CNT dispersions, produces materials with a high degree of interconnection and surface area, obtaining good catalytic properties of entirely made CNT-CE. By this method, the best efficiency reported by Roy et al.[127] is $4 \%$. When the nanotubes are directly self-assembled from a bottom-up technique, as it is the directly spinning from the CVD, the degree of interconnection and alignment between themselves is higher than a buckypaper. This is directly reflected by its superior electrical, thermal and mechanical properties.[58] CNTf sheets has been used as planar $\mathrm{CE}$ reaching efficiencies from 4 to $6.6 \%$.[130] A record efficiency of $9.5 \%$ is presented in this thesis using CNTf as planar CE.

This CNTf has been widely exploited as current-collector, active material and counter electrode for fibre-like devices. Peng's group has been pioneer in the implementation of this material in this application; a summary of the evolution is presented in the Figure 2.19. Firstly, they reported the use of the CNT fibre in an allcarbon DSSC, presented in the Figure 2.19a. The absence of semiconductor material leads to very low $P C E$, but still good progress towards the wearable, flexible device were done in this work.[128] Introducing the standard mesoporous $\mathrm{TiO}_{2}$ micro-layer as a coating of the fibre (see Figure 2.19b), resulted in a performance of 2.6\%.[76] Finally, using a Ti-wire (anodised for obtaining a $\mathrm{TiO}_{2}$ nanotube coating as working electrode) a flexible and stretchable fibre-like DSSC was obtained, reporting a performance of $7 \%$ [129] (see Figure 2.19).

\section{CNT-CE thikness-efficiency dependence: S-shaped I-V curves}

One common factor for different nanocarbons, including CNTs, is their carbon thickness-efficiency dependence.[125, 131] The thickness of the carbon CE is directly proportional to the number of catalytic active sites and series resistance of the electrode and it is directly related to the so called S-shaped I-V curves.

An example of this S-shape is presented in the Figure 2.18d for low CE deposition times. This shape reduces the fill factor of the device and therefore the efficiency of the cell. The low thickness of the CE material produces two additional effects. Firstly, the increase in series resistance of the $J-V$ curve (from the inverse slope of the curve close to the $V_{o c}$ ) that limits the photocurrent at $I_{s c}$ conditions. And secondly, the lack of regeneration of the electrolyte limiting the regeneration of the dye molecules, thus the charge injection and the performance of the cell. The increase in the CNTs deposition time, i.e. CE material thickness, leads to an improved regeneration and an enhancement in the fill factor (from 0.1 to 0.6 ).

Other possible mechanisms, like imbalanced carrier mobilities or injection/ extraction barriers also explain the $S$-shaped $I-V$ curves, $[132,133]$ but these effects are more typical for organic photovoltaic devices $(O P V)$ rather than DSSCs.

\subsubsection{CNT-doped electrolytes}

The electrolyte plays a fundamental role in the operating of the DSSC devices. The incorporation methods of the CNTs to the DSSC electrolyte and its consequences will be described in this section. A schematic of the inplementation and the interaction between CNTs and electrolyte is presented in the Figure 2.20.

The solar cell efficiency crucially depends on the diffusivity of iodide species (iodide/triiodide molecules) in the electrolyte. The effective diffusion coefficient, 
attributed to $I_{3}^{-}$, is deduced from measurements of the limiting current in an electrochemical cell.[134, 135] In addition, the saturation current increases with increasing the weight-in fraction of $I_{2}$ and the concentration of iodide by incrementing the ionic conductivity.[134, 135] Both phenomena are typically interpreted in terms of a Grotthuss-type mechanism.[134, 135]

Grotthuss mechanism, proposed more than two centuries ago, explains the hydrogen ions diffusion inside water. It is the usual mechanism given for facilitated proton mobility. Briefly, the process is similar to an auto-dissociation of the water molecule into $\mathrm{H}^{+}$and $\mathrm{OH}^{-}$and hopping of the proton from one molecule to the next neighbouring molecule.

Although cation-anion pair formation has been an alternative proposed explanation,[136] Grotthuss-like mechanism is accepted for the diffusion of $I^{-}$in liquid iodine solution following the reaction sequence:

$$
I_{3}^{-}+I^{-} \rightarrow I^{-}-I_{2} \ldots I^{-} \rightarrow I^{-} \ldots I_{2}-I^{-} \rightarrow I^{-}+I_{3}^{-}
$$

This allows the electrical conduction like a relay mechanism, with net transport of charge without any net transport of mass.

In this context, recent studies have shown the impact and importance of the presence of longer iodide chains for the diffusion of iodide/triiodide species during the regeneration process of the electrolyte, as well as the importance of the presence of neutral $I_{2}$.[137] The charge transfer in the polyiodide system depends ultimately on the concentration of iodine and the distance between donors $\left(I_{2 n+1}^{-}\right.$, i.e. $\left.I_{3}^{-}, I_{5}^{-}, I_{7}^{-}\right)$ and acceptor $\left(I_{2}\right)$.[138] This will be of critical importance since it is widely reported the favourable polyiodides formation in presence of nanocarbon electrolytes.[139141]

The standard liquid electrolyte for DSSC is composed of $I_{2}$ dissolved in butylmethylimidazolium iodide (BMII) and volatile solvents (acetonitrile and valeronitrile). It also includes some additives, such as, guanidinium thiocyanate, 4-tertbutylpyridine, lithium iodide, which lower charge recombination. These chemical compounds are anchored to the $\mathrm{TiO}_{2}$ surface that is uncovered by dye molecules, to prevent the electrolyte $/ \mathrm{TiO}_{2}$ recombination, increasing the charge collection $\left(J_{s c}\right)$ and the $V_{o c}$ of the device.

The long-term stability of the DSSCs is one of the main bottlenecks of this technology. Enhancing the stability of these devices pass through eliminating the solvent from the list of components of the electrolyte. It is the main responsible of lowering the durability and stability of the device by electrolyte leakage and dye desorption processes among others. Some alternatives are proposed recently, for instance, replacing the solvent based ionic liquids with ionic liquids with zero volatility,[142] or by using solid hole-transport materials,[142] polymer electrolytes,[143] water-based electrolytes[144, 145] or solid-state electrolytes.[142]

Among the possible modifications, using nanocarbons as additive or filler in electrolytes have been proved as an effective method of enhancing the characteristics of DSSCs.[146] The list of carbonaceous materials used for this application and the highest efficiency reported can be summarised in carbon black[147-149] achieving $P C E=8.4 \%$,[150] carbon nanotubes[141, 151-153] achieving PCE $=8.2 \%$, carbon nanohorns[154] achieving $P C E=7.8 \%$, and graphene-based material[87, 155-159] 
achieving $P C E=9.5 \%$.[160] The efficiencies are not directly comparable because these materials have been implemented into liquid, solid, quasi-solid electrolytes, but they represent the potential of the nanocarbons for this application. They act as a multipurpose component and each of them present some advantages and problems.

The proposed advantages of using nanocarbon as dopants for DSSC that leads to an improved device performance can be summarised as:

- Increase in the ionic diffusivity of the species in the electrolyte.

- Enhanced catalytic activity towards $I^{-} / I_{3}^{-}$redox couple.

- Improved dye regeneration (reduced diffusion path of the redox mediator).

- Reduction of the internal device resistance.

Therefore the CNTs dispersed in electrolyte play a dual-role. Firstly, as an electrical dopant of the conductive properties of the electrolyte, and secondly, often overlooked, as a catalytic material acting as an extension of the CE material. Both mechanisms are depicted in the schematic of the Figure 2.20.

Probably, the main disadvantage of using nanocarbons as fillers in DSSC electrolytes lays in obtaining reproducible and stable dispersions of nanocarbons. In addition, dispersion is often enabled by acid functionalisation, $[43,161]$ which is obtained by oxidising the sidewall of the CNTs with nitric acid and or sulfuric acid to increase their dispersion in polar solvents and polymer matrices. However, surface oxidation of MWCNT is often detrimental to their superior electrical and thermal properties.[62]

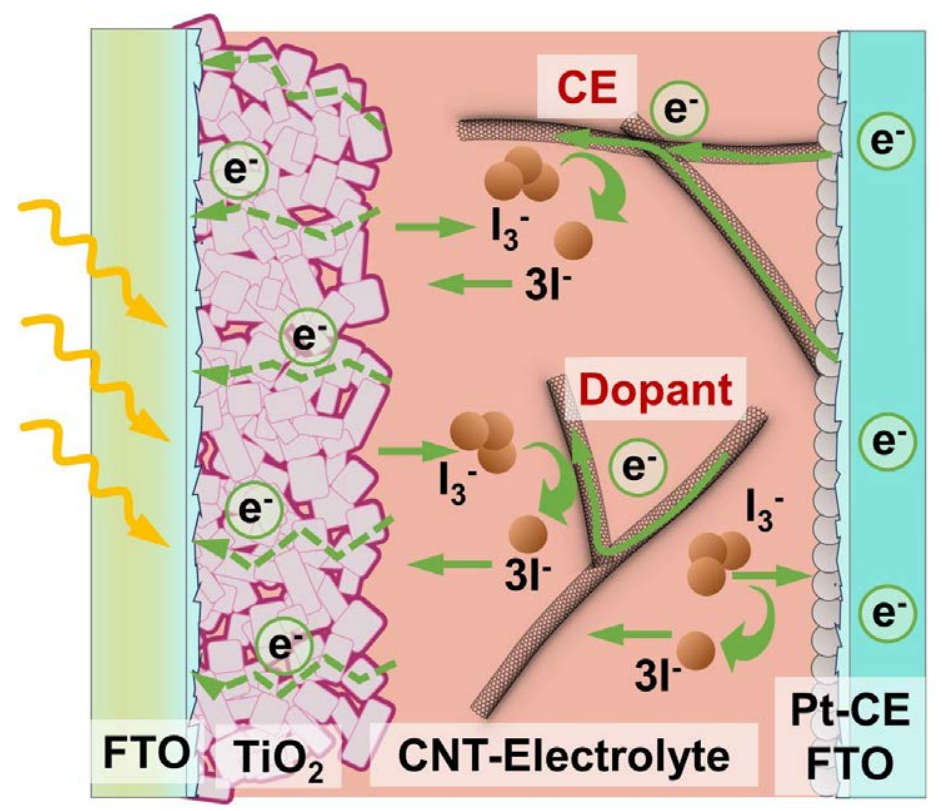

FIGURE 2.20: Schematic of the dual-role of dispersed CNTs in DSSC electrolytes.

Another difficulty for the practical implementation of carbon-doped electrolyte refers to their viscosity. Typically, the two electrodes that forms a DSSC are separated by a tens-of-microns thick spacer. The resulting space between electrodes is 
later filled with the electrolyte. The viscosity of the electrolyte plays an important role in the filling process and in the wettability of the electrodes. When the electrolytes are loaded with fillers, they tend to become more viscus, hampering this fundamental step during the fabrication of the device. This problem can be solved for solid electrolytes in some cases by high temperature injection, or more recently, by screen printing deposition technique. On the other hand, the increased viscosity of electrolytes will prevent the electrolyte leakage, facilitating the fabrication process, performance and long-term stability of the final device.

TABLE 2.4: Efficiency (PCE) obtained after integration of CNTs in different electrolytes for DSSC. Efficiencies have been rounded to the first decimal place.

\begin{tabular}{|c|c|c|c|c|}
\hline Type of electrolyte & CNT Type & $\begin{array}{l}\text { CNT } \\
(\% w t)\end{array}$ & $\begin{array}{l}\text { PCE } \\
(\%)\end{array}$ & Ref. \& Year \\
\hline $\begin{array}{l}\text { CNTs dispersed in standard } \\
\text { electrolyte (liquid solvent-based) }\end{array}$ & MWCNT & 0.2 & 4.2 & $\begin{array}{l}\text { Lee et al. } \\
\text { [153] } 2009\end{array}$ \\
\hline- & SWCNT & 0.2 & 3.6 & \\
\hline- & ref & - & 3.2 & \\
\hline $\begin{array}{l}\text { CNTs-PEO composite elec- } \\
\text { trolyte (quasi-solid electrolyte) }\end{array}$ & MWCNT & 1 & 3.5 & $\begin{array}{l}\text { Akhtar et al. } \\
\text { [141] } 2010\end{array}$ \\
\hline- & ref & - & 1.9 & \\
\hline $\begin{array}{l}\text { CNT-P(EO/EM/AGE) Gel elec- } \\
\text { trolyte (quasi-solid electrolyte) }\end{array}$ & MWCNT & 1 & 3.4 & $\begin{array}{l}\text { Benedetti et } \\
\text { al. [140] } 2012\end{array}$ \\
\hline- & ref & - & 2.4 & \\
\hline $\begin{array}{l}\text { GO/CNT-MPN Gel electrolyte } \\
\text { (quasi-solid electrolyte) }\end{array}$ & MWCNT & 0.25 & 7.1 & $\begin{array}{l}\text { Neo et al. } \\
\text { [139] } 2014\end{array}$ \\
\hline- & ref & - & 6.5 & \\
\hline $\begin{array}{l}\text { CNT/PANi-PAA/PEG Gel elec- } \\
\text { trolyte (quasi-solid electrolyte) }\end{array}$ & CNT & 0.4 & 8.2 & $\begin{array}{l}\text { Jin et al. } \\
\text { [162] } 2018\end{array}$ \\
\hline- & ref & - & 5.7 & \\
\hline $\begin{array}{l}\text { CNT-Ionic liquid physisorbed } \\
\text { (solid-state electrolyte) }\end{array}$ & MWCNT & 0.5 & 3.6 & $\begin{array}{l}\text { Chang et al. } \\
\text { [151] } 2012\end{array}$ \\
\hline - & ref & - & 1.2 & \\
\hline $\begin{array}{l}\text { CNT-EMII/PMII composite } \\
\text { electrolyte (solid-state electrolyte) }\end{array}$ & SWCNT & $\mathrm{N} / \mathrm{A}$ & 3.5 & $\begin{array}{l}\text { Lee et al. } \\
{[152] 2010}\end{array}$ \\
\hline- & ref & - & 0.4 & \\
\hline $\begin{array}{l}\text { CNTs-PAN composite elec- } \\
\text { trolyte (solid-state electrolyte) }\end{array}$ & MWCNT & 5 & 3.9 & $\begin{array}{l}\text { Akthar et al. } \\
\text { [163] } 2011\end{array}$ \\
\hline- & ref & - & $\mathrm{N} / \mathrm{A}$ & \\
\hline
\end{tabular}

It has also been proposed that nanocarbons can restrict interaction between macromolecules, acting as a crystall growth inhibitor of polymer electrolytes, thus preventing a drop in ionic conductivity.[159]

A summary of CNT-doped electrolyte DSSCs are collected in the Table 2.4. These nanocarbon-loaded DSSCs can be divided according to the final viscosity of the electrolyte: liquid, quasi-solid and solid-state electrolytes. Consequently, the performances achieved are not directly comparable because every one of these materials are facing different challenges. 
Regarding the liquid state electrolytes, Lee et al.[153] compares the behaviour of high quality MWCNTs with less graphitic SWCNT material, reporting an enhancement of circa 20 and $10 \%$ with respect to the reference material when using MWCNTs and SWCNTs respectively. In this case, the quality factor of the material is an interesting point to have into account, concluding the purity of the dopant plays a role in the degree of improvement of the electrolyte.

Quasi-solid-state electrolytes are typically obtained by avoiding the incorporation of solvents or by the gelation process with polymers followed by evaporation of the solvent.

By the using of polymer nanocomposites electrolytes, Akhtar et al.[141] has demonstrate the improvement in ionic conductivity of the electrolyte and in the overall photo-conversion-efficiency of the DSSC in polymeric nanocomposite electrolyte. They have found the optimum mixing ratio $(\mathrm{CNT} / \mathrm{PEO})$ corresponding to $1 \%$, and correlated the enhancement in ionic conductivity with the formation of $I_{3}^{-}$ and polyiodide $\left(I_{m}^{-}\right)$species, due to the absorption of $I_{2}$ by the CNTs. It is well known that this species are the ones responsible in the high conductivity of the composite.

Gel polymer electrolyte mixed with fuctionalised MWCNTs was investigated Benedetti et al.[140] finding results in line with the previous authors. They reported an increase in the ionic conductivity of the electrolyte leading to an enhanced photogenerated current, an also a decrease in $I_{m}^{-}$species. The formation of $I_{m}^{-}$species can be followed by Raman spectroscopy. The ratio $I_{m}^{-} / I_{3}^{-}$shows the lowering of $I_{m}^{-}$species with the increase in loading of CNTs, and the authors point out the relation of this fact with the increase in viscosity of the electrolyte. In light of these improvements, the photo-conversion-efficiency of $3.5 \%$ is reported for CNT loaded electrolyte, $1 \%$ higher than the undoped electrolyte.

The solubility enhancement by acid functionalisation treatment is probed for gel polymer electrolytes, and that is an interesting result by itself. Neo et al.[139] introduces a combination of GO and MWCNTs into the MPE-gel electrolytes. A maximum efficiency of 7.1 was achieved in comparison with $6.5 \%$ of the same electrolyte without nanocarbons. The enhancement is explained by a good dispersion of the nanocarbons in the electrolyte, that increases the exposed surface area of the MWCNTs (percolation theory), enhancing the catalysis of the electro-chemical reduction of the $I_{3}^{-}$specie, therefore playing the role of CE material. At the same time, it reduces the ionic diffusion length of the redox species.

More recently, Jin et al.[162] have shown a gel-type quasi-solid-state electrolyte, modified with CNTs, with high performances up to the value of $8.2 \%$. Their mesoporous gel electrolyte not only exceed the standard gel-type quasi-solid electrolyte performance, but also its structure prevents from leakage problems enhancing the long-term stability of the device. Moreover, an enhancement in the conductivity of the electrolyte, and $V_{o c}$ and $J_{s c}$ are explained by a superior catalytic performance towards the $I^{-} / I_{3}^{-}$redox couple, acting at the same time as supported counterelectrode.

All-solid-state electrolytes are introduced in the DSSC field seeking the longterm stability of the devices. Chang et al.[151] have shown the improvement in photovoltaic performance by incorporating ionic liquid-physisorbed MWCNT. They demonstrate the linear relation between the increment in the diffusion limiting current of the $I_{3}^{-}$specie and the short circuit current density of the device when CNTs are added in the range $0-0.5 \% w t$. Also a similar approximation was followed by 
Lee et al.[152] by doping the electrolyte with SWCNTs. After optimising the $I_{2}$ content and EMII/PMII proportions, a PCE $=3.5 \%$ was reached, eight times higher as compared with the undoped ionic liquid. Akhtar et al.[163] reporter a thermal polymerised of CNTs and PAN for solid-state DSSC, leading to a performance of $3.9 \%$. Thanks to the polymerisation of the CNTs, an improvement in the ionic conductivity is achieved and therefore, better performance and characteristics are reported in comparison with the bare-CNTs.

There is a current lack of understanding of the CNTs contribution to increase the PCE. In view of the reviewed literature result, some possible mechanisms have been proposed in this section as possible hypothesis of this general improvement. Summarising,

1. The CNTs increase the ionic conductivity of the ionic liquid/electrolyte (percolation theory).

2. The CNTs interact with the redox mediator modifying the chemical environment of the electrolyte. It increase the concentration of polyiodides and thus the electrolyte conductivity by Grotthus mechanism.

3. The CNTs, when transport the charges through the electrolyte, interact with the electrolyte reducing the triiodide species and acting as CE.

\subsubsection{CNT/semiconductor hybrids as photoanodes}

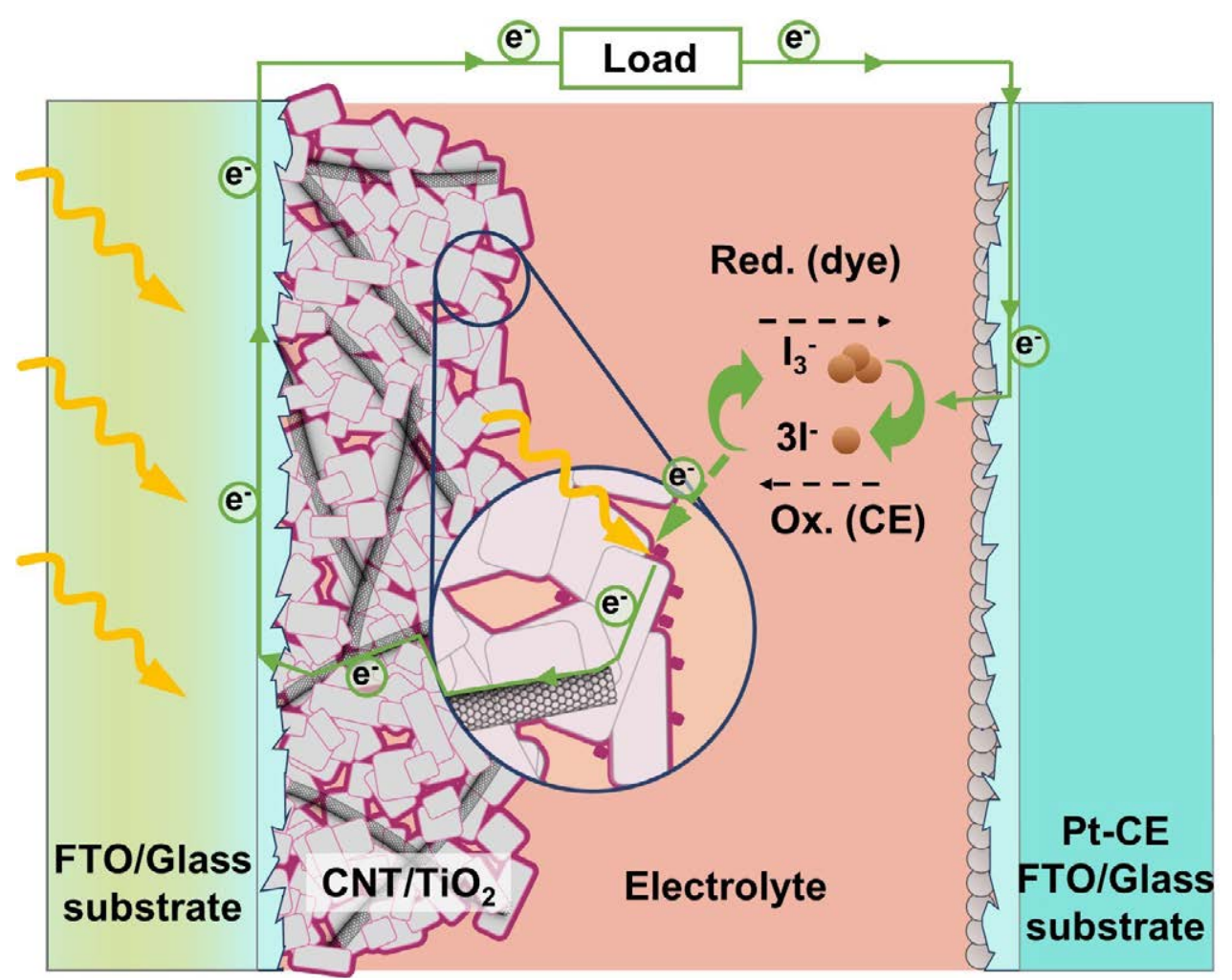

FIGURE 2.21: Schematic of a DSSC incorporating CNTs in the photoanode part of the device. 
There are different semiconductors fulfilling the required properties to be used in DSSC.[164, 165] Some of these semiconductors are n-type, like the $\mathrm{TiO}_{2}$ and the $\mathrm{ZnO}$. ZnO photoanodes have been widely researched[166] and record efficiencies of $7-7.5 \%[167,168]$ have been reported. This value represent approximately half of the performance achieved with the $\mathrm{TiO}_{2}$.

P-type semiconductors, $[169,170]$ for instance $\mathrm{NiO},[171-174]$ are commonly investigated, but the record efficiency reported is $2.5 \%$.[172] Although, p-type semiconductors present low performance, its main interest lays in the fabrication of $\mathrm{np}$ type DSSC, also called tandem cells.[175]

Nevertheless, because the most used material is $\mathrm{TiO}_{2}$ by its good performance and stability, in this section, we will focus on the hybrids produced by CNT and $\mathrm{TiO}_{2}$.

The photoanode of a dye sensitised solar cell is the component responsible of photo-generating the electrons. It is composed by transparent conductive substrate, a semiconductor layer of mesoporous anatase titania and the dye molecules (sensitiser) attached to the surface of the titania.

The nanocarbons can play a different role on the photoanode configuration. Firstly, replacing the transparent conductive oxide (TCO); secondly, as a dopant of the semiconductor layer increasing the PCE through an increased charge extraction from the titania; and thirdly, as a scaffold for the semiconductor material.[176, 177] A schematic of the incorporation of CNTs to the photoanode is depicted in the Figure 2.21.

The idea behind introducing nanocarbons in the photoanode has been discussed in several works before.[176-179] The general working principle is depicted in the Figure 2.22a. CNTs, thanks to its high electron mobility and the appropriate band alignment between $\mathrm{TiO}_{2}$ and $\mathrm{CNTs}$, allows the photogenerated charges to be injected into the CNTs and rapidly extracted from the semiconductor layer. This hypothesis has been confirmed by photoelectrochemical and transient absorption methods on TCO/SWCNT electrodes coated with $\mathrm{TiO}_{2}$ sol-gel by doctor blade technique, which has shown that the back electron transfer (recombination rate) between injected charges and the sensitiser becomes slower.[176]

The energy band alignment between $\mathrm{CNT}$ and $\mathrm{TiO}_{2}$ semiconductor produced by sol-gel has been performed before by Chen et al.[179] A Schottky rectifying contact have been measured and the charge transfer mechanism between these materials is presented in the Figure 2.22b, where the transport under dark and light conditions is compared. As it is presented, the potential barrier formed at the $\mathrm{FTO} / \mathrm{TiO}_{2}$ material is smaller compared with the $\mathrm{CNT} / \mathrm{TiO}_{2}$ heterojunction under dark conditions (TCO presented in the upper part and CNT at the bottom-left). In contrast, when the material is under high illumination power, the charge transfer from the $\mathrm{TiO}_{2}$ to the CNTs is much more favourable (energy band diagram at the bottom-right part).[179] This facilitates the charge extraction through the CNTs increasing the mean free path inside the $\mathrm{TiO}_{2}$ layer and decreasing the recombination component at the $\mathrm{TiO}_{2}$ surface.

It is of great importance controlling and understanding the electrical properties at the carbon/semiconductor interfaces. In a first approximation, this junctions can be described by the theoretical position of the work function between both materials, 4.2-4.3 eV for $\mathrm{TiO}_{2}$ or $\mathrm{ZnO}$, and $5 \mathrm{eV}$ [180] for CNTs. This qualitatively 
agree with the rectifying behaviour observed under $I-V$ measurements, but other effects might contribute to the the shift of the materials work function leading to wide range of Schottky barrier heights (SBH), even to metallic contacts.[181] Among these effects can be included the surface contamination, the formation of oxide layers or metallic element diffusion into the semiconductor layers. Besides, the different synthesis techniques of the materials can produce a high degree of qualities of materials, meaning a different amount of defects, electronic surface states and carrier concentrations. All these affects severely the properties of the carbon/semiconductor junctions.
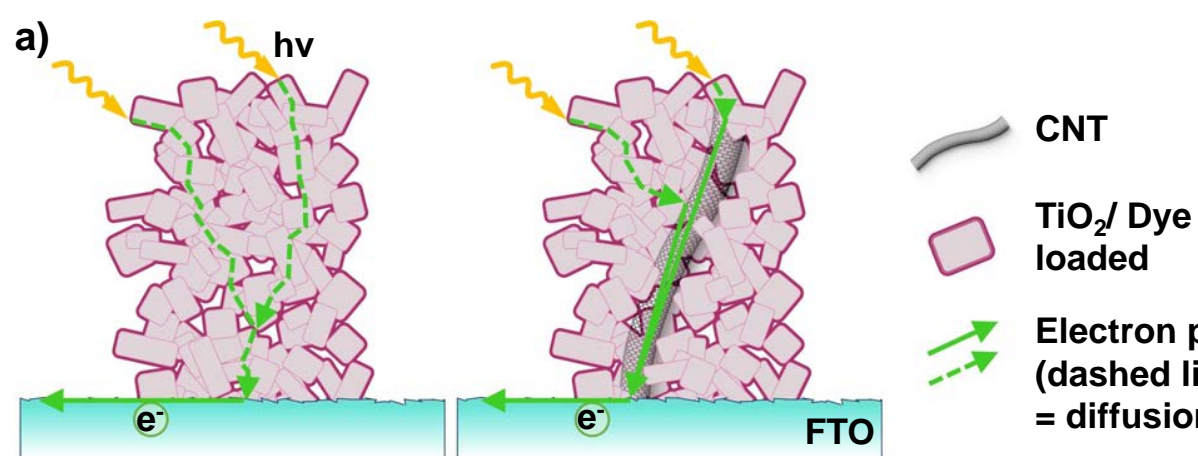

b)
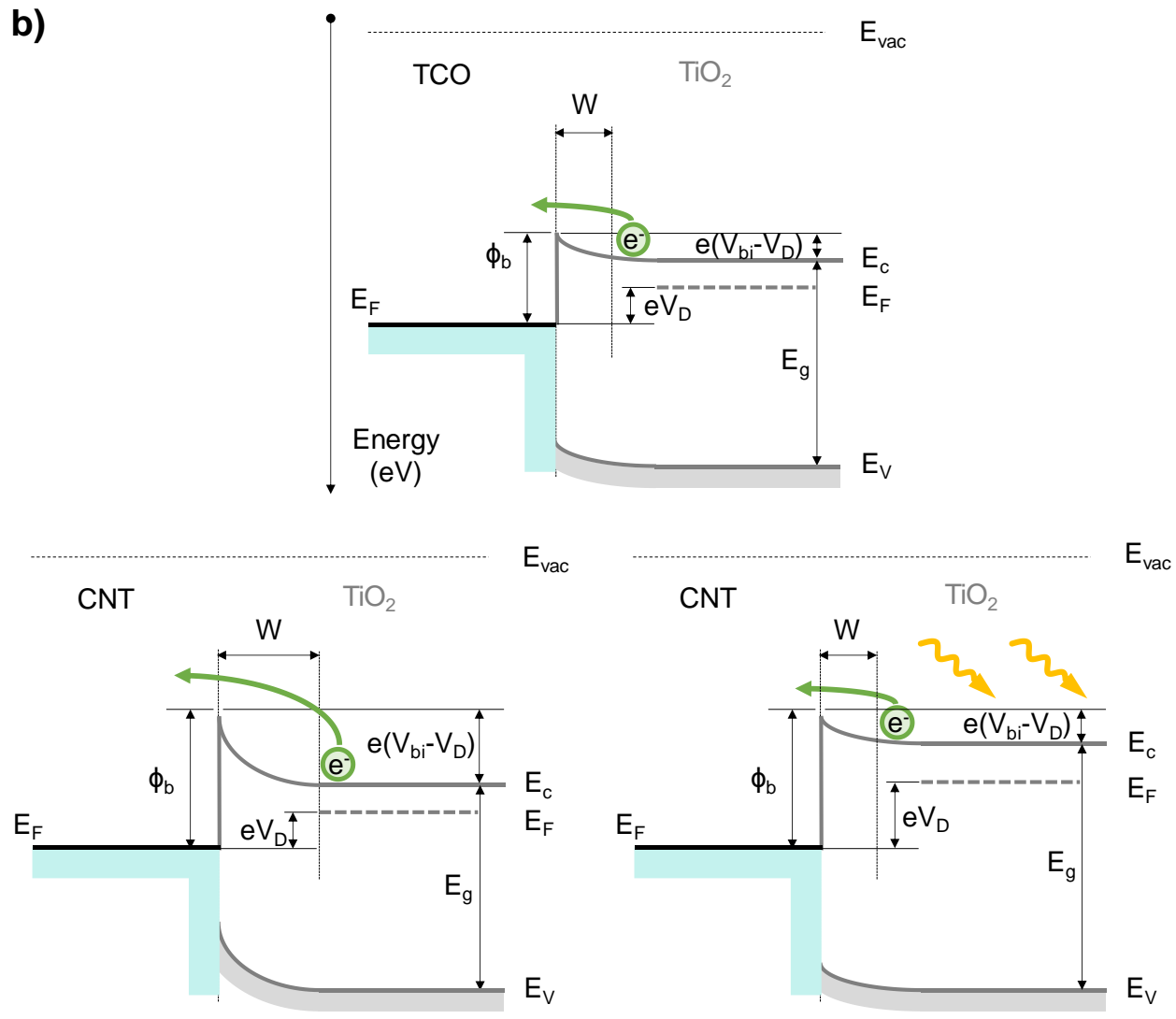

FIGURE 2.22: Schematic of a) the charge collection in $\mathrm{TiO}_{2}$ photoanodes with and without CNTs and $b$ ) the electronic band structure for the TCO and CNT metal connection (left and centre) and $\mathrm{CNT} / \mathrm{TiO}_{2}$ heterojunction at high illumination power. Adapted from Chen et al.[179] 
It is important to highlight the need to perform a clear interfacial characterisation to experimentally confirm the electronic band positioning (ideally under ambient conditions) for better understanding of this junctions to improve their fabrication and produce more efficient charge transfer processes.

In this context, the role of these junctions is to help separating the the photogenerated charges. This has been studied in relation to photovoltaic applications confirming the enhanced electron collection and transport, although under high applied voltage the CNTs can act as leakage centres.[179, 182]

\section{Integration routes of CNTs into photoanode electrodes:}

There have been used alternative routes for including CNTs in the photoanode materials. Blending CNT with the semiconductor,[183] spray depositing on top of a substrate, growing by chemical (hydrothermal)[184] or physical (sputtering) techniques, or creating hybrid nanostructures by electrospinning,[177] are some of the different methods yet experimented. Representative results of these methods are summarised in the Table 2.5 and in the Figure 2.23.

The idea of using 1D nanomaterials to create a direct-charge transport path was also implemented by Yang et al.[177] producing a $\mathrm{TiO}_{2} / \mathrm{CNTs}$ hybrid layer by electrospinning. The results concluded by this investigation shows that the CNTs increase the performance in these materials, achieving efficiency of $10.2 \%$, compared with the $6.1 \%$ for the undoped nanostructured $\mathrm{TiO}_{2}$.

The highest reported efficiency has been obtained by the virus templated selfassembly $\mathrm{CNTs} / \mathrm{TiO}_{2}$ (Figure 2.23a). This suggest that the ordered CNT material acting as a scaffold could imply a great improvement for the working electrode configuration.[185] Moreover, this work also compares the the behaviour of SW- and MW- CNTs under the same conditions. Two conclusions can be extracted regarding the nature of the nanocarbons: i) semiconducting CNTs provides better performance than metallic ones and ii) single layered CNT or few layer CNT shows better performance than MWCNT. A higher diffusion length and short-circuit current density is measured with semiconducting and SW- or few-layered -CNTs. They attributed this improvement to the non-continuous band structure in SWCNTs and semiconducting CNTs, where the electrons can be transferred quickly to the FTO current collector. In contrast, for continuous band structure (metallic CNTs) electrons can stay at continuous energy levels near the Fermi level, increasing recombination with the dye or back reaction to triiodide in the electrolyte.

This back reaction between CNTs at the photoanode and the redox couple in the electrolyte should be avoided. Minimising the exposure between CNTs and electrolyte is achieved by the successful encapsulation of CNTs by the $\mathrm{TiO}_{2}$ nanoparticles. Interestingly, the amount of CNTs used as dopant in photoanodes ranges 0.05$5 \%$. This values seems rational because are values close to the percolation threshold of CNTs.[44] This mass fractions of CNTs also reduces the exposure areas of CNTs in the hybrids, decreasing then the chances back reaction between electrolyte and photogenerated carriers collected by the CNTs. 
a)

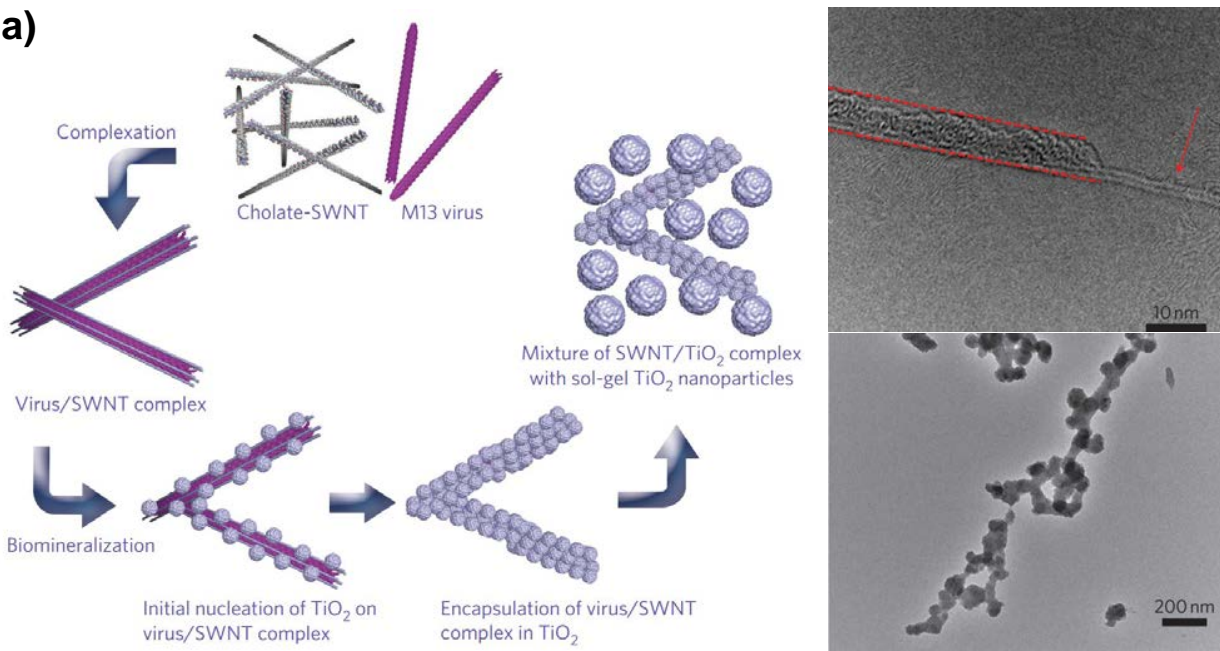

b)

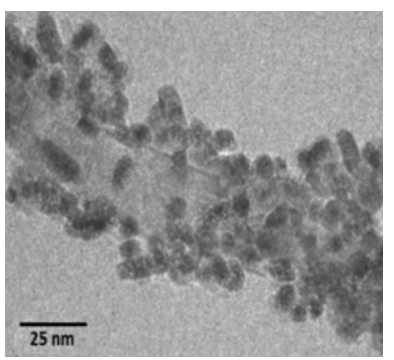

c) $\mathrm{TiO}_{2}$ Coating

d)
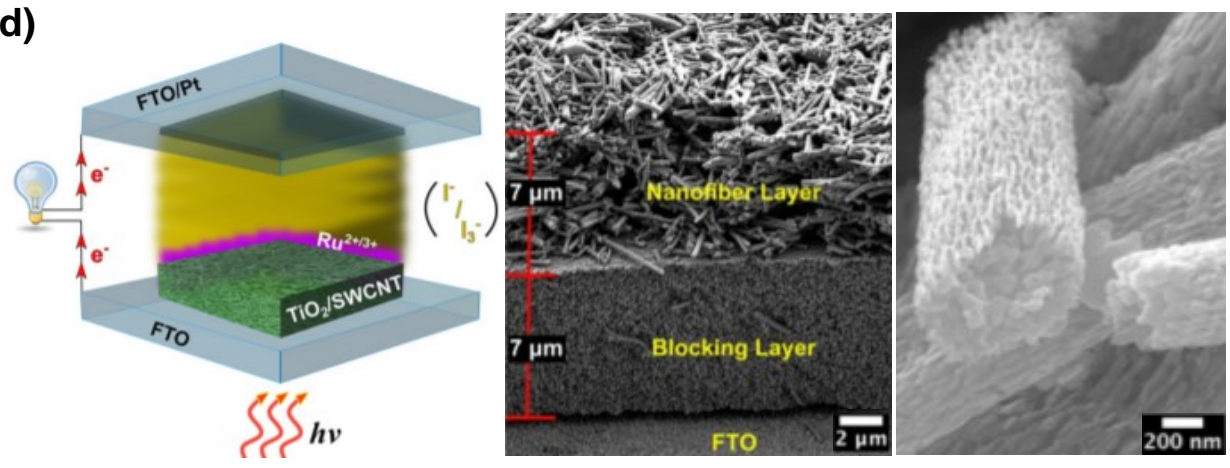

FIGURE 2.23: a) Schematic diagram and TEM images of virusenabled SWNT $/ \mathrm{TiO}_{2}$ DSSCs. Modified from Dang et al.[185] b) TEM image of $\mathrm{CNT} / \mathrm{TiO}_{2}$ anode by sol gel technique. Modified from Koli et al.[186] c) Schematic and SEM image of CVD grown CNT and $\mathrm{TiO}_{2}$ deposited by hydrothermal technique. Modified from Kilic et al.[184] d) Schematic of the $\mathrm{TiO}_{2}-\mathrm{NF} / \mathrm{SWCNT}$ composite photoanodes including an SEM image of the cross-section and a High-resolution image of the $\mathrm{TiO}_{2}-\mathrm{NF}$. Modified from Macdonald et al.[187] 
TABLE 2.5: PCE records of CNT-Photoanodes for different processing routes.

\begin{tabular}{|c|c|c|}
\hline Processing Route & PCE (\%) & Ref. \& Year \\
\hline CNT & 10.6 & 85] 2011 \\
\hline Electrospinning $+\mathrm{TiO}_{2} 0.1 \% \mathrm{MWCNT}$ & 10.24 & Yang et al.[177] 2013 \\
\hline CNT mixing $-0.25 \%$ & 9 & Dembele et al.[183] 2013 \\
\hline $\mathrm{CNT}+\mathrm{TiO}_{2}$ (hydrothermal) & 7 & Kilic et al.[184] 2016 \\
\hline CVD growth of CNT + sol ge & 3.7 & Chen et al.[179] 2012 \\
\hline Electrophoretic deposition and sol gel $\mathrm{TiO}_{2}$ & 0.13 & Brown et al.[176] 2008 \\
\hline
\end{tabular}

\subsection{Objectives of the present work}

In the quest of new materials for novel energy harvesting applications, as DSSC in this work, carbon nanotube fibre presents a combination of properties that fulfil the initial requirements for fabricating more efficient devices. The objective of this thesis is thus to perform a systematic study of the implementation of the CNTf as current-collector of the working and counter electrodes of DSSC devices and analysis of the charge transfer processes in presence of this material. Although there is a large amount of work reported using carbon nanotubes in these line of work, very few are focused on implementing macroscopic CNT assemblies in conventional DSSC architectures. The study is mainly of experimental nature, supported by different electronic, spectroscopic and photo-electrochemical techniques.

\section{Appendix: origin of $\mathrm{V}_{\mathrm{oc}}$ in DSSC devices}

The average steady-state electron density under illumination at $O C P$ is governed by the electron injection (from the dye to the mesoporous $\mathrm{TiO}_{2}$ and extracted by the anode current-collector) and the "electron back reaction" of the electrons with the $I_{3}^{-}$, where

$$
\frac{d n}{d t}=v_{i n j}-v_{b r}=0
$$

being the $v_{i n j}$ and $v_{b r}$ the global volume rates of injection and "back reaction" in the device. The global rate of electron injection $\left(\mathrm{cm}^{-3} \mathrm{~s}^{-1}\right)$ is defined as

$$
v_{i n j}=\frac{\eta_{l h} \eta_{i n j} I_{0}}{d}
$$

where $\eta_{l h}$ is the light harvesting efficiency, $\eta_{i n j}$ is the injection efficiency, $I_{0}$ the fraction of the incident photon flux absorbed by the sensitised mesoporous oxide (corrected for reflection losses) and $d$ the thickness of the mesoporous oxide film.

The involved back reation is

$$
I_{3}^{-}+2 e^{-} \rightarrow I^{-}
$$


and it depends on the reactant concentrations and the rate constants for electron transfer. The global rate of back reaction can be defined in formal terms as

$$
v_{b r}=k_{b r} n^{v_{n}}\left[I_{3}^{-}\right]^{v_{I_{3}}}
$$

where $k_{b r}$ is the rate constant for the back reaction of electrons and $v$ terms represents the reactions order with respect to electrons and $I_{3}^{-}$ions.

During this reaction, different intermediate steps involving the formation of $I_{2}^{-}$ radicals take place, therefore different rate constant appears during the reaction. The chemistry of the iodine reactions with Pt electrodes has been study since long time ago, and further details can be found in the work of Dané et al.[188] Besides that, the rate constant of the transferred electrons from the oxide also varies depending if they are transferred from conduction band or surface states located in the bandgap of the oxide. At this point, some simplifying assumptions are made in the model. Only the conduction band electrons are involved in the back reaction and the reaction orders regarding electrons and tri-iodide are taken equal 1 . Thus, from the steady state condition 2.10, the steady-state density of electrons under illumination and OCP condition is a linear function of the light, it remains:

$$
n_{c}=\frac{\eta_{l h} \eta_{i n j} I_{0}}{k_{b r}\left[I_{3}^{-}\right] d}=\frac{\eta_{l h} \eta_{i n j} I_{0} \tau_{0}}{d}
$$

where the $\tau_{0}=1 / k_{b r}\left[I_{3}^{-}\right]$is the electron lifetime in the conduction band determined by the back reaction with the $I_{3}^{-}$. In dark conditions, the electrons are in equilibrium with the redox system characterised by its redox-Fermi energy $\left(E_{F, \text { redox }}\right)$. The equilibrium electron density in the conduction band is defined by the redox-Fermi energy and the conduction band energy $\left(E_{c}\right)$ and it is given by the Boltzmann limit of the Fermi Dirac distribution (for a non-degenerate semiconductor).

$$
n_{c, e q}=N_{c} \mathrm{e}^{-\left[\frac{E_{c}-E_{F, \text { redox }}}{k_{b} T}\right]}
$$

where $N_{c}$ is the density of conduction band states.

Under light conditions, electrons in the $\mathrm{TiO}_{2}$ are no longer in equilibrium with the redox system. Following the steady-state electron density defined in 2.10, the injection of electrons leads to a photostationary state. In this situation, the carrier density $\left(n_{c}\right)$ defines the electron quasi-Fermi level $\left({ }_{n} E_{F}\right)$.

$$
n_{c}=N_{c} \mathrm{e}^{-\left[\frac{E_{\mathcal{C}}-n E_{F}}{k_{b} T}\right]}
$$

In the practice, an indirect measurement of the conduction band population is the measurement of the open circuit voltage $\left(V_{o c}\right)$. The $V_{o c}$ developed by the DSSC correspond to the change in the Fermi level of the oxide induced by illumination and represented in the Figure 2.24. It is given by

$$
q U_{\text {photo }}=E_{c}-{ }_{n} E_{F}=k_{b} T \ln \left(\frac{n_{c}}{n_{c, e q}}\right)
$$




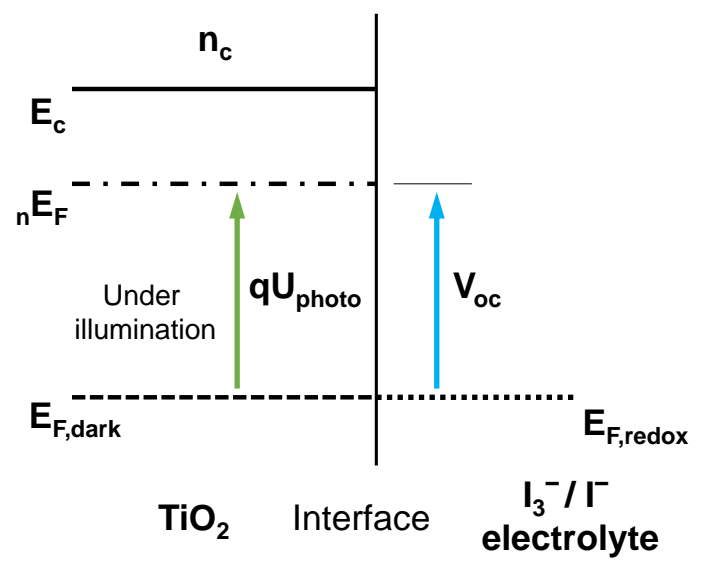

FIGURE 2.24: $V_{o c}$ origin in the DSSC under illumination. Adapted from K. Kalyanasundaram.[189]

Under dark-equilibrium conditions, the electron density corresponds to $\approx 10^{3} \mathrm{~cm}^{-3}$ in the conduction band. When the DSSC is illuminated, this carrier density increases up to $\approx 10^{16} \mathrm{~cm}^{-3}$, corresponding to the typical photovoltage of $0.75 \mathrm{~V}$. Finally, from the Equation 2.14 and 2.17, the photovoltage should be given by

$$
q U_{\text {photo }}=k_{b} T \ln \left(\frac{\eta_{l h} \eta_{i n j}}{n_{c, \text { eq }} d}\right)+k_{b} T \ln \tau_{0}+k_{b} T \ln I_{0}
$$

This previous equation (2.18) predicts that the photovoltage should increase $59 \mathrm{mV}$ for every decade of illumination intensity at $298 \mathrm{~K}$. In practice, values as high as $110 \mathrm{mV} \mathrm{dec} \mathrm{c}^{-1}$ are common due to non-idealities inherent to the devices. Empirical non-ideality factor $(m>1)$, provides the light intensity dependence of the $V_{o c}$ and it can be obtained from

$$
\frac{d U_{\text {photo }}}{d \log _{10} I_{0}}=m \frac{2.303 k_{b} T}{q}
$$




\section{Chapter 3}

\section{Experimental Techniques}

In this chapter, the description of the material synthesis, device fabrication and characterisation techniques common to the following chapters, as well as some important control experiments, are presented.

\subsection{Synthesis and fabrication of materials and devices}

\subsubsection{Synthesis of Carbon Nanotube Fibres (CNTf)}

The carbon nanotube samples used for this thesis were produced at IMDEA Materials in the "Multifunctional Nanocomposite Group" built in-house vertical CVD reactor. The reactor is presented in the Figure 3.1a.[190] This fibres were synthesised using the direct spinning method from the gas-phase during growth of CNTs by floating catalyst chemical vapour deposition.[191] Ferrocene, thiophene, and butanol (or toluene) were used as catalyst, promoter, and carbon source, respectively. Thiophene (extra purity $\geq 99 \%$ ) and ferrocene (purity $=98 \%$ ) were obtained from Acros Organics and 2-butanol (purity $>99 \%$ ) from Sigma Aldrich. Ferrocene was purified by a sublimation/recrystallisation process. The reaction was carried out in hydrogen atmosphere at $1250{ }^{\circ} \mathrm{C}$, using a S/C (sulfur/carbon) ratio chosen so as to produce CNTs with few layers.[192] The overlap of fibres and densification with liquid produces a unidirectional non-woven film (fabric) in which the individual fibres are highly aggregated and integrated as a free-standing material.

The CNTfs can be collected in almost any attachable substrate to the spinning engine, but, unless specifically mention, our samples were collected directly on a paper substrate. The standard collected samples have an area of $10 \times 30 \mathrm{~cm}$. But other geometries such as monofilaments or meter-long sheets of fibre can be also produce. Couple of examples, such as the as-collected CNTf on paper bobbin and a meter size CNTf sample, are presented in the Figure $3.1 b, c$ respectively.

Controlling the time of collected fibre, we are able to produce fibres of different mass and thicknesses. Collection times between seconds and 60 minutes were used, leading to thicknesses of material ranging between hundreds of nanometres to tens of microns. Another important parameter is the winding rate, being an important parameter for tunning the mechanical properties of the fibres.[190] All samples were collected at a winding rate between 5 and $40 \mathrm{~m} / \mathrm{min}$.

The as-collected material from the CVD reactor has very high porosity and low density structure, due to the air trapped in the CNT network. When this material is 
put in contact with non-polar liquids, for example solvents, the structure is rapidly filled by capillary forces. Over time this solvent evaporates, and after this process it produces a denser structure. This densification process is represented in the Figure 3.1d, in which the different stages of the process are shown. This is typically performed pouring some drops of ethanol or isopropanol absolute grade over the surface of the CNTf and waiting until its evaporation.

a)

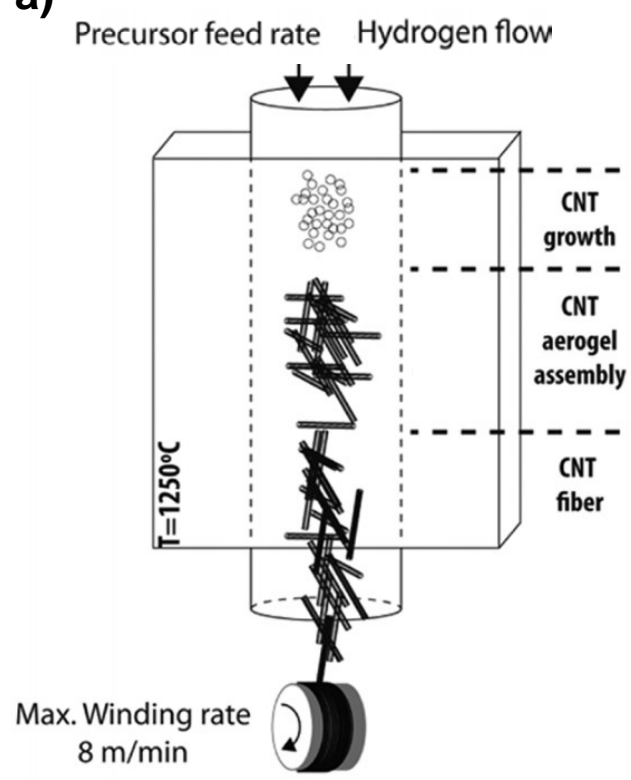

d)
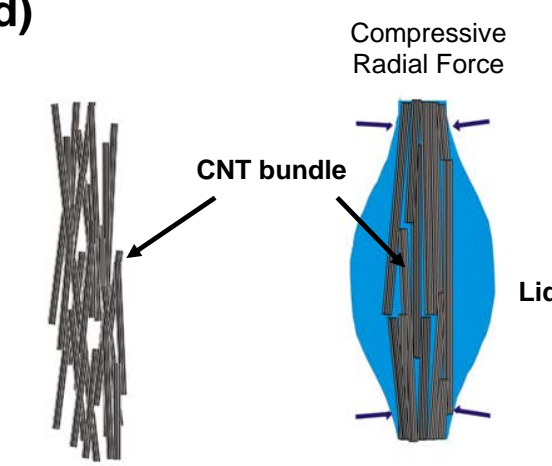

(1)

Uncondensed

fiber
Compressive

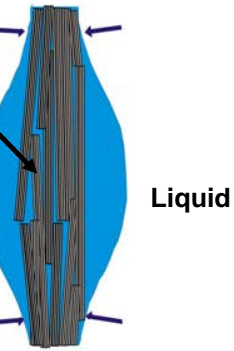

(2)

Liquid/vapour

surface minimisation

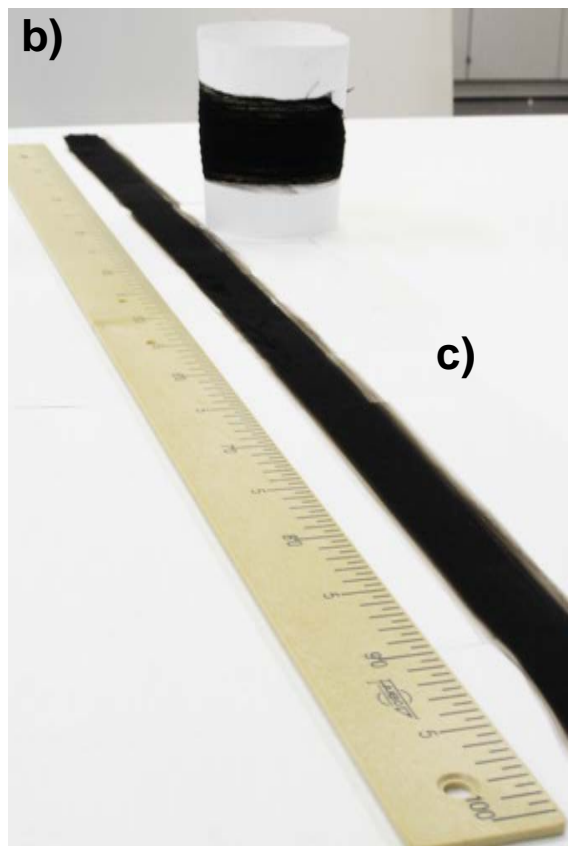

Inter-bundle Capillary Forces

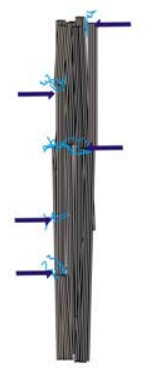

(3)

Liquid

evaporation

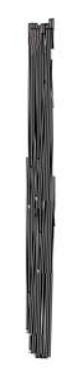

(4)

Condensed

fiber

FIGURE 3.1: a) CNTf CVD reactor.[190] b) CNT fibre as-collected from the CVD reactor and c) CNTf 1 metre length sheet. d) Densification process of the CNTf (Modified from[193]).

When the interfacial properties between nanomaterials are studied, it is important to prove that they are homogeneous along the same piece of material.

During the synthesis process of the CNTf, a distribution of different CNTs are present in the fibre leading to an overall semimetallic behaviour. The number of CNTs coming out from the cross-section of an individual CNT fibre filament has been estimated as $10^{5}$. Also, it is assumed that the distribution type of CNTs during 
the synthesis is constant because the synthesis conditions are constant. Now, considering the high number of CNTs present in the material, the macroscopic behaviour of the CNTf sheet is the average of the individual constituents and homogeneous through the different areas. Moreover, the homogeneity of all pristine CNTf sheets are ensured by electronic microscopy (SEM, TEM) inspection in a first stage before any other use and also checked by Raman spectroscopy.

\subsubsection{Semiconductor synthesis methods}

\section{Sol-gel synthesis method}

Small crystalline coating is deposited on top of the CNTf by sol-gel method for later acting as nucleating material. The chemical synthesis route was reproduced according to previous works.[194] The starting solution contains zinc acetate dihydrate $\left(\mathrm{Zn}\left(\mathrm{CH}_{3} \mathrm{COO}\right)_{2} \cdot 2 \mathrm{H}_{2} \mathrm{O}\right)$ as metal precursor, monoethanolamine (MEA) as complexing agent and interfacial active agent and absolute ethanol as solvent. First, MEA is dissolved in ethanol in a concentration of $1.2 \mathrm{~mol} / \mathrm{l}$ and stirred for $10 \mathrm{~min}$, and then the zinc precursor was added. The molar ratio of [Zn2+]:[MEA] was 1:1.5. The solution was stirred until a clear solution was obtained. At that moment, the solution was cast onto the CNTf film placed on the ceramic crucible. After solvent evaporation, the samples are heat treated in air at $300^{\circ} \mathrm{C}$ for $15 \mathrm{~min}$.

\section{Hydrothermal synthesis method}

The hydrothermal growth of $\mathrm{ZnO}$ nanowires consist on an aqueous solution of zinc nitrate $\left(\mathrm{Zn}\left(\mathrm{NO}_{3}\right)_{2}\right)$ and hexamethylenamine (HMTA) 0.5 and $1.5 \mathrm{M}$ respectively in ethanol and water.[195] The seed-coated CNTf was taken from the ceramic crucible and placed inside a teflon vessel of a steel autoclave. The CNTf was kept immersed in the solution for five hours at $90^{\circ} \mathrm{C}$. Finally, the hybrid CNTf/ZnO-NW's hybrid samples are washed with deionized water and dried for $30 \mathrm{~min}$ at $50^{\circ} \mathrm{C}$.

\section{Atomic Layer Deposition synthesis method (ALD)}

ALD is able to meet the needs for atomic layer control and conformal deposition using sequential, self-limiting surface reactions. The schematic of the process is presented in the Figure 5.17a, where a cycle of the process is represented. It consists on injecting alternative pulses of gas metal precursor and oxidising agent that reacts into a metal oxide monolayer of material.

The ALD process has been applied to CNTf material with the aim of obtaining 3D-bulk heterojunctions, in which the continuous conductive structure of the CNTf is preserved, and to add new functionalities by the presence of the $\mathrm{MO}_{x}$ coating.

Among different precursors (zinc acetate - ZnAc, diethyl zinc - DEZ, dimethyl zinc - DMZ, zinc chloride $\mathrm{ZnCl}_{2}$ or metallic $\mathrm{Zn}$ ), the diethyl zinc (DEZ) is by far the most common $\mathrm{Zn}$ precursor used for the $\mathrm{ZnO}$ ALD process and it has been the one selected for this work. Also, in addition to water, a wide range of oxygen sources are available, including $\mathrm{O}_{2}, \mathrm{O}_{3}$ and $\mathrm{N}_{2} \mathrm{O}$. Normally, for obtaining the same growth rate than water, higher deposition temperatures are required for this oxydising agents. 
The oxydising phase is commonly assisted by plasma in a plasma-enhanced ALD (PEALD) process, which helps to grow layers with higher degree of stoichiometry and lower temperature by the higher reactivity of the plasma oxygen source.[196]

The chemical reaction in the ALD process with DEZ and water precursors can be express as follows:

$$
\mathrm{C}_{2} \mathrm{H}_{5}-\mathrm{Zn}-\mathrm{C}_{2} \mathrm{H}_{5}+\mathrm{H}_{2} \mathrm{O} \rightarrow \mathrm{ZnO}+2 \mathrm{C}_{2} \mathrm{H}_{6}
$$

This reaction has been explained by two half-reactions, one first taking place at the surface of the material during the precursor injection and a second half-reaction when the water pulse is injected:[197, 198]

DEZ half-reaction:

$$
\text { surface }-\mathrm{OH}+\mathrm{C}_{2} \mathrm{H}_{5}-\mathrm{Zn}-\mathrm{C}_{2} \mathrm{H}_{5} \rightarrow \text { surface }-\mathrm{O}-\mathrm{Zn}-\mathrm{C}_{2} \mathrm{H}_{5}+\mathrm{C}_{2} \mathrm{H}_{6}
$$

Water half-reaction:

$$
\text { surface }-\mathrm{O}-\mathrm{Zn}-\mathrm{C}_{2} \mathrm{H}_{5}+\mathrm{H}_{2} \mathrm{O} \rightarrow \text { surface }-\mathrm{O}-\mathrm{Zn}-\mathrm{OH}+\mathrm{C}_{2} \mathrm{H}_{6}
$$

The efficiencies of these half-reactions depends on the experimental conditions, e.g. temperature. These have consequences over the different concentration of -OH groups, which also influences the electrical properties of the final materials.

For the ALD process using the CNTf as substrate, the CNTf were transferred to glass or FTO/glass substrates, followed by absolute isopropanol densification. Later, the substrates were placed under vacuum during 24 hours before the $\mathrm{MO}_{x}$ deposition.

The CNTf/ $\mathrm{MO}_{x}$-ALD materials used in this thesis where synthesised by two different methods in collaboration with two different groups. The same precursor but different reaction conditions and oxidising agents were used for these two processes. For the $\mathrm{TiO}_{2}$ deposition, all the experimental conditions have been previously detailed.[199] For the ZnO deposition, the ALD conditions are provided below.

The first set of samples were produced by Dr. Nina Kemnade (Prof. Dominik Eder group) in a Savannah 100 ALD system (Cambridge Nanotech). Once the samples were place in the reaction chamber, this was kept purging in $\mathrm{N}_{2}(20 \mathrm{sccm})$ for 10 min while reducing the pressure to 0.2 torr. The $\mathrm{N}_{2}$ flow was subsequently reduced to $10 \mathrm{sccm}$ before first $\mathrm{H}_{2} \mathrm{O}$ vapour (oxygen source) and then the metal precursor (Diethylzinc from Sigma Aldrich) were injected into the chamber in pulses of $0.03 s$ and $0.05 s$ respectively. After each injection, the samples were exposed to each reactant for $10 \mathrm{~s}$ before subjected to another purging of $15 \mathrm{~s}$. The deposition temperature was varied between $90^{\circ} \mathrm{C}$ and $200^{\circ} \mathrm{C}$ and the injection cycle repeated 100 times for obtaining $20 \mathrm{~nm}$ thick layer, corresponding to a growth rate of $0.2 \mathrm{~nm} /$ cycle.

The second set of samples were produced by Dr. Manuel Rodriguez (Prof. Daniel Granados group) in a Ultratech Fiji ALD system (Cambridge Nanotech). The optimised reaction temperature was $200{ }^{\circ} \mathrm{C}$. The ALD cycle starts by applying a pulse of $0.06 \mathrm{~s}$ of DEZ (Zn precursor), followed by a purging time of $10 \mathrm{~s}$. The $\mathrm{O}_{2}$ oxidising agent is circulated in the chamber $(30 \mathrm{sccm})$ during a $300 \mathrm{~W}$ plasma is applied for $40 \mathrm{~s}$. The ALD cycle was repeated between 20 and 900 times for obtaining thickness in the range 5-100 $\mathrm{nm}$ at a growth rate of $0.11 \mathrm{~nm} / \mathrm{cycle}$. 


\subsubsection{CNTf/polymer composite fabrication}

Detailed in Appendix A.

\subsubsection{Fabrication of symmetric electrochemical cells and Dye Sensitised Solar Cells (DSSC) including control experiments}

For the preparation of DSSCs and by extension, for the preparation of the symmetric devices, the method and all the details developed by Prof. Gratzël group have been followed.[200]

Schematics of the preparation of these devices are presented in the Figure 3.2. Also, a detailed step-by-step about the fabrication of each step is provided below.

A symmetric cell consist on an two-electrode electrochemical cell that is commonly used for studying catalytic processes in the field of the DSSC. They are explain in detail in the Chapter 4 "CNTf Counter-Electrodes for DSSC".

The preparation of the DSSC, following the flow chart in the Figure $3.2 b$, is detailed as follows: Fluorine-doped tin oxide (FTO, $15 \Omega / s q$, XOP Glass company) were used as substrates for both the photoanodes and counter-electrodes. The standard washing procedure consist on immersing each electrode in water, water and soap and isopropanol, each step during $15 \mathrm{~min}$ in a sonicator. After that, the electrodes are subjected to $U V-\mathrm{O}_{3}$ treatment for $18 \mathrm{~min}$ (Model No. 256-220, JelightCompany, Inc).

All chemical products were of highest purity available, bought from Sigma Aldrich and used without any further treatment. The ruthenium dye N-719, acetonitrile and tert-butanol were used for the preparation of the dye solution $(0.5 \mathrm{mM}$ in ACN:TBA 1:1 v/v). The electrolyte composition was 0.6 M 1-butyl-3-methyl imidazolium iodide, $0.1 \mathrm{M}$ guanidine thiocyanate, $0.5 \mathrm{M}$ 4-tert-butylpyridine, $0.03 \mathrm{M}$ iodine in acetronitrile/valeronitrile $(85: 15 \mathrm{v} / \mathrm{v})$ solvent.

To construct the photoelectrodes, the pre-cleaned FTO substrates were pretreated by immersion into a $0.4 \mathrm{mM} \mathrm{TiCl}_{4}$ solution (titanium(IV) chloride solution, $0.09 \mathrm{M}$ in $20 \% \mathrm{HCl}$ from Sigma) and annealed at $400{ }^{\circ} \mathrm{C}$ for $30 \mathrm{~min}$. After cooling down, a transparent $\mathrm{TiO}_{2}$ paste (Ti-Nanoxide T/SP, Solaronix) was doctor bladed on the electrodes and dried at $125^{\circ} \mathrm{C}$ for $6 \mathrm{~min}$. This procedure has been repeated twice. An additional opaque layer of $\mathrm{TiO}_{2}$ has been employed as scattering layer by doctor blading a large particle $\mathrm{TiO}_{2}$ paste $(18 \mathrm{NR}-\mathrm{AO}, \mathrm{Dyesol})$. Then, the electrodes were annealed at $325^{\circ} \mathrm{C}$ for $5 \mathrm{~min}, 375^{\circ} \mathrm{C}$ for $5 \mathrm{~min}, 450^{\circ} \mathrm{C}$ for $15 \mathrm{~min}$, and finally, at $500{ }^{\circ} \mathrm{C}$ for $15 \mathrm{~min}$. The active area of the cells has been set at $0.196 \mathrm{~cm}^{2}$, by using a pre-cut $5 \mathrm{~mm}$ circle template during the doctor blading procedure.

For standard Pt reference CEs, a solution of $0.5 \mathrm{mM}$ chloroplatinic acid hexahydrate in isopropanol was drop-casted on pre-cleaned FTO substrates and baked at $400{ }^{\circ} \mathrm{C}$ for $20 \mathrm{~min}$. In all cases, the counter-electrodes were drilled before the washing procedure to produce two holes of $1 \mathrm{~mm}$ diameter. The sealing of the devices was performed using a $25 \mu \mathrm{m}$ thickness frame of Surlyn thermoplastic (Meltonix 1170-25, Solaronix) between the electrodes, which were then placed at a hot press at $150{ }^{\circ} \mathrm{C}$ for $50 \mathrm{~s}$. After filling the cell with the electrolyte, this was encapsulated/sealed with a small portion of the same polymer. 


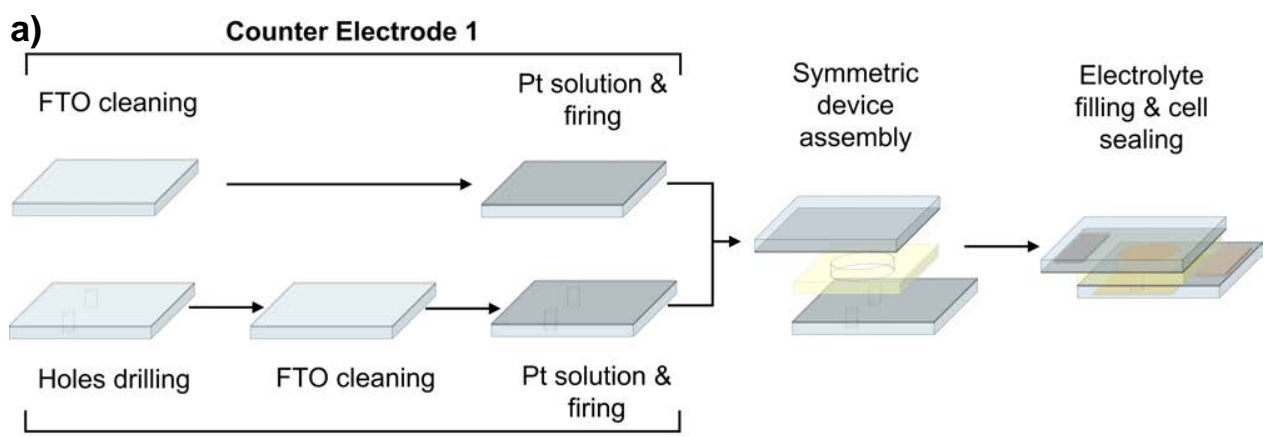

Counter Electrode 2

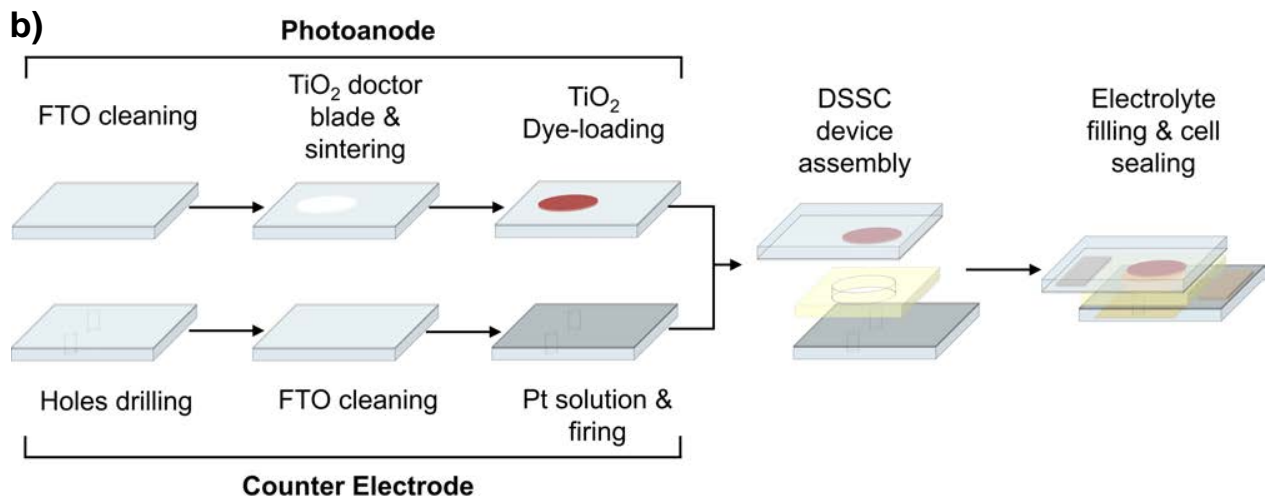

FIGURE 3.2: Schematic process of CNTf a) symmetric cell and b) DSSC fabrication.

During this thesis, when device modifications are proposed, as many devices are prepared until a reproducible result is obtained. At this point, at least three devices are prepared as well as two standard reference devices. From this five devices, the best result obtained is presented and studied in detail.

\section{Pt symmetric cell study and construction parameters influence}

In this section, control experiments using symmetric Pt cells showing the effect of temperature, separator thickness and electrolyte are presented. This background data enable to accurate assignation of processes in CNTf cells avoiding some misleading interpretations or wrong experimental conditions.

The EIS results corresponding to 25 and $100 \mu \mathrm{m}$ separator thickness are presented in the Figure 3.3a. When using pure quasi-solid BMIi electrolyte, the electrolyte present a low ionic conductivity and therefore, the resistance obtained are quite large. In both cases, the EIS results show the two typical interfaces in these symmetric cells. One at higher frequency representing the charge transfer between the counter-electrode and a second interface at low frequency $(<1 \mathrm{~Hz})$ representing the bulk ionic diffusion inside the cell. Two cells for each condition are presented, and the overlapping curves indicates the high precision of the assembly method and the high reproducibility. Diminishing the spacer thickness, the charge transfer interface shifts towards higher frequency, also the resistance $\left(R_{C T}\right)$ decrease by a factor of three. The diffusion of the ions is expected to be higher for thinner spacer because the diffusion path has decreased. This is suggested by the shape of the beginning 
of the semicircle corresponding to the bulk ionic diffusion but the number of points does not allow to perform a proper fitting to quantify this effect.

The influence of the temperature in a range from RT to $120^{\circ} \mathrm{C}$ was measured for $100 \mu \mathrm{m}$ separator and pure quasi-solid electrolyte (Figure 3.3b). The characteristic information from every curve - charge transfer resistance $\left(R_{C T}\right)$ and maximum frequencies $\left(f_{\max }\right)$ of the ionic diffusion and the charge transfer processes, are obtained and plotted versus the temperature in the Figure 3.3c. An exponential behaviour is obtained for the frequency shift of both processes towards higher values (notice here the $\log$ scale). Also the decrease of the charge transfer resistance follows an exponential trend and decreases its magnitude with the temperature. The diffusion behaviour is the expected and explained by the decrease of the viscosity of the quasi-solid electrolyte with the increase of the temperature. This behaviour is well known and can be modelled by the assumption that the electrolyte fluid obeys the Arrhenius equation. This behaviour has been reported before in very similar electrolytes.[201] The charge transfer between counter electrode and electrolyte also follows an exponential law. Initially, the charge transfer is occurring between the traces of iodide present in the quasi-solid ionic liquid. This charge transfer has to overcome a potential barrier and, as soon as the thermal energy is increased, the charges have higher kinetic energy to overcome this barrier. Ultimately, the redox rate of reaction mediated by the charge transfer also follows an Arrhenius equation, thus, it also depends exponentially on the temperature.
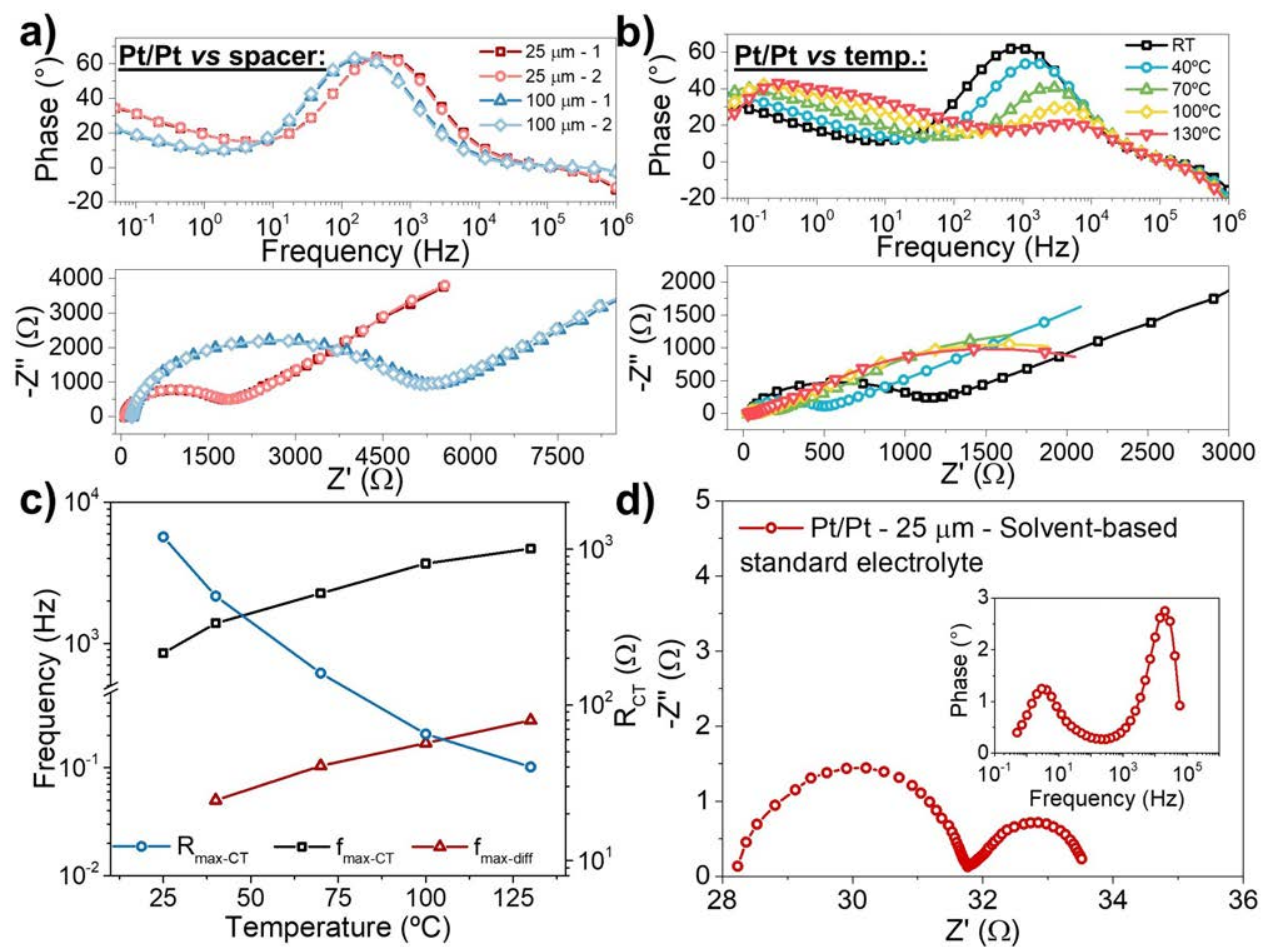

d)
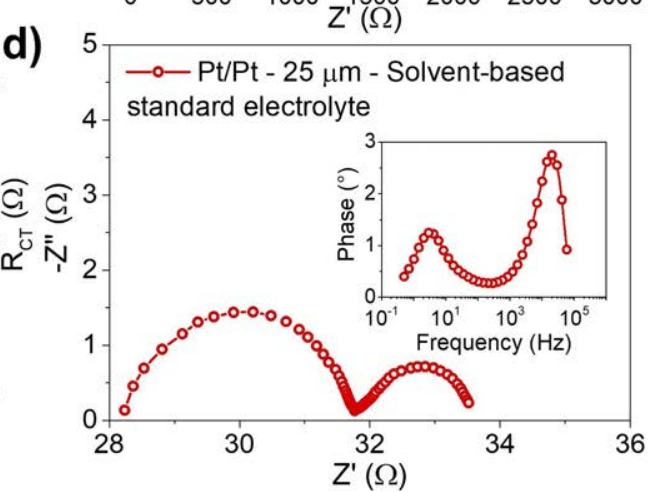

FIGURE 3.3: Pt symmetric cells EIS study. a) 25 vs $100 \mu \mathrm{m}$ separator thickness influence. b) Temperature dependence Nyquist and Bode phase plots, and c) maximum frequencies and charge transfer evolution. d) Nyquist plot with solvent-based standard electrolyte. Bode phase plot presented as inset.

Finally, EIS analysis of the Pt-symmetric cell, $25 \mu m$ thickness spacer, with the 
standard solvent based electrolyte (used for liquid based DSSCs), is presented in Figure 3.3d. Due to the presence of the acetonitrile and valeronitrile solvents, as well as the higher concentration of iodide/triiodide in the solution, the electrolyte is now a much more conductive media. A $R_{C T}$ of $c a .4 \Omega$ is obtained with a maximum characteristic frequency of $10^{4} \mathrm{~Hz}$. The $R_{\text {diff }}$ is $<2 \Omega$ with a $f_{\max }=3 \mathrm{~Hz}$. Similar behaviour is commonly obtained in literature for similar geometry of symmetric cells with the same materials leading to a highly efficient DSSCs.[202] This confirms that the materials (Platinum, electrolyte and electrodes) are in perfect conditions and the reference materials are working totally as expected. This ensures the results will be comparable with the previously reported in literature.

\subsubsection{Architectures according to the number of $\mathrm{TiO}_{2}$ layers: transparent and reflective photoanodes}

Different device architectures has been proposed since the very early stages of the DSSC technology. The low absorption of the titania/dye in the red region and also, with the aim of maximising the path length of the light inside the device, different working electrode multilayer geometries including light scattering materials have been proposed.[203, 204] One of the most used configurations because of the excellent efficiency achieved is proposed by Ito et al.[200]

Schematics comparing devices with and without scattering layer are presented in the Figure 3.4a. As it is depicted at the left part, when thin films of $\mathrm{TiO}_{2}$ nanoparticles are used, the electrodes are semi-transparent, allowing the light to pass trough the device (a TEM image of the $\mathrm{TiO}_{2}$ nanoparticles - anatase phase, is presented in the Figure 3.4b). An additional layer of $\mathrm{TiO}_{2}$ larger size particles - rutile phase, can be added to the working electrode geometry. These larger particles of $\mathrm{TiO}_{2}$, close to the micrometer in diameter (see TEM image in the Figure 3.4c), act as a light scattering layer, producing the internal reflection of the light inside the device.[205] This effect is represented in the right part of the schematic (Figure 3.4a). Although the scattering layer presents lower surface area, and no dye adsorbed, the light path increases inside the cell and the photoexcitation and photocurrent increases proportionally. The comparison in sizes between nano- and micro-particles $\mathrm{TiO}_{2}$ can be clearly observed in the TEM image of the Figure 3.4c (as they are marked with a red and yellow square).

The transmittance of three different $\mathrm{TiO}_{2}$ working electrodes has been measured by UV-VIS spectroscopy and the spectra are shown in the Figure 3.4d. One and two layers of $\mathrm{TiO}_{2}$ nanoparticles deposited on to FTO substrates by doctor blade technique presents a transmittance higher to $80 \%$ over $700 \mathrm{~nm}$ wavelength, later the transmittance decrease to $50 \%$ in the visible range until the absorption edge is found in the UV region (due to the bandgap absorption of $3.3 \mathrm{eV}$ ). When the scattering layer is added to the 2-layer nanoparticles, the named "3 layers" transmittance spectra is obtained. Under these circumstances, the transmittance is lower than $30 \%$ for the whole measured wavelength range, indicating that a $50 \%$ approximately is reflected inside the cell. 
a) Transmitted light
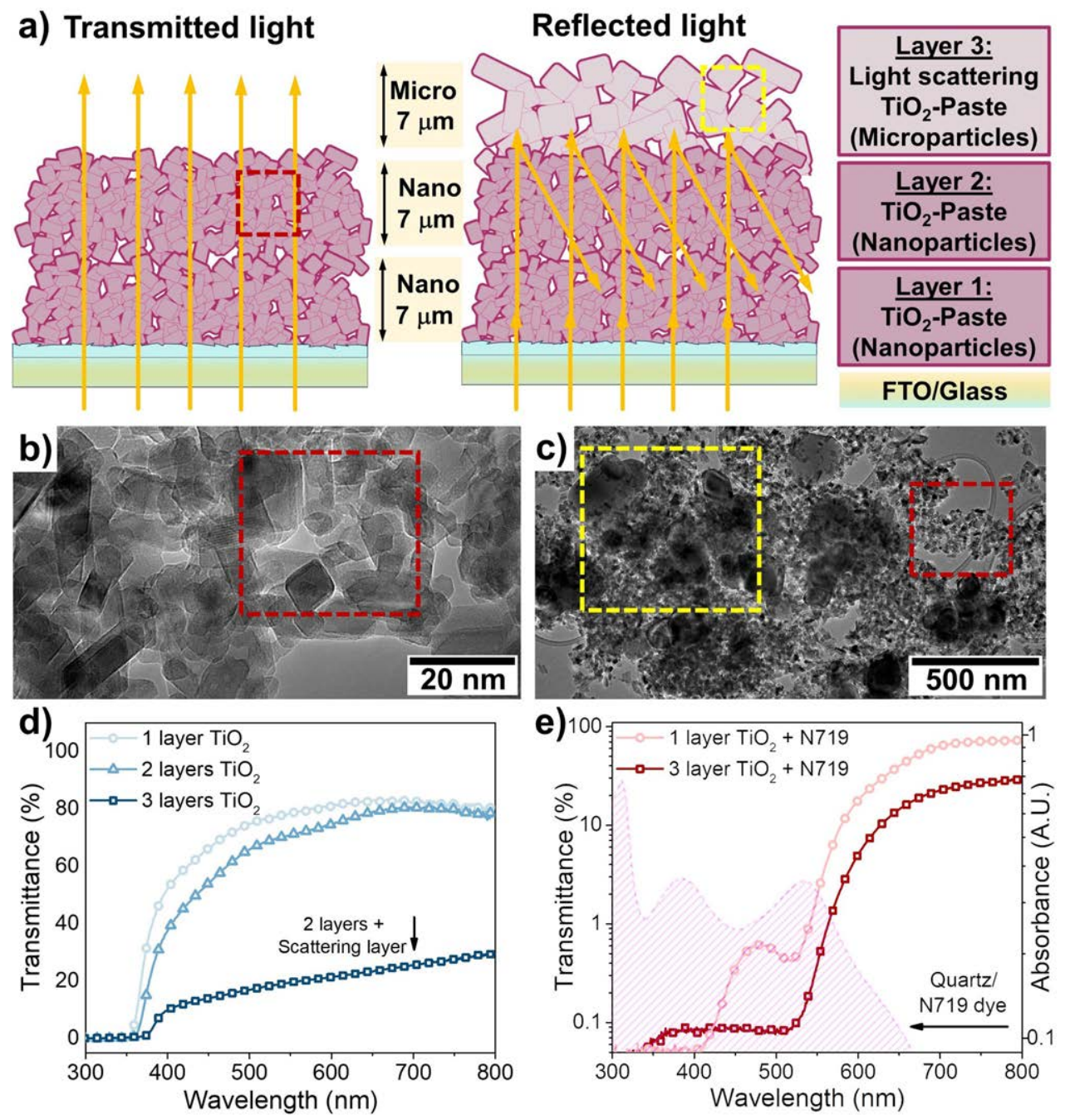

FIgURE 3.4: a) Photoanode schematics showing 1, 2 and $3 \mathrm{TiO}_{2}$ layers, i.e. 7, 14 and $21 \mu \mathrm{m}$ respectively. The light transmitted and reflected depending on the materials used is also represented. Approximate thicknesses of the layers are also indicated. b) TEM image of the transparent $\mathrm{TiO}_{2}$ nanoparticles used as active material. A red square is drawn in the image and over the schematic for better identification of the material. c) TEM image of the opaque $\mathrm{TiO}_{2}$ microparticles used as light scattering layer. A yellow square is drawn in the image and the right part of the schematic. d) UV-VIS transmittance spectra of the 3 different $\mathrm{TiO}_{2}$ working electrode configurations without dye. e) UV-VIS transmittance spectra of the semi-transparent (1 layer) and opaque (2 layer + scattering layer) $\mathrm{TiO}_{2}$ working electrode with N719

dye loaded. The absorbance of the N719 dye is also represented.

The transmittance spectra for the 1 and 3 layers working electrodes, after the N719 adsorption process, are shown in the Figure 3.4e. The transmittance is presented in logarithmic scale for better observation of the absorption bands of the dye in the visible range. The absorption spectra of N719 dye, deposited on to quartz substrate, is also presented in this graph. $\mathrm{TiO}_{2}$ and $\mathrm{TiO}_{2}+\mathrm{N} 719$ dye absorbance spectra are in agreement with previous investigations.[203-206] 


\subsection{Physical characterisation}

In this section, the basic experimental details of the characterisation related to the morphology and structure of the samples and materials are provided.

\subsubsection{Profilometry}

The CNTf and $\mathrm{TiO}_{2}$ layer thicknesses of DSSC devices were characterised with

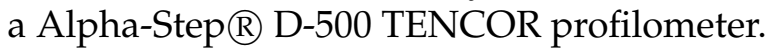

The procedure for measuring the CNTf thickness consist on: once the CNTfs are stacked over a FTO substrate, the edges of the CNTf sheet were trimmed off to keep a sharp thickness of approximately 1-2 $\mathrm{mm}$ at the edges. Still the CNTf electrodes present a surface roughness in the range of the micron, mainly produced by the fibrous nature of the macroscopic material. The average thickness will be the one measured over a range of 1-2 $\mathrm{mm}$.

\subsubsection{Scanning and Transmission Electron Microscopy (SEM, TEM)}

Scanning electron microscopy (SEM) was carried out with a FIB-FEGSEM Helios NanoLab 600i (FEI) at an accelerating voltage of $5 \mathrm{kV}$ and a Talos F200X (FEI) TEM at $300 \mathrm{kV}$.

Selected area electron diffraction (SAED): this technique consist on selecting an area of the sample by using a small aperture of the TEM equipment and acquiring the electron diffraction pattern. It is possible to obtain the pattern of a very specific region of the material as for instance, nanometric-size nanoparticles from an ALD coating or thin CNTs bundles from a macroscopic fibre.

\subsubsection{Energy Dispersive X-ray Spectroscopy (EDX)}

Elemental analysis of samples was determined by energy dispersive X-ray spectroscopy (INCA, Oxford Instruments) at $10 \mathrm{kV}$ accelerating voltage in the above mention equipment.

\subsubsection{X-ray Photoelectron Spectroscopy (XPS)}

X-ray photoelectron spectroscopy (XPS) data was collected in a SPECS GmBH electron spectroscopy system provided with a PHOIBOS $150+\mathrm{MCD}$ analyser.

$X P S$, also known as Electron Spectroscopy for Chemical Analysis (ESCA) is one of the most extended surface analysis techniques. It consists on the sample radiation with monoenergeric X-rays. The emitted electrons from the surface of the sample are collected as a function of their energy. The chemical environment and oxidation state of the different elements presents in the sample can be obtained.

For proper measurement of the materials by XPS technique, it is important to avoid the presence of surface contaminants. For that, all the samples have been degassed during 24 hours. The obtained spectra of carbon materials were calibrated 
according to the position of the C1s C-C bond located at $284.8 \mathrm{eV}$. For the samples coated with $\mathrm{ZnO}$ material, the position of the $\mathrm{Zn} 2 \mathrm{p}_{3 / 2}$ located at $1021.8 \mathrm{eV}$ were used for the calibration. Energies referred to the Fermi level.

\subsubsection{Powder X-ray Diffraction (XRD)}

The crystal structure and average crystal size were determined by X-Ray diffraction (XRD) using an Empyrean, PANalytical diffractometer, with $\mathrm{Cu} \mathrm{K} \alpha$ radiation.

XRD data was also obtained from SAXS/WAXS (small- and wide- angle X-ray scattering) syncrothron measurements. The experimental setup used at the BL11 at ALBA synchrotron facilities is described as follows: the WAXS patterns are acquired with a wavelength of 1 Angstrom by a 2D-CCD detector, and due to experimental considerations and geometries, these patterns correspond to diffraction cakes of approximately $60^{\circ}$. This implies that for an anisotropic material, that is the case of CNTf, it may present different crystallisation depending on the orientation, i.e. preferential growth as we reported before with polymeric materials in presence of the CNTf.[207]

\subsubsection{Raman spectroscopy}

Raman spectroscopy was performed with a Renishaw PLC spectrometer using a laser of $532 \mathrm{~nm}$ of wavelength at 5-10 $\mathrm{mW}$ depending on the sample.

Raman spectroscopy is a common technique for characterising carbon based materials. It has special interest after hybridisation process with other materials, for instance, semiconductors. Raman analysis can shed some light on the structure and interaction between both materials. Moreover, the influence of the ALD process over the CNTf substrate, i.e. structural disorders induced during the growing, residual stresses on the CNT surface, or electrical doping effects, can be investigated.

In a CNT Raman spectrum, there are some characteristic bands that are identified as the G-band is present around $1580 \mathrm{~cm}^{-1}$, the $\mathrm{D}$ band at about $1350 \mathrm{~cm}^{-1}$, the $\mathrm{D}^{\prime}$ at about $1620 \mathrm{~cm}^{-1}$, and the 2D (also known as $\mathrm{G}^{\prime}$ ) at about $2685 \mathrm{~cm}^{-1}$. G and $\mathrm{G}^{\prime}$ are characteristic bands of all graphitic materials with sp2 bonds, while D and $\mathrm{D}^{\prime}$ comes from defects and disorder of the structure.[208, 209]

\subsubsection{Ultraviolet-Visible spectroscopy (UV-VIS)} Corp).

Ultraviolet-Visible spectroscopy was performed with a UV-2700 (Shimadzu

- Electrode dye loading by analysing the dye-solution concentration variation:

For obtaining a precise value of the dye loading in DSSC working electrodes, the variation of concentration of the initial dye solution is a reliable approach.

The variation of dye adsorption at the electrode can be analysed by UV-Vis technique according to the methodology described elsewhere.[210] Basically, the $\mathrm{TiO}_{2}$ film (the photoanode electrode) was immersed in a vial with $5 \mathrm{~mL}$ of $0.5 \mathrm{mM}$ 
N719/ acetonitrile (ACN) solution. Samples of the solution were withdrawn via micropipette before and after the 24 hours dyeing time. Typically, half a day is enough time for the dye saturation of the $\mathrm{TiO}_{2}$ surface, and this adsorption trend is typically observed for organic materials on porous metal oxides.[98] The changes of the absorbance value at $530 \mathrm{~nm}$ were measured with an UV-Vis spectrophotometer. The amount of $\mathrm{N719}$ at equilibrium on the $\mathrm{TiO}_{2}$ films was calculated using the mass balance equation 3.1:

$$
q^{*}=\frac{\left(C_{0}-C_{e}\right) V}{m}
$$

where $q^{*}\left(m g g^{-1}\right)$ is the equilibrium amount of $\mathrm{N} 719$ adsorbed on the $\mathrm{TiO}_{2}$ film, $C_{0}$ and $C_{e}\left(m g L^{-1}\right)$ are the dye concentrations at initial and equilibrium states, respectively. The volume $V(L)$ is the volume of the solution, and $m(g)$ is the mass of the $\mathrm{TiO}_{2}$ film.

Besides, for extremely low concentration, the UV-Vis absorbance follows BeerLambert law (equation 3.2), where the absorbance $(A)$ is proportional to the concentration $\left(C\left[g L^{-1}\right]\right)$ of attenuating species in the solution, the path length $(l[\mathrm{~cm}])$ of the beam light through the material and the absortivity, also called molar attenuation or extinction coefficient $\left(\epsilon\left[\mathrm{L} \mathrm{g}^{-1} \mathrm{~cm}^{-1}\right]\right)$ of the material:

$$
A=\epsilon l C
$$

As an example, the data presented in the Chapter 6 is analysed here. The Figure 6.11 shows the absorption spectra of N719 before and after 24 hours of dye loading of the $\mathrm{TiO}_{2}$-ALD electrodes with and without CNTf. The spectra exhibit typical absorption bands of $\mathrm{N} 719$ at around 375 and $530 \mathrm{~nm}$. The adsorption concentrations of N719 is typically estimated using the absorption band of $530 \mathrm{~nm}$ which is assigned to a $\pi-\pi^{*}$ charge transfer transition. For this calculation, the molar extinction coefficient at $535 \mathrm{~nm}$ of $\mathrm{N} 719$ was taken as $\epsilon_{535}=14 \times 10^{3} \mathrm{~L} \mathrm{~mol}^{-1} \mathrm{~cm}^{-1}$ according to the literature.[211] Moreover, the absorption edge for the ACN solvent is position at $300 \mathrm{~nm}$ and therefore, only the dye is taken into account for this calculation. Finally, a quartz cuvette with a path length of $2 \mathrm{~mm}$, filled with $1 \mathrm{~mL}$ of ACN and $20 \mu \mathrm{L}$ of the dyeing solution (concentration dilution ratio $=51$ ) were the experimental conditions for the different samples. With this previous conditions, $0.5 \mathrm{mM}$ N719 in ACN, $0.2 \mathrm{~cm}$ path length, $\epsilon_{535}=14 \times 10^{3} \mathrm{~L} \mathrm{~mol}^{-1} \mathrm{~cm}^{-1}$, and according to the equation 3.2, the absorbance is calculated as $A=1.4$. From the following relation 3.3

$$
\frac{C^{\prime}}{C_{0}}=\frac{A^{\prime}}{A_{0}}
$$

and taking into account the dilution factor, the concentrations of the solutions before and after dyeing the electrodes can be calculated. The N719 solution has almost the same absorbance before and after the $\mathrm{TiO}_{2}$-ALD dyeing process, $C_{0}^{T i O_{2}-A L D}=1.99 \mathrm{mM}$ and $C_{e}^{T i O_{2}-A L D}=1.93 \mathrm{mM}$. When the CNTfs is added, the surface area of the electrode is increased proportionally to the $250 \mathrm{~m}^{2} \mathrm{~g}^{-1}$, the concentrations calculated from the absorbance spectra remains: $C_{0}^{\mathrm{CNTf} / T \mathrm{TO}_{2}-A L D}=$ $2.25 \mathrm{mM}$ and $C_{e}^{C N T f / T i \mathrm{O}_{2}-A L D}=1.63 \mathrm{mM}$. 
Now, applying the mass balance equation, with an estimated amount of material equal to $2 \mathrm{mg}$ and $14 \mathrm{mg}$ for the $\mathrm{TiO}_{2}-\mathrm{ALD}$ and $\mathrm{CNTf} / \mathrm{TiO}_{2}-\mathrm{ALD}$ respectively, and $5 \mathrm{~mL}$ of volume, the amount of dye adsorbed on the $\mathrm{TiO}_{2}$ is obtained as $q_{\mathrm{TiO}_{2}-A L D}^{*}=$ $0.15 \mathrm{mmol} \mathrm{g}^{-1}$ and $q_{\mathrm{CNTf} / \mathrm{TiO}_{2}-\mathrm{ALD}}^{*}=0.22 \mathrm{mmol} \mathrm{g}^{-1}$. These results confirms the adsorption of the dye at the recrystallised surface of the $\mathrm{TiO}_{2}$, as it is clearly observed from the Figure 6.11.

\subsection{Mechanical properties characterisation}

The mechanical properties of the CNTf/PVDF nanocomposites presented in the Appendix A, were characterised as follows.

The tensile experiments were performed in Instron 5966 at a fixed strain rate of $1 \mathrm{~mm} / \mathrm{min}$. Dog bone shape samples were cut. The final sample dimensions were: length: $10 \mathrm{~mm}$; width: $2 \mathrm{~mm}$ and thickness: 0.2 and $0.02 \mathrm{~mm}$.

\subsection{Electrical properties characterisation}

The electrical and electrochemical measurements and analysis techniques used during this thesis are here described. The measurements were carried out with a Source-Measure Unit (SMU Keithley 2450) and two different potentiostats (Biologic SP-200; Metrohm Autolab PGSTAT204).

\subsubsection{Linear Sweep Voltammetry (LSV)}

In a linear sweep voltammetry (LSV), the voltage is varied linearly from a lower limit to an upper limit. $I-V$ curve is obtained when the current response is plotted as function of voltage applied. The characteristics obtained through LSV method are different depending on the sample and analysis applied.

\section{Resistivity measurements}

Must be distinguished between the two-probe and four-probe methods for measuring resistivity. The appropriate method should be selected according the to the material properties, shape, precision required, etc. Two-point probe is usually easier to implement, but should be taken into account that each contact work as current and as a voltage probe. Therefore, the resistance of the material or device under testing (DUT) is obtained from the total resistance $R_{T}$

$$
R_{T}=V / I=2 R_{W}+2 R_{C}+R_{D U T}
$$

Where $R_{W}$ is the wire resistance, $R_{C}$ is the contact resistance and $R_{D U T}$ is the resistance of the material or device. Clearly, it is hard to obtain a precise value of $R_{\text {DUT }}$. Some multi-contact two-probe measurements are sometimes used to overcome the lack of precision of this method. Transferred length method (TLM) is an example of them. The total resistance is measured for several contact separations 
and plotted versus the spacing $d$. The sheet resistance $\left(R_{s q}\right)$ of the material and the contact resistance can be obtained by this method. The resistance is given by

$$
R_{T}=\frac{R_{s q} d}{Z+2 R_{C}}
$$

More precise values for $R_{D U T}$ can be directly obtained from four-probes method. The voltage now is measure from two additional contacts. Although the voltage path also includes $R_{W}$ and $R_{C}$, now the current flowing through them is almost negligible due to the high input impedance of the voltmeter (higher than $10^{12} \Omega$ ). The device resistance is therefore obtained by $R=V / I$, and the resistivity by normalising with the contact area $\rho=R A$. Schematics of two- and four-probe measurements used in this thesis are presented in the Figure 3.5.

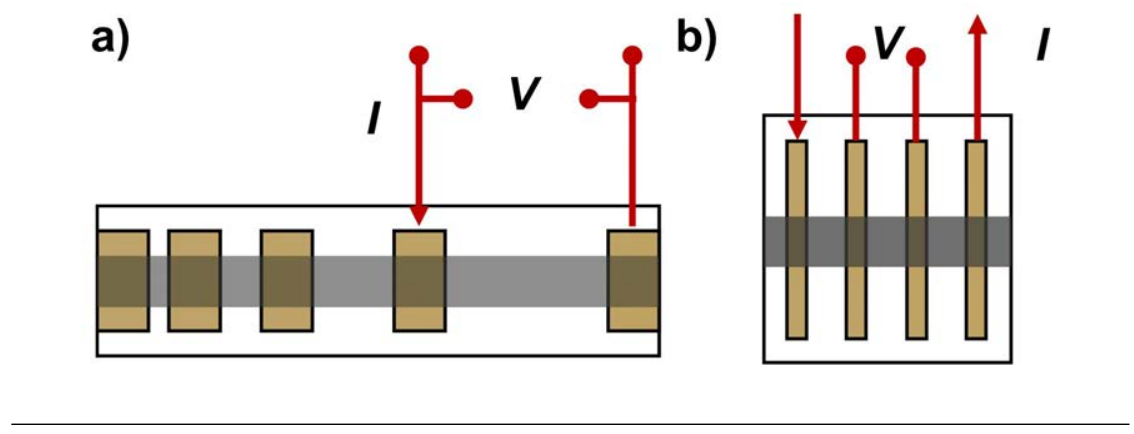

FIGURE 3.5: a) Two-probes contact resistance and b) four-probes conductivity measurements.

\section{Tafel plot}

The Tafel plot is the semi-logarithmic representation of a LSV measurement. It is quite common in the electrical characterisation of materials such as, solid state interfaces, and also a widely used tool for investigating the electrocatalytic activity of counter electrodes for DSSCs.

Theoretically, the Tafel curve, can be divided in three zones: low, middle and high potential. The region at low potential $(|U|<120 \mathrm{mV})$ is the polarisation zone. At middle potential (with a steep slope) is the Tafel zone. At higher potential (close to horizontal) is the diffusion zone. An example curve is presented in the Figure 3.6.

Important information can be extracted related to the catalytic activity of materials when appropriate analysis is carried out. From the Tafel zone, the exchange current density $\left(J_{0}\right)$ is obtained, and the steeper the slope the higher the $J_{0}$. An increase in the $J_{0}$ leads to a higher catalytic activity towards the triiodide reduction. It can be also calculated from Equation 3.6,

$$
J_{0}=\frac{R T}{n F R_{C T}}
$$

From the diffusion zone, the diffusion limiting current $\left(J_{\text {lim }}\right)$ is obtained. This current is limited from the ability of the ions to diffuse in the electrolyte. The higher the $J_{\text {lim }}$ the faster the diffusion of the redox couple in the electrolyte, which means 
improved catalytic activity of the counter electrode. The relation with the diffusion coefficient is given by Equation 3.7,

$$
D_{\text {diff }}=\frac{\delta}{2 n\left[I_{3}^{-}\right] F} J_{l i m}
$$

where $J_{\text {lim }}$ is the polarisation diffusion-limited current, $\delta$ is the distance between electrodes of the symmetric cell, $n$ the number of transferred electrons in the $I_{3}^{-}+2 e^{-} \rightarrow 3 I^{-}$redox reaction, $\left[I_{3}^{-}\right]$is the equilibrium concentration of triiodide ions in the electrolyte and $F$ is the Faraday constant.

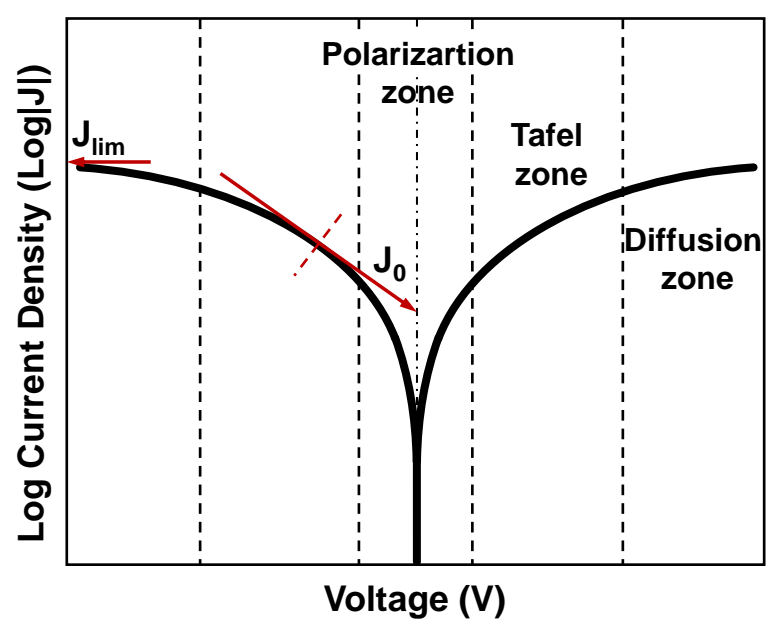

FIGURE 3.6: Tafel plot of the $J-V$ curve showing the different zones and the extracted parameters.

\subsection{Photovoltaic devices: photo-electro-chemical characteri- sation}

\subsubsection{Light source and Air Mass (Solar simulator)}

With the aim of comparing the results between different laboratories, standard test conditions were ensured for the characterisation of the solar cell devices. The photocurrent-voltage characteristics of the DSSCs were measured at 1 sun illumination $\left(1000 \mathrm{~W} / \mathrm{m}^{2}\right)$, calibrated with a KG5-filtered Silicon reference cell (Newport/Oriel, Model 91150V). A 150 W Xe-arc lamp (Newport/Oriel, model 66475150XV-R22) and the AM 1.5G filter was used to irradiate the devices. The lamp is equipped with a set of neutral density filters mounted on a filter-wheel that can be interchanged for intensity variation experiments.

\subsubsection{Current-density-Voltage curves for DSSC $(J-V)$}

$J-V$ curves were obtained in the range -0.9 to $0.2 \mathrm{~V}$ and the selected scan rate for all the samples was $100 \mathrm{mV} / \mathrm{s}$ using the potentiostat PGSTAT204 (Metrohm Autolab) or the Keithley 2450 under the same configuration. 
The standard technique for characterising the performance of solar cells is the current-density-voltage $(J-V)$ curve. $J-V$ curve is a normalisation of the current extracted from the solar cell by the area of the photoactive material. This current voltage characteristics are well described by the thermionic emission theory for Schottky heterojunctions. Figure 3.7 illustrates a typical $J-V$ curve with the characteristic parameters marked and the power output represented.

The analysis of this curve consists on the determination of the following parameters:

Short-circuit current $\left(I_{s c}\right)$ : current extracted from the cell at zero bias potential. It is function of the light intensity.

Open-circuit potential $\left(V_{o c}\right)$ : voltage generated by the cell at open-circuit conditions, i.e. when the current is equal zero.

Maximum power point ( $m p p)$ : corresponds to the coordinated of the maximum power extracted from the cell $\left(P_{m m p}=V_{m p} J_{m p}\right)$. For any given bias voltage, the power output is the product of the measured cell current and the voltage, $P(V)=I(V) V$.

Fill Factor $(F F)$ : is the ratio between the maximum power and the power obtained for short-circuit current and open-circuit voltage.

$$
F F=\frac{P_{t h}}{P_{m p}}=\frac{I_{s c} V_{o c}}{I_{m p p} V_{m p p}}
$$

Efficiency $(P C E)$ : is the ratio of the maximum power extracted to the incident power on the surface of the solar cell:

$$
P C E=\frac{P_{m p p}}{P_{\text {in }}}=\frac{P_{t h} * F F}{P_{\text {in }}}=\frac{I_{s c} V_{o c} F F}{P_{\text {in }}}
$$

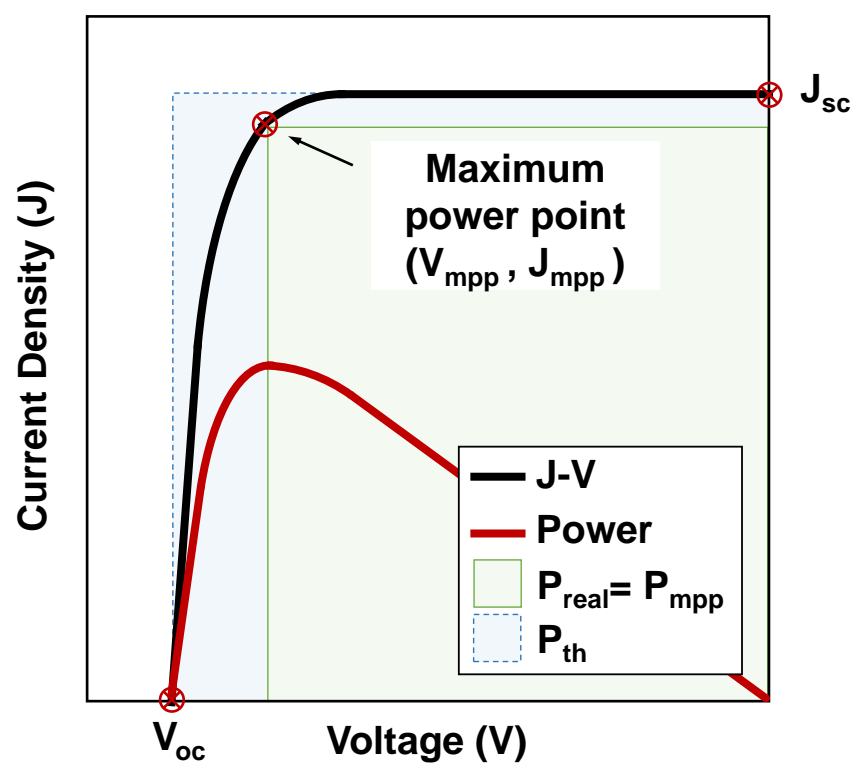

FIGURE 3.7: Solar cell $J$ - $V$ curve under light conditions, power output and main characteristic. 


\section{Series resistance characterisation}

The series and shunt resistance $\left(R_{s}\right.$ and $\left.R_{s h}\right)$ of a solar cell are important parameters that influences the $F F$, therefore, provides and idea about the losses in the device.

For the calculation of the $R_{s h}$ several method are discussed in the literature.[212] Among them, the simplest is the inverted slope of the dark current in the vicinity to zero bias voltage. Alternatively, in some cases, an online script[213] running a more precise method (Lambert W-function) has been used for the calculation of this parameter.

Also, exist several techniques that provides different accuracy over data obtained.[212, 214] Comparing these techniques, one of the most precise and robust is the light intensity variation. This technique is time-consuming and requires for the appropriate setup, thus, it is suggested to be consider for calculating the data when other more straightforward techniques presents very high error or are impossible to be performed. The analysis method is depicted in the Figure 3.8a and consist on:[215]

1. I- $V$ curves of the device are taken at different light intensities.

2. A point is marked at a fixed $\Delta I$ from the short circuit current on every curve. $\Delta I=3 m A$ was the selected value according to the ranges of intensities of the obtained $I-V$ curves.

3. The marked points are fitted with a straight line. $R_{s}$ is obtained directly from the slope of this fitting line and is given by:

$$
R_{S}=\left|\frac{\Delta V}{\Delta I_{S C}}\right|
$$

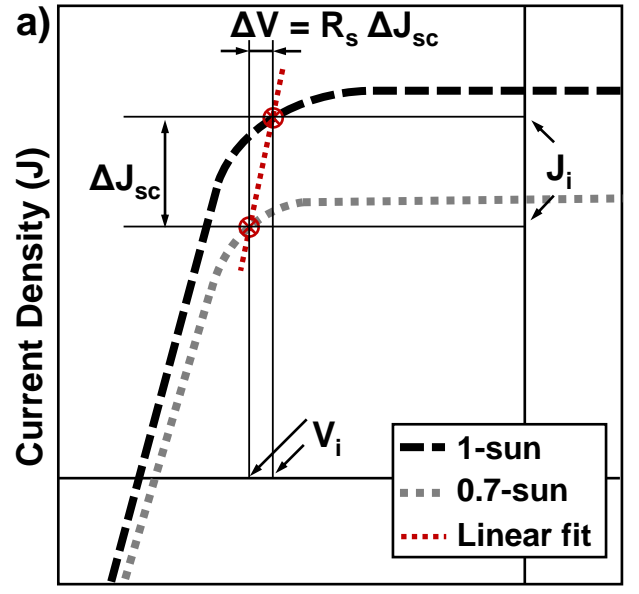

Voltage (V)

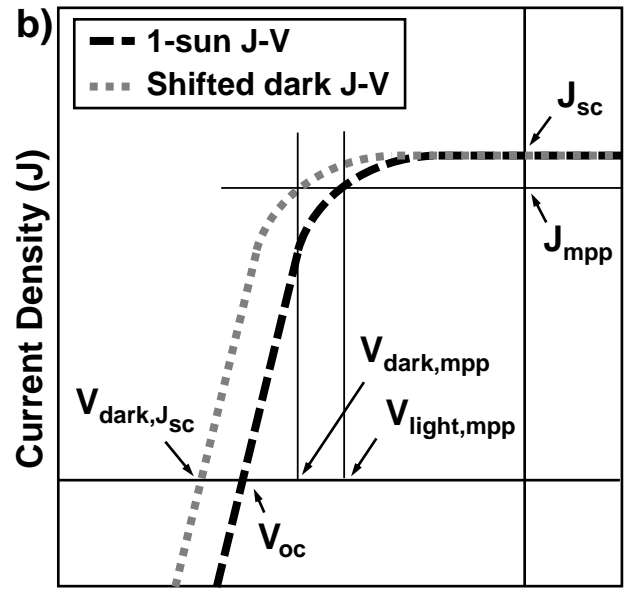

Voltage (V)

FIGURE 3.8: a) Representation of two $I-V$-curves of the same device under different illumination intensities. b) Representation of 1-sun light $I-V$ curve and $J_{S c}$-shifted dark $I-V$ curve. Adapted from Pysch et al.[214] 
An alternative accurate method to calculate $R_{s}$ is the comparison of one-sun $I-V$ curve with the translated dark curve (Figure 3.8b). The method compares the voltage variation at the maximum power point (mpp) of the 1-sun illuminated $I-V$ and the dark $I-V$, which is vertically shifted by the short circuit current density $\left(J_{s c}\right)$ value. The advantage of this method is extracting the data from a single dark-light measurement. The analysis method is depicted in the Figure $3.8 \mathrm{~b}$ and the series resistance is calculated by[214]

$$
R_{S, \text { light }}=\frac{V_{\text {dark,mpp }}-V_{\text {light }, m p p}-\left(\left|J_{s c}\right|-\left|J_{m p p}\right|\right) R_{S, \text { dark }}}{\left|J_{m p p}\right|}
$$

where $R_{S, \text { dark }}$ represents the dark series resistance which is calculated by

$$
R_{S, \text { dark }}=\frac{V_{d a r k, J_{s c}}-V_{o c}}{\left|J_{s c}\right|}
$$

\subsubsection{Cyclic Voltammetry $(C V)$}

$C V^{\prime}$ 's were performed using the potentiostat PGSTAT204 (Metrohm Autolab). Unless mention, all $C V$ curves were performed at $50 \mathrm{mV} / \mathrm{s}$.

Similar technique to the linear sweep voltammetry. Typically performed in a three-electrode cell for referencing to a baseline. The potential of the working electrode is swept between two values at a linear rate. Despite the $L S V$, when the voltage reaches the upper set potential, it is reverted and swept back to the initial potential. $\mathrm{Pt}$ wire is commonly used as reference material.

This technique is widely used in DSSC research for characterisation of dyes, redox mediators and charge transfer processes at interfaces. The redox potential, as well as interface charge transfer rates and diffusion coefficient can be estimated.

$C V$ is the standard method for measuring the redox mediator potentials. The different between anodic $\left(V_{p, a}\right)$ and cathodic peaks $\left(V_{p, c}\right)$ voltages gives the formal potential of the redox couple in the solution, also called half-way potential and defined by the equation 3.13:

$$
V_{p-p}=\frac{V_{p, a}+V_{p, c}}{2}
$$

\subsubsection{Electrochemical Impedance Spectroscopy (EIS)}

EIS measurements were performed both under dark and 1 sun illumination, at open circuit voltage biased, using a potentiostat (PGSTAT204, Metrohm Auto$\mathrm{lab}$ ) with the frequency response analyser module FRA32M. The selected range of frequency for the experiments was $0.05-10^{6} \mathrm{~Hz}$, with $10 \mathrm{mV}$ of amplitude, and 70 points per experiment. All EIS data were fitted and analysed using the Nova v1.11 software.

Electrochemical impedance technique is based on studying the response of an alternative current $(\mathrm{AC})$ signal when varying the frequency. The opposition to electronic flow (i.e. impedance, $Z$ ) is measured for a range of frequencies. It provides useful information about the different interfacial phenomena present in the system. 


\section{Chapter 4}

\section{CNTf Counter-Electrodes for DSSC}

This chapter is adapted from the work submitted to Carbon, co-authored with Dr. Juan J. Vilatela and Dr. Rubén D. Costa. All experiments were performed and analysed by the author, except the $\mathrm{CV}$ experiments related to the stability of the electrodes performed by Dr. M. Bidikoudi.

Firstly, the properties of the CNTf that make it an interesting candidate as counter-electrode and current-collector in DSSC are presented.

Secondly, the catalytic activity of the CNTf for the iodide/triiodide redox couple as a substitute of the standard Pt-CE is investigated. Moreover, new insights about the interaction between liquid electrolytes and mesoporous nanocarbon membranes (e.g. the CNTf), are elucidated by electrochemical impedance spectroscopy. Some preliminary results towards identifying catalytic centres about the nature of the catalytic activity are summarised. Finally, the interaction between the electrolyte and its redox mediator with the CNTf and its effects on stability and reactivity, are analysed.

The third section includes the integration of the CNTf in the full DSSC device. The CNTf features are optimised, leading to higher efficiency than reference Pt-CE material. One of the best efficiencies in carbon-based DSSC is demonstrated after assembly a full device under the previously reported optimum standard conditions. In summary, the demonstration of the CNTf serving as a catalytic, flexible, electrically conductive counter-electrode material is presented in this section.

\subsection{CNT fibre properties: motivation for DSSC counter-electrodes/current-collectors}

CNTf materials are used as current-collector in many different applications such as sensors, supercapacitors, or batteries. Their high electrochemical stability and electronic conductivity make them attractive CE in DSSC. In this chapter, the attention is focused in this application of CNTf as CE for DSSC. These devices are promising solar-energy conversion schemes that have achieved conversion efficiencies (PCE) as high as $13 \%$ at laboratory scale[82] - i.e., photoactive area of $<0.5 \mathrm{~cm}^{2}$, and DSSC modules with PCE of around 7-8 \%.[216-218]

In spite of their high efficiency, there are still several challenges towards the real commercialisation of DSSC technology. Some of these challenges are related to the counter-electrode material, typically Platinum. Firstly, the limited worldwide supply and its increasing expensive price; secondly, the stability of the $\mathrm{Pt}$, which 
is easily corroded by iodide-based electrolytes or poisoned by different chemical compounds or gases.[219] Also, Pt-based counter-electrode materials, often require a conductive substrate as support, which acts as current-collector. Therefore, currentcollector materials are another important part of this electrochemical devices, where the catalyst is supported in.

For the present study CNTf consisting on multiwalled CNTs have been used, which are synthesised by CVD method as described in the Chapter 2 "Background". The as-produced CNTf has an impurity content of approximately 10-15\%, consisting of Fe nanoparticles used as catalyst among other residue materials[56, 192, 220] (see Figure 4.1a,b). By thermogravimetrical analysis of a pristine sample (see Figure 4.1c), the composition is determined as: a) organic material with a considerable high stability in air up to $450{ }^{\circ} \mathrm{C}$, and b) a solid residue after firing up to $900{ }^{\circ} \mathrm{C}$.

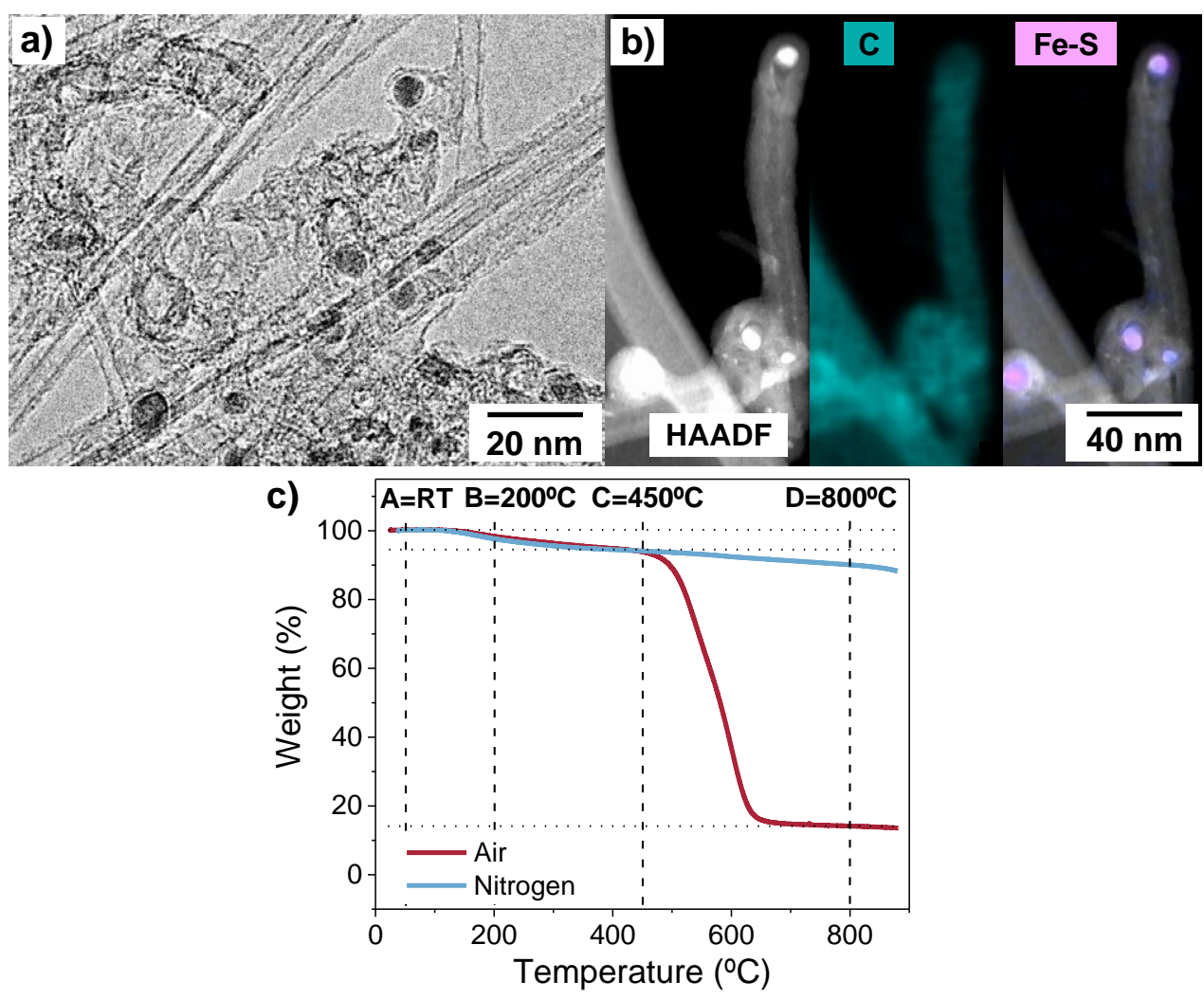

FIGURE 4.1: a) CNTf TEM image showing CNTs and catalyst nanoparticles. b) Compositional EDX mappings obtained by TEM with HAADF detector. (Image courtesy of V. Reguero.) c) Thermogravimetrical analysis of the CNTf in air (red line) and nitrogen (blue line).

Analysing more in detail the decomposition, for a standard sample of CNTf, it is possible to differentiate four regions. Four key temperatures are highlighted:

- room temperature (RT), at which the as-made CNTf is used for most of the applications, is marked $(A)$;

- $200{ }^{\circ} \mathrm{C}(B)$ is the temperature at which the water is completely removed from the surface of the CNTf; 
- $450{ }^{\circ} \mathrm{C}(\mathrm{C})$ corresponds to the onset of a strong lost in weight in the material (air conditions);

- $800{ }^{\circ} \mathrm{C}(D)$ is the temperature at which the solid residue is measured.

In air conditions, the weight loss between temperatures $A B$ is in the range of $2 \%$. Between temperatures $B C$, the degradation of the non-graphitic carbon (sp3) is produced, it corresponds to $3 \%$ of the initial weight. The strong mass reduction measured between $C D$, corresponding to $80 \%$ of the weight, is the degradation of the graphitic carbon (CNTs).[192] The final solid residue is composed by Fe-S inorganic nanoparticles used as catalyst during the synthesis. Notice that, under inert atmosphere $\left(\mathrm{N}_{2}\right.$ or $\left.\mathrm{Ar}\right)$, the weight loss is lower than $10 \%$ at $900{ }^{\circ} \mathrm{C}$, indicating the high thermal stability of the pristine material. The non graphitic carbon is formed at lower temperatures during the synthesis of the CNTf, but also, it is adsorbed contamination over the CNTf from the environment.
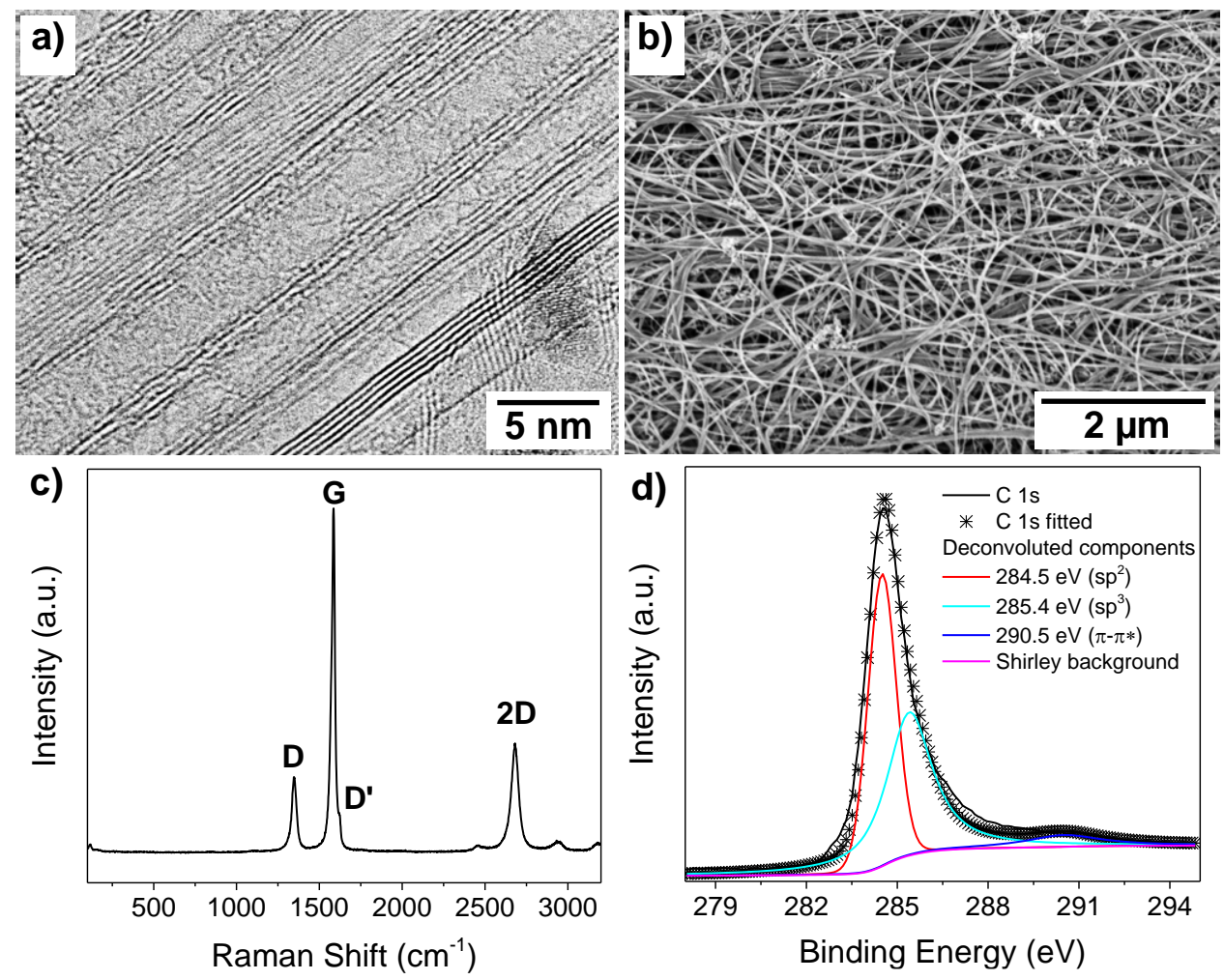

FIGURE 4.2: Structure of CNTf electrodes: a) High resolution transmission electron micrograph, evidencing the high degree of graphitisation of the few-layer CNTs. b) Scanning electron micrograph showing the network structure of interconnected CNT bundles giving rise to a large porosity. c) 10x average Raman spectrum representative of the material and d) XPS spectra of CNTf as proof of the high purity of the pristine CNTf material.

An exceptional characteristic of the CNTf is its structure comprising high conductivity, high porosity and high crystallinity arising from the association of CNTs into bundles (see Figure 4.2a,b). This high crystallinity can be observed, for example, as a strong intensity in X-ray scattering from (002) graphitic basal planes.[221] These 
properties stem partly from the constituent CNTs, consisting predominantly of fewlayer CNTs (1-5 layers), which provides high surface areas.[58] Also, the Raman spectrum (Figure 4.2c) shows that the constituent nanotubes are highly graphitic, as indicated by the low $D / G$ ratio $>0.3$ and strong resonance of the $G$ and $2 D$ bands. The asymmetric $G$ peak and its shoulder at low frequencies $\left(G^{-}\right)$are indicative of a few layers CNTs. Additionally, the synthesis at $1250{ }^{\circ} \mathrm{C}$ in $\mathrm{H}_{2}$ atmosphere produces constituent CNTs with a high perfection in terms of $s p 2$ bond conjugation, as noted in X-ray photoelectron spectroscopy (XPS) measurements that indicate a strong plasmonic band from the delocalised $\pi$ electron cloud - Figure 4.2d. Moreover, the CNTf has shown very high electrochemical stability in ionic liquids and aqueous electrolytes,[58] which can be traced back to the high degree of graphitisation of the constituent CNTs by HRTEM, Raman spectroscopy and XPS (Figure 4.2).

A key feature of this material is the high length of the CNTs constituents from $100 \mu \mathrm{m}$ to $1 \mathrm{~mm}$ scale,[222] and the high degree of graphitisation. This has direct influence on the high conductivity typically reported for this material.

Individual layers of CNTf were stacked and measured by $I-V$ two or fourprobe technique depending on the experiment (see inset in Figure 4.3, further details in Chapter 3 "Experimental Techniques"). Four-probe measurement provides more reliable values of the material properties because it avoids the contribution of the contact resistance. A control experiment is presented comparing the same CNTf sample measured with 2 and 4 probes (see Figure $4.3 b$ ).

Longitudinal conductivities have been obtained as high as $4-5 \times 10^{5} \mathrm{~S} \mathrm{~m}^{-1}\left(\rho_{L}=\right.$ $\left.1.8 \times 10^{-5} \Omega m\right)$, for low thickness samples, and $9 \times 10^{5} \mathrm{~S} \mathrm{~m}^{-1}\left(\rho_{L}=2.2 \times 10^{-5} \Omega m\right)$, when the thickness is increased (see Figure 4.3a). $\approx 200 \mathrm{~nm}$ is the average thickness for a CNTf monolayer obtained by profilometry measurements of the different samples. Normalising by the area of the measured sample ( $4 \times 4 \mathrm{~mm}$ approximately), the sheet resistance $\left(R_{s q}\right)$ goes from $90 \Omega / s q$ for the monolayer CNTf to $3.5 \Omega / s q$ when 32 layers of CNTf are stacked.

Moreover, the maximum current density has been obtained as $6.7 \times 10^{3} \mathrm{~mA} \mathrm{~cm}^{-2}$ for 12 stacked layers of CNTf (sample presented in the inset of Figure 4.3b). This characteristic has been determined by $I-V$ curve measurement until the short-circuit of the measurement due to the suddenly burnt (overheating) of the CNTf filament by Joule heating effect. This value is in the range of the reported values in literature for similar material.[55] The maximum current density will be crucial for determining the maximum current of the harvesting devices when very thin CNTf currentcollectors are used.

Due to the fibril nature of the CNTf, a sheet formed by these fibres might present some anisotropic properties. In this context, the longitudinal and transversal conductivities (in-plane) may differ. Since CNTf sheets used in this work are unidirectional, i.e. CNTfs collected under the same orientation, the longitudinal conductivity is expected to be higher in the $c$-axis direction of the fibres, and to show additional resistances in transversal direction. A rough comparison by two-probe measurement was performed resulting in a transversal conductivity $25 \%$ lower than the longitudinal one. 

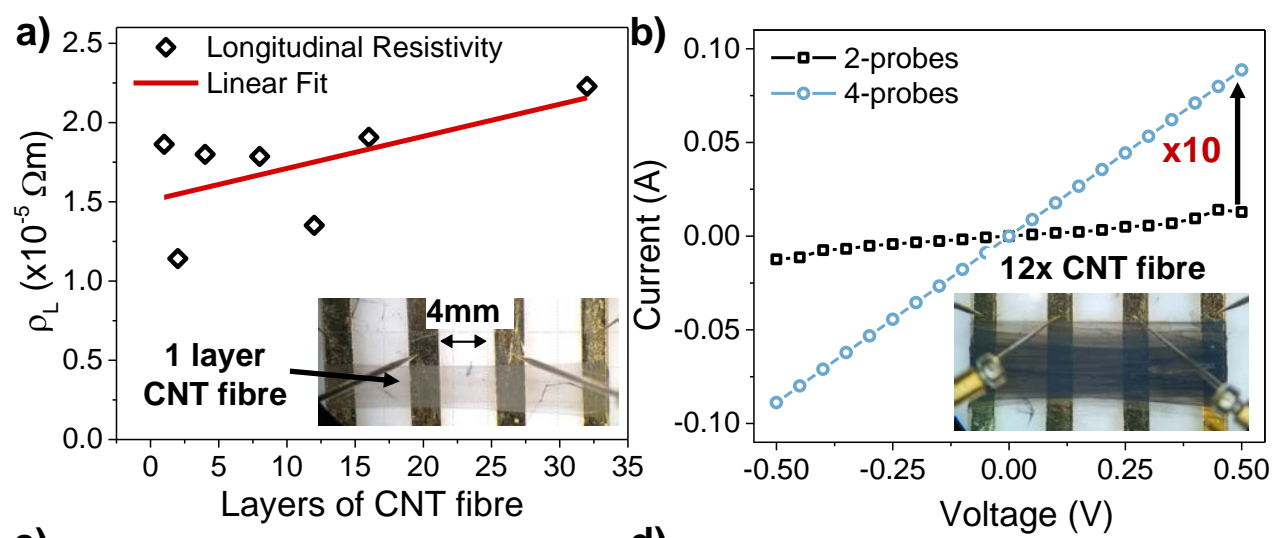

c)

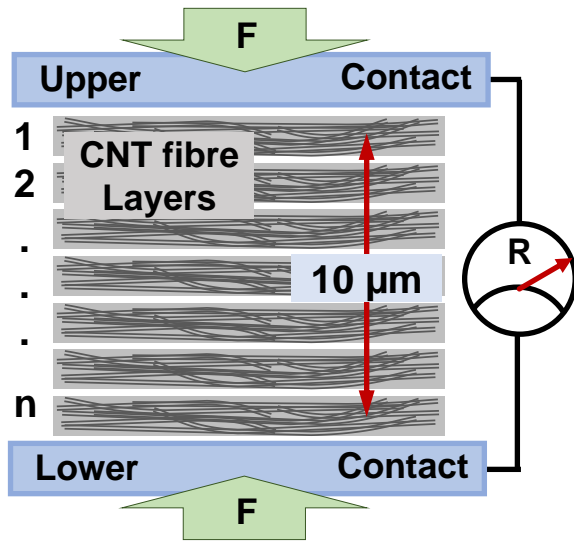

d)

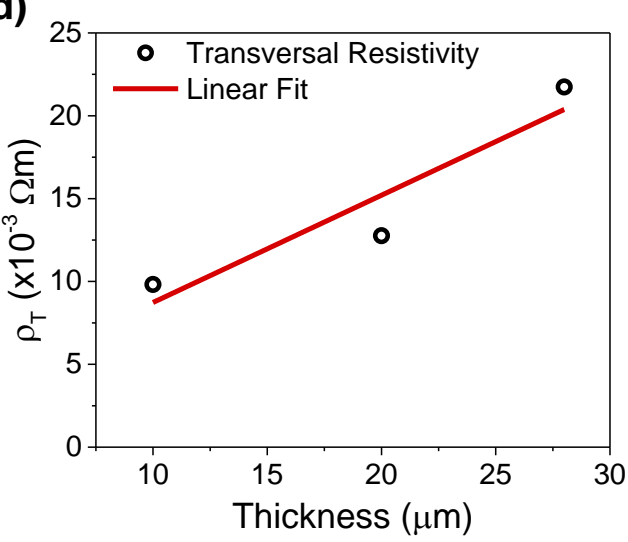

FIGURE 4.3: Electrical characterisation of the CNTf: a) Four-probe resistivity of the CNTf as a function of the number of stacked layers. b) Comparison between $I-V$ curves acquired by two- (black) and fourprobes (blue) technique. c) Schematic view of the $z$-conductivity measurement. d) z-conductivity vs CNTf electrode compression (thickness).

When stacking several layers of CNTf, also additional contact resistances are introduced between them. The z-conductivity (through the thickness of the material) was measured by two probe-technique due to the the geometry of the sample. This experiment consists on measuring the resistance between two metallic pistons pressing top and bottom surfaces of the CNTf material. A schematic of the measurement is shown in the Figure 4.3c. The z-conductivity has been estimated as $\mathrm{ca} .50 \mathrm{~S} \mathrm{~m}^{-1}$ for $28 \mu \mathrm{m}$ thickness of CNTf, and $100 \mathrm{~S} \mathrm{~m}^{-1}$ when force is applied, which results in a thickness of $10 \mu \mathrm{m}$. Notice that when the CNTf is compressed the interconnection between the stacked layers increase and the interfacial resistance decreases. In contrast to previous references,[223] no recovery or hysteresis have been observed during this measurements related to piezoresistive effects, probably due to the low thickness of the material (circa $10 \mu \mathrm{m}$ ). The longitudinal conductivity is at least one order of magnitude higher than z-conductivity.

This can be explained by the in-plane orientation of the CNTs. The carrier movement along the CNTs finds very low resistance, besides this is favoured by the high length of the CNTs. In plane conduction is only disrupted when carriers jump from one CNT to the next one by tunneling effect. On the contrary, when conducting in z-direction, there is almost no CNTs with this orientation due to the sample preparation procedure, and in practice, the length of the CNTs is barely not 
affecting the conductivity in this direction. Many questions remains open about the conducting mechanism and the physics behind the CNTf, but this is out of the scope of this thesis.

Note that the standard transparent conductive oxides current-collectors for DSSC's typically ranges from 10 to $15 \Omega / s q$ (for FTO or ITO substrates). Therefore, the longitudinal conductivity of the CNTf is sufficient to use this material as current-collector.

For DSSC applications, the conductivity of the substrate enhances the electron transfer between the current-collector and the counter-electrode material. Similarly, adhesion between both materials influences the performance of the device. Commonly, some components are added to the CE to improve its adhesion. For instance, carbon materials are commonly dispersed in organic binder (polymers) to increase the adhesion between the substrate and the $\mathrm{CE}$, however, this typically increases the resistivity and blocks the porosity of the catalytic material.[224] In this sense, it is of interest to consider the CNTf material as both CE and CC. Among other benefits, this material reduces interfacial resistances.

A further relevant property of the CNTf to consider as CE material is their specific surface area (SSA), which for the sample used in this work is $250 \mathrm{~m}^{2} \mathrm{~g}^{-1}$ [225] (this will be later explained in detail).

\subsection{Study of the catalytic activity of CNTf: symmetric elec- trochemical cells}

\subsubsection{Electrolyte diffusion and reactivity at the CNTf electrode}

The combination of properties of nanocarbons strongly suggest that these materials can be considered as attractive counter-electrodes/current-collectors for planar DSSC's if the catalytic behaviour for the regeneration of the electrolyte redox mediator is adequate.

Recent reviews have summarised the different types of materials investigated to replace Pt, among which, carbon-based CEs clearly stand out.[97, 226-228] For instance, carbon nanotubes (CNTs), [105, 229, 230] carbon nanohorns, [231, 232] carbon fibres,[233] carbon black,[106] mesoporous carbon,[234] graphite and graphene, $[107,235,236]$ have been demonstrated as good CEs materials for DSSCs. Among them, CNT films deposited from dispersions via spray-coating and spin-coating, that are the closest materials to the CNTf, have achieved PCE of 6-7\%.[230, 237]

This work presents a study on the intrinsic catalytic activity of the CNT fibre towards the $I^{-} / I_{3}^{-}$redox system. Although there are other redox based electrolytes used with carbon, for instance, cobalt complexes,[87-89] in this chapter the attention is focused on the $I^{-} / I_{3}^{-}$mention system. This redox mediator, in combination with the N719 ruthenium sensitiser, provides the most well known figures of merit of DSSC devices.[200] For this reason, this system is widely used for validating new materials and performing accurate comparisons.

First of all, a reproducible method for preparing the CNTf symmetric devices was developed. Extensive optimisation was required to assemble symmetric devices 
(substrate | CNTf $\mid$ electrolyte | CNTf $\mid$ substrate) without shortcircuits and other artefacts. The tested substrates consist on glass, flexible polymer films or transparent conductive substrates (i.e., glass+fluorine tin oxide - FTO). Initial experiments following the standard procedure with this material (see Chapter 3 "Experimental Techniques"), resulted in a high percentage of the devices shortcircuited. The different approaches to increase the CNTf symmetric cells reliability were: i) decreasing the area of the devices; ii) increasing the distance between the electrodes, i.e. thicker separators (sealant); and iii) increasing the viscosity of the electrolyte (reducing or eliminating the amount of solvent).

A schematic of the symmetric cell indicating the components involved and some real cells are presented in the Figure 4.4.

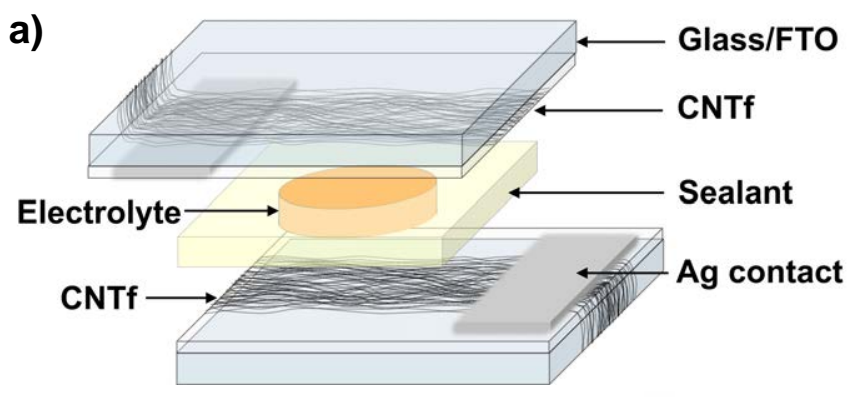

b)

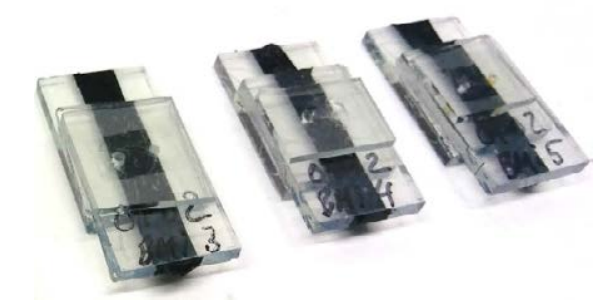

FIGURE 4.4: a) Schematic and b) digital photograph of CNTf symmetric cells used for the study of the catalytic activity of the CE material.

The final procedure for preparing symmetric cells based on CNTf consisted on:

1. The CNTf, initially collected on paper substrate, is cut into strips of $5 \times 30 \mathrm{~mm}$. One of these strips of CNTf is manually transferred to the glass, FTO-glass or PET substrate and densified with solvent. The resulting FTO/CNTf electrode is used directly as a counter-electrode.

2. The symmetric cells are prepared by assembling two counter-electrodes of CNTf (or two platinised-FTO substrates for the reference) with a $100 \mu \mathrm{m}$ thickness thermoplastic separator (Surlyn), also used as sealant. When this thermoplastic is hot-pressed, the melted polymer acts as an adhesive and ensures the stability of the device. It prevents leakage of the electrolyte and thus enables the study of the device under the same conditions during a time span of several days.

3. 1-butyl-3-methylimidazolium iodide (BMII) with iodine content between $0.01-$ $0.2 \mathrm{M}$ was used as electrolyte (no content of solvent) and injected in the cells through two pre-drilled holes of $1 \mathrm{~mm}$ diameter. 
4. The pre-drilled holes are finally sealed with another thermoplastic-covered $1 \mathrm{~cm}^{2}$ glass piece heated at $160^{\circ} \mathrm{C}$.

Notice that the large separation between electrodes $(100 \mu \mathrm{m})$ and the high viscosity electrolyte were chosen to avoid short-circuits between CNTf electrodes.

The catalytic activity in symmetric cells was studied using linear sweep voltammetry (LSV) and electrochemical impedance spectroscopy (EIS). This configuration enables to study electrochemically the catalytic process $I^{-} \rightarrow I_{3}^{-}$. Cyclic voltammetry is typically used in DSSC research for characterisation of dyes, redox mediators and charge transfer processes between materials. The redox potential, as well as interface charge transfer rates and diffusion coefficients can be estimated from such measurements. Two different configurations have been studied and compared: glass | CNTf | electrolyte | CNTf | glass (CNTf-cell) vs FTO $|\mathrm{Pt}|$ electrolyte $|\mathrm{Pt}| \mathrm{FTO}$ (Pt-cell).

A cyclic voltammetry curve of the CNTf-cell with electrolyte containing $0.2 \mathrm{M}$ of iodine is presented in the Figure 4.5a. With this high iodine concentration the possible catalytic reaction is observed clearer. The positive and negative peaks can be assigned to the oxidation and reduction of $I_{3}^{-} / I^{-}$and $I_{2} / I_{3}^{-}$, respectively, confirming the redox capability of the CNTf electrode. From this curve, the difference between the potential value of the oxidation peak and the reduction peak $\left(E_{p p}\right)$ of CNTf is $430 \mathrm{mV}$ is measured, which is slightly smaller than the reported for Pt (ca. $500 \mathrm{mV}$ ).[238] Smaller $E_{p p}$ value reveals that this material can provide facile electron transfer to and from the electrolyte.

The electrocatalytic activity of the CNTf electrode towards the $I^{-} / I_{3}^{-}$redox couple was also evaluated in more representative electrolyte conditions, corresponding to BMII ionic liquid with $I_{2} 0.03 \mathrm{M}$, which corresponds to the typical concentration used in standard DSSC electrolyte. As can be observed in the figure $4.5 \mathrm{~b}$ is still possible to clearly resolve the redox peaks. The peak maximums for the reduction of $I^{-} / I_{3}^{-}$are located at $0.09 \mathrm{~V}$ (Pt-cell) and $0.22 \mathrm{~V}$ (CNTf-cell). It was found that CNTf$\mathrm{CE}$ exhibited a higher absolute current peak and more positive reduction peak potential than the $\mathrm{Pt}-\mathrm{CE}$. The porous structure of CNTf electrode provides much larger surface area and a larger quantity of catalytic active sites, leading to a higher current density than the platinised FTO electrode.

To further evaluate the behaviour of the CNTf-CE the iodide content was varied $(0.05-0.2 \mathrm{M})$. The characteristics of the symmetric cells are extracted and presented in the Table 4.1. The difference in the cathodic potential close to $0.1 \mathrm{~V}$ for Pt-CE and $0.3 V$ for the CNTf is attributed to the nature of the catalytic centres. It indicates, the CNTf-CE needs an extra input of energy for oxidising the redox species of the electrolyte at the counter-electrode relative to $\mathrm{Pt}$. The shift in position of the cathodic peaks can also be due to the concentration of mediator species in the electrolyte. The CNTf is likely to generate a different ionic environment, as it will be confirmed later, shifting the effective potential of the electrode surface.

The steady-state polarisation diffusion-limited current $\left(J_{\text {lim }}\right)$ can be measured when the potential is far past from the cathodic or anodic peaks of the reaction. At this point, the electron transfer is so favourable that electrons transfer to the redox specie (molecule) as soon as they approach the electrode. Thus, the rate of electron transfer (current) depends only on the rate at which the redox species can diffuse to or from the electrode. For $0.05 \mathrm{M} \mathrm{I}_{2}$, the $J_{\text {lim }}$ for CNTf-cells and Pt-cells are $2.4 \times 10^{-3}$ and $0.96 \times 10^{-3}$, respectively. While for $0.2 \mathrm{M} \mathrm{I}_{2}, 5.4 \times 10^{-3}$ and $2.9 \times 10^{-3} \mathrm{~mA} \mathrm{~cm}^{-2}$, 
respectively. This indicates the ease of charge transfer $(C T)$ from the CNTf to the electrolyte. The increase in $J_{\text {lim }}$ is attributed to the high surface area of the CNTfs. In presence of CNTf material, the electrolyte/CNTf interface is much higher compared to the Pt electrode. This interfacial area is estimated from the area of the electrode and the surface area of the material, and for the CNTf electrode presents 3000-times higher area than the $\mathrm{Pt}$ (in a standard $0.2 \mathrm{~cm}^{2}$ electrode). Due to the high surface area of the CNTf, a chemical composition gradient is expected to appear at the surface of the CNTf electrodes. This interface further enhances the ionic diffusion between electrodes due to polarisation forces resulting from the accumulation of the redox species, as it has been reported before for other nanocarbons.[154, 239, 240]

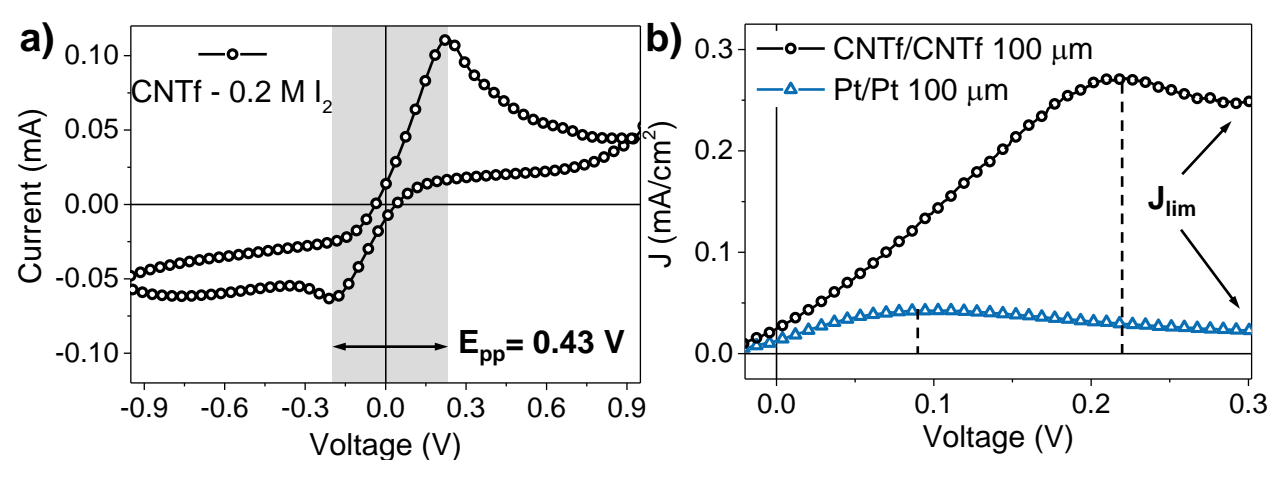

FIGURE 4.5: Demonstration of the catalytic activity of the CNT fibre. a) $C V$ experiment of a CNTf symmetric cells with $0.2 \mathrm{M}$ iodide. b) $L S V$ curves of Pt- and CNTf-cells with 0.03 M iodide in BMII electrolyte.

Moreover, knowing the $J_{\text {lim }}$, the diffusion coefficient $\left(D_{\text {diff }}\right)$ is also determined from the $L S V$ measurements. The $D_{\text {diff }}$ in presence of CNTf $\left(0.2 \mathrm{M} \mathrm{I}_{2}\right.$ concentrations) is calculated as $D_{I_{3}^{-}}=6.2 \times 10^{-11} \mathrm{~cm}^{2} \mathrm{~s}^{-1}$ while for Pt electrode is $D_{I_{3}^{-}}=$ $0.21 \times 10^{-11} \mathrm{~cm}^{2} \mathrm{~s}^{-1}$ according to equation 4.1. The slow diffusion is due to the quasisolid state electrolyte nature - solvent free. But, the important finding is the increased diffusivity of the species in the CNTf system. It will have a direct impact over the device performance. During the regeneration of the dye molecules in DSSC, the redox species in the electrolyte have to diffuse between anode and cathode. The higher the diffusivity of the species, the better the regeneration of the dye molecules.

$$
D_{\text {diff }}=J_{\lim } \frac{\delta}{2 n\left[I_{3}^{-}\right] F}
$$

where $J_{\text {lim }}$ is the polarization diffusion-limited current, $\delta$ is the distance between electrodes of the symmetric cell, $n$ the number of transferred electrons in the $I_{3}^{-}+2 e^{-} \rightarrow 3 I^{-}$redox reaction, $\left[I_{3}^{-}\right]$is the equilibrium concentration of triiodide ions in the electrolyte and $F$ is the Faraday constant.

All the extracted parameters from the LSV measurements related to the catalytic activity of the CNTf material are summarized in the Table 4.1. 
TABLE 4.1: Obtained parameters from $C V$ and $L S V$ for symmetric cells.

\begin{tabular}{|c|c|c|c|c|c|c|}
\hline \multirow{2}{*}{$\begin{array}{l}\text { Iodide } \\
\text { Concentration } \\
{[M]}\end{array}$} & \multicolumn{2}{|c|}{$\begin{array}{l}E_{p, c} \\
{[\mathrm{~V}]}\end{array}$} & \multicolumn{2}{|c|}{$\begin{array}{c}J_{\text {lim }} \text { at } 0.7 \mathrm{~V} \\
{\left[\mathrm{x} 10^{-3}{\left.\mathrm{~mA} \mathrm{~cm}^{-2}\right]}^{-2}\right.}\end{array}$} & \multicolumn{2}{|c|}{$\begin{array}{c}D_{I_{3}^{-}} \\
{\left[\times 10^{-11} \mathrm{~cm}^{2} \mathrm{~s}^{-1}\right]}\end{array}$} \\
\hline & CNTf & $\mathrm{Pt}$ & CNTf & $\mathrm{Pt}$ & CNTf & $\mathrm{Pt}$ \\
\hline 0.05 & 0.28 & 0.09 & 2.43 & 0.96 & 6.2 & 0.21 \\
\hline 0.2 & 0.32 & 0.12 & 5.46 & 2.92 & 3.5 & 1.75 \\
\hline
\end{tabular}

More than 150 cyclic voltammetry cycles has been performed on a CNTf symmetric device to confirm the electrochemical stability of the material as counterelectrode/current-collector in presence of the $I^{-} / I_{3}^{-}$electrolyte. Besides, this experiment rules out possible false conclusions due to some artefacts from individual curves. The high stability of the material under the applied voltage is demonstrated in presence of the redox couple at least in the analysed interval. The $C V$ results and analysis are presented in the Figure 4.6.
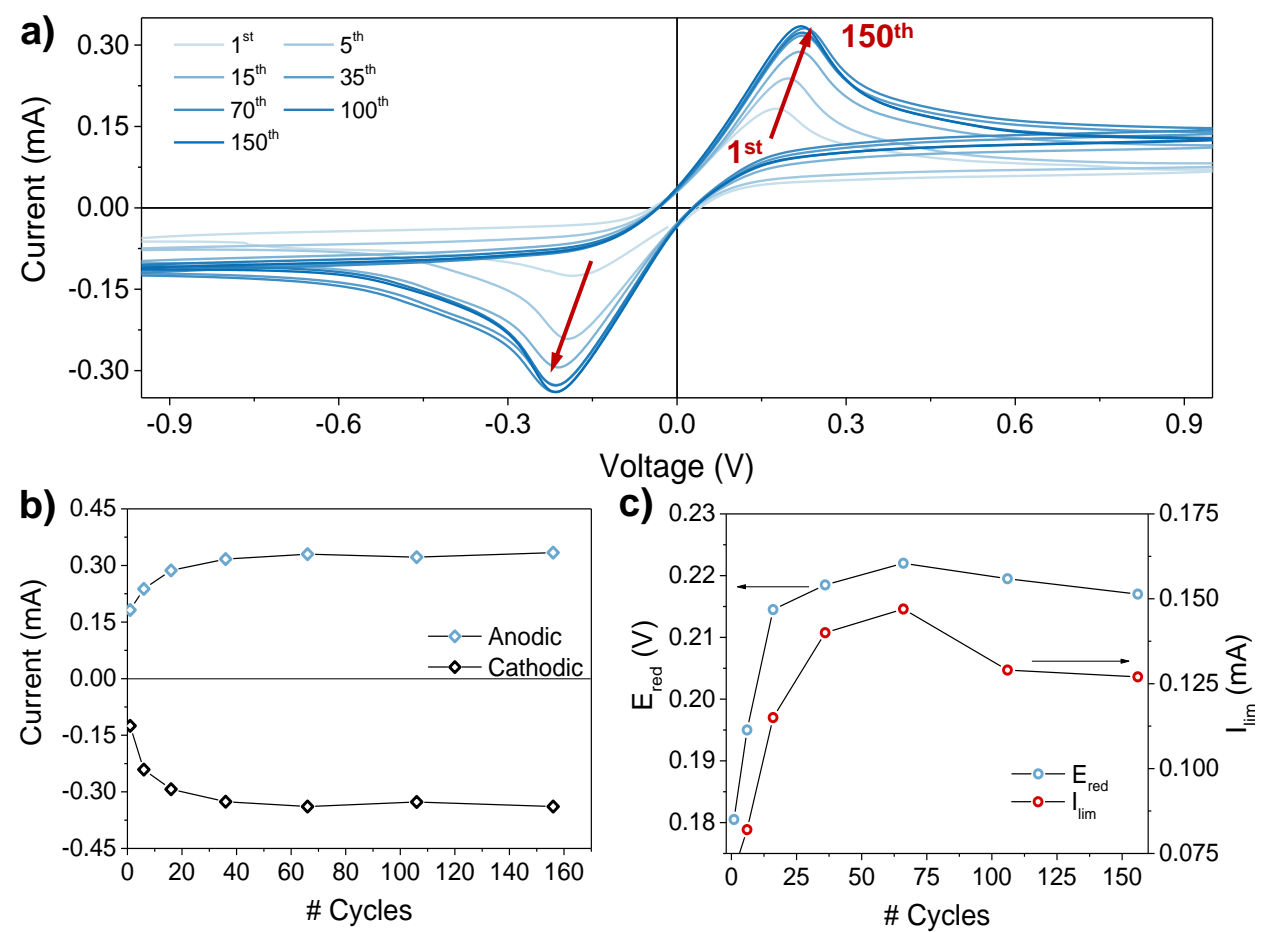

FIGURE 4.6: a) $C V$ curves showing the electrochemical stability of the CNTf-CE after the initial cycles. b) Anodic and cathodic currents and c) diffusion limiting current density and reduction potential evolution extracted from the $C V$ experiment.

The negative peak around $-0.2 \mathrm{~V}$ is assigned to the reduction of triiodide $I_{3}^{-}+2 e^{-} \rightarrow 3 I^{-}$, and the anodic peak that appears at positive voltage represents the reverse reaction. There is an increase of $c a .50 \mathrm{~mA}$ in the diffusion limiting current during the initial 20-30 cycles. After that, it achieves a stable performance showing $I_{\text {lim }}=0.11 \mathrm{~mA}$. The reduction potential of the reaction $\left(E_{r e d}\right)$, determined as mid-point between anodic and cathodic peaks, is stable after the initial ten cycles, reaching a value of $E_{r e d}=0.21 \mathrm{~V}$. This experiment confirms the stability of the 
CNTf electrode and establish the reduction potential and diffusion limiting current of CNTf electrodes, which are important parameters for characterising the behaviour of counter-electrodes for DSSCs.

The high catalytic activity of the CNTf has been demonstrated in the previous results, but still the chemical catalytic reaction in presence of this material is not fully understood. Still further fundamental investigation about the number of reactions involved, the formed intermediate species or the nature of catalytic centres, must be performed.[241]

The mesoporous nature of this material, leads to a huge interface interacting with the electrolyte. The active sites (or catalytic centres) where the catalytic reaction takes place can be assumed to be proportional to the surface of the CNTf, which is 3000 times higher than for Pt on other planar substrates.

The high surface area, in addition to a different chemical interaction between iodide species and the CNTs, will produce a higher concentration of triiodide species in the electrolyte. This different chemical interaction between iodide species and the CNTs is further developed in the next paragraphs.

Some insights into the catalytic process can be inferred from characterisation of the electrolyte species in the vicinity of the electrolyte. The evolution of the iodide electrolyte in presence of CNTf as function of time was followed by Raman spectroscopy and presented in the Figure 4.7. These samples have been recovered from freshly prepared an measured devices under same circumstances, i.e., subjected to the standard device LSV and EIS characterisation set of experiments. After the time indicated for each sample, the device was disassembled and the Raman spectrum of the $\mathrm{CE}$ in presence of the electrolyte was adquired. The polyiodides/triiodide ratios $\left(I_{m}^{-} / I_{3}^{-}\right)$were calculated from the intensities of the peaks at 142 and $110 \mathrm{~cm}^{-1}$ for a $0.1 \mathrm{M} \mathrm{I}_{2}$ concentration in BMII electrolyte and the trend is presented as inset.

The concentration of polyiodide species in the electrolyte rapidly increases in presence of the CNTf as function of the elapsed time. This experiment confirms an increase of the local concentration of $I_{3}^{-}$species at the CNTf/electrolyte interface that is possibly related with the reduction potential in presence of the CNTf. This result will have important implications over the characteristic of the device: for the short circuit current, because it is directly related with the ionic conductivity of the electrolyte; and for the open circuit voltage, because it is directly related with the redox potential of the redox couple.

It has been reported before the increase of triiodide and polyiodides content in presence of CNTs.[141] There is discrepancy in this matter, because the lower content of polyiodides in relation to triiodide has also been reported in presence of CNTs/electrolyte dispersions.[140] Some consideration must be taken with this comparison because of the unique morphology of the CNTf material. It is not the same the interaction between electrolyte and a carbonaceous membrane material, than the interaction between dispersed nanocarbons in electrolyte. There are for instance variations between crucial parameters of the electrolyte, e.g. the viscosity, which has a strong influence over the ionic conductivity of the electrolyte. As it has been demonstrated in previous works, the higher the viscosity of the system, the poorer would be the collisions of ionic species, giving rise to a smaller generation of polyiodides.[242] 
In a DSSC, the $I_{3}^{-}$are the charge limiting species in the electrolyte, and the diffusion of these ions affect the current of the device.[139] Moreover, increasing the interaction between the electrolyte and the catalytic material in a mesoporous membrane, further decreases the ionic diffusion length of the redox species. Other studies focused in electrolyte doping with carbon nanoforms also report an increment in polyiodides species[141, 155, 163] that are responsible of the increase in the ionic conductivity of electrolyte.[160] The consequence is a higher steady-state diffusion limited current $[146,160]$ by increasing the charge transport via Grotthus-type mechanism.[134] Worth to mention that this trend in polyiodides formation was not observed in presence of Pt-CE.

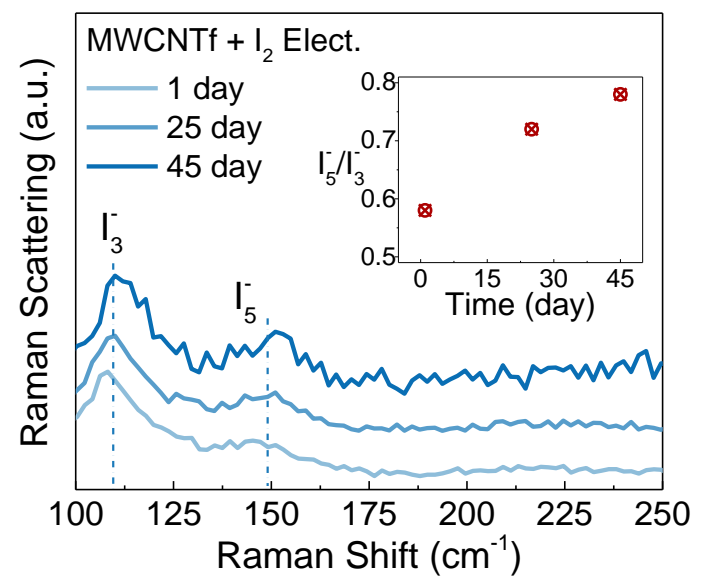

FIGURE 4.7: Triiodide/pentaiodide Raman signal in presence of CNTf electrode with high content of $I_{2}$ as function of time. Triiodide/pentaiodide ratio presented as inset.

Several authors report the enhancement in electrical conductivity by chemically iodine doping of CNTs.[243, 244] They report that, this CNTs p-type doping[245] is produced by intercalation and superficial deposition of iodine atoms that forms $I_{3}^{-}$and $I_{5}^{-}$molecules.[246] A strong interaction has been confirmed in CNT ropes subjected to $\mathrm{I}_{2}$ vapour at moderate high temperature, which according to XRD produce SWCNT lattice distortion due to intercalation.[244] Polyiodides $\left(I_{3}^{-}, I_{5}^{-}\right)$ superficial interaction also present characteristic resonance peaks by Raman spectroscopy located at 117 and $154 \mathrm{~cm}^{-1}$ respectively (under $514 \mathrm{~nm}$ excitation).[245]

The Raman spectra of CNTf in presence of $0.03 \mathrm{M}$ iodide electrolyte - after several months of stabilisation time, is presented in the Figure 4.8a. Raman spectra of the same sample after thoroughly washing with solvent and of the pristine material are also shown for comparison.

After washing the electrodes, the CNT fibre still presents an intense signal of triiodide molecules, attributed to the CNTf doping. According to previous reports, the triiodide might be superficially adsorbed or intercalated between graphite layers, but still some experiments for confirmation of the precise mechanism are needed. A small electrical p-doping effect over the CNTs can be observed by upshift of $c a .2$ and $1 \mathrm{~cm}^{-1}$ of $G$ and $2 D$ peaks-centre respectively, consistent with previous reports.[247] However, it is unclear if the single exposure of CNTfs leads to intercalation, which would have implications for diffusion. The results shows a 
very strong interaction between $I_{m}^{-}$and CNTf. Although there is no established relation between the CNTf possible doping and the catalytic behaviour of the CNTf, there is a clear evolution of the electrolyte and CNTf electrode over time and this phenomenon needs further investigation.
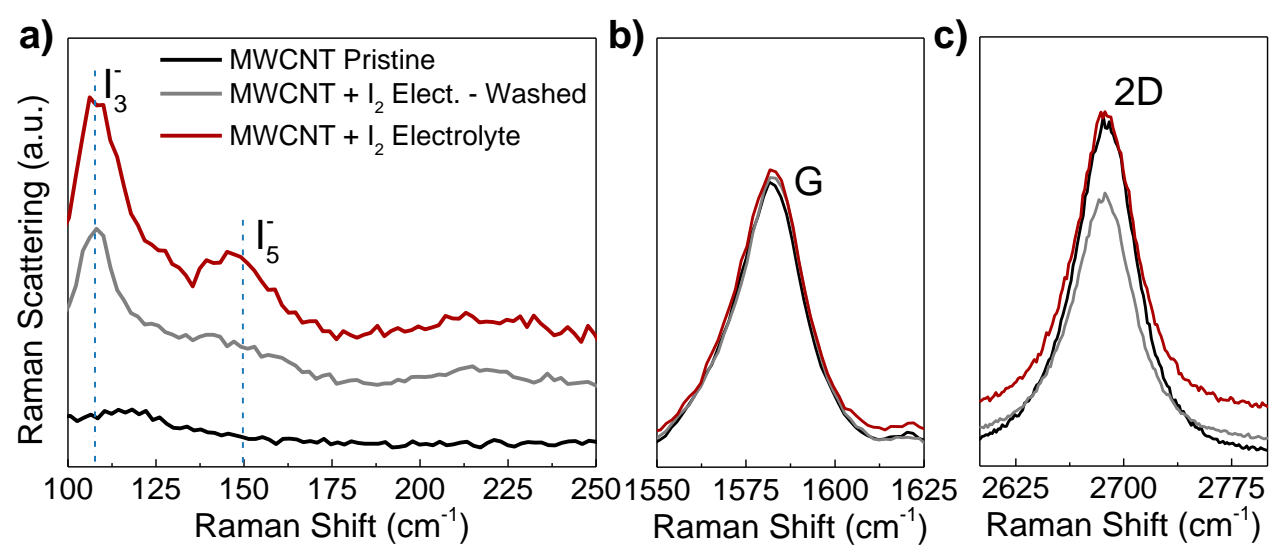

FIGURE 4.8: CNTf- $I_{2}$ electrical doping effect: a) triiodide/pentaiodide Raman signal in presence of CNTf electrode before and after thoroughly washed. b) Raman $G$ and c) $2 D$ bands shifted by electrical doping effect.

\subsubsection{Electrochemistry in presence of nanocarbon porous membranes: $E I S$ study}

Further insights of the catalytic behaviour of CNTf were obtained from a comparative EIS study with the standard catalytic reference material - Platinum. With this technique, by supplying a small $A C$ voltage and varying the frequency, the different electrical interfaces present in the material are identified. A schematic of the CNTf symmetric device with the electrical interfaces is presented in the Figure 4.9a. Also the equivalent circuit typically used for fitting symmetric cells is shown in the Figure $4.9 \mathrm{~b}$. Two $R C$ elements and a series resistance model the material response. A low frequency interface, in the range $1-10^{-2} \mathrm{~Hz}$, corresponding to the bulk diffusion of the electrolyte, and another at high frequency $\left(10^{2}-10^{4} \mathrm{~Hz}\right)$, representing the charge transfer between electrolyte and counter electrode, are the commonly expected interfaces in this system. The maximum frequency of the process as well as the charge transfer resistance are dependent on geometrical parameters of the system, i.e. separation between electrodes or effective area. These are also dependent on physical properties of the electrolyte, as for instance, viscosity - solvent-based, quasi-solid or solid electrolytes.

Figure 4.9c,d shows the Nyquist and Bode phase plots. The charge transfer resistance $\left(R_{C T}\right)$ and the ion diffusion coefficients $\left(D_{\text {diff }}\right)$ extracted from this technique are provided below. The Nyquist plot of Pt-cells exhibits two semicircles centred at $200 \mathrm{~Hz}$ attributed to charge transfer process at the Pt/electrolyte interface[239] with $R_{C T}$ values of $5054 \Omega$, and at $<0.1 \mathrm{~Hz}$ associated to the diffusion of ions in the bulk electrolyte. In contrast, Nyquist plot of CNTf-cells shows three semicircles centred at $1 \mathrm{MHz}, 0.1 \mathrm{kHz}$, and $<0.1 \mathrm{~Hz}$. 
Up to date, there is still debate about the nature of the high frequency processes. Specifically, several authors have discussed the assignment of the peak at $1 \mathrm{MHz}$ either to $\mathrm{CT}$ process between adjacent CNTs or to the presence of a high mesoporosity that promotes trapping-detrapping of chemical species, creating a sort of electrochemical double layer capacitance.[105-109] In order to shed light onto the nature of this feature of the CNTf, two experiments were performed to independently test the role of mesoporosity and of catalytic activity.
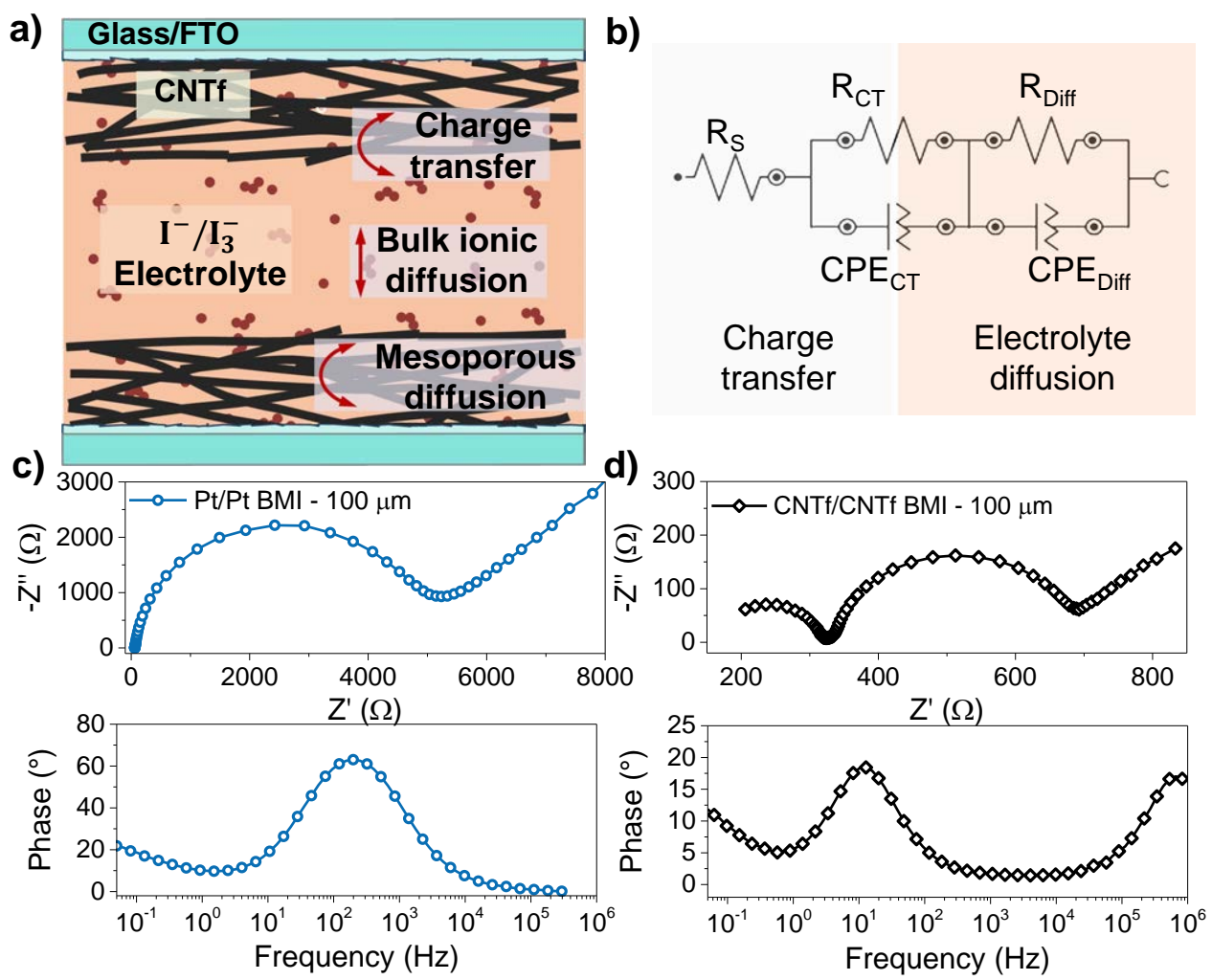

FIGURE 4.9: a) CNTf symmetric cell schematic showing the three identified interfaces. b) Equivalent circuit typically used for fitting EIS data for symmetric cells composed by one counter electrode material and standard electrolyte. c) and d) Nyquist (top) and Bode phase (bottom) plots for Pt- and CNTf-cells respectively.

The first experiment consisted on assembling symmetric devices using a polymeric mesoporous membrane as interlayer (FTO I Pt l electrolyte I membrane l electrolyte $|\mathrm{Pt}| \mathrm{FTO})$. A schematic of this configuration is presented in Figure 4.10a. This membrane is a commercial product (Celgard) which is often used as separator for Li-ion batteries. It consists on $20 \mu \mathrm{m}$ thickness polymer sheet with $20 \mathrm{~nm}$ average pore size (see $S E M$ picture included in the Figure $4.10 \mathrm{~b}$-inset). For preparing these devices, the membrane was sandwiched in between two platinised-FTO-glass electrodes following the same preparation than the rest of the symmetric devices. In these cells, in contrast to the previous symmetric cells, a lower viscosity electrolyte was used in order to allow diffusion through the nanoporous structure of the membrane.

The LSV measurements (Figure 4.10b) of this cell present similar characteristics to the one presented in the Figure $4.5 \mathrm{~b}$, but in this experiment solvent-based 
electrolyte was used and therefore much higher $J_{s s}$ is obtained (ca. $8-10 \mathrm{mAcm}^{-2}$ for $0.2 \mathrm{~cm}^{-2}$ area). As shown in Figure 4.10c,d, these cells featured a similar narrow semicircle at a high frequency of $1 \mathrm{MHz}$ associated to a resistance of $15 \Omega$. This confirms that the unexpected interface found at very high frequency $(1 \mathrm{MHz})$ is related to the diffusion of the species interacting with the mesoporosity of the membrane. Besides, since the polymeric mesoporous membrane is an insulating membrane, these results confirm that the high frequency peak is not related to the electron movement in the mesoporous membrane, as suggested for CNT membranes by other authors.[101]

a)
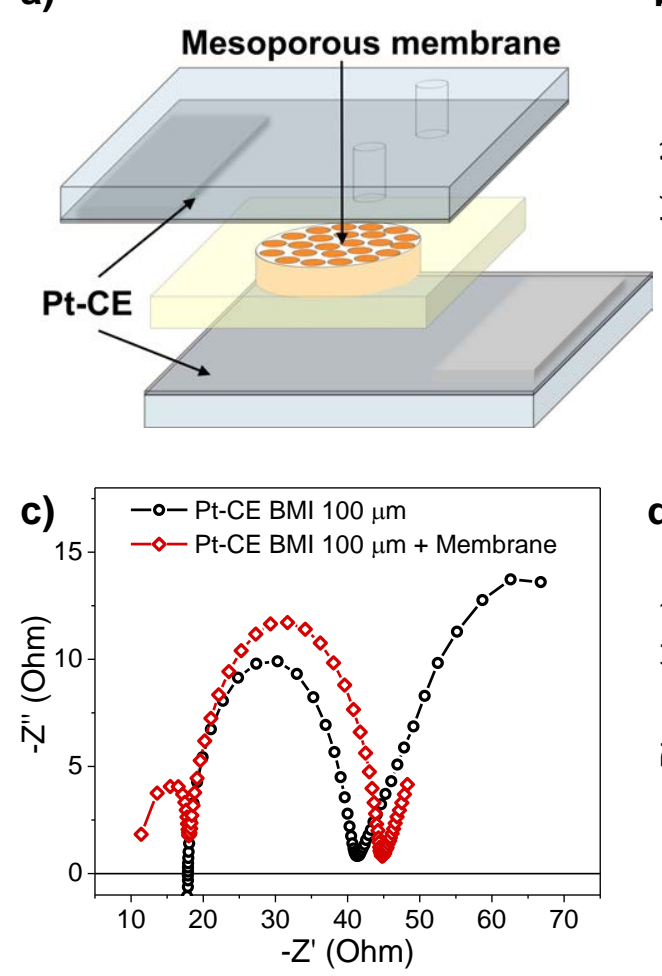

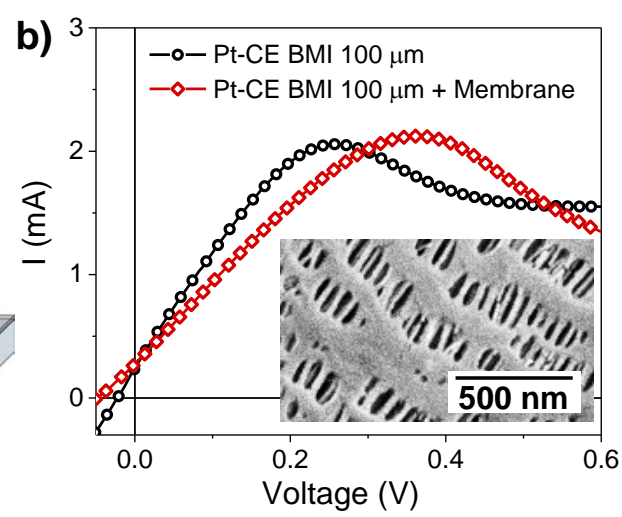

d)

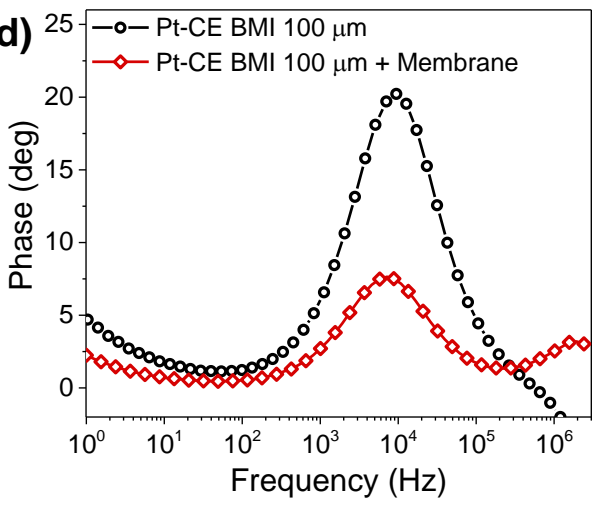

FIGURE 4.10: a) Schematic of the DSSC with the mesoporous membrane as host and separator of the electrolyte. b) LSV curves of Ptcells with and without porous membrane. Inset: SEM image of the mesoporous membrane. c) Nyquist and d) Bode phase plots of the same cells showing the manifestation of the high frequency peak in presence of the mesoporous membrane.

A second experiment using a non-reactive electrolyte was also performed in CNTf- and Pt- symmetric cells. For this experiment, 1-butyl-3-methylimidazolium hexafluorophosphate or $B M I-P F_{6}$ diluted in acetonitrile solvent was used as electrolyte; the same assembly procedure and characterisation than in previous devices were followed. For the CNTf symmetric cell, two semicircles can be observed in the Nyquist plot - Figure 4.11a, each of them resulting in an approx. resistance of $20 \Omega$. The Bode phase plot of this cell shows two peaks centred at $15 \mathrm{kHz}$ and $200 \mathrm{~Hz}$. Because there is no charge transfer or catalytic reaction with this electrolyte, the EIS features observed with this electrolyte can only be attributed to capacitive and diffusive processes. These two interfaces are associated to the mesoporous ion diffusion (high frequency peak) and the bulk ion diffusion (low frequency peak) caused by 
the gradient of accumulated ions at the electrode interface with the CNTs acting as a capacitor. Two different characteristic lengths can be expected in the material; one at the nanoscale derived from the diffusion within the mesopores and a larger one, at the micron-scale, derived from the distance between electrodes. The high diffusivity of the solvent-based electrolyte, explains the low resistance associated to these interfaces. The result obtained with CNTfs was compared with Pt symmetric cells. The EIS results (Figure 4.11b) show diffusive element, typically identified as a straight line for a diffusion process in the Nyquist plot, with a much higher resistance compared to the CNTf material. This higher resistance is a consequence of the larger separation between electrodes in the Pt cell and also, a much smaller specific surface area (SSA) of the flat Pt layer, as previously discussed.
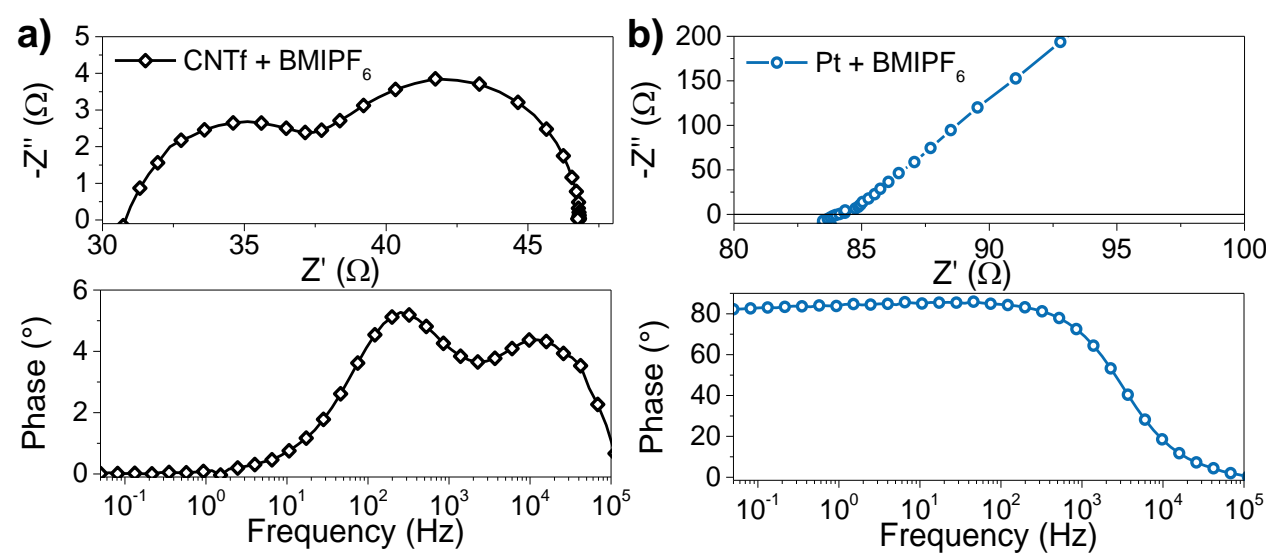

FIGURE 4.11: Nyquist (top) and Bode phase (bottom) plots of cells with $B M I-P F_{6}$ (non-catalytic active ionic liquid) for a) CNTf- and b) Pt-symmetric cells.

These results demonstrate that the high-frequency and narrow semicircle observed in CNTf symmetric cells is ascribed to the mesoporosity of the CNTfs. This allows us to directly compare Pt- and CNTf-cells with respect to the semicircles associated to the catalytic CT process. In line with the $L S V$ experiments, the $R_{C T}$ of CNTf-cells (348 $\Omega$ ) is approximately fifteen times lower than that of the Pt-cells $(5054 \Omega)$. These high resistances are expected since the concentration of iodine in the electrolyte is very low and the high viscosity of the solvent-free electrolyte (see control experiments in the Chapter 3 "Experimental Techniques"). But still it is surprising the difference in $R_{C T}$ between materials. The high SSA of the CNTf electrode allows the interaction of the electrolyte with a high number of catalytic active sites, thus increasing the final charge transfer of the electrode (diminishing the resistance).

Finally, EIS measurements were performed at $100{ }^{\circ} \mathrm{C}$, in order to resolve the bulk ionic diffusivity peak by lowering in the viscosity of the electrolyte - Figure 4.12. Common to both cells, the Nyquist plots show narrower semicircles than those noted at room temperature. Noteworthy, the high frequency peak associated to the mesoporosity of the fibres is not present. The decrease in viscosity of the electrolyte also leads to formation of lower concentration of polyiodides species, as it has been reported before.[140] Therefore, this high frequency interface could be also related to the presence of higher chain length polyiodides with higher electrostatic charge and volume. Due to the quicker trapping-detrapping process at the inner surface of the porosity of the fibre, the reduction in polyiodides concentration and the 
lower resistance associated at higher temperature, the interface becomes invisible. In fact, this interface will be negligible when liquid electrolyte is used in experiments of DSSC devices.

Moreover, by fitting EIS data for several curves at intermediate temperatures for Pt symmetric cells shows that the diffusive process of the electrolyte follows the Arrhenius equation (see also the complete Pt symmetric cell analysis in Chapter 3 "Experimental Techniques"). Same results have been reported with similar electrolytes before.[201] Compared to the CNTf symmetric cells, the diffusion process is not as clearly observed as for the Pt-cell at the intermediate range of temperatures. This suggest a higher activation energy of the process but it is not possible to perform the fitting for the EIS frequency range analysed.

As expected, the increase of temperature leads to an increase in the diffusion of species due to lowering of the viscosity of the electrolyte. The movement of anions in the bulk is now clearly identified with the semicircle formed at low frequencies $(<0.2 \mathrm{~Hz})$ - Figure 4.12. The diffusion parameter $\left(D_{\text {diff }}\right)$ in the bulk electrolyte can be obtained from EIS data by the approximation given by the Equation 4.2:[240, 248]

$$
D_{\text {diff }}=(1 / 2.5) * \delta^{2} * \omega_{\max }
$$

where $\delta$ is the distance between electrodes of the symmetric cell and $\omega_{\max }$ is the peak frequency of the low frequency arc. Under the high applied temperature, $D_{\text {diff }}$ values of CNTf-cells are higher than those of Pt-cells - i.e., $27.8 \times 10^{-10} v \mathrm{~s}$ $14.7 \times 10^{-10} \mathrm{~m}^{2} \mathrm{~s}^{-1}$. The higher diffusivity of the CNTf is likely originated from the closer proximity between electrodes, although it is noted that higher concentration of triiodide and polyiodide species could have a similar effect by increasing ionic conductivity.
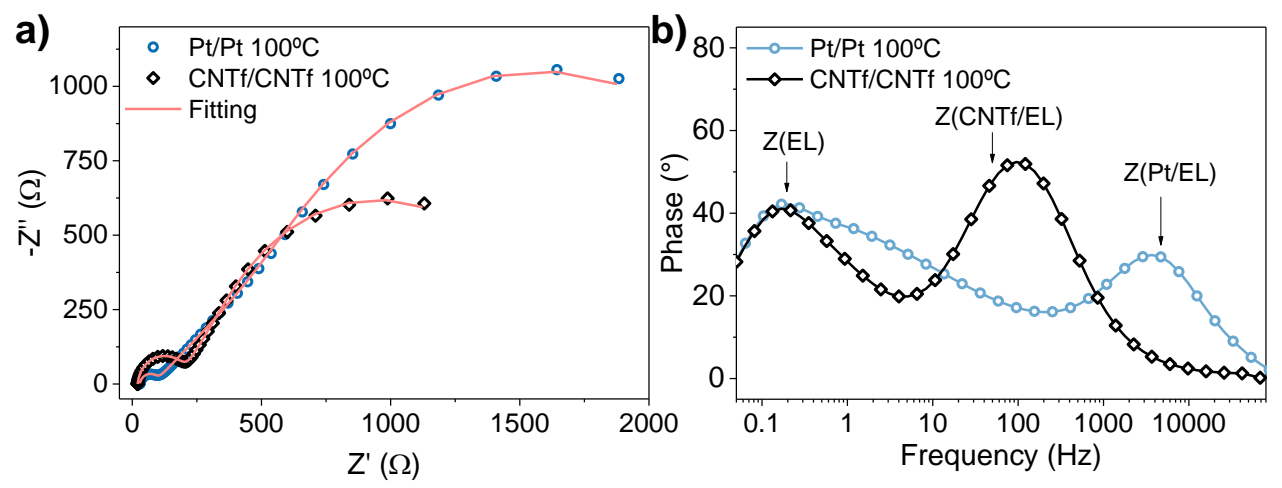

FIGURE 4.12: a) Nyquist and b) Bode phase plots of CNTf- and Ptsymmetric cells with BMII electrolyte at $100{ }^{\circ} \mathrm{C}$.

Summarising, there are several parameters that have been demonstrated as key aspects of the catalytic performance of CNTfs. The high catalytic activity of the CNTf material is due to a higher number of catalytic active sites on account of is high surface area. This surface area and a strong interaction with the electrolyte also produce an increased concentration on polyiodides species. On the other hand, the mesoporous structure of the CNTf electrode increases the effective diffusion path of the electrolyte, decreasing ionic diffusivity of the electrolyte. Further understanding 
of the nature of the catalytic sites could enable the use of doping or similar strategies to increase catalytic activity and thus reduce electrode thickness. Note that, with respect to conductivity, sub-micron CNTf electrodes could be used to replace $\mathrm{Pt}$ and FTO, but the catalytic activity would need to be increased by a factor of 10 for effectively replacing both materials.

This extensive electrochemical study has enabled the first clear identification of the different processes involved in the use of CNTf electrodes as CE, i.e. bulk diffusion, diffusion inside the mesoporous electrode and charge transfer at the CNTf surface. These results provide clear directors for a further understanding of the fundamental catalytic properties of nanocarbons and tools to develop strategies to improve their performance in multiple photoelectrochemical processes.

\subsection{CNTf counter-electrode/current-collector: photovoltaic per- formance}

\subsubsection{CNTf-CE thickness optimisation and influence over the performance of the device}

In view of the catalytic activity showed by the CNTf towards the regeneration of iodide/triiodide redox couple, in this section, the potential of using the CNTf in a full DSSC device is investigated.

For the preparation of DSSC, the standard procedures and material were followed.[200] These are explained in detail in the previous Chapter 3 "Experimental Techniques". The preparation of a DSSC comprise the following steps:

1. Cleaning and degreasing the substrates (FTO-glass).

2. Photoanode construction:

(a) Depositing the "blocking layer" (nm-size compact layer of $\mathrm{TiO}_{2}$ ). This involves the calcination of a pre-deposited layer of $\mathrm{TiCl}_{4}$.

(b) Depositing by doctor blade technique 1, 2 or 3 layers of the commercial semiconductor paste, followed by drying and sintering steps.

(c) Immersing the $\mathrm{TiO}_{2}$ photoelectrode in the dye solution.

3. Cathode: depositing the CE catalytic active material.

- For reference: platinising the substrate $(\mathrm{nm}$-size particles of $\mathrm{Pt})$ by a calcination process of a $\mathrm{H}_{2} \mathrm{PtCl}_{6}\left(\mathrm{H}_{2} \mathrm{O}\right)_{6}$ pre-deposited by chemical procedures.

- Depositing CNTf material.

4. Assembling the device by sandwiching and hot-pressing a thermoplastic film $\left(\right.$ Syrlyn ${ }^{\circledR}$ ) between both electrodes.

5. Filling the DSSC with the electrolyte solution.

6. Encapsulating the DSSC.

As described above, the CNTf-CE were prepared following the procedure describe in the previous section, i.e. sandwiching CNTf strips $(5 \times 30 \mathrm{~mm})$ between the photoanode and a plain FTO-glass substrate. The final device architecture with its 
components is presented in the scheme of the Figure 4.13a. A photograph of a standard DSSC is also presented.

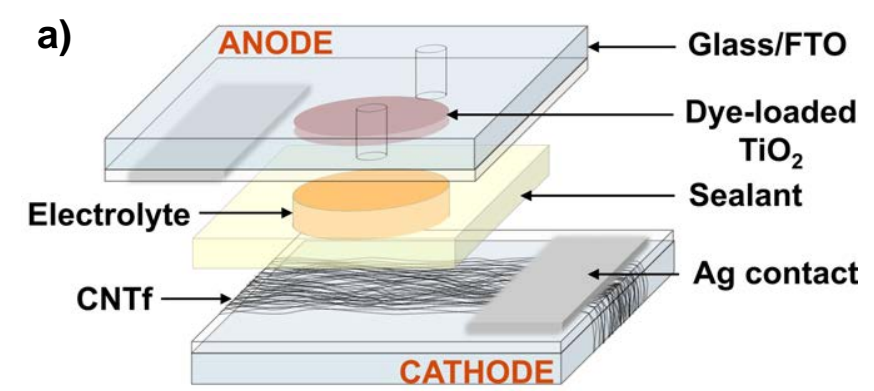

b)

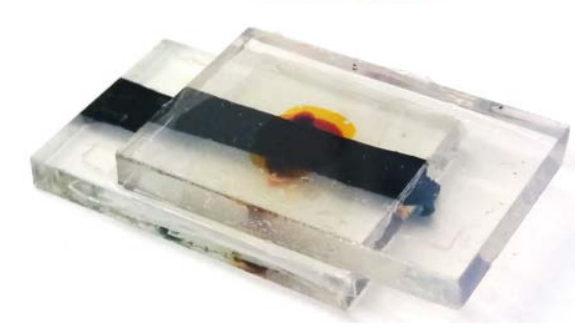

FIGURE 4.13: a) Schematic and b) digital photograph of a typical CNTf-CE DSSC.

The first set of experiments consist in the study and optimisation of CNTf-CE thickness. The data are summarised in the Figure 4.14.

Stacking several layers of CNTf was a straightforward and reproducible way of increasing the CE thickness. To provide a comparable result, independent to our specific CNTf synthesis procedure and setup, the thickness of the stacked electrodes (after condensation step) have been measured by profilometry (further details in Chapter 3 "Experimental Techniques") and obtained a quick correspondence between the number of layers and the final thickness of the CNTf-CE. The extracted information is presented in the Figure 4.14a.

This devices were assembled with the simplest device architecture, i.e. using a working electrode with compact $\mathrm{TiO}_{2}$ layer and $7 \mu \mathrm{m}$ thickness of $\mathrm{TiO}_{2}$ commercial paste. Average $J-V$ figures-of-merits $V_{o c}, J_{s c}, F F$ and $P C E$ were obtained for the devices. The efficiency $(P C E)$ is defined as the cell's maximum power output $\left(P_{\max }\right)$ divided by the input power from the light source and the fill factor is defined as $F F=P_{\max } /\left(V_{o c} I_{s c}\right)$. The extracted performance for $1 \mu \mathrm{m}$ thickness CNTf-CE resulted in a high $J_{s c}\left(15.4 \mathrm{~mA} \mathrm{~cm}^{-2}\right)$ and $V_{o c}(0.72 \mathrm{~V})$ values, but a much lower $F F(44 \%)$ due to a pronounced S-shape form of the $J-V$ curve. These characteristics lead to a $c a$. $5 \%$ efficiency, $3 \%$ lower than with the reference Pt-CE. This obtained S-shape $J-V$ curve for the lower thickness CNTf is presented in the Figure 4.14b, and its origin and influence over the device performance will be discussed later. For a $5 \mu \mathrm{m}$ thick CNTf-CE, the device showed FF $(60 \%), J_{s c}\left(17.7 \mathrm{~mA} \mathrm{~cm}^{-2}\right)$, and $V_{o c}(0.75 \mathrm{~V})$, leading to an overall efficiency of $8 \%$, which is comparable to that of DSSCs with Pt-CEs $\left(J_{s c}\right.$ of $14.5 \mathrm{~mA} \mathrm{~cm} \mathrm{~cm}^{-2}, V_{o c}$ of $0.75 \mathrm{~V}, F F$ of $73 \%, P C E$ of $8 \%$ ). Finally, thicker CNTf-CE $(10 \mu \mathrm{m})$ provide devices with similar figures-of-merit - Figure 4.14b and Table 4.2. Although the $J_{s c}$ is slightly higher for thinner CE, the $F F$ has increased for the thicker CNTf-CE. As the FF is limiting factor aimed to optimise by increasing the thickness of the material, the thicker configuration will be considered as the best from 
this point forward. This easy strategy to increase the performance by increasing the thickness of the CEs is in agreement with some previous reports of CNTs-CE.[249]

The efficiency of the device saturates in the range 8-10 $\mu \mathrm{m}$ but increasing the thickness still presents some drawbacks. Although the as-produced material shows a very high compressibility due to the high porosity and low density - see reference[250] of an analogous material, more than $15 \mu \mathrm{m}$ results on a too thick CE. For DSSC devices assembled with a $25 \mu \mathrm{m}$ spacer, the available free space inside the device is already exceeded. This leads to a bad sealed device after the hot pressing step and thus, a low performance. Another difficulty in the fabrication process of the device derived from the thickness of the CNTf membrane relates to the hindered electrolyte filling step, but this will be discussed in detail in the Chapter 7 "CNTf Electrodes for DSSC: Engineering Aspects".
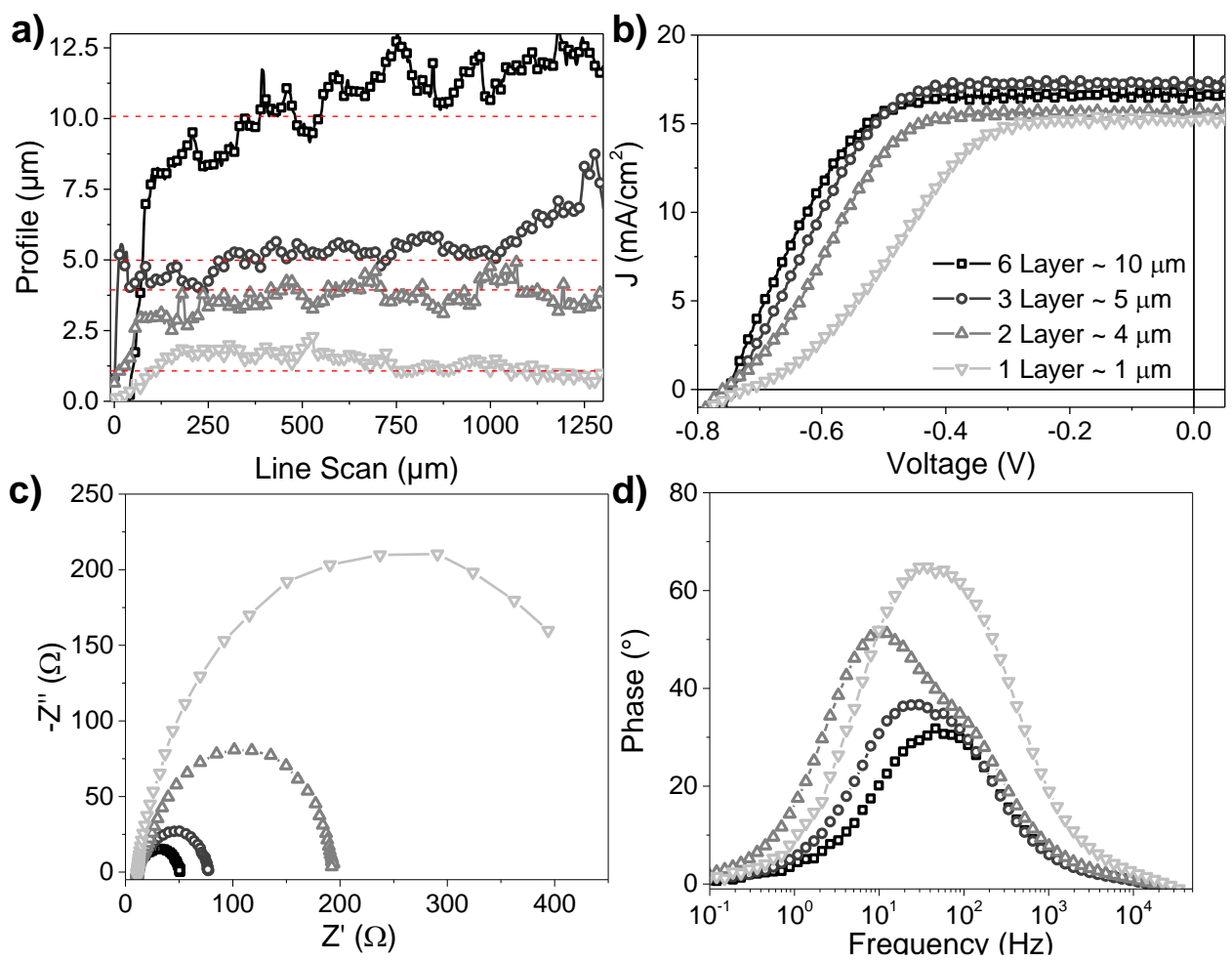

FIGURE 4.14: a) Profilometry measurements of the CNTf electrodes. Red dot-line indicates the average values for the different samples. b) $J-V$ curves showing the increase in FF upon increasing the thickness of the CNTf-CEs. c) Nyquist b) and Bode phase plots of CNTf-DSSCs with different $C E$ thicknesses.

EIS of DSSC devices could in principle provide useful information about the charge kinetics inside the device, to charge injection and recombination components in the device, and to the conducting of the DSSC components (substrates, semiconductor, electrolyte).

For a standard device with $\mathrm{Pt}$ catalyst and $\mathrm{I}^{-} / \mathrm{I}_{3}^{-}$redox couple under one full sun illumination (AM 1.5G conditions) there are three well-assigned peaks.[9295] In the bode-phase plot, at low frequency $2-10^{-2} \mathrm{~Hz}$, the peak corresponds to Nernstian diffusion whitin the electrolyte. The mid-frequency peak centred at $10^{2} \mathrm{~Hz}$ is related to the $\mathrm{TiO}_{2} /$ dye/electrolyte interface. Finally, the peak at higher 
frequency, typically at $10^{4} \mathrm{~Hz}$, corresponds to the charge transfer impedance at the catalyst/electrolyte interface.

TABLE 4.2: DSSC characteristics of CNTf-CE different thicknesses.

\begin{tabular}{lllll}
\hline \multirow{2}{*}{ Devices } & $V_{o c}$ & $\begin{array}{l}J_{s c} \\
m A \mathrm{~cm}^{-2}\end{array}$ & $\begin{array}{l}F F \\
\%\end{array}$ & $\begin{array}{l}P C E \\
\%\end{array}$ \\
\hline Pt-CE & 0.75 & 14.5 & 73 & 7.9 \\
$1 \mu \mathrm{m}$ CNTf-CE & 0.72 & 15.4 & 44 & 4.9 \\
$4 \mu \mathrm{m}$ CNTf-CE & 0.74 & 15.5 & 53 & 6.1 \\
$5 \mu \mathrm{m}$ CNTf-CE & 0.75 & 17.7 & 60 & 7.9 \\
$10 \mu \mathrm{m}$ CNTf-CE & 0.75 & 17 & 64 & 8.1 \\
\hline
\end{tabular}

EIS results for the different CNTf-CE thicknesses are presented in Figure 4.14 $c$,d. For all these thicknesses, instead of these three peaks, the impedance results are characterised by one unique interface at approximately $100 \mathrm{~Hz}$. A direct comparison with the previous results in symmetric cells prompts us to conclude that this represents two processes, namely i) CT process at the CNTf/electrolyte interface and ii) $C T$ process at the photoanode/dye/electrolyte interface. The convolution between the two phenomena in the Nyquist plot, hampers direct comparison with Pt-based DSSCs and therefore a further analysis is not possible to be conducted from this plot. However, it has been noted that there are small changes in the peak position.

\section{Analysing the origin of the S-Shaped $J-V$ curve:}

The origin of the S-shape of the curves has been widely discussed in photovoltaic devices in the past. For instance, this effect has been observed in DSSCs using nanocarbon-based CEs and it has been ascribed to the lack of balance between dye and electrolyte regeneration processes, as well as an increased in the series resistance for lower thicknesses.[106, 129, 249] The predominant strategy to circumvent these issues is by increasing the thickness of the CEs,[249] as described in the previous experiment. But it is of interest to understand how the increase in efficiency is affected by the increase in through thickness conductance, number of catalytic sites, diffusion lengths, all of which are affected by CNTf electrode thickness.

Firstly, the series resistance effect of the device is analysed. In this particular case, the CNTf-CEs are assembled on top of FTO substrates (acting also as CC), thus, no matter the CNTf thickness, there is a minimum conductivity corresponding to the FTO substrate. Although the conductivity of the substrate can be slightly improved by the addition of the CNTf material, for a $0.2 \mathrm{~cm}^{2}$ there is no such a big influence over the device performance. Control experiments, substituting the FTO substrate $8 \Omega / s q$ by $15 \Omega / s q$ underneath the CNTf, show that there is almost no different over the performance. Even for Pt reference devices both provides very similar performance.

The series resistance of the device can be obtained from the slope of the $J-V$ curve. Looking closely the different slopes from the Figure $4.14 \mathrm{~b}$, one could notice that the slope is not so different when the current increases, and the drop in performance is more related to a horizontal translation of the $J-V$ curve. This observation in addition to the lost in short circuit current density suggest that the cause of this phenomena is related to dye/electrolyte regeneration issues. Therefore, it is concluded that although it is commonly associated to the S-shape of $J-V$ curves, 
excessive $R_{s}$ values limit the photocurrent at short-circuit condition $\left(I_{s c}\right)$ but do not directly produce the characteristic S-shaped.

Moreover, the charge transfer between the carbon catalytic material and the FTO current collector could be playing also a role. One of the before mention effects of increasing the CNTf thickness is to decrease the interfacial resistance between FTO and CNTf, as well as increasing the transversal conductivity of the CNTf through the thickness.

To better understanding the catalytic mechanism of the CNTf and the S-shape of the $J-V$ curves, two experiments were carried out. Firstly, by gas phase functionalisation of the CNTf; and secondly, by light soaking of the CNTf- and Pt-CE DSSC.

The functionalisation of the CNTf, by uniformly introducing defects over the surface of the material, could help to understand the catalytic behaviour. If this activity is related to CNTs defects, i.e. graphitic edges, as it has been reported before,[113, $114,251]$ the S-shape should decrease (better $F F$ ).

For studying this effect, $1 \mu \mathrm{m}$ and $10 \mu \mathrm{m}$ thick CNTf-CE are compared with the same material after 20 and $60 \mathrm{~min}$ of $\mathrm{O}_{3}$ treatments as reported elsewhere.[62] Note that $20 \min \mathrm{O}_{3}$ treatment is sufficient time for introducing high amount of defects distributed along the surface of the material.[62]

The obtained $J-V$ curves are presented in the Figure 4.15. There is a clear decrease on the device performance by ozone functionalisation of the CNTf. When sub-optimised thickness ( $1 \mu \mathrm{m} \mathrm{CNTf}$ ) is subjected to $\mathrm{O}_{3}$ treatment, the $V_{o c}$ as well as the S-shaped $J-V$ curves characteristic of this low thickness remains constant, $F F=45 \%$ and $V_{o c}=0.75 \mathrm{mV}$. On the contrary, there is a clear descent of the short circuit current, from 16.8 to $15.2 \mathrm{~mA} \mathrm{~cm}^{-2}$.
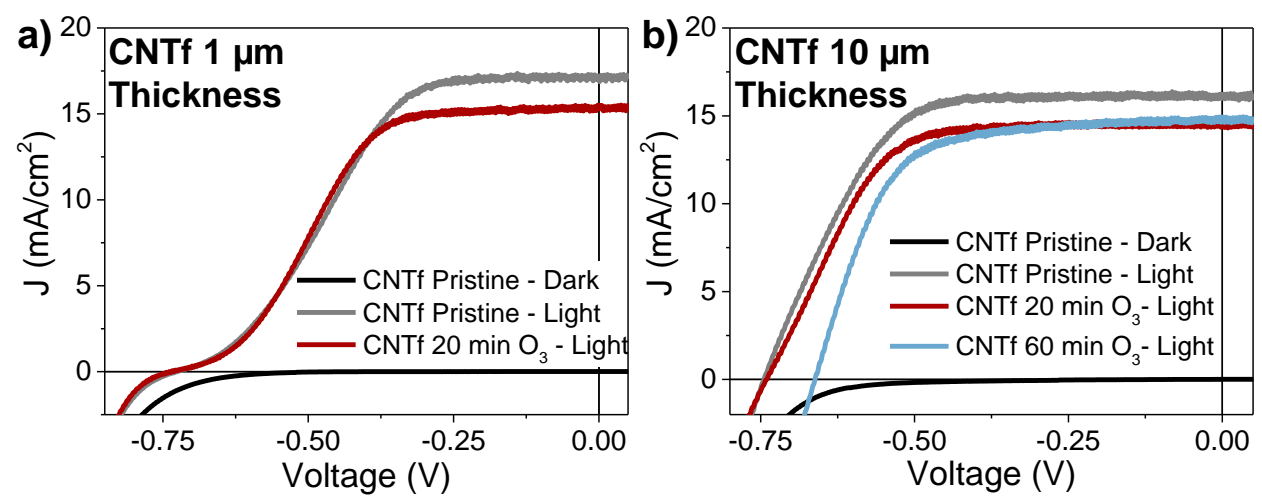

FiguRE 4.15: $J-V$ curves of a) $1 \mu \mathrm{m}$ and b) $10 \mu \mathrm{m}$ CNTf-CE ozone treated during 20 and $60 \mathrm{~min}$ functionalisation time.

Similar trend is observed for optimised $10 \mu \mathrm{m}$ thick CNTf-CE, from the initial $P C E=7.7 \%$ to $7 \%$ after $20 \operatorname{min~}_{3}$ treatment and $6.4 \%$ after $60 \mathrm{~min} \mathrm{O}_{3}$ treatment. Although the gas functionalisation is a diffusion limited process, one hour is enough time to achieve a uniform degree of functionalisation through the entire thickness of the electrode. The first drop after the first treatment is affecting mainly the short circuit current, although small S-shape start to be visible. The lower dye regeneration process has decreased its efficiency because less charges are injected into the mesoporous titania. Thus, the reduction in the catalytic activity can be most the probable 
cause suggesting that the CNTf present now a lower number of catalytic active sites. One hour of $\mathrm{O}_{3}$ treatment is a very long time, and the high density of defects is probably affecting not only the surface properties, but also the electrical conductivity of the material. With the new highly functionalised surface of the CNTf, the redox potential of the electrolyte, as well as the surface chemistry must be highly altered, producing the visible decrease in open circuit voltage of the device.

It is possible to conclude that the functionalisation treatment, and therefore the surface defects on the CNTf, are not the origin of the catalytic activity. In fact, with this treatment, the catalytic activity is diminished. Other mechanisms have to be investigated in relation to the effect of the functionalisation of the CNTf electrode. For instance, via $\mathrm{O}_{3}$ treatment, the amorphous carbon at the surface of the CNTf is removed, the electrical properties of the material itself are modified, and the encapsulated Fe nanoparticles inside the CNTs are exposed. It is proposed as future work to design experiments to isolate and test these individual effects.

The second experiment performed was light soaking. This is a well known procedure for increasing the initial performance of the devices after illuminating for a certain amount of time.[252-254]. With this procedure, some conclusions about the trend of the catalytic activity and the S-shape of the $J-V$ curves can be obtained.

This phenomena occurs when the UV light component promotes charges from the valence band to the conduction band of the oxide, filling the trap states that typically mediates the charge diffusion process of these devices, this would lead to an increase in the conduction band edge.[255] Other proposed mechanisms, the improved injection would be consequence of increasing the density of acceptor states in the semiconductor induced by charging of the surface of the semiconductor and rearrangement of charge species at the dye/semiconductor heterojunction.[256] Also, an increase in the injection rate from the dye is produced. Still, there is no consensus about this mechanism and some other has been proposed.

Freshly prepared CNTf- and Pt-CE DSSC devices were subjected to $30 \mathrm{~min}$ light soaking experiment, acquiring one $J-V$ curve per minute (see Figure 4.16). The cells were constructed with a $7 \mu \mathrm{m}$ thick $\mathrm{TiO}_{2}$ working electrode and standard electrolyte. The standard Pt-CE reference cell and the thinnest thickness CNTf-CE (1 $\mu \mathrm{m})$ were compared.

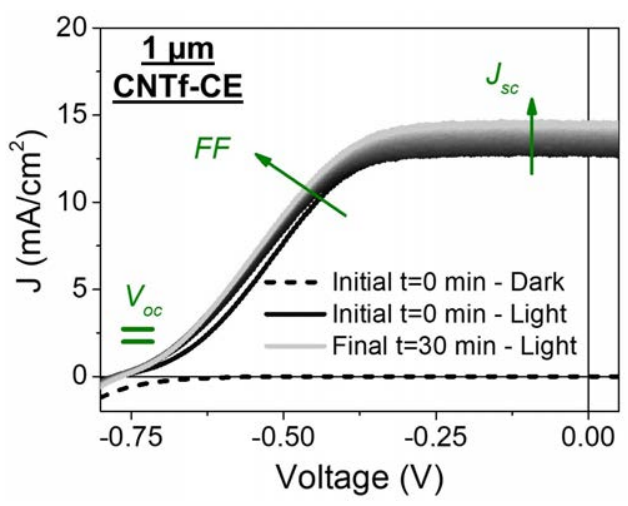

FIGURE 4.16: Light soaking experiment. $J-V$ curves of $1 \mu m$ thick CNTf-CE DSSC device 
Comparing the initial state of the device with final $J-V$ curve, a significant increase in the $J_{s c}$ and $F F$ is observed, the $V_{o c}$ remains almost constant. This improvement in the characteristics leads to an increase in the efficiency from 4.6 to $5.2 \%$, entailing an improvement superior to $10 \%$. The $V_{o c}$ remains constant during the light soaking time as expected due to the thermodynamic nature of this parameter. A small improvement on the FF parameter is observed after the initial couple of minutes, but, above all an increase in the short circuit current - from 12.7 to $14.6 \mathrm{~m} \mathrm{~A} \mathrm{~cm}^{-2}$, is the responsible of the improvement in efficiency. The increase in short circuit current is explained by the improvement of the conductive properties of the semiconductor layer.

This increase in performance suggest that, before the light soaking, the dye molecules are not injecting charges at their most efficient rate, possibly because a lack of regeneration related to a insufficient number of catalytic centres.[253]

To conclude, after analysing the functionalised CNTf-CE DSSC and the light soaking effect over these devices, the S-shape of the $J-V$ curves is more accentuated for short functionalisation times and directly disappear under a strong reduction in the open circuit voltage of the device. Moreover, the photocurrents produced with electrodes subjected to this treatment are visibly reduced. This result points out that the carbon edge defects introduced by gas-phase functionalisation are not the responsible of the catalytic activity of the CNTf-CE. Hence, it is conclude that the emergence of S-shape is produced by a lack in the catalytic activity of the effective mass introduced in the device.

\section{Electrochemical stability of the CNTf-CE under operation:}

Light soaking experiment also provides important information about the shortterm electrochemical stability of the CNTf as CE. Same experiment than before was performed with fully optimised CNTf-CE DSSCs (10 $\mu \mathrm{m}$ thickness).

The $J-V$ curves trend of the Pt-CE (Figure 4.17a) shows that the overall efficiency has decreased from 8.7 to $7.9 \%$ during the initial $30 \mathrm{~min}$. This drop is due to an small decrease in the $V_{o c}$, from 0.75 to $0.73 V$, and the diminish in the $F F$, from 0.72 to 0.66 .

For the $10 \mu \mathrm{m}$ thick CNTf-CE J-V curves, it indicates a stabilisation in the performance of the device. The efficiency remains almost constant, firstly slightly increasing the device performance and later reaching a stable performance. The initial increase in efficiency response to an improvement in the $F F$ of the device after the initial minutes, from 0.62 to 0.66 ; however, the short circuit current slightly decreases from 17.7 to $16.7 \mathrm{~mA} \mathrm{~cm}^{-2}$. The improvement in FF characteristic, reducing the Sshape of the $J-V$ curve, is consistent with the previous experiment with lower CNTf thickness.

As it is mention before, with the increase of extra available states for charge transfer[256] during the light soaking time, also the recombination component of the charge collection efficiency increases[256] and therefore, this charge injection into the semiconductor in presence of CNTs-CE presents a small decrease over time as it is observed in the Figure $4.17 \mathrm{~b}$.

The light soaking experiments indicates that the S-shape is partially recoverable and, thus suggesting that the catalytic activity of the CNTf-CE achieves an optimum steady-state after several minutes of working state of the device. 

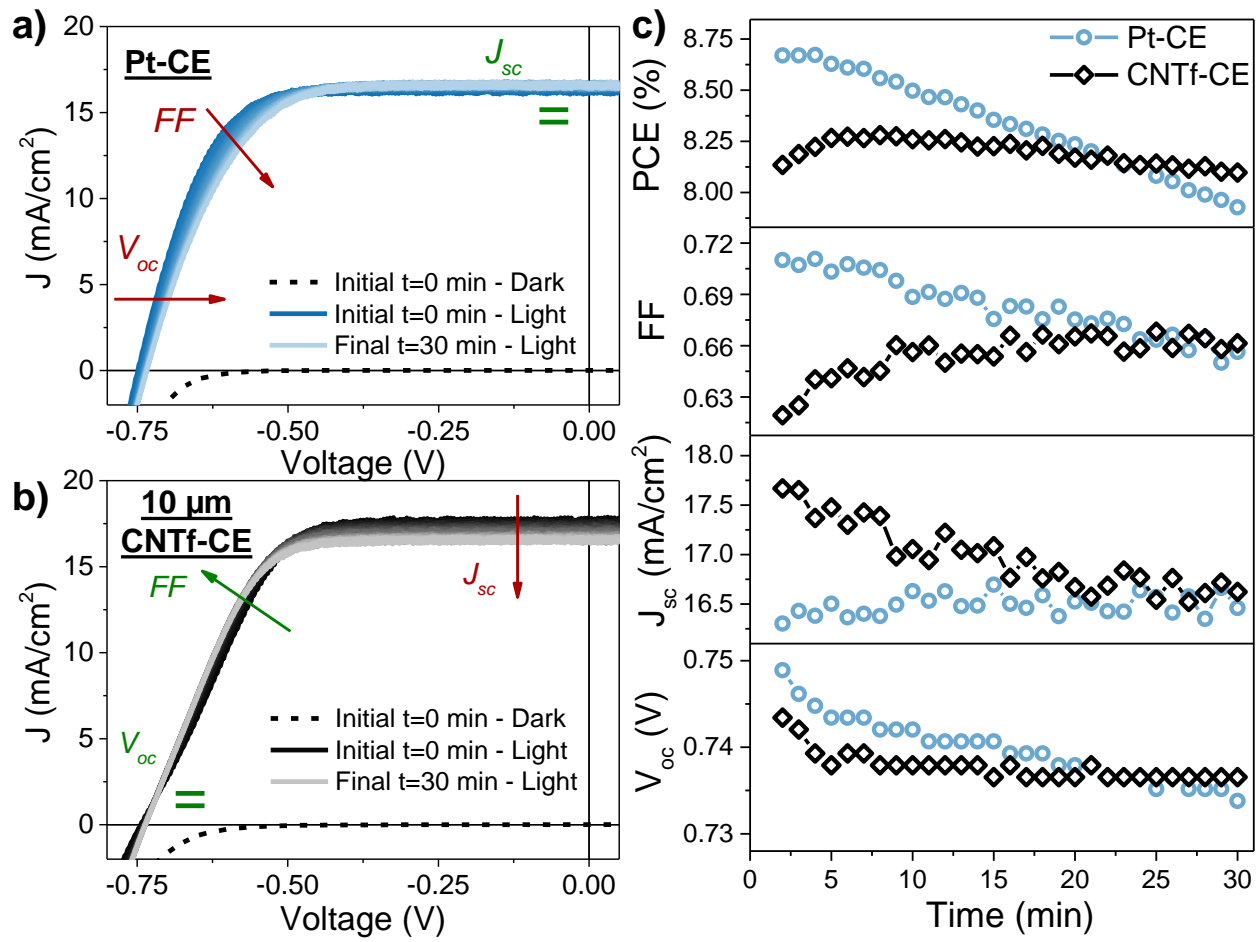

FIGURE 4.17: Light soaking experiment for a) Pt-CE and b) $10 \mu \mathrm{m}$ thick CNTf-CE DSSC devices. c) Characteristic parameters as a function of time.

\subsubsection{CNTf-CE towards high efficiency DSSC}

\section{Photoanode optimisation towards record efficiency with CNTf-CE:}

The results on the optimisation of CNTf-CE morphology presented before correspond to DSSC with one layer of transparent $\mathrm{TiO}_{2}$ nanoparticles ( $7 \mu \mathrm{m}$ thick) as photoanode. Next, the thickness and morphology of $\mathrm{TiO}_{2}$ was optimised. This was done by increasing the thickness of the transparent nanoparticles $\mathrm{TiO}_{2}$ layer and adding a mesoparticles $\mathrm{TiO}_{2}$ scattering layer (see Chapter 3 "Experimental Techniques"), typically done for DSSCs.[200, 203, 204]

Increasing the thickness of the nanostructurated $\mathrm{TiO}_{2}$ layer leads to an increase of surface area and dye loading as indicated by the darker colour of the titania layer (Figure 4.18a). The corresponding electrode thickness determined by profilometry (Figure 4.18b) show that two layers lead to an average thickness of 13-14 $\mu \mathrm{m}$, which is in the range reported to produce the best device performance.[200]

Figure $4.18 \mathrm{c}, \mathrm{d}$ present $J-V$ curves for CNTs- and Pt-CE using three configurations of $\left.\mathrm{TiO}_{2}: \mathrm{i}\right)$ thin $(7 \mu \mathrm{m})$ and ii) thick $(14 \mu \mathrm{m})$ transparent mesoporous $\mathrm{TiO}_{2}$ films, and iii) full photoanodes $(21 \mu \mathrm{m})$ consisting of a $14 \mu \mathrm{m}$ bottom transparent layer and a $7 \mu \mathrm{m}$ top light-scattering layer, all sensitised with N719 dye and filled with standard liquid electrolyte. The measurements are obtained under dark and 1 sun illumination (AM 1.5G), while Table 4.3 summarises the most important figures-ofmerit. 
The CNTf-DSSCs showed lower $V_{o c}$ values ( $c a .50-100 \mathrm{mV}$ ) than those of the PtDSSCs. Nevertheless, $J_{s c}$ values are significantly enhanced in CNTf-DSSCs relative to equivalent reference devices - i.e., $17 \mathrm{~mA} \mathrm{~cm}^{-2}$ vs $19.5 \mathrm{~mA} \mathrm{~cm} \mathrm{~cm}^{-2}$ and $20 \mathrm{~mA} \mathrm{~cm}^{-2}$ vs $23.8 \mathrm{~mA} \mathrm{~cm}^{-2}$ for $14 \mu \mathrm{m}$ and $20 \mu \mathrm{m} \mathrm{Pt}$ - and CNTf-DSSCs, respectively; while FF values are comparable at around 60-70\%. Overall, CNTf-DSSCs featured slightly superior efficiencies to those of the Pt-DSSCs for all $\mathrm{TiO}_{2}$ photoanode configurations, reaching values as high as $9.5 \%$ compared to $9 \%$ in Pt-DSSCs.

a)

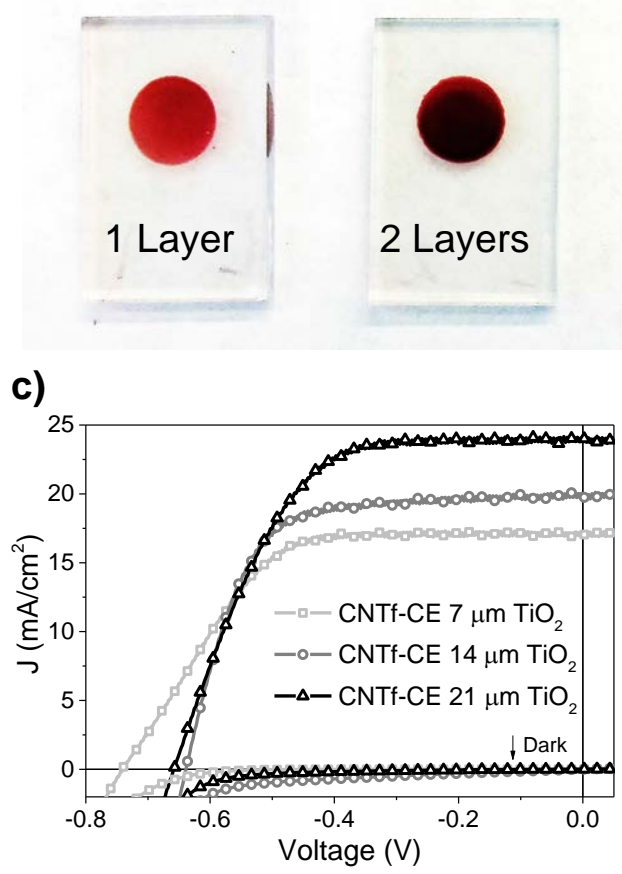

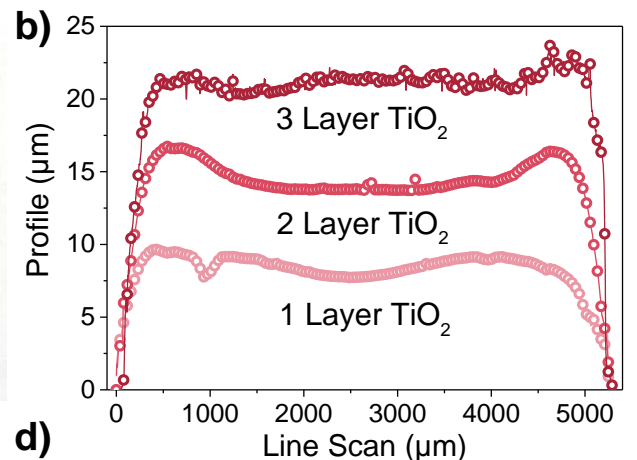

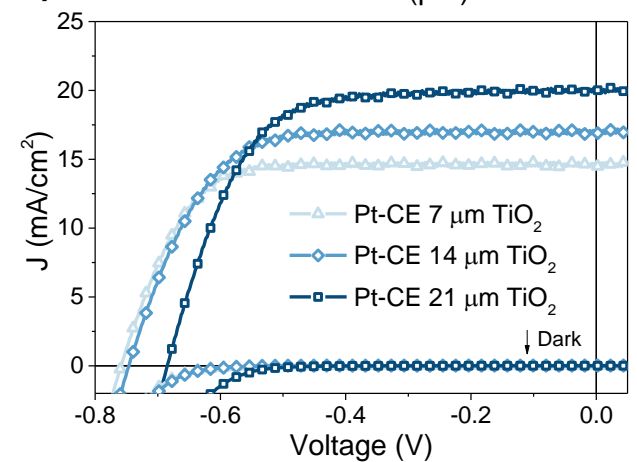

FIGURE 4.18: a) Digital image comparing the dye loading for different thicknesses of the photoanode. b) Profilometry measurements showing increment of the number of $\mathrm{TiO}_{2}$ layers. c) CNTf-DSSCs and d) Pt-DSSCs $J-V$ curves showing the comparison of the performance of devices with $10 \mu m$ CNTf-CE in combination with different $\mathrm{TiO}_{2}$ photoanodes.

TABLE 4.3: DSSC photovoltaic characteristics for Pt- and CNT-CE with different thicknesses of titania layer.

\begin{tabular}{|c|c|c|c|c|}
\hline Devices & $\begin{array}{l}V_{o c} \\
V\end{array}$ & $\begin{array}{l}J_{s c} \\
m A c m^{-2}\end{array}$ & $\begin{array}{l}F F \\
\%\end{array}$ & $\begin{array}{l}P C E \\
\%\end{array}$ \\
\hline Pt-CE $7 \mu m \mathrm{TiO}_{2}$ & 0.75 & 14.5 & 73 & 8 \\
\hline $\mathrm{Pt}-\mathrm{CE} 14 \mu \mathrm{m} \mathrm{TiO}_{2}$ & 0.74 & 17 & 69 & 8.7 \\
\hline Pt-CE $21 \mu m \mathrm{TiO}_{2}$ & 0.68 & 19.5 & 67 & 9.1 \\
\hline CNTf-CE $7 \mu m \mathrm{TiO}_{2}$ & 0.75 & 17.7 & 60 & 8.1 \\
\hline CNTf-CE $14 \mu m \mathrm{TiO}_{2}$ & 0.64 & 20 & 69 & 8.8 \\
\hline CNTf-CE $21 \mu m \mathrm{TiO}_{2}$ & 0.6 & 23.8 & 60 & 9.5 \\
\hline
\end{tabular}

The results show interesting differences in $V_{o c}$ and $J_{s c}$ when Pt- with CNTf-CE devices are compared. This is tentatively attributed to changes of the redox potential due to the catalytic behaviour of the CNTf as it has been shown in the previous 
section. Also, the possible adsorption of electrolyte additives, such as, e.g. 4-tertbutylpyridine, leading to a local decrease of the $\mathrm{pH}$ has been reported as cause of possible variation in the open circuit voltage of the device.[106] Regarding the CNTf device, the decrease in the voltage can be also related with a possible increase in the concentration of the triiodide and polyiodides in the electrolyte, as it has been demonstrated in the previous section (Figure 4.8 and 4.7). It is possible that the faster regeneration of the electrolyte also shifts the equilibrium between redox couple species, leading to an electrolyte with higher content of the triiodide. This increment of triiodide concentration might affect the current developed by the device, possibly explained by the reduction of the charge transfer resistance in the counterelectrode, the higher steady-state diffusion current density and the higher diffusion coefficient in presence of the CNTf.

The effect of reducing the distance between CNTf-CE membrane and working electrode must be considered. This could lead to a quasi-zero distance regeneration of the dye, thus increasing the short circuit current of the device by higher injection and regeneration process.

In summary a combination of a $14 \mu m$-thick nanoparticles $\mathrm{TiO}_{2}$ layer and a $7 \mu \mathrm{m}$ microparticles $\mathrm{TiO}_{2}$ layer produce a device performance close to the limit for this system with CNTf-CE. This configuration leads to a balance between dye absorption-injection, and charge transport in $\mathrm{TiO}_{2}$. Increasing the thickness of $\mathrm{TiO}_{2}$ also leads to longer diffusion path of the electrons through the active layer. During this longer path, the recombination of charges at the surface of the semiconductor with the electrolyte or other excited dye molecules will increase. Moreover, the regeneration of the dye becomes more difficult in a thicker electrode.

The impedance spectra of the best-performimg DSSCs $\left(21 \mu m\right.$ thick $\left.\mathrm{TiO}_{2}\right)$ is shown in Figure 4.19a,b,c. The Bode-phase plots are very useful to distinguish the number of interfaces that are present in the system. Under one full sun illumination $(\mathrm{AM} 1.5 \mathrm{G})$ and $V_{o c}$ conditions, the standard device with $\mathrm{Pt}$ catalyst and $\mathrm{I}_{3}^{-} \rightarrow \mathrm{I}^{-}$ redox couple shows three peaks. At low frequency $2-10^{-2} \mathrm{~Hz}$ the peak corresponds to Nernstian diffusion whitin the electrolyte. The mid-frequency peak centred at $10^{2} \mathrm{~Hz}$ is related to the $\mathrm{TiO}_{2}$ /dye/electrolyte interface. Finally, the peak at higher frequency, typically at $10^{4} \mathrm{~Hz}$, corresponds to the impedance at the catalyst/electrolyte interface. When CNTs are used as catalyst, in general, the impedance behaviour is totally different. The Bode-phase plot for CNTf-CE shows a convolution of the high-frequency and mid-frequency domain, therefore, for this type of cell, it is more difficult to separate the contributions of the CNTf/electrolyte (CNTf/EL) interface and the $\mathrm{TiO}_{2} /$ dye/electrolyte $\left(\mathrm{TiO}_{2} /\right.$ dye/EL).

Nevertheless, fitting of EIS with an equivalent circuit enables a comparison of the processes occurring at the two types of CEs. The three semicircles observed by EIS results for the Pt-CE DSSC (Figure $4.19 \mathrm{~d}$ ) are fitted with three $R C$ elements (parallel resistor and non-ideal capacitor, i.e. constant phase element) and a series resistance, following the conventional method.[129, 240] For reference, the semicircle at the right-hand side of the spectra corresponds to ion diffusion in the electrolyte, whereas the intermediate semicircle is related to the charge transfer at the $\mathrm{TiO}_{2} /$ dye/electrolyte interface. The semicircle at the left-hand side corresponds to the charge transfer between $\mathrm{Pt}$ and the redox couple in the electrolye. Comparing dark and light curves, when the recombination increases in the system, the intermediate semicircle is enlarged (higher $R_{\mathrm{TiO}_{2}}$ ). 
For the CNTf-CE the standard equivalent circuit does not produce a good fitting. Instead, two RC elements are used (Figure 4.19d) leading to a much better result with minimum error. This alternative equivalent circuit represents better the electrical interfaces in the CNTf device. Since the CNTf electrode is in the rage of $10 \mu \mathrm{m}$, it fills all the internal space of the DSSC, and most likely, both electrodes, $\mathrm{CE}$ and photoanode, are in close proximity. The consequence is that there is no bulk diffusion for the electrolyte because it is embedded in the porosity of the CNTf membrane. This interpretation is supported by the absence of diffusion peak at low frequency in the Bode phase plot.

Accordingly an increase in catalytic activity of the CE is reflected by the $C T$ resistance $\left(R_{C T}\right)$ lowering observed as the semicircle located at the left-hand side of the spectra becomes smaller. The semicircle that appears at the right-hand side of the spectra is related to the charge recombination and accumulation at the $\mathrm{TiO}_{2} /$ dye/ electrolyte interface.
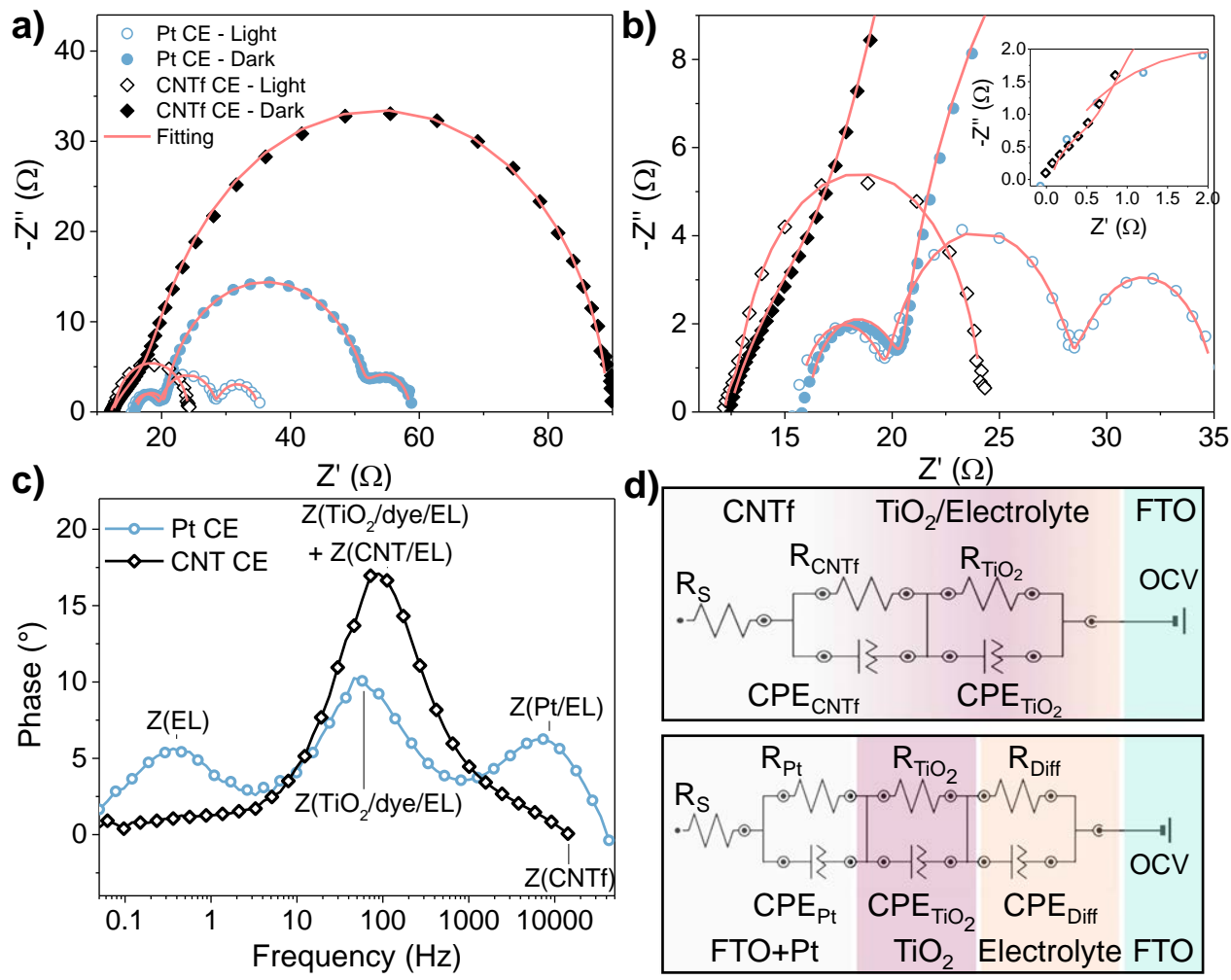

FIGURE 4.19: Comparison of Pt- and CNTf-DSSC EIS measurements. a) and b) Nyquist plots under light and dark conditions. c) Bode phase plots under illumination. d) Equivalent circuits for CNTf- and reference $\mathrm{Pt}-\mathrm{CE}$.

After the proper fitting of the EIS results under dark and light conditions, direct comparison between the impedance characteristics provides some extra information about the conduction mechanisms underlying the devices with different $\mathrm{CE}$ material.

The series resistance is the first element of the equivalent circuit. The value in the $x$-axis where the first semicircle begins in the left hand side correspond to $R_{s}$. Although it is similar for both devices, is smaller for the CNTf/FTO substrate, 
$R_{s-P t}=15.6 \Omega$ and $R_{s-C N T f}=12.2 \Omega$, demonstrating also the improved conductivity of the CNTf current-collector.

The charge transfer resistance between catalytic CNTf and Pt is hard to compare because of the convolution of the $C T$ and bulk diffusion phenomena in the CNTf material. Still, the $R_{C T}$ for the Pt under light conditions is $4 \Omega$, overlapping with the semicircle under dark conditions. For the CNTf, the $R_{C T}=0.6 \Omega$ is obtained under light conditions by fitting the initial points of the EIS spectra (see inset Figure $4.19 \mathrm{~b}$ ). Although there are no enough points to obtain accurate fitting, $R_{C T}$ for the CNTf-CE is visibly smaller than the Pt-CE CT, therefore implying a resistance lower than $4 \Omega$.

Another important parameter is the charge collection efficiency $-\eta_{\text {coll }}$, which reflects on the efficiency of the photoanode under operation conditions in the full DSSC. $\eta_{\text {coll }}$ is obtained from the electron transport resistance $\left(R_{w}\right)$ at the $\mathrm{FTO} / \mathrm{TiO}_{2}$ interface under illumination and the resistance to charge recombination $\left(R_{k}\right)$ across $\mathrm{FTO} / \mathrm{TiO}_{2}$ and $\mathrm{FTO} /$ electrolyte interfaces under dark conditions according to:

$$
\eta_{\text {coll }}=1-\frac{R_{w}}{R_{k}}
$$

$\eta_{\text {coll }}$ for both the Pt- and CNTf-CE resulted on $72 \%$ and $85 \%$ respectively. These values are calculated from the $R_{w}=8.8 \Omega$ and $R_{k}=31.3 \Omega$ for the Pt-CE and $R_{w}=11.6 \Omega$ and $R_{k}=73.5 \Omega$ for CNTf-CE. Although both transport and recombination resistances are higher for the CNTf-CE DSSC, the device still shows higher performance due to a much higher resistance towards recombination under dark conditions. The differences in $\eta_{\text {coll }}$ can originate from both differences in device construction, particularly with respect to the thickness and separation of the various layers, as well as from the interaction of electrodes with the species in the electrolyte. Both can affect electrolyte diffusion, for example, and thus the kinetics of dye regeneration.

TABLE 4.4: EIS parameters of DSSC with Pt- and CNT-CE.

\begin{tabular}{llllllll}
\hline CE Material & $R_{s}(\Omega)$ & $R_{C T}(\Omega)$ & $R_{\text {TiO }_{2}}(\Omega)$ & $R_{\text {diff }}(\Omega)$ & $R_{w}(\Omega)$ & $R_{k}(\Omega)$ & $\eta_{\text {coll }}(\%)$ \\
\hline $\mathrm{Pt}$ & 15.6 & 4 & 10 & 8 & 8.8 & 31.3 & 72 \\
CNTf & 12.2 & 0.6 & & $-12-$ & 11.6 & 73.5 & 85 \\
\hline
\end{tabular}

\section{CNTf-CE DSSC light power dependence:}

The performance of DSSCs under different irradiation power can provides further information about the charge dynamics in the cell. This dependence is also of technological relevance, for example with respect to the ability of DSSCs to generate power at low power illumination intensities, recently reaching $30 \%$ under 0.1 sun with Co based sensitisers and a Cu redox shuttle.[257]

Normally, when the light intensity is increased it produces a net increase in the output current. This occurs up to a point where the kinetics of the system can not sustain the regeneration rate needed and thus, the cell becomes less efficient. In order to understand losses due to regeneration of photo-generated carriers during device operation, the characteristics of the device (PCE, FF, $J_{s c}$ and $\left.V_{o c}\right)$ were measured as a function of the light intensity. The results are presented in Figure 4.20 and the extracted characteristics from these curves are presented in the Figure 4.20c. As 
it can be observed, the trend for both material is very similar. For the majority of the constructed devices, the $F F$ and $V_{o c}$ is higher when using Pt-CE. On the contrary, the extracted charge $\left(J_{s c}\right)$ is always between 5-10\% larger when used CNTf. This balance leads to similar efficiencies for both materials.
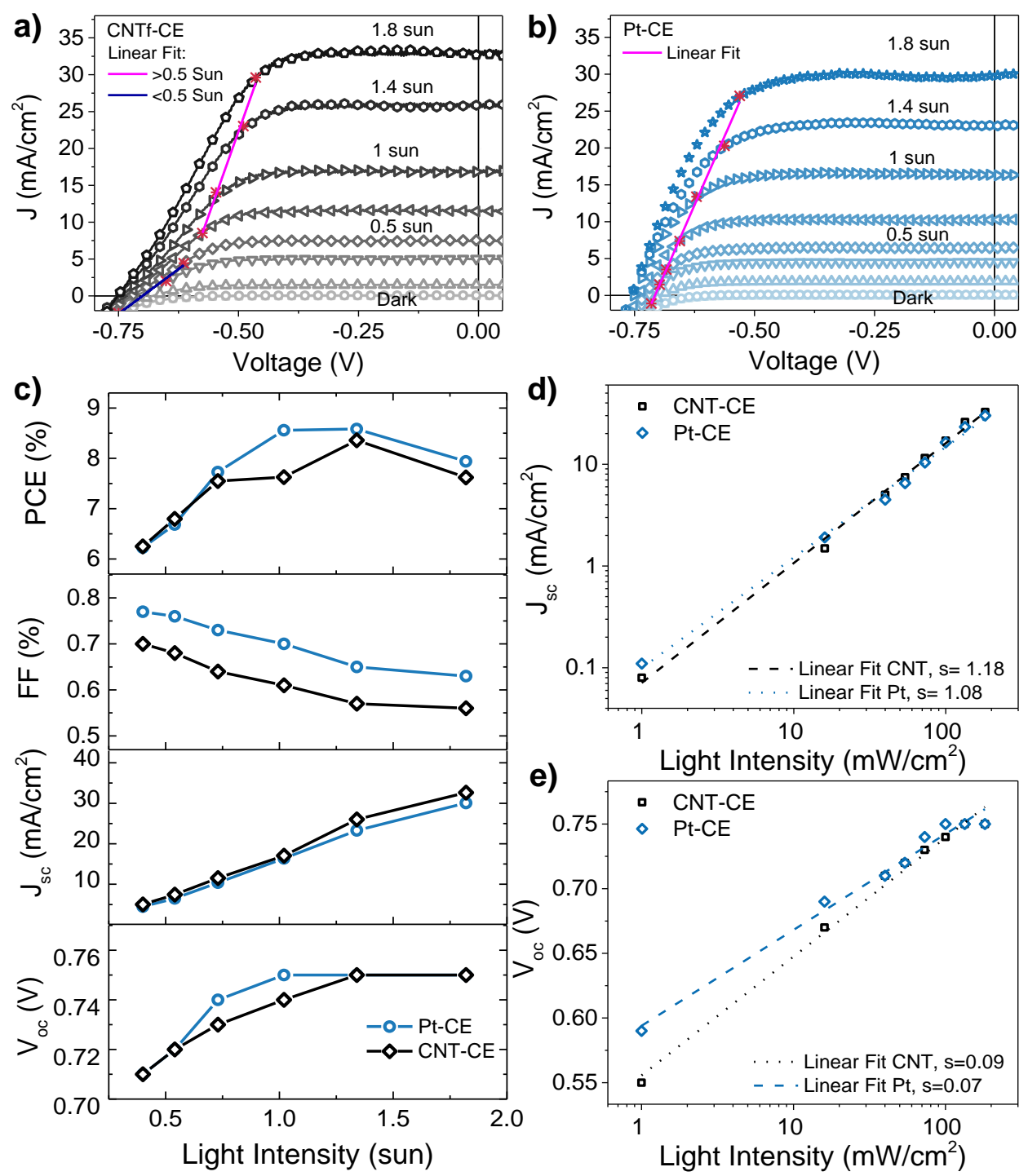

FIgURe 4.20: a) and b) $J-V$ curves of CNTf-CE and Pt-CE devices respect to the light intensity. c) $P C E, F F, J_{s c}$ and $V_{o c}$ parameter power dependence. d) $\log _{-} J_{s c}$ and e) $V_{o c}$ versus the logarithm light intensity comparing both Pt- and CNTf-CE.

The dependence of short circuit current $\left(J_{s c}\right)$ on light intensity is shown in Figure 4.20d. $J_{s c}$ follows a power law dependence of on intensity, i.e. $J_{s c} \propto I^{\alpha}$, with values of $\alpha$ of 1.08 and 1.17 for DSSCs made with the Pt- and CNTf-CE respectively. A value of $\alpha$ closer to unit indicates that the charge collection efficiency is independent of the light intensity, and thus not limited by electron and hole mobility or transport in the systems. This could suggest the influence of the light intensity over the CNTf electrical or catalytical properties. 
Figure 4.20e shows the $V_{o c}$ against the logarithm of light intensity with DSSCs made with the Pt- and CNTf-CE. If the recombination occurs only by conduction band electrons of the $\mathrm{TiO}_{2}$ and the redox reaction, considered first order, the recombination and the carrier concentration increase linearly with the light intensity and, it results in a slope of $59 \mathrm{mV} \mathrm{dec}^{-1}$ at $298 \mathrm{~K}(\mathrm{~m} \approx 1)$, see Appendix at the end of the Chapter 2 "Background". The slope obtained with the Pt-CE is $75 \mathrm{mV} \mathrm{dec}-1$ ( $m V$ per decade), which is a value close to the ideal behaviour and correspond to an ideality factor $m=1.18$. This slope is in agreement with previous reported values $\left(76 \mathrm{mV} \mathrm{dec}^{-1}\right)$ [258] for the same $\mathrm{TiO}_{2}$ /electrolyte system. In contrast, CNTf-CE

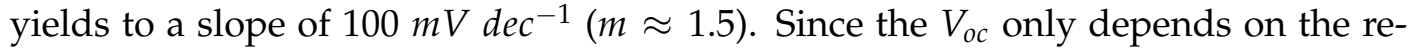
dox potential of the electrolyte and the quasi-Fermi level of the $\mathrm{TiO}_{2}$, relatively high value of $m$ is likely due to non linear recombination kinetics for the back reaction of conduction band electrons with the $I_{3}^{-}$ions. Since the photoanode is the same in both cells, it is possible to discard electron transfer via surface states and/or shifts in the conduction band under illumination.[258] Instead, the hypothesis is that the increment in local concentration of $I^{-}$ions in the surface of the mesoporous CNTf$\mathrm{CE}$ affects constant rates of the regeneration reaction and thus the recombination kinetics.

An important result comes from looking closer the series resistance $R_{s}$ evolution extracted from the $J-V$ curves at different light intensities. The series resistance indicates the drop of voltage and therefore, energy lost, because of resistive elements inside the cell. The calculation of this characteristic is detailed in the Chapter 3 "Experimental Techniques" based on the method of the light intensity variation.[215]

For the standard Pt-CE series resistance behaviour of the cell follows a linear relationship with the light intensity, and $R_{s}=6.71 \Omega \mathrm{cm}^{2}$ is obtained (Figure $4.20 \mathrm{~b}$ ). This value and behaviour is in agreement with typical values reported[214] and mainly related with the sheet resistance of the current collector in this devices. Being the comparison performed on identical devices (in terms of photoanode and electrolyte materials, manufacturing process) only the substitution of the $\mathrm{Pt}$ for the CNTf material has to be considered for the comparison, and therefore, all changes in the $R_{S}$ are entirely attributed to the introduction of this material. In the Figure 4.20a, it can be observed the non-linear relationship of the $R_{s}$ with the light intensity. Two regions can be distinguish: i) the one at low light intensity $(<0.5$ sun) presents a $R_{s}=20.59 \Omega \mathrm{cm}^{2}$; ii) at higher light intensities ( $>0.5 \mathrm{sun}$ ), the $R_{s}$ decreases a factor of 20 down to value $R_{s}=1.013 \Omega \mathrm{cm}^{2}$. This decrease in resistance explains the increase of output current above 1 sun illumination, being the current in the CNTf device $10 \%$ higher than in the Pt device at 1.8 sun - 33 versus $29 \mathrm{~mA} \mathrm{~cm}^{-1}$ respectively. Moreover, these differences in resistance and behaviour indicate the $\mathrm{CNTf}$ is acting not only as counter-electrode in the DSSC but also as current-collector due to the lower resistance of the CNTf versus the FTO substrate. The large drop in $R_{S}$ for CNTf-CE at higher light intensities is not due to thermal effects over the conductivity of the $\mathrm{CE}$. All the measurements are performed at $\approx 25^{\circ} \mathrm{C}$ to ruled out this factor. Moreover, the longitudinal conductivity of CNTf electrodes in fact decreases with increasing the temperature.

A possible reason for decreases in $R_{s}$ would be an increased CNTf conductivity due to doping by iodide species. A 30 -fold increment of electrical conductivity and carrier concentration of CNTfs by iodine doping have been reported.[243, 259, 260] The iodine doping effect has been also observed in our devices and the data is presented in the "catalytic activity" section of this chapter, Figure 4.8. Although this 
doping occur after some time of the electrode exposed to the electrolyte, the high intensity of the light during the experiment could accelerate this effect. Speculatively, as light power is increased, the concentration of polyiodides in the electrolyte increases, and thus, the number of dopants through charge transfer at the CNTf-CE.

Concluding, the origin of this interesting and beneficial effect needs further investigation. Experiments at a wider range of light intensity could help for understanding better the mechanisms and limits of the CNTf-CE. Besides that, look into the nature of the catalysis of the CNTf will help to understand better the limitations of the material.

\section{DSSC device variability in presence of CNTf-CE:}

In this subsection the variability between devices is discussed.

First of all, the variability related to the manual fabrication process of this devices has to be considered. The devices are produced one by one without any automation, e.g. there is no screen printing machine for producing identical sets of anodes. Therefore, every step will inevitably introduce some small variations that makes unique every device. Apart from that, the variability of the materials itself introduce another source of error. For standard commercial materials, this is not a big issue and is fair to assume that they have a good reproducibility. But when materials are synthesised as part of the investigation (i.e. CNTf), this is a factor that must be considered.

For, the standard $\mathrm{Pt}$ reference cells present a high degree of reproducibility. This is mainly attributed to the low roughness of the Pt-CE. The variability between this devices is below to $5 \%$.

On the contrary, the manual procedure for manufacturing CNTf-CE DSSCs is a delicate process. Firstly, the CNTf material and synthesis procedure has to be highly reliable for avoiding material inconsistencies. Large batches of material were produced under the same conditions (for minimising this issue). The second source of error during the manufacturing of CNTf-CE devices is the sealing, encapsulation and filling of electrolytes. As an example, since the CNTf is highly porous, during the hot pressing step, some part of the fibre can be infiltrated by the thermoplastic, this leads to geometrical variations in the effective area of the device. Also, due to the roughness of the CNTf electrode and the high thickness of the material, the complete electrolyte infiltration is more difficult.

The typical variability of the CNTf-CE DSSC devices is presented in the Figure 4.21. Three sets of devices are presented after ruling out short-circuited devices (30-50 \%), and the $J-V$ curves are shown in the Figure 4.21a. The different configuration are detailed as follows:

- $5 \mu \mathrm{m}$ thin $\mathrm{CNTf}$ and 1 layer of $\mathrm{TiO}_{2}$ deposited by doctor blade technique $(7 \mu \mathrm{m})$. "1 layer - CNTf $5 \mu m "$

- $10 \mu \mathrm{m}$ thick CNTf and 1 layer of $\mathrm{TiO}_{2}$ deposited by doctor blade technique (7 $\mu \mathrm{m})$. "1 layer - CNTf $10 \mu m "$

- $10 \mu \mathrm{m}$ thick CNTf and 2 layers of $\mathrm{TiO}_{2}$ deposited by doctor blade technique (14 $\mu \mathrm{m})$. "2 layer - CNTf $10 \mu \mathrm{m} "$ 
The characteristics extracted from the $J-V$ curves are presented in the Figure $4.21 \mathrm{~b}$. The $V_{o c}$ is quite reproducible between devices with the same configuration. This is expected because of the thermodynamic nature of this characteristic. On the contrary, $J_{s c}$ shows a huge variability. The $J_{s c}$ is proportional to the thickness of the layer due to the higher number of dye molecules absorbed. Moreover, as it has been mention before, this parameter is quite dependent on the degree of filling of the cell by the electrolyte. For thicker photoanodes ( 2 layers of $\mathrm{TiO}_{2}$ ), the electrolyte filling presents more problems and the variability of the $J_{s c}$ due to this process also increases. The fill factor of the first two sets $\left(1\right.$ layer $\left.\mathrm{TiO}_{2}\right)$ is lower than for the "2 layers" configuration. Also, it should be notice that the thin CNTf $(5 \mu \mathrm{m})$ and thick CNTf $(10 \mu \mathrm{m})$ are not directly comparable in terms of FF due to the small S-shaped presented. Finally, the variation of efficiency is lower for thin photoanodes. The variation in performance increases proportionally to the variation of previous characteristics, besides, there are more sources of error preparing thicker photoanode devices.
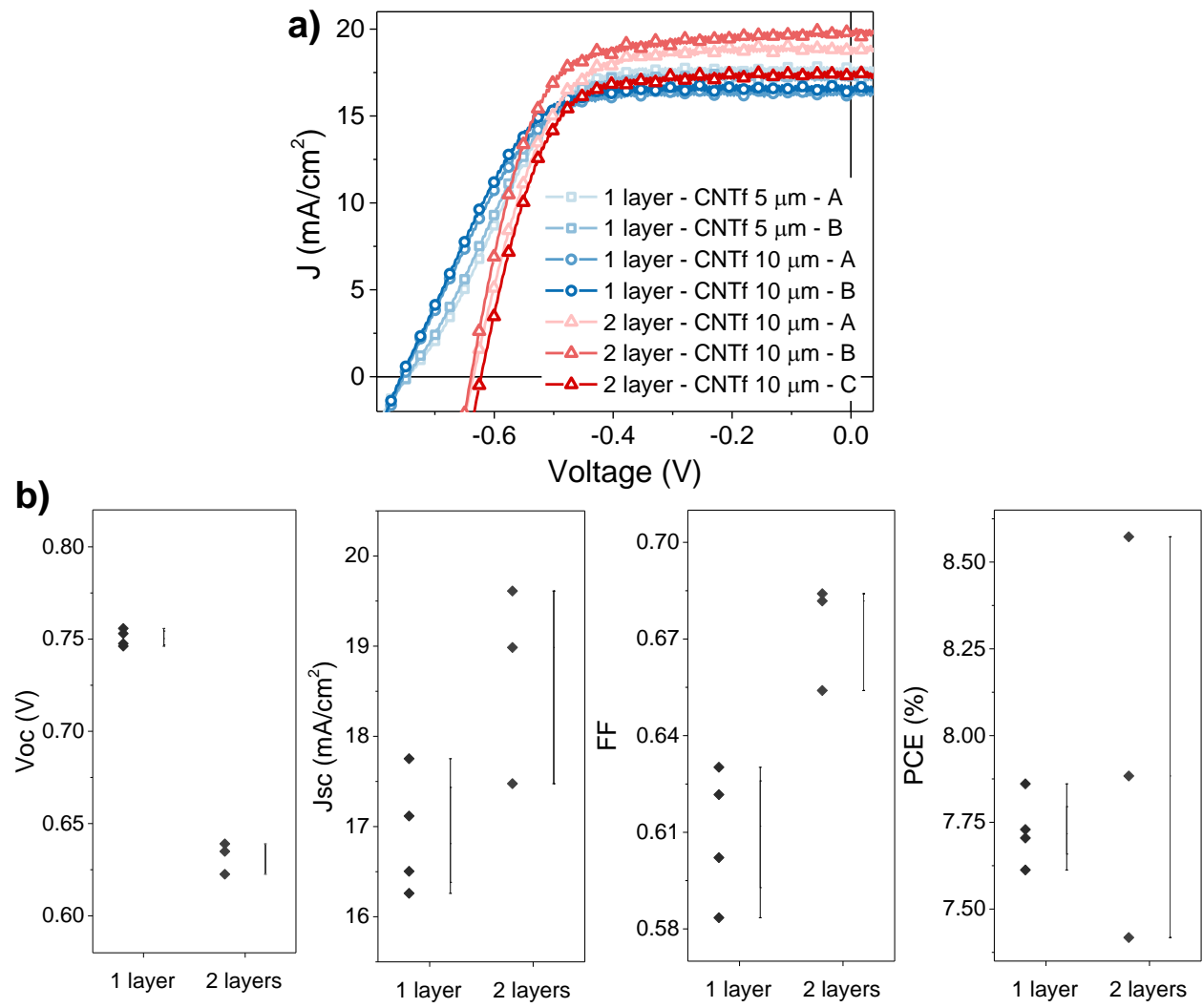

FIGURE 4.21: a) $J-V$ curves of several CNTf-CE DSSC devices with 1 and 2 layers of $\mathrm{TiO}_{2}$ by doctor blade. b) Device characteristics grouped by the device architecture. The vertical line represents the range of the obtained values for the characteristics.

Although the reproducibility presented in this section is representative of the obtained devices during this thesis, the aim of this section is not showing the reproducibility of the results, if not explaining the different factors affecting the variability when the CNTf-CE are used. 
Pt-CE material has been established as reference for DSSC devices by its superior catalytic activity and electrical conductivity, it still represents some drawbacks, among them the stability itself. Pt electrode is easily corroded by presence of solventbased iodide electrolyte[261] and also suffers form poisoning effect in presence of air.[262] Although there should not be air inside the device, when solvent electrolyte start to evaporates from the cell, this volume is inevitably filled with air, adding extra detrimental effects to the device stability. Besides this degradation issues of the $\mathrm{Pt}-\mathrm{CE}$, exist other less corrosive electrolytes and also strategies for protecting the material - e.g. Pt alloy CE[263] or alternative dye molecules,[264] therefore, stabilities as high as $95 \%$ of the initial performance has been reported.[265] On the other hand, many Pt-free counter electrodes has been studied as candidates that does not present this degradation issues, see for instance review about stability on this materials by Yun et al.[219]

As a first approximation for studying the stability of the CNTf-CE DSSC, the performance of the devices presented in previous sections, as well as the reference devices, were measured after 30 days and 6 months of their assembly. As it is described in the Chapter 3 "Experimental Techniques", the sealing procedure is performed by hot pressing a small portion of thermoplastic that covers the holes in the electrode through where the electrolyte is injected. This method allows some solvent evaporation, and therefore, the results presented in this section must be taken as lower limit values in terms of stabilities for this materials. The devices were kept in dark and RT condition between measurements.

The CNTf-DSSCs showed a comparable stability to the reference Pt-CE during the first month in the range of $10 \%$ drop in efficiency. After that, the CNTf-DSSCs show superior stability for all the different photoanode thickness studied, reaching nearly $70 \%$ after 6 months for the $7 \mu \mathrm{m}$ thick photoanode and close to $60 \%$ for the full configuration $(21 \mu \mathrm{m}) . J-V$ curves of the comparative between devices and a column chart summarising the stability data of the performance for different $\mathrm{TiO}_{2}$ photoanodes are presented in the Figure 4.22.
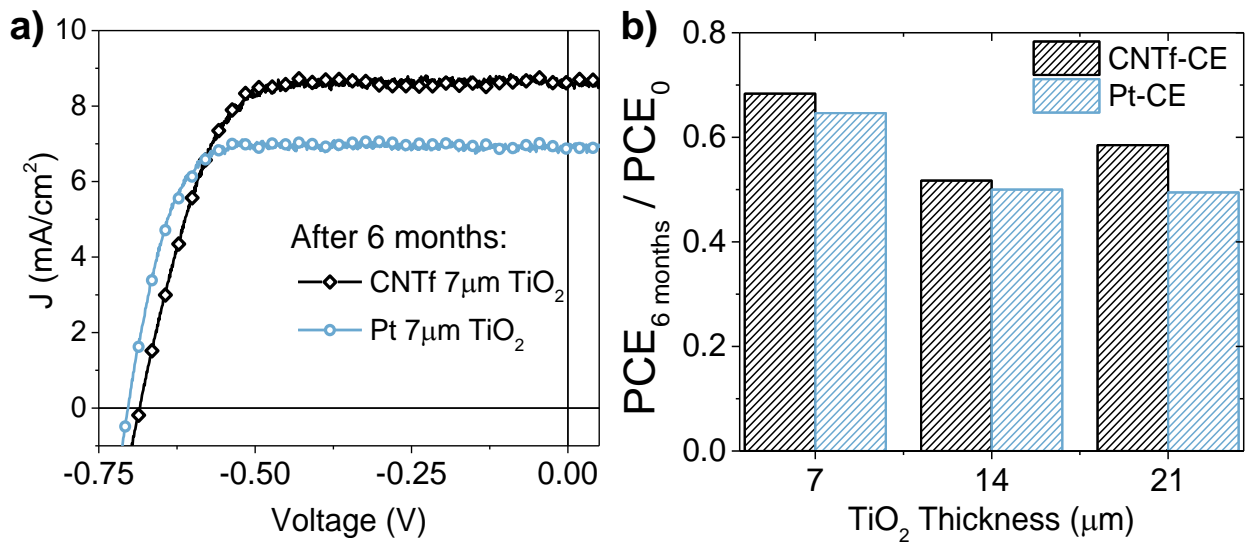

FIGURE 4.22: a) $J$ - $V$ curves of Pt- and CNTf-DSSCs after 6 months. b) Column chart representing the relative stability of 6-month old devices with respect to fresh measurements, highlighting the better performance of the CNTf-DSSCs.

The higher stability of the CNTf-CE can be related to two factors. Firstly, the 
electrolyte stability is higher in presence of the CNTf membrane. With its high porosity and surface area, the CNTf enhances the stability of the electrolyte by reducing the possible leakage and evaporation. This is in agreement with previous studies using polymeric membranes for accommodating the electrolyte and increasing the stability.[266, 267]

The degradation of DSSC devices has been related with the lowering in performance of the N719-dye.[268] A blue shift in the absorbance of the N719 spectra has been observed after 1000 hours under storage conditions. This blue shift and the lowering in performance has been attributed to a ligand exchange reaction between the dye thiocyanato $\left(\mathrm{SCN}^{-}\right)$ligand and the iodide component.[268] The chemical environment of the electrolyte in presence of the CNTf, as well as the preferential interaction of the iodide specie with the CNTf, as has been explained in previous sections, could be acting as a protecting agent of the electrolyte solution. Similar stabilisation effects has been reported before in presence of CNTs,[183] although further experiments must be performed for clarifying the fundamental effects underlying this improvement.

\subsubsection{CNTf-CE/TiO 2 -photoanode microcontacts: spacer-free DSSC}

The standard procedure for assembling a DSSC device involves the hot pressing of the working- and counter-electrodes using a pre-cut thermoplastic film as adhesive after melting. Apart from this function, the film acts as a spacer between the parallel electrodes, avoids the short-circuit between electrodes and serves as gasket for accommodating the liquid electrolyte inside the device. Typically, a $25 \mu \mathrm{m}$ thick polymer film (Surlyn) is used. Therefore, in theory, the layer of active material that forms the working electrode is separated from the counter-electrode by a gap of several microns through, as schematically shown for a Pt-CE DSSC device in Figure 4.23a.

Considering the thicker thickness of CNTf-CE and the reduction of thermoplastic thickness upon melting, the final DSSC architecture must comprise regions where the photoanode and counter-electrode are in contact (Figure 4.23b).

The final dimension of the cell after hot-pressing remains ca. $20 \mu \mathrm{m}$. Therefore, taking into account the active layer thickness (from 7 to $20 \mu \mathrm{m}$ ) and the thickness of the CNTf-CE (approx. $10 \mu \mathrm{m}$ ), local microcontacts between both electrodes are possible.

However, note that in this configuration the electrolyte also fills the voids of the CNTf porous membrane. Previous results from the group have demonstrate that the porous volume fraction in the CNTf is approximately $80 \%$, enough space for accommodating the electrolye and making possible the operation of the DSSC. The high short-circuit current density observed for CNTf-CE DSSCs stems partially from the high interaction between CNTf-CE and electrolyte. A high $J_{s c}$ has been reported for the same photoanode architecture $\left(\mathrm{TiO}_{2} / \mathrm{N} 719\right)$ [269] and also for similar nanocarbon based systems suggesting that similar high interface could be overlooked in previous researchs.[124, 155, 157]

These microcontacts at the interface between the CNTf and the $\mathrm{TiO}_{2}$ can be detected by obtaining the $J-V$ curve of the device prior to filling with ionic electrolyte (Figure 4.23c). Probably, they are likely occurring in the majority of the devices and 
it has been confirmed that these microcontacts do not hinder the proper operation of these devices.

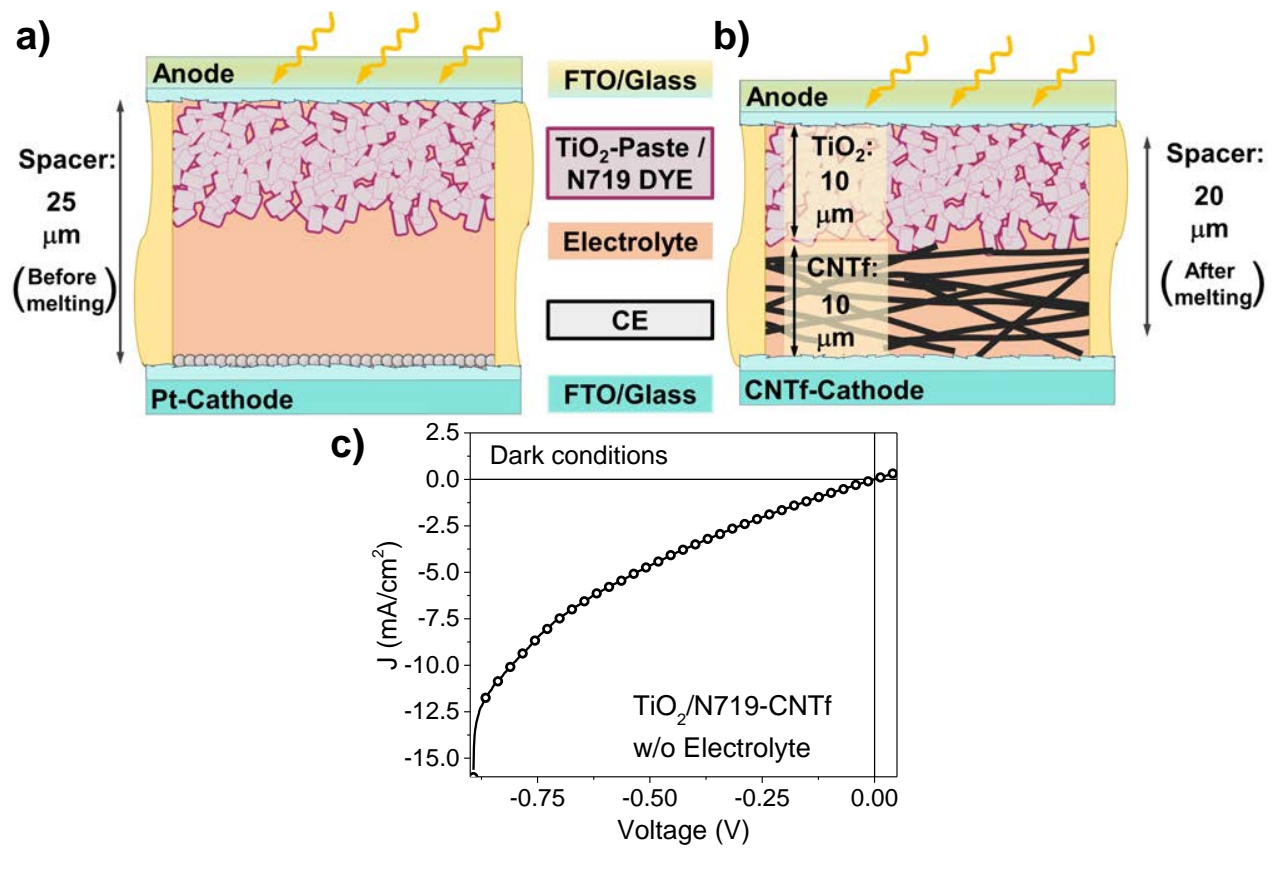

FIGURE 4.23: Schematic of the a) Pt- and b) CNT-CE configurations where the micro-contacts between $\mathrm{CNTf}$ and $\mathrm{TiO}_{2}$ are represented after assembly. c) $J-V$ curve in dark conditions obtained before introducing the liquid electrolyte.

Additional solid-state measurements have been performed directly contacting the surface of the $\mathrm{TiO}_{2}$ with the CNTf, using the CNTf directly as current-collector. Using a pre-cut thermoplastic film is possible to leave uncovered the surface of the $\mathrm{TiO}_{2}$ semiconductor for avoiding the sortcircuit with the $\mathrm{TiO}_{2}$ current collector - FTO in these experiments. A schematic of this measurement is presented in the Figure 4.24a. The Tafel measurement showing a slightly rectifying behaviour is presented in the Figure 4.24. But more importantly, confirming that the $\mathrm{TiO}_{2} / \mathrm{CNTf}$ direct contact could still lead to high current density as it is observed for the CNTf-CE.

a)

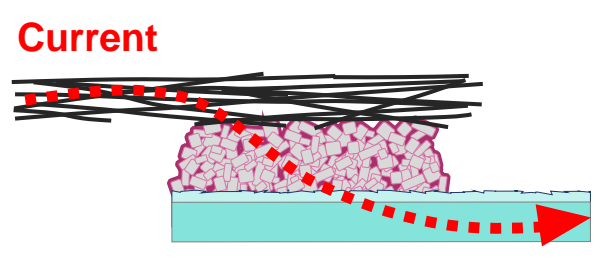

b)

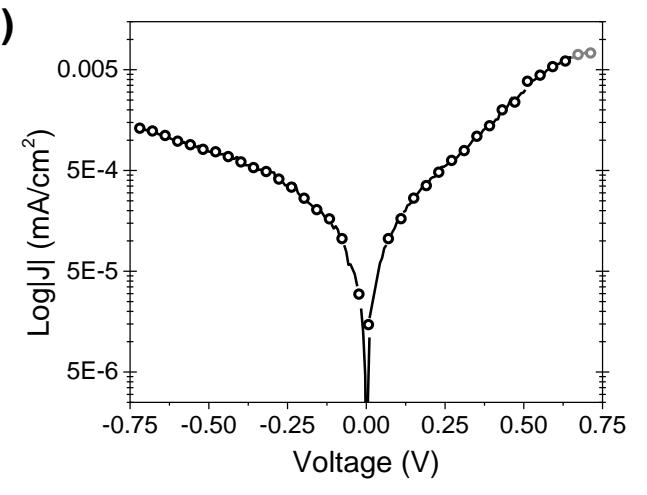

FIGURE 4.24: a) Schematic of a on-purpose produced heterojunction prepared for measuring the properties of the interface. b) Dark J-V measurement through the $\mathrm{CNTf} / \mathrm{TiO}_{2}$ interface. 
It is very interesting that devices works in spite of shorts. With respects to charge dynamics it implies that the charges under working conditions still follows the $\mathrm{TiO}_{2} /$ FTO injection path and these microcontacts are not acting as recombination points (at least with a predominant contribution). The low conductivity of the mesoporous sintered titania, in addition to the internal polarisation created at the solar cell when the device is under illumination explain this behaviour.

There are also some technological aspects that make this system interesting. For instance, it means that the porous $\mathrm{CE}$ can acts as a direct $\mathrm{CC}$ but also hosts the electrolyte, thus enabling the use of the CNTf as direct electrolyte impregnated membrane.[270]

\subsection{Summary and conclusion}

In this chapter, a comprehensive electrochemical impedance spectroscopic (EIS) study to shed light onto the catalytic features of CNTf-CE and the role of its mesoporosity is presented. Besides, the stability of this material is studied by $C V$ and compared with the standard Pt. An important contribution has been done according to the electrochemistry between the CNTf and the standard electrolyte for DSSC. The interaction between both components alters the chemical environment of the device. Starting from different diffusion coefficients, related to the variation in concentration of conductive species in the electrolyte, and finishing by the possibly stabilisation of the electrolyte by taking the CNTf the role of protecting agent from the dye/electrolyte interaction. Still important question regarding the catalytic nature of the carbon material remains open. Investigations about the introduced defects over the surface of the material by functionalisation treatment indicates that the carbon edges are not the responsible of the catalytic activity.

In addition, the preparation and assembly of a planar CNT-CE DSSC is described. The optimisation of the CNTf thickness is studied, with special attention to the S-shape limiting factor of the performance. Its connection with the critic mass of catalytic CNTf material has been found, allowing to outperformed the efficiency of the reference Pt material. Later, the device architecture was optimised using the CNTf acting as both CE and current-collector leading to PCE as high as $9.5 \%$ and stability of $c a .70 \%$ after 6 months, superior to reference Pt-CE (9\% and of $c a .60 \%$ stability after 6 months).

This performance is consequence of the combination of high electrical conductivity and high specific catalytic surface area of the samples used in this work. These features in relation with the interaction of the iodide based electrolyte and the CNTf, lead to a different chemical environment inside the cell, also responsible of the enhanced behaviour of the CNTf-CE material.

Finally, the configuration of $10 \mu \mathrm{m}$ CNTf-CE/CC for DSSC is discussed taking into account the high interaction between the porous membrane and the electrolyte. This confirguration has been observed leading to microcontacts between electrodes, but interestingly, the device works in spite of shorts. 



\section{Chapter 5}

\section{Fundamental Properties of CNTf/MOx Heterojunctions}

Parts of this chapter are adapted from the work published in the journal ChemPlusChem, co-authored with Dr. Juan J. Vilatela. Unless mentioned, all experiments have been performed and analysed by the author.

Chapter 5 discusses the synthesis, structure and electrical properties of CNTf/MO $\mathrm{MO}_{x}$ hybrid materials. Large area samples of heterojunctions between $\mathrm{ZnO}$ and CNTf arrays are synthesised by two different methods.

Firstly, a two-step sol-gel/hydrothermal chemical method is used to grow ZnO-Nanowires (NWs) in-situ directly onto the CNTf array. It results in a bulk heterojunction, consisting of a 2-5 $\mu \mathrm{m}$ layer of $\mathrm{ZnO}$ crystals in contact with the built-in current-collector CNTf fabric, with preferential orientation of the $\mathrm{ZnO} c$-axis perpendicular to the CNTf sheet. The electrical properties of the heterojunction correspond to a Schottky junction with barrier height of $0.26 \mathrm{eV}$ and an ideality factor of 2.9. Photoconductivity measurements demonstrate charge transfer through the CNTf/ZnO interface, leading to a gain factor of approximately 100 and a responsivity as high as $5000 \mathrm{~mA} / W$, under UV irradiation.

Secondly, atomic layer deposition (ALD), a physical deposition technique that offers precise control of the synthesised materials, was used to produce CNTf/MO $\mathrm{MO}_{x}$ hybrids $\left(\mathrm{ZnO}\right.$ and $\left.\mathrm{TiO}_{2}\right)$ but with the semiconductor as a conformal coating and much thinner thicknesses. The structure, chemical interaction between both materials and electrical properties, were also investigated. The relation between the thickness of the deposited $\mathrm{MO}_{x}$ layers and the electrical behaviour of the hybrids is discussed. Besides, the charge transfer behaviour is further confirmed by UVphotocurrent measurements.

\subsection{Chemical synthesis route of $\mathrm{ZnO} / \mathrm{CNTf}$ based heterojunc- tions}

A porous network of CNTs can acts as a large-area scaffold for the semiconductor and be used as current-collector to harvest carriers generated in the semiconductor by photovoltaic,[271] photochemical[272] and piezoelectric processes.[273] Such CNT-based electrodes usually have the added benefit of being flexible as a consequence of a network structure and small thickness of the constituent nanotubes 
(bending stiffness scales with thickness to the fourth power). In the quest for making robust, flexible and large-area devices based on nanocarbon/semiconductor hybrids, some challenges remain in terms of the quality of nanocarbon/semiconductor junction that can be produced using simple large-area processes, and in the precise determination of interfacial properties.

\subsubsection{In-situ double step sol-gel/hydrothermal method}

For the preparation of ZnO-NWs large area heterojunctions with the CNTf as a substrate/current-collector, a double-step process[195] (sol-gel+hydrothermal) was applied based on the parameters reported elsewhere.[194] All the experimental synthesis conditions are provided in the Chapter 3 "Experimental Techniques".

Small pieces of CNTf $(5 \times 5 \mathrm{~cm})$ were cut out from the initial CNTf sheet $(30 \times 10 \mathrm{~cm})$, manually detached from the paper and transferred to a ceramic crucible (or other substrates) for $\mathrm{ZnO}$ seed deposition. A solution of zinc acetate dihydrate in absolute ethanol is poured with a syringe over the CNTf substrate. After solvent evaporation, the material is calcined in air at $300{ }^{\circ} \mathrm{C}$ for $15 \mathrm{~min}$ and in some cases recrystallised in Ar atmosphere.

The next step consists in the hydrothermal growth of $\mathrm{ZnO}$ nanowire from an aqueous solution of zinc nitrate and hexamethylenamine.[195] The seed-coated CNT fibre was taken from the ceramic crucible and placed inside a teflon vessel of a steel autoclave. The CNTf was kept immersed in the solution for five hours at $90{ }^{\circ} \mathrm{C}$. Finally, the hybrid CNTf/ZnO-NW hybrid samples are washed and dried to remove any residue of the synthesis process.
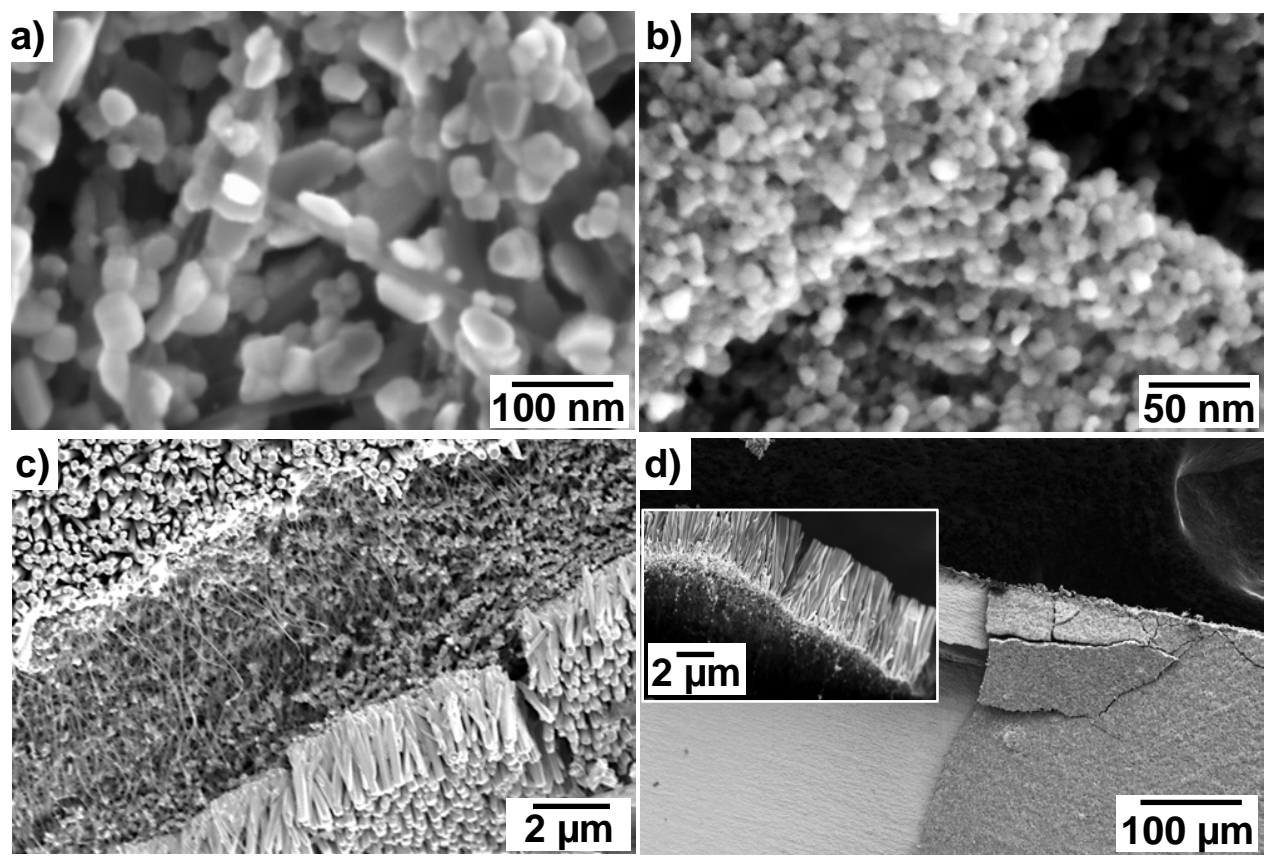

FIGURE 5.1: a) $50 \mathrm{~nm}$ and b) $10 \mathrm{~nm} \mathrm{ZnO}$ seeds decorating the CNTs bundles. c) and d) $\mathrm{ZnO}-\mathrm{NW}$ s layers respectively grown by hydrothermal from the previous seed layers. 
The $\mathrm{ZnO}$ particles grown by sol-gel method act as seeds for the growth of $\mathrm{ZnO}$ structures by the subsequent hydrothermal process. The influence of the seed concentration and size is studied by varying the synthesis conditions. A more homogeneous, uniform and mechanically robust $\mathrm{ZnO}$ layer is obtained when low concentration of $\mathrm{ZnO}$ seeds is used. Figure 5.1a,c corresponds to a sample with lower concentration obtained after $500{ }^{\circ} \mathrm{C}$ Ar crystallization, leading to $\mathrm{ZnO}$ seeds with diameter $>50 \mathrm{~nm}$, and which result in a uniform $\mathrm{ZnO}-\mathrm{NWs}$ coating with high mechanical stability. In contrast, when the concentration of seeds is too high, without any further recrystallisation, they tend to cover all the surface of the CNTf, forming a highly dense conformal layer of small nanoparticles, $10 \mathrm{~nm}$ approximately (see Figure 5.1b). It results in a very high number of nucleation points that produces later the formation of a too compact layer of $\mathrm{ZnO}$ that easily detaches from the seed layer (see Figure 5.1d).

\subsubsection{Morphology and crystal structure of the CNTf/ZnO-NWs}

The obtained material after the hydrothermal synthesis is presented in the Figure 5.2b. The SEM images of the plan view and cross section of typical samples show a large and homogeneous area of the CNTf sheet covered by ZnO nanostructures. These nanostructures grow as tapered hexagonal prisms normal to the plane of the CNTf sheet as can be observed by the SEM images. The final structure will be composed by the CNTf sheet coated with the layer of ZnO-NWs at both sides (see Figure 5.2b right).

a)

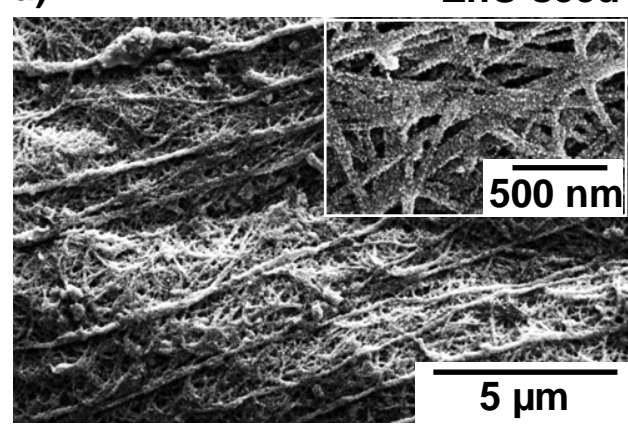

ZnO seed deposition

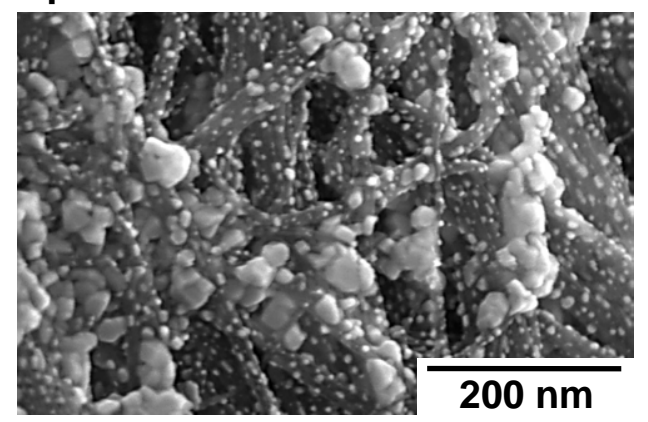

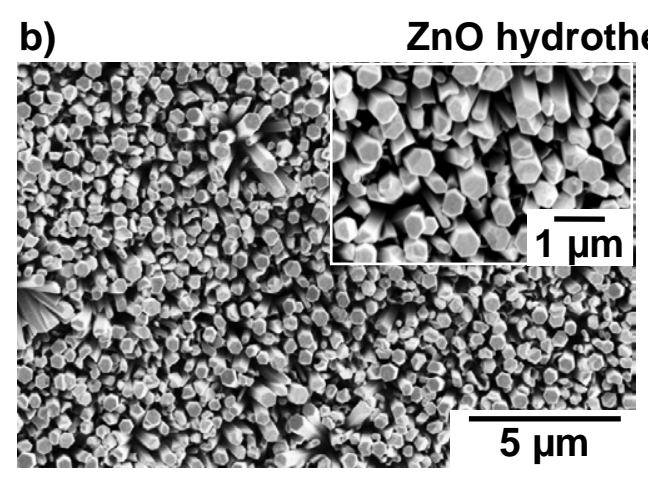

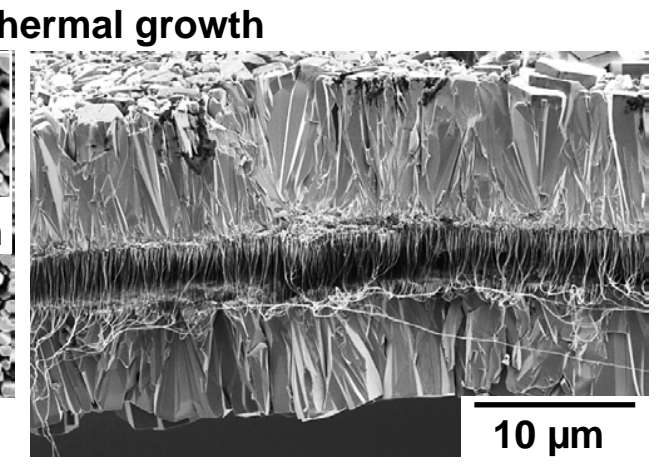

FIGURE 5.2: SEM images of ZnO growth over CNTf substrate by a) first step sol-gel process, b) second step hydrothermal method. Planview (left) and cross section (right) of $\mathrm{ZnO}$ nanorod and the interface with the CNTf. 
The synthesis method can be applied to produce relatively large samples. Figure 5.3 shows an example of a $10 \mathrm{~cm}^{2}$ hybrid with $\mathrm{ZnO}$ coverage throughout all its surface, except for the area that was purposely masked to establish electrical contact on the CNT network. The ZnO-NWs crystals have a typical diameter of $500 \mathrm{~nm}$ and length of approximately 2-5 $\mu \mathrm{m}$, according to the electron micrographs.

Considering the porous nature of the CNTf substrate, the size of the interface between $\mathrm{ZnO}$ and CNTf in a $10 \mathrm{~cm}^{2}$ is as high as $1.5 \mathrm{~m}^{2}$. This value can be roughly estimated from the data of the amount of material collected, the linear density and the surface area of the CNTf. The calculation can be summarised as follows: the sample used in this work is a CNTf sheet of $30 \times 10 \mathrm{~cm}\left(300 \mathrm{~cm}^{2}\right)$, collected by winding fibre for $30 \mathrm{~min}$ at $6 \mathrm{~m} / \mathrm{min}$. From here, the length of the collected CNTf, $180 \mathrm{~m}$, is extracted. The linear density for this CNTf, under this synthesis conditions, is approximately $1 \mathrm{mg} / \mathrm{m}$. For the collected length of $180 \mathrm{~m}$, there is therefore $180 \mathrm{mg}$. With this mass of material and the surface area of $250 \mathrm{~m}^{2} / \mathrm{g}$, the exposed surface area of the CNTf sheet, $45 \mathrm{~m}^{2}$ is calculated. Finally, for a $10 \mathrm{~cm}^{2}$ sample of $\mathrm{ZnO} / \mathrm{CNTf}$, the exposed area is divided by a factor of 30 (original size of the CNTf sheet and final size of the sample, $300 / 10=30$ ), finally obtaining $45 \mathrm{~m}^{2} / 30=1.5 \mathrm{~m}^{2}$.

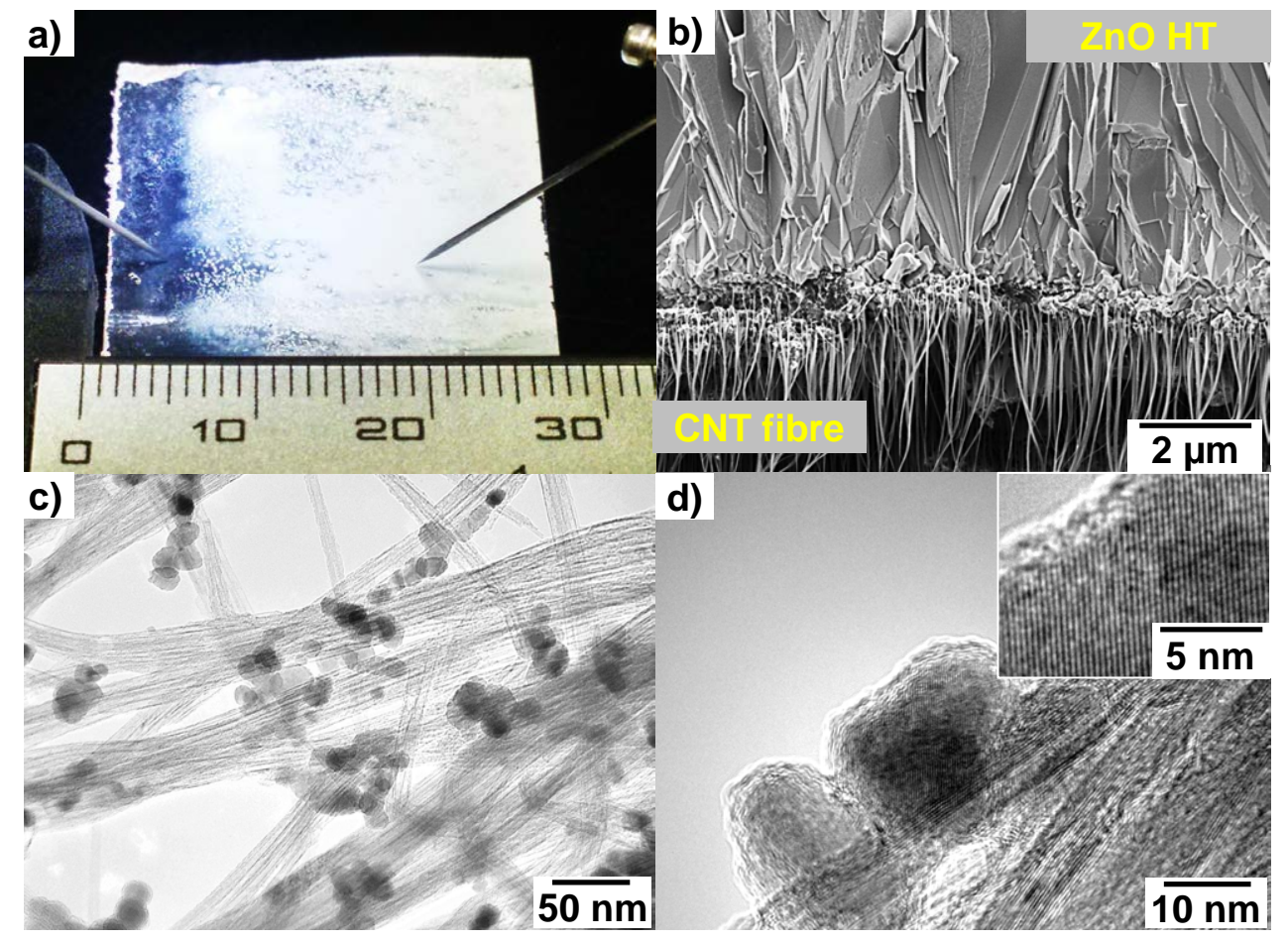

FIGURE 5.3: a) Digital photogragh of the $10 \mathrm{~cm}^{2} \mathrm{CNTf} / \mathrm{ZnO}$ hybrid. b) SEM and c, d) TEM images showing the direct contact between the $\mathrm{CNT}$ and $\mathrm{ZnO}$ particles.

More importantly, in the resulting interface, the CNTs and ZnO are in close proximity as observed by electron microscopy and HRTEM (see Figure 5.3b,c,d). The ZnO sol-gel nanoparticles are firmly attached to the CNTf and hence will act as anchoring points for the ZnO-NWs. Therefore, it is expected to have a good interconnection between $\mathrm{ZnO}$ and CNTf electrode. In view of of the close contact between 
the two phases, the material should behave as a bulk electronic heterojunction between $\mathrm{ZnO}$ and CNTs and thus, it can be used to study interfacial charge transfer processes of interest in various applications.[274]

Additional EDX characterisation about the CNTf/ZnO nanoparticle interface is presented in the Figure 5.4. By Z-contrast imaging in transmission mode, the nanoparticles are easily detected and by EDX compositional mapping. It is further confirmed that $\mathrm{ZnO}$ nanoparticles are actually the material decorating the CNTf surface.

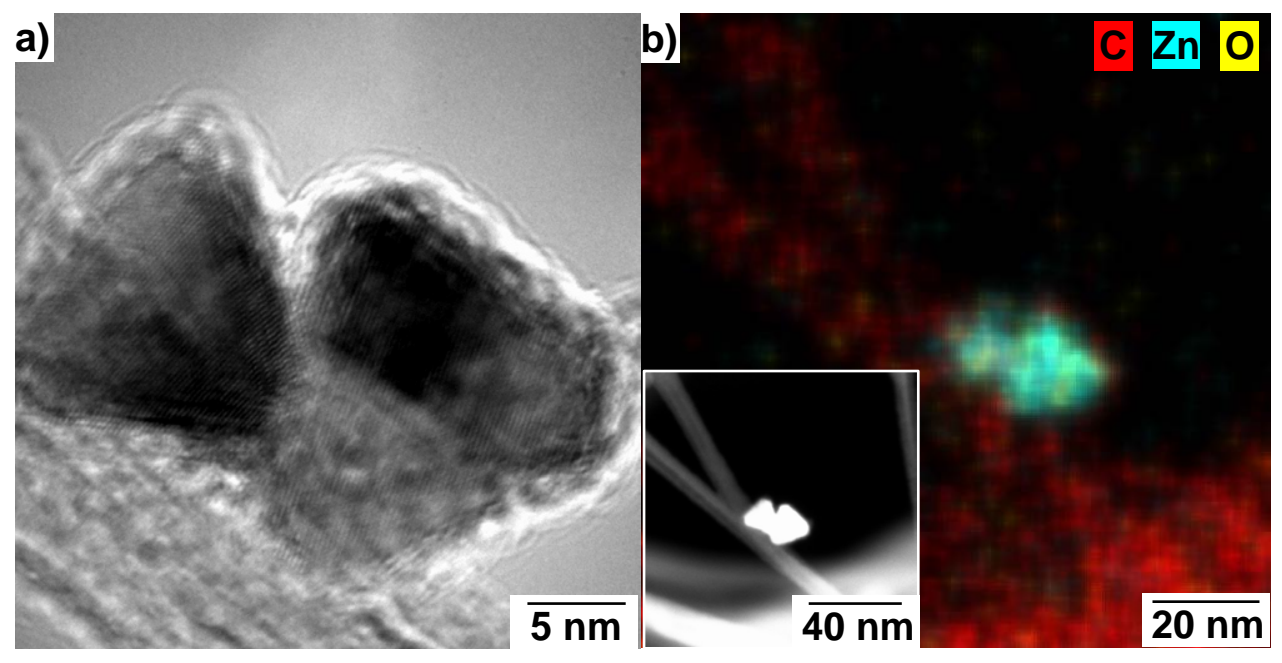

FIGURE 5.4: a) TEM image and b) EDX mapping at the interface between CNTf/ZnO sol-gel nanoparticles. Inset: Z-contrast TEMHAADF image.

The electrical properties of the final CNTf/ZnO heterojunction will depend on the properties of the individual components. It is well known the dependence of the electrical properties on the crystallinity of the semiconductors. Therefore, the crystallinity of the $\mathrm{ZnO}$ after the sol-gel process and the hydrothermal growth is analysed by XRD and TEM electron diffraction.

The orientation and size of the $\mathrm{ZnO}$ sol-gel nanoparticles have been investigated by combination of XRD analysis, Scherrer equation, and electron microscopy image analysis. The wide-angle $x$-ray scattering (WAXS) 2D-pattern is presented in the Figure 5.5a. The main diffractions of the $\mathrm{ZnO}$, as well as the (002) corresponding to the graphite plane distance in the CNTs are clearly observed. By azimuthal integration of each individual diffraction, and representing the intensity versus the azimuth angle $(\phi)$, a small alignment of the sol-gel particles with the $\mathrm{ZnO}$ basal plane parallel to the CNTs $c$-axis is obtained (see Figure 5.5b).

By radial integration of the entire range of the collected WAXS pattern, the XRD diffraction pattern presented in the Figure 5.5c is obtained, showing a crystal structure characteristic of the $\mathrm{ZnO}$-wurtzite lattice (JCPDS card number: 36-1451) and observing the characteristic (002) peak of the graphite. The crystal domain sizes, calculated with the Scherrer equation by fitting the (002) diffraction of the $\mathrm{ZnO}$ (see Figure 5.5d), are in the interval from 8 to $50 \mathrm{~nm}$. This is in agreement with the observed particles by electron microscopy imaging. 

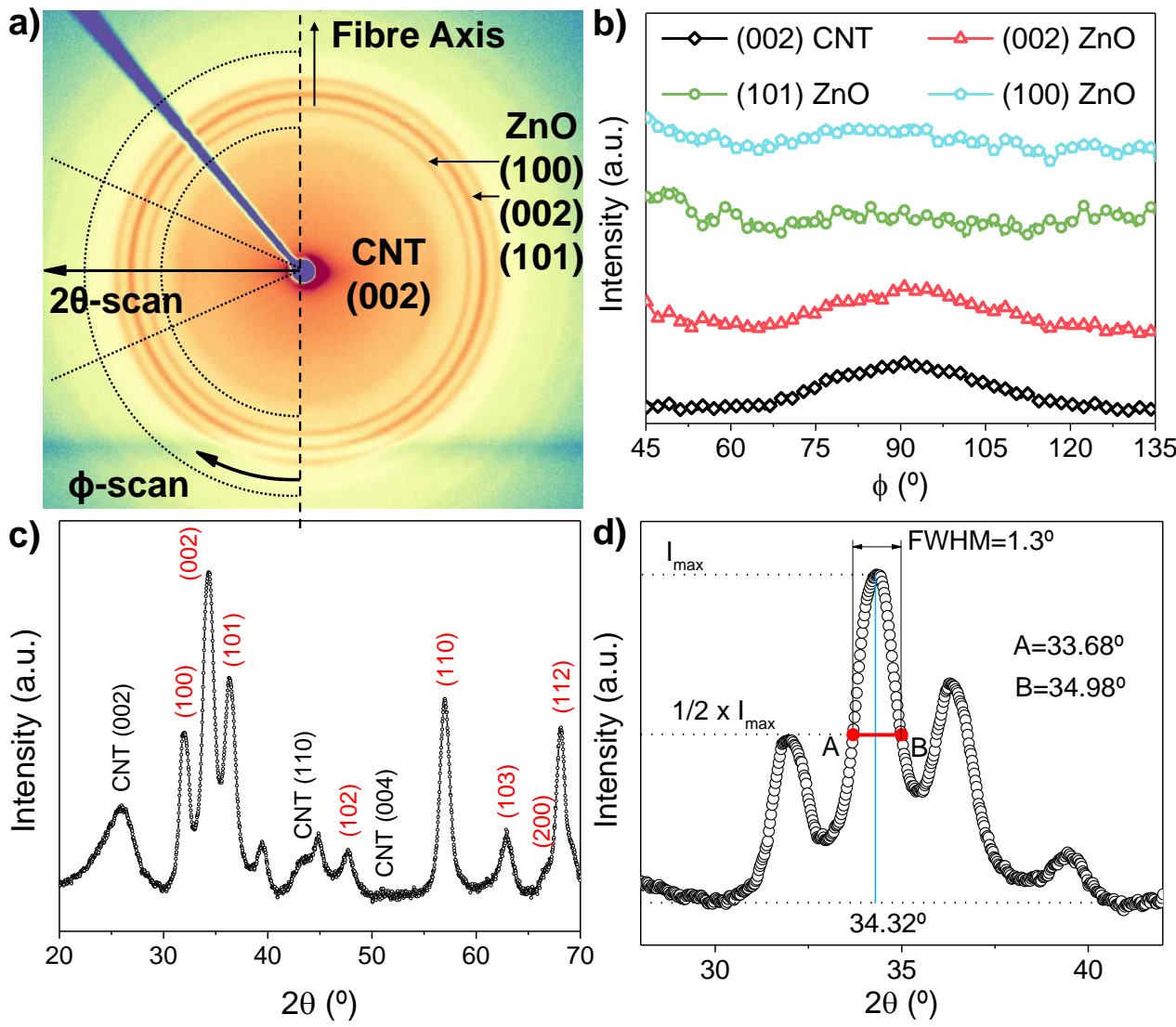

FIGURE 5.5: a) WAXS pattern of ZnO sol-gel nanoparticles decorating CNTf, b) azimuthal integration of the WAXS, c) radial integration and d) Scherrer equation study applied to the (002) $\mathrm{ZnO}$ diffraction.

The orientation and crystallinity of the $\mathrm{ZnO}-\mathrm{NWs}$ grown by the hydrothermal second step of the synthesis was also analysed by XRD measurements. Their orientation relative to the CNTs was determined by taking $X$-ray diffraction patterns with the sample at different orientation relative to the X-ray beam. Considering the symmetry of the samples, the relevant comparison is with the beam perpendicular and parallel to the plane of the CNTf sheet, thus parallel and perpendicular to [001] $\mathrm{ZnO}$, respectively. Schematics of these different configurations are included in the Figure 5.6. The first measurement is in transmission mode, and it was obtained from radially integrated wide-angle X-ray scattering (WAXS) 2D patterns (these data were collected at NCD BL11 ALBA Light Source using a wavelength of 1 angstrom). The other orientation corresponds to a standard powder XRD configuration in reflection mode with the planar sample resting on the sample holder.

XRD measurements confirm that the ZnO-NWs have wurtzite structure, with the $c$-axis predominantly perpendicular to the CNTs (Figure 5.6), and an average $\mathrm{ZnO}$ crystal size of $80 \mathrm{~nm}$ in the $c$-direction and $40 \mathrm{~nm}$ in the basal plane. The average crystal size domains of $\mathrm{ZnO}-\mathrm{NW}$ s were determined using the Scherrer equation after correcting for instrumental broadening. These values are smaller than the nanorod sizes observed by SEM, which indicates that the nanowires contain a few crystalline domains and therefore grain boundaries. The $\mathrm{ZnO}$ lattice parameters 
$\mathrm{a}=\mathrm{b}=0.324 \mathrm{~nm}, \mathrm{c}=0.520 \mathrm{~nm}$ were directly extracted from the interplanar distances of the (100) and (002) planes and are consistent with HR-TEM images. Moreover, these parameters are consistent with previous reports $(0.324 \mathrm{~nm}$ and $0.521 \mathrm{~nm}$ from JCPDS card number: 36-1451).

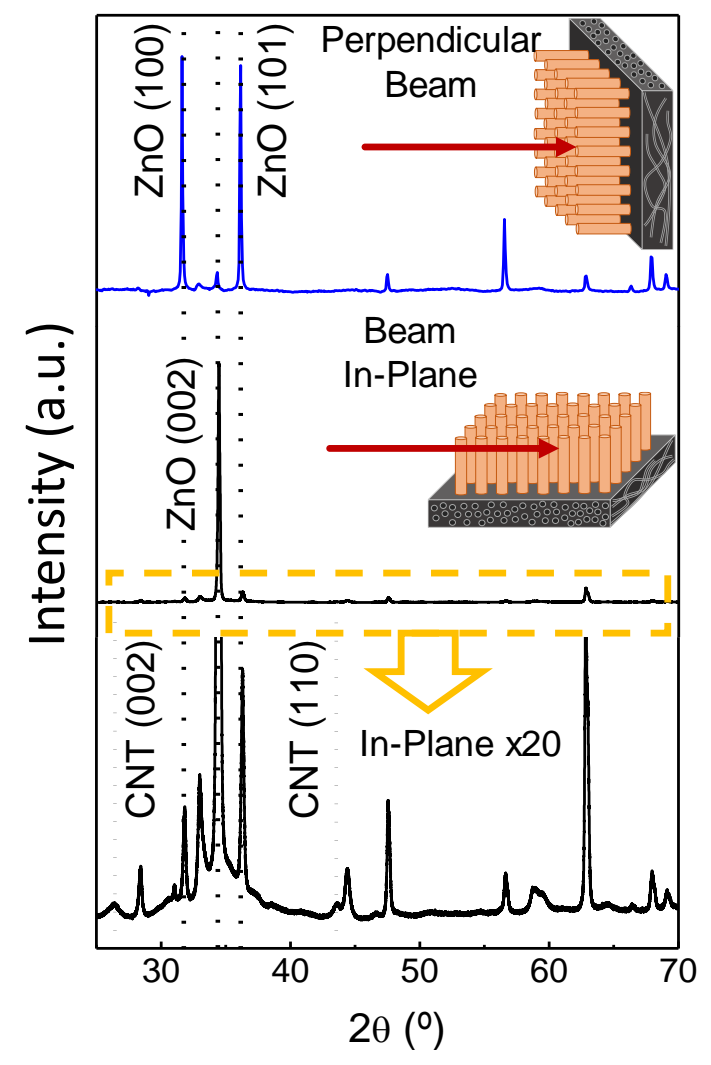

FIGURE 5.6: XRD patterns of the material with the incident beam perpendicular and in-plane with the CNTf substrate.

Although the sol-gel nucleant nanoparticles show a slightly preferential orientation, the final orientation of the large $\mathrm{ZnO}$ structures is most likely a result of crystals blocking each other in the plane during hydrothermal growth and thus tilting perpendicular to the substrate to continue growing. This is concluded by direct observation of intermediate stages of the $\mathrm{ZnO}-\mathrm{NW}$ growth, in addition to the well known and confirmed preferential growth of these structures along the $c$-axis.

Further structural analysis was carried out by high resolution TEM microscopy. The interplanar spacings of both materials, CNTf and ZnO-NWs, are obtained by fast Fourier transform of the HR-TEM image. The ZnO-NWs are highly crystalline and the interplanar spacing along the $c$-direction was determined as $0.520 \mathrm{~nm}$. For the CNTf, the measured interplanar spacing between graphite planes is $0.34 \mathrm{~nm}$, very close to the expected value of $0.335 \mathrm{~nm}$.

The formation of large heterojunctions facilitates the study of their morphology and interfacial processes, but it produces a "bulk" description of a very large nanostructured material, and thus falls on sample uniformity for the correct interpretation of the results. 
a)

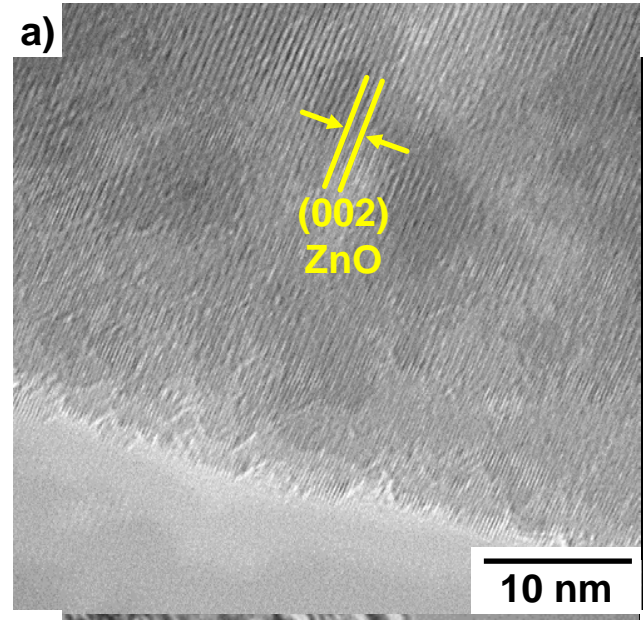

c)

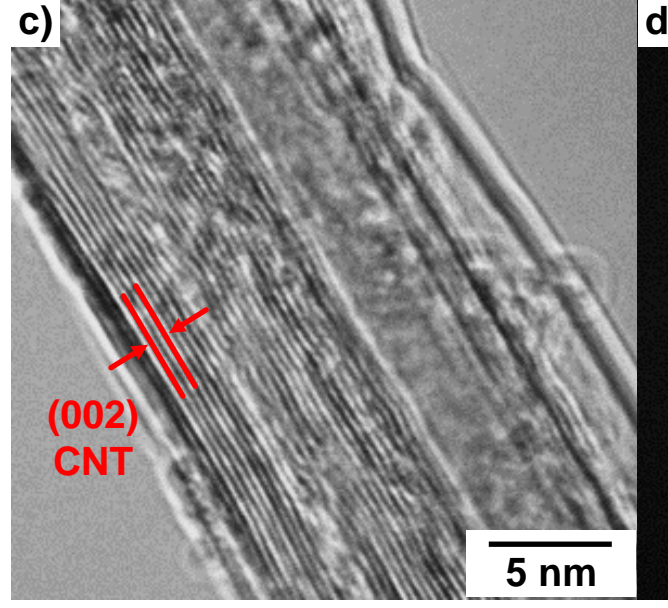

b)

d)

FIGURE 5.7: TEM images and TEM-FFT are presented for a hydrothermal ZnO-NW (up) and a CNT bundle (down).

Several Raman spectra are presented as demonstration of the homogeneity of the pristine material in the Figure 5.8a.

Raman spectrum of the CNTf/ZnO hybrid is presented in the Figure 5.8b. The hybrid material reveals the typical spectrum of the carbon nanotubes,[208] there is almost no shift in the position of the $G\left(1583 \mathrm{~cm}^{-1}\right), \mathrm{D}\left(1350 \mathrm{~cm}^{-1}\right)$ or $2 \mathrm{D}\left(2685 \mathrm{~cm}^{-1}\right)$ observed, compared with the pristine CNTf. An increase of the D/G ratio, from $0.2 \pm 0.01$ to 0.45 , suggests some degree of functionalisation of the CNTf as a consequence of the hybridization process. The main Raman active modes of $\mathrm{ZnO}$ are also clearly observed, including those parallel to the $c$-axis, $A_{1}$ at $373.7 \mathrm{~cm}^{-1}$ and $B_{1}^{(l o w)}$ $B_{1}^{(h i g h)}$ at $284 \mathrm{~cm}^{-1}$ and perpendicular $E_{2}^{(\text {low })}$ at $100 \mathrm{~cm}^{-1}, E_{2}^{(h i g h)}-E_{2}^{(\text {low })}$ at $333 \mathrm{~cm}^{-1}$, $E_{1}(\mathrm{TO})$ at $410 \mathrm{~cm}^{-1}, E_{2}^{(h i g h)}$ at $438 \mathrm{~cm}^{-1}, E_{1}(\mathrm{LO})$ at $590 \mathrm{~cm}^{-1}$. Their positions are in agreement with reports on hydrothermal $\mathrm{ZnO}$ single crystals[275] and no changes in peak position are found due to an abnormal presence of defects or residual strains. 

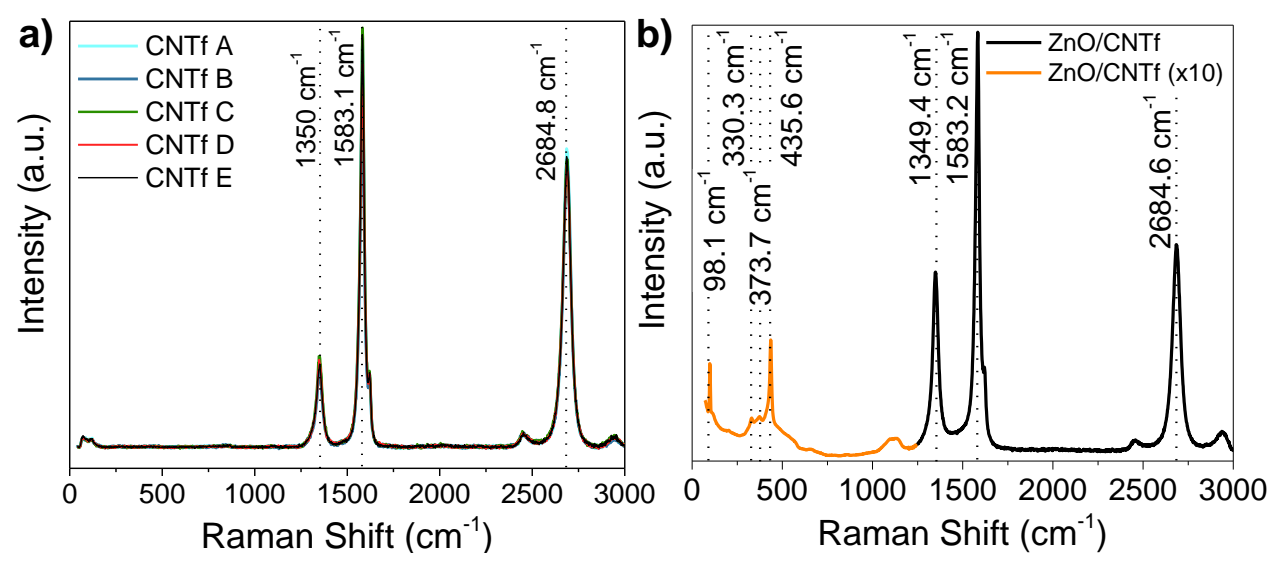

FIGURE 5.8: Raman spectra of the a) pristine CNTf and b) CNTf/ZnO hybrid.

\subsubsection{Electrical characterisation of the CNTf/ZnO-NWs heterojunction}

The properties of the hybrid material have been evaluated by directly carrying out current-voltage $(I-V)$ and capacitance-voltage $(C-V)$ measurements across the CNTf/ZnO interface. This characterisation was carried out using a microprobe station (see Figure 5.9b) combined with a 2450 Keithley source-measure unit. An optical image of the plan-view and a schematic of the contacts for electrical measurement are presented in the Figure 5.9a,b. Because the CNTf sheets have a very high in-plane conductivity, between $500-1000 \mathrm{~S} / \mathrm{cm}$, this contact could be made far from the $\mathrm{ZnO}$. In this sense, the CNTf can be treated as a continuous network electrode, i.e. the current-collector is directly built into the hybrid material as presented in the schematic of Figure 5.9c.

The irregular surface morphology of the CNTf/ZnO-NWs material implies that metal evaporation to produced contacts often leads to short circuiting. A more reproducible two-point microprobe technique (direct contact) has been used instead.

A further challenge in analysing non-planar heterojunctions is to determine their dimensions. The $\mathrm{ZnO}$ layer thickness $(5 \mu \mathrm{m})$ can be taken as the length of the conductive channel between contacts, but accurate determination of the junction cross-sectional area $(S)$ is complicated in a system with a highly irregular morphology, such as the one in this study. We take $S$ to be equal to the contact area $(A)$ corresponding to the projected area of the microprobe tip calculated according to the classical Hertz theory of non-adhesive elastic contact (schematic presented in Figure $5.9 \mathrm{~d}$ ). The radius of the contact area between a sphere and a flat plate under a semi-elliptic pressure distribution is given by[276]

$$
a=\sqrt[3]{\frac{3}{4} F R_{T i p}\left[\frac{1-v_{\mathrm{ZnO}}}{E_{\mathrm{ZnO}}}+\frac{1-v_{W}}{E_{W}}\right]}
$$

where $R_{\text {Tip }}$ is the radius tungsten needle, $50 \mu \mathrm{m}$ (from manufacturer datasheet), and $E_{W}=311 \mathrm{GPa}$,[277] $E_{\mathrm{ZnO}}=100 \mathrm{GPa}$, [278] $v_{W}=0.28$,[277] $v_{\mathrm{ZnO}}=$ 
0.35[279] are the elastic modulus and Poisson's ratio of the Tungsten and $\mathrm{ZnO}$ respectively. The resulting contact radius is $a \approx 7.5 \mu \mathrm{m}$ and the contact area is $A=S \approx$ $180 \mu \mathrm{m}^{2}$.

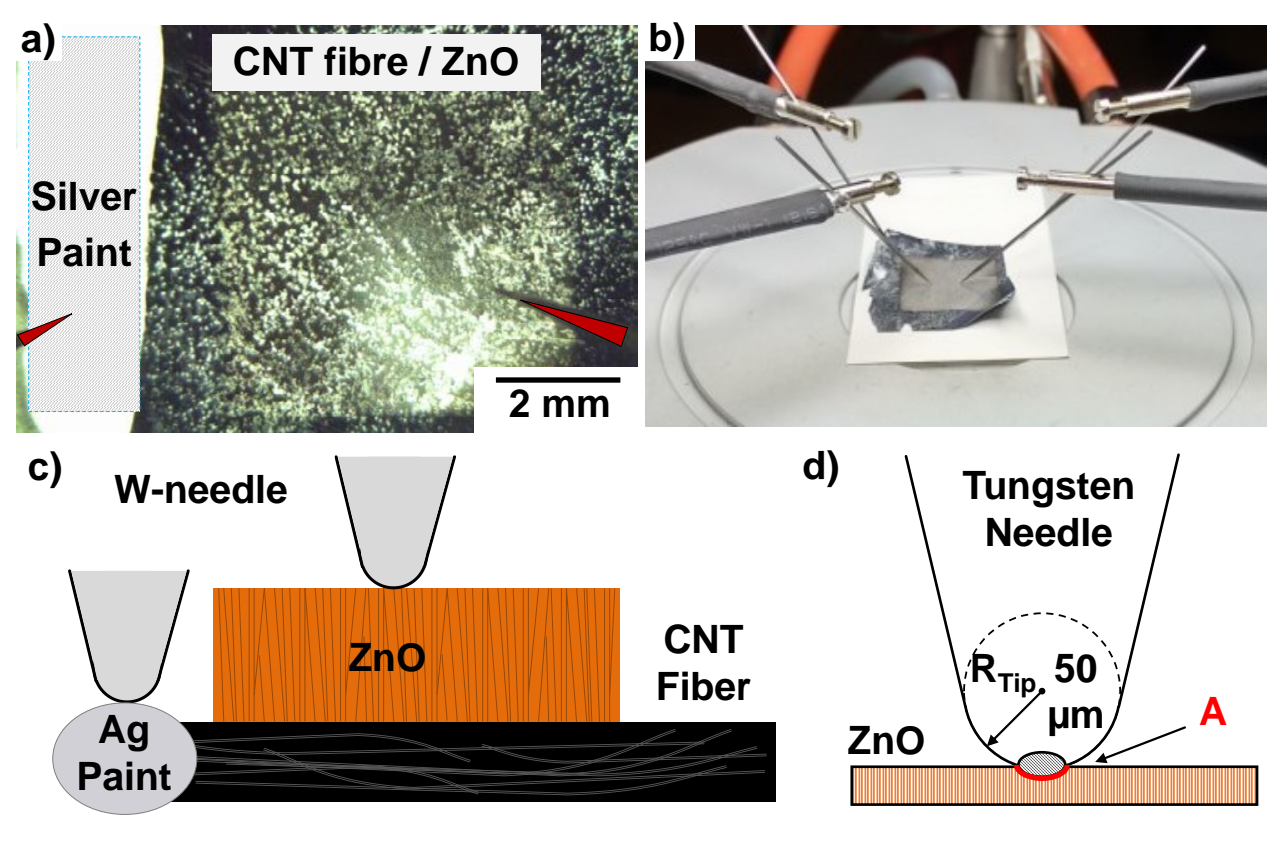

FIGURE 5.9: Details of the configuration used for the electrical measurements: a) Plan view optical image of the CNTf/ZnO heterojunction and b) digital photograph of the probe station and sample. c) Schematic diagram of the samples for the electrical measurements. d) Schematic of the contact area between the $\mathrm{W}$-needle of the probe station and the CNTf/ZnO sample.

The measured electrical properties across the CNTf/ZnO interface were found to be sensitive to the pressure of the contact on the $\mathrm{ZnO}$ layer. However, gradually increasing pressure, it was found to lead to converging $I-V$ curves. Control experiments are presented in the Figure 5.10. As a confirmation of the metallic junction between the CNTf and the tungsten needles, both contacts were performed on top of the CNTf, which led to the characteristic curve of a metallic junction (Figure 5.10a), notice the low resistance as compared to the other control experiments due to the absence of $\mathrm{ZnO}$ along the current path. Indium metal is a well known metallic contact for $\mathrm{ZnO}$ semiconductor,[280] therefore, the measured Schottky contact is sure to be obtained from the $\mathrm{CNTf} / \mathrm{ZnO}$ interface. Finally, the direct contact of the tungsten needle on the $\mathrm{ZnO}$, performed the same characteristic as for the indium contact, confirming the metallic junction between $\mathrm{W}$ and $\mathrm{ZnO}$, and once again, the Schottky barrier between $\mathrm{ZnO}$ and CNTf. Notice also the lower current of the tungsten needle as compared to the metallic indium, this is attributed to the smaller size of the tungsten tip and lower contact area.

Good ohmic contacts were obtained using a tungsten micro-needle to contact the metal oxide. The CNTf is essentially a continuous conductive network and therefore it can be contacted with standard silver paint in an area purposely left free of $\mathrm{ZnO}$. During the electrical measurements, the CNTf was grounded and the bias voltage was applied to the top electrode. 
Figure 5.11a shows an example of a typical $I-V$ curve for the CNTf/ZnO junction. It shows evident rectifying behaviour, similar to a Schottky diode, with current rapidly increasing at forward bias above a threshold voltage, $V_{\text {th }}=0.58 \mathrm{~V}$, and a much smaller current at reverse bias, increasing in magnitude only at very large negative voltages after breakdown of the semiconductor at $-4 \mathrm{~V}$. The threshold voltage has been directly obtained by linear extrapolation of the forward bias current of the device. Several semi-logarithmic $I-V$ measurements has been included as inset in the Figure 5.11a. It shows the formation of such Schottky junctions throughout the whole hybrid material and in various samples tested.

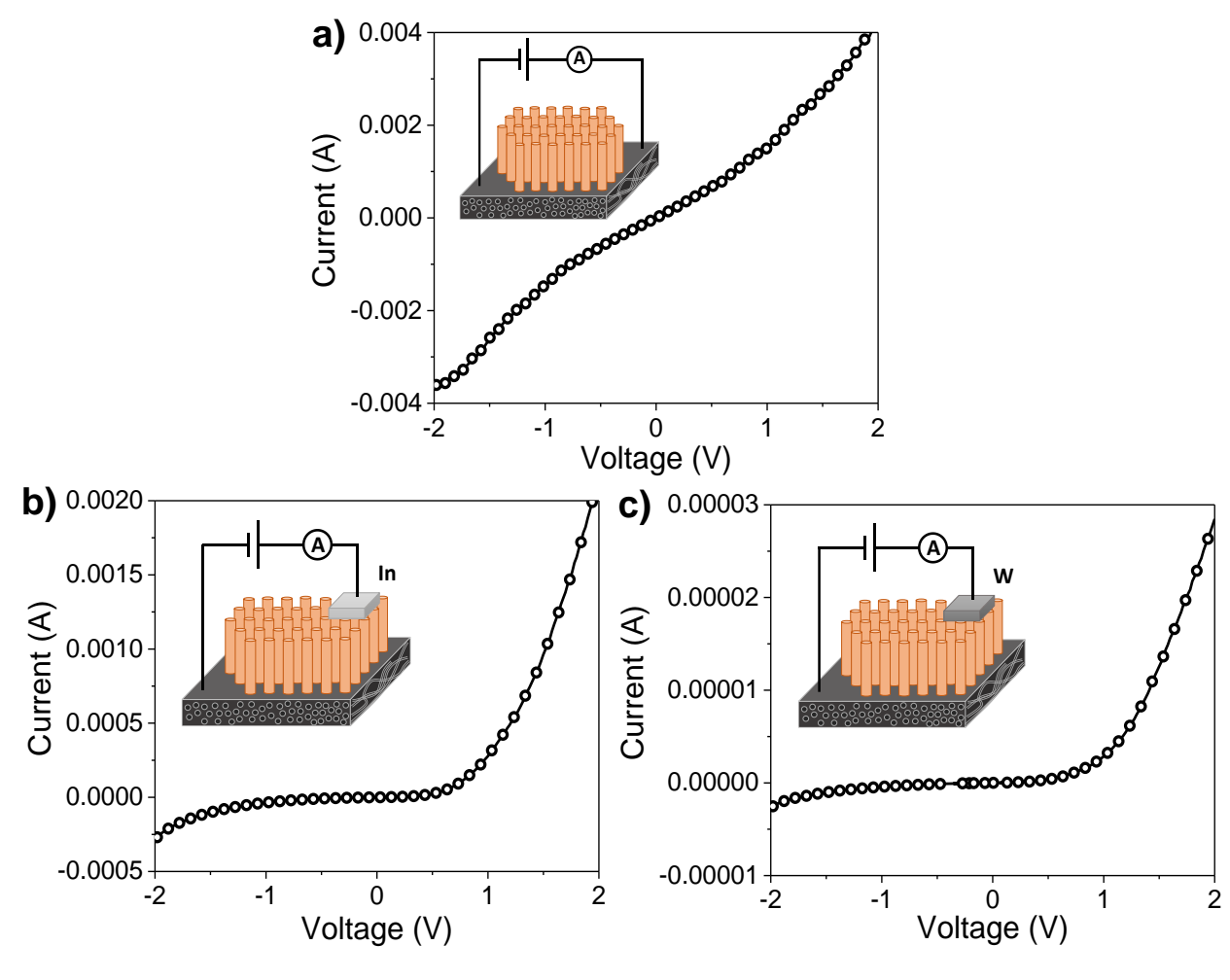

FIGURE 5.10: $I-V$ characteristics of the CNTf/ZnO interface. a) Contacting both terminals in the CNTf, b) contacting the $\mathrm{ZnO}$ with indium, c) contacting $\mathrm{ZnO}$ with tungsten.

Figure $5.11 \mathrm{~b}$ presents the $C-V$ and $C^{-2}-V$ (inset) measurements of the heterojunction interface. $C-V$ measurements were carried out at room temperature and at high AC frequency $(1 \mathrm{MHz})$ with small amplitude $(10 \mathrm{mV})$ at different reverse bias. Under reverse bias conditions the semiconductor is depleted of free carriers, thus capacitance takes a low value and remains constant. At forward bias, when the applied voltage equals the built in voltage, $V_{b i}$, capacitance starts to increase as the interface is repopulated of free carriers. The built-in potential can be obtained from linear extrapolation of $C^{-2} v s V$ to $C^{-2}=0$, which gives $V_{b i}=0.58 \mathrm{~V}$.

The density of carriers can be extracted from Figure 5.11b using the depletion approximation and the Mott-Schottky equation[281]

$$
\frac{1}{C^{2}}=\frac{2\left(V_{b i}-V\right)}{\epsilon q A^{2} N_{D}}
$$


which after rearrangement can be expressed as

$$
N_{D}=\frac{2}{q \epsilon_{Z n O} \epsilon_{0}} \frac{\Delta V}{\Delta\left(\frac{A}{C}\right)^{2}}
$$

where $C$ is the capacitance, $V$ is the bias voltage, $q$ is the electronic charge, $\epsilon$ is the permittivity of $\mathrm{ZnO}$ and $A$ is the area of contact.
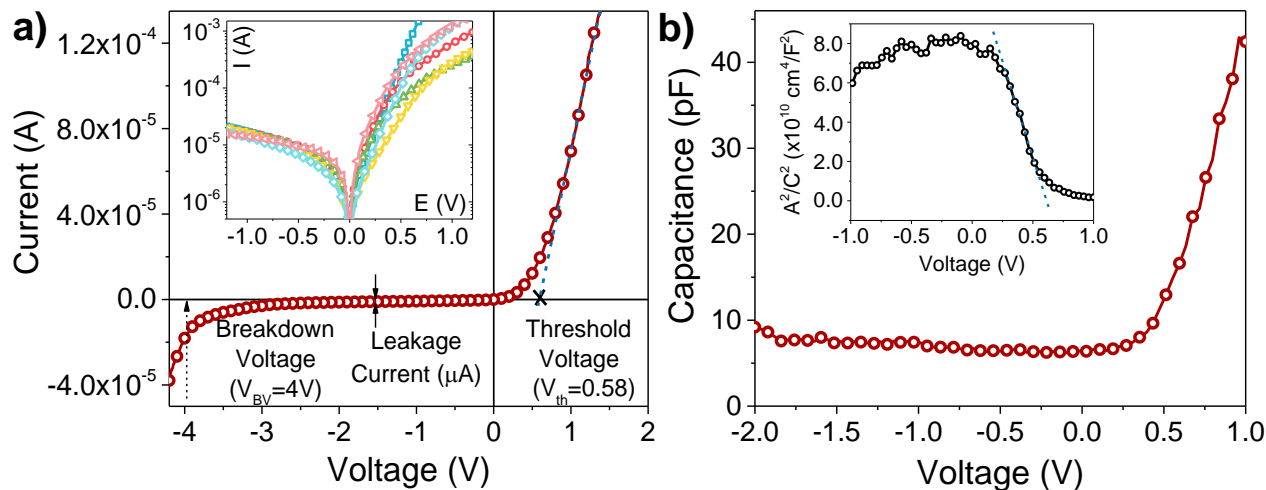

FIGURE 5.11: a) $I-V$ characteristics (inset: semi-logarithmic curves of different samples) and b) $C-V$ measurement of the CNTf/ZnO heterojunction showing rectifying behaviour typical of a Schottky barrier junction. Inset: $(S / C)^{2}$ graph.

The carrier concentration $N_{D}$ obtained from the slope of $C^{-2}$ vs $V$ comes out as $8.1 \times 10^{17} \mathrm{~cm}^{-3}$, which is in agreement with reported values for hydrothermal $\mathrm{ZnO} \cdot[282,283]$

Next, the transport properties across the interface are analysed. The starting point is determining the type of transport mechanism at the heterojunction, among tunnelling, thermionic emission, or a combination, and which can be determined based on the tunnelling energy parameter[284]

$$
E_{00}=\frac{q h}{4 \pi} \sqrt{\frac{N_{D}}{\epsilon m_{e}^{*}}}
$$

Thermionic emission dominates for low doping densities and/or relatively high temperatures, when the tunnelling energy parameter $E_{00} / k T \ll 1$.[285] Conversely, tunnelling predominates when $E_{00} / k T \gg 1$. Intermediate values of $E_{00} / k T \sim$ 1 correspond to a combination of both mechanisms.

As a consequence of the relatively low doping density of $\mathrm{ZnO}\left(N_{D}=8.12 \times 10^{17}\right.$ $\mathrm{cm}^{-3}$ ), at room temperature, $E_{00} / k T=0.44<1$, and the junction is therefore dominated by thermionic emission. Thus, the current flowing through the metal/ semiconductor junction at forward bias can be described according to thermionic emission theory by [284]

$$
I=I_{S}\left[\mathrm{e}^{\left(\frac{q V_{e f f}}{\eta k T}\right)}-1\right]
$$




$$
I_{S}=A R^{*} T^{2}\left[\mathrm{e}^{-\left(\frac{q \phi_{b}}{\eta k T}\right)}\right]
$$

where $\eta$ is the ideality factor, $I_{S}$ is the reverse saturation current ( $J_{0}$ for current density), $V_{e f f}$ is the effective voltage across the junction and $R^{*}$ is the effective Richardson constant for $\mathrm{ZnO}$ calculated, from $R^{*}=4 \pi q m^{*} k^{2} / h^{3}=32 \mathrm{Acm}^{-2} \mathrm{~K}^{-2}$. $\phi_{b, e f f} / q=V_{e f f}=V-I R_{s}$ is used to take into account the resistance of the semiconductor $R_{s}$, and when $V>3 k T / q$, Eq. (5.5) can be expressed as

$$
I=I_{S}\left[\mathrm{e}^{\left(\frac{q\left(V-I R_{S}\right)}{\eta k T}\right)}\right]
$$

Figure 5.12a presents a semi-log current density versus voltage plot $(\log (J)-$ $V)$. A value of $\eta$ is often directly extracted from the slope $(s=q /(\eta k T))$ in such plot.[281] In the voltage range 0.01-0.1 V, $\eta$ comes out as 3.2. However, $\eta$ clearly takes higher values as the voltage increases and the semiconductor resistance becomes more important $(\eta=9.3$ for 0.1-0.3 $V)$.

An alternative method to extract diode parameters from $I-V$ measurements was proposed by Cheung et al.[286] The ideality factor and series resistance can be obtained from the intercept and slope of a plot of $d V / \ln J v s J$, with Eq. (5.7) modified as

$$
\frac{\mathrm{d} V}{d \ln J}=R_{S} A J+\eta\left(\frac{k T}{q}\right)
$$
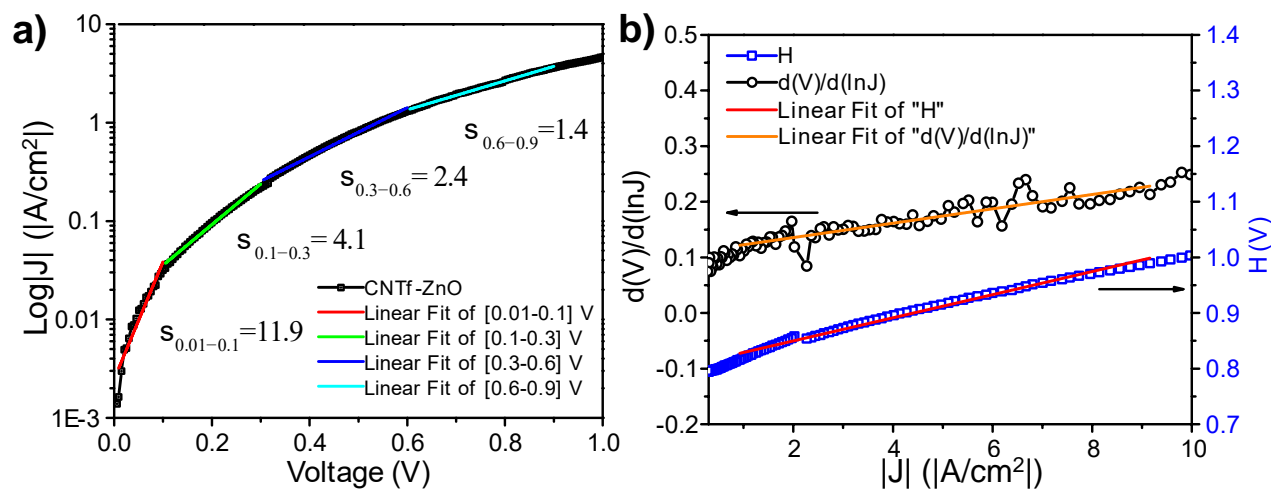

FIGURE 5.12: a) Semilog-J- $V$ characteristic for the CNTf/ZnO and b) Cheung method: $d(V) / d \ln J$ and $H$ function vs $V$.

Figure $5.12 \mathrm{~b}$ presents such plot for the same voltage and current range as in Figure 5.12a. The experimental data follow the linear dependence expected from Eq. (5.8) and confirm that an accurate description of the transport properties across these junctions requires consideration of the semiconductor resistance according to Eq. (5.7). The value of $\eta$ now comes out as 2.9 and $R_{s}$ as $20 \mathrm{k} \Omega$. $\eta$ is in the range observed for high-quality graphene/semiconductor junctions,[287] close to heterojunctions with cleaved highly oriented pyrolytic graphite $(\eta=1.25-2)[288]$ and similar to literature reports on $\mathrm{CNT} / \mathrm{ZnO}$ junction devices $(\eta=3)$.[289] Although the $C-V$ analysis for the extraction of the doping concentration is suitable for close-to-ideal Schottky diodes $(\eta<2)$, some non-idealities are tolerable.[290] For $\eta \gg 2$ additional 
capacitance contributions are included in the analysis, but whose effect is in slightly reducing the doping concentration relative to the value obtained from Eq. (5.3),[290] thus not affecting significantly the results and interpretation presented above.

$R_{s}$ gives a low resistivity of $\rho_{Z n O}=70 \Omega \mathrm{cm}$, which is in the range of high-quality solution processed $\mathrm{ZnO}$ thin films $(2.9-138,000 \Omega \mathrm{cm}),[291]$ although smaller than previous reported values on hydrothermal $\mathrm{ZnO}\left(10^{3}-10^{5} \Omega \mathrm{cm}\right) \cdot[292]$

The treatment of Cheung et al.[286] also enables determination of the Schottky barrier height $(S B H)$ by defining a function

$$
H(J) \equiv V-\frac{k T \eta}{q} \ln \left(\frac{J}{R^{*} T^{2}}\right)
$$

Plotting the $H(J)$ vs $J$ and using the obtained value of $\eta$, the $S B H$ can be obtained from

$$
H(J)=R_{S} A J+\eta \phi_{B}
$$

This method (Figure 5.12b) gives a value of $\phi_{b, e f f}=0.263 \mathrm{eV}$, which is between the $S B H$ determined directly from the $I-V$ curve $(0.61 \mathrm{eV})$ and the theoretical value obtained from the Mott-Schottky rule $(0.1 \mathrm{eV})$ (see Eq. 5.13 in the latter $S B H$ calculation). The discrepancy between values of $S B H$ from Mott-Schottky $(C-V)$ and Cheung $(J-V)$ is attributed to the resistance of the thick $\mathrm{ZnO}$ layer, which is only accounted for in the latter method. When the resistance of the $\mathrm{ZnO}$ layer is significant, as in the samples in this study, the $S B H$ value according to equation 5.10 is more appropriate. This will also be the case in thick $\mathrm{ZnO}$ layers produced hydrothermally, and which are common in other nanocarbon-ZnO hybrids used for energy harvesting[73] and photocatalysis.[272]

The Schottky barrier height $(S B H)$ of the junction, $q \phi_{b}$, can be also estimated from

$$
\phi_{b}=q V_{b i}+\delta_{s c}
$$

where $\delta_{s c}$ is the depth of the location of the Fermi level with respect to the conduction band of the $\mathrm{ZnO}-\mathrm{NWs}$, which can be calculated from equation:[281]

$$
\delta_{s c}=k T \ln \left[2 n^{-1}\left(\frac{2 \pi m_{n}^{*} k T}{h^{2}}\right)^{3 / 2}\right]
$$

where $k$ is the Boltzmann constant, $T$ is the absolute temperature, $h$ the Plank constant, $n\left(=N_{D}\right)$ is the electron doping concentration and $m_{n}^{*}=0.24 m_{0}$ [293] is the effective mass of electrons in $\mathrm{ZnO}$, with $m_{0}$ approximately $9.1 \times 10^{-31} \mathrm{~kg}$. It was found that $\delta_{s c}=0.03 \mathrm{eV}$ and therefore the $S B H$ is $0.61 \mathrm{~V}$. This value is very close to $V_{\text {th }}$ from $I-V$ at forward bias $0.58 \mathrm{eV}$, hence why sometimes it is directly taken as the SBH.[294]

For reference, it was calculated the $S B H$ for electron injection from the semiconductor to the CNT predicted by the Mott-Schottky rule

$$
q \phi_{b}=q\left(\phi_{C N T}+\chi_{s c}\right)
$$


where $\phi_{C N T}=4.4 \mathrm{eV}[295]$ is the work function of the CNTfs and $\chi_{s c}$ the electron affinity of the semiconductor, in the range 4.1[296]-4.5[297] for $\mathrm{ZnO}$. The $S B H$ obtained by capacitance measurements falls in the range of possible values calculated with Eq. (5.13), although it is higher than that value obtained using the mid value of the literature data $(\approx 4.3 \mathrm{eV})$.

This leads to a predicted barrier height of $\phi_{b}=4.4-4.3=0.1 \mathrm{eV}$, which is substantially lower than the experimental value obtained. This discrepancy is attributed to the well-known limitations of the Mott-Schottky rule, for instance, the exclusion of surface states in this theory.[280, 298]

Finally, a schematic representation of band alignment of the resulting CNTf/ $\mathrm{ZnO}-\mathrm{NWs}$ junction before and after contact, is obtained from $I-V$ and $C-V$ measurements (Figure 5.13). The depletion layer width, calculated from $w=\sqrt{\frac{2 \epsilon V_{b i}}{q N_{D}}}=$ $17.9 \mathrm{~nm}$, is also shown for reference.

a)
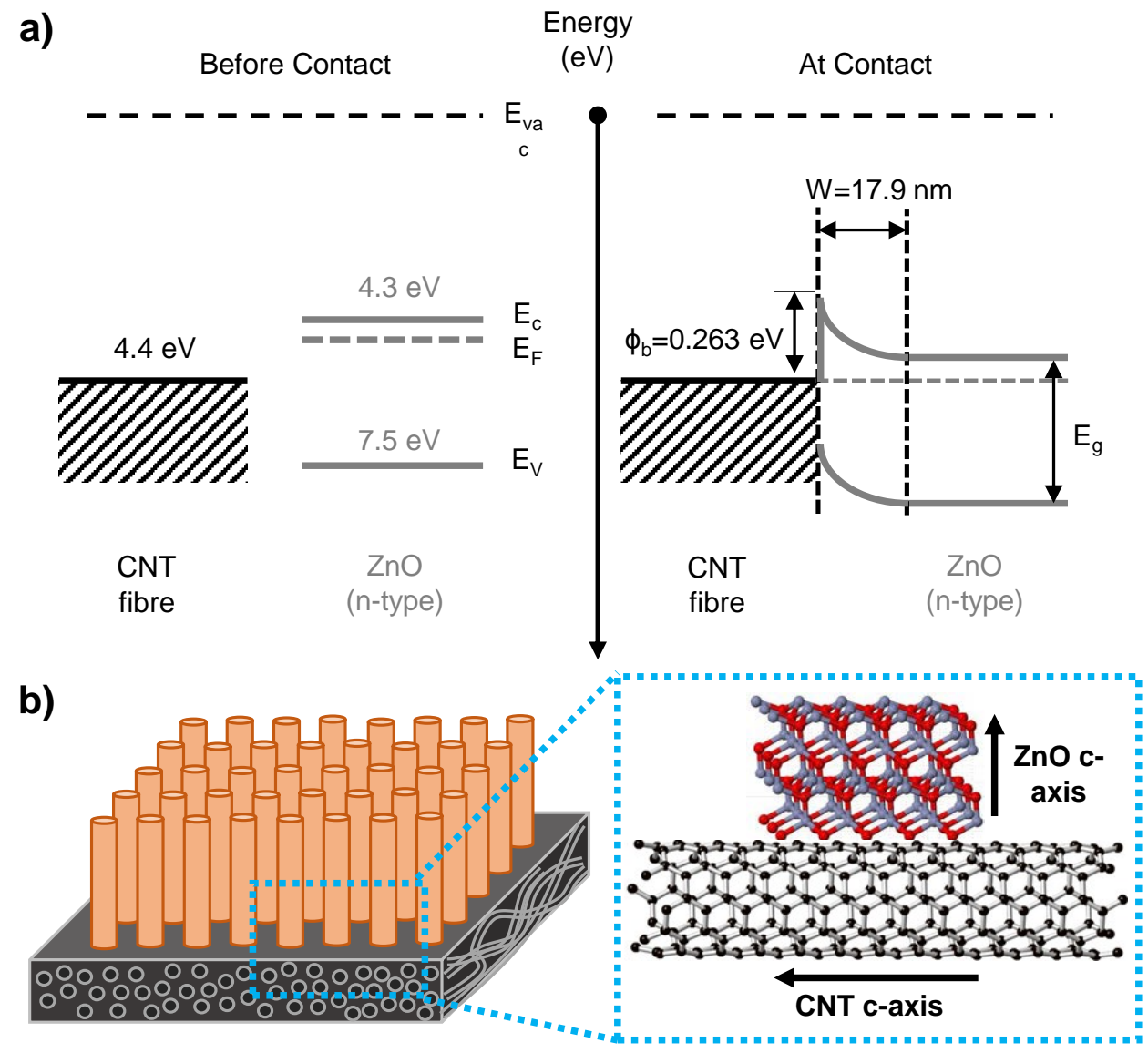

FIGURE 5.13: a) Schematic of the energy levels in the CNTf/ZnO heterojunction before and after contact. b) Schematic showing the $\mathrm{ZnO}$ orientation representation according to SEM and XRD data.

Table 5.1 summarises parameters of interest obtained for the CNTf/ZnO-NWs Schottky heterojunctions presented in this section. It includes the rectification ratio $(R R)$ at $1.4 \mathrm{~V}$, the saturation current density, and the shunt resistance determined as the differential resistance in the vicinity of zero bias. 
TABLE 5.1: Extracted parameters for the CNTf/ZnO Schottky heterojunction.

\begin{tabular}{lllllllll}
\hline$V_{b i}$ & $\begin{array}{l}N_{D} \\
(\mathrm{C})\end{array}$ & $\begin{array}{l}\phi_{b} \\
(\mathrm{eV})\end{array}$ & $\begin{array}{l}\phi_{b, e f f} \\
(\mathrm{eV})\end{array}$ & $\begin{array}{l}R_{s} \\
(\mathrm{k} \Omega)\end{array}$ & $\begin{array}{l}R_{s h} \\
(\mathrm{k} \Omega)\end{array}$ & $\begin{array}{l}J_{0} \\
\left(\mathrm{~mA} / \mathrm{cm}^{2}\right)\end{array}$ & $\begin{array}{l}\eta \\
-\end{array}$ & $\begin{array}{l}R R \\
-\end{array}$ \\
\hline 0.58 & $8.12 \times 10^{17}$ & 0.6 & 0.263 & 20 & 300 & 273 & 2.9 & 144 \\
\hline
\end{tabular}

The electrical contact between carbon material and $\mathrm{ZnO}$ has been reported in the past as a rectifying behaviour,[294] but, up to the author knowledge, it has not been characterised before for CNTf.

This parameters in Table 5.1 are expected to be representative of hybrid samples produced using the method discussed in this section. However, as it has been indicated before, there is substantial uncertainty in determining the effective interfacial area of these samples, as well as in all meso(nano)porous hybrids. This will affect the value of $A_{e f f}$, whose main effect is on current densities and $N_{D}$, but not on ideally factor or rectification ratio.

\subsubsection{Charge transfer at the interface: photocurrent measurements}

Photoconductivity measurements were performed on the CNTf/ZnO heterojuntion in order to study the charge transfer between both materials. In the set-up used the CNTf acts as current-collector, or back contact, and FTO on $\mathrm{ZnO}$ as front contact (see inset Figure 5.14a).

The samples are irradiated with sources of different wavelengths (365 $\mathrm{nm}$ UVA lamp and 400-532-650 $\mathrm{nm}$ lasers) and the photocurrent is measured. Tafel plots of the pristine CNTf and CNTf/ZnO heterojunction are shown in Figure 5.14.

Although CNTs can exhibit photocurrent signals, for example due to the Bolometric effect upon light-induced sample heating,[299] control experiments ruled these out (see Figure 5.14a). No photocurrent effect is obtained for the pristine CNTf independently from the wavelength used. Both forward and reverse currents are equal to the dark condition in all cases, confirming also that no heating effect has been produced at these power densities conditions.
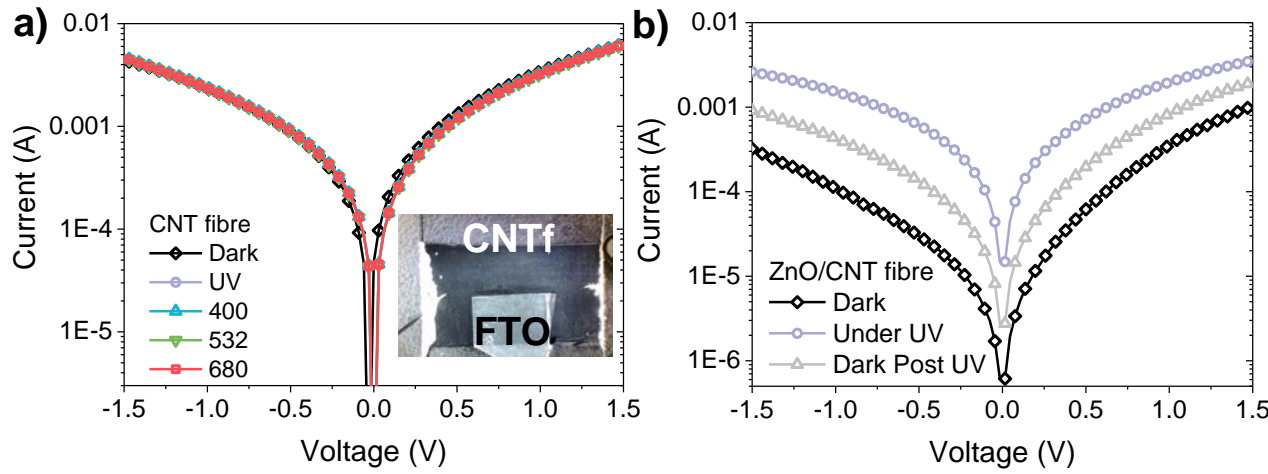

FIGURE 5.14: Tafel plots of a) the pristine CNTf under dark, 365, 400, 532, $680 \mathrm{~nm}$ radiation and b) CNTf/ZnO heterojunction under dark, UV light and after UV exposure. Inset: set-up showing the a sample connected through the Ag and FTO upper contact. 
For the CNTf/ZnO characteristics, the rectifying behaviour of the heterojunction is observed under reverse bias in dark conditions. After $30 \mathrm{~s}$ of UV light exposure, and the increase of the current is clearly observed. The decay of the photocurrent when the light is switched off is not instantaneous, and the curve 30 second after switching off the UV light is also presented.

Figure 5.15a shows the photocurrent response of the CNTf/ZnO-NWs heterojunction at $1 \mathrm{~V}$ bias voltage after $15 \mathrm{~s}$ exposure for the different irradiation wavelengths. The photocurrent increases exponentially as the light source wavelength approaches the absorption edge of $\mathrm{ZnO}$ near $375 \mathrm{~nm}$. Thus, under the configuration used, the recorded photocurrent corresponds to photogenerated electrons transferred from $\mathrm{ZnO}$ to the CNTf.

The photocurrent signal originates exclusively from $\mathrm{ZnO}$ and considering that the interfacial charge transfer resistance is smaller than the bulk resistance of $\mathrm{ZnO}$, the photocurrent data can be conveniently used to characterise other optoelectronic properties of the $\mathrm{ZnO}$ layer. Figure 5.15b presents a typical photocurrent profile obtained at $100 \mathrm{mV}$ forward bias under steady state transient measurements. Upon illumination of the heterojunction with UV light (365 $\mathrm{nm}$ and $300 \mu \mathrm{W} / \mathrm{cm}^{2}$ ), photocurrent increases rapidly but then starts to level off after a few minutes. The photoresponse can be quantified as the photo-to-dark current ratio, which comes out as 95. Responsivity, obtained by normalising photocurrent by effective irradiation power gives $733 \mathrm{~mA} / \mathrm{W}, 5000 \mathrm{~mA} / \mathrm{W}$ when fully saturated from the unexcited state (value in Figure 5.15a), which is much higher compared to that of commercial silicon photodiodes (500 $\mathrm{mA} / W)$ and graphene photodetectors $(10 \mathrm{~mA} / W)$,[300] but lower than devices based on individual electrospun $\mathrm{TiO}_{2}$ nanofibres $(90000 \mathrm{~mA} / W)$ under UV-irradiation.[301]
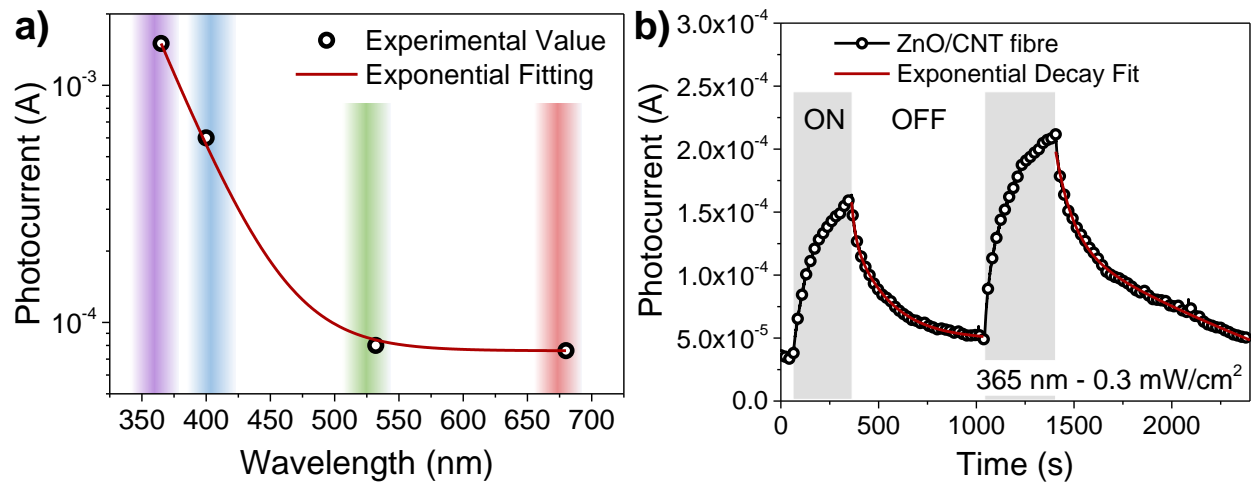

FIGURE 5.15: a) Current response to different wavelengths radiations after several cycles of 15 seconds exposure. b) Steady state photocurrent response at $100 \mathrm{mV}$ bias under UV light.

In the absence of trap states, the steady state photocurrent flowing through the heterojunction depends linearly on the lifetime $(\tau)$ and mobility $(\mu)$ of photocarriers. It can be expressed as

$$
I_{p h}=I_{\text {light }}-I_{\text {dark }}=\frac{e G(\mu \tau) V A}{L}
$$




$$
\sigma_{p h}=e G\left(\mu_{n} \tau_{n}+\mu_{p} \tau_{p}\right)
$$

where $G$ is the generation rate, given by

$$
G \approx \frac{\eta_{q} I_{0}}{h v}(1-R) \alpha
$$

with $\eta_{q}$ the quantum efficiency $\left(\eta_{q}=1\right.$, for $h v>E_{g a p}$ and no photogating effects), $I_{0}$ the intensity of light at the surface of the material $\left(300 \mu \mathrm{Wcm}^{-2}\right)$, $h$ the Planck constant, $v$ frequency of light, $R$ the reflectance coefficient $(R=0.1$ for $\lambda=365 \mathrm{~nm})$ [302] and $\alpha$ the absorption coefficient $\left(\alpha=130.000 \mathrm{~cm}^{-1}\right.$ for $\lambda=$ $365 \mathrm{~nm}$ ).[303] These values give a generation rate $G=6.4 \times 10^{19} \mathrm{~cm}^{-3}$.

Knowing $G$, it is possible to calculate $\mu \tau=0.04 \mathrm{~cm}^{2} V^{-1}$, which has been a useful parameter when comparing transport of photogenerated carriers in $\mathrm{ZnO}$ materials produced under a range of synthesis conditions.[291] It comes out as $0.04 \mathrm{~cm}^{2} \mathrm{~V}^{-1}$, which is in the range observed for solution processed $\mathrm{ZnO}$ thin films.[291]

From steady state photocurrent measurements the decay constant times can be calculated. It is accepted the adsorption-desorption of oxygen molecules mediated with the photoconductive gain. Therefore two different phenomena have to be taken into account. Firstly, the trapping of photogenerated carriers on the defects of the material. Secondly, the desorption of oxygen molecules. With these two processes in mind, two different time constants can be obtained trough the fitting by a biexponential curve as:

$$
y=y_{0}+A_{1} e^{\frac{-t}{\tau_{1}}}+A_{2} e^{\frac{-t}{\tau_{2}}}
$$

The total time constant of decay is defined as:

$$
\tau_{\text {total }}=\frac{A_{1} \tau_{1}^{2}+A_{2} \tau_{2}^{2}}{A_{1} \tau_{1}+A_{2} \tau_{2}}
$$

$\tau_{\text {total }}=189.84 \mathrm{~s}$ was obtained, being the different fitting parameters: $A_{1}=$ $2.87 \times 10^{-5} A, \tau_{1}=19.76 \mathrm{~s}, A_{2}=8.22 \times 10^{-5} A, \tau_{2}=196.5 \mathrm{~s}$ (Reduced chi-sqr $=$ $7.45 \times 10^{-13}$ and Adj R-sqr $\left.=0.998\right)$.

\subsubsection{Electromechanical behaviour of $\mathrm{CNTf} / \mathrm{ZnO}$ flexible electrodes and sensing applications}

Finally, a flexible devices to study their electrical properties under mechanical stimuli has been fabricated. Figure 5.16a shows photographs of a electromechanical device and the two individual electrodes comprising it. One electrode is the CNTf/ZnO-NWs hybrid, and the other is pristine CNTfused for contacting the ZnO surface, this has to be highly conductive and tough/flexible to adhere to the irregular $\mathrm{ZnO}$ surface.

The piezoresistivity of the device has been tested by applying a constant pressure for 10 seconds and releasing the force $(18 \mathrm{~N})$ while the voltage was acquired. 
Several cycles were measured for ensuring the response of the material. A photograph and a schematic of the experimental setup is shown in the Figure 5.16b.

A control device with only two CNTf electrodes was also tested to understand the contribution of the metal oxide to electrostatic voltage generation,[289] as well as to discriminate piezoresistive effects from the CNT fibre.[223] For the control device, no voltage variation is obtained. Moreover, with the selected device geometry and test, the piezoresistive variation of the pristine CNTf is below $5 \%$ of the initial resistance.

The CNTf/ZnO-NWs voltage variation is presented at the Figure 5.16c. Upon loading, there is a gradual increase in voltage, which is associated with a timedependent change in electrical resistance, attributed to the deformation of the various interfaces in the system, as confirmed by inspection of the displacement of the DMA cross-head over time. Releasing the pressure produces the corresponding opposite effect. Superimposed on this effect is a sharp transient voltage ( $5 \mathrm{mV}$ on average), only observed upon compressive loading and which is of opposite sign as the piezoresistive effect mentioned above. It acts over a time-frame of few $m s$, which is agreement with measurements in similar systems.[73, 304] Such transient voltage is interpreted as the instantaneous piezoelectric polarisation of $\mathrm{ZnO}$ and the corresponding charge flow through the CNT current collector, a mechanism that can in principle be exploited as energy harvesting by transforming mechanical vibrations into electrical current.[17]
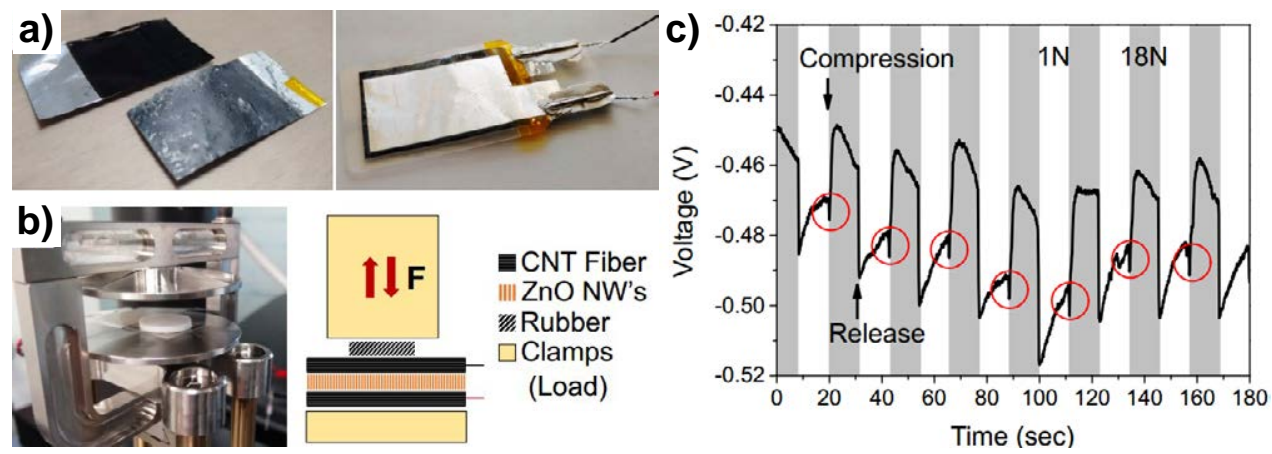

FIGURE 5.16: a) Energy harvesting assembly parts and device. b) Energy harvesting set-up image and schematic. c) Voltage variation vs time for CNTf/ZnO hybrid material.

\subsection{Physical synthesis route of $\mathrm{CNTf} / \mathrm{MOx}$ heterojunctions}

\subsubsection{Atomic layer deposition synthesis technique}

The results presented in the previous section confirm the formation of heterojunction in $\mathrm{CNTf} / \mathrm{MO}_{x}$ hybrids and have helped to solved numerous challenges associated with the complex system combining large areas/interfaces with low dimensional features. In this respect, it is of interest to reduce the thickness of the $\mathrm{MO}_{x}$ and increase coverage of the CNTf. For that purpose, atomic layer deposition is a powerful technique that allows to coat the material conformally, no matter the internal geometry of the substrate, in theory. 
A schematic of the ALD process is presented in the Figure 5.17a. The deposition conditions and chemical reactions are further explained in the Chapter 3 "Experimental Techniques". Briefly, it consists on injecting the metal precursor (diethylzinc - DEZ) in a vacuum chamber where the material is placed. This precursor interacts with the substrate forming a monolayer of material, followed by the purging of the chamber for removing the excess of material. After this first-half of cycle, the oxidising reactant is injected. The reactant interacts with the metal precursor forming the metal oxide material, and this second-half of a cycle finishes with the purging of the oxidising agent. Increasing the number of cycles will therefore increase the thickness of the $\mathrm{MO}_{x}$ material.

a)

Repeat ALD cycle $\mathbf{N}$ times

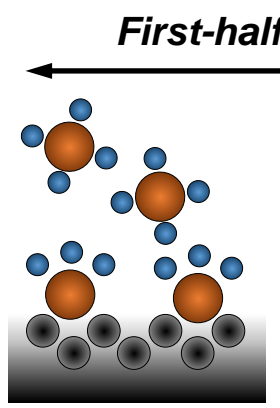

Precursor

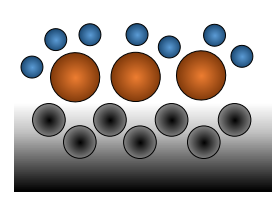

Purge

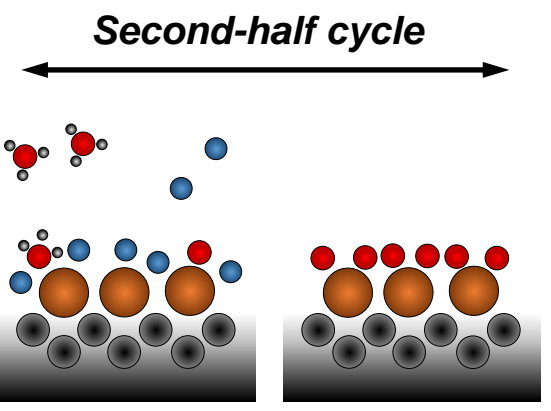

Reactant

Purge

b)

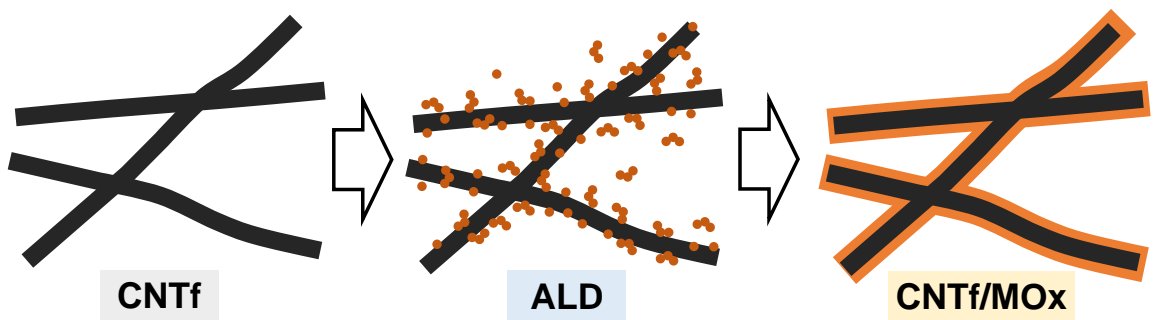

c)

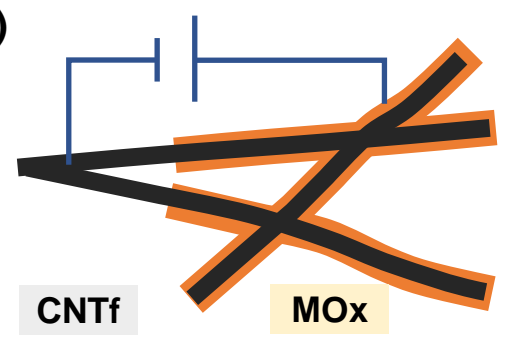

d)

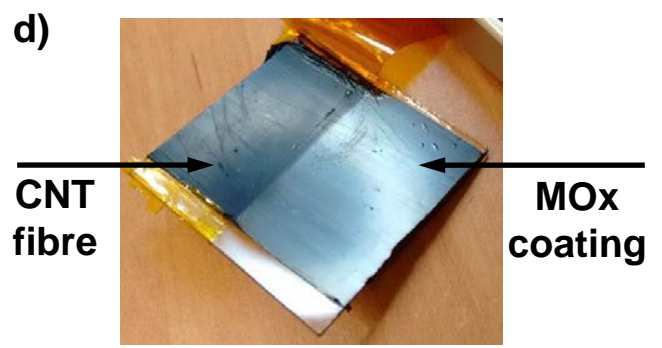

FIGURE 5.17: CNTf/ $\mathrm{MO}_{x}$ heterojunctions by ALD technique. a) Atomic scale process of one ALD cycle during the synthesis/deposition of the $\mathrm{MO}_{x}$ coating over the CNTf. b) Schematic of the conformal deposition over the CNT bundles structure preserving its inner interconnection. c) Schematic of the remote electrical connection of the CNTf across the $\mathrm{MO}_{x}$ interface. d) Digital image of unmasked CNTf electrode after growing $\mathrm{ZnO}$ in half of its surface.

The interest in producing conformal layers by ALD is that the continuous conductive structure of the CNTf is preserved. As represented in the Figure 5.17b, the carbon material forms a conductive path with very low resistivity and, at the same time, it is the scaffold for the semiconductor layer and because ALD is a gas phase 
method, the precursor can in principle penetrate through all the open porosity of the material. CNTf ends up coated with a conformal layer but with an intact conductive structure. Hence, the CNTf can be remotely electrically contacted from a non-coated area (see schematic Figure 5.17c).

Furthermore, the CNTf sheet deposited over glass or FTO substrates can be masked from the precursor deposition to obtaining partially coated materials. This facilitates electrical measurements of these heterojunctions. An example of CNTf/ $\mathrm{ZnO}$-ALD large heterojunction $(2 \times 2 \mathrm{~cm})$ is presented in Figure $5.17 \mathrm{~d}$.

The ALD method for CNTf was developed by the research group and collaborators for $\mathrm{ZnO}$ and in parallel for $\mathrm{TiO}_{2}$.[295]
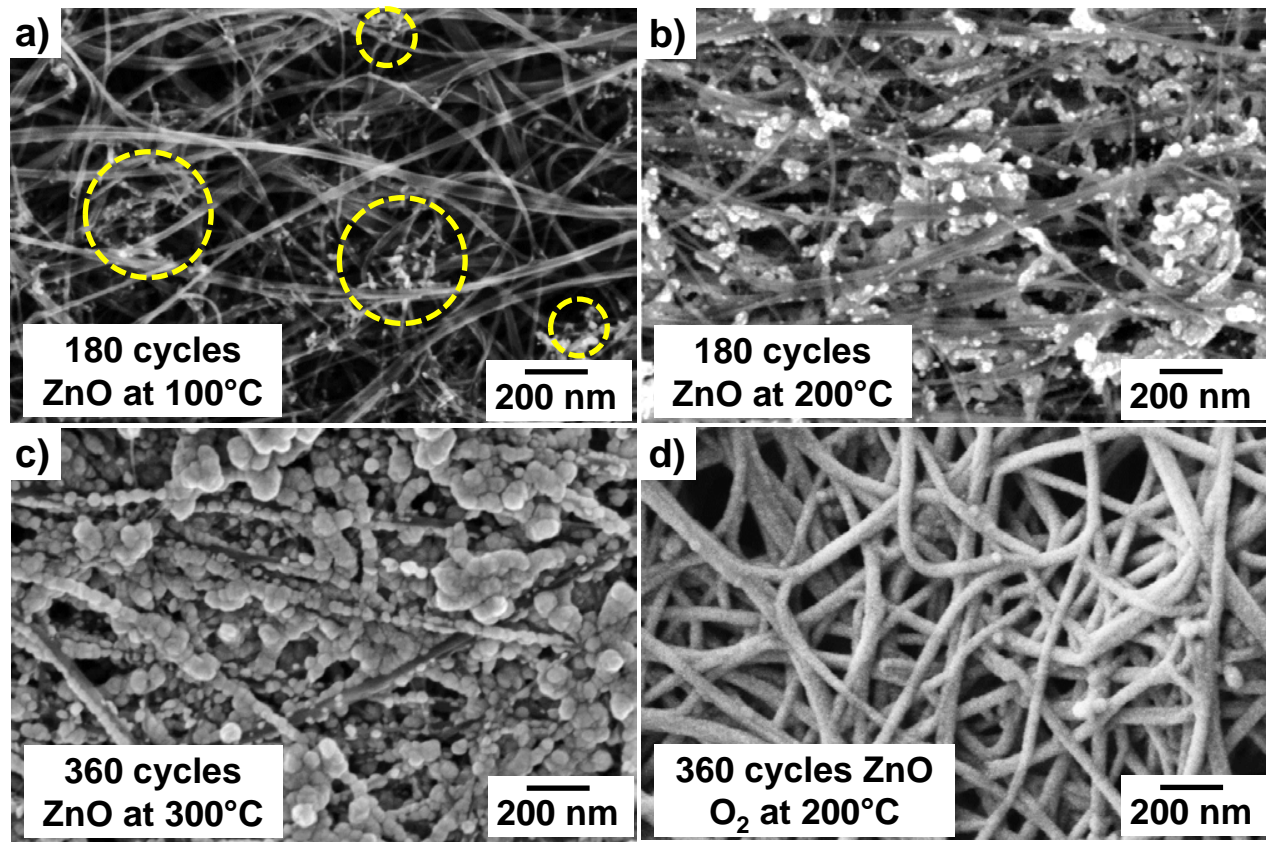

FIGURE 5.18: ZnO ALD optimisation over the CNTf substrate: a) $100{ }^{\circ} \mathrm{C}$ deposition temperature presenting $\mathrm{ZnO}$ aggregates - marked with yellow circles. b) $200^{\circ} \mathrm{C}$ deposition temperature showing non uniform $\mathrm{ZnO}$ growth. c) $300^{\circ} \mathrm{C}$ deposition temperature showing non uniform coating. d) $\mathrm{O}_{2}$-plasma enhanced deposition at $200{ }^{\circ} \mathrm{C}$ result-

ing in a good conformal coating.

As it is not frequent to grow these materials on top of CNTs, an optimisation of the parameters was carried out. This process for the $\mathrm{ZnO}$ is summarised in Figure 5.18. The process was tried at low temperature resulting in no $\mathrm{ZnO}$ material deposited using oxygen as oxidising reactant. Increasing the temperature to $100{ }^{\circ} \mathrm{C}$ the $\mathrm{ZnO}$ start to be deposited at some points forming particles agglomerates (see yellow circles in Figure 5.18a). Further increase of the temperature, up to $200{ }^{\circ} \mathrm{C}$, leads to bigger size agglomerates. At $300{ }^{\circ} \mathrm{C}$ the $\mathrm{ZnO}$ start to form a layer of big and coarse particles. More importantly, the material is not coating uniformly the surface of the CNTs, remaining partially uncoated areas of the CNTf substrate. Finally, using $\mathrm{O}_{2}$ plasma enhanced atomic layer deposition (PE-ALD),[305] the coatings resulted in conformal and smooth layers of $\mathrm{ZnO}$ (see Figure 5.18d). 
For growing $\mathrm{MO}_{x}$ with ALD technique, some wettability between the precursor and the substrate is needed. The as-made CNTf is a hydrophobic material, but this wettability with the $\mathrm{ZnO}$ precursor can be mainly produced by interaction with defects of the carbon substrate, adsorbed molecules or hydrophilic functional groups. Other option for increasing the interaction between materials is to increase the temperature of the process.

Successful conformal coatings were also obtained by the utilisation of water vapour as oxidising agent, obtaining a morphology of the final material identical to the one presented with the $\mathrm{O}_{2}$ plasma (see Figure 5.18d). These results confirms that the oxidant also plays an important role of functionalising the CNTf surface for the adhesion of the $\mathrm{MO}_{x}$ precursor. In this respect, it shares features with water vapour and ozone treatments developed to enhance hydrophilicity.[62, 306]
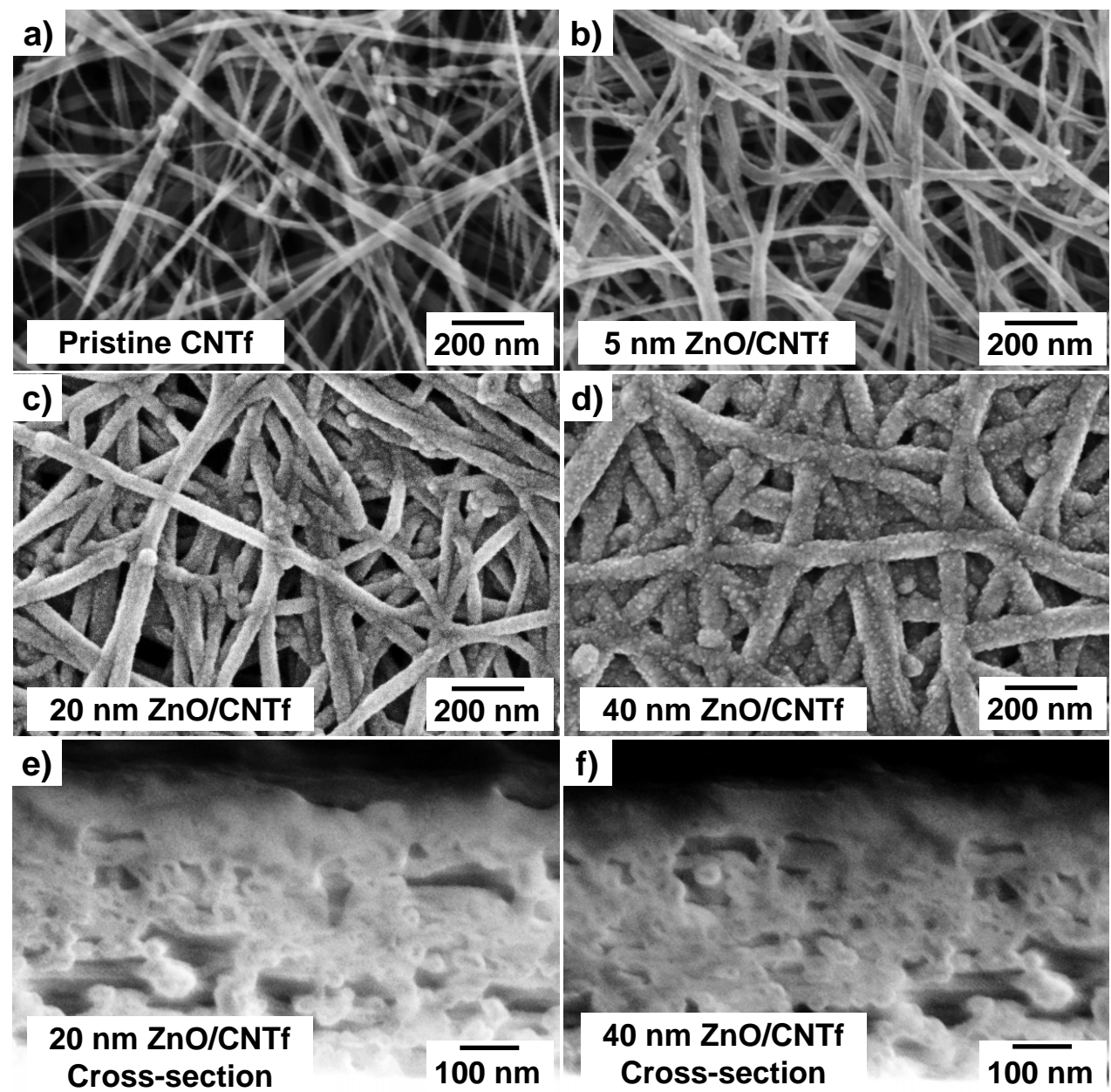

FIGURE 5.19: SEM images of a) pristine CNTf, b-d) CNTf coated by ALD with 5, 20, $40 \mathrm{~nm}$ of $\mathrm{ZnO}$ respectively, and e,f) cross-sections of 20 and $40 \mathrm{~nm}$ samples.

Moreover, by controlling the number of cycles, it is possible to tune the thickness of the final material, at a rate of $0.11 \mathrm{~nm} /$ cycle. Figure 5.19 presents plain-views and cross sections of different thicknesses as an example. The thicknesses grown and used during this study were 5, 20, 40, 50, $100 \mathrm{~nm}$, although not all of them were 
analysed and presented for every experiment. There is however a challenge in determining the thickness and surface uniformity of the $\mathrm{MO}_{x}$ coating.

SEM micrographs of the pristine CNTf are included for comparison in the Figure 5.19a. Under the voltage used ( $3 \mathrm{keV}$ ) there is electron transmission, observed for example where the bundles of CNTs overlap. In contrast, when the CNTf is coated with a nominal $5 \mathrm{~nm}$ of $\mathrm{ZnO}$, this feature is not observed, indicating the presence of the thin layer of $\mathrm{MO}_{x}$ material (Figure 5.19b). 20 and $40 \mathrm{~nm}$ are presented in plainview and cross-section in Figure 5.19c-f respectively. The cross section from thin films of CNTf $(<1 \mu \mathrm{m})$ coated with 20 and $40 \mathrm{~nm}$ of $\mathrm{ZnO}$ were obtained by focus ion beam milling of the sample. The ZnO-ALD coating is observed conformally coating the surface of the bundles as a clear change in contrast between materials. The coating is observed for both thicknesses to be uniformly deposited through all the cross section of $<1 \mu m$ thick CNTf substrate.

An important observation from these results is obtained looking closer the porosity of the material. From the plain view and cross section (Figure 5.19) is concluded that the volumetric porosity of the CNTf decreases as the thickness of the ALD layer is increased.

\subsubsection{Morphology, structure and interfacial characterisation of the CNT fibre/ ZnO-ALD material}

Although this chapter is focus on CNTf/ZnO heterojunctions, ALD was also used to produce other heterojuctions $\left(\mathrm{CNTf} / \mathrm{TiO}_{2}\right)$ and these structures and electrical properties were studied in our group.[295] Since both materials are used in this thesis, both are discussed. The general morphology of $\mathrm{CNTf} / \mathrm{MO}_{x}-\mathrm{ALD}$ junctions is proposed in Figure 5.20.

The $\mathrm{ZnO}$ (left) and $\mathrm{TiO}_{2}$ (right) are continuous and homogeneous through all the surface of the CNTf substrate. This can be observed at different magnification scales, from the micro- and nano-scale (Figure 5.20a-c) to the macroscopic size, observing the same morphology through all the surface of a sample of $10 \mathrm{~cm}^{2}$. From TEM imaging (Figure 5.20c,d) it is concluded that the $\mathrm{ZnO}$ and $\mathrm{TiO}_{2}$ present different crystallisation and growing mechanisms. $\mathrm{ZnO}$ obtained layer is completely crystalline, on the contrary the $\mathrm{TiO}_{2}$ deposition results in a totally amorphous material. The crystallinity of the material is confirmed by selected area electron diffraction (SAED). In the Figure 5.20d both SAED patterns (insets) correspond to the area presented in the TEM images. The $\mathrm{ZnO}$ crystallises as small particles (5-10 $\mathrm{nm}$ diameter), growing in close proximity, forming the continuous layer. For the $\mathrm{TiO}_{2}$, the material is deposited in a completely amorphous phase providing a much smoother surface of the oxide.

The detailed microstructure of the $\mathrm{ZnO}$ nanoparticles was investigated by TEM. The interface between the nanoparticles and the CNT bundles after the deposition of the initial $5 \mathrm{~nm}$ of material is presented in the Figure 5.21. A bundle of CNTs decorated with $\mathrm{ZnO}$ nanoparticles was selected (Figure 5.21a) and the diffraction pattern of this region was acquired (Figure 5.21b). The SAED pattern of the interface area presents the structure of the CNT bundle and the $\mathrm{ZnO}$ coating together. The CNT bundle presents a turbostatic graphitic structure and two very intense diffraction spots perpendicular to the bundle axis from CNTs (002) planes of the graphitic structure with a calculated interplanar distance of $0.34 \mathrm{~nm}$. This diffraction spots are 
perpendicular to the CNT bundle axis, as marked with a red arrow. The first ring of diffraction spots correspond to the three main planes of the $\mathrm{ZnO}$-wurtzite structure - (100), (002) and (101), marked with a yellow arrow.

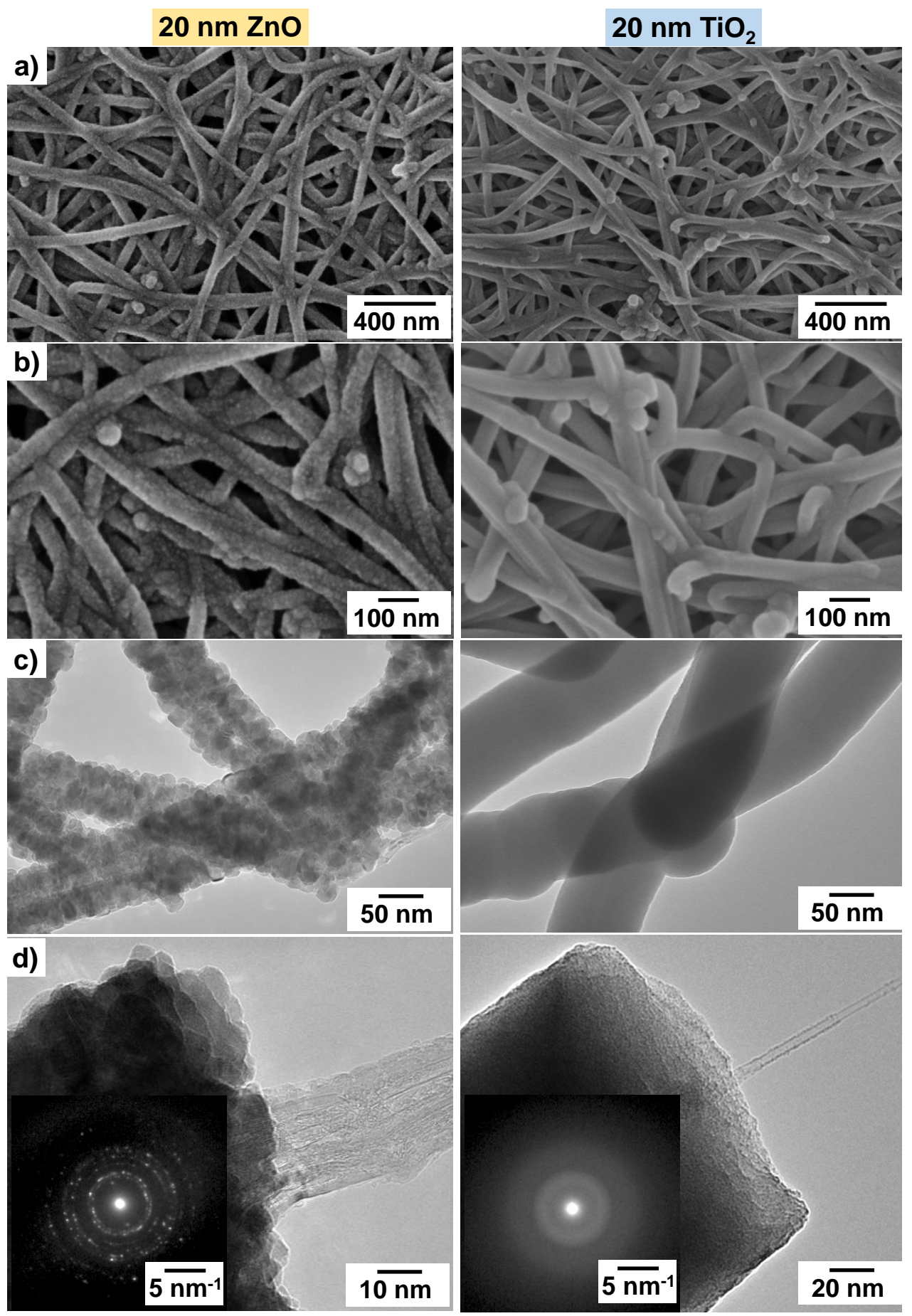

FIGURE 5.20: Comparison between $\mathrm{ZnO}$ (left) and $\mathrm{TiO}_{2}$ (right) conformally growth over the CNTf by ALD. SEM images at a) 20,000x magnification and b) 100,000x magnification. c) TEM images showing a rough nanocrystal-formed $\mathrm{ZnO}$ coating besides the smooth $\mathrm{TiO}_{2}$ coating. d) TEM images and diffraction patterns showing the crystalline structure obtained for $\mathrm{ZnO}$ and an entirely amorphous structure for the $\mathrm{TiO}_{2}$. 


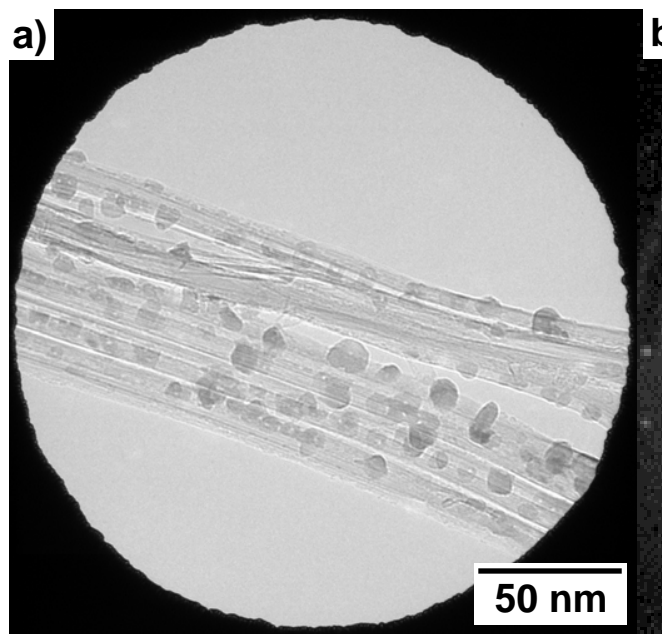

b)

c)

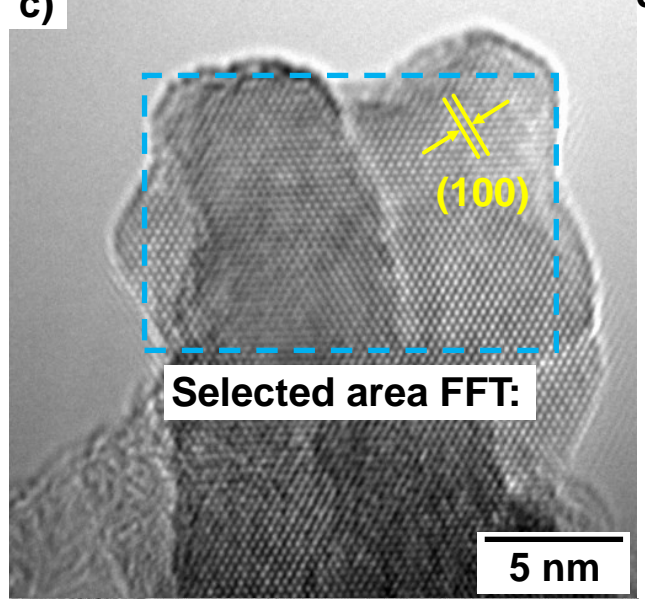

d)
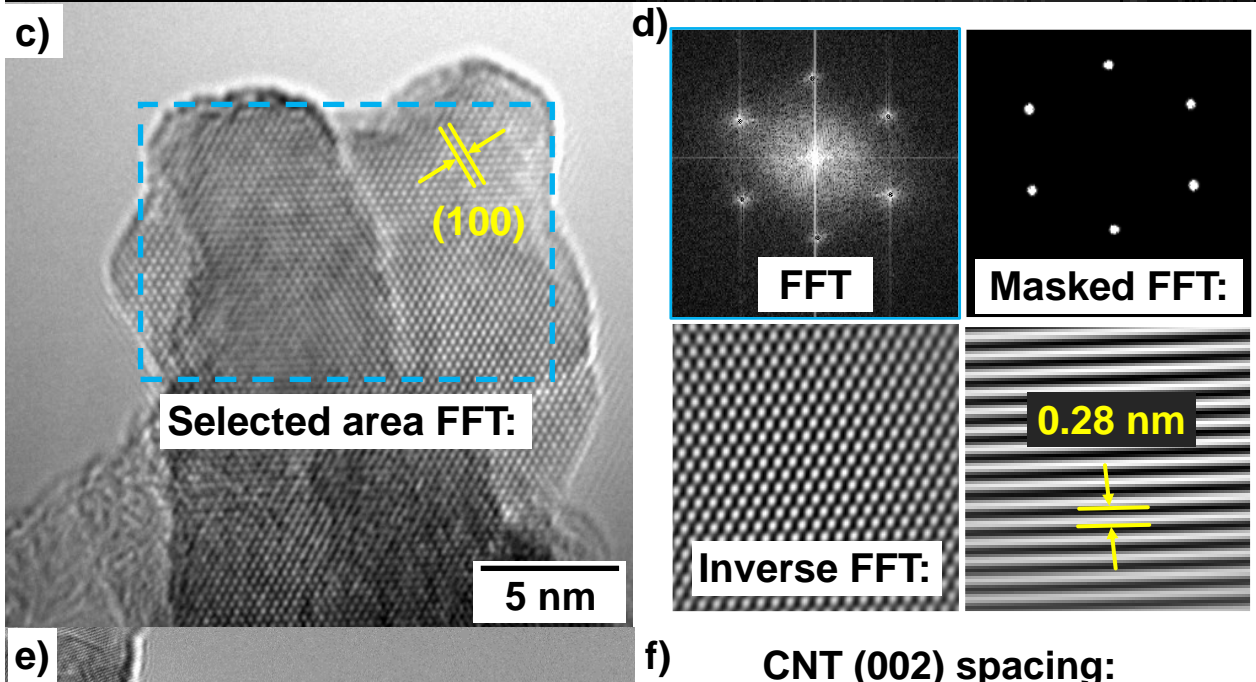

f) CNT (002) spacing:
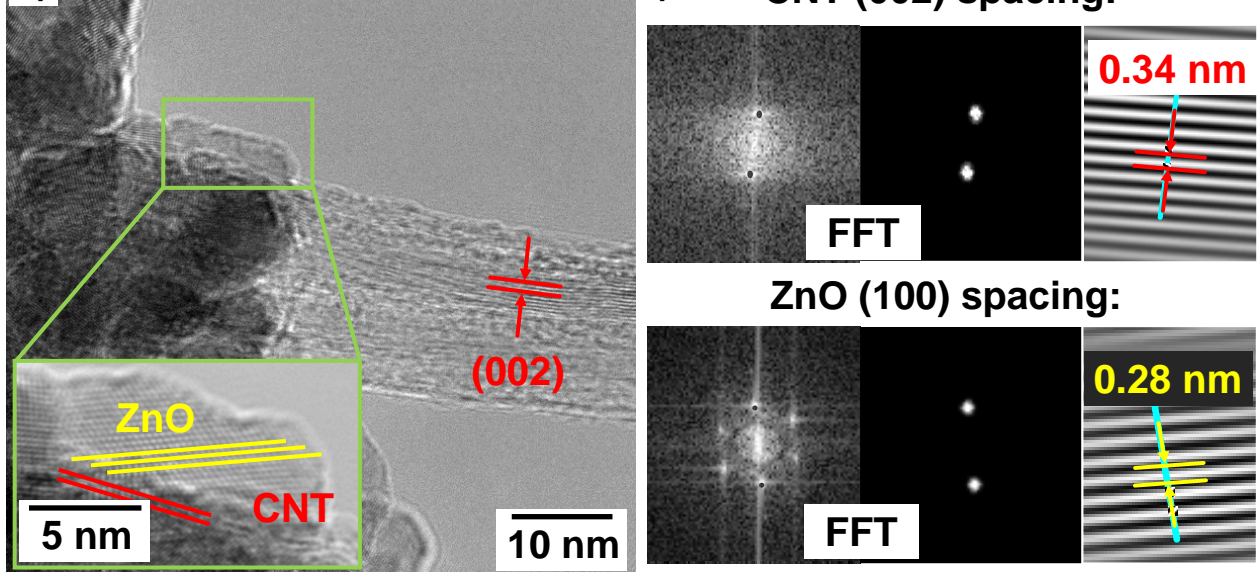

ZnO (100) spacing:

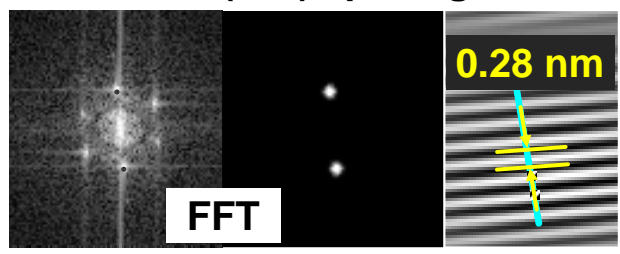

FIGURE 5.21: TEM structural analysis of the CNTf/ZnO-ALD herterojunction. a) Low-resolution image of a CNTf bundle coated with ZnO-ALD nanoparticles after the initial $5 \mathrm{~nm}$ of material. b) Selected area electron diffraction (SAED) pattern of region presented in $a$. c) HR-TEM image of untreated $\mathrm{ZnO}$ nanocrystal in the (011) direction showing the lattice distance in (100) plane. d) Interplanar distance analysis of the $\mathrm{ZnO}$ nanocrystal: corresponding fast Fourier transform (FFT) pattern and same pattern after masking and subtracting the noise, inverse FFT and 2D-instensity profile from where the interplanar distance is obtained. e) TEM image of an additional particle in contact with a CNT bundle. Magnification of the interface showed as inset. f) Interplanar distance analysis of the $\mathrm{ZnO}$ nanocrystal and the 
A closer view of the $\mathrm{ZnO}$ nanoparticles (Figure 5.21c) shows the high crystallinity of the as-deposited material. The fast Fourier transform (FFT) of the selected area in Figure 5.21c is included in Figure 5.21d (upper left image). After masking, filtering and performing the inverse FFT, the atomic distribution presented in Figure 5.21d shows that, although with irregular shape, the entire nanoparticle is monocrystalline. From calculations using the intensity profile of this generated lattice, the bond length of atoms within the $\mathrm{ZnO}(100)$ planes are $0.28 \mathrm{~nm}$.

Several regions of the material were analysed to elucidate a possible relation between the CNTf orientation and the $\mathrm{ZnO}$ crystal growth orientation. As an example, the region presented in Figure 5.21e was analysed. The crystalline orientation between $\mathrm{ZnO}$ and CNT bundle was obtained by calculating orientation of particles referenced to the CNTf axis (along (002) graphite planes), but there is no any preferential orientation observed between these two materials after analysing several particles.
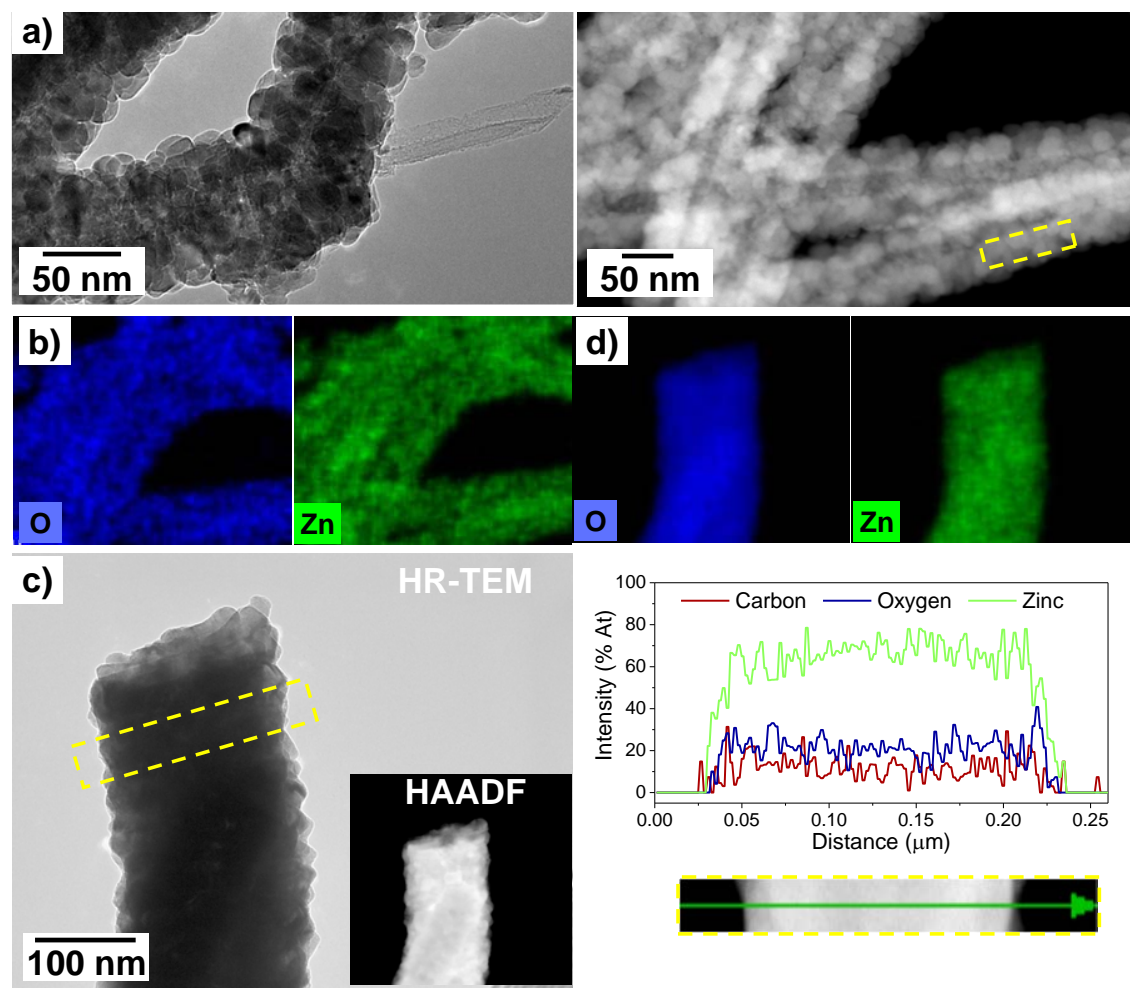

FIGURE 5.22: TEM-EDX analysis of 20 and $100 \mathrm{~nm}$ thickness $\mathrm{ZnO}-$ ALD coating. a) $20 \mathrm{~nm}$ HR-TEM and HAADF images. b) EDX compositional mappings. c) $100 \mathrm{~nm}$ HR-TEM and HAADF images. d) EDX compositional mappings and line-scans showing the uniform $\mathrm{Zn}$ and O content. Selected regions represented with a yellow rectangle in images $a$ and $c$.

The uniformity of the hybrid material was confirmed by energy-dispersive $X$ ray spectroscopy (EDX) of the nanocrystals by TEM technique. Thin $(20 \mathrm{~nm})$ and thick $(100 \mathrm{~nm})$ thickness $\mathrm{ZnO}$ coating are compared in the Figure 5.22. Two clear regions of both materials were selected and they are presented in HR-TEM imaging and HAADF modes. Compositional mappings of those areas were acquired and 
they are presented in the Figure $5.22 \mathrm{~b}$,d; a strong signal of Zn element appears homogeneously distributed along the samples. The presence and distribution of the $\mathrm{ZnO}$ was further verified by semi-quantitative estimation by EDX.[307] The scanned areas are marked with a yellow rectangle in the Figure 5.22a,c. For the $100 \mathrm{~nm}$ thickness it is presented as a magnified inset under the corresponding compositional line-scan in Figure 5.22c,d.

Both materials presents a uniform surface coated with $\mathrm{ZnO}$ nanoparticles. The thicker material presents lower transmission of electrons and the inner CNTs bundles are not observed.

Further insights about the crystal structure of the CNTf coated with $\mathrm{ZnO}$ were also obtained by X-ray diffraction (XRD) in reflection mode, and by synchrotron Xray diffraction in transmission mode (Figure 5.23).
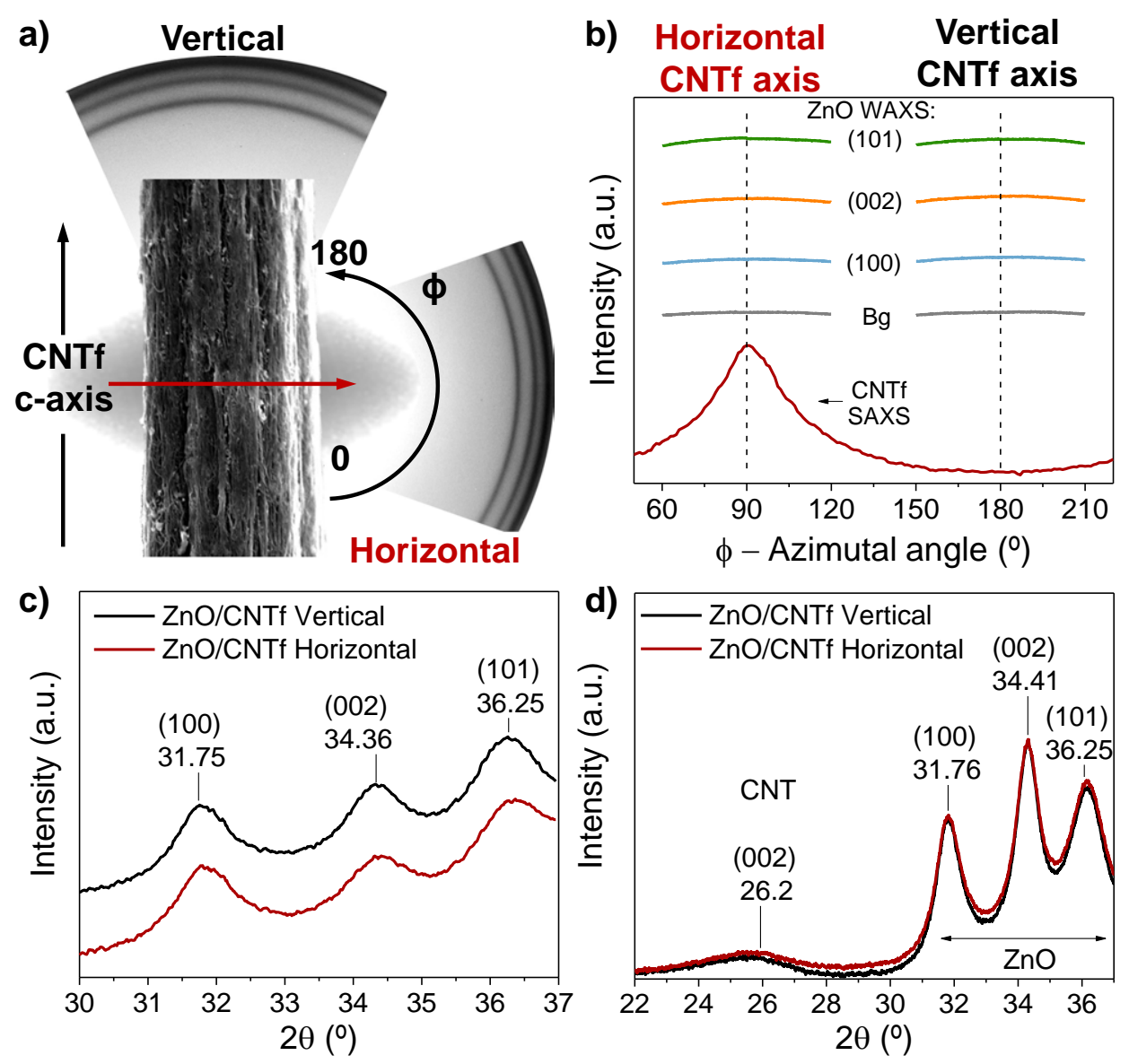

FIGURE 5.23: a) 2D-SAXS/WAXS patterns of the CNTf/ZnO-ALD heterojunction with the CNTf $c$-axis in vertical direction. b) Azimuthal integration of the CNTf SAXS pattern and ZnO WAXS diffractions in vertical and horizontal direction. c) Radial integration of the WAXS patterns. d) Conventional XRD measurements in reflection mode.

The horizontal and vertical orientations of the CNTf (respect to the $c$-axis of the fibre) were analysed in transmission mode, providing the 2D patterns shown in the Figure 5.23a. Notice the shadows that appears in horizontal direction, closer to the 
CNTf. This part of the pattern corresponds to the SAXS region, and these shadows are characteristics of the oriented CNTf in transversal direction to its $c$-axis.

The 2D patterns were analysed by integrating in azimuthal and radial directions the signal. The azimuthal integration of the different signals that appear in the patterns along vertical and horizontal directions are presented in the Figure 5.23b. By integrating the intensity of one diffraction along the azimuthal angle $(\phi)$, it is possible to observe if there is any preferential crystalline orientation. The orientation of the CNTf is obtained from the SAXS pattern, showing a FWHM of approximately $30-40^{\circ}$, corresponding to a low orientation degree CNTf typical of large tows of material.[53] As it is possible to observe in this graph, there is no preferential orientation of any of the $\mathrm{ZnO}$ crystal reflections. This confirms that the $\mathrm{ZnO}$ conformal coating is produced by small nanoparticles that are not preferentially orientated at the nanoscopic level at the interface with the CNTf. As such, these results rule out epitaxy or a strong interaction with specific crystal planes favouring nucleation.

The vertical and horizontal WAXS patterns are also azimuthally integrated (Figure 5.23c). The intensity ratio among the peaks, as well as their broad profiles, are indicative of the nanoparticle morphology of the crystals, in agreement with conventional powder XRD patterns. The diffraction peaks were assigned to $\mathrm{ZnO}$ wurtzite. Once again, the XRD patterns indicates a polycrystalline structure with broad peaks due to the small dimension of the nanoparticles. Three main diffraction peaks are observed at $31.76^{\circ}, 34.41^{\circ}$ and $36.25^{\circ}$. These peaks can be indexed respectively as (100), (002) and (101) crystallographic planes of the wurtzite $\mathrm{ZnO}$ structure (JCPDS No. 36-1451) and in agreement with the previous experiment, the intensity relation shows no clear preferential growth of the crystals.

The hybrid materials have been analysed by Raman spectroscopy showing differences depending on the oxidising agent used during the synthesis. The Raman spectra of both materials are presented in the Figure 5.24a,b, before and after $\mathrm{ZnO}$ deposition. The measured spectra signals are in agreement with the literature,[209] but in this Figure 5.24a,b, the attention is focused in the D-G peaks of the material.

From this comparison, the higher oxidising capability of the $\mathrm{O}_{2}$ plasma synthesis conditions against the water vapour is concluded. The ratio $D / G$ between from $\mathrm{H}_{2} \mathrm{O}$ vapour change from 0.21 to 0.34 when ALD process is carried out (Figure 5.24a). On the contrary, this ratio increases almost a factor of two when the $\mathrm{O}_{2}$ plasma is applied - from 0.06 to 0.67, presented in Figure 5.24b. The CNTf surface modification by both routes leads to creation of preferential sites for the anchoring of the chemical precursors. For a proper comparison and reducing additional artefacts, the spectra were acquired with low intensity and same parameters for all samples, further details in Chapter 3 "Experimental Techniques". Although here are compared 20 and $40 \mathrm{~nm}$ hybrids, the result is consistent with different thicknesses of the deposited layers.

For a $\mathrm{ZnO}$ monocrystal prepared by hydrothermal method, vibrations parallel to $c$-axis, $A$ and $B$ modes, are presents: $A_{1}$ at about $378 \mathrm{~cm}^{-1}, B_{1}^{(\text {low })}-B_{1}^{(\text {high })}$ at about $284 \mathrm{~cm}^{-1}$. Vibrations perpendicular to $c$-axis show more active $E$ modes; $E_{2}^{(\text {low })}$ at about $100 \mathrm{~cm}^{-1}, E_{2}^{(h i g h)}-E_{2}^{(\text {low })}$ at about $333 \mathrm{~cm}^{-1}, E_{1}(\mathrm{TO})$ at about $410 \mathrm{~cm}^{-1}$, $E_{2}^{(h i g h)}$ at about $438 \mathrm{~cm}^{-1}, E_{1}(\mathrm{LO})$ at about $590 \mathrm{~cm}^{-1}$.[275] The Raman scattering from $\mathrm{ZnO} / \mathrm{CNTf}$ in the $\mathrm{ZnO}$ region was very weak, but its main reflection $E_{2}^{(\text {high }}$ could nevertheless be resolved after optimisation of measurement conditions for the $40 \mathrm{~nm}$ 
thickness $\mathrm{ZnO}$ layer (see Figure 5.24c). The low intensity and broadening of the peaks is attributed to the small size effect of the nanoparticle layer.[308] Similar Raman spectra have been reported for ALD-ZnO layers at different temperatures,[309] showing the same small contribution of the $E_{2}^{(h i g h)}$ mode for higher thicknesses and independently of the growth temperature range.
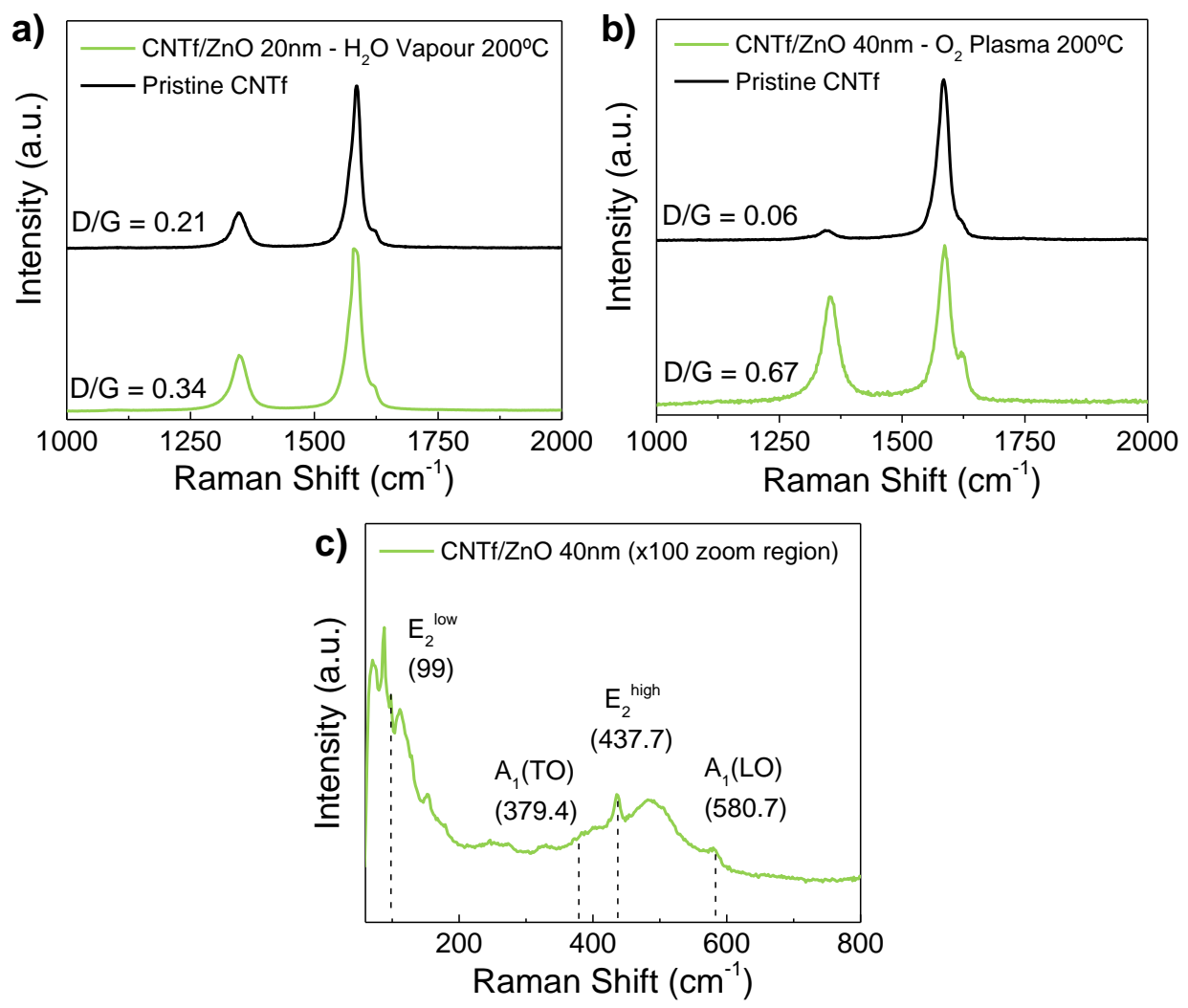

FIGURE 5.24: Raman spectra of the CNTf/ZnO-ALD heterojunction produced by a) water vapour and b) oxygen plasma oxidising agents.

c) Magnified region of the $\mathrm{ZnO}$ Raman spectrum.

The fracture surface of a 1-2 $\mu \mathrm{m}$ thick CNTf/ZnO-ALD $40 \mathrm{~nm}$ sample - synthesised from $\mathrm{H}_{2} \mathrm{O}$ vapour oxidising agent, is presented in the Figure 5.25a. Although the bulk $\mathrm{ZnO}$ is a brittle material, its morphology and low dimensionality still allows a high degree of deformation of the structure. The fracture surface presents a clear degree of deformation in the direction of the forces responsible of breaking the material. A closer look reveals the strained CNTf/ZnO region (Figure 5.25b) shows the high toughness of the hybrid material. Some cracks are observed in the coating of the semiconductor layer (marked with a yellow circle), but still the material presents a high structural integrity demonstrating the robust mechanical interface between both materials. This SEM image attests the intimate contact between the $\mathrm{MO}_{x}$ coating and the CNTs surface, which probably constitutes the basis for the electronic interactions between the components. The CNTf, acting as scaffold, ensures the electrical path inside the semiconductor. Because of the lower electrical resistance of the CNTs, the phenomena occurring at the surface of the semiconductor will be independent in most cases of the observed cracks at the surface, e.g. electrochemical or photoelectrical phenomena. 

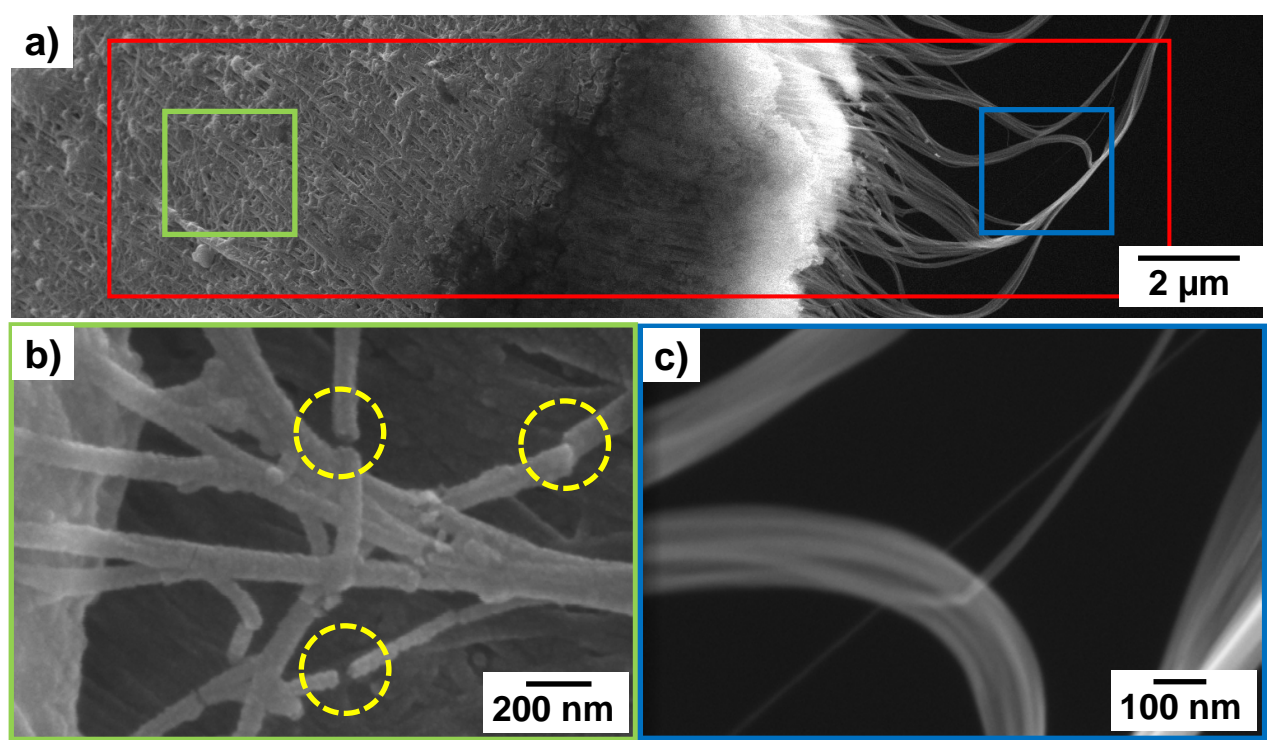

d)

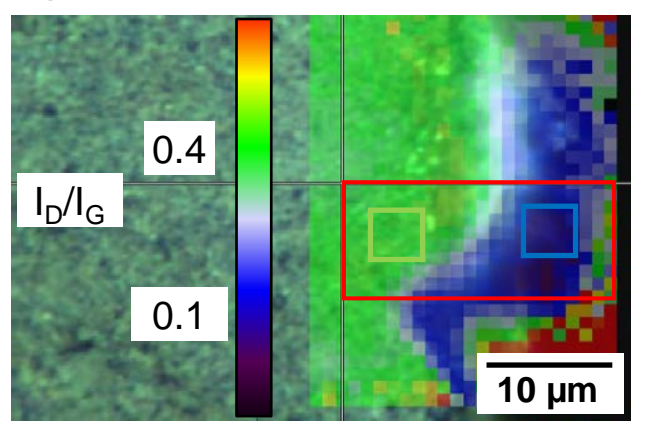

e)

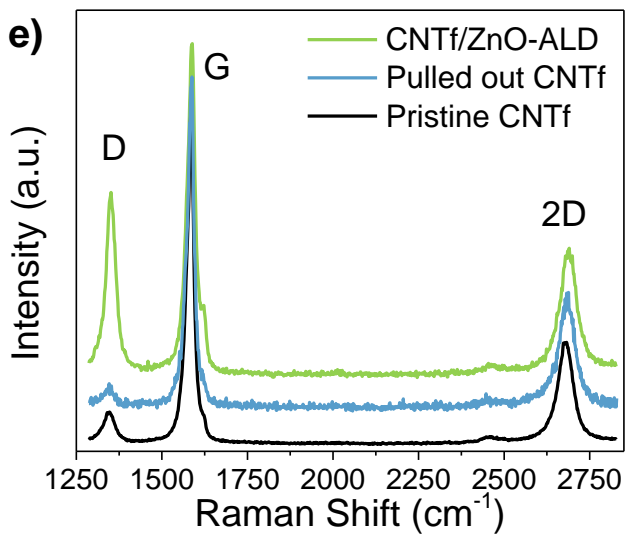

FIGURE 5.25: a) SEM images at the fracture surface of the CNTf/ZnO-ALD $40 \mathrm{~nm}$ hybrid. b) Closer look of the CNTf bundles coated with $\mathrm{MO}_{x}$ partially cracked and strained. c) SEM of the CNTf pulled out from the fracture surface.

For a better understanding of the CNTf/ZnO interaction, a Raman mapping of 20x50 $\mathrm{mm}^{2}$ (over 500 spectra) was performed at the fracture surface interface. This region is marked with a red square in the Figure 5.25a. The representation of the $\mathrm{D} / \mathrm{G}$ ratio is overlapped with the optical microscopy image of the selected analysed region. Two clear different regions can be observed in the mapping. The area where the CNTs are coated and hybridised with the $\mathrm{ZnO}$ semiconductor presents a D/G ratio of approximately 0.4 , whereas the exposed CNTs coming out from the fracture surface shows a $\mathrm{D} / \mathrm{G}$ ratio below 0.1 . These two regions are the analogous to the ones presented in the Figure $5.25 \mathrm{~b}, \mathrm{c}$.

The low D/G ratio of the CNTf at the fracture surface is explained by considering that the CNTs are pulled out from inside the CNTf/ZnO hybrid material. Part of the outer and defective CNTs that compose the CNT bundles could remain inside the conformal $\mathrm{ZnO}$ structure. After the fracture, defect-free CNTs of the CNT bundles are exposed leading to a less defective Raman signal (lower D/G ratio). This would explain the "purity" and low level of defects of the CNTf coming out the 
fracture surface. Moreover, it would also support the strong and robust interaction present at the CNTs/ZnO interface.

Apart from the $D / G$ ratio variation by the oxide deposition, the shift of $G$ and $2 \mathrm{D}$ peaks has been analysed. Changes in the graphitic electronic structure can be induced either by mechanical strain of the carbon lattice or by charge doping. Electron transfer at the interface between both components, similar to electron/hole doping of the CNTs, results in noticeable shifts in the CNT Raman modes.

An additional Raman mapping with the same parameters was acquired far from the fracture area. The spectra were analysed and fitted their $G$ and 2D peaks with Lorentzian shapes. The peak centre positions resulted from the fitting have been plotted in Figure 5.26, showing an average shift of approx. $1 \mathrm{~cm}^{-1}$ for the $G$ peak (from $1582.1 \pm 0.2 \mathrm{~cm}^{-1}$ to $1583.2 \pm 0.5 \mathrm{~cm}^{-1}$ ) and $2 \mathrm{~cm}^{-1}$ for the 2D peak (from $2680.4 \pm 0.4 \mathrm{~cm}^{-1}$ to $2682.5 \pm 0.8 \mathrm{~cm}^{-1}$ ). The ratio $\Delta 2 \mathrm{D} / \Delta \mathrm{G}>2$ indicates the presence of residual strain induced in the CNTf by the ZnO-ALD layer, probably by differences in thermal expansion coefficients during the deposition process. Charge doping effect has been reported to provide quasi-linear behaviour between $G$ and $2 D$ peaks shift, but with a lower proportionality factor (typically $<1$ ).[310, 311] Therefore, CNTf is being affected by the hybridisation process, where the growth of metal oxides nanoparticles onto the CNTf surface produces a compression force on the CNTf bundles.

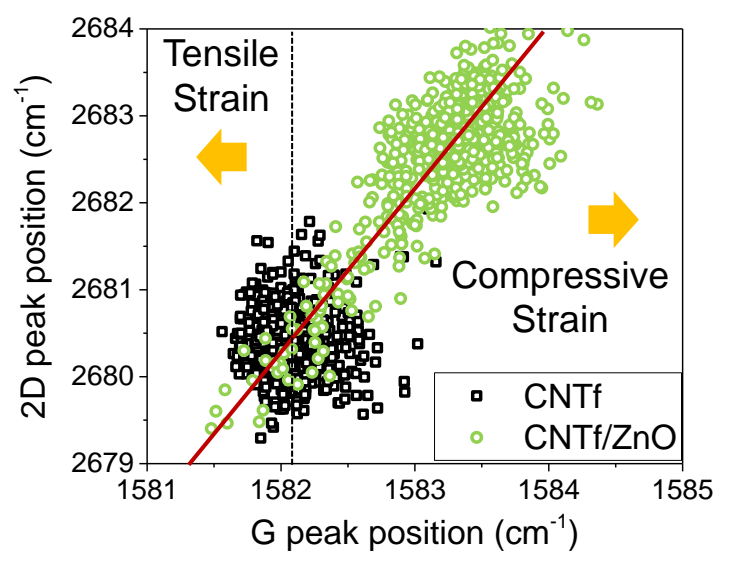

FIGURE 5.26: CNTf 2D and G Raman modes extracted from fitting a 500 spectra Raman mapping before and after the ZnO-ALD process.

Residual compression state has been measured for similar hybrids $\mathrm{CNTf} / \mathrm{TiO}_{2}-$ ALD by x-ray diffraction microstrain measurements.[295] The previous Raman spectroscopy mappings also support the same phenomenon. Moreover, this compression stress ensures a close interface between the $\mathrm{ZnO}$ and the CNTf that will have consequences over the charge transfer phenomena at the interface and the hybrid mechanical properties in general.

A schematic explaining the origin of the residual stresses is shown in the Figure 5.27. When the precursor diffuses along the surface of the CNTf it forms small clusters of atoms that grows until they find the neighbour particle. When the particles collide some tangential forces are generated and transferred to the substrate generating a radial compression state and probably axial compression. Moreover, 
the synthesis process carried out at $200{ }^{\circ} \mathrm{C}$ might generate residual axial compression stresses over the CNTf due to mismatch between coating and substrate thermal expansion coefficients.

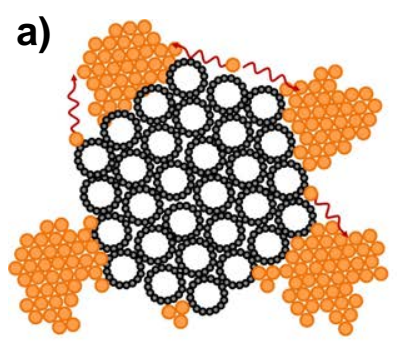

b)

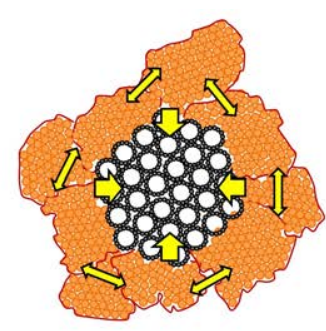

c)

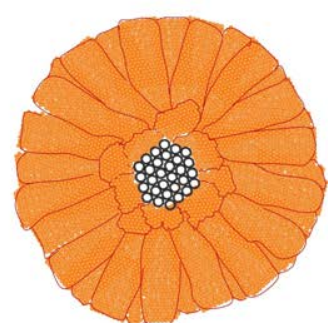

FIGURE 5.27: Schematic of the $\mathrm{MO}_{x}$-ALD layer growth and the consequent CNTf compression stress state. a) Particles nucleation and growth by precursor diffusion and preferential adsorption during the initial cycles. b) Initial conformal layer formation after few nanometers, and stress state represented by the yellow arrows due to thermal expansion variations during the process. c) Schematic of a final thicker conformal layer on top a CNTf bundle.

\subsubsection{Chemical environment and electronic structure of the $\mathrm{CNTf} / \mathrm{ZnO}$ - ALD material}

The chemical environment and electronic structure of the CNTf/ZnO-ALD hybrids were analysed by XPS, which provides information about the composition and oxidation state of materials and thus about interfacial interactions between components. Moreover, the different functional groups present at the surface of the pristine CNTf, as well as the ones created during the ALD technique can also be analysed by this technique.

Figure 5.28 shows the XPS survey spectra of the CNTf and the CNTf/ZnOALD hybrids materials. The pristine CNTf is compared with the CNTf treated with 10 minutes of $\mathrm{O}_{2}$ plasma at $200{ }^{\circ} \mathrm{C}$, corresponding to the conditions applied during the ALD treatment process (Figure 5.28a). 5 and $50 \mathrm{~nm}$ thickness ZnO-ALD deposited over the CNTf has been compared in the Figure 5.28b. The C1s and O1s peaks appear at approximately $284.4 \mathrm{eV}$ and $533 \mathrm{eV}$ for all samples.[59, 62] For the hybrid materials, the Zn2p peaks are also observed at $1022 \mathrm{eV}$.

The oxygen to carbon relative content has been obtained from the peak-areas ratio after the fitting and deconvolution of every individual peak. For the pristine and plasma treated CNTf, the ratio increase from 0.18 o 0.76 respectively. This indicates a high density of functional groups introduced by the plasma treatment used as part of the optimised ALD process, responsible of the enhanced attachment of the ALD precursor over the CNTf and the nucleation of the $\mathrm{MO}_{x}$. This implies that the interface between ZnO-Nanoparticles (NPs) and the CNTs will have a high density of anchoring points between the $\mathrm{ZnO}$ and the $\mathrm{CNTs}$, later responsible of better mechanical and electrical interconnection of both materials. For the hybrid materials, there is a $\mathrm{C} 1 \mathrm{~s}$ signal, which decreases with increasing $\mathrm{ZnO}$ thickness. This also implies that the carbon signal observed in the $50 \mathrm{~nm} \mathrm{ZnO}$ sample originates from 
adsorbed carbon contaminants including possibly the subproducts of the ALD synthesis technique (-CH groups from the diethylzinc precursor) that remain trapped into the $\mathrm{MO}_{x}$ structure.[198]
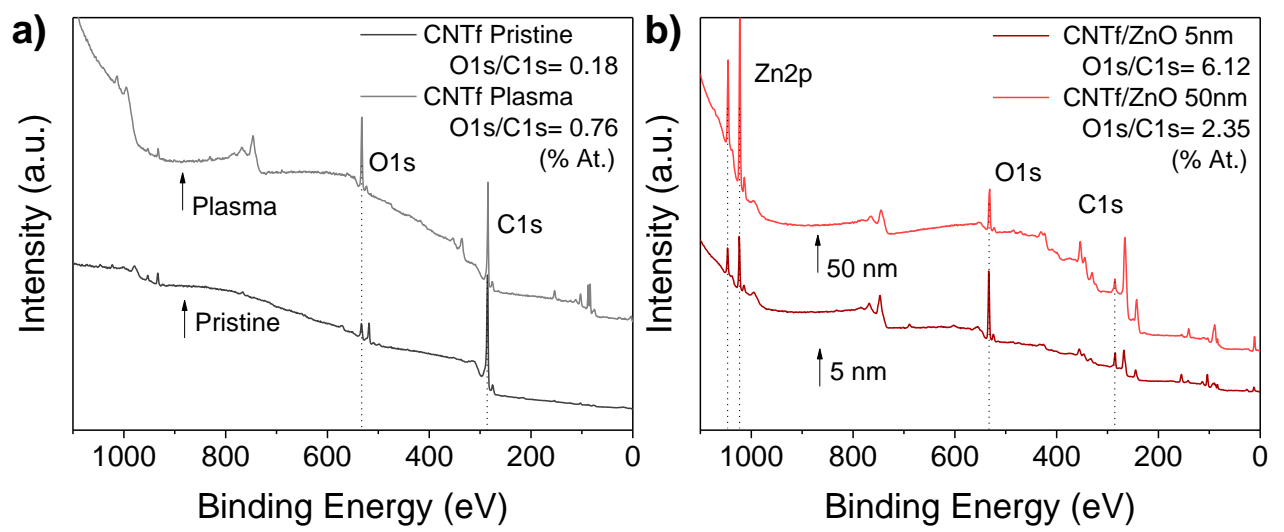

FIGURE 5.28: Survey XPS spectra of a) pristine CNTf and oxygen plasma treated CNTf, and b) 5 and $50 \mathrm{~nm} \mathrm{ZnO}$ deposited over the CNTf. The O1s, C1s and Zn2p emissions are marked as well as the peak area ratios.

Figure 5.29 shows a closer look of the XPS spectra for the C1s core level with their corresponding fitted components. The C1s peak of the pristine CNTf (Figure 5.29a) presents a main components located at $284.4 \mathrm{eV}$, corresponding to $\mathrm{sp} 2$ hybridised carbon in the CNT structure $(\mathrm{C}=\mathrm{C})$. Two additional components are included for the deconvolution of this peak, one located at $284.8 \mathrm{eV}$ related to the C-C (sp3 carbon) and C-H bonds, and another $285.5 \mathrm{eV}$ attributed to C-O groups.[59, 62] These emissions are mainly attributed to residual amorphous carbon produced at final stages of the CVD synthesis process. The oxygen plasma treatment has an strong influence over the structure of the CNTf material (Figure 5.29b). The reduction of the $s p 3$ carbon component is due to the plasma creating defects at the surface of the CNTs, similar to gas phase ozone treatment using a UV lamp.[62] These defects increases the amount of $s p 3$ carbon (C-C bonds) in the material, observed as an increment of the $284.8 \mathrm{eV}$ emission.

For the $5 \mathrm{~nm} \mathrm{ZnO}$ hybrid material, the $\mathrm{C} 1 \mathrm{~s}$ emission (Figure 5.29c) shows a predominant $\mathrm{C}-\mathrm{O}$ component $(285.5 \mathrm{eV})$, in agreement with literature[312] and mentioned before, probably related to confined residual components of the ALD precursor deposition (DEZ - diethylzinc).[198] Due to the low thickness of the semiconductor, and perhaps the incomplete coating of the surface, there is probably still contribution from emission of the CNTf substrate (sp2 and sp3 components). Although the chemical interaction between carbon and zinc has been reported in the past by some authors, $[313,314]$ there is no evidences in our material of the corresponding $\mathrm{C}-\mathrm{O}-\mathrm{Zn}$ bond at $283.7 \mathrm{eV}$. When the thickness of the $\mathrm{ZnO}$ is increased, there is no more contribution of the CNTf substrate to the XPS spectra (Figure $5.29 \mathrm{~d}$ ). The C-O component is still present, as well as a peak $290 \mathrm{eV}$ attributed to $\mathrm{O}-\mathrm{C}=\mathrm{O}$ bonds[192, 315] from surface groups. 
a)

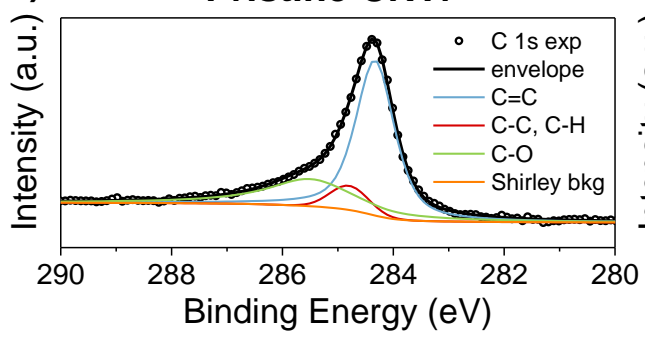

c) CNTf/ZnO-ALD $5 \mathrm{~nm}$

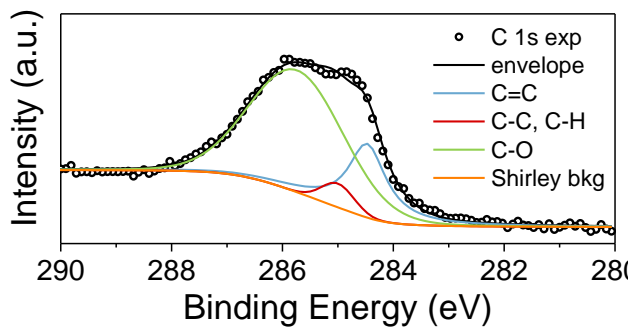

b) CNTf Plasma

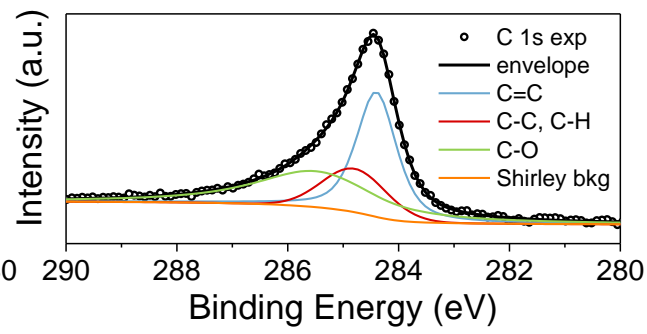

d) CNTf/ZnO-ALD $50 \mathrm{~nm}$

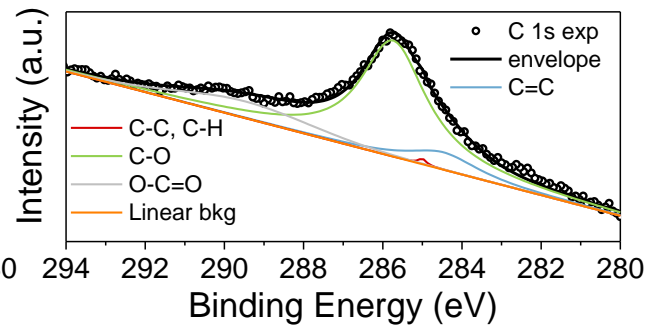

FIGURE 5.29: XPS C1s core level of a) CNTf substrate, b) oxygen plasma treated CNTf, c) $5 \mathrm{~nm}$ and d) $50 \mathrm{~nm}$ CNTf/ZnO-ALD hybrid materials.

The O1s core level is also presented in detail in the Figure 5.30. For the pristine CNTf, O-C bonds (533 eV) are present due to amorphous carbon residue as mentioned before. Still the intensity of the O1s emission is small as it is observed from the O1s/C1s ratio presented in the Figure 5.28a. There is no other contribution observed when the CNTf substrate is oxidised by plasma treatment, although the intensity of this signal shows an important increment in magnitude.

For the hybrid materials (Figure 5.30c,d), after depositing $5 \mathrm{~nm}$ of $\mathrm{ZnO}$, the O1s peak shows a wider envelope and two more components can be deconvoluted. The C-O peak appears very similar in CNTf and $5 \mathrm{~nm}$ ZnO/CNTf, suggesting that is mainly due to impurities. According to previous studies of ZnO by ALD,[198, 312] residual O-H bonds, located at $532.2 \mathrm{eV}$, can easily be formed in oxygen vacancies. More importantly, $\mathrm{Zn}=\mathrm{O}$ bonds normally located at $530.4 \mathrm{eV}$ start to appear in the spectra, although of low intensity. The low intensity of the $\mathrm{Zn}=\mathrm{O}$ peak may suggest that the initial layers (or nanometres) of material deposited by ALD could be not fully oxidised. This would lead to $\mathrm{Zn}_{(1-x)} \mathrm{O}$ or even metal zinc clusters,[309] as similarly reported before for other metals.[316] When the deposited $\mathrm{ZnO}$ thickness is increased (Figure 5.30d), the ratio between $\mathrm{O}=\mathrm{Zn}$ and $\mathrm{O}-\mathrm{H}$ signals is increased, indicating a higher degree of oxidation. This is a important result suggesting differences in composition of the $\mathrm{ZnO}-\mathrm{ALD}$ layer when is deposited at different thicknesses over the CNTf substrate.

Notice here that the difference in $\mathrm{ZnO}$ thicknesses correspond to longer ALD process. This time varies in several hours from one sample to other. That time at $200{ }^{\circ} \mathrm{C}$ could explain some chemical differences as well as produce some recrystallisation of the $\mathrm{ZnO}$ thicker layers. 
a)

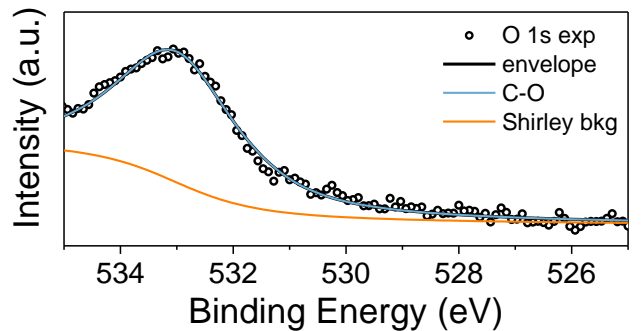

c) CNTf/ZnO-ALD $5 \mathrm{~nm}$

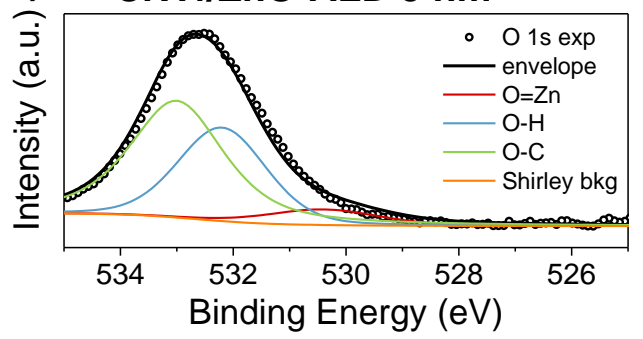

b) CNTf Plasma

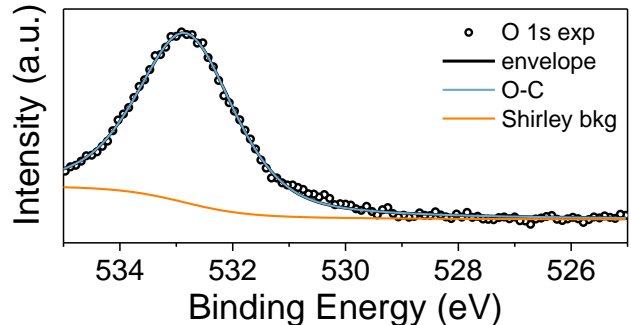

d) $\mathrm{CNTf} / \mathrm{ZnO}$-ALD $50 \mathrm{~nm}$

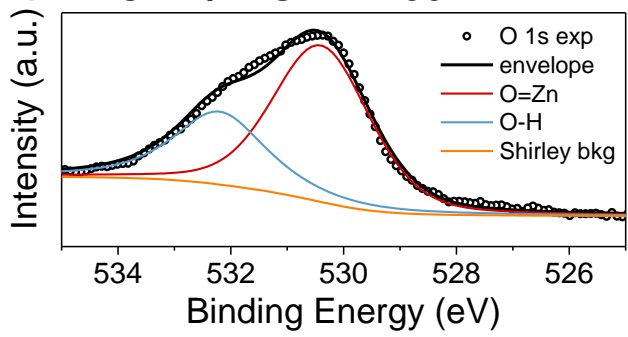

FIGURE 5.30: XPS O1s core level of a) CNTf substrate, b) oxygen plasma treated CNTf, c) $5 \mathrm{~nm}$ and d) $50 \mathrm{~nm}$ CNTf/ZnO-ALD hybrid materials.

Finally, the core levels of the Zn2p are analysed for the 5 and $50 \mathrm{~nm}$ CNTf / ZnOALD hybrids and presented in the Figure 5.31a. The peaks related to the $\mathrm{Zn} 2 \mathrm{p}_{3 / 2}$ and $\mathrm{Zn} 2 \mathrm{p}_{1 / 2}$ are clearly observed at 1021.6 and $1044.4 \mathrm{eV}$ respectively. The oxidation state of the $\mathrm{ZnO}$ can be attributed from the position of the $\mathrm{Zn} 2 \mathrm{p}_{3 / 2}$ emissions. However, it is difficult to distinguish the oxidation state of $\mathrm{Zn}$ according to the $\mathrm{Zn} 2 \mathrm{p}_{3 / 2}$ peak, since the binding energy of the metallic $\mathrm{Zn}^{0}(1021-1022 \mathrm{eV})$ overlaps with that of the $\mathrm{Zn}^{2+}$ in $\mathrm{ZnO}(1022-1022.5 \mathrm{eV}) \cdot[317,318]$
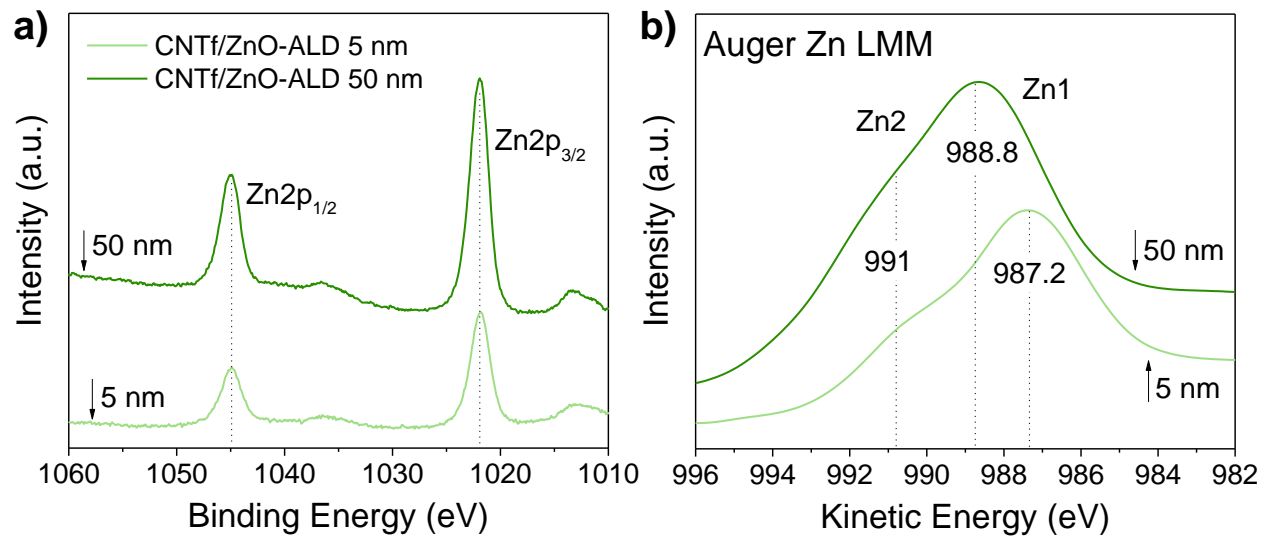

FIGURE 5.31: a) XPS Zn2p core levels and b) Auger Zn LMM lines of CNTf/ZnO-ALD hybrid materials.

Apart from the higher intensity of the $50 \mathrm{~nm} \mathrm{ZnO}$ hybrid, some differences are observed in the Zn LMM Auger lines (Figure 5.31b) of both materials, which are more sensitive to differences in chemical environment than Zn2p peaks. The lower kinetic energy peak (Zn1: 987.2 and $988.8 \mathrm{eV}$ ) are attributed with $\mathrm{Zn}$ bonded with oxygen, whereas the shoulder at higher kinetic energy (Zn2: $991 \mathrm{eV}$ ) indicates the 
presence of metallic $\mathrm{Zn}$ originated from zinc interstitial and/or oxygen vacancies. The kinetic energy for the LMM peak of $\mathrm{Zn}^{0}$ is ranged in 991.8-992.5 $\mathrm{eV}$, and $\mathrm{Zn}^{2+}$ is in the range 987.7-988.5 $\mathrm{eV}$.[318] The peak ratio $\mathrm{Zn2} / \mathrm{Zn} 1$ (metallic to oxidised $\mathrm{Zn}$ ) are apparently more than 0.5 for 50 and $5 \mathrm{~nm}$ coatings, and although there are evident differences between both materials, for proper quantitative determination, more precise measurements with standard calibrants are required. This result implies different chemical environment for the $\mathrm{ZnO}$ material produced at low number of cycles against large ALD treatments.

\subsubsection{Electrical characterisation and photocurrent measurements of the CNTf/ZnO-ALD heterojunction}

The electrical properties of the CNTf/ZnO-ALD hybrids were characterised by solid state $I-V$ measurements. One of the difficulties of measuring such a thin $3 \mathrm{D}$-interfaces is ensuring the proper contacts between materials. For measuring the metal-semiconductor interface, a proper electrical contact between both materials must be performed, and for that, tungsten needles mounted in a probe station were used. The measurement procedure involves firstly contacting one of probe-station needles (negative electrode) with the CNTf material. For ensuring that, the tip is pressed until it goes through the thickness of the material, breaking the semiconductor layer and therefore ensuring the CNTf (metallic) connection (see Figure 5.32a). Secondly, for the semiconductor electrical contact, a live electrical measurement using the tungsten needle while approaching the surface of the semiconductor was followed. With this approach, it is possible to ensure the contact without breaking the thin semiconductor layer. At the same time, considering the elasticity of the CNTf substrate, due to its foam-like structure at the nanoscale, the contact at the surface of the semiconductor was sequentially increased to understand the effect of the pressure over the conduction properties of the heterojunction. A schematic of the material and this measurement approach as well as an optical image is presented in the Figure 5.32a.

Data from a control experiment showing the $I-V$ characteristics of a pristine CNTf while increasing the pressure (P1 to P6) are presented in Figure 5.32b. Although the pressure was finely adjusted by decreasing the z-height of the contact with a micropositioner, there was no set-up in place to measure the corresponding force applied. As shown, the current increases slightly due to a better electrical contact, and resistance goes about from 180 to $400 \Omega$. This variation is mainly attributed to the diminished contact resistance between the tungsten needle and the CNTf. This control experiment provides a magnitude for the changes in CNTf/contact resistance.

The same experiment was performed over a CNTf/ZnO-ALD heterojunction with a semiconductor thickness of $20 \mathrm{~nm}$, enough for ensuring a uniform conformal coverage at the surface of the CNTf. A continuous measurement of $I$ vs $t$ (time) was performed during $300 \mathrm{~s}$, sweeping the voltage at the same time between +2 and $2 \mathrm{~V}$ with an scan rate of $500 \mathrm{mV} / \mathrm{s}$ (Figure 5.32c). During this measurement, the pressure at the semiconductor contact was slightly increased from P1 to P6. The calculated resistance shows a resistance variation of two orders of magnitude range. This experiment also shows the stability of the measurement under fixed conditions, with resistance being stable for a constant contact pressure. 
An individual $I-V$ curve is extracted from every range of pressure from the previous experiment, they are presented in the Figure 5.32d. These characteristics shows a non-ohmic behaviour due to the presence of the semiconductor at the interface.

a)
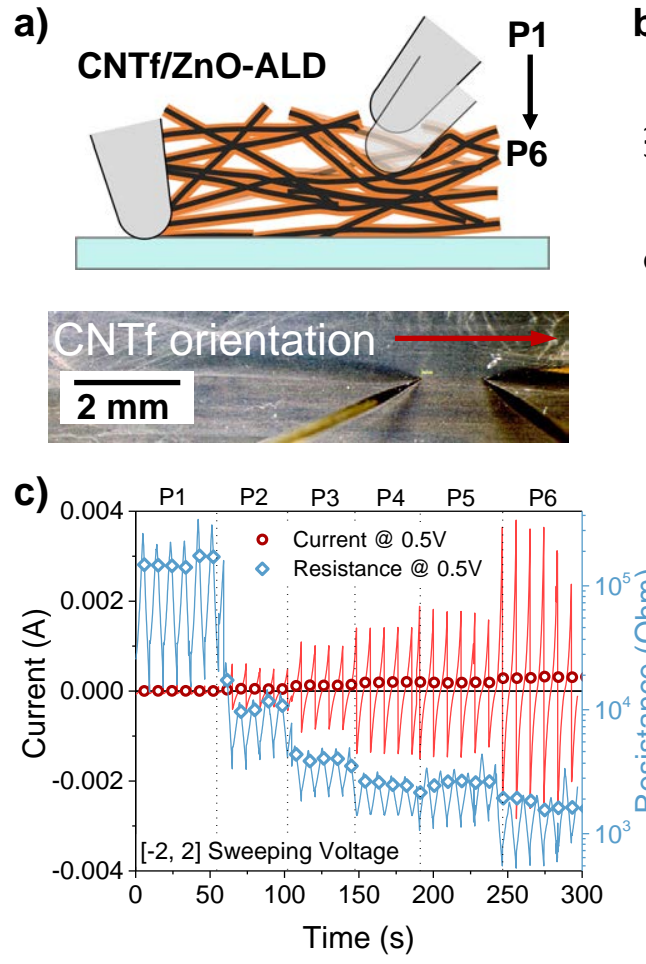
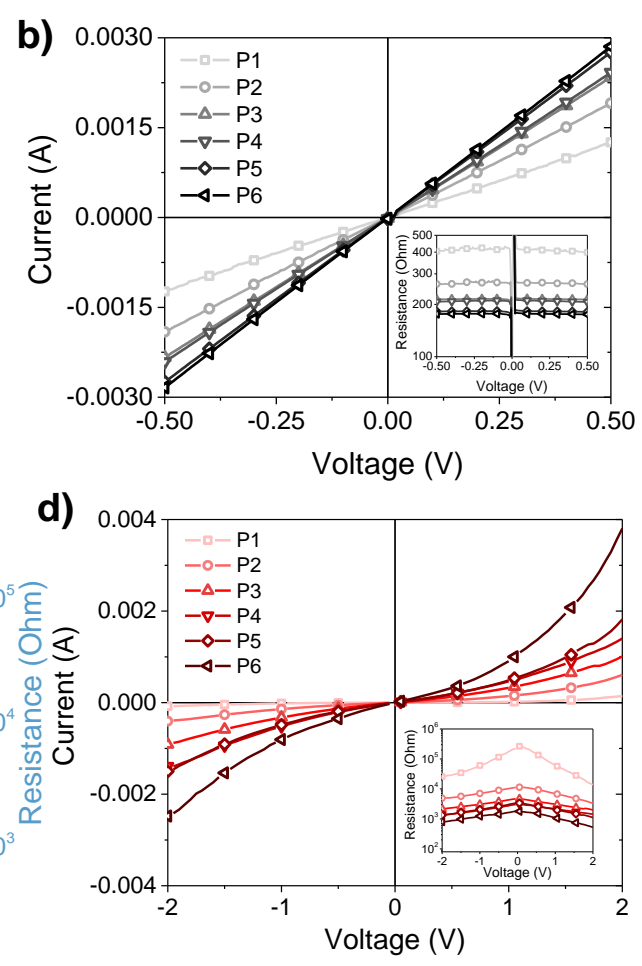

FIGURE 5.32: a) Micro-contacts scheme representing the increase in pressure and optical microscope image of a CNTf/ZnO-ALD heterojunction. $I-V$ variation as a function of pressure for $\mathrm{b}$ ) pristine CNTf and c,d) CNTf/ZnO-ALD heterojunctions. Calculated resistance presented as an inset.

To gain further insights into the electrical properties of the hybrids, this experimental method was applied to samples with different semiconductor thickness. The $\mathrm{I}-\mathrm{V}$ curves for 5, 20, 50, $100 \mathrm{~nm} \mathrm{ZnO}-\mathrm{ALD}$ thicknesses are included the Figure 5.33a. As it is observed from these curves, the low thickness ZnO-ALD hybrids leads to metallic (ohmic) contacts, whereas the thicker ZnO layers (50-100 $\mathrm{nm}$ ) show an evident rectifying behaviour. The rectification ratios are 5.6 and 4.5 for $100 \mathrm{~nm}$ and for $50 \mathrm{~nm}$ respectively (both calculated between -1 and $1 V$ from Figure 5.33b).

Due to the low resistance of this heterojunction, in comparison to the previous section, the thermionic emission theory can be used for obtaining the SBH and ideallity factor of the junction. $\eta=30$ and $V_{b i}=0.54 V(0.7-1.5 V$ range $)$ are obtained for $50 \mathrm{~nm}$ coating, and $\eta=50$ and $V_{b i}=1.1 \mathrm{~V}(1.2-1.5 \mathrm{~V}$ range) are obtained for $100 \mathrm{~nm}$. These values are far from the reported range of an ideal Schottky diode behaviour.[280] It is important considering the low dimension of the $\mathrm{ZnO}$ layers for 5 and $20 \mathrm{~nm}$. In this range, the charge depletion region of the material $(w)$ would be close to the physical size of the thin ZnO-layers, it can be estimated considering a carrier concentration $8 \times 10^{19} \mathrm{~cm}^{-3}[319]$ as $3 \mathrm{~nm}$ and $3.7 \mathrm{~nm}$ for $50 \mathrm{~nm}$ and $100 \mathrm{~nm}$ respectively. The obtained characteristics obtained from the electrical characterisation 
are summarised in the Table 5.2. Apart from the physical properties of the junction, the formation of electrical pinholes induced by current in such a thin layers could explain the low resistive behaviour of the thinner ALD layers.[320, 321] The presence of defects also increase when decreasing thickness, increasing the leaking current and the possible appearance of metallic conducting channels through the layer.
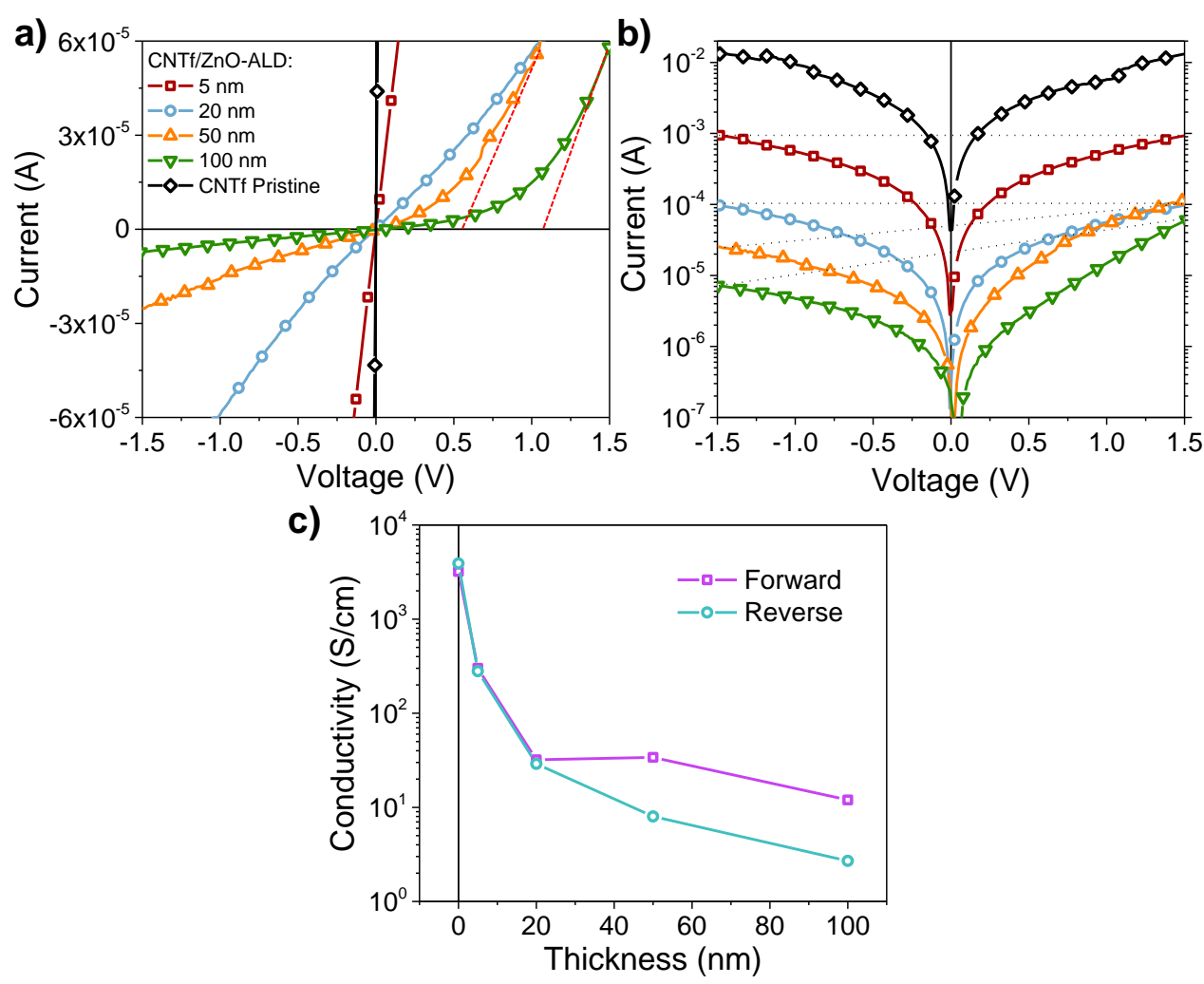

FIGURE 5.33: a) $I-V$, b) Tafel curves and c) conductivities of the CNTf/ZnO-ALD heterojunctions for the different ZnO-ALD thicknesses.

TABLE 5.2: Extracted parameters for the CNTf/ZnO-ALD hybrids heterojunction.

\begin{tabular}{llllll}
\hline Sample & $\begin{array}{l}\text { Conductivity } \\
(\mathrm{S} / \mathrm{cm})\end{array}$ & $\eta$ & $\begin{array}{l}V_{b i} \\
(V)\end{array}$ & $\begin{array}{l}w \\
(\mathrm{~nm})\end{array}$ & $\begin{array}{l}\mathrm{RR} \\
-\end{array}$ \\
\hline Pristine CNTf & 3200 & - & - & - & - \\
CNTf/ZnO-ALD 5 nm & 300 & - & - & - & - \\
CNTf/ZnO-ALD 20 nm & 32 & - & - & - & - \\
CNTf/ZnO-ALD 50 nm & 34 & 30 & 0.54 & 3 & 4.5 \\
CNTf/ZnO-ALD 100 nm & 12 & 50 & 1.1 & 3.7 & 5.6 \\
\hline
\end{tabular}

The CNTf/ZnO-ALD hybrid conductivities have been calculated from the I$\mathrm{V}$ measurements, directly from currents at $1 \mathrm{~V}$ forward biased and $0.5 \mathrm{~V}$ reverse biased. The obtained values are presented as an inset of the Figure 5.33c. The same approximation than in the previous section was applied for calculating the effective area of the measurement $\left(180 \mathrm{\mu m}^{2}\right)$ and the electric channel was directly taken as the total hybrid material thickness $(\approx 10 \mu \mathrm{m})$. The obtained conductivities are 3200, 300, 
32,34 and $12 \mathrm{~S} / \mathrm{cm}$ for the pristine material, and 5,20,50, $100 \mathrm{~nm}$ respectively. This values are in the range of previous reported similar ZnO-ALD material.[196, 322]

The conductivity of the ZnO by ALD method has been reported very sensitive to the deposition technique conditions. In previous studies, increasing the deposition temperature from 100 to $170{ }^{\circ} \mathrm{C}$, the conductivity increases from 0.43 to $260 \mathrm{~S} / \mathrm{cm}$, measured by Hall measurements, at a drain voltage of $25 \mathrm{~V}$ and for $130 \mathrm{~nm}$ thickness of ZnO-ALD layer. The values are consistent with the results obtained. In this study, although the deposition temperature is $200^{\circ} \mathrm{C}$, for the thickest layer, the conductivity ranges one order of magnitude below the reported values. Moreover, in other research, the enhanced conductivity has been attributed to an increase concentration of $-\mathrm{OH}$ groups that increases the carrier concentration.[319] This is in agreement with the XPS presented results, where the concentration of -OH groups is also higher for the $5 \mathrm{~nm}$ compared to the $50 \mathrm{~nm}$.

More importantly, the metallicity of the electrical junction has been shown very dependent on the carrier concentration of the material. The variation in thermal processing conditions have shown an increase in carrier concentration from $10^{16}$ to $10^{20} \mathrm{~cm}^{-3}$. This is also related with a variation in the rectifying ratio of a $\mathrm{ZnO}$ junction of six orders of magnitude.[280,319]

\subsubsection{Charge transfer study of the interface}

As it has been explained for the previous photocurrent measurements, the photon-sensing properties of the $\mathrm{ZnO}$ photodetectors are primarily governed by the oxygen molecule adsorption/desorption behaviours on the metal oxide surface. With this type heterojunctions, where the surface of the $\mathrm{ZnO}$ has been maximised, there is a huge increase in accessible surface for the chemical reactions to take place. Moreover, using the CNTf as inner current-collector for the semiconductor, the electrical signal generated by the surface chemical reactions is extracted much more efficiently.

UV-photocurrent measurements are used as confirmation of charge transfer phenomena at the CNTf/ZnO-ALD interface, but apart from that, it also serves as prove of the material for other promising applications like UV-photodetectors. CNTs/ZnO-ALD materials has already been tested as UV n-type and p-type photodetectors depending on the ALD reaction cycles and the degree of coverage.[323]

A schematic of the CNTf/ZnO-ALD photocurrent measurement is presented in Figure 5.34a. In this measurement, the hybrid material deposited over a glass substrate is measured through the metal-semiconductor interface. $1 \mathrm{~cm}^{2}$ FTO is used as upper electrode for allowing the illumination during the interface characterisation. This electrode allows the illumination of the semiconductor and at the same time to measure its photocurrent response. Philips UVA-lamp, $365 \mathrm{~nm}$ radiation and $0.3 \mathrm{~mW} / \mathrm{cm}^{2}$ power was used; notice here the low power intensity of the illumination source, important parameter for calculating the responsivity of the material. A bias voltage of $0.1 \mathrm{~V}$ was applied for better resolution of the acquired signal.

The materials with different $\mathrm{ZnO}$ thicknesses - 20, 50, $100 \mathrm{~nm}$, have been illuminated during $300 s$ and later waited until its complete recovery. The I- $t$ curves are presented in the Figure 5.34b. A consistent behaviour is obtained for the three hybrids, showing an increase in the current under UV-light exposure and decreasing 
slowly close to initial value after switching off the light. It is well known the that $\mathrm{ZnO}$ is n-type semiconductor with electrons as major charge carriers. Under dark conditions, oxygen species are chemisorbed onto the surface of the $\mathrm{ZnO}$ nanoparticles capturing the free electrons in the conduction band of the n-type semiconductor. This results in an increase in the thickness of the electron depletion layer at the physical interface. However, under UV-light illumination, electron-hole pares are generated in the $\mathrm{ZnO}$ nanoparticles, an the photon generated holes recombine with the adsorbed oxygen species. As a result, the adsorbed oxygen species are removed and release electrons to the conduction band of $\mathrm{ZnO}$.
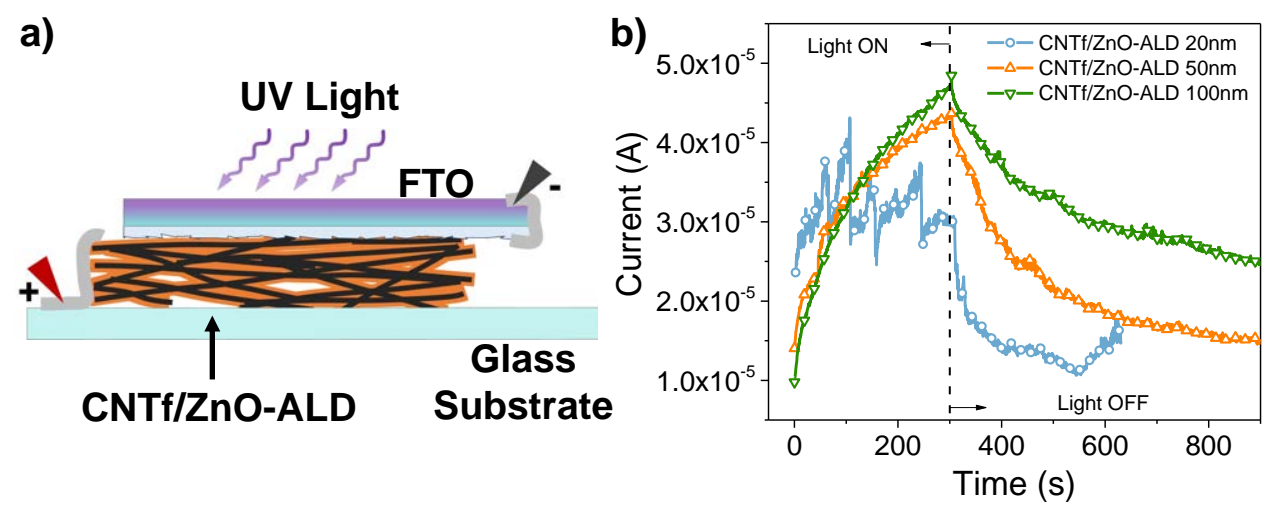

FIGURE 5.34: a) Schematic of the UV-light photocurrent contacts and measurement. b) CNTf/ZnO-ALD heterojunction UV photocurrent measurements as function of the semiconductor thickness.

Some differences can be found between the curves. Firstly, the initial currents, prior illumination, are proportional to the conductivity of the material. Thinner semiconductor coating leads to lower interfacial resistance and higher current $(I$ at $t=0$ ). Secondly, the $20 \mathrm{~nm} \mathrm{ZnO}$ hybrid shows a lower increase in the current conductivity under illumination. This suggest that the depletion region at the interface of this material is almost inexistent, an no charge accumulation is observed. With this thin semiconductor thickness, the electrons are directly transferred to the CNT forming a quasi-metallic interface, therefore, the effect of the oxygen accumulation at the surface is not causing a noticeable variation on the conduction state of the material. The third important difference between the hybrids with different $\mathrm{ZnO}$ thickness is related to the decay times of the current. This time is much sorter for the thin semiconductor coating $\left(0.0048 \mathrm{~s}^{-1}\right.$ decay rate calculated from a single exponential for $20 \mathrm{~nm})$ compared to the thicker coating $\left(0.0233 \mathrm{~s}^{-1}\right.$ decay rate for $\left.100 \mathrm{~nm}\right)$, confirmed by the intermediate value of the middle thickness coating, $0.0073 \mathrm{~s}^{-1} \mathrm{de}-$ cay rate for $50 \mathrm{~nm}$. The values are consistent with the previous reports for similar materials, where the increase in conductivity is explained by a n-type doping of the nanocomposite produced by oxygen adsorption at the surface of the material.[323]

Finally, the photoresponse of the materials (photo-to-dark current ratios) are obtained approximately as 3,3.2 and 5, for the 20,50 and $100 \mathrm{~nm}$ respectively, that are in agreement with the higher current injection provided by larger number of excited carriers. The responsivity of the materials, obtained by normalising the photocurrent $\left(J_{\text {light }}-J_{\text {dark }}\right)$ by the effective irradiation power $\left(300 \mu \mathrm{W} / \mathrm{cm}^{2}\right)$, is obtained from the curves presented in Figure 5.34b. 100, 143 and $182 \mathrm{~mA} / \mathrm{W}$ are the obtained for the 20, 50 and $100 \mathrm{~nm}$ respectively. 


\subsection{Summary and conclusion}

In this chapter, the attention is focus on the structure and electrical properties of a novel material, CNTf hybridised with $\mathrm{ZnO}$ semiconductor, that can potentially be employed for multiple applications.

In first place, micrometer-size $\mathrm{ZnO}$ layers are synthesised by a double step chemical route. We have successfully reported the Schottky barrier formation between these materials. Meanwhile, the SBH value is smaller than the expected according to the energetics of the materials. The heterojunctions are better fitted by the Cheung analisys, providing results that take into account the high resistance at the interface. With this analysis, ideality factor of 2.9, effective $\mathrm{SBH}$ of $0.26 \mathrm{eV}$ and rectifying ratio of 144 are obtained. The charge transfer properties through the interface have been successfully investigated by photocurrent measurements, leading to a responsivity of the material close to $5000 \mathrm{~mA} / \mathrm{W}$. As an alternative application, the piezorresistive behaviour of the $\mathrm{CNTf} / \mathrm{ZnO}$ heterojunction is analysed distinguishing the different contributions of piezoresistive and piezoelectric phenomena taking place under mechanical deformation on the material.

For further understanding of the CNTf hybridisation and charge transfer phenomena, alternative nanometer-size layers of $\mathrm{ZnO}$ were synthesised by atomic layer deposition (ALD), a physical method that provides higher reproducibility in terms of the chemistry and structure of the metal oxide.

$\mathrm{CNTf} / \mathrm{MO}_{x}$-ALD heterojunctions have shown the tuning of the electrical properties of the CNTf by the number of cycles or by the different $\mathrm{MO}_{x}$ deposited on it. For instance, metallic or Schottky behaviour at the interface have been demonstrated, as well as a wide range of conductivities for the hybrid material. This tunability of the properties of ALD hybrids has been explained by the combination of three factors. Firsty, the ALD number of cycles lead to very thin layers of oxide nanoparticles that partially covers the surface of the CNTf. Increasing the number of cycles also increases the coverage percentage of the surface, until finally obtaining fully conformal coatings at the surface of the CNTf. Secondly, the oxidation state of the initial nanometers of material can be slightly different to the obtained for thicker oxide layers. Partially reduced $\mathrm{Zn}_{(1-x)} \mathrm{O}$ could be formed for the initial few nanometer size coatings, probably related to the different chemical environment of the initial layers in presence of the CNTf substrate. Thirdly, different electronic structure is obtained for thin $(<20 \mathrm{~nm})$ and thick $(>20 \mathrm{~nm})$ layers, where for very thin layers, the charge depletion region is in the range of the physical dimension of the semiconductor. Moreover, the close work function of both materials suggest the low height of the formed Schottky barrier. Experimental insights for this conclusion have been found by XPS, Raman, XRD and morphological study of the hybrid material.

The combination of these factor makes the ALD 3D-bulk heterojunctions very interesting materials for different applications that combine electrochemical or photoelectronic effects.

The easy charge transfer has been demonstrated by UV-photocurrent measurements, demonstrating the tunability of the properties by the ALD number of cycles and the potential for UV-photodetectors applications. This application serves as a good example for testing the material. Moreover, the photoresponse under low power UV-light is highly improved by the high surface area accessible for this interaction, easy modifiable by the thickness of the $\mathrm{ZnO}$ layer. 



\section{Chapter 6}

\section{CNTf/MOx Photoanodes for DSSC}

There is vast literature on the use of nanocarbons in n-type DSSC,[176-179] in some cases even taking the role of the common $\mathrm{TiO}_{2} \cdot[128,324]$

Inspired by these works and by the promising results discussed in Chapter 4, in this Chapter the implementation of the CNTf in the working electrodes (photoanodes) of the n-type-DSSC is presented. Several architectures are investigated in order to study the performance of DSSC devices with CNTf in different parts of the photoanode, as shown schematically in Figure 6.1. The overall goal is the comprehension of porous nanocarbon electrodes (CNTf in this case) as part of the photoanodes, as well as to add new functionalities to the DSSC devices and boost the performance of the overall device.

Firstly, the use of the neat CNTf decorated with N719 dye molecules has been tried.

Secondly, the CNTf/MO $\mathrm{MO}_{x}$ hybrids by ALD method presented in the previous chapter are also researched as photoanodes materials for DSSC. The large heterojuctions of these materials are potentially good candidates for acting as scaffolds for the dye molecules, or as injection layers in combination with the mesoporous $\mathrm{TiO}_{2}$ layers. With the aim of understanding the effect of electronic recombination $v s$ dye injection, the CNTfs $/ \mathrm{MO}_{x}$-ALD thickness dependence is investigated also as a function of $\mathrm{TiO}_{2}$-paste thickness. This material is compared with the the CNTf/ZnONWs heterojunctions presented in the previous section. At the same time, the mechanical stability of the $\mathrm{CNTf} / \mathrm{TiO}_{2}$-commercial paste is demonstrated by reducing the cracks at the surface of the electrode.

For concluding this chapter, the role of the thickness of the CNTfs is investigated using semi-transparent CNTf as photoanode. The relation between the CNTf thickness and electron charge recombination is analysed.

The "road-map" of the anode architectures studied along this chapter, in addition to the hypothesis investigated and a schematic of every specific photoanode configuration, are included in the Figure 6.1. 


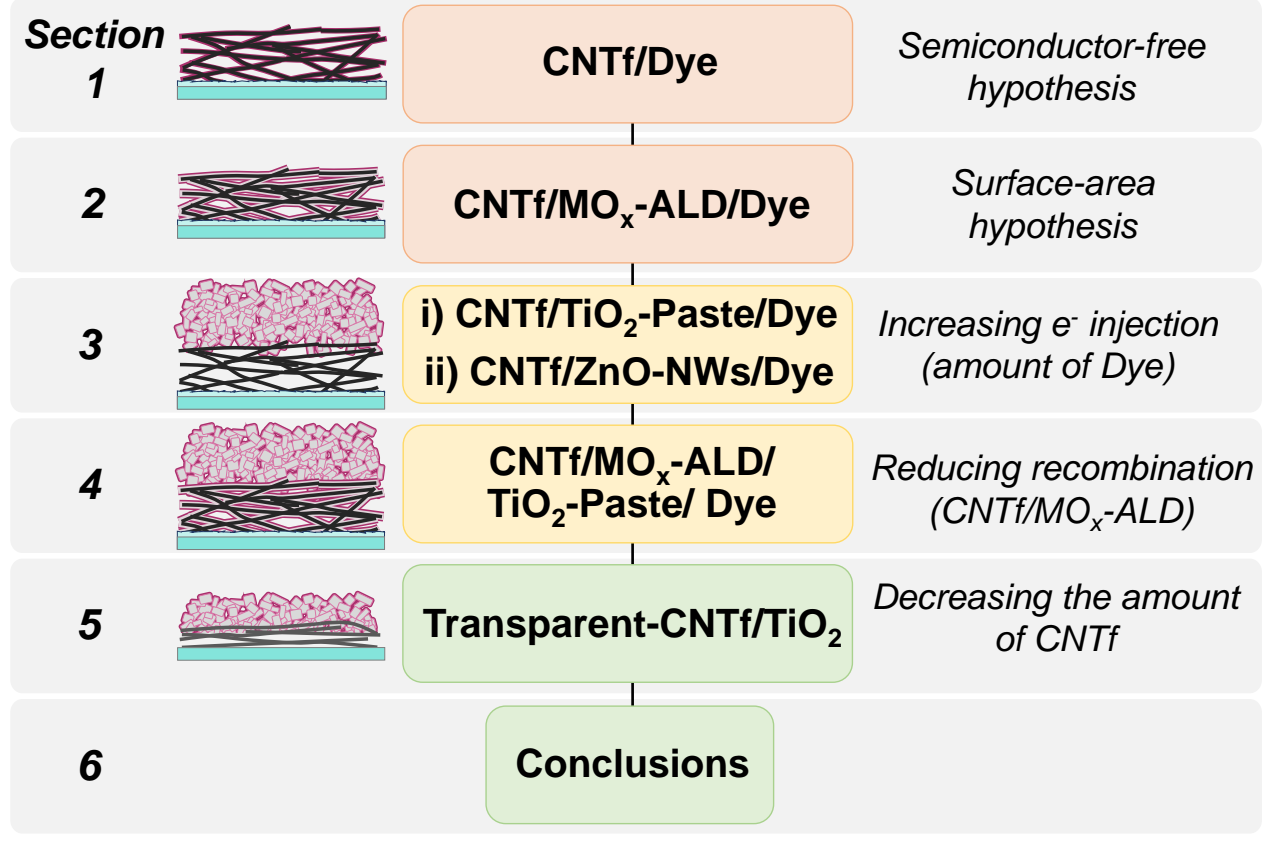

FIGURE 6.1: "Road-map" summary followed in this chapter towards CNTfs photoanodes for DSSC.

\subsection{CNTf/Dye semiconductor-free photoanodes}

Typically, the photovoltaic field has been developed around the planar technology based on the silicon solar cells. Inherited from this technology, the substrates and materials used have been always rigid materials. With the emergence of new applications as the portable electronics, wearables and highly integrated devices, new materials with new added properties are sought. In this context, the CNTf presents excellent electrical properties combined with high surface area and great mechanical properties.

The CNTf have been proposed as replacement of the $\mathrm{TiO}_{2}$ nanoparticle active layer in a DSSC in the past.[128, 324] The motivation for replacing the $\mathrm{TiO}_{2}$ with CNTf would be the faster electron transfer through the nanotubes in comparison with the metal oxide, where grain boundaries between sintered $\mathrm{TiO}_{2}$ nanoparticles decrease the mobility of the electrons in this material and at the same time increase the recombination. Finally, simpler devices architectures can be proposed by replacing the $\mathrm{MO}_{x}$ by tougher material as the CNTf.

The working principle consist in the photoexcitation of the dye molecules by visible light illumination, followed by charge injection from the lowest unoccupied orbital to the conduction band of the CNTf, where it could be rapidly extracted. This process is schematically represented in the Figure 6.2. The rest of the processes in the solar cell would work in the same way than a conventional cell: the dye molecules need to be regenerated by the redox couple mediated electrolyte, and the redox couple needs the regeneration on the surface of the CE. 
This process has been reported for CNTf that were referred to as semiconducting, but which in fact are semi-metallic and have a composition similar to that of the materials in this thesis.

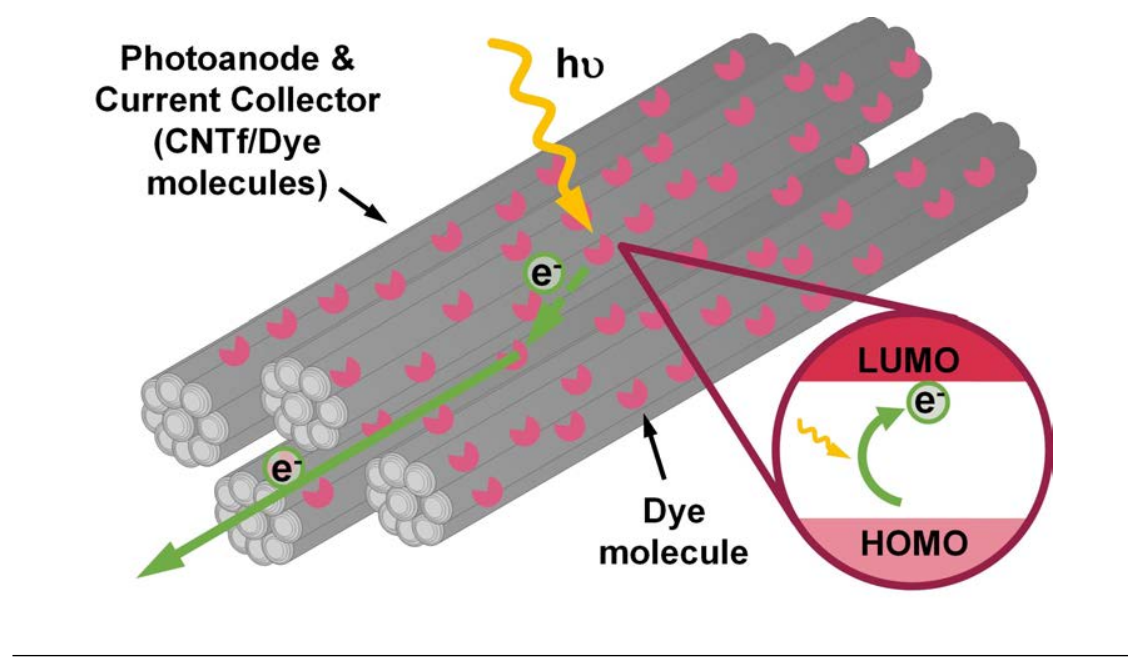

FIGURE 6.2: Schematic of a CNTf decorated with N719 dye molecules as photoanodes.

First of all, for the preparation of the active electrodes - CNTf/N719 dye loaded molecules, the pristine fibre supported on FTO substrates was immersed in a N719/ acetonitrile solution $(0.5 \mathrm{mM})$ for one day, followed by solvent evaporation. The dye adsorption is confirmed by Raman spectroscopy measurements. In the Figure 6.3 the Raman spectra of the pristine CNTf, the N719 deposited on FTO substrate and the CNTf dye loaded (CNTf/N719) is presented. In a closer look of the spectra (Figure 6.3b), the most representative bands of the dye are found together with CNTf spectra: the peaks at $454,699,1018,1467 \mathrm{~cm}^{-1}$.
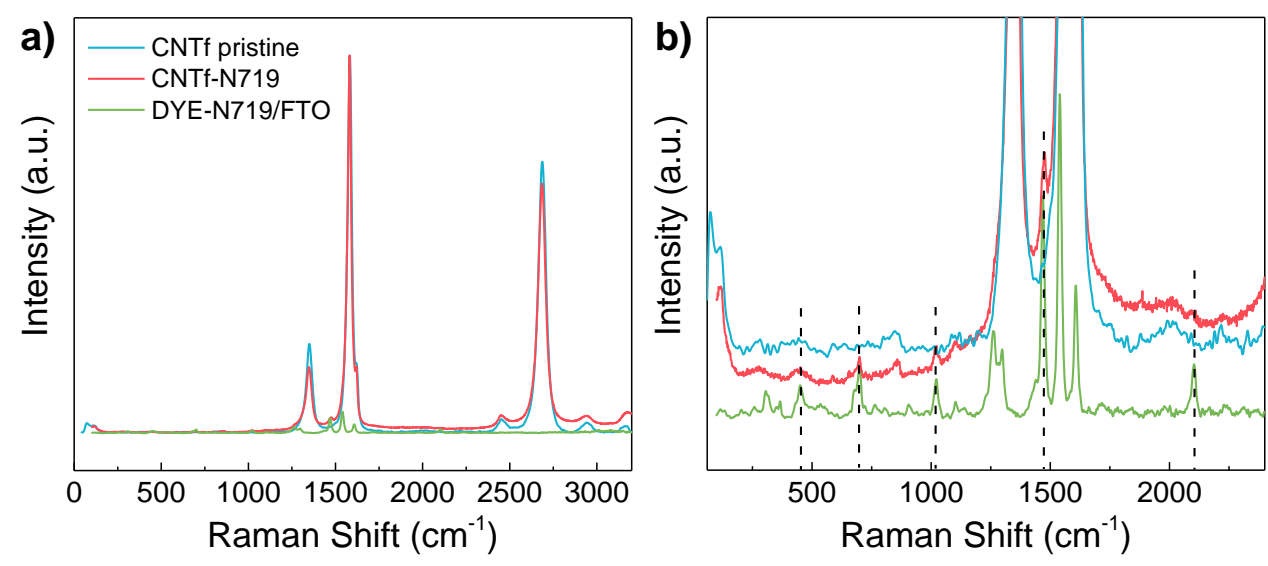

FIGURE 6.3: Raman spectra of pure dye N719 deposited on FTO substrate, pristine CNTf and CNTf decorated with N719 dye molecules.

The legend applies to all the figure.

Once the CNTf is effectively loaded with the sensitiser, a complete DSSC is assembled using $\mathrm{Pt}$ as counter electrode and an iodide-based liquid electrolyte, following the standard procedure detailed before. The schematic of the device and 
the resulting $J-V$ curves are presented in the Figure 6.4. This device configuration shows almost no current or voltage generated under illumination. This behaviour is attributed to the presence of sort-circuits inside the device. The CNTf is a flexible material and, when it is in contact with solvent electrolyte, the liquid infiltration can potentially produce swelling of the CNTf, leading to short-circuits between the CNTf and the Pt/FTO substrates. A similar problem was reported by Cai et al.[128] in fibre-like devices composed by the same material (CNTf/dye).

To avoid sort-circuits a non-electrically conductive porous membrane is used as separator between electrodes. This membrane enables ionic transport needed for the regeneration of the electrolyte.

The measured $J-V$ curves of these Pt I membrane ICNTf-N719 devices under illumination are presented in the Figure $6.4 \mathrm{~b}, \mathrm{c}$ (labelled as "membrane") and both devices present the same parameters, $V_{o c}=0.015 \mathrm{~V}$ and $J_{s c}=0.8 \mathrm{~mA} \mathrm{~cm}^{-2}$. Interestingly, similar characteristics are reported for previous reports using CNTf electrodes directly as photoandoes $\left(V_{o c}=0.025 \mathrm{~V}\right.$ and $\left.J_{s c}=0.8 \mathrm{~mA} \mathrm{~cm}^{-2}\right)$.[128]

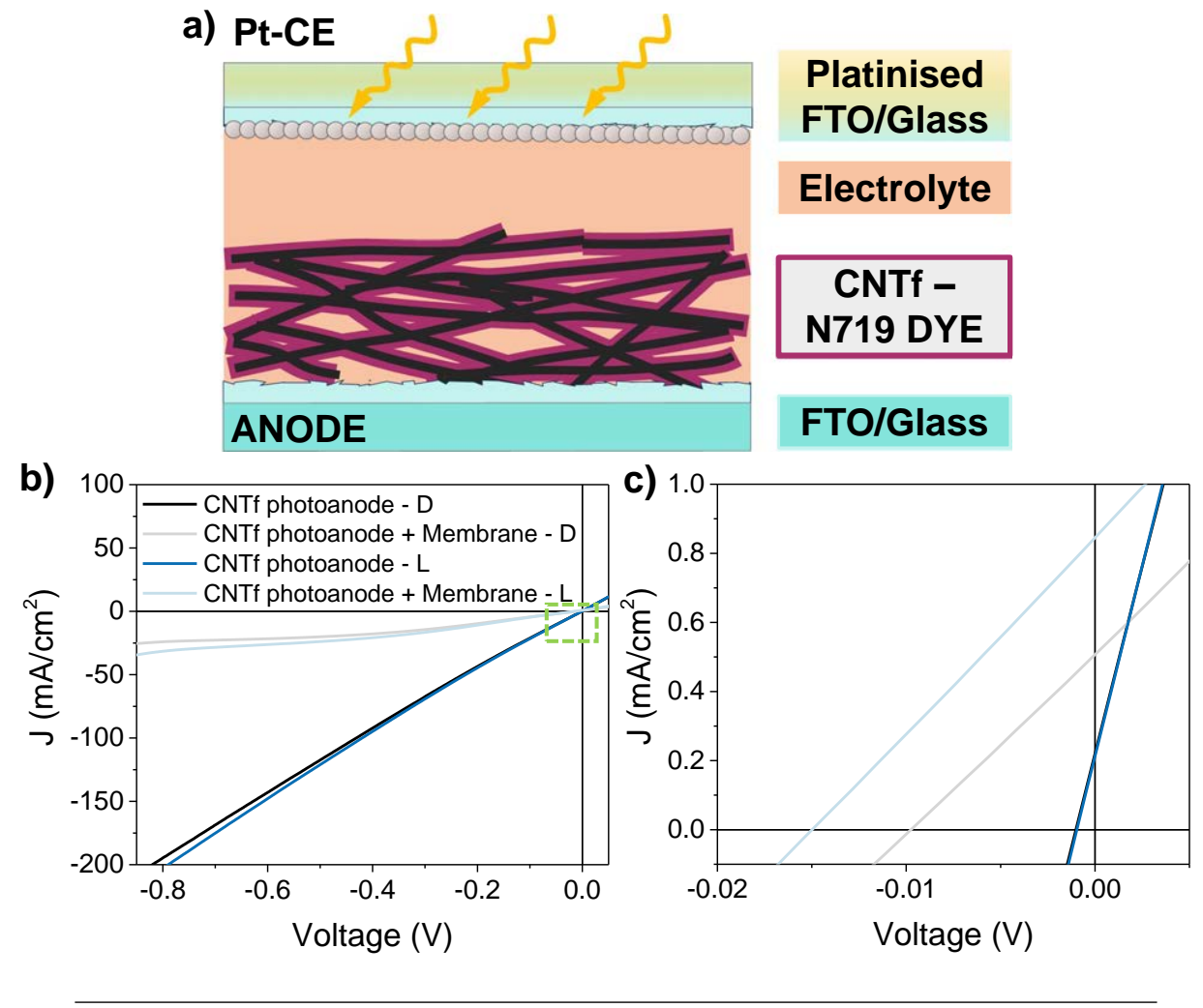

FIGURE 6.4: a) Schematic and b) $J-V$ curves of the CNTf decorated with N719 dye molecules as photoanodes for DSSC. c) Magnification of the area of interest of the $J-V$ curves. The legend of graph $b$ applies also for the graph presented in $c$.

Under bias potential, the measured dark current is partially suppressed (Figure 6.4b) compared with the CNTf without separator membrane which confirms that sorts have been suppressed, but both results show a device behaviour far from the expected diode behaviour of an operational DSSC. As it is introduced in the background section of this thesis, the slope of the dark current close to zero bias (inverse of the shunt resistance, $R_{s h}$ ) also indicates the prevention of sort-circuit in the device. 
The $R_{\text {sh }}$ for the CNTf anode is $\approx 50 \Omega$, and $85-95 \Omega$ for the CNTf-membrane, whereas for high efficient devices this value ranges $10^{4}-10^{6} \Omega$. The low $R_{s h}$ obtained in these devices is directly related to the high reverse saturation current observed (leakage current).

The high leakage current is due to the strong interaction between electrolyte and active photoanode material, undesired in the photovoltaic device. Introducing the insulating membrane decreases $R_{s h}$ but still with a huge leakage current, hindering the proper working of the device.

Previous studies have shown that a low shunt resistance (high leakage current) can be induced by photoelectron recombination, which leads to decreases in $J_{s c}, V_{o c}$ and especially in FF.[325] Typical solution for reducing recombination mechanism is by passivising the metallic materials that compose the working electrode of the device. This would suggest that the low FF observed in Figure 6.4c is due to recombination at the photoanode, which can to some extent be expected based on the highly efficient recombination observed in CNTf-CEs (Chapter 4 "CNTf CounterElectrodes for DSSC").

Note also that, for a $10 \mu \mathrm{m}$ thick CNTf photoanode, light penetration through the black CNTf is limited to the initial couple of micrometers from the surface, therefore, electron injection is limited only to the top surface of the material. This would lead to lower carrier injection due to the limited illuminated volume of the electrode in comparison to a standard $\mathrm{TiO}_{2}$ electrode. In contrast, the electrolyte is free to recombine through the hole thickness $(7$ to $10 \mu \mathrm{m}$ ) of the porous photoanode.

In conclusion, the $J-V$ curves of these devices show the ineffective configuration produce CNTf/dye working electrodes. The high recombination mechanism, possibly in combination with the low carrier injection due to the limited illuminated volume of the electrode, hampers the operation of the device. A typical solution for recombination issues at the surface of metallic surface is the passivisation of the surface. Passivasing the CNTf electrode by $\mathrm{MO}_{x}$-ALD could lead to lower recombination effect and higher injection by adsorbed dye molecules.

\subsection{CNTf scaffolds: high surface area photoanodes by ALD}

Large-area heterojunctions by physical or chemical methods studied in the previous Chapter 5 "Fundamental Properties of CNTf/MOx Heterojunctions" can be potentially exploited as photoanodes for DSSC applications.

The initial hypothesis for using these materials as photoanodes for photovoltaic applications is based on the high surface that can be created between the current-collector and the $\mathrm{MO}_{x}$ in a small area. Thanks to the high surface area of the CNTf, as high as $250 \mathrm{~m}^{2} / \mathrm{g}$,[225] current-collector/ $\mathrm{MO}_{x}$ interfaces 3 orders of magnitude higher than conventional electrodes can be obtained. The hierarchised structure of the CNTf is responsible of the high surface area of this electrodes. The author's representation of the $\mathrm{CNTf} / \mathrm{MO}_{x}$ hybrid material is presented in the Figure 6.5. Notice that by ALD method every individual bundle that forms a single CNTf is covered by a conformal coating of $\mathrm{MO}_{x}$ leading to a large CNTf $/ \mathrm{MO}_{x}$ interface. 


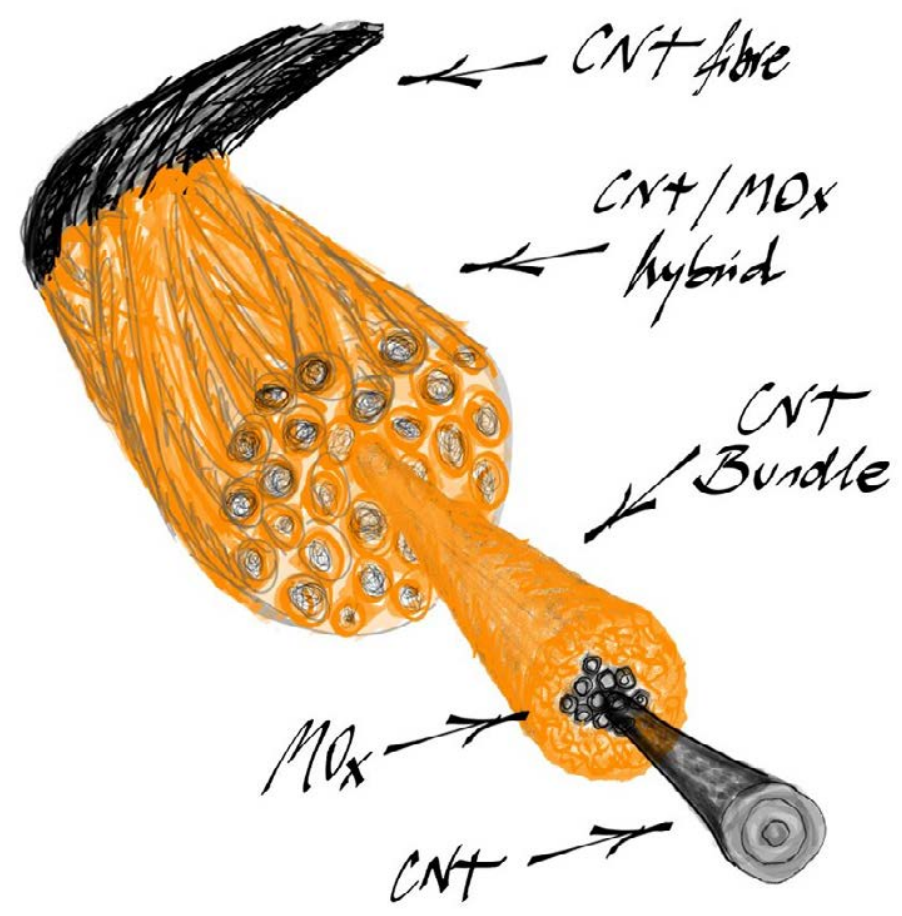

FIGURE 6.5: Author's representation of the CNTf/MO $\mathrm{M}_{x}$ hybrid material leading to a large interface of both continuous phases. The $\mathrm{MO}_{x}$ conformal coating covers every CNT bundles remaining the CNTs as an internal current-collector.

A small portion of the $\mathrm{CNTf} / \mathrm{MO}_{X}$-ALD material (several interconnected CNT bundles) is schematically depicted in the Figure 6.6a. The CNTf acts as a scaffold for a sub-micrometer layer of semiconductor and at the same time as currentcollector, this would lead to lower recombination because, firstly, the electrolyte would has less time to recombine at the $\mathrm{TiO}_{2}$ surface, and secondly, the charges would find lower path to reach the current-collector and less grain boundaries for getting trapped and recombine (lower $\left.R_{\operatorname{dif} f\left(e^{-}\right)}\right)$. Diminishing the diffusion length of the charges inside the semiconductor in an extended large heterojunction is where this material $\left(\mathrm{CNTf} / \mathrm{TiO}_{2}-\mathrm{ALD}\right)$ could represent an advantage against the conventional DSSC structure $\left(\mathrm{FTO} / \mathrm{TiO}_{2}\right)$.

A schematic representing an individual CNT bundle conformally coated with $\mathrm{MO}_{x}$, showing the charge injection and extraction processes, is presented in the Figure 6.6b. The dye molecule excitation and electron injection into the $\mathrm{MO}_{x}$ are depicted. The electron has to diffuse through the $\mathrm{MO}_{x}$ layer, typically tens or hundreds of nanometres thick (depicted in the schematic with a dashed line). The electron, after diffusing through the semiconductor thickness, is extracted by the internal CNTf scaffold acting as current-collector of the photogenerated carriers. This also takes advantage of the high electrical conductivity of the CNTf. Note that whereas the electrical conductivity of $\mathrm{TiO}_{2}$ is around $10-30 \mathrm{~S} / \mathrm{cm}$, the longitudinal conductivity of the CNTf is around 500-1000 S/ $\mathrm{cm}$ (as shown in the Chapter 4).

With respect to the morphology of these hybrids photoanodes, the CNTf $/ \mathrm{MO}_{x}$ interface remains fixed to the $250 \mathrm{~m}^{2} / \mathrm{g}$ [225] of the CNTf, whereas the amount of adsorbed dye is proportional to the exposed area of $\mathrm{MO}_{x}$. In conventional porous $\mathrm{TiO}_{2}$ electrodes, the $S S A$ is around $100 \mathrm{~m}^{2} / \mathrm{g}$.[326] 

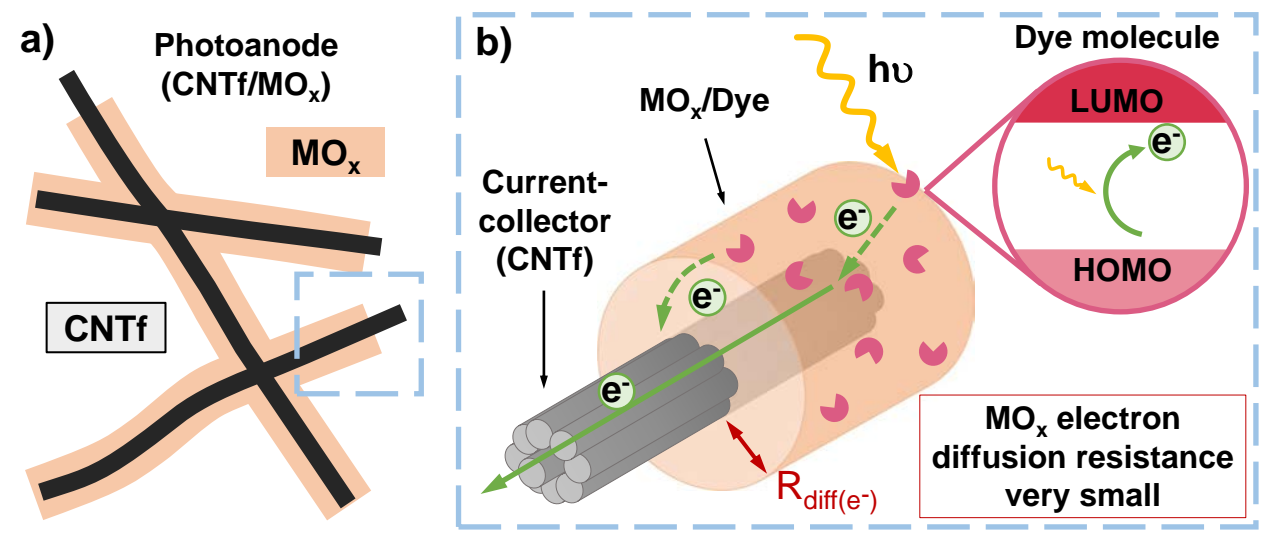

FIGURE 6.6: Sketches of a) the CNTf bundles interconnected inside the $\mathrm{MO}_{x}$ material. b) CNTf bundle conformally-coated by ALD method with $\mathrm{MO}_{x}\left(\mathrm{TiO}_{2}\right.$ or $\left.\mathrm{ZnO}\right)$, working as a photoanode for DSSC. The $\mathrm{MO}_{x}$ is decorated with dye molecules and the charge extraction is produced through the CNTf current-collector.

A first set of samples were produced in which the hybrids were used directly after ALD deposition. In these samples the crystal structure of the $\mathrm{TiO}_{2}$ layer is amorphous, concluded by TEM electron diffraction. The conformal growth of the $\mathrm{TiO}_{2}$ around the CNTf-bundles was further confirmed by this technique (see Figure 6.7a). At high-resolution TEM the morphology structure does not reflect any periodicity (Figure 6.7b). No diffraction spots or rings are observed in the 2D-XRD micrograph confirming the amorphous structure of the hybrid $\mathrm{CNTf} / \mathrm{TiO}_{2}$ (see Figure 6.7c). In the same HR-TEM image, an individual CNT can be observed coming out from the broken fibre cross-section. Besides, by EDX mapping, the composition of the materials is confirmed titanium oxide as expected (see inset Figure 6.7a and compositional graph in $d$ ).

A set of devices were prepared in order to measure the performance when nanometre size $\mathrm{TiO}_{2}$ is used as active anode material. A layer of $\mathrm{TiO}_{2}-\mathrm{ALD} 100 \mathrm{~nm}$ thick was deposited following the procedure described in the Chapter 5 "Fundamental Properties of CNTf/MOx Heterojunctions".

$\mathrm{CNTf} / \mathrm{TiO}_{2}$-ALD hybrids were used directly as DSSC photoanodes, and a schematic of this devices is presented in the Figure 6.8a. The devices were measured under simulated AM 1.5G sun light and fabricated according to the standard procedure.

The photoanodes were place in the dye solution overnight in order to get loaded with the N719 dye molecules. The electrodes after 16 hours in the dye solution are presented in the Figure 6.8b. Compared to the conventional 10-20 $\mu \mathrm{m}$ thick photoanodes, presented in Chapter 4 "CNTf Counter-Electrodes for DSSC", the colour of these electrodes after the dyeing step is not the characteristic red intense colour of a high dye loaded electrode. This is indicative of a very small amount of dye adsorbed.

A reference device was fabricated with the $100 \mathrm{~nm} \mathrm{TiO}_{2}$-ALD material and four devices were fabricated for the $\mathrm{CNTf} / \mathrm{TiO}_{2}-\mathrm{ALD}$. Two representative devices are shown in the Figure 6.8c, besides, an electron microscopy image of the surface of the CNTf coated with the $\mathrm{TiO}_{2}$ by ALD is presented in the Figure 6.8d. 
The obtained $J-V$ curves are presented in the Figure 6.8e. All cells present the same behaviour, with no significant positive current generated under illumination. In the vicinity to zero bias, the $\mathrm{CNTf} / \mathrm{TiO}_{2}-\mathrm{ALD}$ devices, present similar characteristics to the device presented in the previous section without the semiconductor deposited. The measured $R_{s h}$ for the reference device is in the range of $3.3 \mathrm{M} \Omega$, while for the hybrid photoanodes it ranges 30-60 $\Omega$.
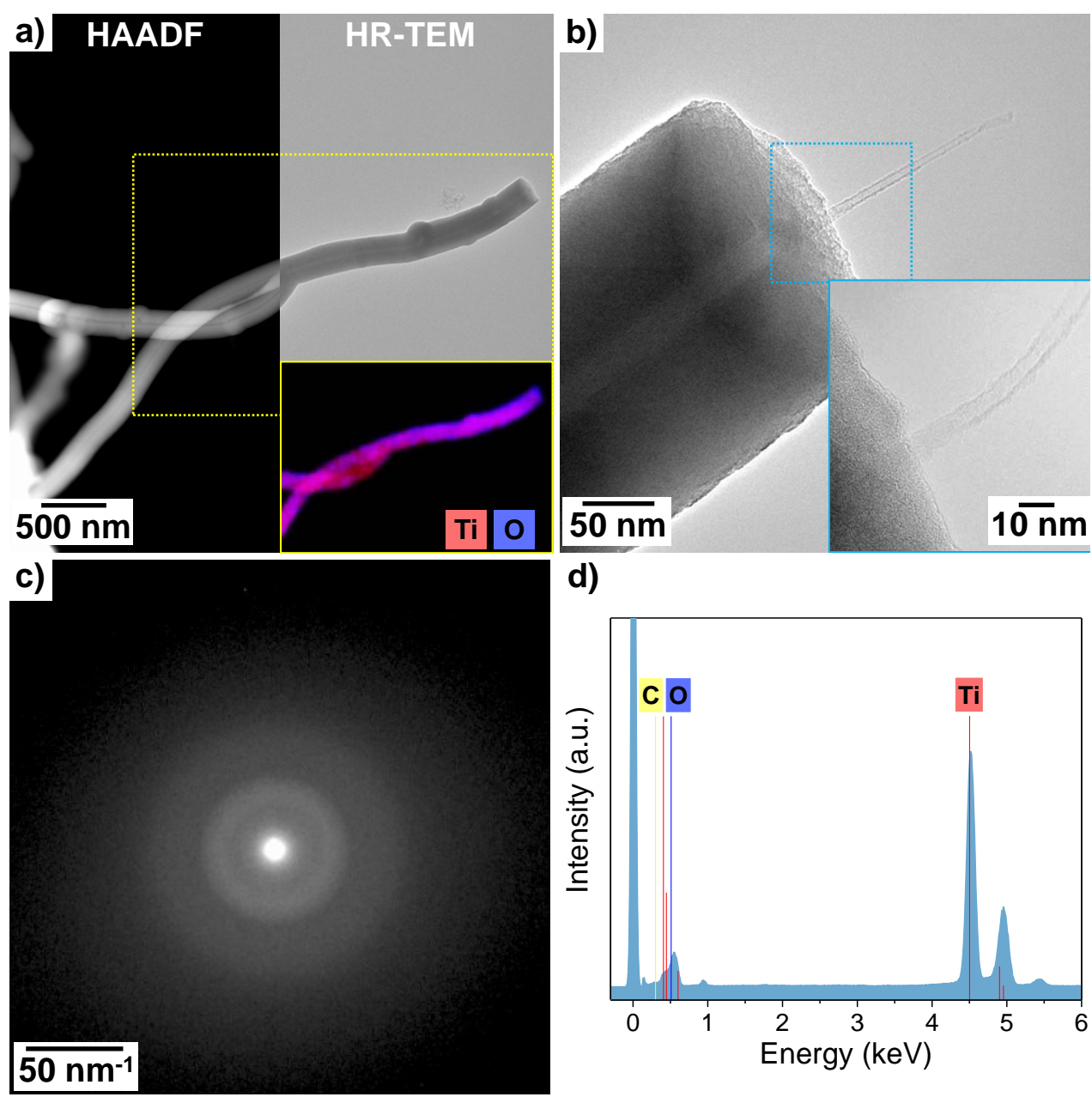

FIGURE 6.7: As-made $\mathrm{CNTf} / \mathrm{TiO}_{2}$-ALD analysed by a) HR-TEM imaging in conventional mode and HAADF, inset: EDX-mapping. b) HR-TEM at the interface of the CNTs and the $\mathrm{TiO}_{2}$. c) 2D-XRD pattern obtained by TEM. d) Compositional graph by EDX technique.

There are two possible reasons that explain why these devices are not working. On the one hand, there are no photogenerated charges in the device; on the other hand, there are no extracted charges due to recombination at the photoanode.

First of all, there has to be confirmed that the electrode is loaded with the N719-dye. For that, Raman spectroscopy of the electrode in combination with UVVIS spectroscopy is measured. The results are presented in the Figure 6.9.

By UV-VIS technique, the absorbance of solution after the desorption of the dye is analysed. For analysing the desorption of the dye, two dye-loaded electrodes, one coated with standard $\mathrm{TiO}_{2}$-commercial paste and other of CNTf coated with 
$\mathrm{TiO}_{2}-\mathrm{ALD}$, are placed during 24 hours in a vial with a solution of $\mathrm{NaOH}: \mathrm{H}_{2} \mathrm{O} 0.1 \mathrm{M}$. The action of the basic solution produced the desorption of the dye from the $\mathrm{TiO}_{2}$ surface. This method is well known and typically used with for this purpose, not only with $\mathrm{TiO}_{2}$ layers from commercial paste [327] but also, with $\mathrm{MO}_{x}$ produced by ALD.[328, 329]

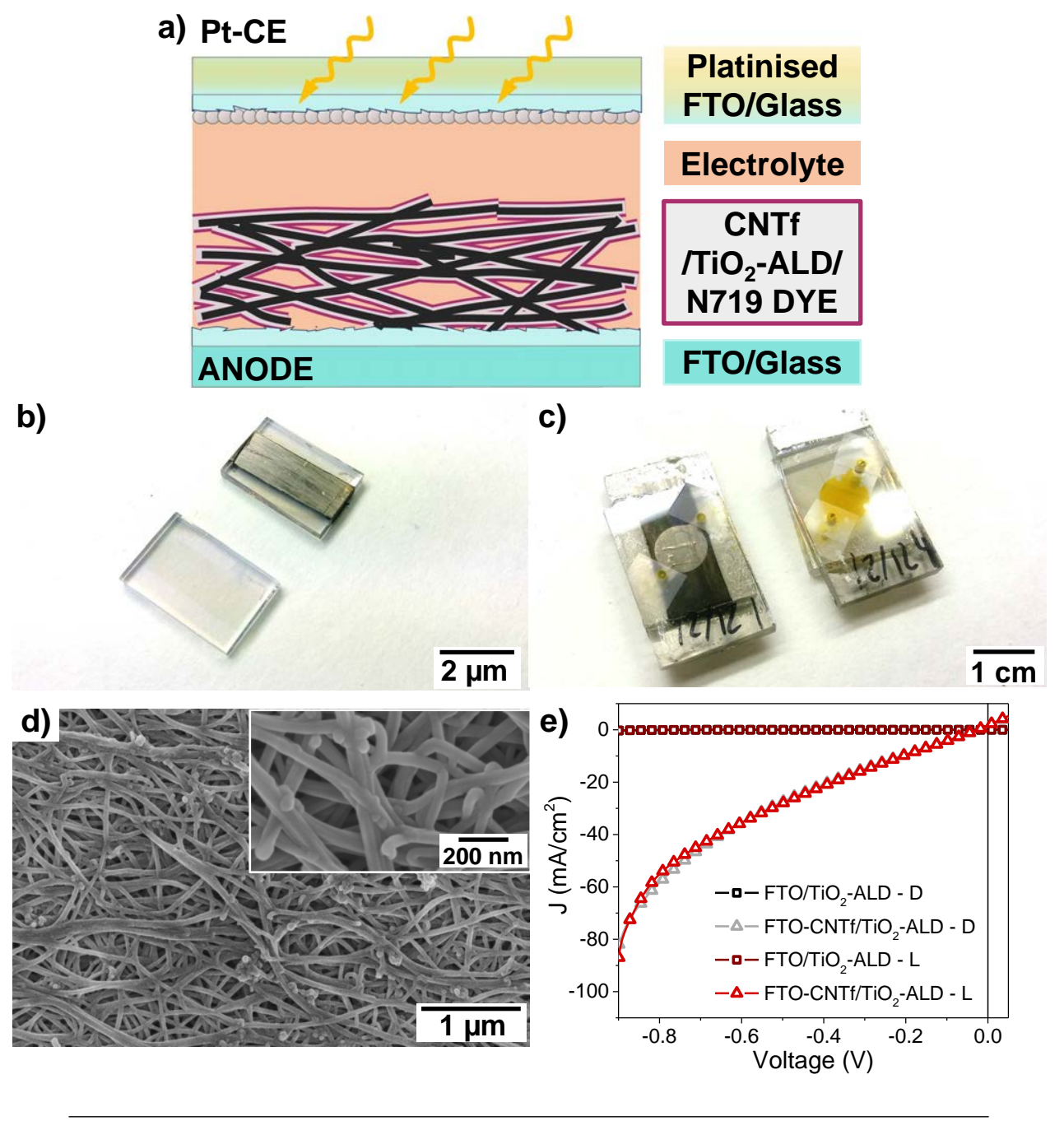

FIGURE 6.8: a) Schematic of DSSC based on CNTf/TiO 2 -ALD photoanodes. b) FTO and CNTf substrates coated with $100 \mathrm{~nm} \mathrm{TiO}_{2}$ by ALD. c) DSSC devices (CNTf and FTO as reference) assembled with the ALD deposited electrodes. d) SEM image of the CNTf substrate deposited with $\mathrm{TiO}_{2}$ where the conformallity of the layer can be observed. e) $J-V$ curves of the $\mathrm{CNTf} / \mathrm{TiO}_{2}$-ALD conformally-coated as photoanodes for DSSC.

In the Figure 6.9a is presented the desorption process before and after 24 hours. The UV-Vis absorbance spectra of $1 \mathrm{~mL}$ of the solution, after the desorption process, is measured for both electrodes, and the resulting spectra are presented in the Figure $6.9 \mathrm{~b}$. The absorbance spectrum obtained for the $\mathrm{CNTf} / \mathrm{TiO}_{2}-\mathrm{ALD}$ is almost identical to the pristine $\mathrm{NaOH}$ solution. On the contrary, the solution from electrode coated with commercial paste presents the absorbance spectrum typical of the N719 dye. 
For corroborating the lack of dye molecules on the surface of $\mathrm{CNTf} / \mathrm{TiO}_{2}-\mathrm{ALD}$, the electrodes were also analysed by Raman spectroscopy before the desorption process. The Raman spectra of both electrodes compared with the N719-dye spectrum are presented in the Figure 6.9c. The characteristic signals of the CNT ( $D$ and $G$ bands) are present, as well as the anatase $\mathrm{TiO}_{2}$ signals for the $\mathrm{CNTf} / \mathrm{TiO}_{2}-\mathrm{ALD}$ electrode, but none of the peaks associated to the N719 are found in this spectrum (see the magnified region of the spectra in Figure $6.9 \mathrm{~d}$ ). We can conclude that no dye is present in the $\mathrm{CNTf} / \mathrm{TiO}_{2}$-ALD samples and therefore, no photocurrent is generated.
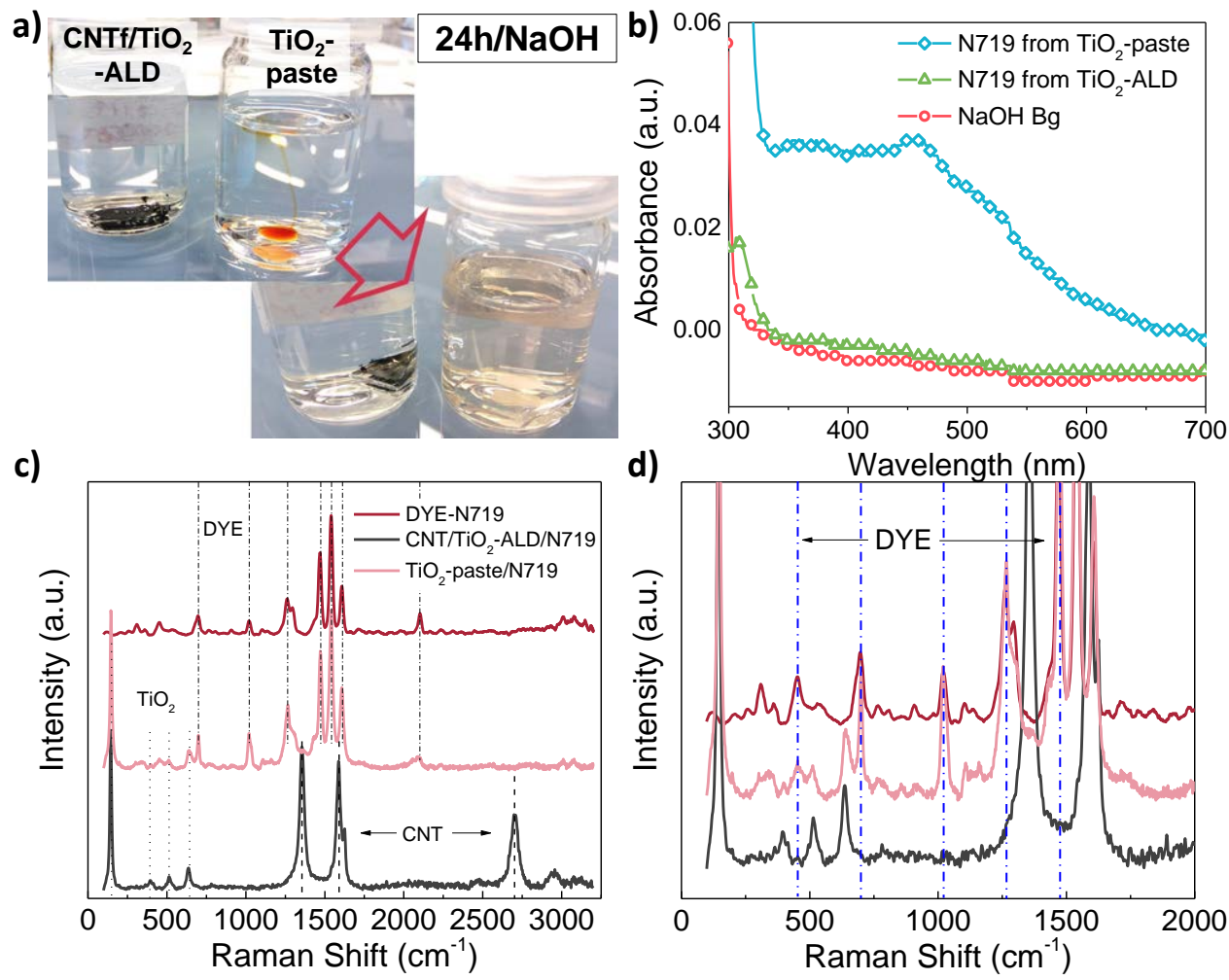

FIGURE 6.9: a) Dye desorption with $\mathrm{NaOH}$ solution from $\mathrm{TiO}_{2}$ commercial paste and $\mathrm{CNTf} / \mathrm{TiO}_{2}$-ALD. b) Absorbance spectra for the $\mathrm{NaOH}$ solutions after desorption. c) Raman spectra of the same electrodes before desorption compared with the pure N719 dye spectrum and d) magnified region of the spectra. The legend of graph $c$ applies also for the graph presented in $d$.

In view of these results a crystallisation protocol was established. The recrystallisation treatment consisted in keeping the ALD samples at $400{ }^{\circ} \mathrm{C}$ for 1 hour in a $\mathrm{N}_{2}$ (Ar) atmosphere.[295] The inert gas atmosphere is important to avoid degradation of the CNTf sample, which in the presence of a $\mathrm{MO}_{x}$ can occur at temperatures as low as $350^{\circ} \mathrm{C}$.

The TEM images and 2D-XRD pattern for recrystallised samples are presented in the Figure 6.10. After the recrystallisation process the CNTf preserves its macroscopic structure, as it is shown in the Figure 6.10a. At higher magnification, crystalline planes are observed along all the semiconductor coating (see Figure 6.10b). In contrast to the non-recrystallysed material, the 2D-pattern presents crystalline diffraction spots characteristics of the $\mathrm{TiO}_{2}$, confirming the success of the treatment. 
In the Z-contrast image, the internal channels produced by the CNTf-bundles inside the conformal $\mathrm{TiO}_{2}$ are still observed, indicating that the heat treatment does not modify the initial morphology of the sample (Figure 6.10d).
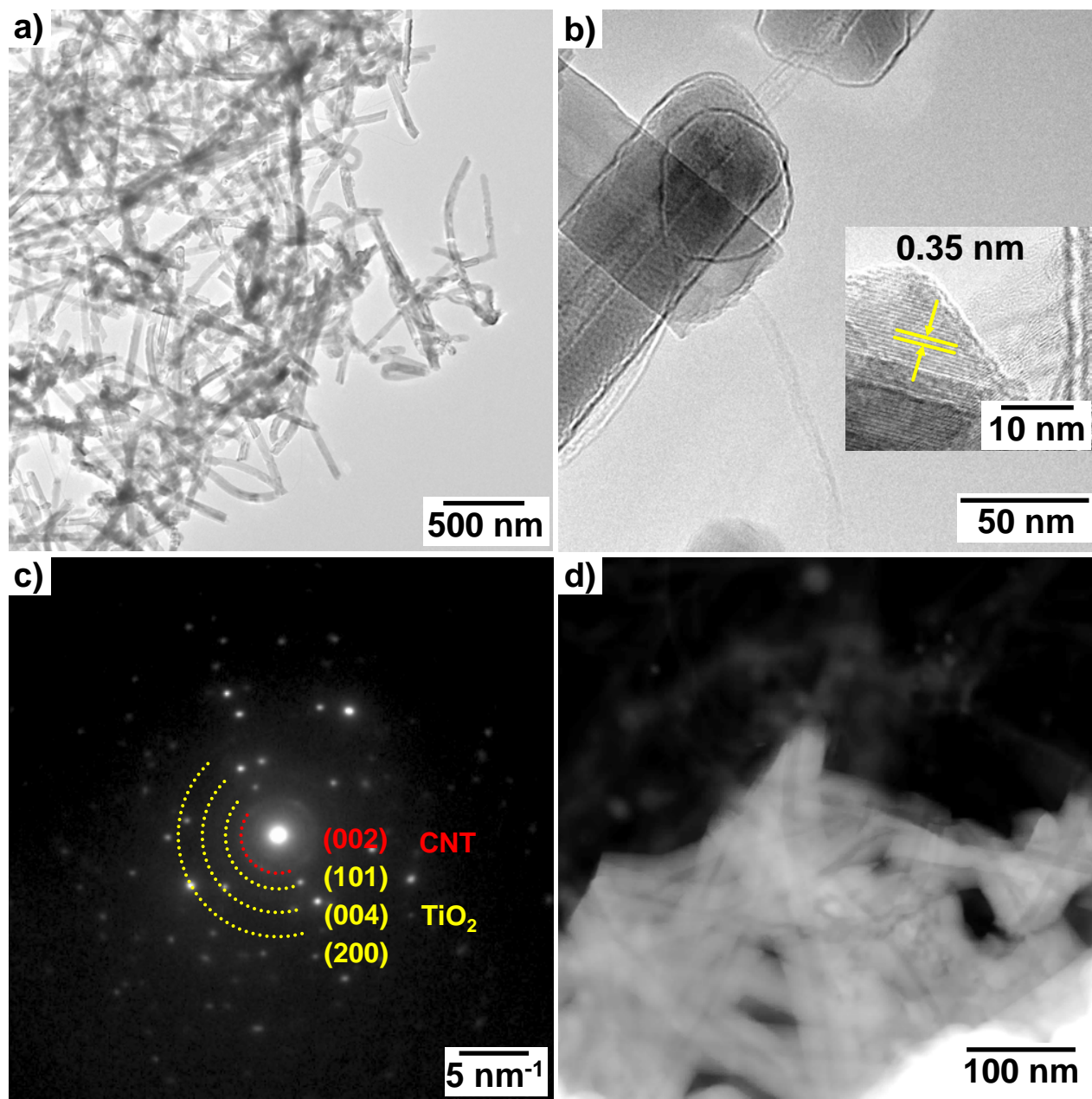

FIGURE 6.10: Recrystallised CNTf/ $\mathrm{TiO}_{2}$-ALD analysed by a,b) HRTEM imaging (images a-b courtesy of Alicia Moya). c) 2D-XRD pattern obtained by TEM. d) Z-contrast TEM image.

For an effective dye loading of the semiconductor, preferential crystallographic facets of the anatase crystal structure must be exposed from the surfaces.[330, 331] In this line of work, a lot of efforts have been done by the scientific community trying to optimise the synthesis routes and maximising the exposure of different crystallographic planes.[332] There are also other important factors for exposing preferential crystallographics planes of the $\mathrm{TiO}_{2}$, for instance, it retards the recombination process between dye and $\mathrm{TiO}_{2}$. This is due to an improvement in the electron transfer between the dye and (001)-facets.[333] Also, the light scattering inside the cell, and therefore the photogenerated charge, is improved for this preferential orientation.[334]

The most typical material used for DSSC is $\mathrm{TiO}_{2}$ anatase phase. It has been reported before the dominant (101) facet of this structure, being the $94 \%$ of the exposed surface area.[332] Other facets, as the (001) with higher surface energy, have been presented as candidates for higher dye loading by their strong ability to absorb 
organic groups of the dye molecules, possibly for surface energy minimisation reasons. Therefore, engineering the surface by recrystallisation of the $\mathrm{TiO}_{2}$ must lead to improved performance of DSSC devices.

After recrystallising the $\mathrm{TiO}_{2}$ produced by ALD, the adsorption of dye at the surface of the semiconductor has increased, as confirmed by Raman spectroscopy and quantitatively by UV-VIS (see Figure 6.11).

For obtaining a quantitative value of the dye loading, the variation of concentration of the initial solution is a reliable approach.[210] The method and calculation are detailed in the Chapter 3 "Experimental Techniques".

Briefly, the amount of N719 at equilibrium in the $\mathrm{TiO}_{2}$ films is calculated using the mass balance equation 3.1. Besides, for low concentrations, the UV-Vis absorbance follows Beer-Lambert law (equation 3.2), where the absorbance $(A)$ is proportional to the concentration $\left(C\left[g L^{-1}\right]\right)$ of attenuating species in the solution. The Figure 6.11 shows the absorption spectra of N719 before and after 24 hours of dye loading of the $\mathrm{TiO}_{2}$-ALD electrodes with and without CNTf. These results confirm the adsorption of the dye at the recrystallised surface of the $\mathrm{TiO}_{2}$ in presence of the CNTf.

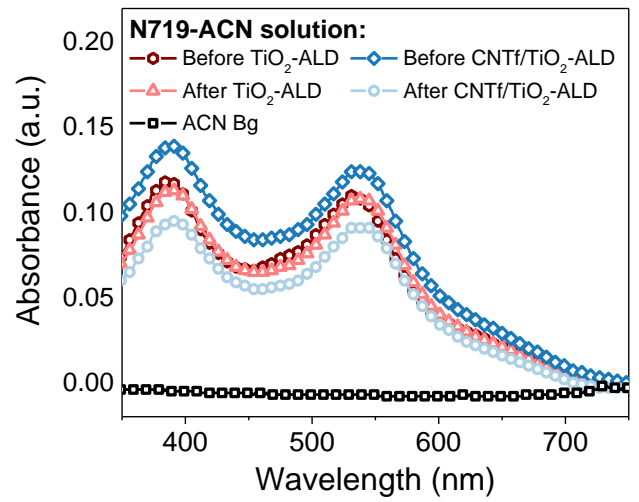

FIGURE 6.11: Absorption spectra for N719 solution before and after dye loading of the electrodes.

After demonstrating the dye loading at the recrystallised surface of $\mathrm{TiO}_{2}$ electrodes, more devices were prepared with this material and the configuration described in the Figure 6.8. Two CNTf/ $\mathrm{TiO}_{2}$-ALD DSSC devices are compared with one reference device produced by the same ALD technique (Figure 6.12).

The dark current in the devices fabricated with CNTf is 100 times higher than the reference and no positive current is obtained under illumination. The shunt resistances of these devices are compared with those of devices before recrystallisation treatment (Table 6.1). 2-4 times higher shunt resistance is obtained for the recrystallised $\mathrm{TiO}_{2}$-ALD layers. The increase in the dark current, compared to the reference device, is related to the high conductivity of the CNTf electrode. The current density is 2 times lower than the devices before recrystallisation. This is attributed to an increase of the shunt resistance of the $\mathrm{CNTf} / \mathrm{TiO}_{2}-\mathrm{ALD}$ during the recrystallisation treatment. Moreover, the onset of the dark current occurs at a lower forward bias compared to the reference device. As mentioned before, the shunt resistance 
decreases when the current follows alternative paths or reactions to the desired $\mathrm{TiO}_{2}$ conduction, for instance by recombination sites at the anode.

A closer look of the reference $\mathrm{TiO}_{2}$-ALD DSSC is presented in Figure 6.12b showing photogenerated current. $J_{s c}$ and $V_{o c}$ are quite low due to the small amount of $\mathrm{TiO}_{2}$ material and N719 dye molecules. The latter confirms that the amount of dye or the carrier injection, although is not very high, is measurable for ALD layers. In contrast, when the CNTf current-collector is used, this charge is not extracted.
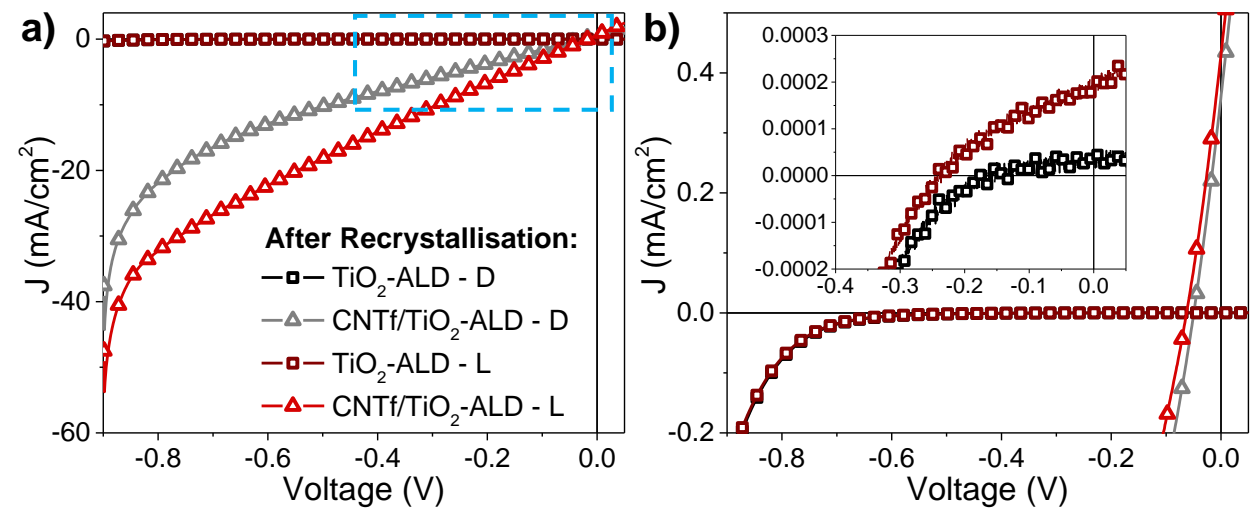

FIGURE 6.12: a) $J-V$ curves of recrystallised DSSC devices prepared with $\mathrm{FTO} / \mathrm{TiO}_{2}-\mathrm{ALD}$ and $\mathrm{CNTf} / \mathrm{TiO}_{2}$-ALD photoanodes. b) Magnification of the selected region with a blue dashed line square. Inset: $\mathrm{TiO}_{2}$ reference cell. The legend of graph $a$ applies also for the graph presented in $b$.

TABLE 6.1: Shunt resistance comparison before and after $\mathrm{TiO}_{2}$-ALD recrystallisation treatment.

\begin{tabular}{c|cc}
$R_{s h}\left(\Omega \mathrm{cm}^{2}\right)$ & $\mathrm{FTO} / \mathrm{TiO}_{2}-\mathrm{ALD}$ & $\mathrm{CNTf} / \mathrm{TiO}_{2}-\mathrm{ALD}$ \\
\hline Before recrystallisation & $3.3 \times 10^{6}$ & 60 \\
After recrystallisation & $6.6 \times 10^{6}$ & 234
\end{tabular}

The results presented in the Figure 6.12 allow to conclude that $\mathrm{CNTf} / \mathrm{TiO}_{2}$ ALD is not an efficient configuration for light harvesting. As it has been shown before, the output generated current is the balance between the injected number of electrons by the dye molecules minus the electrons lost by recombination mechanism. The carriers injected when using ALD layers are not extracted from the CNTf electrode. Under this configuration, the photogenerated carriers by the dye molecules attached to the $\mathrm{TiO}_{2}$ surface of the material produced by ALD are not enough to compete with the recombination process.

A priori, this large recombination could occur at the $\mathrm{TiO}_{2} /$ electrolyte interface or at the CNTf/electrolyte interface in regions of the photoanode where the CNTs are not covered by $\mathrm{TiO}_{2}$.

Following these observations, the structure of $\mathrm{CNTf} / \mathrm{MO}_{x}$ samples was analysed in more detail. Note that this samples produced by ALD are a new type of hybrid recently developed by the research group[199] and thus their structure is still under study and further optimisation. 
Indeed, close inspection of typical $\approx 10 \mu m$-thick $\mathrm{CNTf} / \mathrm{MO}_{x}$-ALD used as photoanodes show that the $\mathrm{MO}_{x}$ layer does not cover all the thickness of the electrode, which occurs for both $\mathrm{ZnO}$ and $\mathrm{TiO}_{2}$ and most likely other oxides.

The observation of $\mathrm{CNTf} / \mathrm{TiO}_{2}$-ALD FIB milled cross-section (Figure 6.13) confirms an inhomogeneous coating through the CNTf thickness. A compact layer of $\mathrm{TiO}_{2}$ is observed at the surface of the CNTf electrode depicted in the Figure $6.13 \mathrm{~b}$ at higher magnification. However, directly discriminating the $\mathrm{CNTf}$ and $\mathrm{TiO}_{2}$ phases is challenging.
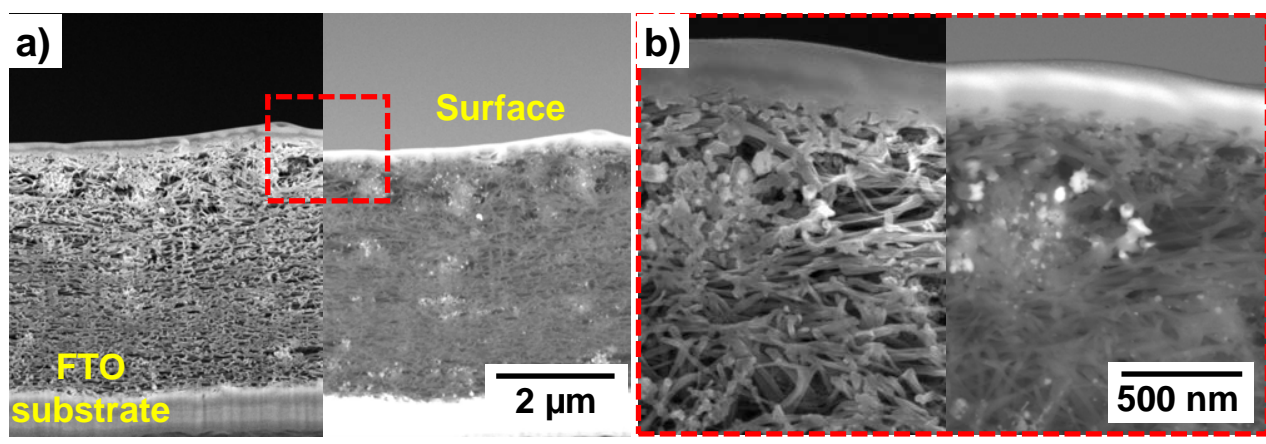

FIGURE 6.13: SEM images of a) $\mathrm{CNTf} / \mathrm{TiO}_{2}$-ALD hybrid crosssection. b) Magnified area of the upper surface marked with a red square. Images were taken in secondary electrons (left) and backscattered electrons modes (right).

Similar analysis for the ZnO-ALD hybrid also show that the top surface has a higher content of the $\mathrm{ZnO}$ coating layer (Figure 6.14). The cross-section of the CNTf/ZnO-ALD hybrid was analysed by SEM imaging and EDX compositional line-scans along the cross section. These results are presented in the Figure 6.14b,c.

Note that $\mathrm{Zn}$ has a higher atomic number and thus produces higher EDX intensity that leads to a more accurate spatial map. It is clearly observed in the compositional line-scan (Figure 6.14b) that the content of $\mathrm{Zn}$ and $\mathrm{O}$ decrease as function of depth. In fact, it is mainly localised in the initial 2-3 microns in dept from the surface. This result is in agreement with the samples presented in the previous chapter, Figure 5.19e,f, where the CNTf seems fully coated for $1 \mu \mathrm{m}$ CNTf thick. The FTO substrate signal (mainly Sn) is also clearly observed in the scan. The rest of the metal traces are derived from the CNTf synthesis process with no interest for the present discussion.

Finally, three regions were selected from the cross-section for higher magnification observation. The regions highlighted with coloured squares in the Figure 6.14a are presented as high magnification SEM Z-contrast images in the Figure $6.14 \mathrm{c}$. The $\mathrm{ZnO}$ layer can be observed as a brighter material conformally coating the CNTf in the upper part of the cross-section.

The evidences shown before suggest that partial exposure of the CNTf might be the source of the large leakage current and low efficiency observed.

This was further studied by producing photoanodes with standard $\mathrm{TiO}_{2}$ paste, instead of ALD-grown, which therefore lead to the same charge generation and injection dynamics as in a standard photoanode. 

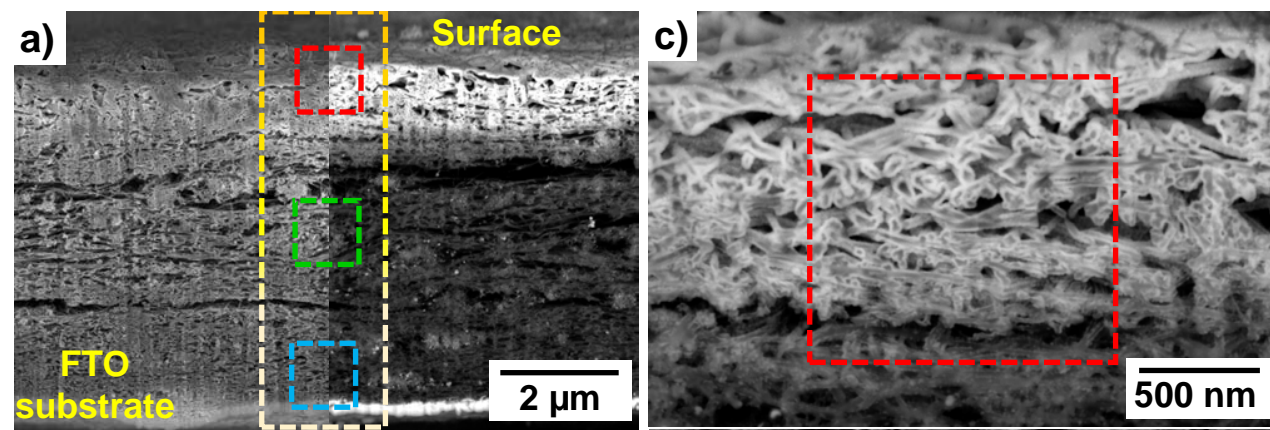

b)
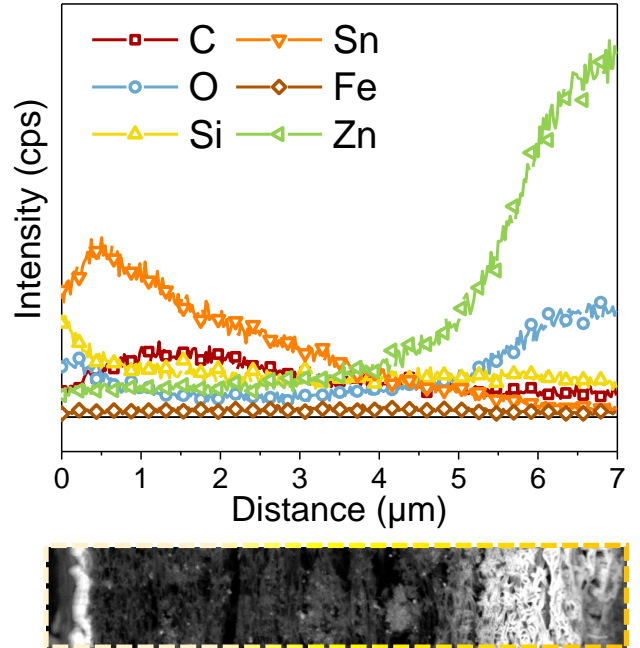

FTO substrate

Surface

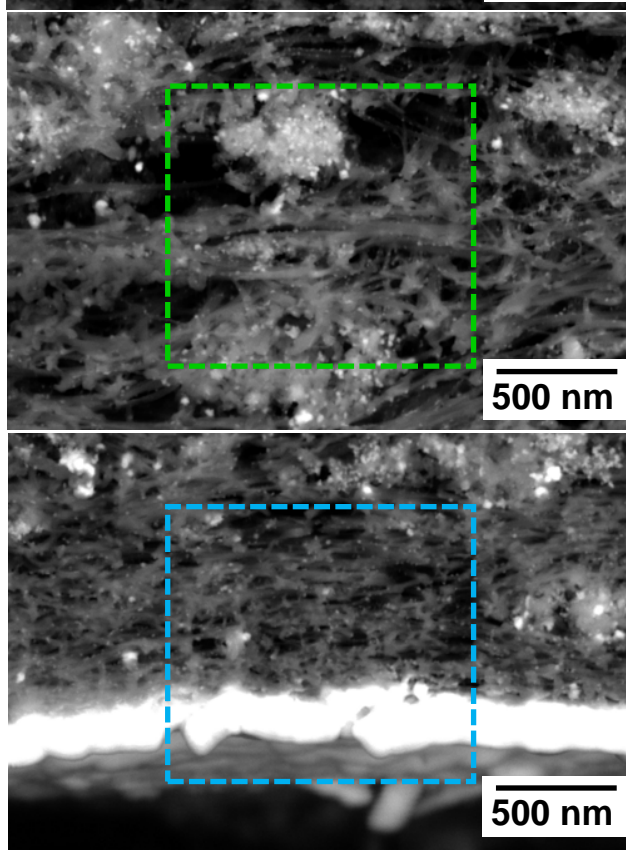

FIGURE 6.14: a) SEM images of CNTf/ZnO-ALD hybrid crosssection. b) EDX-SEM compositional analysis on the selected region (yellow rectangle) as a function of depth. c) SEM magnified images of the upper (red), middle (green) and lower (blue) regions of the

CNTf/ZnO-ALD hybrid.

\subsection{CNTf-photoanodes: increasing oxide thickness and dye loading}

The standard reference configuration used in previous Chapter 4, based on a Pt-CE and one layer of $\mathrm{TiO}_{2}$ paste deposited by doctor-blade technique (approx. $7 \mu \mathrm{m}$ thickness), was here reproduced with the $10 \mu \mathrm{m}$ thickness CNTf. A schematic of this configuration is presented in the Figure 6.15a.

Interestingly, cracks were consistently observed in these $\mathrm{FTO} / \mathrm{CNTf} / \mathrm{TiO}_{2}-$ paste electrodes after the sintering process (see Figure 6.15b). The cracks at higher magnification were analysed by SEM technique, and top view of the crack region is presented as an example in the Figure 6.15c.

Commonly, these cracks appear when the heating ramp during the sintering process is not fully optimised. But in this case, the heating ramp during all this 
thesis has provide best results when preparing reference cells. Hence, a possible explanation of the cracks development would be the difference in thermal expansion between both materials, which have similar thicknesses. The thermal expansion coefficients (CTE) for $\mathrm{CNTs}$ and $\mathrm{TiO}_{2}$-anatase are reported in the range of [-0.15 to -1.5] $\times 10^{-5} K^{-1}[335,336]$ and [3.5 to 8.5] $\times 10^{-6} K^{-1}$ [337] respectively. This CTE mismatch combined with the brittleness of the $\mathrm{TiO}_{2}$ material $\left(2.8 \mathrm{MPa} \mathrm{m} \mathrm{m}^{1 / 2}\right.$ fracture toughness)[338] and its crystallisation when annealed at $500{ }^{\circ} \mathrm{C}$, must be the responsible causes of the cracks appearance at the surface of the $\mathrm{TiO}_{2}$.
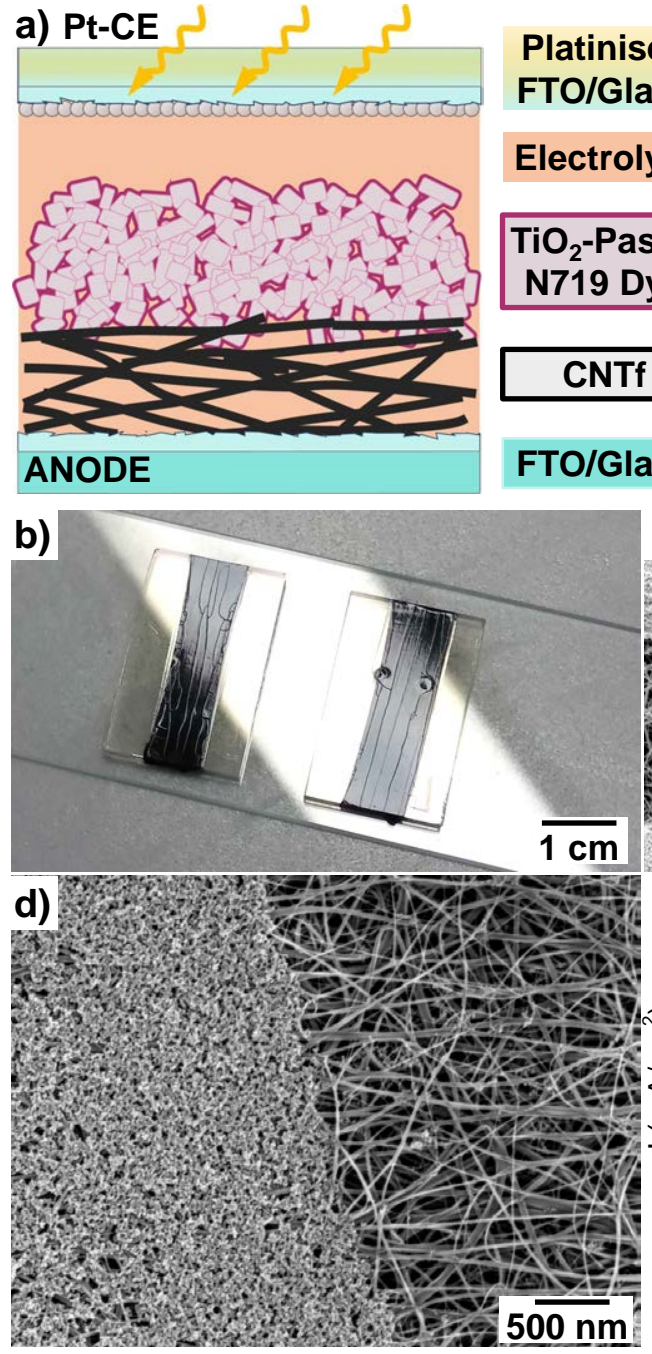

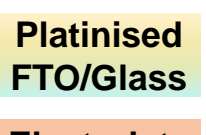

Electrolyte

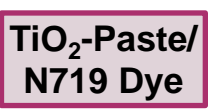

CNTf

FTO/Glass
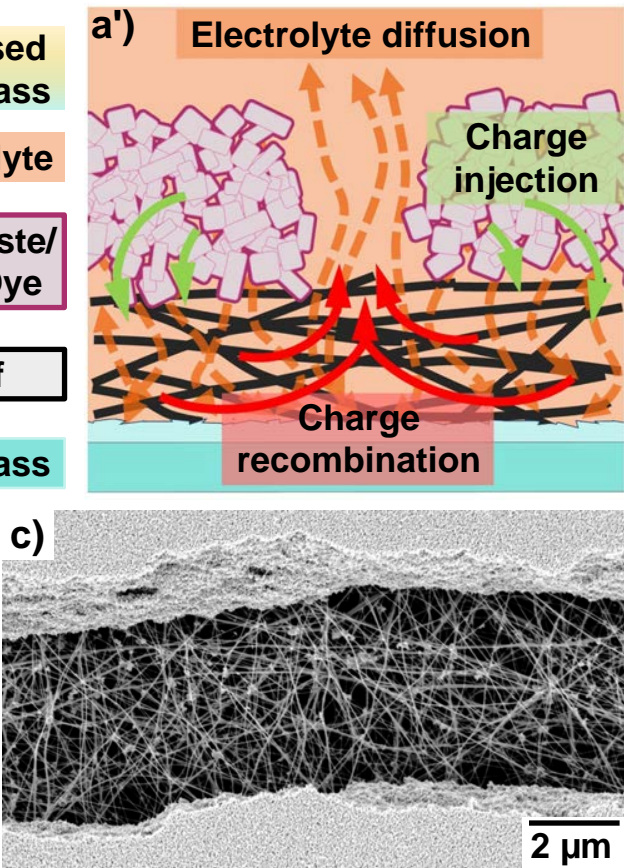

e)

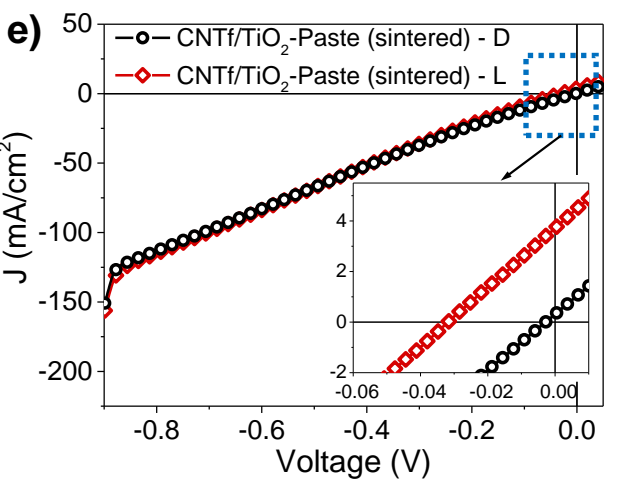

FIGURE 6.15: a) Schematic of a DSSC with the CNTf as currentcollector at the photoanode and a') charge recombination mechanism at the CNTf current-collector favoured by the existence of cracks at the surface of the $\mathrm{TiO}_{2}$ layer. b) Photographs of $\mathrm{CNTf} / \mathrm{TiO}_{2}-$ commercial-paste after sintering. Here the cracks can be observed. c) SEM image at the crack region. d) Top-view SEM image at high magnification of the $\mathrm{CNTf} / \mathrm{TiO}_{2}$ nanoparticles. e) Representative $J-V$ curves under light $(\mathrm{L})$ and dark $(\mathrm{D})$ conditions of these devices. Inset: magnified region of the $J-V$ curve.

The cracks are several microns width, leading to the CNTf electrode exposure. Still the local interconnection between the $\mathrm{TiO}_{2}$ nanoparticle layer is in close contact 
with the CNTf bundles (Figure 6.15d), ensuring effective paths of electron charge transfer between the two materials.

A set of devices were assembled and tested with these photoanodes, and the $J-V$ curves are shown in the Figure 6.15e. These curves show a huge dark leakage current, close to $150 \mathrm{~mA} \mathrm{~cm}^{-2}$ and a $R_{s h}=23 \Omega \mathrm{cm}^{2}$. Compared to the previous results obtained with the thin layers produced by ALD, the sort-circuit current density under light is now positive, in the range of $4 \mathrm{~mA} \mathrm{~cm}^{-2}$, but still very low $F F$ and $V_{o c}$ are measured. It also rules out the possibility of short circuits leading to such high leakage current.

Although with the characteristics of these cells is not possible to say that they are properly working, the increase in the $\mathrm{TiO}_{2}$ thickness leads to a higher charge injection into the device. This unexpected result suggests that there is a high recombination process at the surface of the CNTf even when very small areas of the CNTf are exposed. The schematic presented in Figure 6.15a' represents the open crack at the $\mathrm{TiO}_{2}$ surface which makes the electrolyte much more accessible to the photoanode CNT electrode, leading to a higher recombination and a much lower net extracted current. Indeed, a nanometre-size compact layer of $\mathrm{TiO}_{2}$ is deposited over the FTO substrate for avoiding the recombination at the electrode (detailed in $\mathrm{TiCl}_{4}$ treatment in the Chapter 3 "Experimental Techniques").

A solution for the CTE mismatch between materials is usually to decrease the thickness of the semiconductor layers, or to avoid the thermal stresses by reducing the sintering temperature and heating ramp.

For preparing thinner layers of $\mathrm{TiO}_{2}$, the commercial pastes used in previous experiments have been diluted with ethanol solvent $\left(1: 5 \mathrm{TiO}_{2}\right.$ paste:ethanol dilution ratio). Layers in the range 1-2 $\mu \mathrm{m}$, depending on the number of applied layers ( $\mathrm{x} 1$, $\mathrm{x} 2$ and $\mathrm{x} 3$ layers by doctor blade).

Moreover, two different paste formulations have been used (HT-high temperature and LT-low temperature sintering) with the aim of reducing the sintering temperature and therefore the thermal stresses. Three different sintering conditions were studied $500{ }^{\circ} \mathrm{C}$ for the HT-paste, and $200{ }^{\circ} \mathrm{C}, 100{ }^{\circ} \mathrm{C}$ for the LT-paste, besides the non-sintered LT-paste have been obtained.

As an indicator of the recombination process evolution the $R_{s h}$ is calculated for all the devices and presented in the Table 6.2. Representative $J-V$ curves are summarised in the Figure 6.16.

TABLE 6.2: Shunt resistance $\left(\Omega \mathrm{cm}^{2}\right)$ comparison of low and high temperature recrystallised $\mathrm{TiO}_{2}$ diluted paste.

\begin{tabular}{c||c|ccc} 
& High temperature & \multicolumn{3}{|c}{ Low temperature } \\
\# Layers & $500^{\circ} \mathrm{C}$ & $\mathrm{RT}$ & $100^{\circ} \mathrm{C}$ & $200{ }^{\circ} \mathrm{C}$ \\
\hline \hline 1 & 140 & 15 & 10 & 25 \\
2 & 520 & - & - & - \\
3 & 90 & 33 & 166 & 35
\end{tabular}

Using diluted pastes the porosity of the CNTf electrodes is infiltrated and saturated with $\mathrm{TiO}_{2}$ nanoparticles, procuring a more interconnected material instead of two clear separated layers of $\mathrm{CNTf}$ and $\mathrm{TiO}_{2}$. The schematic of this final device is presented in the Figure 6.16a. After the sintering process, the electrodes prepared at 
high temperature (HT), still present cracked surface, no matter the number of layers applied. On the contrary, when low temperature (LT) paste is applied, regardless of the sintering temperature, no cracks are observed at the surface of these electrodes. The optical microscopy images of the surface of these electrodes are shown in the Figure 6.16b.
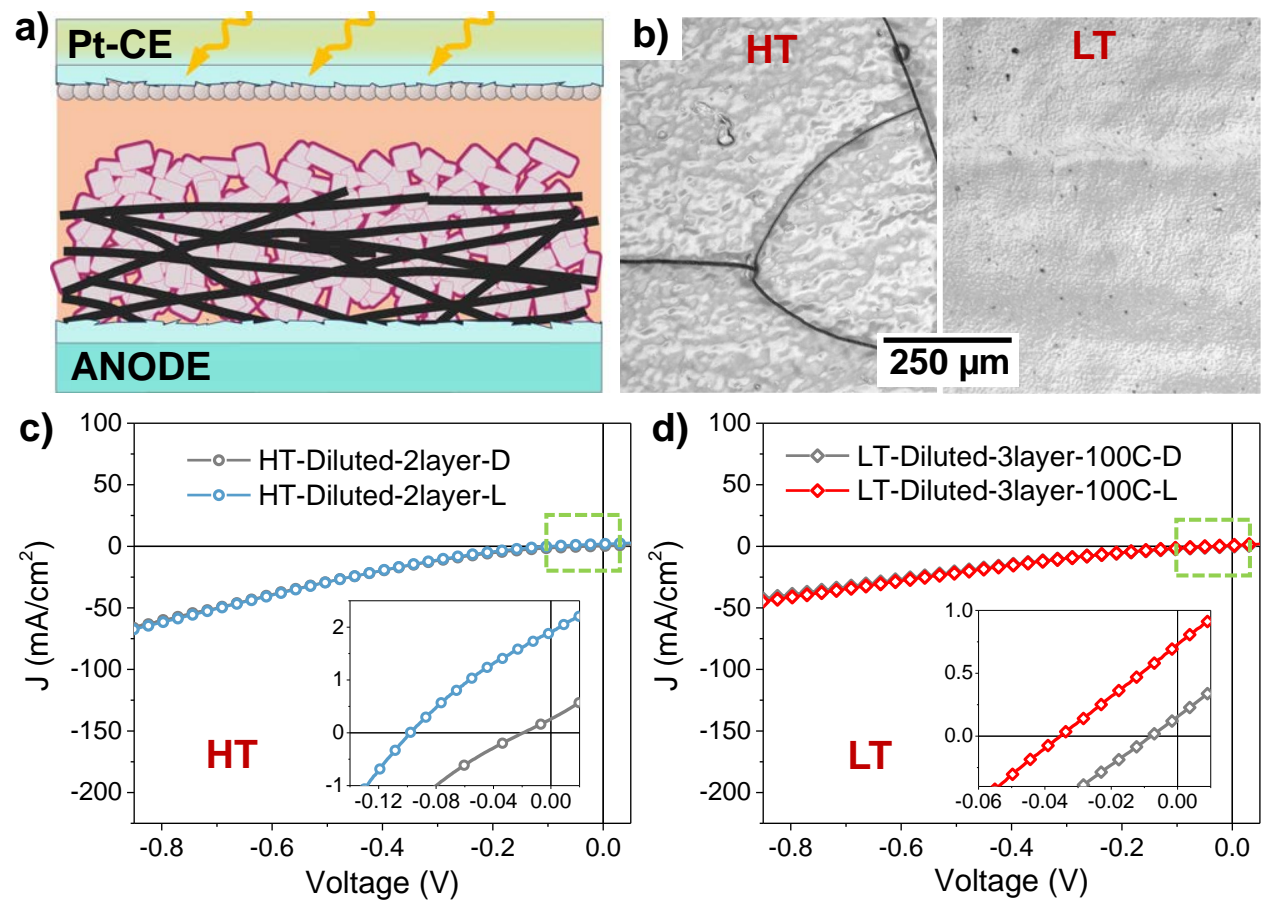

FIGURE 6.16: a) Schematic of a conventional DSSC with the CNTf as current-collector and using a low viscosity (diluted) $\mathrm{TiO}_{2}$ paste filling the porosity of the CNTf electrode. b) Optical microscope images of the surface of both electrodes showing the presence of cracks for the high temperature paste (left) and the absence of them with low temperature paste (right). c) and d) are representative $J-V$ curves under light (L) and dark (D) conditions of these devices. Inset: magnified region of the $J-V$ curve.

The representative $J-V$ curves of the devices showing higher leakage current (low shunt resistance) are presented in the Figure 6.16c,d. In summary, none of the devices present $J-V$ curves expected from a proper working solar cell, although small current and voltage is obtained (see inset graphs). Large leakage current is obtained independently of the paste composition or number of layer deposited.

Comparing the electrodes without cracks produced by low temperature diluted paste, the thinner layers of $\mathrm{TiO}_{2}$ present approximately 2-fold higher dark current with a $R_{s h}$ of $10-25 \Omega \mathrm{cm}^{2}$.

This result clearly indicates that recombination occurs at the surface of the CNTf current-collector independently of the cracks at the surface of the $\mathrm{TiO}_{2}$. The electrolyte penetrates through the nanoparticle layers interacting with the CNTf and leading to high recombination.

The different $J-V$ curves obtained in the previous sections are summarised in the graph presented in the Figure 6.17. The obtained trend suggest the increase of 
shunt resistance (decrease of leakage current) when the degree of surface coating of CNTf is icreased.

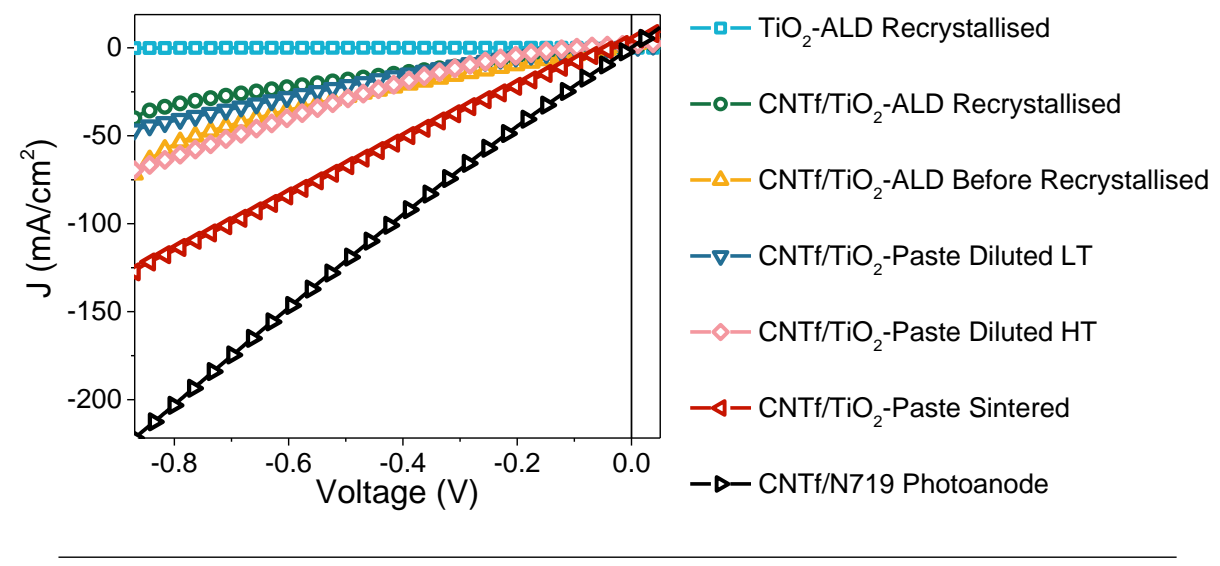

FIGURE 6.17: Summary of the $J$ - $V$ curves of the different photoanode configurations studied in the previous chapters.

Two possible modification are proposed with the aim of reducing the recombination component at the surface of the CNTf:

- Using an extra layer of conformal $\mathrm{TiO}_{2}$-ALD coating the CNTf in order to reduce the recombination in combination with a higher injection from a thicker $\mathrm{TiO}_{2}$ layer. The $\mathrm{TiO}_{2}$-ALD would act as a passivising layer of the CNTf, analogous to the "blocking layer" for standard FTO substrates.

- Reducing the amount of CNTf in order to unbalance the injection/ recombination process towards a less recombining system.

\subsection{CNTf/MO $\mathrm{MO}_{\mathrm{x}}-\mathrm{ALD}$ as photoanode blocking layers}

The final set of photoanodes explore the possibility of combining $\mathrm{CNTf} / \mathrm{MO}_{x^{-}}$ ALD coated material with a micrometric $\mathrm{TiO}_{2}$ nanoparticles layer. The ALD coating can be seen as playing the role of passivising material for avoiding the recombination of the photogenerated carriers. Although on flat substrates, several works have reported the use of thin layers deposited by ALD as efficient blocking layer.[210, 339-341]

In addition, using the standard blocking layer deposition treatment is not feasible because this layer is deposited from a $\mathrm{TiCl}_{4}$ solution at $0.4 m \mathrm{~m}$ in $\mathrm{H}_{2} \mathrm{O}$. Due to the hydrophobic nature of the CNTf, this aqueous-based process is not feasible.

An alternative solution is to use the $\mathrm{MO}_{x}$ layers deposited by ALD technique as blocking layers. Must be emphasised, the term blocking layer refers to the ability of this layer to inhibit the recombination of the injected electrons into the currentcollector. Hence, the required properties for this material are, firstly, to grow as a compact layer and to achieve complete coverage of the surface of the currentcollector; secondly, to favour the injection of the electrons from the nanoporous $\mathrm{TiO}_{2}$ into the compact layer, and also, from the compact layer into the anode currentcollector material (CNTf in our case). 
A schematic of this configuration is presented in the Figure 6.18a, where this mention hypothesis of protecting the CNTf current-collector with the $\mathrm{MO}_{x}$-ALD material is also depicted.

Two series of electrodes were prepared for using them as photoanodes with this configuration. One set of CNTf electrodes was prepared with ZnO-ALD, and the other one with $\mathrm{TiO}_{2}$-ALD. Also, two different thicknesses of ALD coating (20 and $100 \mathrm{~nm}$ ) were deposited.
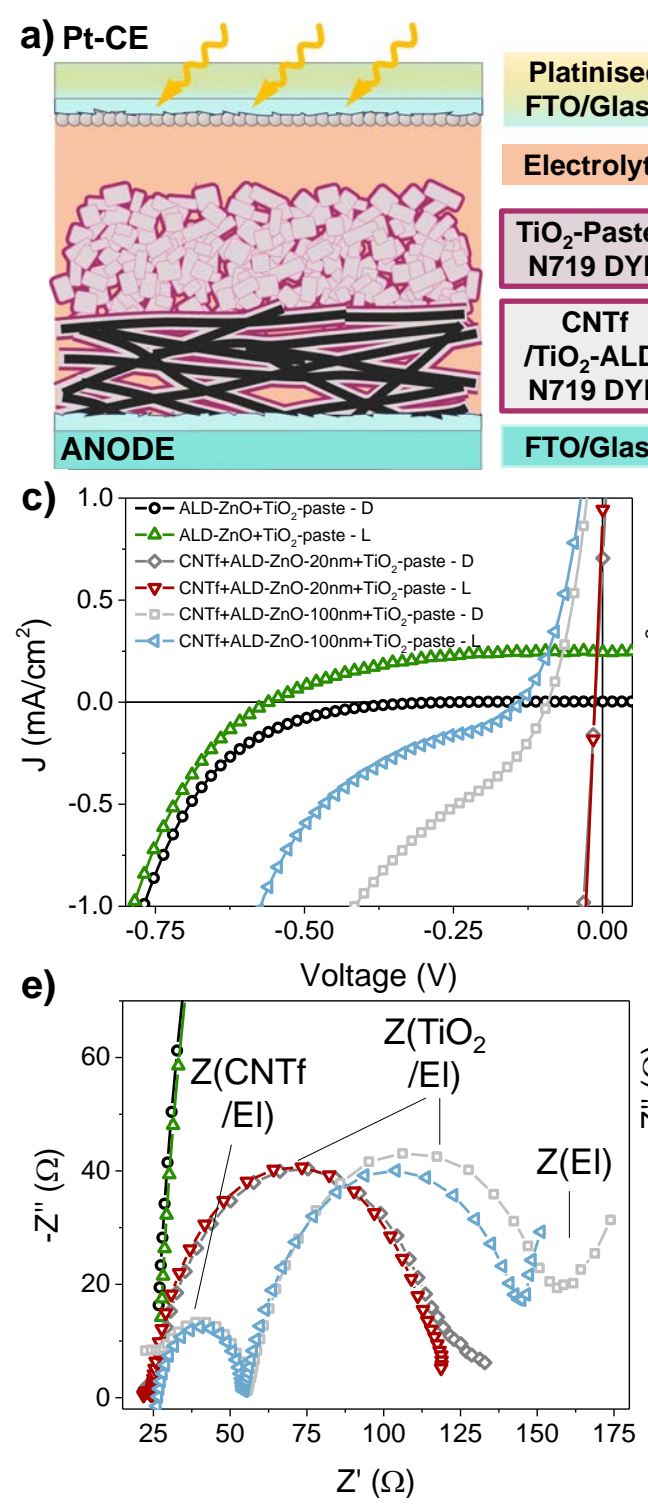

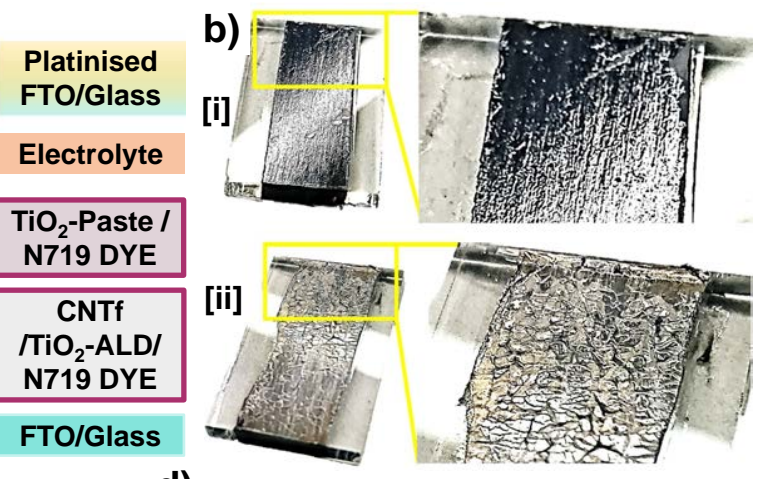

d)

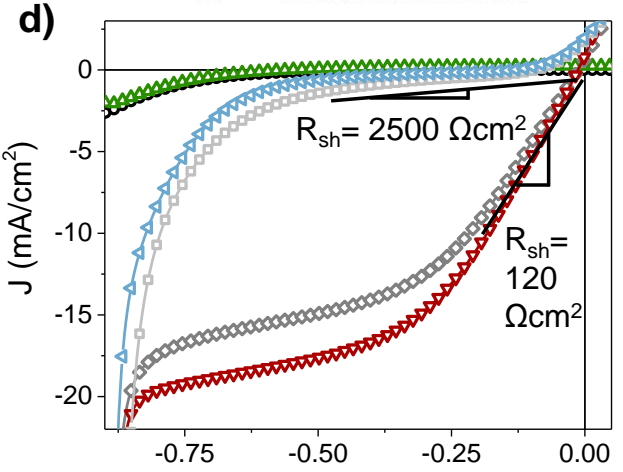

f)

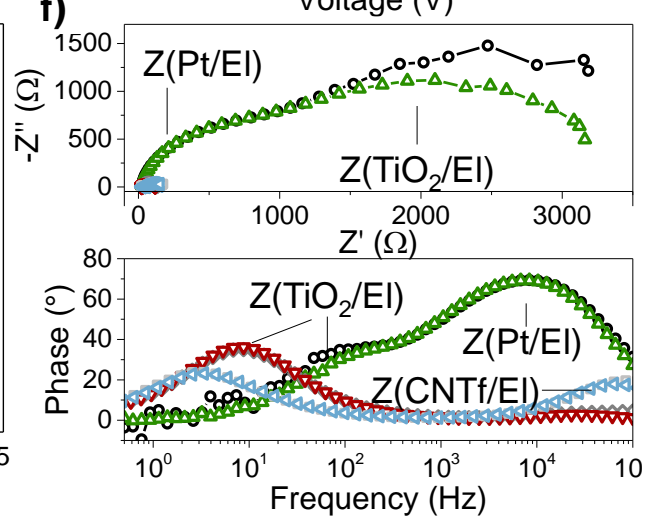

FIGURE 6.18: a) DSSC schematic with the CNTf/MO $\mathrm{MO}_{x}-\mathrm{ALD} / \mathrm{TiO}_{2}-$ paste photoanode: CNTf conformally coated with $\mathrm{MO}_{x}$ by ALD and with a $\mathrm{TiO}_{2}$-paste layer deposited by doctor-blade technique. b) Digital photographs of $\mathrm{CNTf} / \mathrm{MO}_{x}-\mathrm{ALD} / \mathrm{TiO}_{2}$-paste photoanodes: [i] ZnO-ALD and [ii] $\mathrm{TiO}_{2}$-ALD. c) J-V curves of the DSSC devices prepared with $\mathrm{MO}_{x}$-ALD blocking layer photoanodes. d) $J-V$ curves presented in a larger current range. e) Magnified region of the Nyquist curves presented in f) (up) of the DSSC devices, also presented Bode phase plot (down). The legend in graph $c$ applies for the graphs. 
The resulting electrodes end up with the CNTf current-collector coated with a nanometre-size conformal layer of $\mathrm{MO}_{x}$ by ALD, and directly on top, $2 \mu \mathrm{m}$ of mesoporous titania deposited by doctor blading few $\mathrm{ml}$ of commercial $\mathrm{TiO}_{2}$-paste diluted in solvent (Figure 6.18b). As can be observed in these images, the $\mathrm{TiO}_{2}-\mathrm{ALD}$ coated electrode is completely full of cracks (see Figure 6.18b-[ii]), and these electrodes are not suitable for preparing devices with them. Surprisingly, the photoanode coated with $\mathrm{ZnO}$ by ALD totally prevents the developing of cracks in the mesoporous $\mathrm{TiO}_{2}$ layer. The not uniform appearance of this electrode is attributed to the light reflection at the rough surface of the hybrid CNTf, although no cracks are observed after optical microscopy analysis (see Figure 6.18b-[i]).

This incompatibility between $\mathrm{TiO}_{2}$-ALD and $\mathrm{TiO}_{2}$-paste is attributed to the morphology of the ALD layer. As it is presented in previous sections, $100 \mathrm{~nm} \mathrm{TiO}_{2}-$ ALD produces a thicker continuous layer on top of the CNTf electrode, possibly consequence of CNTf surface porosity saturation. Hence, the thermal stresses crack the $\mathrm{TiO}_{2}$ layer. On the contrary, $\mathrm{ZnO}-\mathrm{ALD}$ leads to a conformal coating of the CNTf that improves the matching between $\mathrm{CNTf}$ substrate and $\mathrm{TiO}_{2}$-paste thermal expansion coefficients.

DSSC devices were assembled and measured in the solar simulator at 1 sun (AM 1.5G). The obtained $J-V$ curves are included in the Figure $6.18 \mathrm{c}, \mathrm{d}$. For the reference device, low characteristics, $V_{o c}$ close to $0.6 \mathrm{~V}, J_{s c}$ in the range of $0.3 \mathrm{~mA} \mathrm{~cm}^{-2}$, $F F=47 \%$, leads to a final efficiency of $P C E=0.07 \%$. This reference device was also measured illuminating from the anode side, obtaining the performance without the losses produced by the Pt-CE and electrolyte light absorption. Short-current density and efficiency, four times higher than illuminating from the $\mathrm{CE}$ side, have been obtained $\left(P C E=0.22 \%, J_{s c}=1.2 \mathrm{~mA} \mathrm{~cm}^{-2}\right)$. The $J-V$ curves corresponding to CNTf coated with 20 and $100 \mathrm{~nm}$ ZnO-ALD blocking layer are presented in the Figure $6.18 \mathrm{c}, \mathrm{d}$. A dark current density ca. $-18 \mathrm{~mA} \mathrm{~cm}^{-2}$ and $-0.5 \mathrm{~mA} \mathrm{~cm} \mathrm{~cm}^{-2}$ are obtained at $-0.5 \mathrm{~V}$ for 20 and $100 \mathrm{~nm}$ respectively. The performance of the cells using CNTf current-collector is lower compared with the reference device. Higher current density is obtained but with a very low open circuit voltage.

Observing the $J-V$ curves in a wider range (Figure 6.18d), a clear trend between ALD-thickness and leakage current is observed. The leakage current is drastically diminished increasing the ALD thickness from 20 and $100 \mathrm{~nm}$. The $R_{s h}$ for $100 \mathrm{~nm}$ ALD layer can be calculated extrapolating the current from the -[0.2-0.4] $V$ range obtaining $R_{s h}=2500 \Omega \mathrm{cm}^{2}$, whereas for $20 \mathrm{~nm} R_{s h}$ is in the range of $120 \Omega \mathrm{cm}^{2}$. In contrast with the $R_{s h}=0.3 \mathrm{M} \Omega \mathrm{cm}^{2}$ for the reference device, this suggest that there is still recombination at the surface of the CNTf current-collector.

EIS was also performed and analysed based on the equivalent circuit explained in previous chapters. The Nyquist and Bode plots are presented in the Figure 6.18e,f. The reference device shows a high cell resistance and the typical interfaces are resolved. For the CNTf-photoanode devices, the cell resistances $\left(R_{\text {cell }}\right)$ measured were 100 and $125 \Omega$ for 20 and $100 \mathrm{~nm}$ thickness ZnO-ALD layer respectively. In case of $100 \mathrm{~nm}$ ALD, the interfaces were characterised showing $R_{C T}=27 \Omega$ with a characteristic $f_{\text {max }-C T}=10^{5} \mathrm{~Hz}$ and $R_{\mathrm{TiO}_{2}}=95 \Omega$ with a frequency $f_{\max -\mathrm{CT}}=3-4 \mathrm{~Hz}$, also the bulk diffusion $\left(R_{d i f f}\right)$ is identified, however there are no enough points for allowing the fitting of this interface. For $20 \mathrm{~nm}$ thickness layer, the extracted characteristics were $R_{C T}=2.5 \Omega$ with a characteristic $f_{\max -\mathrm{CT}}=3 \times 10^{4} \mathrm{~Hz}$ and $R_{\mathrm{TiO}_{2}}=90 \Omega$ with a frequency $f_{\max -\mathrm{CT}}=9 \mathrm{~Hz}$. 
From the comparison of these two devices, the charge collection efficiency can be obtained. Since the standard N719 dye and iodine-based electrolyte are used, the quantum yields of dye regeneration $\left(\eta_{\text {reg }}\right)$ and charge injection $\left(\eta_{i n j}\right)$ can be assumed to be close to unity.[342] Differences in light harvesting efficiency (LHE) can be discarded because the same materials are used and the variation in the nanometre scale for the ALD blocking layers can be considered negligible in terms of optical properties. LHE is defined by the equation 6.1:

$$
L H E=1-T-R
$$

where $T$ is transmittance and $R$ is reflectance.

The main impact of the $\mathrm{TiO}_{2}$-ALD blocking layers thickness on the charge collection efficiency $\left(\eta_{\text {coll }}\right)$ is here discussed. The charge collection efficiencies were calculated on the basis of electron transport resistance $\left(R_{w}\right)$ and resistance to charge recombination $\left(R_{k}\right)$ and the equation 6.2:

$$
\eta_{\text {coll }}=1-\frac{R_{w}}{R_{k}}
$$

where $\left(R_{w}\right)$ refers to the $\mathrm{FTO} / \mathrm{TiO}_{2}$ interface under illumination and $\left(R_{k}\right)$ refers to the $\mathrm{FTO} /$ electrolyte interface under dark conditions.

$\eta_{\text {coll }}$ for both 20 and $100 \mathrm{~nm}$, resulted on $4 \%$ and $14 \%$ respectively. These values are calculated from the $R_{w}=95 \Omega$ and $R_{k}=98 \Omega$ for the $20 \mathrm{~nm}$ ALD layer and $R_{w}=95 \Omega$ and $R_{k}=110 \Omega$ for the $100 \mathrm{~nm}$ ALD layer. This indicates the high recombination component at the CNTf electrode and its improvement by introducing ALD blocking layers. Exposed CNTf surface is still source of huge recombination with the electrolyte. For thick CNTf electrodes (10 $\mu \mathrm{m}$ in these devices), the ALD layer is not completely covering the surface of the CNTf throughout its cross-section.

Three differentiated behaviours are obtained for the three cells. The reference cell is behaving as a standard DSSC, and its low performance is related to the low injection (thin $\mathrm{TiO}_{2}$ active layers). For the CNTf-ALD blocking layers, the heterojunction interface between these materials is close to $10^{3}$ times higher compared to the $2 D$ active layer of the reference cell. Therefore, the resistance between the CNTf/ZnO-ALD is much smaller. This is in agreement with the cell resistances observed in EIS plots (Figure 6.18f). For CNTf/ZnO-20 nm/ $\mathrm{TiO}_{2}$-paste cell, the ALD layer is not effectively protecting the electrode from electrolyte recombination. For $\mathrm{CNTf} / \mathrm{ZnO}-100 \mathrm{~nm} / \mathrm{TiO}_{2}$-paste cell Nyquist impedance is representative of a standard DSSC device but providing very poor characteristics. Further optimisation is needed for obtaining the ratio between ALD blocking layer and CNTf thickness for obtaining a complete coating throughout the entire CNTf electrode thickness.

\subsubsection{CNTf/ZnO-NWs photoanodes}

Similar results were obtained when replacing the porous $\mathrm{TiO}_{2}$ layer with $\mathrm{ZnO}$. ZnO-NWs show faster electron transport than polycrystalline structures of $\mathrm{TiO}_{2}$,[343] combined with large SSA $\left(65 \mathrm{~m}^{2} \mathrm{~g}^{-1}\right)$,[344] on the contrary when they are used as photoanode (active material) lead to lower efficiency. As a reference value, the first time reported $\mathrm{ZnO}$-anodes for DSSC by Law et al.[343] PCE $=1.5 \%$ was achieved. Here, these devices are used to complement the results obtained with $\mathrm{TiO}_{2}$. 
Conventional reference cells were prepared with $\mathrm{ZnO}$ pastes by doctor-blade technique according to the procedure described elsewhere.[200] The characteristics were measured in the solar simulator, at 1 sun intensity (AM 1.5G). The resulting $\mathrm{ZnO}$ layers after the dying step are used as reference cells, (Figure 6.19a). The average thickness of these layers, obtained by profilometry measurements, are $\approx 2.5 \mu \mathrm{m}$ for the hydrothermal reference electrodes.

The CNTf hybrid samples used for these devices consisted on the large heterojunctions investigated in the previous chapter. Figure $6.19 \mathrm{~b}$ shows the CNTf deposited on aluminium foil, coated with $\mathrm{ZnO}-\mathrm{NW}$ s by hydrothermal and transfer to a FTO substrate. And the material after dyeing step is presented in Figure 6.19c. CNTf is directly in contact with $\mathrm{ZnO}-\mathrm{NWs}$ (Figure 6.19d). The ZnO-NWs crystalls has an approximate length of 2-4 $\mu \mathrm{m}$ and half a micron in diameter. The CNTf substrate presents a thickness of $c a$. 1-2 $\mu \mathrm{m}$. The aluminium foil is directly used as external current-collector to avoid extra resistance components.

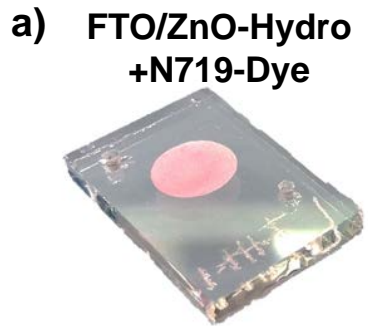

c)

c) CNTf/ZnOHydro+N719-Dye

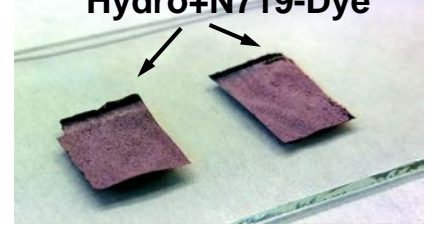

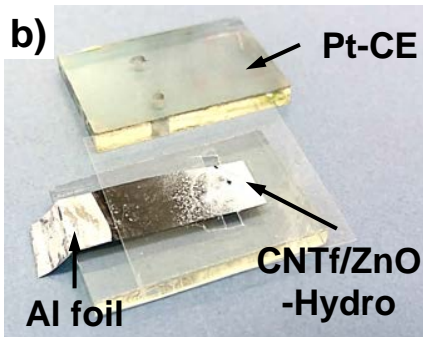
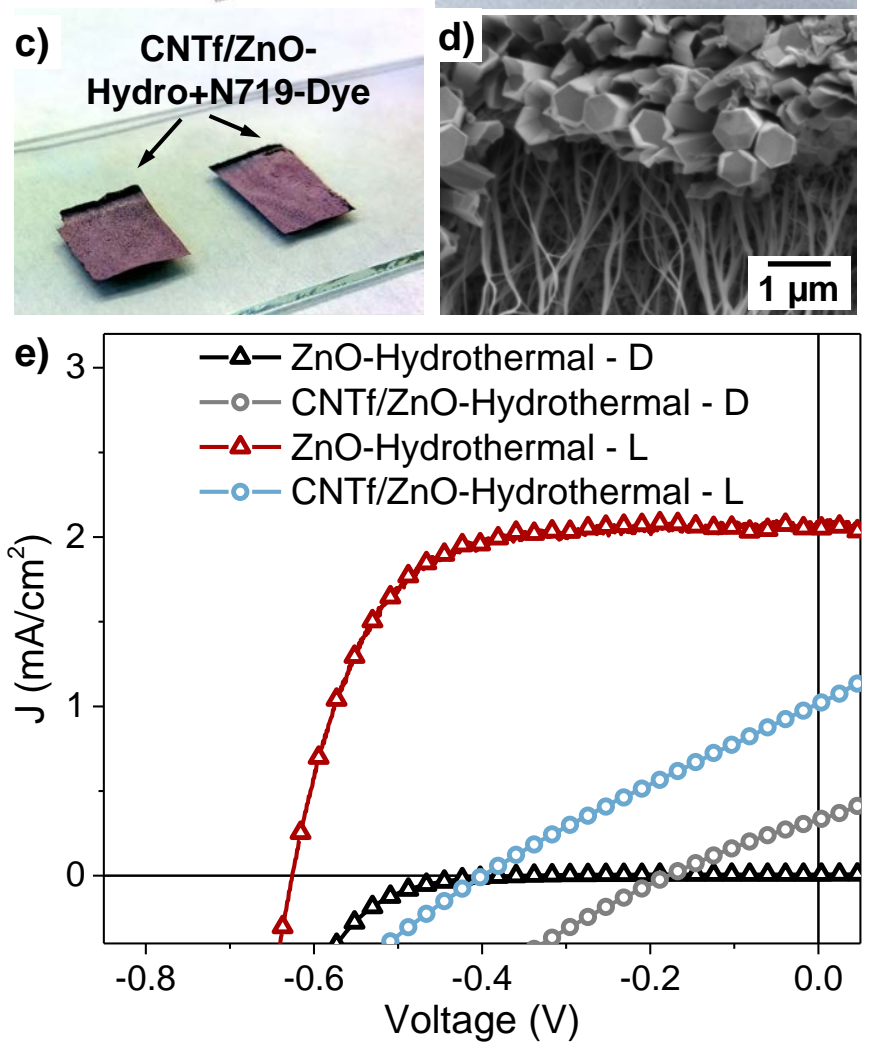

FIGURE 6.19: a) ZnO-NWs/FTO reference electrodes after dye adsorption. b) CNTf/ZnO-NWs photoanode produced by double step hydrothermal + sol-gel process. c) CNTf/ZnO-NWs after dyeing step. d) SEM image of the interface between CNTf and ZnO-NWs.

e) $J-V$ curves under dark and light conditions of these devices. 
The $J-V$ curves under dark and light conditions corresponding to these devices are presented in the Figure 6.19e. For the reference device, the obtained characteristics are $P C E=0.87 \%, V_{o c}=0.62 \mathrm{~V}, J_{s c}=2.1 \mathrm{~mA} \mathrm{~cm}^{-2}$ and $F F=65 \% . R_{s h}$ is in the range of $200 \mathrm{k} \Omega \mathrm{cm}^{2}$. This obtained characteristics are in the range of previous reports.[343]

In contrast, for the CNTf/ZnO-NWs hybrids, lower efficiency ( $P C E=0.1 \%$ ) and open circuit voltage $\left(V_{o c}=0.4 \mathrm{~V}\right)$ are obtained. The short-circuit current density is more than $1 \mathrm{~mA} \mathrm{~cm}^{-2}$, much higher than what is obtained in the previous section with the ALD materials. Moreover, the low FF (26\%) and $R_{\text {sh }}$ obtained $\left(625 \Omega \mathrm{cm}^{2}\right)$ are still an indicator of the suboptimal operation of the device. This is explained by the high series resistance and recombination component of the hybrid material and also confirmed by the high leakage current and small shunt resistance of the device.

Interestingly, the extracted current using the CNTf as photoanode currentcollector suggest that the ratio injection/recombination in these devices is higher than in the previous $\mathrm{ALD} / \mathrm{TiO}_{2}$ devices. This is because, firstly, the lower thickness of CNTf electrode leading to less accesible CNTf/electrolyte accessible surface, and secondly, the $\mathrm{ZnO}$ sol-gel nanoparticle coating possibly is acting as a passivising layer against the $\mathrm{CNTf} /$ electrolyte recombination.

\subsection{CNTf as transparent photoanode current-collector}

As soon as the CNTf is thick enough, there is no resistive problems with the extraction of the photogenerated charges from the device. On the contrary, the interaction between the electrolyte and the CNTf electrode leads to high charge recombination. To further change the balance between injection and recombination, semi-transparent CNTf electrodes are used with non-conductive substrates (glass) to evaluate its influence over the recombination process.

When using an opaque anode as in previous sections, the device can only be illuminated from the counter-electrode side. One of the advantages of transparent substrates is the chance of illuminating from the anode side or from both sides at the same time with a back-reflector, thus increasing the carrier photogeneration. Firstly, the effect of the transparency and resistive losses of the CNTf electrodes must be considered.

In the Figure 6.20, the transparency $(\approx$ transmittance, $T)$ and sheet resistance $\left(R_{s q}\right)$ of the CNTf are presented versus the stacked number of CNTf-layers, where these layers refer to individual CNTf monofilaments. It shows that increasing the thickness of the CNTf rapidly decrease the transparency of the CNTf. For instance, 1 layer of CNTf leads to a transmittance of approx. $75 \%$ (at $550 \mathrm{~nm}$ ) and a $R_{s q}=$ $95 \Omega / s q$. On the contrary, when more than 20 layers of CNTf are stacked, the electrode becomes basically opaque $(T \approx 5 \%)$ and $R_{s q}=5-10 \Omega / s q$. At the moment, obtaining highly conductive electrodes based on CNTf, is a tradeoff with the transparency of the material.

Several CNTf electrodes with less than $<1 \mu m$ thickness (semi-transparent) were deposited on glass substrates. Later, they were coated by doctor-blade technique with $7 \mu \mathrm{m}$ of $\mathrm{TiO}_{2}$ commercial paste and, after the sintering, coated by a 
layer of 4-times-diluted $\mathrm{TiO}_{2}$ paste for healing the cracks that appear in the previous thicker $\mathrm{TiO}_{2}$ layer. After the dyeing step, the electrodes still present a high transparency. Representative electrodes are presented in the Figure 6.21b.

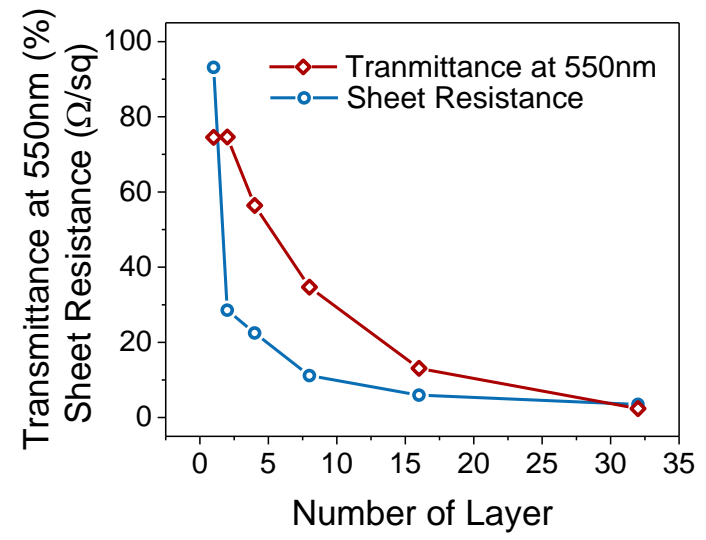

FIGURE 6.20: Sheet resistance $\left(R_{s q}\right)$ and transmittance $(T)$ vs the stacked number of CNTf layers.

The schematic and digital photograph of a semi-transparent device using CNTf as semi-transparent current-collector are presented in the Figure 6.21a and c respectively. A thin CNTf film with sub-micron thickness $(<1 \mu \mathrm{m})$ is the only currentcollector of the device.

$J-V$ curves of two of these devices are presented in the Figure 6.21d. The characteristics of the device with superior performance are $V_{o c}=0.59 \mathrm{~V}, J_{s c}=$ $5 \mathrm{~mA} \mathrm{~cm}{ }^{-2}, F F=26 \%$ and PCE $=0.8 \%$. The $R_{s}$ and $R_{s h}$ of these devices are 33 and $13100 \Omega \mathrm{cm}^{2}$ respectively. The low thickness of the CNTf current-collector has some implications on the device performance, typically affecting the fill factor of the $J-V$ curve and lowering the efficiency. Indeed some control experiments with semi-transparent CNTf supported on FTO substrate have shown similar $V_{o c}$ and $J_{s c}$ but with higher FF and PCE. This confirms the lower recombination mechanism with semi-transparent CNTf substrates.

For the glass/CNTf-photoanodes, the Nyquist impedance under illumination (Figure 6.21e) presents only one semicircle with a characteristic frequency of $f_{\max }=$ $4725 \mathrm{~Hz}$ and the cell resistances $\left(R_{\text {cell }}\right)$ measured was circa $60 \Omega$. Under dark condition, a broad peak was identified with a resistance of approximately $R=260 \Omega$ and a characteristic frequency $f_{\max }=400 \mathrm{~Hz}$. The broadening of this peak is indicative of a convoluted secondary component attributed to the charge transfer resistance between electrolyte and $\mathrm{Pt}-\mathrm{CE}$ at higher frequency $\left(10^{4} \mathrm{~Hz}\right)$.

The device under illumination shows the carrier saturation of the conduction band of the titania, lowering the resistance of this semiconductor layer. Bearing this in mind, the observed peak under dark conditions in EIS plots (Figure 6.21e) is attributed to the charge transfer between $\mathrm{TiO}_{2} /$ electrolyte. Whereas in measurements under illumination, this semicircle becomes much smaller and the charge transfer between CNTf and electrolyte is now observed. The large variation between dark and light conditions could be explained by a lowering in the charge recombination therefore increasing the charge collection efficiency. 
The recombination mechanism has been drastically reduced by introduction of semi-transparent CNTf. This is further confirmed by the much higher $R_{s h}$. On the contrary, the sheet resistance of such a thin material is hampering the charge extraction by increasing the series resistance of the device. It is well known the limitations of the $R_{S}$ over the $F F$ of the device. At this point, it is possible to conclude that there is an existing between optical transparency and low $R_{s q}$ for CNTf.

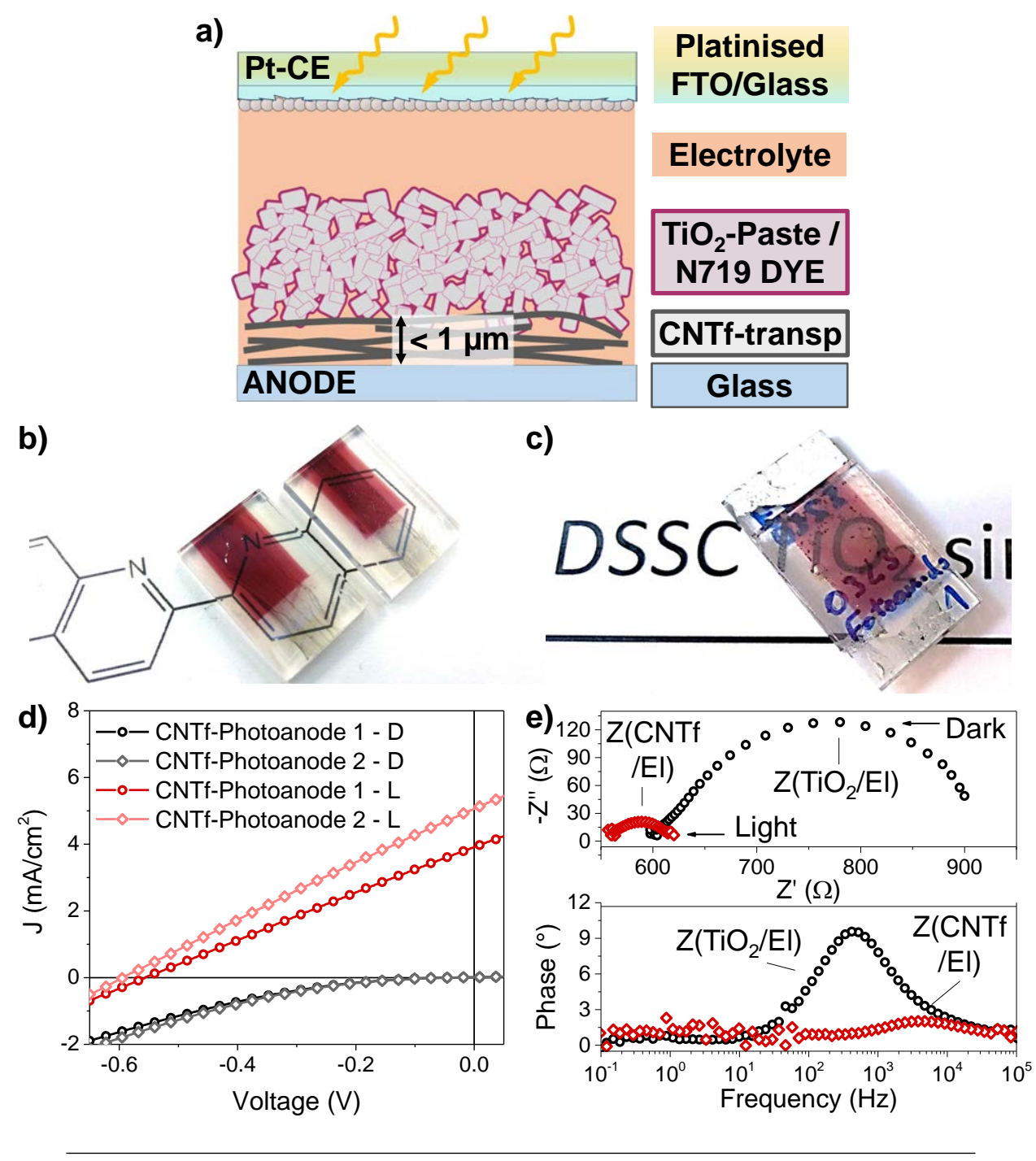

FIGURE 6.21: a) Schematic of a DSSC implementing transparent submicrometric CNTf current-collector photoanode. b) Digital photograph of the semitransparent Glass $/ \mathrm{CNTf} / \mathrm{TiO}_{2}$ photoanodes. c) DSSC device assembled with semitransparent Glass $/ \mathrm{CNTf} / \mathrm{TiO}_{2}$ photoanode and FTO/Pt-CE. d) $J-V$ curves of representative devices at 1 sun illumination intensity. d) Nyquist (up) and Bode phase (down) plots of one device under dark (D) and light (L) conditions.

\subsection{Summary and conclusion}

Inspired in some previous works, the use of the neat CNTf decorated with N719 dye molecules has been investigated as DSSC devices. But, none of the tested 
devices presents the typical characteristics of a solar cell and no power was generated. This result suggest that there is charge recombination between the liquid electrolyte and the surface of the CNTf that inhibit the proper operation of the device.

The hypothesis of the high surface area 3D electrodes was investigated by depositing conformal layers of $\mathrm{TiO}_{2}$ by ALD onto CNTf substrates. The photogenerated carrier injection $v s$ recombination mechanism was analysed by tunning the amount of emitter material (thickness of $\mathrm{TiO}_{2} /$ N719-dye) and its interaction with the CNTf. Low performance was achieved with this configuration due to the low photocarrier generation and high recombination observed. The surface area of this electrode in combination with the highly catalytic activity demonstrated in previous chapters explains this behaviour.

The ALD material proved to be a good passivisation layer (blocking layer) for the CNTf electrode. The leakage current of the device has been drastically reduced, although further optimisation is needed for obtaining fully passivised CNTf substrates. Obtaining homogeneous $\mathrm{MO}_{x}$ deposited throughout the CNTf substrate thickness is required for obtaining fully operative devices.
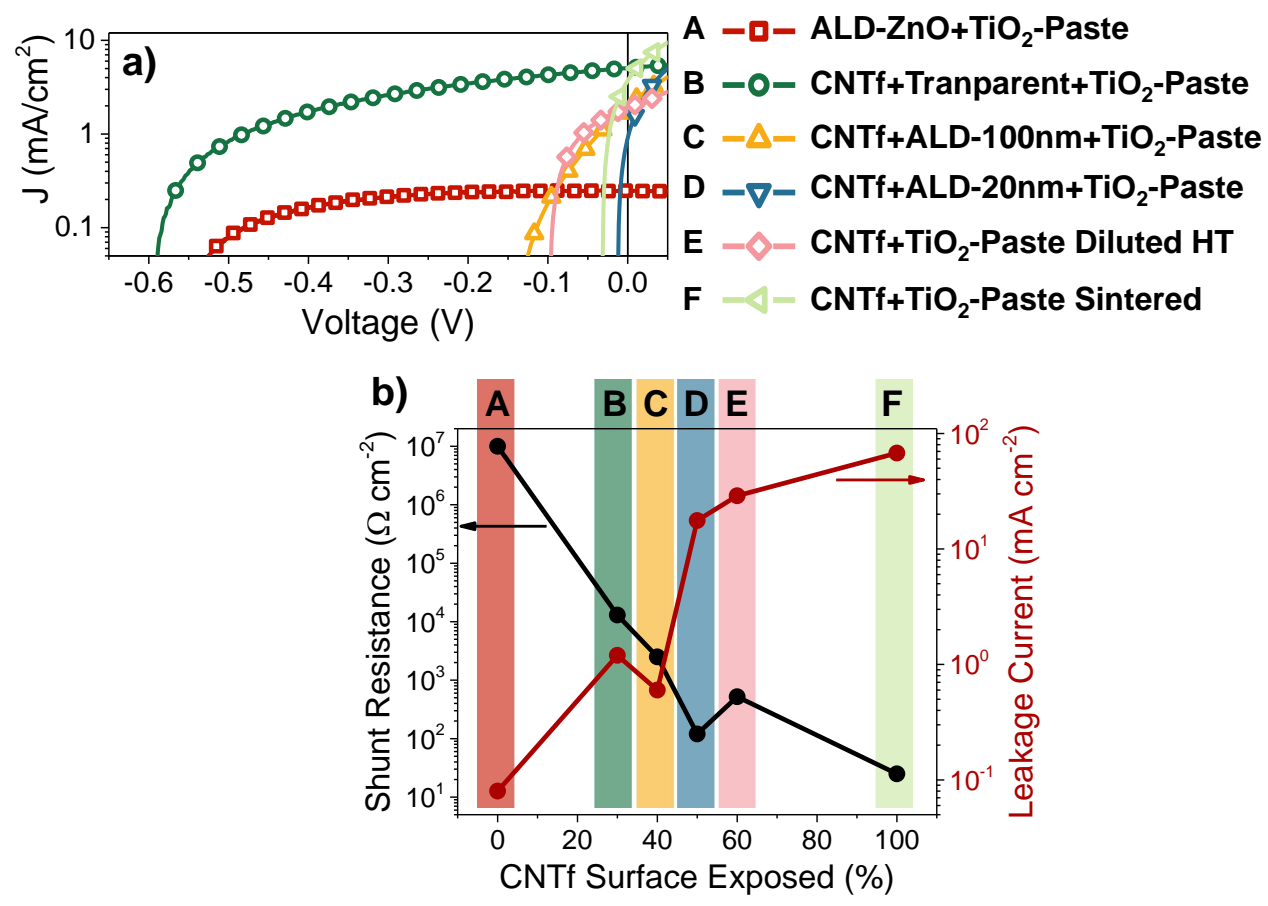

FIGURE 6.22: Recombination mechanism according to the degree of CNTf surface exposure in the photoanode. a) $J-V$ curves of the main operative CNTf-photoanodes. b) Shunt resistance and leakage current according to the CNTf surface exposure.

The competition between carrier generation- injection and recombination mechanism was also investigated by reducing the thickness of the CNTf to semi- transparent electrodes. Decreasing the available CNTf surface, thus the number of recombination centres, the recombination mechanism is diminish obtaining good current and voltage characteristics but very low FF. This drawback is attributed to the high sheet resistance of semi-transparent CNTf electrodes. 
Concluded from these previous architectures, the degree of exposed CNTf surface proved to be a critical parameter. The main operative devices are summarised here by their $J-V$ curve (Figure 6.22). The different approached strategies show the direct relation between CNTf exposed surface and recombination component. The passivisation of the CNTf surface led to higher shunt resistances, lower leakage currents and better overall performance of the devices.

Proposed future work should combine the results obtained by the passivisation of the CNTf substrate by $\mathrm{MO}_{x}$-ALD (after optimisation) in combination to semi-transparent electrodes. Moreover, the increment of the conductive properties of semi-transparent electrodes would lead to better performance DSSC devices. The starting point for low recombination CNTf devices is clearly depicted by the results obtained in this chapter.

Finally, some alternative solutions for avoiding the recombination mechanism are proposed. For instance, using non-reactive electrolytes with the CNTf, hence avoiding this source of recombination. Other possibility would be using water based electrolytes with low wettability with the CNTf electrodes, thus reducing the physical contact between these two materials. 


\section{Chapter 7}

\section{CNTf Electrodes for DSSC: Engineering Aspects} CEs.

This chapter analyses engineering aspects of DSSC devices based on CNTf-

Firstly, it discusses fabrication challenges and opportunities arising from the porous nature of these CEs. It considers the performance of CNTf-CEs devices against standard DSSCs, but also presents results of relevance towards the development of DSSC with augmented mechanical properties.

Next, an example of a device architecture that overcome the intrinsic optical absorption of CNTs is presented. vestigated.

Finally, device size effects of counter-electrode and working-electrode are in-

\subsection{Progress towards innovative DSSC architectures}

In the previous Chapter 4 the CNTf took the role of CE and current-collector. But in view of the mechanical toughness of this material, it is of interest to use it in free-standing form. Such arrangement leads itself to the fabrication of flexible, easy-to-process, tough electrodes DSSC.

\subsubsection{Electrolyte infiltration}

Typically, DSSC at laboratory scale are filled with the electrolyte through very small holes ( $<1 \mathrm{~mm}$ diameter) drilled at the TCO current-collector substrate. By capillary forces the electrolyte flows and fills the gap between both electrodes (see the schematic presented in the Figure 7.1a). This gap between electrodes is in the range of tens of microns (20-100 $\mu \mathrm{m})$. Visual observation of the electrolyte flow shows that complete coverage of the device area takes around 1 second, although the infiltration of mesoporous $\mathrm{TiO}_{2}$ layer would take longer.

When the CNTf-CE is used, there is no gap between the mesoporous CNTf membrane CE and the photoanode. The porosity of the CNTf material provides strong capillary forces for the infiltration of liquids and polymers, but also implies longer time to fully saturate the device with electrolyte, as well as the possibility of forming air voids (see Figure 7.1b). 
a)

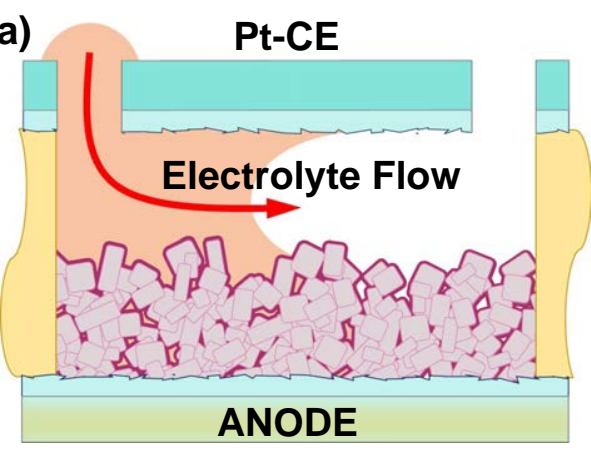

b)

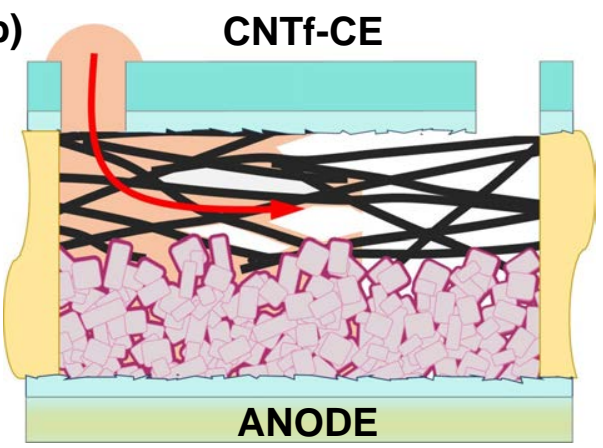

FIGURE 7.1: Schematic showing the electrolyte flow inside the DSSC by capillary forces for a) Pt-CE and b) CNTf-CE.

In contrast with the Pt-CE DSSC, observation of the CNTf-CE DSSC shows that the electrolyte spreads/wicks much slower, taking 6 minutes to cover all the visible area (Figure 7.2). In the infiltration of porous media by a fluid, there is often formation of bubbles or air pockets in the pores. Indeed partial infiltration of cells with CNTf-CE is thought to be main the main source of variability in these devices.

As a first approximation, the bulk wicking rate is considered that is driven exclusively by the capillarity of the CNTf-CE following a similar behaviour. Previous studies have shown that the wicking rate of a droplet on a CNT fibre fabric can be described by a modified Lucas-Washburn equation 7.1 of the form[345]

$$
\frac{S}{S_{0}} \cdot\left(\ln \frac{S}{S_{0}}-1\right)=-1+\left(\frac{2 \pi r \gamma \cos \theta}{3 \eta^{*} S_{0}}\right) \cdot t
$$

where $S_{0}$ is the initial wicking area covered by the droplet at time zero, $S$ the wicking area at time $t, r$ the effective capillary radius of the fibre, $\gamma$ the liquid surface tension, $\theta$ the Young-Laplace contact angle and $\eta^{*}$ the liquid viscosity.

This equation can be used to predict the infiltration time for the electrolyte to completely saturate the CNTf membrane. Approximating the electrolyte viscosity to the one of the pure acetonitrile solvent $(110 \mathrm{~m} \mathrm{~Pa} \mathrm{~s}),[346]$ the initial contact angle of 25.8 ${ }^{\circ}$ [347] (neglecting dynamic and secondary contact angles[348]) and average capillary radius for CNTf $(r)$ between 50-70 $\mathrm{nm}$ according to previous studies,[102, 349] the predicted time from the Eq.7.1, is in the range 10 minutes that is in agreement with the experimental observations presented in the Figure 7.2.

Notice that this capillarity radius represents the average pore radius encountered by the liquid flow, but in reality the pores are highly irregular and reflect a wide distribution of interbundle separations.[102] This implies that initial filling of macropores can proceed faster than the bulk wicking rate.

In order to accelerate the flow of fluids in porous media, while also removing air pockets, vacuum is often applied at an outlet. This has been widely studied in vacuum infusion of fabrics by viscous epoxies in the fabrication of structural composites, for example.[350] Sometimes, the electrolyte flow is assisted with vacuum,[200] to achieve better CNTf infiltration and saturation of the space between the electrodes and, more importantly, of the mesoporous titania layer. On the contrary, this approach is not convenient with conventional solvent based liquid electrolytes. 
If vacuum is applied for filling the cell, the high volatile solvents present in the electrolyte will evaporate becoming a denser electrolyte, changing the concentration of additives in solution and producing a less efficient electrolyte.

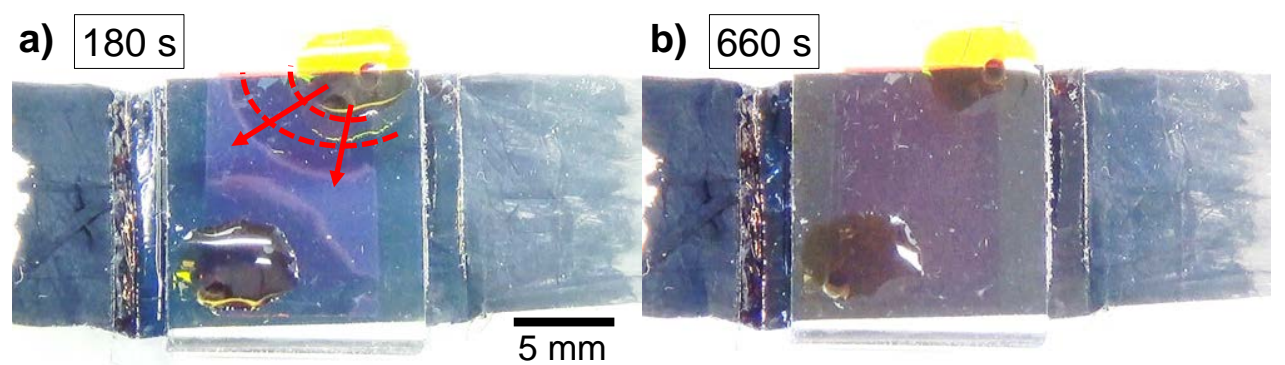

FIGURE 7.2: Optical images showing the electrolyte flow inside the CNTf-CE cell by capillary forces for a) $t=180 \mathrm{~s}$ and b) final $t=660 \mathrm{~s}$.

Although a characteristic time for the electrolyte filling step is defined in this section $(\approx 10 \mathrm{~min})$, not all the devices achieved the complete saturation and some devices still show air pockets after this infiltration time. The devices presenting lack of electrolyte provides lower performance than the fully saturated ones. This is attributed to a lower short circuit current possibly related to a lack of dye regeneration. However, the $V_{o c}$ and $F F$ of the devices is approximately the same independently the degree of electrolyte saturation.

This work highlight the importance of porosity on device fabrication and associated performance.

\subsection{Towards Glass/TCO-free electrodes}

The CNTf layer used as CE is sufficiently conducting to act as CC and is strong/stiff enough to be self standing, flexible and even leading to structural properties. These properties of CNTf not only are sufficient for replacing the TCO at the $\mathrm{CE}$, but also open the possibility for the CNTf to host the electrolyte in its internal pores. The electrolyte filled CNTf would act as a composite membrane, leading to a substantially simplify the DSSC assembly process.[270]

This aspect was studied by fabricating CNTf free-standing devices produced by making direct contact between an electrolyte filled CNTf with the $\mathrm{TiO}_{2}$ photoanode as presented in the schematic in the Figure 7.3a. A thin polymeric film is hotpressed between electrode, avoiding the photoactive area, to keep permanently assembled the device. The electrical contact can be made directly on the CNTf and the device tested as a DSSC (see Figure 7.3b,c).

The device performance was characterised at 1 sun (AM 1.5G conditions). I$V$ curves were recorded every 10 seconds starting a few minutes after finishing the device assembly (Figure 7.4a). 


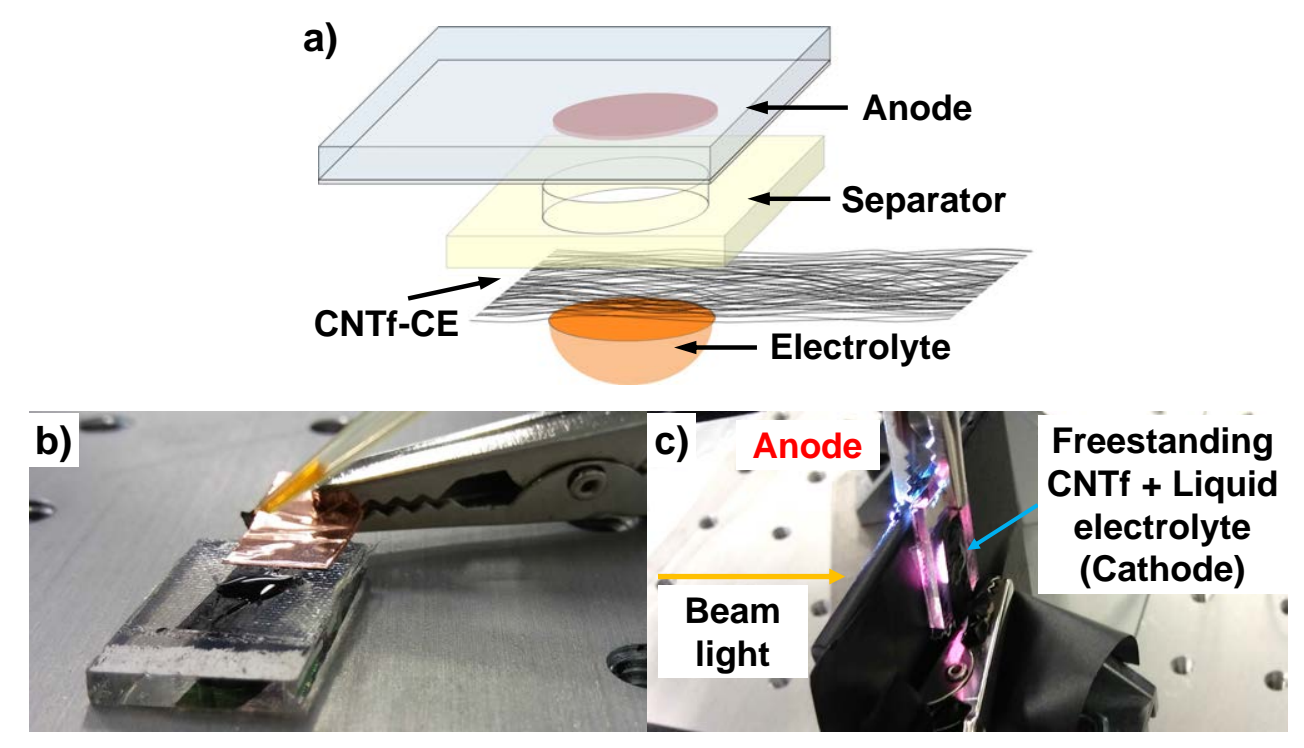

FIGURE 7.3: a) Schematic of the proposed architecture for a freestanding-CNTf membrane used as both CE and current-collector without further substrate. b) Example of a free standing CNTf-CE device. d) Characterising a free-standing CNTf-CE DSSC in the solar simulator with the electrical contact made directly on the CNTf.

The initial performance is close to $3 \%$, with $I_{s c}=1.8 \mathrm{~mA}$ and $V_{o c}=0.7 \mathrm{~V}$. As shown in Figure 7.4b, the efficiency after three minutes drops more than $50 \%$ of the initial performance. Assuming a linear decay, the data show rates of $-0.7 \% P C E / \mathrm{min}$ (efficiency loss per minute) and $-0.3 \mathrm{~mA} / \mathrm{min}$ (short circuit current loss per minute). Indeed, extrapolating the efficiency and current to the initial point of assembly, 3-5 minutes before the first data point, a performance of $6-7 \%$ is predicted, which is in line with results on previous chapters.

This efficiency drop is directly linked with the evaporation of the solvent under air exposure as was confirmed by direct observation and by re-filling the cells. The standard for DSSC used here is composed of acetonitrile and valeronitrile, both solvents with low boiling point. Solvent evaporation increases the electrolyte viscosity and reduces ionic conductivity. In this respect, note that DSSC with quasi-solid electrolyte, that is the case of BMII electrolyte after evaporating the solvent, leads to below $3 \%$ (examples included in the Chapter 2 "Background").

With this in mind and seeking better performance and stability, further research with different solvent based electrolyte or solid-state electrolyte are proposed as future work.

In order to prevent from liquid electrolyte leakage and evaporation, CNTf-CE was deposited on thin layer of polyethylene terephthalate (PET) film of $200 \mu m$-thick, as presented in the Figure 7.5a.

A set of devices of $0.2 \mathrm{~cm}^{2}$ is prepared using the CNTf-CE encapsulated with a layer of PET $200 \mu m$-thick as substrate. A representative $J$ - $V$ curve of these devices is shown in Figure 7.5b. The obtained efficiency is in the range of $5 \%$, the $V_{o c}=0.78 \mathrm{~V}$, the $J_{s c}=11.25 \mathrm{~mA} \mathrm{~cm}^{-2}$ and the $F F=56 \%$. 


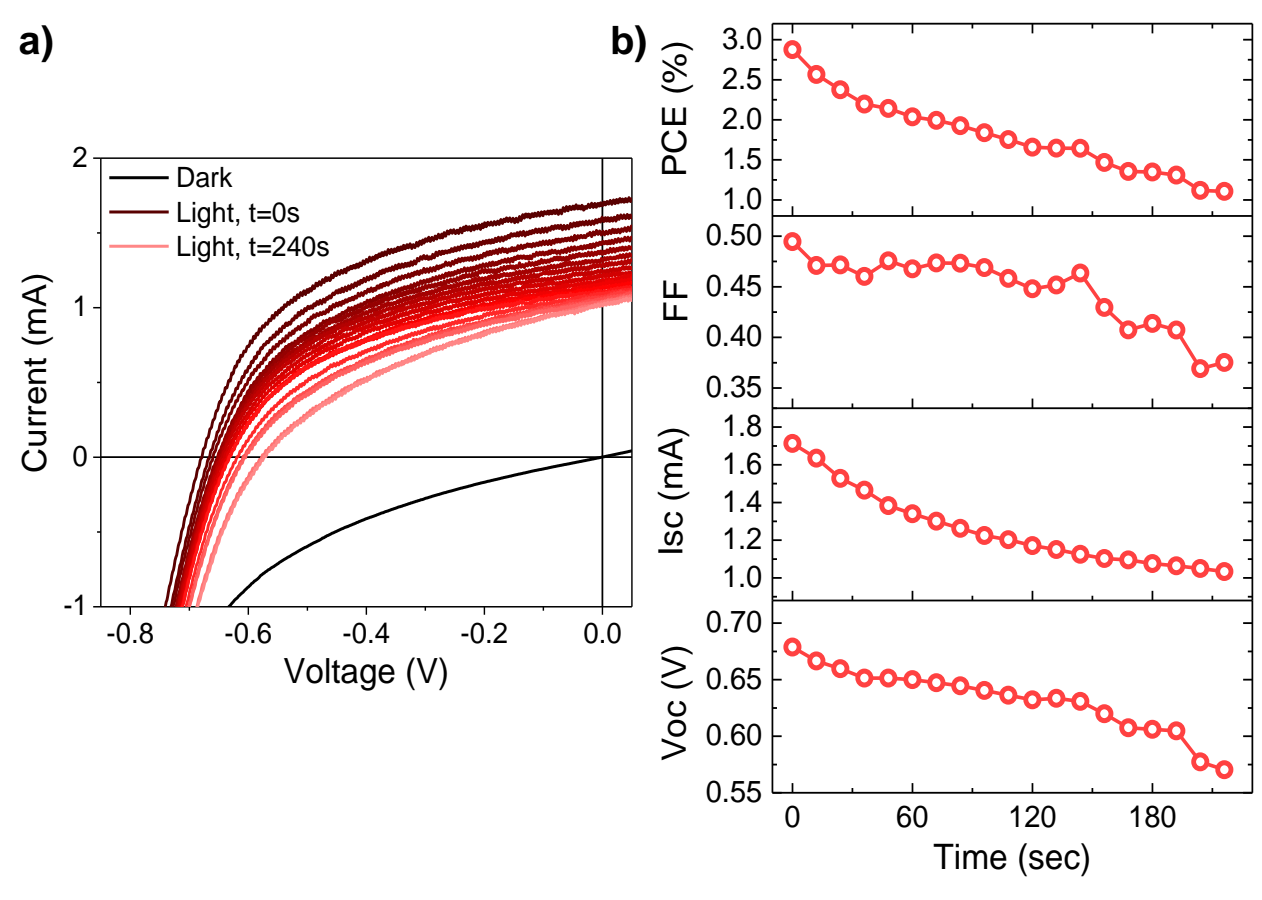

FIGURE 7.4: Photovoltaic characteristics of the free-standing CNTfCE DSSC. a) $J-V$ curves for the initial minutes of light soaking after saturating the CNTf with liquid electrolyte. b) DSSC characteristics evolution as function of the light soaking time.

This last device configuration, avoiding the direct evaporation of the electrolyte, resulted in a stable performance. A stable $J-V$ curve during the initial five minutes was measured. Stability over time similar to the CNTf-CE supported on FTO was confirmed after measuring a constant performance during the following days.
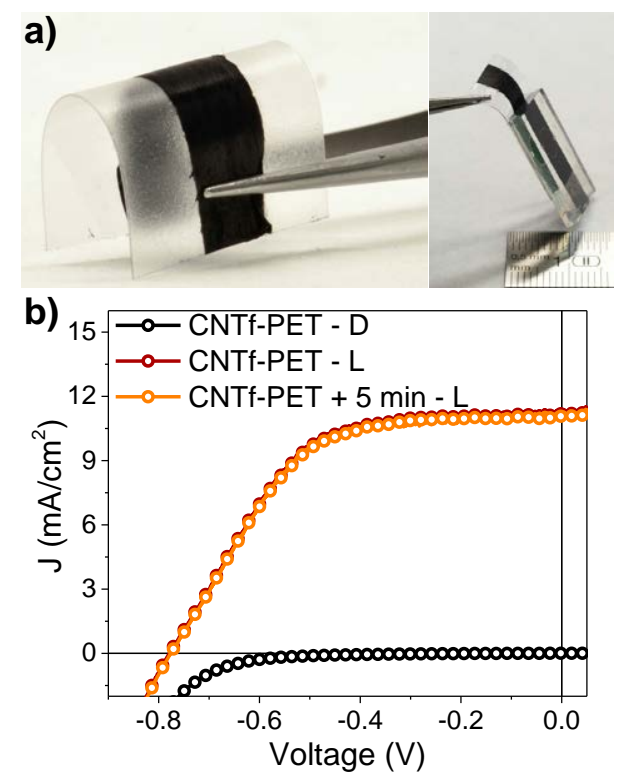

FIGURE 7.5: a) Flexible CNTf/PET CE and assembled DSSC. b) Representative $J-V$ curves of the CNTf/PET device. 


\subsection{PVDF/CNTf-CE nanocomposite for tough electronics DSSC devices}

The previous results show that a thin polymer layer over the CNTf-CE can successfully prevent electrolyte evaporation or leakage. If provided a flexible photoanode, that can be produced as reported for instance elsewhere,[351] this will easily lead to a flexible DSSC with CNTf-CE.

However, it is also of interest to consider the possibility of the DSSC becoming a semi-structural element. With this objective in mind, structural materials (e.g. carbon fibre, CF) have been used as CE for DSSC,[77] and the mechanical properties of some devices have probed to be sufficient to behave as structural photovoltaics in its elastic regime.[352]

CNTf/PVDF nanocomposite samples were used as CE for DSSC applications and compared with a standard structural material, carbon fibre reinforced polymer composite (CFRP) (schematics depicted in Figure 7.6a,b). Fabrication and characterisation details are presented in the Appendix A. Summarising, CF composites are materials composed by carbon fibres reinforcing an epoxy matrix. These materials are nowadays widely used for structural elements due to their light weight and high mechanical performance. The CNTf nanocomposite presents similar structure and mechanical properties but showing higher electrical performance and much larger surface area. For the CNTf acting as a multifunctional material, with structural properties and at the same time CE for DSSC, their catalytic performance must be investigated.

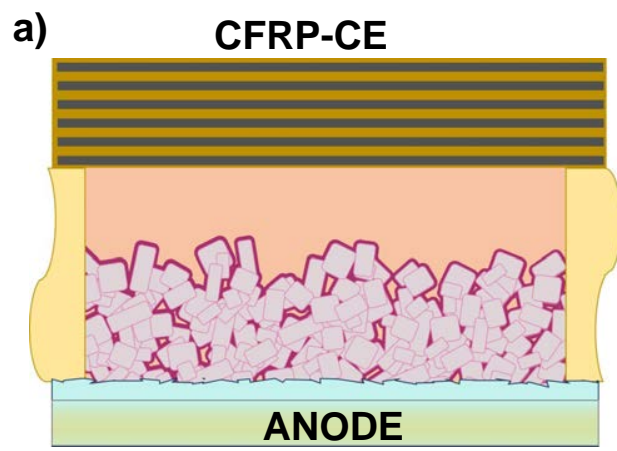

b) CNTf/PVDF-CE

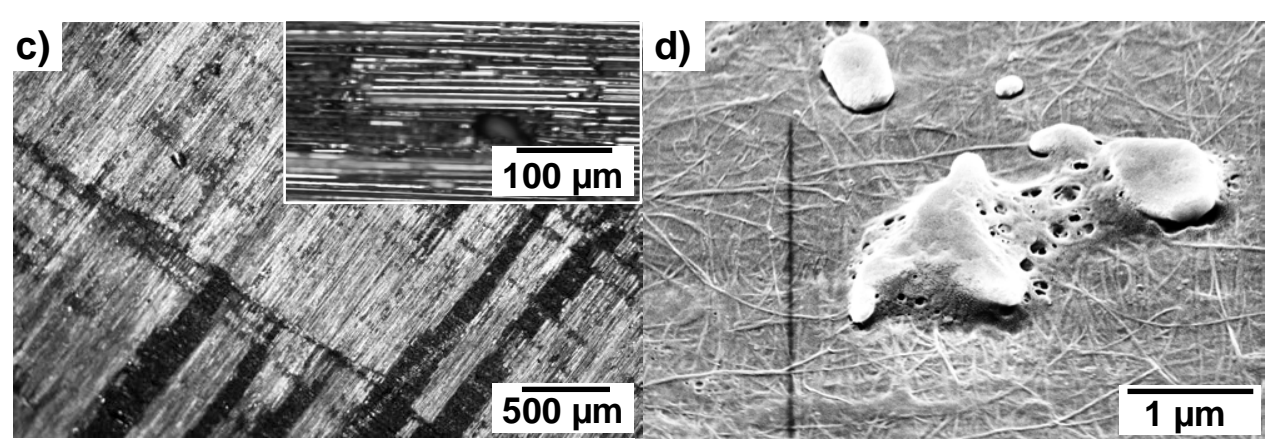

FIGURE 7.6: DSSC schematics implementing a) CF composite and b) CNTf nanocomposite as CE. c) Optical image of the surface of CF composite and d) SEM image of the surface of CNTf nanocomposite. 
Besides, the CNTf nanocomposite presents a thin superficial coating of polymer but electrical measurements show that this surface is still conductive. Similarly, the CF composite, due to the manufacturing process, also ends up with an external resin skin, although in this case this layer is thicker and insulating. This superficial polymer is removed by surface sanding for few second before any further measurement. Images of the surface of CF composite and CNTf nanocomposite used as CE are presented in the Figure $7.6 \mathrm{c}, \mathrm{d}$.
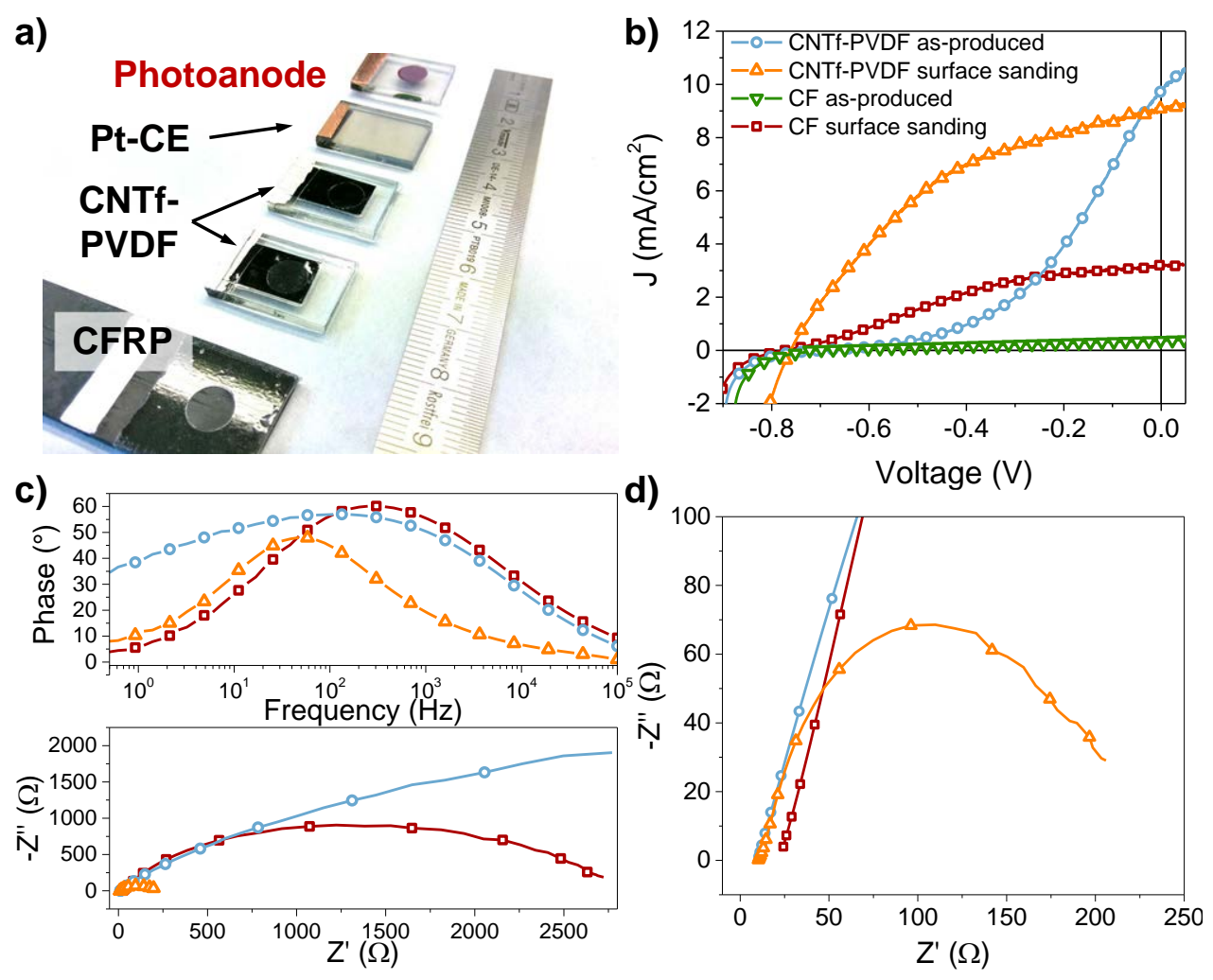

FIGURE 7.7: a) CNTf nanocomposite compared with CFRP and Pt counter-electrodes for DSSC. b) J-V curves of these materials. c) Bode phase and Nyquist plots. d) Nyquist plot magnified region showing the sanded CNTf nanocomposite. The legend in graph $b$ also refers to graphs $c$ and $d$.

The performance of CNTf/PVDF $15 \% w t$ is compared with a piece of a cured laminate of standard carbon fibre/epoxy composite (CFRP) as CE. This materials, in addition to the standard $\mathrm{Pt}-\mathrm{CE}$ material and $\mathrm{a} \mathrm{TiO}_{2}$ photoanode, are presented in the Figure 7.7a.

The $J-V$ curves of both materials are presented in the Figure 7.7b. The devices are prepared following the standard procedure but using office clamps for the nonpermanent assembly of the device. The area of $0.2 \mathrm{~cm}^{2}$ for the CE-CC materials is ensured by masking with Scotch tape the electrodes. The CF material presents a performance of approximately $0.9-1 \%$ with average characteristics of $J_{s c}=3.2 \mathrm{~mA} \mathrm{~cm}^{-2}$, $V_{o c}=0.78 \mathrm{~V}$ and $F F=35 \%$. For the as-made CNTf nanocomposite a similar low performance $(0.8 \%)$ is obtained but attributed to a different behaviour. The observed S-shape leads to a $F F$ of $10-12 \%$, with a similar $V_{o c}=0.7 \mathrm{~V}$ but a $J_{s c}=10 \mathrm{~mA} \mathrm{~cm}^{-2}$ 
significantly higher. This high sort circuit current suggests the higher catalytic activity of the CNTf in comparison to the CF but also lack of catalytic active sites due to the saturated porosity and reduced surface area of the CNTf by the polymer infiltration. The CNTf, after a similar sanding process than than the applied to the $\mathrm{CF}$ composite electrode, results in a performance substantiallyhigher, in the range of $3.5 \%$. This improvement is attributed to an enhanced FF up to $45 \%$ and slightly increased $V_{o c}=0.76 \mathrm{~V}$ because of the reduction of the S-shape of the material. This suppression of the S-shaped profile is an important result confirming that the Sshaped curve is obtained due to the lack of catalytic active sites.

Finally, this improved performance of the CNTf nanocomposite in comparison to the CFRP are supported by the EIS measurements depicted in the Figure $7.7 \mathrm{c}, \mathrm{d}$. The cell resistance of the CNTf nanocomposite CE (after sanded) is one order of magnitude smaller than the $\mathrm{CF}$ composite attributed to the higher electrical conductivity of the CNTf nanocomposite. These impedance results are similar to those of the pristine CNTf material, depicting a unique electrical interface that convolutes the charge transfer process at the $\mathrm{CE}$ and the electron transfer at the $\mathrm{TiO}_{2}$ /electrolyte interface.

\subsection{Overcoming issues with transparency enabled by mechan- ical properties}

Besides the rationalised CNTf-CE design, the intrinsically poor optical transmittance of CNTs can easily be overcome. By assembling two photoanodes facing each other in a sandwich-like design, the free-standing CNTf-CE can act as unipolar or unifacial counter-electrode/current-collector without losing performance for each cell. This new architecture is presented in the Figure 7.8a.

As shown in the Figure 4.13b, the remaining bottleneck aspect of using CNTfCEs is the significant loss of transparency, limiting the possibility of illuminating the DSSCs from both sides with a back-reflector as in one of the possible conventional configurations of solar cells. However, the mechanical, electrical, and catalytic features of CNTf-CEs allow to introduce the free-standing CNTfs sandwiched between two photoanodes (see Figure 7.8b).

The device consisted in two $5 \mathrm{~mm}$ diameter photoanodes $\left(0.4 \mathrm{~cm}^{2}\right.$ photoanode surface in total) and $\approx 20 \mu \mathrm{m}$-thick CNTf-free-standing-CE. The optimised thickness of the CNTf from previous Chapter 4 is here multiplied by two to ensure that there is enough mass of CNTf (proportional to the number of cataytic centres) and thus avoid the previous mention S-shaped J-V curve. $60 \mu \mathrm{m}$ Surlyn gasket was placed separating the CNTf film from each photoanode (see Figure 7.8a). For measuring the unipolar device, both faces of the cell where individually characterised in the solar simulator under the standard procedure. For measuring both sides at the same time, a concave mirror was used to illuminate the cell from both sides, and a wire was used to connect both photoanodes in parallel configuration - front and back. The position where the back-reflected light had an intensity of 1 sun was also determined with the silicon reference.

Without interconnecting the photoanodes, each cell features similar $F F$ of $62 \%$, photocurrents $(I)$ of 2 and $2.6 \mathrm{~mA}$, and $V_{o c}$ of $0.74 \mathrm{~V}$ and $0.63 \mathrm{~V}$. Upon connecting the anodes in parallel and illuminating both sides under 1 sun (AM 1.5G) conditions, the 
device response features an overall I of $5 m A, V_{o c}$ of $0.65 V$ and FF of $60 \%$, leading to an overall device PCE of $5 \%$, similar to that of each independent cell.

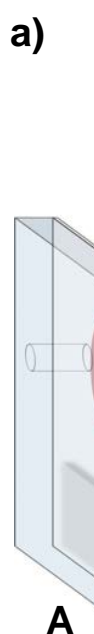

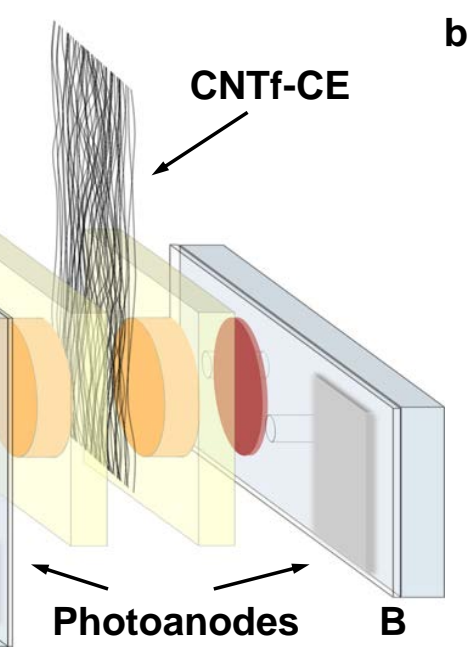

b)
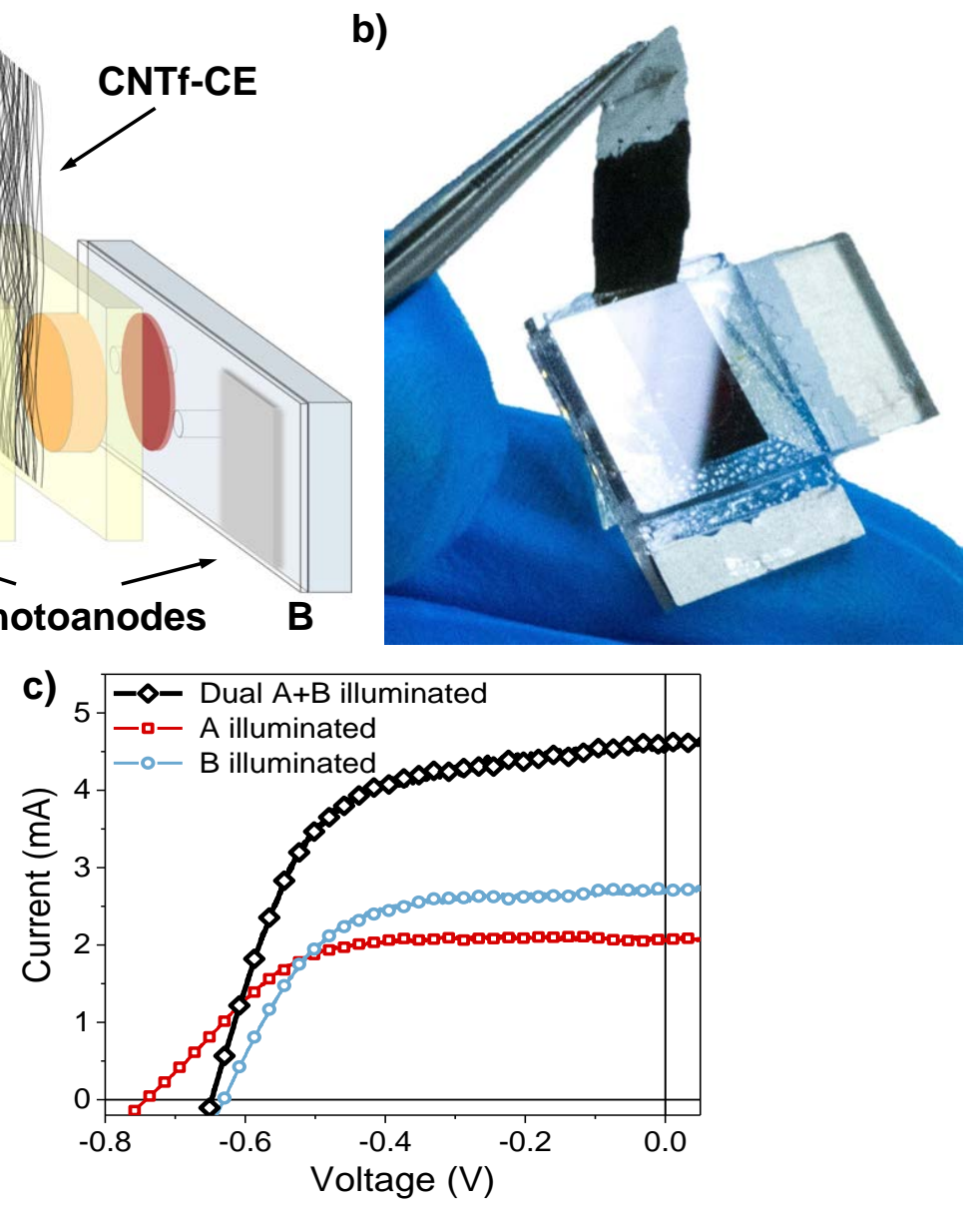

FIGURE 7.8: a) Schematic, b) digital photograph and c) $I-V$ curves of DSSCs with individual and dual side illumination.

As it is observed in the Figure 7.8c, both individual cell has slightly different behaviour. This is common to all tested devices and attributed to the lack of symmetry of the device. Due to the CNTf is a flexible substrate, it is almost impossible to keep both distances the same, photoanode-A and photoanode- $B$ to the CE. Likewise, the differences in electrolyte saturation between both anodes lead to inhomogeneous working of both sides when they are independently measured. For avoiding that, filling with electrolyte both sides of the cell helps to completely saturates both anodes.

A final important consideration of this architecture is related with the lower losses of illuminating two photoanodes versus one photoanode from both sides (standard configuration). When a conventional DSSC is illuminated from both sides with a back reflector, the light has to travel through the electrolyte layer. The standard electrolyte based on $I^{-} / I_{3}^{-}$redox couple has and important absorbance band centred in $475 \mathrm{~nm}$, thus, the back illumination of the cell involves the loss of almost half of the visible light spectrum compared with the front illumination. In the case of the unipolar device, the light reaching the front or back anode of the cell has the same intensity and components with no losses due to the absorption of the electrolyte. 


\subsection{Electrical aspects: proof-of-concept mini-module}

\subsubsection{Photoanode size-dependence effect of planar CNTf-CE DSSC}

Scaling up DSSC devices involve the study of the resistive effects related to the size increment. In general, increasing the size of the device affects severely the efficiency.[353-355] It leads to a severe impact on the FF and, to a lesser extent, on the $J_{s c}$ of the device. This represents one of the main difficulties of increasing the area of the PV devices. This is typically explained in terms of power dissipation of the solar cell due to the electrode series resistance, in particular, the series resistance of the front electrode TCO (FTO in our case). The power losses per unit of area $\left(P_{R}\right)$ due to the series resistance are given by

$$
P_{R}=R_{s} A J_{m p p}^{2}
$$

where $A$ is the cell area, $R_{S}$ is the series resistance, and $J_{m p p}$ is th current density at the maximum power point.

When the size (area) of a solar cell increases, the resistance of the currentcollector increases proportionally. At some point, the losses due to this additive series resistance must be considered.
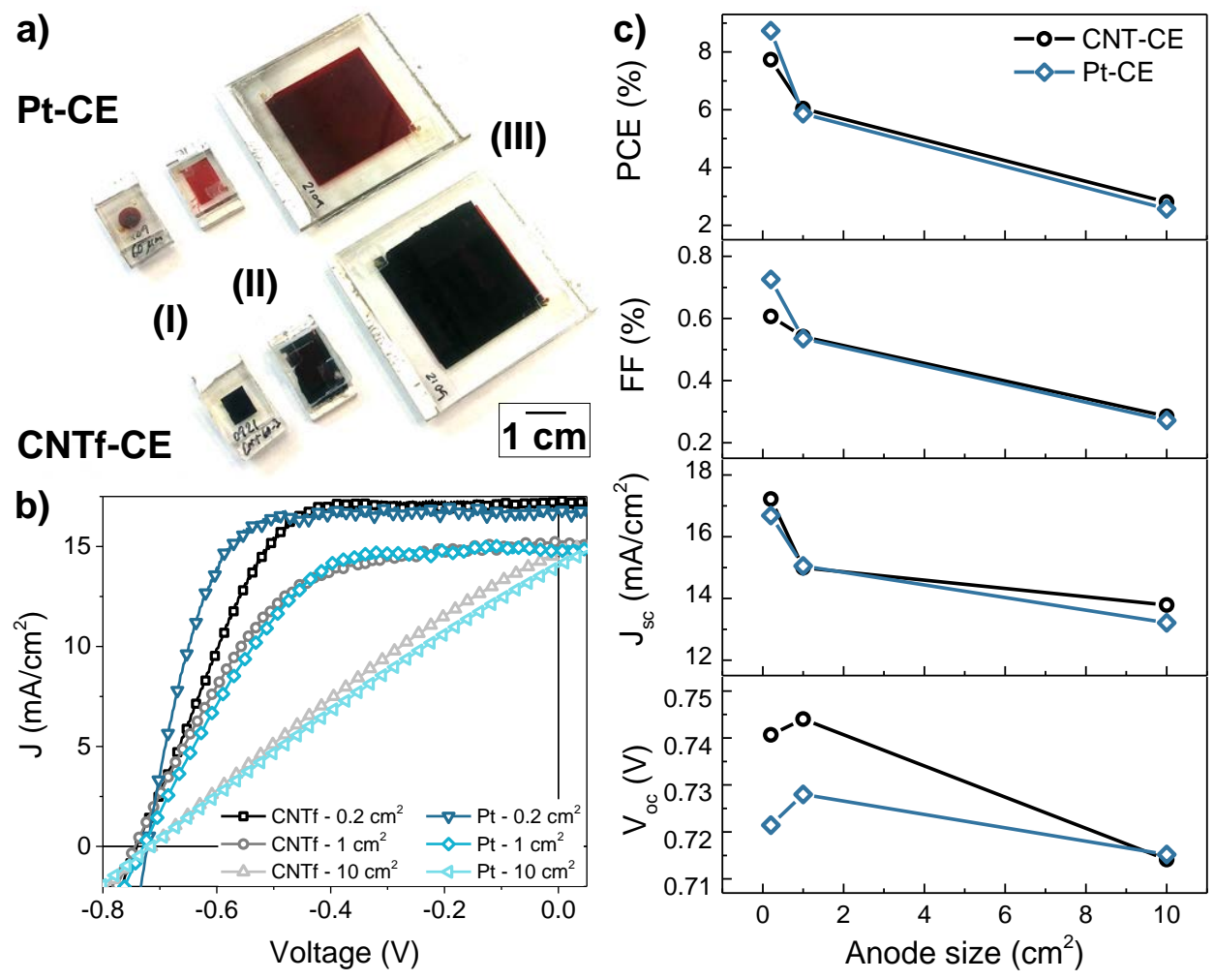

FIGURE 7.9: a) Photograph showing the three different sizes of photoanode studied, (I) $=0.2 \mathrm{~cm}^{2}$, (II) $=1 \mathrm{~cm}^{2}$ and (III) $=10 \mathrm{~cm}^{2} . \mathrm{b}$ ) $I-V$ curves of CNTf- and Pt-CE for these mentioned areas. c) DSSC characteristic $v s$ pohotoanode area. 
This mention drop in performance as function of the photoanode size is shown in the Figure 7.9, where a comparison between different size DSSCs is presented (Figure 7.9a). J-V curves corresponding to (I) 0.2 , (II) 1 and (III) $10 \mathrm{~cm}^{2}$ devices (Figure $7.9 \mathrm{~b}$ ) shows a clear trend of the $J_{s c}$ and $F F$ decreasing linearly with the increment of active size (see Figure 7.9c). On the contrary, the $V_{o c}$ remains almost constant, generating 740 and $720 \mathrm{mV}$ for the $0.2 \mathrm{~cm}^{2}$ cells and 728 and $735 \mathrm{mV}$ for the $10 \mathrm{~cm}^{2}$, CNTfand $\mathrm{Pt}-\mathrm{CE}$ respectively. This is in agreement with previous references showing the same trend of the characteristics in organic solar cells.[353-355] Notice that, the drop of performance from the initial $0.2 \mathrm{~cm}^{2}$ to $1 \mathrm{~cm}^{2}$ for the CNTf and the Pt reference, from $P C E_{C N T f-0.2 \mathrm{~cm}^{2}}=7.7 \%$ and $P C E_{P t-0.2 \mathrm{~cm}^{2}}=8.7 \%$ to $P C E_{C N T f-1 \mathrm{~cm}^{2}}=6.2 \%$ and $P C E_{P t-1 \mathrm{~cm}^{2}}=5.8 \%$.

To further understand the size dependence effect of the photoanode, the series resistances are calculated. For the calculation of $R_{s}$, a method based on the voltage difference between dark and 1-sun $J$ - $V$-curves was applied to the $0.2 \mathrm{~cm}^{2}$ and $1 \mathrm{~cm}^{2}$ $J-V$ characteristics. $R_{s}$ for the $10 \mathrm{~cm}^{2}$ devices have been calculated from $J-V$ curves at different light intensities. The description of this methods and analysis are explained in the Chapter 3 "Experimental Techniques".

The product of the series resistance per area $\left(R_{S} A\right)$ and power losses $\left(P_{R}\right)$ are shown $v s$ the photoanode area in Figure 7.10. With these series resistances, it is possible to calculate the power losses according to the Equation 7.2. It can be concluded that power loss increases proportionally with the series resistance and therefore the size of the device. The CNTf-CE behaves in all aspects as good as Pt, with the benefit of acting as CC. All the extracted characteristics from the photoanode size dependence are presented in the Table 7.1.

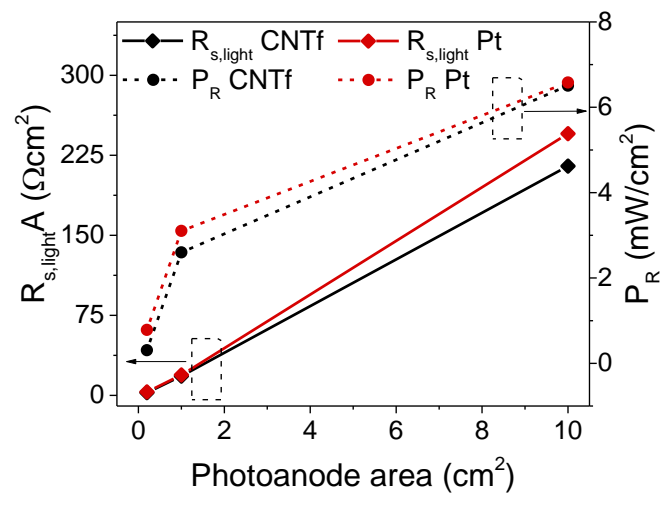

FIGURE 7.10: Series resistance and power loss vs photoanode area.

The solution for this well known size dependence power loss in the PV technology consists on depositing a metallic grid on the surface of the photoanode for decreasing the series resistance of the electrode. This solution is a trade-off between minimising the resistance by decreasing the metal size grid and maximising the transparency by increasing this size. But, this optimisation of the device falls out of the objectives of this thesis. 
TABLE 7.1: $V_{o c}, J_{s c}, F F, P C E, P_{R}$ and $R_{s, l i g h t} A$ characteristics of different size photoanode DSSC devices.

\begin{tabular}{|c|c|c|c|c|c|c|c|}
\hline Devices & $\begin{array}{l}\text { Size } \\
\mathrm{cm}^{-2}\end{array}$ & $\begin{array}{l}V_{o c} \\
V\end{array}$ & $\begin{array}{l}J_{s c} \\
m A c^{-2}\end{array}$ & $\begin{array}{l}F F \\
\%\end{array}$ & $\begin{array}{l}P C E \\
\%\end{array}$ & $\begin{array}{l}R_{s, \text { light }} A \\
\Omega \mathrm{cm}^{2}\end{array}$ & $\begin{array}{l}P_{R} \\
m W c^{-2}\end{array}$ \\
\hline \multirow{3}{*}{ CNTf-CE } & 0.2 & 0.74 & 17.2 & 60 & 7.7 & 2.6 & 0.3 \\
\hline & 1 & 0.74 & 15 & 55 & 6.1 & 18 & 2.6 \\
\hline & 10 & 0.72 & 6.2 & 26 & 1.2 & 214.8 & 6.5 \\
\hline \multirow{3}{*}{ Pt-CE } & 0.2 & 0.72 & 16.7 & 72 & 8.7 & 2.4 & 0.7 \\
\hline & 1 & 0.72 & 15.1 & 53 & 5.8 & 19.1 & 3.1 \\
\hline & 10 & 0.73 & 5.7 & 25 & 1.1 & 245.3 & 6.6 \\
\hline
\end{tabular}

\subsubsection{CNTf-CE mini-module: resistive losses and size-dependence}

As a further proof of innovative cell assembly methods enabled by the use of these CNTf-CEs, and taking advantage of the large CNTf production capacity on account of the synthesis method - ca. $10 \mathrm{~km} /$ day, a large-area DSSC mini-module was investigated. This was fabricated covering 1 meter long with 10 photoanodes $\left(1 \mathrm{~cm}^{2}\right.$ each) connected in parallel featuring an average PCE comparable to the state-of-theart carbon-based DSSC module.[356] This proposed solution avoids the increase in series resistance associated to bigger size photoanodes.

Technically, this large-area DSSC can be considered as a module because it is composed by several individual cells independently sealed and interconnected in parallel.[189] When manufacturing a PV-module, several connection schemes of the individual cells can be used. In summary, series, parallel connection or a combination of both (monolithic, Z-type, W-type among others) are the different options. Series connection of the cells imply a unique path for the current to travel along the circuit and the final voltage is the additive voltage of each individual cell. In a parallel connection, there are multiple paths for the current to move along the circuit, producing and additive current. This last is the case of the module presented in the Figure 7.11, where the current is additive when all the cells are interconnected.

A proof-of-concept mini-module is presented in the Figure 7.11a. The assembly method consist on fabricating individual photoanodes of $1 \mathrm{~cm}^{2}(1.25 \mathrm{~cm} \times 0.8 \mathrm{~cm})$ and directly placing them along a continuous strip made of CNTf. A layer of thermoplastic film (Surlyn) was used to secure the anodes to the fibre and seal the electrolyte by hot-pressing each photoanode onto the fibre CE/CC. The back side of the cells were sealed with a piece of PET for avoiding the entrance of humidity and the evaporation of solvent.

The mini-module CNTf-CE DSSC was characterised outdoor because the required illuminated area was larger than one metre. Certified silicon reference cell was used for ensuring 1 sun as external illumination power density during all the measurements. The photoanodes were connected in parallel with an external wire for extracting the photogenerated charges. Although the CNTf is acting as CE and CC for every cell, an additional wire was also used for connecting in parallel all the devices. The experimental setup is presented in the photograph and schematic included in the Figure 7.11a,c. 

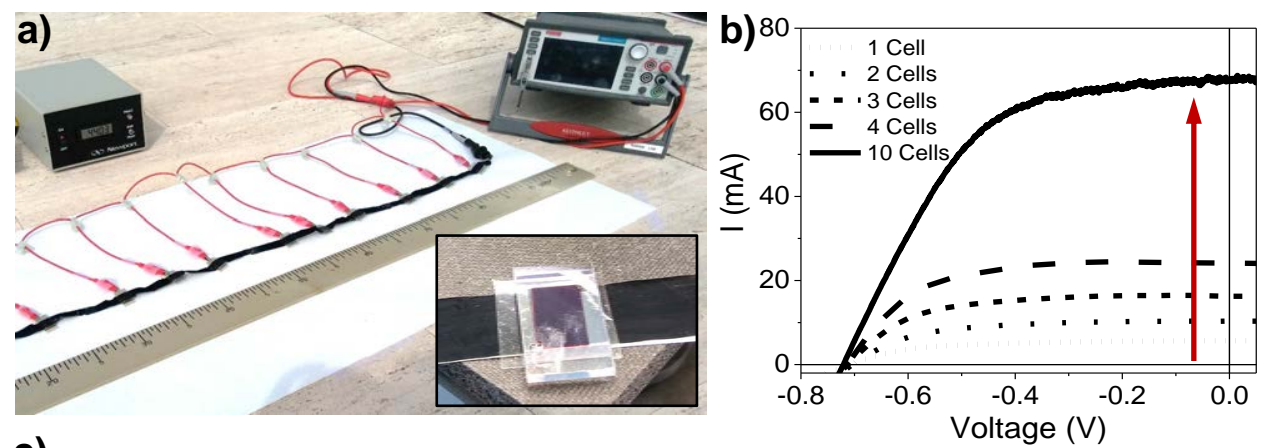

c)

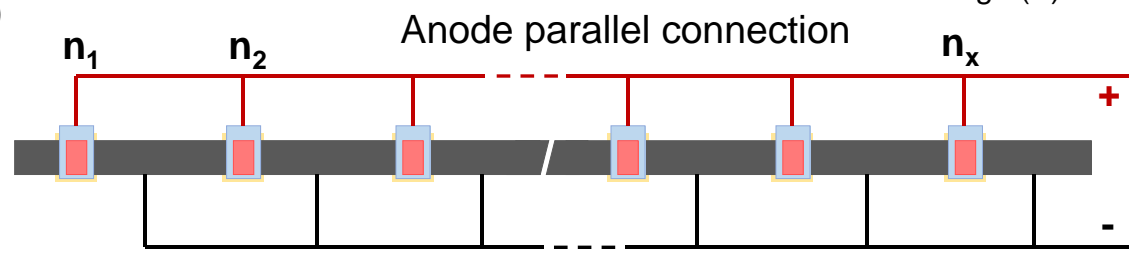

Cathode parallel connection

FIGURE 7.11: a) Digital photograph of a module with 10 sequentially parallel connected DSSCs along 1 metre-long CNTf-CE. Inset: closer view of an individual $1 \mathrm{~cm}^{2}$ CNTf -DSSC of the module and b) $I-V$ curves of the sequentially connected devices. c) Schematic of the topview of the mini-module connection for the measurement.

The individual cells were characterised after visual inspection and rejecting the cells showing lack of electrolyte filling or construction defects. They featured average characteristics of $J_{s c}=10 \pm 2 \mathrm{~mA} \mathrm{~cm}^{-2}, V_{o c}=0.73 \pm 0.3 \mathrm{~V}, F F=60 \pm 5 \%$, and $P C E=4.5 \pm 0.5 \%$.

The mini-module was connected in parallel and characterised under calibrated 1 sun outdoor conditions (Getafe, 10/10/2017). The device features $J_{s c}$ of $7 \mathrm{~mA} \mathrm{~cm}^{-2}$ or $I$ of $69 \mathrm{~mA}$ that results from the sum of each individual cell, $V_{o c}$ of $0.72 \mathrm{~V}, \mathrm{FF}$ of $53 \%$ and an overall PCE of $2.7 \%$ (Figure 7.11b). Noteworthy, this proof-ofconcept device showed PCE values that are comparable to the state-of-art graphenebased DSSC modules,[356] as well as other optimised carbon-based modules (average $P C E=4.5 \%$ ).[356-360]

This proposed photovoltaic architecture has several advantages against other traditional architectures:

- The counter-electrode acts at the same time as current-collector of every individual cell.

- Although the photoanodes are built onto glass substrates, they are interconnected from each other by the CNTf, thus the final module is a flexible unit.

- The continue fibre geometry of the module preserves the mechanical properties of the CNTf, leading potentially to integrating them into composite materials.

- Using CNTf-CE/CC also has some potential industrial manufacturing advantages, for instance, it is more convenient having the conductor material integrated with the current collector in a flexible shape where some conventional manufacturing techniques as roll-to-roll can be applied. 
However the obtained efficiency falls short of that observed in small area (ca. $0.2 \mathrm{~cm}^{2}$ ) cells discussed in the earlier sections and chapters. This is attributed to at least two identified effects:

- The charge extraction is limited by resistive effects of the photoanode. This is presented in the previous section and entails $\approx 35 \%$ of the loss of efficiency.

- The CNTf-strip-architecture limits the charge extraction along the one meter CNTf current-collector (resistive effects).

With the aim to elucidate the influence of the CNTf-CE/CC length on the performance, the resistive effect of the CNTf electrode was tested.

In a first attempt, the CNTf mini-module presented in the Figure 7.11a, was characterised using directly the CNTf as final current-collector (without cathode external wire depicted in Figure 7.11c). Interestingly, the performance achieved by this configuration was equivalent to 3-4 devices.

To understand this behaviour a sequence of measurements over the same device were performed. The experiment consist on increasing the distance between the illuminated cell (1 sun conditions) and the connection of the cathode/currentcollector. This is schematically depicted in the Figure 7.12a, in where the cathode of device under test (DUT) is connected at 1,25, 50 and $100 \mathrm{~cm}$.

The obtained $J-V$ curves are shown in the Figure $7.12 \mathrm{~b}$ and the extracted characteristics summarised in the Figure 7.12c.
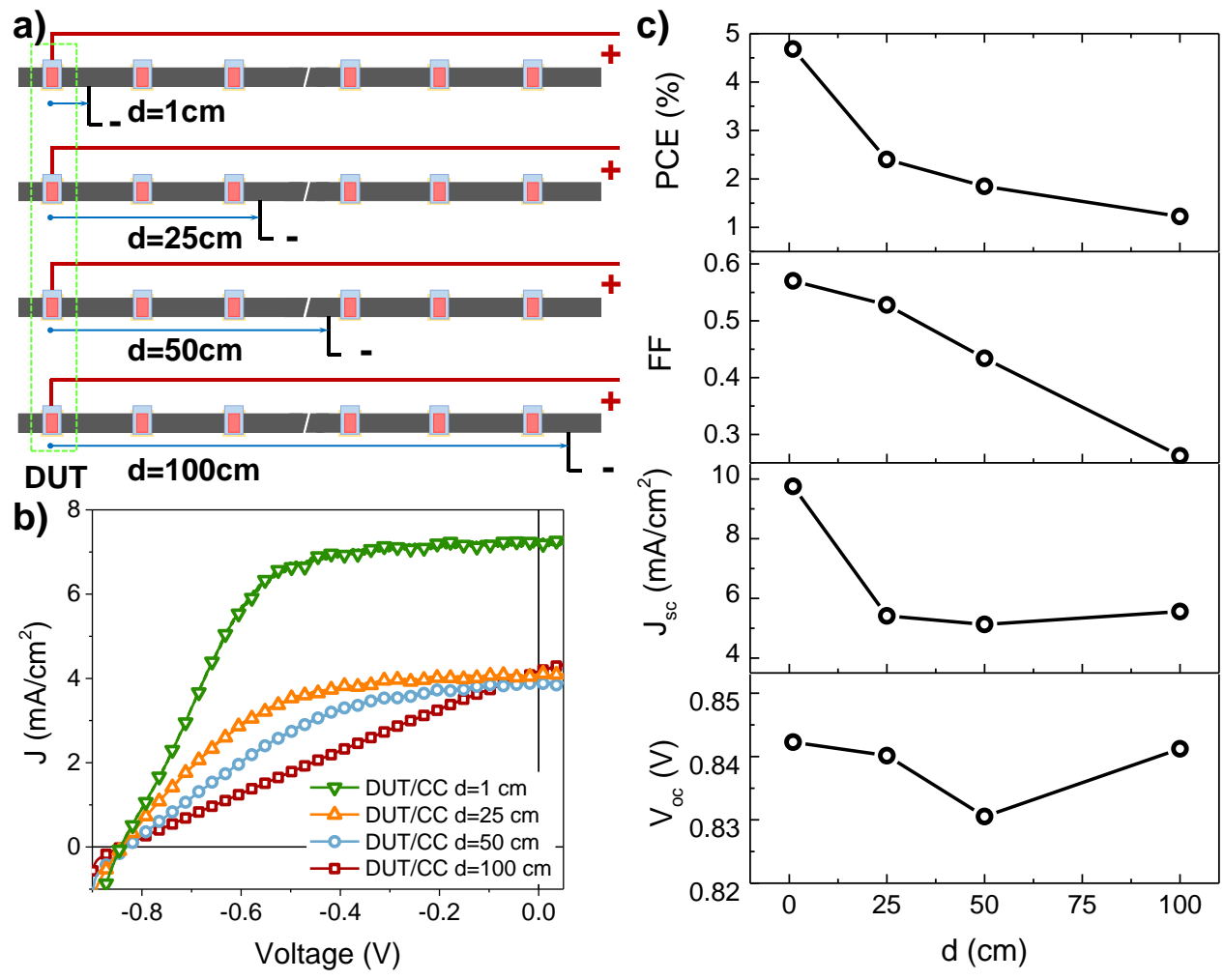

FIGURE 7.12: a) Schematic of the measurement. The current-collector is connected at different distances from the DUT (device under test). b) $J-V$ curves showing the dependence with the distance. c) Characteristics extracted from the $J-V$ curves $v s$ distance. 
The first point to highlight is the significant reduction in short circuit current density. Interestingly, this $J_{s c}$ reduction is not proportional to the distance, it shows a main drop when the current flows through the next assembled device and remains constant for subsequent measurements. Moreover, the constant behaviour of the $V_{o c}$ and the linear decrease of the fill factor suggest that the performance is limited by a purely resistive effect. The series resistance for the four measurements resulted 0.8, $4.5,9,18.3 \Omega \mathrm{cm}^{-2}$ for $1,25,50$ and $100 \mathrm{~cm}$ respectively. The lineal increase in the series resistance explains the efficiency loss by proportionally decreasing the FF of the mini-module. As said before, interconnecting the CNTf-strip/current-collector with an additional wire the series resistance is kept small, thus diminishing this source of resistive losses.

\subsection{Summary and conclusion}

The importance of the electrolyte filling process for CNTf-CE DSSCs has been discussed. Preventing the air pockets and incomplete membrane infiltration is a key point for obtaining the maximum performance of the device. The bulk electrolyte infiltration, as a first approximation, is analysed following Lucas-Washburn equation, showing that infiltration times longer than 10 minutes are possible. This is in agreement with experimental observations.

New device architectures based on the CNTf-CE has been proposed. The freestanding CNTf-CE, although presenting low efficiency related to the low stability of the liquid solvent-based electrolyte, has shown potential benefits in view of large scale manufacturing processes like the roll-to-roll. Further work optimising the electrolyte stability under air conditions is proposed.

The encapsulation with flexible substrate is an alternative manner of stabilising the electrolyte embedded in the CNTf membrane. With this solution, the flexibility of the CNTf is preserved, resulting in flexible CE. In this line, CNTf/PVDF nanocomposites, exhibiting semi-structural mechanical properties, has been used as CE. The CNTf surface has been exposed by sanding procedure and the S-shaped profile of the $J-V$ curve has been reduced (improving efficiency and $F F$ ). This is a direct result proving the relation between exposed catalytic surface of the CNTf and the S-shaped. The controlled surface polymer solution could be used for selectively exposing certain amount of CNTf surface possibly without hampering the overall mechanical properties of the nanocomposite.

One of the new configurations proposed is the bifacial DSSC. This tackles the lack of transparency of the CNTf by sandwiching the CNTf membrane in between two photoanodes. As has been shown, both faces of the device work independently with similar performance, but when connecting them and illuminating both faces, the current of the device is nearly doubled. This solution allows the illumination of the CNTf-CE devices from both sides.

Furthermore, proof-of-concept mini- module has been fabricated using the CNTf as direct CE/CC. Several independent photoanodes connected over 1 metre length CNTf-strip, has shown a total efficiency of $2.7 \%$ close to other optimised nanocarbon based DSSC modules recently reported by other groups. The size dependence effect on these solar modules and large area devices $\left(>10 \mathrm{~cm}^{2}\right)$ has been analysed, attributing power losses to resistive effects. 



\section{Chapter 8}

\section{Conclusions and Future Directions}

The aim of this thesis has been the implementation of the CNTf as currentcollectors for energy harvesting applications, and more importantly, understand the charge transfer processes occurring at the surface of this material.

CNT fibres presents a combination of properties, such as its high conductivity, mechanical properties in the high performance range, open and interconnected porosity, high surface area and chemical and electrochemical stability, that makes the material suitable for combining with liquid electrolytes, semiconductors or polymers, among other materials. Moreover, the mechanical interaction and electrical charge transfer at the interface between these materials are completely different. Because of the noverlty of the CNTf, all these interactions raise many fundamental questions, and providing answers to some of them have been in the objectives of this thesis.

As shown in the background chapter, among the alternative energy harvesting technologies, the photovoltaic technology is nowadays one of the most promising for capturing ambient energy. There are multitude of solar technologies, and DSSC has been the one selected to study in this thesis. They have shown a rapid development during the last two decades but also is a well understood platform for introducing new material for improving the actual performance.

In view of all these elements, the CNTf has been implemented into the different parts of the DSSC. Summarising, by using the CNTf and CNTf nanocomposites as $\mathrm{CE}$ and $\mathrm{CC}$, the properties of the material as well as, the charge transfer phenomena in the catalytic reaction towards iodide/triiodide redox couple have been investigated. Moreover, this CNTf membranes have been used to design new device architectures. On the other hand, the CNTf has been hybridised with semiconductors. The heterojunctions formed by different synthesis techniques has been widely characterised. The hybrids have been implemented as working electrodes (photoanodes) of DSSC and the charge transfer at the $\mathrm{CNTf} / \mathrm{TiO}_{2} /$ dye/electrolyte has resulted in several interesting conclusions.

For studying the catalytic activity of the CNTf, firstly the implementation of the CNTf as CE/CC of planar devices have been optimised. Reliable procedures for fabricating these type of devices (symmetric cells or full DSSC devices) have been developed. Firstly, the catalytic nature have been proven and the high catalytic activity has been associated to the high SSA present in the CNTf. After demonstrating the stability of these electrodes for this application, the role of the mesoporosity of the CNTf have been clarified by EIS measurements. This result is not only important for this material because some other electrochemical interfaces are also observed 
for other nanocarbons (e.g. graphene), and the same conclusion can be extended to similar mesoporous carbon systems.

An important contribution has been done attending to the chemical environment produced by the interaction between the CNTf and the standard electrolyte for DSSC. Starting from different diffusion coefficients related to the variation in concentration of conductive species in the electrolyte, and finishing by the possible stabilisation of the electrolyte by taking the CNTf the role of protecting agent from the dye/electrolyte interaction. This interaction leads to an increase in polyiodides species that improves the diffusion limiting current densities, and therefore the short circuit current of the device. The role of the CNTf-CE as membrane hosting the electrolyte can also be understood as electrolyte filler. This implies that the observations and conclusions observed for CNTs as electrolyte filler can be extrapolated for thick CE made of nanocarbon materials.

There are fundamental question regarding the catalytic nature of the carbon material that remains open. The functionalisation of the CNTf substrate does not lead to an improved efficiency, suggesting the catalytic activity and thus the number of catalytic active sites are not proportional to the carbon edges created. Other alternatives must be explored, for instance, the iron catalyst residue as origin of the catalytic activity. Similar experiments have been reported with ferrocene catalyst residue and, although they reported an improved catalytic activity, this material is oxidised and still is different to the metallic iron observed encapsulated in our CNTf. These metallic clusters should produce highly distorted electronic area at the surface of the CNTf. Precise experiments should be design to further clarify this feature of the CNTf.

The implementation of CNTf-CE into DSSC devices resulted in a successful application. After the optimisation of the CNTf parameters, with special attention to the S-shape limiting factor of the performance, the obtained efficiency of the CNTf devices outperformed the reference $\mathrm{Pt}$ material, obtaining a performance superior to the $9 \%$ and demonstrating the stability after 6 months (> $60 \%$ of the initial performance).

Continuing with the CNTf-CE implementation, new device architectures based on the CNTf-CE has been proposed. The free-standing CNTf-CE, although presenting low efficiency related to the low stability of the liquid solvent-based electrolyte, has shown promising results, with potential benefits in view of large scale manufacturing processes like the roll-to-roll. Further work optimising the electrolyte stability under air conditions is proposed. Moreover, the encapsulation with flexible substrate is an alternative manner of stabilising the electrolyte embedded in the CNTf membrane, as well as preserving the flexibility of the CNTf. Still further work is needed in flexible planar working electrodes for obtaining the full flexible device.

CNTf nanocomposites have been fabricated and used as DSSC-CE. Several mass fractions of CNTf have shown an improved mechanical performance, exceeding the rule of mixtures and in agreement with previous results of the group. A reliable procedure for producing controlled CNTf nanocomposites has been developed and an overall characterisation of the material has been performed. The morphology of the nanocomposite material has been related with the performance as CE for DSSC. Indeed, the infiltration with polymer has resulted in a good procedure for tuning the CNTf exposed surface. The S-shaped profile of the $J-V$ curve has been evaluated. This is a direct result proving the relation between exposed catalytic 
surface of the CNTf and the S-shaped. The obtained performance still is far from the $9 \%$ obtained with optimised Pt devices. The controlled polymer solution can be used for selectively exposing certain amount of CNTf surface, possibly without hampering the overall mechanical properties of the nanocomposite.

One of the new configurations proposed is the bifacial DSSC. This tackles the lack of transparency of the CNTf by sandwiching the CNTf membrane in between two photoanodes. As has been shown, both faces of the device work independently with similar performance, but when connecting them and illuminating both faces, the current of the device is nearly doubled. Furthermore, a proof-of-concept minimodule has been fabricated using the CNTf as direct CE/CC. Several independent photoanodes connected over the same 1 metre length CNTf-strip, has shown a total efficiency of $2.7 \%$ close to other optimised nanocarbon based DSSC modules recently reported by other groups. The size dependence effect on these solar modules $\left(>10 \mathrm{~cm}^{2}\right.$ ) has been analysed, explaining and attributing part of the power losses to resistive effects.

Before implementing the CNTf in the working electrodes of DSSC devices (photoanodes), the synthesis and characterisation of CNTf/semiconductor hybrids have been performed. This is more important since the appearance of novel materials like CNT or graphene, and its increasing use as electrode for similar applications than activated carbon, as for instance energy storage.

Firstly, CNTf hybridised with ZnO semiconductor synthesised by a double step chemical route have been studied. The Schottky barrier formation between these materials have been successfully demonstrated. Meanwhile, the SBH value is smaller than the expected according to the energetics of the materials. The analysed Schottky heterojunctions present an ideality factor of 2.9, effective SBH of $0.26 \mathrm{eV}$ and rectifying ratio of 144 . The wide range of work function values reported for the individual components, even for the same synthesis conditions, makes difficult to extract comparable conclusions with other nanocarbons materials. This gives prominence to the obtained result as one of the first for studies for macroscopic large heterojunctions with CNTf.

On top of that, the charge transfer properties through the interface have been successfully investigated by photocurrent measurements, leading to a responsivity of the material close to $5000 \mathrm{~mA} / W$, a high value related with the large SSA of the CNTf and the large extended interface with the metal oxide. As an alternative application, the obtained $\mathrm{ZnO}-\mathrm{NWs}$ wurtzite crystals, widely studied as piezoelectric nanogenerators, were analysed distinguishing the different contributions of piezoresistive and piezoelectric phenomena taking place under mechanical deformation on the material.

For further understanding of the CNTf hybridisation and charge transfer phenomena, alternative nanometer-size layers of $\mathrm{ZnO}$ were synthesised by atomic layer deposition (ALD), a physical method that provides precise control in terms of the chemistry, structure and thickness of the deposited metal oxide layer. CNTf/MO $\mathrm{M}^{-}$ ALD heterojunctions have shown different electrical properties depending on the number of cycles applied during the synthesis of the $\mathrm{MO}_{x}$. For instance, for $\mathrm{ZnO}$ material, metallic junction has been reported for thin layers $(<20 \mathrm{~nm})$ in contrast to the rectifying behaviour observed for thicker $(>20 \mathrm{~nm}$ ) layers. This tunability of the properties of ALD hybrids has been explained by differences in carrier concentration and oxidation state of the materials upon different thicknesses. Experimental 
insights for this conclusion have been found by XPS, Raman, XRD and morphological study of the hybrid material.

The charge transfer through this interface has been demonstrated by UV photocurrent measurements, supporting the different properties obtained depending on the ALD number of cycles. The photoresponse under low power UV-light is highly improved. This results also suggest the potential application of this material for UVphotodetectors. The electrochemical effects produced by the adsorption of oxygen molecules under light irradiation govern the behaviour of this type of photodetector. The high surface area accessible for this interaction in $\mathrm{CNTf} / \mathrm{MO}_{x}$ hybrids, and thus the properties of the possible photodetector device, could be easily modifiable by the thickness of the $\mathrm{ZnO}$ layer.

These hybrids materials obtained by conformal layer of semiconductor over the CNTf by ALD have been implemented into the working electrodes of DSSC. The high surface area and large 3D heterojunction interfaces has been investigated as photoanodes. The photogenerated carrier injection $v$ s recombination mechanism has been analysed by tunning the amount of emitter material (thickness of $\mathrm{TiO}_{2} / \mathrm{N} 719$ dye) and its interaction with the CNTf. Very low performance was achieved with this configuration and high recombination is the responsible of this performance. Several strategies for avoiding the recombination at the surface of CNTf photoanodes has been explored.

The ALD material has proved to be a good passivisation layer (blocking layer) for the CNTf electrode and, the leakage current of the device has been drastically reduced. Although further optimisation is needed for obtaining fully passivised substrates and homogeneous ALD layers throughout the CNTf substrate cross-section. This optimisation is required for obtaining fully operative CNTf-photoanode devices with an increased performance. Furthermore, the ALD physico-chemical interaction with the CNTf substrate has to be further studied. Surface modification by functionalisation treatments could lead to improve the homogeinity of these hybrids electrodes.

The competition between carrier generation-injection and recombination mechanisms have been further analysed by reducing the thickness of the CNTf to semitransparent electrodes. By decreasing the available CNTf surface, thus the number of recombination centres, the recombination mechanism is diminish. This devices performed good current and voltage characteristics but very low FF. After analysing the electrical and optical properties of the semi-transparent CNTf, this low FF is attributed to the sheet resistance of semi-transparent CNTf electrodes.

Concluded from these previous architectures, the degree of exposed CNTf surface has proved to be a critical parameter. The passivisation of the CNTf surface led to higher shunt resistances, lower leakage current and better overall performance of the device.

Proposed future work should combine the results obtained by the passivisation of the CNTf substrate by $\mathrm{MO}_{x}$-ALD with semi-transparent CNTf electrodes. Moreover, the increment of the conductive properties of semi-transparent electrodes would lead to better performance DSSC devices.

Finally, some alternative solutions for avoiding the recombination mechanism are proposed. For instance, using non-reactive electrolytes with the CNTf, hence avoiding this source of recombination. Other possibility would be using water based 
electrolytes with low wettability with the CNTf electrodes, thus reducing the physical contact between these two materials.

As a final remark, some precautions must be taken when introducing nanocarbons as photoanode dopant. There is a lot of work done in the literature but, it is not commonly reported the high recombination processes associated to these materials. Although, in most cases, an increment in efficiency is reported, other parasitic reactions (recombination) could be occurring when the nanocarbons are not effectively passivised. 



\section{Appendix A}

\section{CNTf/Polymer Nanocomposites}

In this Appendix, CNTf/polymer nanocomposite is presented. This material is presented in the Chapter 7 as $\mathrm{CE}$ with polymeric encapsulation for preventing electrolyte leakage and evaporation. However, this material research is out of the main scope of this thesis, more details about the nanocomposite preparation and characterisation are presented here below.

\section{A.1 CNTf/PVDF nanocomposite fabrication}

Extensive research has been performed in the past with the aim of extracting the outstanding properties of the CNTs from the nano-scale to the micron-scale. One exploited route in the last years is using CNTf as reinforcement in polymeric composite materials.[361] Among different techniques for preparing nanocarbon composites, hot-pressing technique is widely used, $[362,363]$ but still it is challenging to obtain uniform composite materials and reproducible properties.

The CNTf used in this section has been in-situ oriented during its synthesis by the drawing method.[53] The precursor solution and conditions are slightly different (detailed in Chapter 3 "Experimental Techniques"), but the important different lays on the drawn parameter, here close to $40 \mathrm{~m} / \mathrm{min}$ (for previous materials it is $5 \mathrm{~m} / \mathrm{min}$ ). This difference confers higher mechanical properties to the CNTf, tensile strength and elastic modulus, without lowering its porosity, as it is demonstrated in previous work of the group.[102]

Polyvinylidene fluoride (PVDF) is a common thermoplastic that presents good mechanical properties and chemical resistance.[364] It is widely used in many applications as, for instance, wires encapsulation,[364] supercapacitors,[365] batteries[366] or solar cells.[128, 267]

Unidirectional CNTf/PVDF polymer nanocomposites were fabricated according to the method presented in the Figure A.1. The pristine CNTf and polymer materials are shown in the first step of the manufacturing process as raw materials (1). These materials are combined by inverse-sandwiching process as is presented in the second stage of the process (2). The CNTf sheet $(30 \times 5 \mathrm{~cm})$ is used for wrapping the neat PVDF lamina $(3 \times 5 \mathrm{~cm})$. The advantage of this procedure is that the CNTf is firmly secured in place around the lamina of polymer, presented in the third stage of the process (3). At this point, the CNTf and PVDF lamina are independent materials that are later consolidated by hot-pressing technique. The final composite lamina is presented at the last stage of the process (4). 
Due to the considerable amount of interconnected porosity of the dry CNTf, discussed before in previous chapters, and confirmed by specific surface area of the material $\left(250 \mathrm{~m}^{2} / \mathrm{g}\right)$,[58] the voids of the fibre will be filled by the polymer during the hot pressing stage. This inverse-sandwiching process is an innovative and controlled way of producing CNTf/composite materials that allows to fabricate reproducible composite materials with the CNTf. Notice that during this process the CNTf sheets were incorporated into the composite as-synthesised and without any further modification by mechanical or chemical treatments.

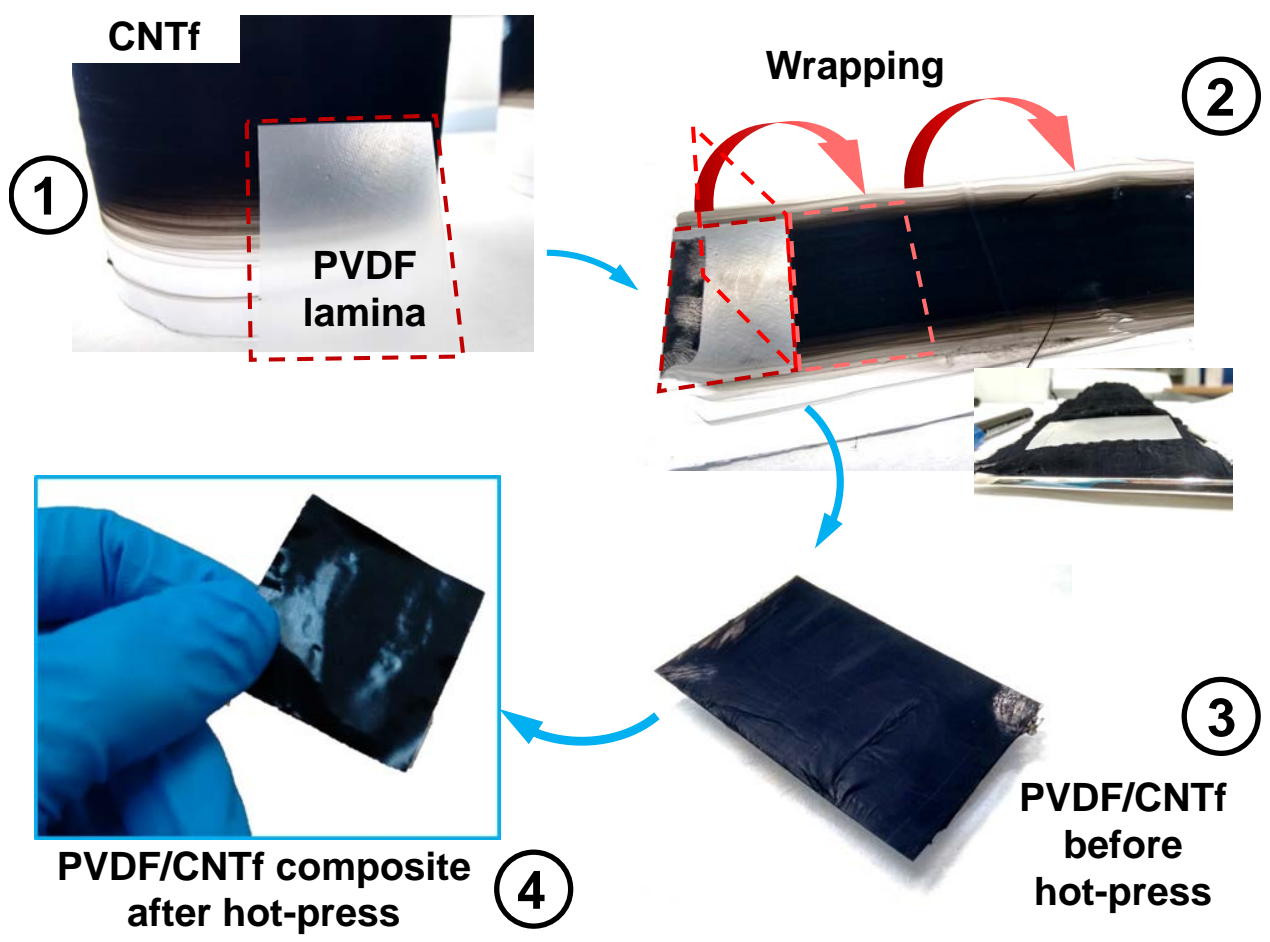

FIGURE A.1: CNTf/PVDF nanocomposites: manufacturing process and materials.

Derived from the composite fabrication process, the final material presents a unusual structure. A schematic of the cross-section of the dry CNTf/PVDf composite (before hot-pressing) is presented in the Figure A.2a, where the lamina of neat polymer is sketched inside the CNTf dry CNTf sheet. When the dry composite material is subjected to the hot-press step $\left(200^{\circ} \mathrm{C}\right.$ and 1 Ton pressure), the melted PVDF flows radially in plane due to the constraining plates of the equipment. The polymer flows freely along $X$ direction (see Figure A.2b) and finds limited movement along $Y$ direction due to the CNTf sheet wrapped around the polymer. Lastly, the polymer flows through the CNTf thickness $(Z)$ infiltrating its porous structure until it reaches the parallel plates of the hot pressing machine.

The hot-press parameters were optimised for the proper infiltration of the CNTf, thus reducing the possible formation of voids or lack of infiltration within the nanocomposite. Among the different fabrication parameters, the pressure holding time is an important one because determines the degree of infiltration of the polymer in the CNTf. An approximated time temperature/pressure holding time superior to $30 \mathrm{~min}$ was applied. 
a) Dry composite cross-section:

b) Hot-press schematic:
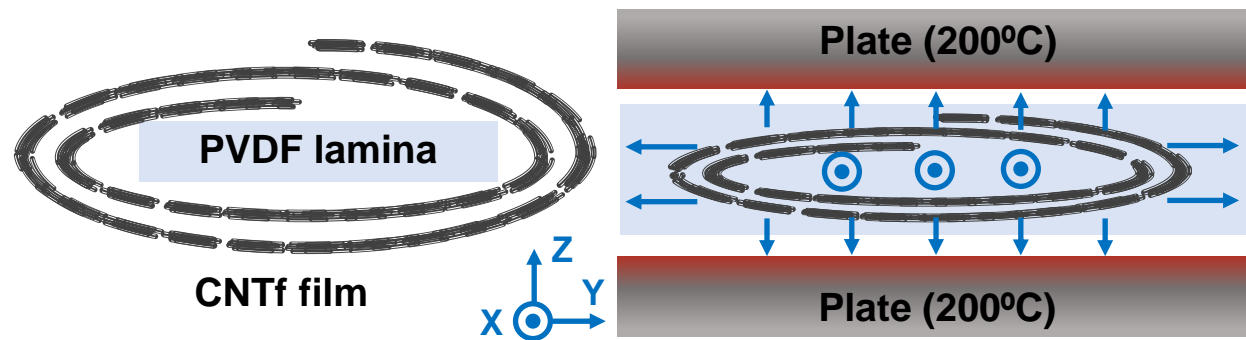

c)

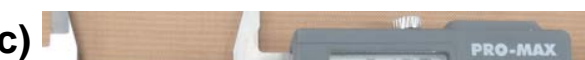

d)

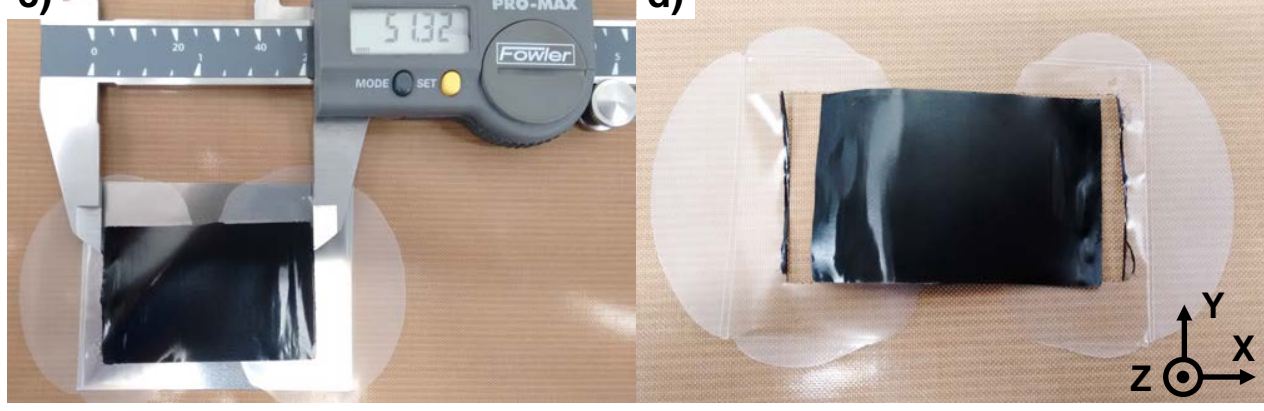

FIGURE A.2: CNTf/PVDF nanocomposite consolidation by hot-press technique.

The as-made composite lamina is presented in the Figure A.2c. An homogeneous composite film of $5 \times 3 \mathrm{~cm}$ is obtained. $200 \mu \mathrm{m}$ thick is fixed using a stainless steel frame round the dry composite during the hot pressing.

Both CNTf and PVDF materials present the main decomposition step at 450$500{ }^{\circ} \mathrm{C}$, thus, thermogravimetrical analysis (TGA) is not a suitable method for mass fraction determination of the composite material. For solving this issue, the pristine materials are weighted before and after the composite preparation, and after trimming the excess of neat polymer from the composite material (Figure A.2d), a precise value of mass fraction can be calculated. The final mass fraction of the composite can be increased either by using a thinner initial layer of polymer, or by subjecting to the hot-press process twice of three times the same composite lamina. During the development of this work, composite laminates up to $45 \mathrm{~cm}^{2}$ and between 1 and $15 w t \%$ were fabricated.

\section{A.2 CNTf/PVDF nanocomposite infiltration and mechanical properties}

Once fabricated the nanocomposite material, its morphology has been analysed. Firstly, it is important to determine the internal porosity of the composite material and degree of infiltration of the CNTfs. For that, the surface and cross-section were inspected by electron microscopy technique (Figure A.3a).

The surface of the composite material presents the morphology of the CNTf, indicative of a nanometric layer of polymer coating the CNTf fibre and saturating the surface porosity of the material (Figure A.3b). For analysing the cross-section, a vertical wall was prepared by removing material by focused ion beam milling in 
two depth steps (Figure A.3c). The initial 3-5 $\mu \mathrm{m}$ of CNTf/PVDF shows distributed nanometric porosity in a range from 20 to $100 \mathrm{~nm}$. The same inspection at $50 \mu \mathrm{m}$ depth from the surface reveals no porosity, this suggest either better infiltration of the CNTf or the absence of this material, directly appearing as a shell of the composite.

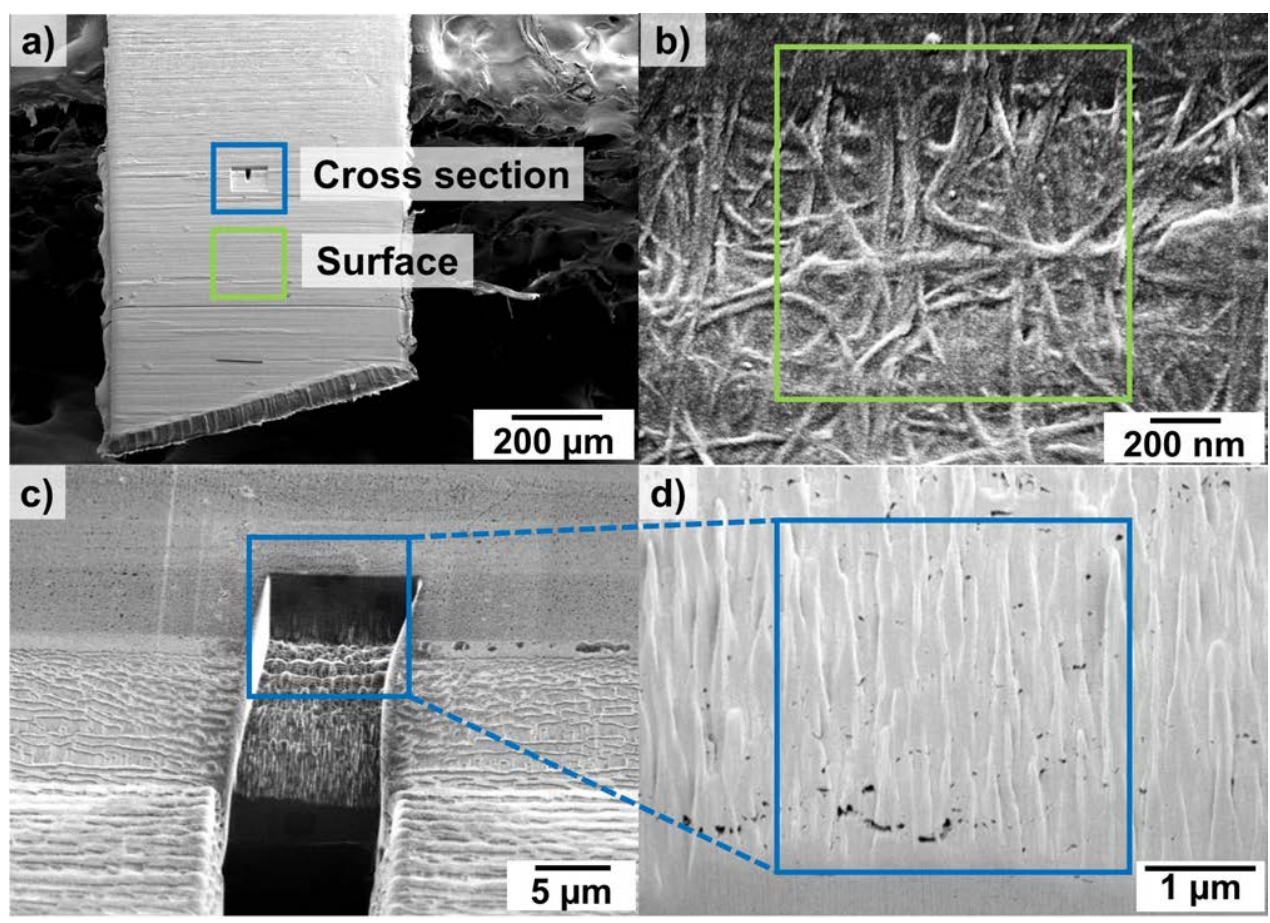

FIGURE A.3: Morphological study of the CNTf/PVDF nanocomposite by electron microscopy.

The internal structure of the CNTf was further investigated by X-ray microtomographic analysis and the data is presented in the Figure A.4. With a resolution of $1 \mu \mathrm{m}$, the absence of micro-porosity was confirmed. The most relevant information is the confirmation of the CNTf appearing as a shell inside the nanocomposite. The superficial morphology of the CNTf composite observed in the previous Figure A.3b, is also similar to the observed at the $3 \mathrm{D}$ reconstructed volume at the outer faces (highlighted in red colour in the 3D reconstruction).

On the contrary, the interior of the sample, i.e. parallel slices to the red plane, shows an homogeneous morphology without any characteristic morphology. This is interpreted as neat PVDF polymer confined between both layers of material. The differences in brightness between the CNTf observed accumulated as a shell of the nanocomposite and the interior PVDF are attributed to differences in the electron density absorption. The CNTf has a solid residue in the range of 12 to $18 \%$ according to TGA analysis. This residue is mainly formed by metallic iron and silicon nanoparticles that remains inside the CNTf as residue from synthesis procedure. Just considering these elements, there is a higher absorbance of the CNTf as compared to the polymer.

The electrical properties of the composite material are relevant for device applications. The conductivity of the nanocomposite is basically the same than for the 


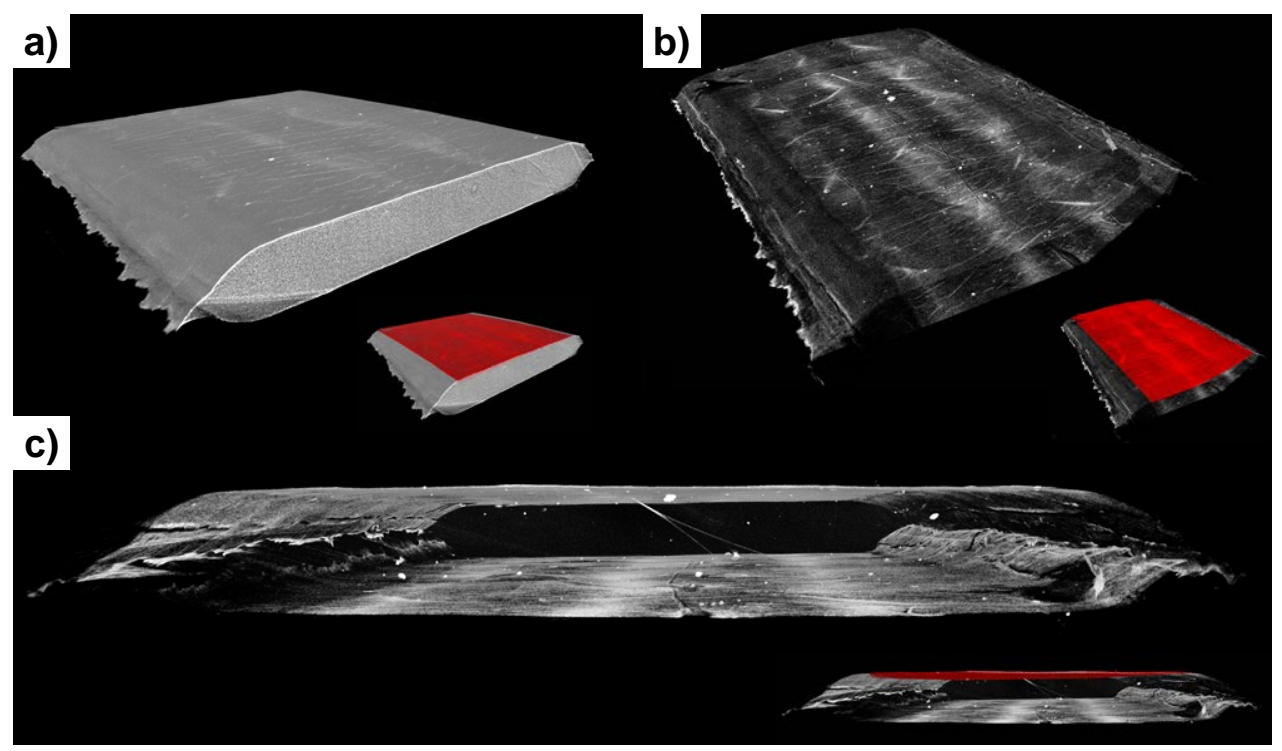

FIGURE A.4: X-ray tomography of the CNTf/PVDF nanocomposite. a) $3 \mathrm{D}$ reconstructed volume of a $3 \times 1 \times 0.2 \mathrm{~mm}^{3}$ nanocomposite volume. b) CNTf shell obtained by signal intensity filtering. c) 3D slice of the volume showing the CNTf shell. Upper plane of the CNTf indicated by a red plane for reference.

pristine CNTf along the longitudinal axis of the fibre. Unlike the composite materials produced by dispersed CNTs in a polymer matrix, CNTf/PVDF composites are composed by oriented and interconnected fibres. The electrical connections inside the composite material are the same than in the pristine CNTf, whereas for a nanocarbon dispersed nanocomposite, other mechanisms such as polymer tunneling conduction plays an important role.[367]

Notice here that, as it is shown in the previous tomography (Figure A.4), there is no contact between the external CNTf faces for a $100 \mu \mathrm{m}$ thick composite lamina. The internal layer of neat polymer prevents the nanocomposite from conducting through the thickness ( $z$-conductivity). It has been observed that when the hot pressing is repeated twice or three times over the same composite lamina, the final thickness decreases down to 20-30 microns, and, at that range, the composite lamina conducts through $z$-direction.

This implies that for lower thicknesses or in other words, diminishing the internal amount of polymer by repeated hot pressing methods, the CNTf/PVDF nanocomposite has an homogeneous distribution through the thickness. More importantly, the conductivity through the thickness (z-direction) can be used as a evaluation of the nanocomposite homogeneity.

Finally, the mechanical properties of the nanocomposite has been tested by uniaxial tensile test. Several mass fractions (1,5 and $15 \%)$ have been fabricated and tested. The samples for the mechanical test were prepared by punching out from the previous nanocomposite CNTf/PVDF laminas several dumbbell-shaped specimens with a standardised die (gauge dimensions of $10 \mathrm{~mm}$ length and $2 \mathrm{~mm}$ in width).

Based on the very low residual porosity reported in this section, the density of the composite material is calculated according to the rule of mixtures, e.g. $1.33 \mathrm{~g} / \mathrm{cm}^{3}$ for the higher mass fraction. This property is assumed to increase linearly for the 
mass fraction range here analysed $(<20 \%)$ [361] up to the limit of the neat polymer $\left(1.78 \mathrm{~g} \mathrm{~cm}^{-3}\right)$ for no content of CNTf. This behaviour is depicted in the inset of the Figure A.5b. The density of the CNTf has been obtained subtracting the volume of porosity of the CNTf ( $\approx 80 \%$ from previous results of the group) to the density of graphite $\left(2.2 \mathrm{~g} \mathrm{~cm}^{-3}\right)$. This CNTf density has to be taken with caution because most probably the material is packed to greater densities during the hot pressing stage.

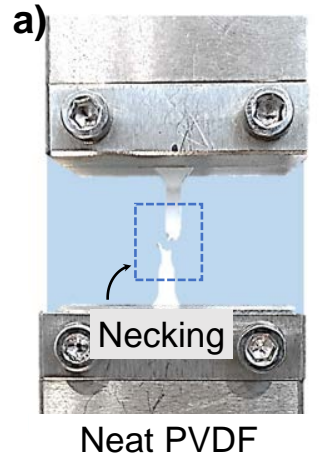

c)

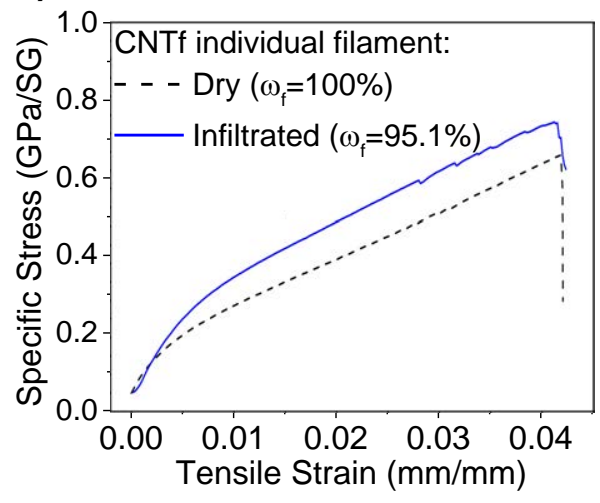

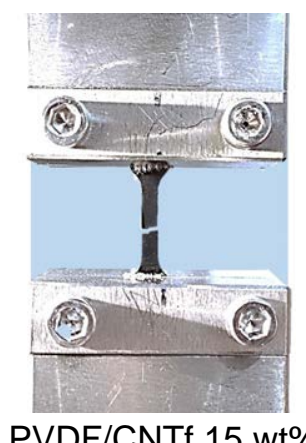

PVDF/CNTf 15 wt\%

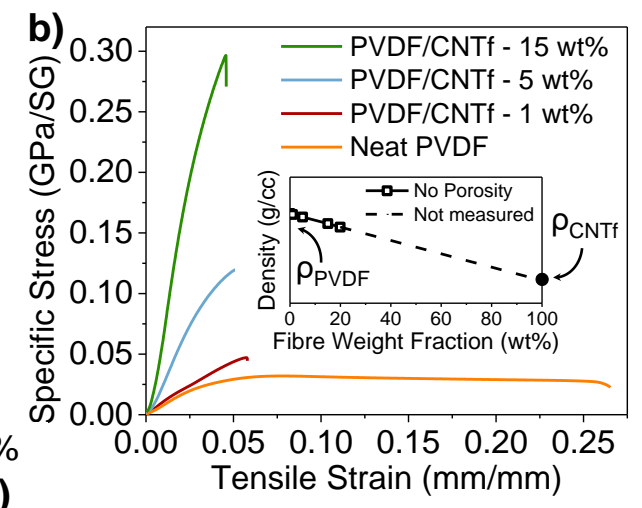

d)

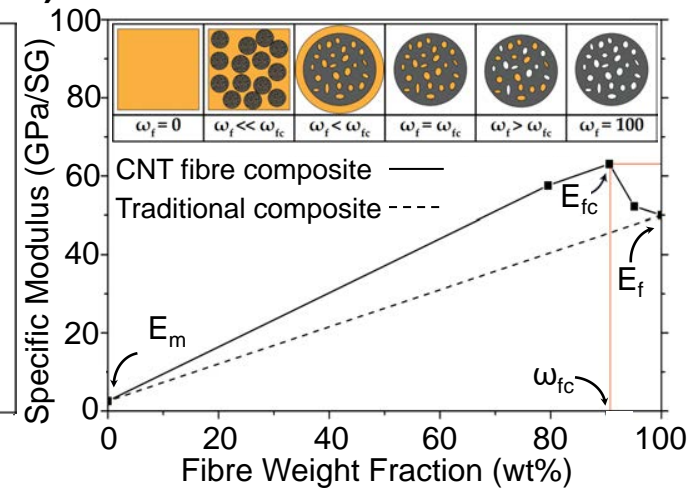

FIGURE A.5: a) Tensile test experiment showing the plastic failure and necking of the neat PVDF (white material) and the failure of the CNTf/PVDF nanocomposte (black material). b) Representative specific stress vs strain curves of the neat polymer and the nanocoposite with different mass fractions. Inset: theoretical composite density variation according to the rule of mixtures. c) Representative curves of the dry CNTf filament and polymer infiltrated. d) Elastic modulus versus weight fraction for a traditional composite (dashed line) and CNTf composite (solid line). Graphs $c$ and $d$ courtesy of B.Mas.[368]

Figure A.5a,b shows the digital images of samples after tensile test and representative stress-strain curves for the neat polymer and the 1, 5, $15 w t \%$ CNTf/PVDF nanocomposites. The obtained characteristics - tensile strength, elastic modulus, elongation-to-break and fracture energy, are presented in the Table A.1. Specific values are presented in the graph but also included without normalisation in the Table A.1. Strength and elastic modulus $(\mathrm{GPa})$ are normalised by the Specific Gravity $(S G)$, which is the density $\left(\mathrm{g} \mathrm{cm}^{-3}\right)$ relative to that of water $\left(\approx 1 \mathrm{~g} \mathrm{~cm}^{-3}\right)$. This unit, $\mathrm{GPa} / S \mathrm{G}$, is equivalent to $\mathrm{N} / \mathrm{tex}$, a typical unit used in the textile industry for fibrous elements, where tex represent the linear density, mass per unit length $(\mathrm{g} / \mathrm{km})$.

Two very different behaviours are obtained for the neat polymer and the CNTf composite. The neat PVDF presents a high elongation ( $>25 \%$ ) and ductile failure, 
after the plastic deformation regimen (necking), characteristic of thermoplastic materials. In contrast the failure of the nanocomposite samples is produced with much lower deformation. This is attributed to the small elongation $(\approx 5 \%)$ of the CNTf compared to the polymer material. Since the nanocomposites are fabricated with oriented fibre, when the maximum strain of the fibres are exceeded the material fails.

TABLE A.1: Mechanical properties of CNTf/PVDF nanocomposite. Specific values included in brackets.

\begin{tabular}{|c|c|c|c|c|c|c|}
\hline Material & $\begin{array}{l}\text { Fibre } \\
\text { weight/ } \\
\text { volume } \\
\text { fraction }\end{array}$ & Density & $\begin{array}{l}\text { [Specific] } \\
\text { Elastic } \\
\text { modulus }\end{array}$ & $\begin{array}{l}\text { [Specific] } \\
\text { Tensile } \\
\text { strength }\end{array}$ & $\begin{array}{l}\text { Elongation } \\
\text { at break }\end{array}$ & $\begin{array}{l}\text { Fracture } \\
\text { energy }\end{array}$ \\
\hline & $\begin{array}{l}w t \% / \\
v o l \%\end{array}$ & $g / c c$ & $\begin{array}{l}G P a \\
{[G P a / S G]}\end{array}$ & $\begin{array}{l}\mathrm{MPa} \\
{[\mathrm{MPa} / \mathrm{SG}]}\end{array}$ & $\%$ & $J / g$ \\
\hline Neat PVDF & 0 & 1.78 & $1.6[0.89]$ & 55 [30.9] & 26.5 & 13 \\
\hline PVDF/CNTf & $1 / 2.2$ & 1.75 & 2 [1.14] & 82 [47] & 5.7 & 2.7 \\
\hline PVDF/CNTf & $5 / 11.1$ & 1.65 & $5.6[3.4]$ & 197 [119.4] & 5.0 & 5.7 \\
\hline PVDF/CNTf & $15 / 33.3$ & 1.33 & $11.7[8.8]$ & 395 [297] & 4.5 & 10 \\
\hline Dry CNTf & 100 & 0.45 & $22.5[50]$ & 275 [610] & 4.2 & 16.7 \\
\hline
\end{tabular}

The results obtained show that both tensile strength and elastic module scales with the mass fraction of CNTf indicating the effective reinforcement of the individual fibres. For $15 w t \%$ loading, the tensile strength is enhanced a factor of 8 and the elastic modulus by a factor of 7.3. Although the lower mass fractions show less impressive improvement, still they follow the expected improvement for this range of reinforcement. Notice that this values are in agreement to previous work reported with similar materials. [361, 369] This improvement is typically predicted by the rule of mixtures, but the values here presented exceed this prediction.

In relation to this, an individual dry CNTf monofilament has been purposely infiltrated with dilute polymer solution so as to produce a composite fibre with a low volume fraction and thus fibre-dominated properties (Figure A.5c). Something that we notice and reported in previous work is that the CNTf composites present an enhanced stress-transfer when infiltrated by low volume fractions of diluted polymer.[368] The higher values of fibre modulus and tensile strength after polymer infiltration indicate that the polymer can effectively transfer stress to regions of the fibre that otherwise shared little load in the dry state.

Figure A.5d depicts a plot of CNTf composite modulus against fibre mass fraction. As shown schematically, the system starts as a dry fibre $\left(w_{f}=100 \%\right)$ and its porosity is gradually filled up by increasing the amount of polymer (decreasing $w_{f}$ ). The fibre/composite modulus increases as the polymer fills up the pores and acts as an adhesive that bridges accross the CNT bundles. This effect peaks at a critical value $\left(w_{f c} \approx 100 \%\right)$ where the enhancement start decreasing as the matrix only adds weight surrounding the fibre.

CNTf composites have properties that depend on the choice of the matrix as occurs in conventional fibres.[368] An effective fibre modulus after polymer infiltration can be used for predict properties of higher fractions. A first approximation 
value can be extracted from the rule of mixtures equation:

$$
E_{C}=E_{f}^{e f f} v_{f}+E_{m}\left(1-v_{f}\right)
$$

where $E_{C}$ is the final modulus of the composite, $E_{f}^{e f f}$ is the effective fibre modulus after polymer infiltration, $E_{m}$ is the matrix modulus and $v_{f}$ is the volume fraction of fibre.

Applying the equation A.1 to the previous data presented in the Table A.1 values in the range of 32-38 $\mathrm{GPa}$ are obtained for 5 and $15 \% w t \mathrm{CNTf}$, therefore confirming the improved stress transfer.

Although these values are exclusive for PVDF polymer, and because of the inconvenience of producing a similar study for every fibre/matrix combination, a model has been recently developed in the group considering the effective fibre modulus reinforcement after polymer infiltration.[368]

Finally, the fracture energy as an indirect manner of measuring the toughness of the material is discussed. The fracture toughness is defined as the resistance to the sudden propagation of a crack of length $a$ at a stress $\sigma$ to give a brittle fracture. This is related to the product of the stiffness of the material and the work to create crack surfaces $\gamma$. The value $\gamma$ depends on the energy absorption processes at the crack tip. Moreover, measuring fracture toughness involves the insertion of notches of known geometries, larger samples and more precise experimental setup.

In contrast, the resistance to failure can be measured by the work of fracture, obtained by the integration of the stress-strain curve up to the breaking point. Although this value will be dependent on sample geometry and testing mode, it provides a useful indication of the fracture resistance. In the Table A.1 the values of fracture energy are compared. The reduced ductility at lower mass fractions of CNTf (1-5 \%) lead to lower fracture energy, in contrast, for the higher mass fraction (15\%) the fracture energy is comparable to the neat polymer material but achieving much higher tensile strength.

The previous description and characterisation of CNTf nanocomposites aims to provide better understanding of the material used in the previous Chapter 7 as CE for DSSCs. 


\section{Bibliography}

[1] Juan J Vilatela and Rebeca Marcilla. “Tough electrodes: carbon nanotube fibers as the ultimate current collectors/active material for energy management devices". In: Chemistry of Materials 27.20 (2015), pp. 6901-6917.

[2] Wanchul Seung et al. "Nanopatterned textile-based wearable triboelectric nanogenerator". In: ACS nano 9.4 (2015), pp. 3501-3509.

[3] Feng-Ru Fan et al. "Transparent triboelectric nanogenerators and self-powered pressure sensors based on micropatterned plastic films". In: Nano letters 12.6 (2012), pp. 3109-3114.

[4] Cynthia Watkins, Bing Shen, and Rama Venkatasubramanian. "Low-gradeheat energy harvesting using superlattice thermoelectrics for applications in implantable medical devices and sensors". In: Thermoelectrics, 2005. ICT 2005. 24th International Conference on. IEEE. 2005, pp. 265-267.

[5] Qiang Zheng et al. "In vivo powering of pacemaker by breathing-driven implanted triboelectric nanogenerator". In: Advanced Materials 26.33 (2014), pp. 5851-5856.

[6] Mahammad A Hannan et al. "Energy harvesting for the implantable biomedical devices: issues and challenges". In: Biomedical engineering online 13.1 (2014), p. 79.

[7] Martin Kaltenbrunner et al. "Ultrathin and lightweight organic solar cells with high flexibility". In: Nature communications 3 (2012), p. 770.

[8] Wonho Lee et al. "Mechanically robust and high-performance ternary solar cells combining the merits of all-polymer and fullerene blends". In: Journal of Materials Chemistry A 6.10 (2018), pp. 4494-4503.

[9] Wansun Kim et al. "Comparative Study of the Mechanical Properties of AllPolymer and Fullerene-Polymer Solar Cells: The Importance of Polymer Acceptors for High Fracture Resistance". In: Chemistry of Materials 30.6 (2018), pp. 2102-2111. eprint: https://doi.org/10.1021/acs . chemmater. 8b00172.

[10] Mohammad $\mathrm{H}$ Malakooti et al. "ZnO nanowire interfaces for high strength multifunctional composites with embedded energy harvesting". In: Energy $\mathcal{E}$ Environmental Science 9.2 (2016), pp. 634-643.

[11] Paul D Mitcheson et al. "Energy harvesting from human and machine motion for wireless electronic devices". In: Proceedings of the IEEE 96.9 (2008), pp. 1457-1486.

[12] G Jeffrey Snyder. "Small thermoelectric generators". In: The Electrochemical Society Interface 17.3 (2008), p. 54.

[13] Ming-Pei Lu et al. "Piezoelectric nanogenerator using p-type ZnO nanowire arrays". In: Nano letters 9.3 (2009), pp. 1223-1227.

[14] Zhong Lin Wang. "Triboelectric nanogenerators as new energy technology and self-powered sensors-principles, problems and perspectives". In: Faraday discussions 176 (2015), pp. 447-458. 
[15] Rodrigo V Salvatierra et al. "ITO-Free and Flexible Organic Photovoltaic Device Based on High Transparent and Conductive Polyaniline/Carbon Nanotube Thin Films". In: Advanced Functional Materials 23.12 (2013), pp. 14901499.

[16] Je-Hyeong Bahk et al. "Flexible thermoelectric materials and device optimization for wearable energy harvesting". In: Journal of Materials Chemistry C 3.40 (2015), pp. 10362-10374.

[17] Zhong Lin Wang and Jinhui Song. "Piezoelectric nanogenerators based on zinc oxide nanowire arrays". In: Science 312.5771 (2006), pp. 242-246.

[18] Alper Erturk and Daniel J Inman. "An experimentally validated bimorph cantilever model for piezoelectric energy harvesting from base excitations". In: Smart materials and structures 18.2 (2009), p. 025009.

[19] Canan Dagdeviren et al. "Recent progress in flexible and stretchable piezoelectric devices for mechanical energy harvesting, sensing and actuation". In: Extreme mechanics letters 9 (2016), pp. 269-281.

[20] Feng-Ru Fan, Zhong-Qun Tian, and Zhong Lin Wang. "Flexible triboelectric generator". In: Nano energy 1.2 (2012), pp. 328-334.

[21] Guang Zhu et al. "Linear-grating triboelectric generator based on sliding electrification". In: Nano letters 13.5 (2013), pp. 2282-2289.

[22] Qingshen Jing et al. "Case-encapsulated triboelectric nanogenerator for harvesting energy from reciprocating sliding motion". In: ACS nano 8.4 (2014), pp. 3836-3842.

[23] Sihong Wang et al. "Freestanding triboelectric-layer-based nanogenerators for harvesting energy from a moving object or human motion in contact and non-contact modes". In: Advanced Materials 26.18 (2014), pp. 2818-2824.

[24] Zhong Lin Wang and Wenzhuo Wu. "Nanotechnology-enabled energy harvesting for self-powered micro-/nanosystems". In: Angewandte Chemie International Edition 51.47 (2012), pp. 11700-11721.

[25] Markus B Schubert and Jürgen H Werner. "Flexible solar cells for clothing". In: Materials today 9.6 (2006), pp. 42-50.

[26] J Liu et al. "Screen Printed Dye-Sensitized Solar Cells (DSSCs) on Woven Polyester Cotton Fabric for Wearable Energy Harvesting Applications". In: Materials Today: Proceedings 5.5 (2018), pp. 13753-13758.

[27] https://solarimpulse.com/, Accessed: 16.08.2018.

[28] Jan Christoph Goldschmidt. "From window to solar cell and back". In: Nature materials 17.3 (2018), p. 218.

[29] Chunyi Zhi and Liming Dai. Flexible Energy Conversion and Storage Devices. John Wiley \& Sons, 2018.

[30] Mildred S Dresselhaus, Gene Dresselhaus, and Peter C Eklund. Science of fullerenes and carbon nanotubes: their properties and applications. Elsevier, 1996.

[31] Bharat Bhushan. Springer handbook of nanotechnology. Springer, 2017.

[32] S Amelinckx, A Lucas, and Ph Lambin. "Electron diffraction and microscopy of nanotubes". In: Reports on Progress in Physics 62.11 (1999), p. 1471.

[33] R Saito et al. "Electronic structure of chiral graphene tubules". In: Applied physics letters 60.18 (1992), pp. 2204-2206.

[34] Xueshen Wang et al. "Fabrication of ultralong and electrically uniform singlewalled carbon nanotubes on clean substrates". In: Nano letters 9.9 (2009), pp. 31373141.

[35] T Dürkop et al. "Extraordinary mobility in semiconducting carbon nanotubes". In: Nano letters 4.1 (2004), pp. 35-39. 
[36] Ch Schoenenberger et al. "Interference and Interaction in multi-wall carbon nanotubes". In: Applied Physics A 69.3 (1999), pp. 283-295.

[37] BQ Wei, R Vajtai, and PM Ajayan. "Reliability and current carrying capacity of carbon nanotubes". In: Applied Physics Letters 79.8 (2001), pp. 1172-1174.

[38] Savas Berber, Young-Kyun Kwon, and David Tománek. "Unusually high thermal conductivity of carbon nanotubes". In: Physical review letters 84.20 (2000), p. 4613.

[39] Min-Feng Yu et al. "Strength and breaking mechanism of multiwalled carbon nanotubes under tensile load". In: Science 287.5453 (2000), pp. 637-640.

[40] BG Demczyk et al. "Direct mechanical measurement of the tensile strength and elastic modulus of multiwalled carbon nanotubes". In: Materials Science and Engineering: A 334.1-2 (2002), pp. 173-178.

[41] Alain Peigney et al. "Specific surface area of carbon nanotubes and bundles of carbon nanotubes". In: Carbon 39.4 (2001), pp. 507-514.

[42] A Martone et al. "Reinforcement efficiency of multi-walled carbon nanotube/ epoxy nano composites". In: Composites science and technology 70.7 (2010), pp. 11541160.

[43] Peng-Cheng Ma et al. "Dispersion and functionalization of carbon nanotubes for polymer-based nanocomposites: a review". In: Composites Part A: Applied Science and Manufacturing 41.10 (2010), pp. 1345-1367.

[44] Zdenko Spitalsky et al. "Carbon nanotube-polymer composites: chemistry, processing, mechanical and electrical properties". In: Progress in polymer science 35.3 (2010), pp. 357-401.

[45] Nanda Gopal Sahoo et al. "Polymer nanocomposites based on functionalized carbon nanotubes". In: Progress in polymer science 35.7 (2010), pp. 837-867.

[46] Juan J. Vilatela. "Nanocarbon-based composites". In: Nanocarbon-Inorganic Hybrids: Next Generation Composites for Sustainable Energy Applications Chapter 8 (2014), pp. 227-254.

[47] Ya-Li Li, Ian A Kinloch, and Alan H Windle. "Direct spinning of carbon nanotube fibers from chemical vapor deposition synthesis". In: Science 304.5668 (2004), pp. 276-278.

[48] Mei Zhang, Ken R Atkinson, and Ray H Baughman. "Multifunctional carbon nanotube yarns by downsizing an ancient technology". In: Science 306.5700 (2004), pp. 1358-1361.

[49] Brigitte Vigolo et al. "Macroscopic fibers and ribbons of oriented carbon nanotubes". In: Science 290.5495 (2000), pp. 1331-1334.

[50] Lars M Ericson et al. "Macroscopic, neat, single-walled carbon nanotube fibers". In: Science 305.5689 (2004), pp. 1447-1450.

[51] Virginia A Davis et al. "Phase behavior and rheology of SWNTs in superacids". In: Macromolecules 37.1 (2004), pp. 154-160.

[52] Virginia A Davis et al. "True solutions of single-walled carbon nanotubes for assembly into macroscopic materials". In: Nature nanotechnology 4.12 (2009), p. 830.

[53] Belén Alemán et al. "Strong Carbon Nanotube Fibers by Drawing Inspiration from Polymer Fiber Spinning". In: ACS Nano 9.7 (2015), pp. 7392-7398.

[54] Rajyashree M Sundaram and Alan H Windle. "One-step purification of directspun CNT fibers by post-production sonication". In: Materials \& Design 126 (2017), pp. 85-90.

[55] Agnieszka Lekawa-Raus et al. "Electrical Properties of Carbon Nanotube Based Fibers and Their Future Use in Electrical Wiring". In: Advanced Functional Materials 24.24 (2014), pp. 3661-3682. 
[56] B Alemán et al. "Carbon nanotube fibers with martensite and austenite Fe residual catalyst: room temperature ferromagnetism and implications for CVD growth". In: Journal of Materials Chemistry C 5.22 (2017), pp. 5544-5550.

[57] Juan J Vilatela and Alan H Windle. "Yarn-like carbon nanotube fibers". In: Advanced Materials 22.44 (2010), pp. 4959-4963.

[58] E Senokos et al. "Macroscopic fibres of CNTs as electrodes for multifunctional electric double layer capacitors: from quantum capacitance to device performance". In: Nanoscale 8.6 (2016), pp. 3620-3628.

[59] Belén Alemán, Maria Vila, and Juan J Vilatela. "Surface Chemistry Analysis of Carbon Nanotube Fibers by X-Ray Photoelectron Spectroscopy". In: physica status solidi (a) (2018), p. 1800187.

[60] Mohammad Naraghi et al. "A multiscale study of high performance doublewalled nanotube- polymer fibers". In: ACS nano 4.11 (2010), pp. 6463-6476.

[61] Peng-Xiang Hou, Chang Liu, and Hui-Ming Cheng. "Purification of carbon nanotubes". In: Carbon 46.15 (2008), pp. 2003-2025.

[62] Daniel Iglesias et al. "Gas-phase functionalization of macroscopic carbon nanotube fiber assemblies: reaction control, electrochemical properties, and use for flexible supercapacitors". In: ACS applied materials \& interfaces (2018).

[63] Material Property Data. http://www.matweb.com, Accessed: 16.08.2018.

[64] Hexcel. Carbon Fibre HexTow AS4A. https://www.hexcel.com, Accessed: 16. 08. 2018.

[65] Dupont. Kevlar 29. http://www.dupont.com, Accessed: 16.08.2018.

[66] Krzysztof Koziol et al. "High-performance carbon nanotube fiber". In: Science 318.5858 (2007), pp. 1892-1895.

[67] Peng Liu et al. "Continuous carbon nanotube-based fibers and films for applications requiring enhanced heat dissipation". In: ACS applied materials $\mathcal{E}$ interfaces 8.27 (2016), pp. 17461-17471.

[68] Dawid Janas, Andrea C Vilatela, and Krzysztof KK Koziol. "Performance of carbon nanotube wires in extreme conditions". In: Carbon 62 (2013), pp. 438446.

[69] AZO Materials. E-Glass Fibre properties. https://www.azom.com, Accessed: 16.08.2018.

[70] Goodfellow. Tables of Properties of Materials. http://www.goodfellow.com, Accessed: 16.08.2018.

[71] Zhitao Zhang et al. "Weaving efficient polymer solar cell wires into flexible power textiles". In: Advanced Energy Materials 4.11 (2014), p. 1301750.

[72] Yong Qin, Xudong Wang, and Zhong Lin Wang. "Microfibre-nanowire hybrid structure for energy scavenging". In: Nature 451.7180 (2008), p. 809.

[73] Hongfang Li et al. "Hydrothermal Deposition of a Zinc Oxide Nanorod Array on a Carbon Nanotube Film as a Piezoelectric Generator". In: RSC Advances 4.82 (2014), pp. 43772-43777.

[74] Xiuhan Li et al. "3D fiber-based hybrid nanogenerator for energy harvesting and as a self-powered pressure sensor". In: ACS nano 8.10 (2014), pp. 1067410681.

[75] Yi Jia et al. "Strong, conductive carbon nanotube fibers as efficient hole collectors". In: Nanoscale research letters 7.1 (2012), p. 137.

[76] Tao Chen et al. "Intertwined aligned carbon nanotube fiber based dye-sensitized solar cells". In: Nano letters 12.5 (2012), pp. 2568-2572.

[77] Alvira Ayoub Arbab et al. "A complete carbon counter electrode for high performance quasi solid state dye sensitized solar cell". In: Journal of Power Sources 343 (2017), pp. 412-423. 
[78] Xiaoyan Wang et al. "TiO2 nanotube arrays based flexible perovskite solar cells with transparent carbon nanotube electrode". In: Nano Energy 11 (2015), pp. 728-735.

[79] Ru Li et al. "Wearable Double-Twisted Fibrous Perovskite Solar Cell". In: Advanced Materials 27.25 (2015), pp. 3831-3835.

[80] Cheong Hoon Kwon et al. "High-power biofuel cell textiles from woven biscrolled carbon nanotube yarns". In: Nature communications 5 (2014), p. 3928.

[81] Woon Seok Yang et al. "High- performance photovoltaic perovskite layers fabricated through intramolecular exchange". In: Science 348.6240 (2015), pp. 1234 1237.

[82] Simon Mathew et al. "Dye-sensitized solar cells with $13 \%$ efficiency achieved through the molecular engineering of porphyrin sensitizers". In: Nature chemistry 6.3 (2014), p. 242.

[83] Martin A. Green et al. "Solar cell efficiency tables (version 51)". In: Progress in Photovoltaics: Research and Applications 26.1 (2018), pp. 3-12.

[84] Brian O'regan and Michael Grätzel. "A low-cost, high-efficiency solar cell based on dye-sensitized colloidal TiO2 films". In: nature 353.6346 (1991), p. 737.

[85] Jeffery L Gray. "The physics of the solar cell". In: Handbook of Photovoltaic Science and Engineering, Second Edition (2011), pp. 82-129.

[86] Wolfgang Tress. "Organic Solar Cells". In: Organic Solar Cells. Springer, 2014, pp. 67-214.

[87] Maria Giannouri et al. "Reduced graphene oxide catalysts for efficient regeneration of cobalt-based redox electrolytes in dye-sensitized solar cells". In: Electrochimica Acta 219 (2016), pp. 258-266.

[88] Dorothea Perganti et al. "Cost-efficient platinum-free DSCs using colloidal graphite counter electrodes combined with D35 organic dye and cobalt (II/III) redox couple". In: Electrochimica Acta 232 (2017), pp. 517-527.

[89] Jennifer A Baker et al. "Development of Graphene Nano-Platelet Ink for High Voltage Flexible Dye Sensitized Solar Cells with Cobalt Complex Electrolytes". In: Advanced Engineering Materials 19.3 (2017).

[90] Andrea Listorti, Brian O'Regan, and James R Durrant. “Electron transfer dynamics in dye-sensitized solar cells". In: Chemistry of Materials 23.15 (2011), pp. 3381-3399.

[91] Meysam Pazoki et al. "Characterization techniques for dye-sensitized solar cells". In: Energy \& Environmental Science 10.3 (2017), pp. 672-709.

[92] R Kern et al. "Modeling and interpretation of electrical impedance spectra of dye solar cells operated under open-circuit conditions". In: Electrochimica Acta 47.26 (2002), pp. 4213-4225.

[93] Juan Bisquert et al. "Theoretical models for ac impedance of finite diffusion layers exhibiting low frequency dispersion". In: Journal of Electroanalytical Chemistry 475.2 (1999), pp. 152-163.

[94] Juan Bisquert. "Theory of the impedance of electron diffusion and recombination in a thin layer". In: The Journal of Physical Chemistry B 106.2 (2002), pp. 325-333.

[95] Motonari Adachi et al. "Determination of parameters of electron transport in dye-sensitized solar cells using electrochemical impedance spectroscopy". In: The Journal of Physical Chemistry B 110.28 (2006), pp. 13872-13880.

[96] Ming Chen and Leng-Leng Shao. "Review on the recent progress of carbon counter electrodes for dye-sensitized solar cells". In: Chemical Engineering Journal 304 (2016), pp. 629-645. 
[97] Sining Yun, Anders Hagfeldt, and Tingli Ma. "Pt-free counter electrode for dye-sensitized solar cells with high efficiency". In: Advanced Materials 26.36 (Sept. 2014), pp. 6210-6237.

[98] Seunghwa Hwang et al. "Dye-Sensitized Solar Cell Counter Electrodes Based on Carbon Nanotubes". In: ChemPhysChem 16.1 (2015), pp. 53-65.

[99] Joseph D Roy-Mayhew and Ilhan A Aksay. "Graphene materials and their use in dye-sensitized solar cells". In: Chemical reviews 114.12 (2014), pp. 63236348.

[100] Fabian Lodermeyer, Rubén D Costa, and Dirk M Guldi. "Implementation of Single-Walled Carbon Nanohorns into Solar Cell Schemes". In: Advanced Energy Materials (2017).

[101] Seon Hee Seo et al. "Influence of electrolyte composition on the photovoltaic performance and stability of dye-sensitized solar cells with multiwalled carbon nanotube catalysts". In: Langmuir 26.12 (2010), pp. 10341-10346.

[102] Guoqiang Yue et al. "Carbon nanotubes hybrid carbon counter electrode for high efficiency dye-sensitized solar cells". In: Journal of Materials Science: Materials in Electronics 27.5 (2016), pp. 4736-4743.

[103] Zhen Huang et al. "Application of carbon materials as counter electrodes of dye-sensitized solar cells". In: Electrochemistry Communications 9.4 (2007), pp. 596-598.

[104] Xiuting Luo, Yaojia Zhang, and Soo Hyung Kim. "Incorporation of Nanostructured Carbon Composite Materials into Counter Electrodes for Highly Efficient Dye-Sensitized Solar Cells". In: Nanoscale Research Letters 13.1 (2018), p. 274.

[105] Seon Hee Seo et al. "Influence of electrolyte composition on the photovoltaic performance and stability of dye-sensitized solar cells with multiwalled carbon nanotube catalysts". In: Langmuir 26.12 (June 2010), pp. 10341-10346.

[106] Takurou N. Murakami et al. "Highly efficient dye-sensitized solar cells based on carbon black counter electrodes". In: Journal of The Electrochemical Society 153.12 (Dec. 2006), A2255-A2261.

[107] Joseph D. Roy-Mayhew et al. "Functionalized graphene as a catalytic counter electrode in dye-sensitized solar cells". In: ACS Nano 4.10 (Oct. 2010), pp. 62036211.

[108] Joseph D Roy-Mayhew et al. "Functionalized graphene sheets as a versatile replacement for platinum in dye-sensitized solar cells". In: ACS applied materials $\mathcal{E}$ interfaces 4.5 (2012), pp. 2794-2800.

[109] Byung-wook Park et al. “Understanding interfacial charge transfer between metallic PEDOT counter electrodes and a cobalt redox shuttle in dye-sensitized solar cells". In: ACS applied materials E interfaces 6.3 (2014), pp. 2074-2079.

[110] Dang Sheng Su, Siglinda Perathoner, and Gabriele Centi. "Nanocarbons for the development of advanced catalysts". In: Chemical reviews 113.8 (2013), pp. 5782-5816.

[111] Maocong Hu, Zhenhua Yao, and Xianqin Wang. “Graphene-based nanomaterials for catalysis". In: Industrial \& Engineering Chemistry Research 56.13 (2017), pp. 3477-3502.

[112] Ana Primo, Vasile Parvulescu, and Hermenegildo Garcia. "Graphenes as MetalFree Catalysts with Engineered Active Sites". In: The journal of physical chemistry letters 8.1 (2016), pp. 264-278.

[113] Craig E Banks et al. "Electrocatalysis at graphite and carbon nanotube modified electrodes: edge-plane sites and tube ends are the reactive sites". In: Chemical Communications 7 (2005), pp. 829-841. 
[114] Craig E Banks and Richard G Compton. "Edge plane pyrolytic graphite electrodes in electroanalysis: an overview". In: Analytical Sciences 21.11 (2005), pp. 1263-1268.

[115] Craig E Banks and Richard G Compton. "New electrodes for old: from carbon nanotubes to edge plane pyrolytic graphite". In: Analyst 131.1 (2006), pp. 1521.

[116] Dimitrios Tasis et al. "Chemistry of carbon nanotubes". In: Chemical reviews 106.3 (2006), pp. 1105-1136.

[117] Manuel Fernando R Pereira et al. "Catalytic activity of carbon nanotubes in the oxidative dehydrogenation of ethylbenzene". In: Carbon 42.14 (2004), pp. 2807-2813.

[118] Philippe Serp, Massimiliano Corrias, and Philippe Kalck. "Carbon nanotubes and nanofibers in catalysis". In: Applied Catalysis A: General 253.2 (2003), pp. 337358.

[119] Ana Primo et al. "Graphenes in the absence of metals as carbocatalysts for selective acetylene hydrogenation and alkene hydrogenation". In: Nature communications 5 (2014), p. 5291.

[120] Easwaramoorthi Ramasamy and Jinwoo Lee. "Ferrocene-derivatized ordered mesoporous carbon as high performance counter electrodes for dye-sensitized solar cells". In: Carbon 48.13 (2010), pp. 3715-3720.

[121] Xiao Gang Luo et al. "Continuous preparation of copper/carbon nanotube composite films and application in solar cells". In: ChemSusChem 9.3 (Feb. 2016), pp. 296-301.

[122] Craig E Banks et al. "Carbon nanotubes contain metal impurities which are responsible for the "electrocatalysis" seen at some nanotube-modified electrodes". In: Angewandte Chemie International Edition 45.16 (2006), pp. 25332537.

[123] Na Guo et al. "Greatly enhancing catalytic activity of graphene by doping the underlying metal substrate". In: Scientific reports 5 (2015), p. 12058.

[124] Jung Gyu Nam et al. "Enhancement of the efficiency of dye-sensitized solar cell by utilizing carbon nanotube counter electrode". In: Scripta Materialia 62.3 (2010), pp. 148-150.

[125] Easwaramoorthi Ramasamy et al. "Spray coated multi-wall carbon nanotube counter electrode for tri-iodide (I3-) reduction in dye-sensitized solar cells". In: Electrochemistry Communications 10.7 (2008), pp. 1087-1089.

[126] Syed Ghufran Hashmi et al. "A durable SWCNT/PET polymer foil based metal free counter electrode for flexible dye-sensitized solar cells". In: Journal of Materials Chemistry A 2.46 (2014), pp. 19609-19615.

[127] Soumyendu Roy et al. "Plasma modified flexible bucky paper as an efficient counter electrode in dye sensitized solar cells". In: Energy \& Environmental Science 5.5 (2012), pp. 7001-7006.

[128] Fangjing Cai, Tao Chen, and Huisheng Peng. "All carbon nanotube fiber electrode-based dye-sensitized photovoltaic wire". In: Journal of Materials Chemistry 22.30 (2012), pp. 14856-14860.

[129] Zhibin Yang et al. "Stretchable, wearable dye-sensitized solar cells". In: Advanced Materials 26.17 (May 2014), pp. 2643-2647.

[130] Zhibin Yang et al. "Aligned carbon nanotube sheets for the electrodes of organic solar cells". In: Advanced Materials 23.45 (2011), pp. 5436-5439.

[131] F Lodermeyer et al. "Facile and quick preparation of carbon nanohorn-based counter electrodes for efficient dye-sensitized solar cells". In: Nanoscale 8.14 (2016), pp. 7556-7561. 
[132] Wolfgang Tress. "Further Origins of S-shaped J-V Curves". In: Organic Solar Cells. Ed. by Wolfgang Tress. Springer International Publishing, 2014, pp. 359376.

[133] Wolfgang Tress. "Interplay Between Electrodes and Active Materials: The Open-Circuit Voltage and S-shaped J-V Curves". In: Organic Solar Cells. Ed. by Wolfgang Tress. Vol. 208. Springer International Publishing, 2014. Chap. Interplay, pp. 315-357.

[134] N Papageorgiou et al. "The performance and stability of ambient temperature molten salts for solar cell applications". In: Journal of the Electrochemical Society 143.10 (1996), pp. 3099-3108.

[135] Ryuji Kawano and Masayoshi Watanabe. "Equilibrium potentials and charge transport of an I-/I 3- redox couple in an ionic liquid". In: Chemical communications 3 (2003), pp. 330-331.

[136] Friedemann Call and Nicolaas A Stolwijk. "Impact of I2 additions on iodide transport in polymer electrolytes for dye-sensitized solar cells: reduced pair formation versus a Grotthuss-like mechanism". In: The Journal of Physical Chemistry Letters 1.14 (2010), pp. 2088-2093.

[137] Ranjit Thapa and Noejung Park. "First-principles identification of iodine exchange mechanism in iodide ionic liquid". In: The journal of physical chemistry letters 3.20 (2012), pp. 3065-3069.

[138] Jihuai $\mathrm{Wu}$ et al. "An all-solid-state dye-sensitized solar cell-based poly (Nalkyl-4-vinyl-pyridine iodide) electrolyte with efficiency of 5.64\%". In: Journal of the American Chemical Society 130.35 (2008), pp. 11568-11569.

[139] Chin Yong Neo, Nishanth Karimbintharikkal Gopalan, and Jianyong Ouyang. "Graphene oxide/multi-walled carbon nanotube nanocomposites as the gelator of gel electrolytes for quasi-solid state dye-sensitized solar cells". In: Journal of Materials Chemistry A 2.24 (2014), pp. 9226-9235.

[140] João E Benedetti et al. "Cross-linked gel polymer electrolyte containing multiwall carbon nanotubes for application in dye-sensitized solar cells". In: Journal of Power Sources 208 (2012), pp. 263-270.

[141] M Shaheer Akhtar et al. "Carbon nanotubes-polyethylene oxide composite electrolyte for solid-state dye-sensitized solar cells". In: Electrochimica Acta 55.7 (2010), pp. 2418-2423.

[142] Jihuai $\mathrm{Wu}$ et al. "Electrolytes in dye-sensitized solar cells". In: Chemical reviews 115.5 (2015), pp. 2136-2173.

[143] Mohd Sukor Su'ait, Mohd Yusri Abd Rahman, and Azizan Ahmad. "Review on polymer electrolyte in dye-sensitized solar cells (DSSCs)". In: Solar Energy 115 (2015), pp. 452-470.

[144] ChunHung Law et al. "Water-based electrolytes for dye-sensitized solar cells". In: Advanced Materials 22.40 (2010), pp. 4505-4509.

[145] Federico Bella et al. "Aqueous dye-sensitized solar cells". In: Chemical Society Reviews 44.11 (2015), pp. 3431-3473.

[146] Shanmuganathan Venkatesan and Yuh-Lang Lee. "Nanofillers in the electrolytes of dye-sensitized solar cells-A short review". In: Coordination Chemistry Reviews 353 (2017), pp. 58-112.

[147] Chuan-Pei Lee et al. "Iodine-free high efficient quasi solid-state dye-sensitized solar cell containing ionic liquid and polyaniline-loaded carbon black". In: Journal of Materials Chemistry 20.12 (2010), pp. 2356-2361.

[148] Bing-Xin Lei et al. "All-solid-state electrolytes consisting of ionic liquid and carbon black for efficient dye-sensitized solar cells". In: Journal of Photochemistry and Photobiology A: Chemistry 216.1 (2010), pp. 8-14. 
[149] Kiranjyoti Mohan et al. "A highly stable and efficient quasi solid state dye sensitized solar cell based on Polymethyl methacrylate (PMMA)/Carbon black (CB) polymer gel electrolyte with improved open circuit voltage". In: Electrochimica Acta 247 (2017), pp. 216-228.

[150] Varishetty Madhu Mohan et al. "Poly (acrylonitrile)/activated carbon composite polymer gel electrolyte for high efficiency dye sensitized solar cells". In: Journal of Materials Chemistry A 1.25 (2013), pp. 7399-7407.

[151] Yu-Hsun Chang et al. "Enhancing photovoltaic performance of all-solid-state dye-sensitized solar cells by incorporating ionic liquid-physisorbed MWCNT". In: Journal of Materials Chemistry 22.31 (2012), pp. 15592-15598.

[152] Chuan-Pei Lee et al. "All-solid-state dye-sensitized solar cells incorporating SWCNTs and crystal growth inhibitor". In: Journal of Materials Chemistry 20.18 (2010), pp. 3619-3625.

[153] Sung Uk Lee, Won Seok Choi, and Byungyou Hong. "A comparative study of dye-sensitized solar cells added carbon nanotubes to electrolyte and counter electrodes". In: Solar Energy Materials and Solar Cells 94.4 (2010), pp. 680-685.

[154] Fabian Lodermeyer et al. "Carbon nanohorn- based electrolyte for dye- sensitized solar cells". In: Energy Environ. Sci. 8.1 (2015), pp. 241-246.

[155] M Shaheer Akhtar et al. "High efficiency solid state dye sensitized solar cells with graphene-polyethylene oxide composite electrolytes". In: Nanoscale 5.12 (2013), pp. 5403-5411.

[156] Momina Khannam, Ratan Boruah, and Swapan Kumar Dolui. "An efficient quasi-solid state dye sensitized solar cells based on graphene oxide/gelatin gel electrolyte with $\mathrm{NiO}$ supported $\mathrm{TiO} 2$ photoanode". In: Journal of Photochemistry and Photobiology A: Chemistry 335 (2017), pp. 248-258.

[157] Shi-Qing Bi et al. "High efficiency and stability of quasi- solid- state dyesensitized $\mathrm{ZnO}$ solar cells using graphene incorporated soluble polystyrene gel electrolytes". In: Journal of Power Sources 272 (2014), pp. 485-490.

[158] Yu-il Kang and Jun Hyuk Moon. "In situ Poly (methyl methacrylate)/Graphene Composite Gel Electrolytes for Highly Stable Dye-Sensitized Solar Cells". In: ChemSusChem 8.22 (2015), pp. 3799-3804.

[159] Shanmuganathan Venkatesan et al. "Graphene Oxide Sponge as Nanofillers in Printable Electrolytes in High-Performance Quasi-Solid-State Dye-Sensitized Solar Cells". In: ACS applied materials E interfaces (2018).

[160] Shanmuganathan Venkatesan et al. "Performance enhancement effects of dispersed graphene oxide sponge nanofillers on the liquid electrolytes of dyesensitized solar cells". In: Carbon 132 (2018), pp. 71-77.

[161] Uday Narayan Maiti et al. "25th anniversary article: chemically modified/doped carbon nanotubes \& graphene for optimized nanostructures \& nanodevices". In: Advanced Materials 26.1 (2014), pp. 40-67.

[162] Xiao Jin et al. "High-efficiency platinum-free quasi-solid-state dye-sensitized solar cells from polyaniline (polypyrrole)-carbon nanotube complex tailored conducting gel electrolytes and counter electrodes". In: Electrochimica Acta 260 (2018), pp. 905-911.

[163] M Shaheer Akhtar et al. "A new carbon nanotubes (CNTs)-poly acrylonitrile (PAN) composite electrolyte for solid state dye sensitized solar cells". In: Electrochimica Acta 56.27 (2011), pp. 9973-9979.

[164] Juan A Anta, Elena Guillen, and Ramon Tena-Zaera. “ZnO-based dye-sensitized solar cells". In: The Journal of Physical Chemistry C 116.21 (2012), pp. 1141311425 . 
[165] Ke Fan, Jiaguo Yu, and Wingkei Ho. "Improving photoanodes to obtain highly efficient dye-sensitized solar cells: a brief review". In: Materials Horizons 4.3 (2017), pp. 319-344.

[166] Rajesh Kumar et al. "Zinc oxide nanostructure-based dye-sensitized solar cells". In: Journal of Materials Science 52.9 (2017), pp. 4743-4795.

[167] Nafiseh Memarian et al. "Hierarchically assembled ZnO nanocrystallites for high-efficiency dye-sensitized solar cells". In: Angewandte Chemie 123.51 (2011), pp. 12529-12533.

[168] Chengkun Xu et al. "Multilayer assembly of nanowire arrays for dye-sensitized solar cells". In: Journal of the American Chemical Society 133.21 (2011), pp. 8122 8125 .

[169] Ludovic Favereau et al. "Engineering Processes at the Interface of p- Semiconductor for Enhancing the Open Circuit Voltage in p-Type Dye-Sensitized Solar Cells". In: Advanced Energy Materials 7.12 (2017).

[170] Fabrice Odobel et al. "New photovoltaic devices based on the sensitization of p-type semiconductors: challenges and opportunities". In: Accounts of chemical research 43.8 (2010), pp. 1063-1071.

[171] Elizabeth A Gibson et al. "A p-Type NiO-Based Dye-Sensitized Solar Cell with an Open-Circuit Voltage of 0.35 V". In: Angewandte Chemie 121.24 (2009), pp. 4466-4469.

[172] Ishanie Rangeeka Perera et al. "Application of the Tris (acetylacetonato) iron (III) / (II) Redox Couple in p-Type Dye-Sensitized Solar Cells". In: Angewandte Chemie International Edition 54.12 (2015), pp. 3758-3762.

[173] Vasilis Nikolaou et al. "Recent advances and insights in dye-sensitized $\mathrm{NiO}$ photocathodes for photovoltaic devices". In: Journal of Materials Chemistry A 5.40 (2017), pp. 21077-21113.

[174] Oliver Langmar et al. "Designing Squaraines to Control Charge Injection and Recombination Processes in NiO-based Dye-Sensitized Solar Cells". In: ChemSusChem 10.11 (2017), pp. 2385-2393.

[175] Andrew Nattestad et al. "Highly efficient photocathodes for dye-sensitized tandem solar cells". In: Nature materials 9.1 (2010), p. 31.

[176] Patrick Brown, Kensuke Takechi, and Prashant V Kamat. "Single-walled carbon nanotube scaffolds for dye-sensitized solar cells". In: The Journal of Physical Chemistry C 112.12 (2008), pp. 4776-4782.

[177] Lijun Yang and Wallace Woon-Fong Leung. “Electrospun TiO2 Nanorods with Carbon Nanotubes for Efficient Electron Collection in Dye-Sensitized Solar Cells". In: Advanced Materials 25.12 (2013), pp. 1792-1795.

[178] Song-Rim Jang, R Vittal, and Kang-Jin Kim. "Incorporation of functionalized single-wall carbon nanotubes in dye-sensitized TiO2 solar cells". In: Langmuir 20.22 (2004), pp. 9807-9810.

[179] Jiazang Chen et al. "Role of carbon nanotubes in dye-sensitized TiO2-based solar cells". In: The Journal of Physical Chemistry C 116.28 (2012), pp. 1484814856.

[180] Masashi Shiraishi and Masafumi Ata. "Work function of carbon nanotubes". In: Carbon 39.12 (2001), pp. 1913-1917.

[181] Emlyn Huw Rhoderick. "Metal-semiconductor contacts". In: IEE Proceedings I-Solid-State and Electron Devices 129.1 (1982), p. 1.

[182] Run Long. "Electronic structure of semiconducting and metallic tubes in TiO2/ carbon nanotube heterojunctions: density functional theory calculations". In: The journal of physical chemistry letters 4.8 (2013), pp. 1340-1346. 
[183] Kadiatou Therese Dembele et al. "Hybrid carbon nanotubes-TiO2 photoanodes for high efficiency dye-sensitized solar cells". In: The Journal of Physical Chemistry C 117.28 (2013), pp. 14510-14517.

[184] Bayram Kilic et al. "Preparation of Carbon Nanotube/TiO 2 Mesoporous Hybrid Photoanode with Iron Pyrite (FeS 2) Thin Films Counter Electrodes for Dye-Sensitized Solar Cell". In: Scientific reports 6 (2016), p. 27052.

[185] Xiangnan Dang et al. "Virus-templated self-assembled single-walled carbon nanotubes for highly efficient electron collection in photovoltaic devices". In: Nature Nanotechnology 6.6 (2011), p. 377.

[186] Valmiki Koli et al. "A simple strategy for the anchoring of anatase titania on multi-walled carbon nanotubes for solar energy harvesting". In: Solar Energy 149 (2017), pp. 188-194.

[187] Thomas J Macdonald et al. “A TiO2 Nanofiber-Carbon Nanotube-Composite Photoanode for Improved Efficiency in Dye-Sensitized Solar Cells". In: ChemSusChem 8.20 (2015), pp. 3396-3400.

[188] LM Dane, LJJ Janssen, and JG Hoogland. "The iodine/iodide redox couple at a platinum electrode". In: Electrochimica Acta 13.3 (1968), pp. 507-518.

[189] Kuppuswamy Kalyanasundaram. Dye-sensitized solar cells. EPFL press, 2010.

[190] Belén Alemán et al. "Strong carbon nanotube fibers by drawing inspiration from polymer fiber spinning". In: ACS Nano 9.7 (July 2015), pp. 7392-7398.

[191] Ya-Li Li, Ian A Kinloch, and Alan H Windle. "Direct spinning of carbon nanotube fibers from chemical vapor deposition synthesis". In: Science (New York, N.Y.) 304.5668 (2004), pp. 276-278.

[192] Víctor Reguero et al. "Controlling carbon nanotube type in macroscopic fibers synthesized by the direct spinning process". In: Chemistry of Materials 26.11 (2014), pp. 3550-3557.

[193] J. J. Vilatela García. "Structure, Properties and Treatments of Carbon Nanotube Fibres (doctoral thesis)". PhD thesis. University of Cambridge, 2009.

[194] Jiaheng Wang et al. "A self-assembly mechanism for sol-gel derived ZnO thin films". In: Smart Materials and Structures 16.6 (2007), p. 2673.

[195] Lionel Vayssieres. "Growth of arrayed nanorods and nanowires of $\mathrm{ZnO}$ from aqueous solutions". In: Advanced Materials 15.5 (2003), pp. 464-466.

[196] Tommi Tynell and Maarit Karppinen. "Atomic layer deposition of ZnO: a review". In: Semiconductor Science and Technology 29.4 (2014), p. 043001.

[197] Jie Ren. "Initial growth mechanism of atomic layer deposition of $\mathrm{ZnO}$ on the hydroxylated Si (1 00 )-2× 1: A density functional theory study". In: Applied Surface Science 255.11 (2009), pp. 5742-5745.

[198] E Guziewicz et al. "ALD grown zinc oxide with controllable electrical properties". In: Semiconductor Science and Technology 27.7 (2012), p. 074011.

[199] Alicia Moya Cuenca. "Hybrid systems based on metal oxide and nanocarbons: electronic properties and applications for photocatalysis". PhD thesis. Universidad Politécnica de Madrid, 2017.

[200] Seigo Ito et al. "Fabrication of thin film dye sensitized solar cells with solar to electric power conversion efficiency over 10\%". In: Thin solid films 516.14 (2008), pp. 4613-4619.

[201] Patrick Ngoy Tshibangu, Silindile Nomathemba Ndwandwe, and Ezekiel Dixon Dikio. "Density, viscosity and conductivity study of 1-butyl-3-methylimidazolium bromide". In: Int. J. Electrochem. Sci 6.6 (2011), p. 2.

[202] Hong Sun, Lu Zhang, and Zhong-Sheng Wang. "Single-crystal CoSe 2 nanorods as an efficient electrocatalyst for dye-sensitized solar cells". In: Journal of Materials Chemistry A 2.38 (2014), pp. 16023-16029. 
[203] Sarmimala Hore et al. "Influence of scattering layers on efficiency of dyesensitized solar cells". In: Solar Energy Materials and Solar Cells 90.9 (2006), pp. 1176-1188.

[204] Kyung-Jun Hwang et al. "Statistical TiO2/dye-mass dependence and dyeregeneration efficiency on dye-sensitized solar cells". In: Nano Energy 16 (2015), pp. 383-388.

[205] Hyung-Jun Koo et al. "Size-dependent scattering efficiency in dye-sensitized solar cell". In: Inorganica Chimica Acta 361.3 (2008), pp. 677-683.

[206] Zhong-Sheng Wang et al. "Significant influence of TiO2 photoelectrode morphology on the energy conversion efficiency of N719 dye-sensitized solar cell". In: Coordination chemistry reviews 248.13-14 (2004), pp. 1381-1389.

[207] Hangbo Yue et al. "Macroscopic CNT fibres inducing non-epitaxial nucleation and orientation of semicrystalline polymers". In: Scientific Reports 5 (2015), p. 16729.

[208] Mildred S Dresselhaus et al. "Raman spectroscopy of carbon nanotubes". In: Physics reports 409.2 (2005), pp. 47-99.

[209] Mildred S Dresselhaus et al. "Perspectives on carbon nanotubes and graphene Raman spectroscopy". In: Nano letters 10.3 (2010), pp. 751-758.

[210] Tae-Young Kim et al. "In situ measurement of dye adsorption on $\mathrm{TiO} 2$ thin films for dye-sensitized solar cells". In: Measurement 46.5 (2013), pp. 1692 1697.

[211] Peng Wang et al. "A high molar extinction coefficient sensitizer for stable dye-sensitized solar cells". In: Journal of the American Chemical Society 127.3 (2005), pp. 808-809.

[212] Yuan Li et al. "Evaluation of methods to extract parameters from currentvoltage characteristics of solar cells". In: Solar Energy 90 (2013), pp. 51-57.

[213] Li Yuan. Open Photovoltaics Analysis Platform. http://opvap.com, Accessed: 30.07.2018.

[214] Damian Pysch, Ansgar Mette, and Stefan W Glunz. "A review and comparison of different methods to determine the series resistance of solar cells". In: Solar Energy Materials and Solar Cells 91.18 (2007), pp. 1698-1706.

[215] RJ Handy. "Theoretical analysis of the series resistance of a solar cell". In: Solid-State Electronics 10.8 (1967), pp. 765-775.

[216] Tzu-Chien Wei et al. "Fabrication of grid type dye sensitized solar modules with $7 \%$ conversion efficiency by utilizing commercially available materials". In: Progress in Photovoltaics: Research and Applications 21.8 (Dec. 2013), pp. 1625-1633.

[217] Songyuan Dai et al. "Design of DSC panel with efficiency more than 6\%". In: Solar Energy Materials \& Solar Cells 85 (2005), pp. 447-455.

[218] Liyuan Han et al. "Integrated dye-sensitized solar cell module with conversion efficiency of 8.2\%". In: Applied Physics Letters 94.32 (2009), p. 13305.

[219] Sining Yun, Peter D Lund, and Andreas Hinsch. "Stability assessment of alternative platinum free counter electrodes for dye-sensitized solar cells". In: Energy \& Environmental Science 8.12 (2015), pp. 3495-3514.

[220] B Alemán et al. "Inherent predominance of high chiral angle metallic carbon nanotubes in continuous fibers grown from a molten catalyst". In: Nanoscale 8.7 (2016), pp. 4236-4244.

[221] H. Yue et al. "Fractal carbon nanotube fibers with mesoporous crystalline structure". In: Carbon 122 (2017), pp. 47-53. 
[222] Víctor Javier Reguero Sanz. "Synthesis, structure and scalability of macroscopic carbon nanotube fibre". PhD thesis. Universidad Carlos III de Madrid, 2017.

[223] Juan C Fernández-Toribio et al. "A composite fabrication sensor based on electrochemical doping of carbon nanotube yarns". In: Advanced Functional Materials 26.39 (2016), pp. 7139-7147.

[224] Syed Ghufran Hashmi et al. "Highly conductive, non-permeable, fiber based substrate for counter electrode application in dye-sensitized solar cells". In: Nano energy 9 (2014), pp. 212-220.

[225] E Senokos et al. "Macroscopic fibres of CNTs as electrodes for multifunctional electric double layer capacitors: from quantum capacitance to device performance." In: Nanoscale 8.6 (Feb. 2016), pp. 3620-3628.

[226] Mojgan Kouhnavard et al. "Carbonaceous materials and their advances as a counter electrode in dye-sensitized solar cells: challenges and prospects". In: ChemSusChem 8.9 (May 2015), pp. 1510-1533.

[227] Munkhbayar Batmunkh, Mark J. Biggs, and Joseph G. Shapter. “Carbon nanotubes for dye-sensitized solar cells". In: Small 11.25 (July 2015), pp. 29632989.

[228] Fabian Lodermeyer, Rubén D. Costa, and Dirk M. Guldi. "Implementation of single-walled carbon nanohorns into solar cell schemes". In: Advanced Energy Materials 7.10 (Jan. 2017), p. 1601883.

[229] Jung Gyu Nam et al. "Enhancement of the efficiency of dye-sensitized solar cell by utilizing carbon nanotube counter electrode". In: Scripta Materialia 62.3 (), pp. 148-150.

[230] Won Jae Lee et al. "Efficient dye-sensitized solar cells with catalytic multiwall carbon nanotube counter electrodes". In: ACS Applied Materials $\mathcal{E}$ Interfaces 1.6 (June 2009), pp. 1145-1149.

[231] F. Lodermeyer et al. "Facile and quick preparation of carbon nanohorn-based counter electrodes for efficient dye-sensitized solar cells". In: Nanoscale 8.14 (Apr. 2016), pp. 7556-7561.

[232] Stefano Carli et al. "Single walled carbon nanohorns as catalytic counter electrodes for Co(III)/(II) electron mediators in dye sensitized cells". In: ACS Applied Materials \& Interfaces 8.23 (June 2016), pp. 14604-14612.

[233] Alvira Ayoub Arbab et al. "A complete carbon counter electrode for high performance quasi solid state dye sensitized solar cell". In: Journal of Power Sources 343 (Mar. 2017), pp. 412-423.

[234] Zhonglin Du et al. "Carbon counter-electrode-based quantum-dot-sensitized solar cells with certified efficiency exceeding 11\%". In: Journal of Physical Chemistry Letters 7.16 (Aug. 2016), pp. 3103-3111.

[235] Ladislav Kavan, Jun-Ho Yum, and Michael Grätzel. “Graphene nanoplatelets outperforming platinum as the electrocatalyst in Co-bipyridine-mediated dyesensitized solar cells". In: Nano Letters 11.12 (Dec. 2011), pp. 5501-5506.

[236] Yu-Yan Li et al. "Graphite with different structures as catalysts for counter electrodes in dye-sensitized solar cells". In: Electrochimica Acta 179 (Oct. 2015), pp. 211-219.

[237] Easwaramoorthi Ramasamy et al. "Nanocarbon counterelectrode for dye sensitized solar cells". In: Applied Physics Letters 90.17 (Apr. 2007), p. 173103.

[238] Abdulla Hilmi, Tharallah A Shoker, and Tarek H Ghaddar. "Universal lowtemperature MWCNT-COOH-based counter electrode and a new thiolate/ disulfide electrolyte system for dye-sensitized solar cells". In: ACS applied materials \& interfaces 6.11 (2014), pp. 8744-8753. 
[239] Qing Wang, Jacques-E. Moser, and Michael Grätzel. "Electrochemical impedance spectroscopic analysis of dye-sensitized solar cells". In: The Journal of Physical Chemistry B 109.31 (Aug. 2005), pp. 14945-14953.

[240] Motonari Adachi et al. "Determination of parameters of electron transport in dye-sensitized solar cells using electrochemical impedance spectroscopy". In: The Journal of Physical Chemistry B 110.28 (July 2006), pp. 13872-13880.

[241] Gerrit Boschloo and Anders Hagfeldt. "Characteristics of the iodide/triiodide redox mediator in dye-sensitized solar cells". In: Accounts of chemical research 42.11 (2009), pp. 1819-1826.

[242] João E Benedetti et al. "A polymer gel electrolyte composed of a poly (ethylene oxide) copolymer and the influence of its composition on the dynamics and performance of dye-sensitized solar cells". In: Journal of Power Sources 195.4 (2010), pp. 1246-1255.

[243] Yao Zhao et al. "Iodine doped carbon nanotube cables exceeding specific electrical conductivity of metals". In: Scientific reports 1 (2011), p. 83.

[244] L Grigorian et al. "Reversible intercalation of charged iodine chains into carbon nanotube ropes". In: Physical review letters 80.25 (1998), p. 5560.

[245] Julien Cambedouzou et al. "Raman spectroscopy of iodine-doped doublewalled carbon nanotubes". In: Physical Review B 69.23 (2004), p. 235422.

[246] Golap Kalita et al. "Iodine doping in solid precursor- based CVD growth graphene film". In: Journal of Materials Chemistry 21.39 (2011), pp. 15209-15213.

[247] Agnes Claye et al. "In situ Raman scattering studies of alkali-doped single wall carbon nanotubes". In: Chemical Physics Letters 333.1-2 (2001), pp. 16-22.

[248] Anneke Hauch and Andreas Georg. "Diffusion in the electrolyte and chargetransfer reaction at the platinum electrode in dye-sensitized solar cells". In: Electrochimica Acta 46 (2001), pp. 3457-3466.

[249] Easwaramoorthi Ramasamy et al. "Spray coated multi-wall carbon nanotube counter electrode for tri-iodide (I3-) reduction in dye-sensitized solar cells". In: Electrochemistry Communications 10.7 (2008), pp. 1087-1089.

[250] Xuchun Gui et al. "Soft, highly conductive nanotube sponges and composites with controlled compressibility". In: ACS nano 4.4 (2010), pp. 2320-2326.

[251] Yuta Ohtani and Hajime Hoshi. "Effect of graphene nanoplatelet edges on the iodide/triiodide redox reaction". In: Electrochemistry Communications 87 (2018), pp. 49-52.

[252] Lei Yang et al. "Initial light soaking treatment enables hole transport material to outperform spiro-OMeTAD in solid-state dye-sensitized solar cells". In: Journal of the American Chemical Society 135.19 (2013), pp. 7378-7385.

[253] Andrea Listorti et al. "The mechanism behind the beneficial effect of light soaking on injection efficiency and photocurrent in dye sensitized solar cells". In: Energy \& Environmental Science 4.9 (2011), pp. 3494-3501.

[254] Lydia Cabau et al. "Light soaking effects on charge recombination and device performance in dye sensitized solar cells based on indoline- cyclopenta dithiophene chromophores". In: Journal of Materials Chemistry A 1.31 (2013), pp. 8994-9000.

[255] Brian A Gregg, Si-Guang Chen, and Suzanne Ferrere. “Enhanced dye-sensitized photoconversion efficiency via reversible production of UV-induced surface states in nanoporous TiO2". In: The Journal of Physical Chemistry B 107.13 (2003), pp. 3019-3029.

[256] Priti Tiwana et al. "The origin of an efficiency improving "light soaking" effect in SnO 2 based solid-state dye-sensitized solar cells". In: Energy \& Environmental Science 5.11 (2012), pp. 9566-9573. 
[257] Marina Freitag et al. "Dye-sensitized solar cells for efficient power generation under ambient lighting". In: Nature Photonics 11.6 (2017), p. 372.

[258] Sandra M Feldt et al. "Design of organic dyes and cobalt polypyridine redox mediators for high-efficiency dye-sensitized solar cells". In: Journal of the American Chemical Society 132.46 (2010), pp. 16714-16724.

[259] Am M Rao et al. "Evidence for charge transfer in doped carbon nanotube bundles from Raman scattering". In: Nature 388.6639 (1997), p. 257.

[260] RS Lee et al. "Conductivity enhancement in single-walled carbon nanotube bundles doped with K and Br". In: Nature 388.6639 (1997), p. 255.

[261] Espen Olsen, Georg Hagen, and Sten Eric Lindquist. "Dissolution of platinum in methoxy propionitrile containing LiI/I2". In: Solar Energy Materials and Solar Cells 63.3 (2000), pp. 267-273.

[262] Anneke Hauch and Andreas Georg. "Diffusion in the electrolyte and chargetransfer reaction at the platinum electrode in dye-sensitized solar cells". In: Electrochimica acta 46.22 (2001), pp. 3457-3466.

[263] Qunwei Tang et al. "Dissolution Engineering of Platinum Alloy Counter Electrodes in Dye-Sensitized Solar Cells". In: Angewandte Chemie 127.39 (2015), pp. 11610-11614.

[264] Gabriela G Sonai et al. "Long-Term Stability of Dye-Sensitized Solar Cells Assembled with Cobalt Polymer Gel Electrolyte". In: The Journal of Physical Chemistry C 121.33 (2017), pp. 17577-17585.

[265] Andreas Hinsch et al. "Long-term stability of dye-sensitised solar cells". In: Progress in Photovoltaics: Research and Applications 9.6 (2001), pp. 425-438.

[266] Kyung Chul Sun et al. "Highly efficient and durable dye-sensitized solar cells based on a wet-laid PET membrane electrolyte". In: Journal of Materials Chemistry A 4.2 (2016), pp. 458-465.

[267] Kyung Chul Sun et al. "A PVdF-based electrolyte membrane for a carbon counter electrode in dye-sensitized solar cells". In: RSC Advances 7.34 (2017), pp. 20908-20918.

[268] Guogang Xue et al. "Degradation mechanisms investigation for long-term thermal stability of dye-sensitized solar cells". In: Int. J. Electrochem. Sci 7 (2012), pp. 1496-1511.

[269] Chin-Cheng Chiang et al. "PtCoFe Nanowire Cathodes Boost Short-Circuit Currents of Ru (II)-Based Dye-Sensitized Solar Cells to a Power Conversion Efficiency of 12.29\%". In: Advanced Functional Materials 28.3 (2018), p. 1703282.

[270] Alfonso Monreal-Bernal, Rubén D. Costa Riquelme, and Juan J. Vilatela García. "Carbon nanotube-based membrane for solar cells". In: European Pat. No. 17382704.9-1501 (23.10.2017).

[271] Feijiu Wang et al. "Considerably Improved Photovoltaic Performance of Carbon Nanotube-based Solar Cells Using Metal Oxide Layers". In: Nature Communications 6 (2015), p. 6305.

[272] Muhammad M Hossain et al. "In situ fabrication of a thermally stable and highly porous conductive solar light-driven ZnO-CNT fiber photocatalyst". In: RSC Advances 6.75 (2016), pp. 71450-71460.

[273] Juan J Vilatela and Rebeca Marcilla. “Tough electrodes: carbon nanotube fibers as the ultimate current collectors/active material for energy management devices". In: Chemistry of Materials 27.20 (2015), pp. 6901-6917.

[274] Dominik Eder. “Carbon Inorganic Hybrids". In: Chemical Reviews 110.3 (2010), pp. 1348-1385.

[275] Ramon Cuscó et al. "Temperature dependence of Raman scattering in ZnO”. In: Physical Review B 75.16 (2007), p. 165202. 
[276] Kenneth Langstreth Johnson and Kenneth Langstreth Johnson. Contact mechanics. Cambridge university press, 1987.

[277] Goodfellow. Tungsten, online catalogue source. www.goodfellow.com, Accessed: 14.09.2017.

[278] S Hoffmann et al. "Fracture strength and Young's modulus of $\mathrm{ZnO}$ nanowires". In: Nanotechnology 18.20 (2007), p. 205503.

[279] Sadao Adachi. Handbook on physical properties of semiconductors. Vol. 2. Springer Science \& Business Media, 2004.

[280] K Ip et al. "Contacts to zno". In: Journal of crystal growth 287.1 (2006), pp. 149 156.

[281] Simon M Sze and Kwok K Ng. Physics of semiconductor devices. John Wiley \& Sons, 2006.

[282] Yuk Fan Hsu et al. "Undoped p-Type ZnO Nanorods Synthesized by a Hydrothermal Method". In: Advanced Functional Materials 18.7 (2008), pp. 10201030.

[283] Nurul Azzyaty Jayah et al. "High electron mobility and low carrier concentration of hydrothermally grown $\mathrm{ZnO}$ thin films on seeded a-plane sapphire at low temperature". In: Nanoscale research letters 10.1 (2015), p. 7.

[284] FA Padovani. "The voltage-current characteristic of metal-semiconductor contacts". In: Semiconductors and semimetals. Vol. 7. Elsevier, 1971, pp. 75-146.

[285] Leonard J. Brillson and Yicheng Lu. "ZnO Schottky Barriers and Ohmic Contacts". In: Journal of Applied Physics 109.12 (2011), p. 121301.

[286] S. K. Cheung and N. W. Cheung. "Extraction of Schottky diode parameters from forward current-voltage characteristics". In: Applied Physics Letters 49.2 (1986), pp. 85-87.

[287] Antonio Di Bartolomeo. “Graphene Schottky diodes: an experimental review of the rectifying graphene/semiconductor heterojunction". In: Physics Reports 606 (2016), pp. 1-58.

[288] S Tongay, T Schumann, and AF Hebard. "Graphite based Schottky diodes formed on Si, GaAs, and 4H-SiC substrates". In: Applied Physics Letters 95.22 (2009), p. 222103.

[289] Zong-Hong Lin et al. "Harvesting Water Drop Energy by a Sequential ContactElectrification and Electrostatic-Induction Process". In: Advanced Materials 26.27 (2014), pp. 4690-4696.

[290] Meredith CK Sellers and Edmund G Seebauer. "Measurement method for carrier concentration in $\mathrm{TiO}_{2}$ via the Mott-Schottky approach". In: Thin Solid Films 519.7 (2011), pp. 2103-2110.

[291] Ahmad Hossein Adl et al. "Effect of sol stabilizer on the structure and electronic properties of solution-processed ZnO thin films". In: Rsc Advances 5.106 (2015), pp. 87007-87018.

[292] A Y Polyakov et al. "Electrical properties of undoped bulk $\mathrm{ZnO}$ substrates". In: Journal of Electronic Materials 35.4 (2006), pp. 663-669.

[293] M Oshikiri et al. "Comparison of the electron effective mass of the n-type $\mathrm{ZnO}$ in the wurtzite structure measured by cyclotron resonance and calculated from first principle theory". In: Physica B: Condensed Matter. International Conference on High Magnetic Fields in Semiconductors 298.1-4 (2001), pp. $472-476$.

[294] R Yatskiv et al. "Electrical and optical properties of graphite/ZnO nanorods heterojunctions". In: Carbon 77 (2014), pp. 1011-1019. 
[295] A Moya et al. "Large area photoelectrodes based on hybrids of CNT fibres and ALD-grown $\mathrm{TiO}_{2}$ ". In: Journal of Materials Chemistry A 5.47 (2017), pp. 2469524706.

[296] Zhi Yang et al. "Developing Seedless Growth of ZnO Micro/Nanowire Arrays towards $\mathrm{ZnO} / \mathrm{FeS} 2 / \mathrm{CuI}$ PIN Photodiode Application". In: Scientific Reports 5 (2015), p. 11377.

[297] Zhong Lin Wang and Jinhui Song. "Piezoelectric nanogenerators based on zinc oxide nanowire arrays". In: Science 312.5771 (2006), pp. 242-246.

[298] K Ip et al. "Carrier concentration dependence of $\mathrm{Ti} / \mathrm{Al} / \mathrm{Pt} / \mathrm{Au}$ contact resistance on n-type ZnO". In: Applied Physics Letters 84.4 (2004), pp. 544-546.

[299] Alexey S Cherevan and Dominik Eder. "Dual Excitation Transient Photocurrent Measurement for Charge Transfer Studies in Nanocarbon Hybrids and Composites". In: Advanced Materials Interfaces 3.16 (2016), p. 1600244.

[300] Michele Buscema et al. "Photocurrent generation with two-dimensional van der Waals semiconductors". In: Chemical Society Reviews 44.11 (2015), pp. 36913718 .

[301] Aday J Molina-Mendoza et al. "Highly responsive UV-photodetectors based on single electrospun $\mathrm{TiO}_{2}$ nanofibres". In: Journal of Materials Chemistry C 4.45 (2016), pp. 10707-10714.

[302] Narges Kiomarsipour and Reza Shoja Razavi. "Hydrothermal synthesis of $\mathrm{ZnO}$ nanopigments with high UV absorption and vis/NIR reflectance". In: Ceramics International 40.7 (2014), pp. 11261-11268.

[303] H A Wahab et al. "Optical, structural and morphological studies of ( $\mathrm{ZnO})$ nano-rod thin films for biosensor applications using sol gel technique". In: Results in Physics 3 (2013), pp. 46-51.

[304] Yu Qiu et al. "Flexible piezoelectric nanogenerators based on $\mathrm{ZnO}$ nanorods grown on common paper substrates". In: Nanoscale 4.20 (2012), pp. 65686573.

[305] Pieter C Rowlette et al. "Plasma-Enhanced Atomic Layer Deposition of Semiconductor Grade ZnO Using Dimethyl Zinc". In: Chemical Vapor Deposition 15.1-3 (2009), pp. 15-20.

[306] Jingjie Luo et al. "A green and economical vapor-assisted ozone treatment process for surface functionalization of carbon nanotubes". In: Green Chemistry 19.4 (2017), pp. 1052-1062.

[307] Sayan Bayan, Biswarup Satpati, and Purushottam Chakraborty. "ZnS nanoparticle decorated $\mathrm{ZnO}$ nanowall network: investigation through electron microscopy and secondary ion mass spectrometry". In: Surface and Interface Analysis 47.1 (2015), pp. 37-44.

[308] PK Giri et al. "Correlation between microstructure and optical properties of ZnO nanoparticles synthesized by ball milling". In: Journal of Applied Physics 102.9 (2007), p. 093515.

[309] Yo-Sep Min et al. "Growth and characterization of conducting ZnO thin films by atomic layer deposition". In: Bulletin of the Korean Chemical Society 31.9 (2010), pp. 2503-2508.

[310] Ji Eun Lee et al. "Optical separation of mechanical strain from charge doping in graphene". In: Nature communications 3 (2012), p. 1024.

[311] Fei Ding et al. "Stretchable graphene: a close look at fundamental parameters through biaxial straining". In: Nano letters 10.9 (2010), pp. 3453-3458.

[312] Semyung Kwon et al. "Characteristics of the $\mathrm{ZnO}$ thin film transistor by atomic layer deposition at various temperatures". In: Semiconductor Science and Technology 24.3 (2009), p. 035015. 
[313] Yunzhe Du et al. "Improvement of bond strength between $\mathrm{ZnO}$ nanorods and carbon fibers using magnetron sputtered $\mathrm{ZnO}$ films as the interphase". In: CrystEngComm 19.5 (2017), pp. 868-875.

[314] S Ben Amor et al. "XPS characterisation of plasma treated and zinc oxide coated PET". In: Applied Surface Science 255.9 (2009), pp. 5052-5061.

[315] Yuan-Li Huang et al. "Effect of extended polymer chains on properties of transparent graphene nanosheets conductive film". In: Journal of Materials Chemistry 21.45 (2011), pp. 18236-18241.

[316] Niancai Cheng et al. "Platinum single-atom and cluster catalysis of the hydrogen evolution reaction". In: Nature communications 7 (2016), p. 13638.

[317] F Moulder John et al. "Handbook of X-ray photoelectron spectroscopy". In: Perkin-Elmer Corporation Physical Electronics Division (1992).

[318] C. D. Wagner et al. NIST X-ray Photoelectron Spectroscopy Database. URL: http: //srdata.nist.gov/xps/ (visited on 07/23/2018).

[319] E Guziewicz et al. "ALD grown zinc oxide with controllable electrical properties". In: Semiconductor Science and Technology 27.7 (2012), p. 74011.

[320] J Ventura et al. "Electrical current induced pinhole formation and insulatormetal transition in tunnel junctions". In: Journal of Physics: Condensed Matter 19.17 (2007), p. 176207.

[321] JM Teixeira et al. "The effect of pinhole formation/growth on the tunnel magnetoresistance of $\mathrm{MgO}$-based magnetic tunnel junctions". In: Journal of Applied Physics 106.7 (2009), p. 073707.

[322] N Huby et al. "Electrical behavior of zinc oxide layers grown by low temperature atomic layer deposition". In: Applied Physics Letters 92.2 (2008), p. 023502.

[323] Yu-Hung Lin et al. "Atomic layer deposition of zinc oxide on multiwalled carbon nanotubes for UV photodetector applications". In: Journal of The Electrochemical Society 158.2 (2011), K24-K27.

[324] Tao Chen et al. "Flexible, light-weight, ultrastrong, and semiconductive carbon nanotube fibers for a highly efficient solar cell". In: Angewandte Chemie International Edition 50.8 (2011), pp. 1815-1819.

[325] Christopher M Proctor and Thuc-Quyen Nguyen. "Effect of leakage current and shunt resistance on the light intensity dependence of organic solar cells". In: Applied Physics Letters 106.8 (2015), 23_1.

[326] Solaronix SA. Application Note - Ti-Nanoxide T/SP. www.solaronix.com, Accessed: 19.03.2018.

[327] Nansra Heo, Yongseok Jun, and Jong Hyeok Park. "Dye molecules in electrolytes: new approach for suppression of dye-desorption in dye-sensitized solar cells". In: Scientific reports 3 (2013), p. 1712.

[328] Thomas W Hamann et al. "Atomic layer deposition of $\mathrm{TiO} 2$ on aerogel templates: new photoanodes for dye-sensitized solar cells". In: The Journal of Physical Chemistry C 112.27 (2008), pp. 10303-10307.

[329] Thomas W Hamann et al. "Aerogel Templated ZnO Dye-Sensitized Solar Cells". In: Advanced Materials 20.8 (2008), pp. 1560-1564.

[330] Jiajie Fan, Weiquan Cai, and Jiaguo Yu. "Adsorption of N719 dye on anatase $\mathrm{TiO} 2$ nanoparticles and nanosheets with exposed (001) facets: equilibrium, kinetic, and thermodynamic studies". In: Chemistry-An Asian Journal 6.9 (2011), pp. 2481-2490.

[331] Jia-De Peng et al. "Hierarchically assembled microspheres consisting of nanosheets of highly exposed (001)-facets TiO 2 for dye-sensitized solar cells". In: RSC Advances 6.17 (2016), pp. 14178-14191. 
[332] Wee-Jun Ong et al. "Highly reactive $\{001\}$ facets of TiO 2-based composites: synthesis, formation mechanism and characterization". In: Nanoscale 6.4 (2014), pp. 1946-2008.

[333] Jia-De Peng et al. "TiO2 nanosheets with highly exposed (001)-facets for enhanced photovoltaic performance of dye-sensitized solar cells". In: Nano Energy 10 (2014), pp. 212-221.

[334] Haimin Zhang et al. "Anatase $\mathrm{TiO} 2$ microspheres with exposed mirror-like plane $\{001\}$ facets for high performance dye-sensitized solar cells (DSSCs)". In: Chemical Communications 46.44 (2010), pp. 8395-8397.

[335] Yutaka Maniwa et al. "Thermal expansion of single-walled carbon nanotube (SWNT) bundles: X-ray diffraction studies". In: Physical Review B 64.24 (2001), p. 241402.

[336] Keiichi Shirasu et al. "Negative axial thermal expansion coefficient of carbon nanotubes: Experimental determination based on measurements of coefficient of thermal expansion for aligned carbon nanotube reinforced epoxy composites". In: Carbon 95 (2015), pp. 904-909.

[337] DR Hummer, PJ Heaney, and JE Post. "Thermal expansion of anatase and rutile between 300 and $575 \mathrm{~K}$ using synchrotron powder X-ray diffraction". In: Powder diffraction 22.4 (2007), pp. 352-357.

[338] ZOU Jian-peng and WANG Ri-zhi. "Crack initiation, propagation and saturation of TiO2 nanotube film [J]". In: Transactions of Nonferrous Metals Society of China 22.3 (2012), pp. 627-633.

[339] Hang $\mathrm{Hu}$ et al. "Atomic layer deposition of $\mathrm{TiO} 2$ for a high-efficiency holeblocking layer in hole-conductor-free perovskite solar cells processed in ambient air". In: ACS applied materials $\mathcal{E}$ interfaces 8.28 (2016), pp. 17999-18007.

[340] Luping Li et al. "Improving performance via blocking layers in dye-sensitized solar cells based on nanowire photoanodes". In: ACS applied materials $\mathcal{E}$ interfaces 7.23 (2015), pp. 12824-12831.

[341] Ladislav Kavan et al. "Electrochemical characterization of TiO2 blocking layers for dye-sensitized solar cells". In: The Journal of Physical Chemistry C 118.30 (2014), pp. 16408-16418.

[342] M Khaja Nazeeruddin et al. "Conversion of light to electricity by cis-X2bis (2, 2'-bipyridyl-4, 4'-dicarboxylate) ruthenium (II) charge-transfer sensitizers $(\mathrm{X}=\mathrm{Cl}-, \mathrm{Br}-, \mathrm{I}-, \mathrm{CN}-$, and $\mathrm{SCN}-)$ on nanocrystalline titanium dioxide electrodes". In: Journal of the American Chemical Society 115.14 (1993), pp. 63826390.

[343] Matt Law et al. "Nanowire dye-sensitized solar cells". In: Nature materials 4.6 (2005), p. 455.

[344] Shouli Bai et al. "Quantum-sized ZnO nanoparticles: synthesis, characterization and sensing properties for NO 2". In: Journal of Materials Chemistry 21.33 (2011), pp. 12288-12294.

[345] A Borhan and KK Rungta. "An experimental study of the radial penetration of liquids in thin porous substrates". In: Journal of colloid and interface science 158.2 (1993), pp. 403-411.

[346] JH Dymond et al. "Transport properties of nonelectrolyte mixtures. IX. Viscosity coefficients for acetonitrile and for three mixtures of toluene+ acetonitrile from 25 to $100^{\circ} \mathrm{C}$ at pressures up to $500 \mathrm{mPa}$. In: International journal of thermophysics 12.3 (1991), pp. 433-447.

[347] Joyashish Debgupta, Bhalchandra A Kakade, and Vijayamohanan K Pillai. "Competitive wetting of acetonitrile and dichloromethane in comparison to 
that of water on functionalized carbon nanotube surfaces". In: Physical Chemistry Chemical Physics 13.32 (2011), pp. 14668-14674.

[348] Luman Zhang et al. "Wettability of carbon nanotube fibers". In: Carbon 122 (2017), pp. 128-140.

[349] Alexander V Neimark et al. "Hierarchical pore structure and wetting properties of single-wall carbon nanotube fibers". In: Nano letters 3.3 (2003), pp. 419 423.

[350] Joaquim Vilà et al. "An in situ investigation of microscopic infusion and void transport during vacuum-assisted infiltration by means of X-ray computed tomography". In: Composites science and technology 119 (2015), pp. 12-19.

[351] Jin-Yun Liao et al. "Oriented hierarchical single crystalline anatase TiO 2 nanowire arrays on Ti-foil substrate for efficient flexible dye-sensitized solar cells". In: Energy \& Environmental Science 5.2 (2012), pp. 5750-5757.

[352] J Ramier et al. "Mechanical integrity of dye-sensitized photovoltaic fibers". In: Renewable Energy 33.2 (2008), pp. 314-319.

[353] Assaf Manor et al. "Origin of size effect on efficiency of organic photovoltaics". In: Journal of Applied Physics 109.7 (2011), p. 074508.

[354] Dhritiman Gupta, Monojit Bag, and KS Narayan. "Area dependent efficiency of organic solar cells". In: Applied Physics Letters 93.16 (2008), p. 384.

[355] Seungkeun Choi, William J Potscavage Jr, and Bernard Kippelen. "Area-scaling of organic solar cells". In: Journal of Applied Physics 106.5 (2009), p. 054507.

[356] Simone Casaluci et al. "Graphene-based large area dye-sensitized solar cell modules". In: Nanoscale 8.9 (2016), pp. 5368-5378.

[357] L Lucera et al. "Printed semi-transparent large area organic photovoltaic modules with power conversion efficiencies of close to $5 \%$ ". In: Organic Electronics 45 (2017), pp. 209-214.

[358] Siew-Lay Lim et al. "Efficient, large area organic photovoltaic modules with active layers processed with non-halogenated solvents in air". In: Organic Electronics 43 (2017), pp. 55-63.

[359] Won Jae Lee et al. "Grid type dye-sensitized solar cell module with carbon counter electrode". In: Journal of Photochemistry and Photobiology A: Chemistry 194 (2008), pp. 27-30.

[360] Yaoguang Rong et al. "Monolithic all-solid-state dye-sensitized solar module based on mesoscopic carbon counter electrodes". In: Solar Energy Materials and Solar Cells 105 (Oct. 2012), pp. 148-152.

[361] Anastasiia Mikhalchan, Thurid Gspann, and Alan Windle. "Aligned carbon nanotube-epoxy composites: the effect of nanotube organization on strength, stiffness, and toughness". In: Journal of Materials Science 51.22 (2016), pp. 1000510025.

[362] Xin Wang et al. "Mechanical and electrical property improvement in CNT/ Nylon composites through drawing and stretching". In: Composites Science and Technology 71.14 (2011), pp. 1677-1683.

[363] Toshio Ogasawara et al. "Mechanical properties of aligned multi-walled carbon nanotube/epoxy composites processed using a hot-melt prepreg method". In: Composites science and technology 71.16 (2011), pp. 1826-1833.

[364] Arkema Inc. New Grades of PVDF for Wire and Cable Applications. URL: https: //www . azom. com/article. aspx?ArticleID=14404 (visited on 07/03/2018).

[365] Evgeny Senokos et al. "Energy storage in structural composites by introducing CNT fiber/polymer electrolyte interleaves". In: Scientific reports 8.1 (2018), p. 3407. 
[366] Brandon Ludwig et al. "Solvent-free manufacturing of electrodes for lithiumion batteries". In: Scientific reports 6 (2016), p. 23150.

[367] Miguel AS Matos et al. "Predictions of the electro-mechanical response of conductive CNT-polymer composites". In: Journal of the Mechanics and Physics of Solids 114 (2018), pp. 84-96.

[368] Bartolomé Mas, Alfonso Monreal-Bernal, and Juan J Vilatela. "A model for understanding the enhancement of the Young's modulus of macroscopic carbon nanotube fibers via polymer infiltration". In: AIP Conference Proceedings (2017).

[369] Min Li et al. "Carbon nanotube film/epoxy composites with high strength and toughness". In: Polymer Composites 38.3 (2017), pp. 588-596. 